LAWRENCE LIVERMORE N A T IO N A L LABORATORY

\title{
Foundations for Improvements to Passive Detection Systems - Final Report
}

S. E. Labov, L. Pleasance, P. Sokkappa, W. Craig, G. Chapline, M. Frank, J. Gronberg, J. G. Jernigan, S. Johnson, J. Kammeraad, D. Lange, A. Meyer, K. Nelson, B. Pohl, D. Wright, R. Wurtz

October 8, 2004 
This document was prepared as an account of work sponsored by an agency of the United States Government. Neither the United States Government nor the University of California nor any of their employees, makes any warranty, express or implied, or assumes any legal liability or responsibility for the accuracy, completeness, or usefulness of any information, apparatus, product, or process disclosed, or represents that its use would not infringe privately owned rights. Reference herein to any specific commercial product, process, or service by trade name, trademark, manufacturer, or otherwise, does not necessarily constitute or imply its endorsement, recommendation, or favoring by the United States Government or the University of California. The views and opinions of authors expressed herein do not necessarily state or reflect those of the United States Government or the University of California, and shall not be used for advertising or product endorsement purposes.

This work was performed under the auspices of the U.S. Department of Energy by University of California, Lawrence Livermore National Laboratory under Contract W-7405-Eng-48. 


\title{
Foundation for Improvements to Passive Detection Systems
}

\section{Final Report}

Simon Labov (P.I.), Lynn Pleasance (Co-I.), Padmini Sokkappa (Co-I.),Bill Craig (Co-I.) George Chapline, Mike Frank, Jeff Gronberg, J. Garrett Jernigan*, Stephen Johnson, Judy Kammeraad, David Lange, Alan Meyer, Karl Nelson, Bert Pohl, Doug Wright, Ron Wurtz

Radiation Detection Center Lawrence Livermore National Laboratory

*University of California, Berkeley

Work performed for:

U.S. Department of Energy (DOE)

Office of Nonproliferation and National Security (NA-20)

Office of Nonproliferation Research and Engineering (NN-22)

Period of Performance: January 1, 2003 - December 31, 2003

Funding: $\$ 1,000,000$

PMIS PROJECT NUMBER: LL243iDP

B\&R CODE: NN2001-32

\begin{abstract}
This project explores the scientific foundation and approach for improving passive detection systems for plutonium and highly enriched uranium in real applications. Sources of gamma-ray radiation of interest were chosen to represent a range of national security threats, naturally occurring radioactive materials, industrial and medical radiation sources, and natural background radiation. The gamma-ray flux emerging from these sources, which include unclassified criticality experiment configurations as surrogates for nuclear weapons, were modeled in detail. The performance of several types of gamma-ray imaging systems using Compton scattering were modeled and compared. A mechanism was created to model the combine sources and background emissions and have the simulated radiation "scene" impinge on a model of a detector. These modeling tools are now being used in various projects to optimize detector performance and model detector sensitivity in complex measuring environments. This study also developed several automated algorithms for isotope identification from gamma-ray spectra and compared these to each other and to algorithms already in use. Verification testing indicates that these alternative isotope identification algorithms produced less false positive and false negative results than the "GADRAS" algorithms currently in use. In addition to these algorithms that used binned spectra, a new approach to isotope identification using "event mode" analysis was developed. Finally, a technique using muons to detect nuclear material was explored.
\end{abstract}




\section{Table of Contents}

Overview 3

$\begin{array}{ll}\text { Source Simulations } & 6\end{array}$

Source and Background Gamma-ray Spectra 14

Gamma-Ray Imaging Performance Analysis 19

Study of Compton vs. Photoelectric Interactions 26

Study of the uncertainty on Compton ring reconstruction 32

Electron tracking device angular and energy resolution 41

Evaluation of the Effectiveness of Algorithms for Source Identification 47

Spectral Analysis of Gamma-ray Event List Data Using K-S Tests 72

A New Method for the Modeling of Gamma-ray Spectra 78

Appendix A1: Gamma-Ray Spectra of Surrogate Threat Sources 87

Appendix A2: Analytic Expressions for the Angular Resolution of Compton Gamma-ray Detectors $\quad 97$

Appendix A3: Comparison of Tools to Determine Isotopic Content 108

Appendix A4: Details of Isotope Identification Evaluation 115

Appendix A5: Remote Muon Scanning for Nuclear Materials Inside Containerships

Appendix A6: Summary published in the Radiation Detection Technologies R\&D Portfolio 


\section{Overview}

Nuclear and radiological weapons contain radioactive materials. These materials emit a constant stream of gamma rays, neutrons, alpha and beta particles. In many situations, the gamma rays and neutrons can be detected remotely. Passive detection systems are those that measure these spontaneous emissions without applying any external stimulation such as X-ray, gamma-ray or particle beams. While some situations may demand active methods, passive systems have the advantage of being less invasive and are less likely to create hazards by exposing people to radiation or triggering detonation if a radiation switch "booby trap" is employed.

Unfortunately, two factors can severely limit the performance of passive detection systems. First, the radioactivity of some sources is low, or limited to emissions that can be easily shielded. Therefore this study created detailed models of the radiation output from potential threats, as well as common legitimate sources of radioactivity including industrial and medical isotopes, and background radiation. For threat sources, standard "criticality test configurations" and simple spherical geometries were used to provide unclassified estimates of the output of improvised or more sophisticated nuclear weapons.

The second factor that limits the performance of passive detection systems is the background radiation. Small quantities of naturally occurring radioactive isotopes are present everywhere in our environment including the earth, rocks, building materials, trees and people. The source of this background varies dramatically with location, time, and viewing direction. Additionally, radiation from legitimate sources can further interfere with passive detection methods. New detection systems under development use imaging and spectroscopy to allow much of this background noise to be subtracted or attenuated from the analysis, thus greatly improving the sensitivity. In this study the sensitivity of these systems is calculated explicitly.

In the study various detection systems were modeled and analyzed to determine their overall performance characteristics. Models were created to track the gamma rays from the sources of interest and the background. These gamma-rays then pass through various shielding configurations, and enter the remote detection system. Interactions in the detector were modeled, and the "measurements" of these interactions were listed in the same way measurements come out of real detector systems. Analysis tools were created to compare the output of various detection systems to determine the probability of detection and the probability of triggering false alarms. Systems modeled so far include several gamma-ray imaging detectors that measure the interactions during Compton scattering to determine some information about the direction of the incoming gamma ray. One class of Compton imagers uses position-sensitive semiconductor-based detectors to measure the energy and location of each interaction. The second class uses scintillating fibers to measure the location, energy and direction of the electron recoil during the Compton scattering. The semiconductor-based system can measure the energy of each interaction very precisely, typically better than $0.5 \%$, but cannot measure the direction of the electron recoil. The fiber-based system measures the recoil, but provides only a very 
crude measurement of the energy, typically $20 \%$. The study compares the resulting imaging performance, and the effect of these limitations on sensitivity.

The study has thus produced source models, detector analysis tools, and a mechanism to determine how well a given system can detect threat sources in the presence of real-world conditions. The source models and analysis tools are now available to anyone who wishes to determine the sensitivity of any specific detection system. These tools are now being used to optimize the next generation of imaging detectors.

Once a radioactive material has been detected, it is important to determine the nature of the material. This study developed several automated algorithms for isotope identification from gamma-ray spectra and compared these to each other and to algorithms already in use. The algorithms tested used the same data libraries that characterize the different isotopes, but used different analysis techniques including multiple linear regression (AutoGADRAS/fittodb), principle component analysis (PCA) and maximum likelihood. Some algorithms like PCA simply classify inputs to the closest representative isotope in the library. Others like linear regression form estimates of composition based on library mixtures and thus are able to recognize unexpected combinations based on similarities to known library elements. These algorithms were tested against spectra produced from our source models, and the performance of each algorithm was evaluated. On these verification tests, GADRAS fittodb performed significantly worse than either of the other two algorithms. A fourth isotope identification technique was also developed that uses individual photon energy measurements rather than the collection of such measurements binned into a spectrum. This "event mode" technique appears very powerful for quickly and accurately determining the presence or absence of a source of interest with very few processor operations.

The project was divided into three tasks. The first task (lead by Lynn Pleasance and Simon Labov) focused on modeling gamma-ray imaging detectors, including the modeling of threat sources and background. The second task (lead by Padmini Sokkappa) evaluated three conventional isotope identification techniques used with NaIbased scintillator detectors, and the third task (lead by Bill Craig) explored isotope identification using unbinned, or "list mode" or "event mode" data. In the first section of this report we describe the source modeling and present quantitative flux and intensity levels that can be used to evaluate detector performance. In the next section we describe the modeling of imaging detector systems, including several detailed reports on the performance for two types of Compton imaging systems. This is followed by the comparison of isotope identification algorithms and the exploration of list-mode techniques.

This report also includes six appendices. The first four support and expand the material in the main body of the report. In Appendix A1 we present detailed spectra, plotted both on a linear scale and on a log scale, showing the flux emitted by the various gamma-ray sources we studied. Appendix A2 is a report showing the derivation of analytic expressions for the angular resolution provided by Compton scattering-based imaging systems. Appendix A3 is a report describing a framework for the comparison of isotope 
identification algorithms. In Appendix A4 we present all the results and correlation matrices supporting the isotope identification comparison study.

In addition to evaluating gamma-ray imaging systems, we looked at other detection systems aimed at unique signatures of fissile material. One concept involved looking for correlated neutrons on a containership. In our calculations we found that the estimated neutron background in the center of the ship may be so high $\left(\sim 1 / \mathrm{cm}^{\wedge} 2 \mathrm{sec}\right)$ that one can't see even 2-neutron correlations. We then explored measuring the $6 \mathrm{MeV}$ muonic "x-rays" generated when cosmic muons are captured by nuclear material. Due to the extremely low rate of cosmically generated muonic "X-rays" we examined approaches to building an accelerator-based muon source that could fit on a ship. This is discussed further in Appendix A5.

We conclude this report in Appendix A6 with the summary of the project that was published in the Radiation Detection Technologies R\&D Portfolio prepared for the NA22 Radiation Detection Program Review in December 2003. 


\title{
Source Simulations
}

\author{
Ron Wurtz, Bert Pohl and Mike Frank
}

\section{Introduction}

In order to evaluate the performance of a simulated gamma ray detector assembly, it is necessary to model spectrophotometrically correct radiation sources.

"Spectrophotometrically correct" means having the correct spectrum at the correct level of flux. The flux is not presented to the detector in bulk, but rather as discrete photon events. Each event is a seven-tuple: the 3-D location along the envelope enclosing the detector, the three direction vectors, and the photon energy, also referred to as $(\mathrm{x}, \mathrm{y}, \mathrm{z}, \mathrm{u}, \mathrm{v}, \mathrm{w}, \mathrm{E})$. The direction vector has absolute value of one. Of particular interest is comparing the performance of detectors in two nearly similar scenarios where one is threatening, the other benign. To this end, the source terms we modeled consist of proxies for realistic threat assemblies, as well as background sources and nuisance sources. It is possible to place any number of these sources in space with some known relation to the detector. The computer code then creates photons at the proper relative fluxes and sends them through the envelope enclosing the detector.

Detailed assembly information for real weapons is classified. This project made a conscious decision to avoid classified models. However, there are a number of critical assemblies in the open literature, notably the International Handbook of Evaluated Criticality Safety Benchmark Experiments (Nuclear Energy Agency, 2001). Parts of these assemblies can be used as proxies for a radioactive threat source or portions of a threat's assembly being transported. The emission from a source is highly dependent on its surface area because of self-shielding.

\section{Software for simulating pre-detector spectra.}

Several different software packages are available for simulating source spectra. It is necessary for the detector simulation project to have simulated spectra without detector response function convolved with it, and so some software packages had to be rejected. We selected GAMGEN + MCNP + TCF in order to leverage the LLNL expertise of Bert Pohl, Mike Frank, and others. GAMGEN and TCF are internal codes. MCNP is the particle transport code, but the list of isotopes that generate the particles is created by GAMGEN. TCF is used to convert the output of MCNP to a useful format. Here is a list of packages we considered, and some comments.

SYNTH version 5. Previous versions are said to be not very good. Version 5.1 released October 2002.

GAMGEN + MCNP + TCF. (Gosnell and Pohl, 1999). Highly shielded or selfshielded sources can take more than a day to run on a PC. Individually tracking the particles can take a long time. 
Gamma Designer (Rowland et al, LLNL). Good fast 1-D code which uses the

GADRAS (see next item) module named ANAD for downscatter. Not appropriate for non-1-D modeling.

GADRAS (Mitchell et al, SNL) will not create spectra free of detector response.

A user uses GAMGEN to age a pure isotope by inputting an isotope and its age. GAMGEN creates a list of all daughter products and their proportions. The input to MCNP is the geometry and composition of the components of the source and its shielding to be modeled. The output, after processing by TCF, contains two tallies, the "twoparagraph format" or ".cdf format", one for the continuum, with variable width bins, and one for the lines, each of which contains the full energy in a zero-width bin. The advantage of these simulations to this project is that the line emission is how the detector sees it, along with downscattering from self-shielding and external shielding. Ultimately, we get a dynamic range of up to $8 \mathrm{e} 16$ from the lowest flux continuum counted to the highest flux line. These files are particularly useful because the photons in lines are counted separately from photons in continuum bins, allowing the end-user to re-bin the data while retaining full flux values for the spectral lines. For this project, the spectral format is further converted into a cumulative distribution function, still in $4 \pi$ flux format, for use in the particle creation and transport of the detector model.

The processes modeled are the following:

Gammas scattered by electrons and nuclei

alpha decay

beta decay, which must be put in as Bremsstrahlung . For us, bremsstrahlung typically only goes into U238, which has the only betas with high enough energy. Betas from Sr90, though intense, are too low energy.

Positron decay gamma de-excitation from metastable states electron capture spontaneous fission. Creates continuum emission from fission daughters, but not line emission.

(n,gamma) if spontaneous fissioning material is modeled as source+neutrons. Our only SF material is Pu240.

Processes not modeled include alpha and fission induced gammas.

For the emitted gamma radiation, the low cutoff is presently $90 \mathrm{keV}$ and high is presently $3300 \mathrm{keV}$. The region $90 \mathrm{keV}$ to $3300 \mathrm{keV}$ corresponds at the low end to where one would set the low end cutoff, as the flux rises steeply in the x-ray band, and on the high end above the thorium chain's $2614 \mathrm{keV}$ line, where the flux is extremely low (one count per hour per keV). Although above 2.614 is low flux, for 4pi imaging spectroscopy, there is hope for detecting $10.8 \mathrm{MeV}$ nitrogen(n,gamma). Because of that hope, we modeled a "neutron source in wool" above $10 \mathrm{MeV}$ to show these nitrogen(n,gamma) lines. These data can be added to simulations where high-energy detection is more important than the time required to accumulate these low-flux photons. Above $3300 \mathrm{keV}, \mathrm{MCNP}$ will occasionally substitute NaI-measured lines for monochromatic lines. This means that 
some high energy tallies must be checked to make sure they do not go into .cdf files as continuum bins.

\section{Source simulations of interest, both pure and linear combinations.}

Because bombs are made of uranium and plutonium, we modeled threat sources of these substances. Threat uranium mixtures are of highly enriched uranium (HEU), uranium with enhancement of U-235, with and without U-232. The presence of U-232 indicates that the uranium was processed through a nuclear reactor, and is therefore interpreted as a marker for Russia and America as its source. Threat mixtures of plutonium are of weapons grade plutonium (WGPu), plutonium with reduced amounts of $\mathrm{Pu}-240$. Sources used in unclassified criticality experiments tend to be shaped as either balls or shells.

All our threat proxies are models of pieces of published NEA criticality experiments "International Handbook of Evaluated Criticality Safety Benchmark Experiments" NEA/NSC/DOC(95)03, run by Pohl and Frank. We selected the following NEA uranium and plutonium parts based on their mass (up to about $10 \mathrm{~kg}$ ) and surface area, as well as their mix of shell and ball parts. The files contain flux/mass spectra in cts/sec/4*pi sr/keV vs $\mathrm{MeV}$ in .cdf format, scalable flux fixed to geometry by mass alone:

$20 \mathrm{yr} \mathrm{Pu}$ shell and its isotopes with wool and cardboard

$20 \mathrm{yr} \mathrm{Pu}$ ball and its isotopes with wool and cardboard

$20 \mathrm{yr} \mathrm{U}$ shell and its isotopes with wool and cardboard

$20 \mathrm{yr} \mathrm{U}$ hemisphere and its isotopes with wool and cardboard

For these simulations, "wool" means a 1x1x1 foot box of wool perfectly surrounding the shell or ball. Wool was selected as a good proxy for the kind of shielding obtained by the nitrogen found in high explosives. The $1 \times 1 \times 1$ foot cardboard enclosure is $0.5 \mathrm{~cm}$ thick. There are additional runs with $7 \mathrm{~cm}$ Fe substituted for the $0.5 \mathrm{~cm}$ cardboard and $1.5 \mathrm{~cm}$ lead substituted for the cardboard.

Here are three lists describing isotopes, geometries, and mixtures for simulated threats. The first is the list of isotopes available for making the mixtures. The second is a list of the ball and shell parts by element. The third is a list of isotopic mixtures from Pohl. There are two geometries for each element. There are three mixtures for $\mathrm{Pu}$, times two geometries makes six Pu "sources". There are four mixtures of U, but you can make them "virgin" by putting in only 235 and 238 (plus 234, a descendant of 238), so that makes eight mixtures, times two geometries, so there are 16 U "sources". Total: 22 "sources" that we can make spectra for. If we add the various kinds of shielding, we have $\mathrm{Pu}$ in $\mathrm{Fe}$ and $\mathrm{Pb}$, and $\mathrm{U}$ in $\mathrm{Fe}$. This makes $18 \mathrm{Pu}$ sources and $32 \mathrm{U}$ sources for a grand total of 50 sources.

\subsection{Isotopes}


The materials were simulated as aged for twenty years so that all the daughter products were present in proportion as would be found after that period of time. If they were modeled at zero years, only the original isotopes would be present. All the isotopes of $U$ and $\mathrm{Pu}$ modeled have half-lives longer than a few days, but they are all unstable. If the age of the materials were modeled to be something different from twenty years, say ten or forty, the proportion of the daughters present would change. The modeled substances (with half-lives in parentheses) whose presence is most dependent on the selection of modeled age are 232-U (70y), 236-Pu (2.9y), 238-Pu (88y), and 241-Pu (14y). We expect the greatest departure from our 20-year model to be seen in variations in strength of the bright spectral line at $2614 \mathrm{keV}$ from the decay of 236-Pu.

20 year old $\mathrm{Pu}$ isotopes.

Pu236

Pu238

Pu239

Pu240

Pu241

$\mathrm{Pu} 242$

Am241 (for Pu mixtures)

20 year old $\mathrm{U}$ isotopes:

$\mathrm{U} 232$

$\mathrm{U} 234$

$\mathrm{U} 235$

$\mathrm{U} 236$

$\mathrm{U} 238$

\subsection{Geometries}

NEA assembly parts with the section ID of the NEA report:

Pu ball (PU-MET-FAST-019, hemis. 7 and $4+$ plug 14. combined, they are one full sphere) $1.70785 \mathrm{~kg}$, outer diameter $=6.3 \mathrm{~cm}$, inner diameter $=2.8 \mathrm{~cm}$, surface area $=125 . \mathrm{cm}^{2}$

Pu shell (PU-MET-FAST-019, hemis. 10 and $1+$ plug 11. combined, they are one full spherical shell) $3.32848 \mathrm{~kg}$, outer diameter $=10.7 \mathrm{~cm}$, inner diameter $=9.32 \mathrm{~cm}$, surface area $=359 . \mathrm{cm}^{2}$

U ball (HEU-MET-FAST-004, hemis. 1. Hemisphere Only!) 10.906 kg, radius $=6.55 \mathrm{~cm}$, surface area $=404 . \mathrm{cm}^{2}$

U shell (HEU-MET-FAST-020, shell 8. Complete spherical shell.) $9.50648 \mathrm{~kg}$, inner radius $=6.75 \mathrm{~cm}$, outer radius $=7.55 \mathrm{~cm}$, surface area $=716 . \mathrm{cm}^{2}$ 


\title{
3.3 Isotopic Mixtures
}

Each part can be modeled as having an initial isotopic mixture as expected to be present depending if it had been removed from a reactor or an existing weapon, and whether it contained uranium-232, signifying that it had been reactor processed by the Americans or Russians. Because these are mixtures, a superposition of spectral flux from isotopes of the same geometry is a proper model of the emission from the surface of the part, and if the sum of the proportions is $100 \%$, then the emission is spectrophotometrically correct. Enrichment refers to percent of U-235, Pu grade refers to ratio of $\mathrm{Pu}-239$ to $\mathrm{Pu}-240$.

\author{
20 yr old WGPu \#1 \\ 20 yr old WGPu \#2 \\ 20 yr old High Burnup Pu (reactor grade) \\ 20 yr old $93.17 \%$ enriched uranium (virgin or processed) \\ 20 yr old $20.107 \%$ enriched uranium (virgin or processed) \\ $20 \mathrm{yr}$ old $52.488 \%$ enriched uranium (virgin or processed) \\ 20 yr old $4.46 \%$ enriched uranium (virgin or processed)
}

\subsection{Nuisances and Background}

Lists of nuisances were developed by looking at GADRAS's canonical and by talking to Alan Sicherman and others working on DTS, the Detection and Tracking System, at LLNL. Particular nuisances are those that share lines with threat sources, such as Ga-67 $(185 \mathrm{keV} \sim 235-\mathrm{U})$ and $137-\mathrm{Cs}(662 \mathrm{keV} \sim \mathrm{Pu}-241661 \mathrm{keV})$. Field workers say that reactor rods being moved in trucks have been nuisances, unfortunately, these contain similar isotopes to threat sources, but may be distinguished by isotopic mixtures and shielding.

spectra run by Pohl, (because of deep penetration, a 1-D model is insufficient):

"dirtball" background (K, Th, Rn, etc). This background must be scaled using the real detector background due to dirt (roughly 50\%). It is modeled to be a typical mixture of thorium decay chain products, arising from natural thorium-232, uranium-238, and potassium-40, downscattered through dirt. The units of background flux are different from the other sources, the background flux must be scaled to the expected background flux given by local background counts and detector window. The background outdoors is also known to have a strong spatial asymmetry in flux with very little contribution from the sky. Also, the flux can vary greatly on small scales because of the variation in emission and shielding by the constituent elements of local dirt, concrete, asphalt, plants, and so on.

"fertilizer" $5 \mathrm{e} 6$ gm, gives $1.5 \mathrm{e}-4 \mathrm{Ci}$, through its own envelope, reduced to a point source. Very little of the initial activity gets through its self attenuation. 
"Co60 in lead" through its own envelope, reduced to a point source. 100 Curies of cobalt-60 through 1000 pounds (approximately $20 \mathrm{~cm}$ ) of lead. Very little of the initial activity gets through the lead.

Small nuisance sources do not have self-shielding, and so line lists, with intensity in proper proportion, are sufficient. We have the following line lists in .cdf format, by way of Dave Banner. Half-lives are indicated in order to estimate how long they can be transported before decaying away:

$$
\begin{aligned}
& \text { 137 Cs } 1 \mathrm{Ci}(30 \mathrm{y}) \\
& \text { 99mTc } 1 \mathrm{Ci}(6 \mathrm{~h}) \\
& \text { 131I } 1 \mathrm{Ci}(8 \mathrm{~d}) \\
& \text { 60Co } 1 \mathrm{Ci}(5.2 \mathrm{y}) \\
& \text { 201Tl } 1 \mathrm{Ci}(72 \mathrm{~h}) \\
& \text { 67Ga } 1 \mathrm{Ci}(3.2 \mathrm{~d}) \\
& \text { 40K } 1 \mathrm{Ci}(1.2 \mathrm{e} 9 \mathrm{y})
\end{aligned}
$$

\subsection{Deep real germanium spectra}

For purposes of comparison, we also obtained real spectra, at high spectral resolution and high signal to noise, from researchers at LLNL. The list and researchers are as follows.

$\mathrm{Pu}$ and $\mathrm{U}$ sources, and a corresponding background (Nakae, LLNL)

Random outdoor spectrum at LLNL (Vetter, LLNL) Indoor lab background, two different sources (Gosnell, LLNL)

The Nakae data were compared against Pohl's dirtball simulation and the simulation was found to have good agreement. These background data have been converted to spectra with $1 \mathrm{keV}$ spectral bins, but because they contain detector response, they cannot be converted to .cdf file format.

\subsection{Variations of real data}

As the models mature, it will be necessary to properly model the variations in time and space as seen in real data. There are three kinds of variations. First there is variation from location to location on the earth's surface. Karl Nelson of LLNL quotes variation in count rates from a single $\mathrm{NaI}$ detector operated on water versus in downtown Las Vegas of factors of 50. Second is the diurnal and annual cycle in intensity in a single location. Zahorowski and Chambers (2002) show annual variations of nearly a factor of two and diurnal variations of nearly the same magnitude. Third are local variations due to local geometry of emitters and absorbers, like dirt, plants, concrete, asphalt, and sky.

We obtained data from Hugh Scott of Savannah River Site from the "Radrover", a NaI detector cruising the roads of Savannah River site, retracing its path on the way out and back. The intensity of the countrate changes on very short spatial scales, of order a meter, and the variation in countrate in the same location is shown to vary by as much as $20 \%$ between two visits going in opposite directions. The countrate at specific locations along the route also changed by as much as a factor of four; it is probable that the highest rates 
occurred as the rover passed a storage facility. Scott has offered to obtain more data to help investigate these variations. We also obtained NaI data from Dan Dietrich of LLNL obtained by driving around LLNL and the backroads near Livermore, but those data were difficult to extract, because the time, location, and spectral information were in different files.

The anecdotal conclusion from these data are that, if intensities from a fixed background are to be modeled, the spatial scale can be a meter or two with intensity variations of a factor of two, and the timescale can be a few minutes with intensity variations of a factor of $20 \%$, and longer timescales give a factor of perhaps $80 \%$, while background intensity from location to location can vary by factors of tens.

\subsection{Flux scaling}

Because presently generating new spectra for different geometries is time-consuming, it will be desirable to change the size of sources by scaling the flux of an object without rerunning the simulation. If the internal geometry of the source is always much larger than a mean free path, all scaling should be possible with identical geometry scaled by surface area. Although they are not one-dimensional models, they can all be scaled up or down somewhat by surface area (with the mass changing by $\left.m_{1} / m_{2}=\left(A_{1} / A_{2}\right)^{3 / 2}\right)$. Densities are assumed to be equal among all isotopes. The mass is in the header of the spectral files.

Flux at large distances will also be reduced by air mass attenuation. By looking at the NIST "mixtures table", we find the scale length of air is about 160 meters. http://physics.nist.gov/PhysRefData/XrayMassCoef/tab3.html particularly: http://physics.nist.gov/PhysRefData/XrayMassCoef/ComTab/air.html

\subsection{Absorbers}

The materials of interest for shielding and absorbers are iron, stainless steel, D38, lead, and wool (as a proxy for any high nitrogen content material). Resources for these absorbers are available at http://physics.nist.gov/PhysRefData/XrayMassCoef/tab3.html

It would be preferable not to do a complete time-consuming model of each and every shielding external to the sources, but instead perform a quick 1-D simulation of the absorption and downscatter through these materials. Such a utility is available with GADRAS, the code available through Dean Mitchell at Sandia. GADRAS incorporates a 1-D code called ANAD that has pre-computed downscatter responses of monochromatic input energy for (Z,Area density). Ordinarily, Pohl uses this module to simulate shielding for other models he has made. Unfortunately, this module also requires the user to specify a detector model from a range of $\mathrm{NaI}$ or Ge detectors, through which the spectrum is ultimately degraded, losing its two-paragraph .cdf nature in the process. Degrading the spectra through a detector is unacceptable for this project, and so the detector modeling group for this project began building a module to do the same kind of pre-computing. This module has not been completed, and tests of the model as-is are inconclusive. 


\section{Software}

The following are lists of both data and code that have been accumulated and created as part of this spectral simulation project.

Files and file formats

Raw spectra from various $\mathrm{NaI}$ and Ge detectors. Also, time-tagged spectra. .cdf files from output of GAMGEN + MCNP + TCF

Photon track files from output of MCNP's PTRAC.

Isotopic mixture files.

$1 \mathrm{keV}$ resolution files to be used as input by the detector modelers.

Spectrum manipulation code: all in IDL, some in c:

Raw spectra readers from real detectors.

Converters from .cdf modeled data files to $1 \mathrm{keV} / \mathrm{bin}$ spectra.

Spectra mixers using a file of isotopic mixtures.

Converter from spectra to cumulative distribution function, the format for extracting individual photons using a random number.

Photon track file readers. 


\title{
Source and Background Gamma-ray Spectra
}

\author{
Jeff Gronberg, Stephen Johnson, David Lange, Doug Wright \\ High Energy Physics group, N Division
}

September 29, 2003

\section{Introduction}

For this study, we have made spectra and integral count rate estimates for a trivial detector configuration with a variety of gamma-ray input sources. The source/background spectra and absolute flux are taken from cumulative distribution files created from MCNP simulations prepared by Ron Wurtz and Mike Frank. All of the source/background configurations given below are available in H4, the GEANT4 simulation implemented by the High Energy Physics group, for gamma-ray detector studies

We determine the signal and background count rates for a $1 \mathrm{~m}^{3}$ cubical detector with an a priori efficiency of $30 \%$, i.e., we arbitrarily assume that for each photon that reaches the detector, there is a $30 \%$ chance of it being detected. We then consider a point source at $50 \mathrm{~m}$ away in vacuum along a line perpendicular to one of the detector faces. For the background file, we assume that the background is isotropic and the detector is perfectly shielded on one face (e.g., mimicking the case where the ground shields the bottom of the detector).

Figures 1- 4 show the source spectra (in both linear and logarithmic scale) with and without $7 \mathrm{~cm}$ iron shielding. Note that the absolute rate is meaningful, it is the rate registered by the hypothetical $30 \%$ efficient one meter cube detector. The histogram bin size in the figures is $1 \mathrm{keV}$.

Table 1 shows the count rate for each source with various cuts on the gamma-ray energy. The total count rate for the background file is 0.4 counts $/ \mathrm{second} / \mathrm{cm}^{2}$ into a surface.

Table 1: Counts per second for $1 \mathrm{~m}^{3}$ detector with $30 \%$ efficiency.

\begin{tabular}{lcccc}
\hline & $\geq 100 \mathrm{keV}$ & $\geq 200 \mathrm{keV}$ & $\geq 500 \mathrm{keV}$ & $\geq 1000 \mathrm{keV}$ \\
\hline \hline Background & 5888.9 & 4785.8 & 2876.7 & 1394.0 \\
\hline HEU & 15.8 & 1.52 & 0.70 & 0.40 \\
HEU $+7 \mathrm{~cm} \mathrm{Fe}$ & 0.128 & 0.116 & 0.086 & 0.056 \\
\hline vHEU & 14.8 & 0.80 & 0.16 & 0.03 \\
vHEU + 7cm Fe & 0.021 & 0.017 & 0.009 & 0.003 \\
\hline WGPU & 89.7 & 51.9 & 3.3 & 0.33 \\
WGPU + 7cm Fe & 0.85 & 0.71 & 0.14 & 0.05 \\
\hline \hline
\end{tabular}



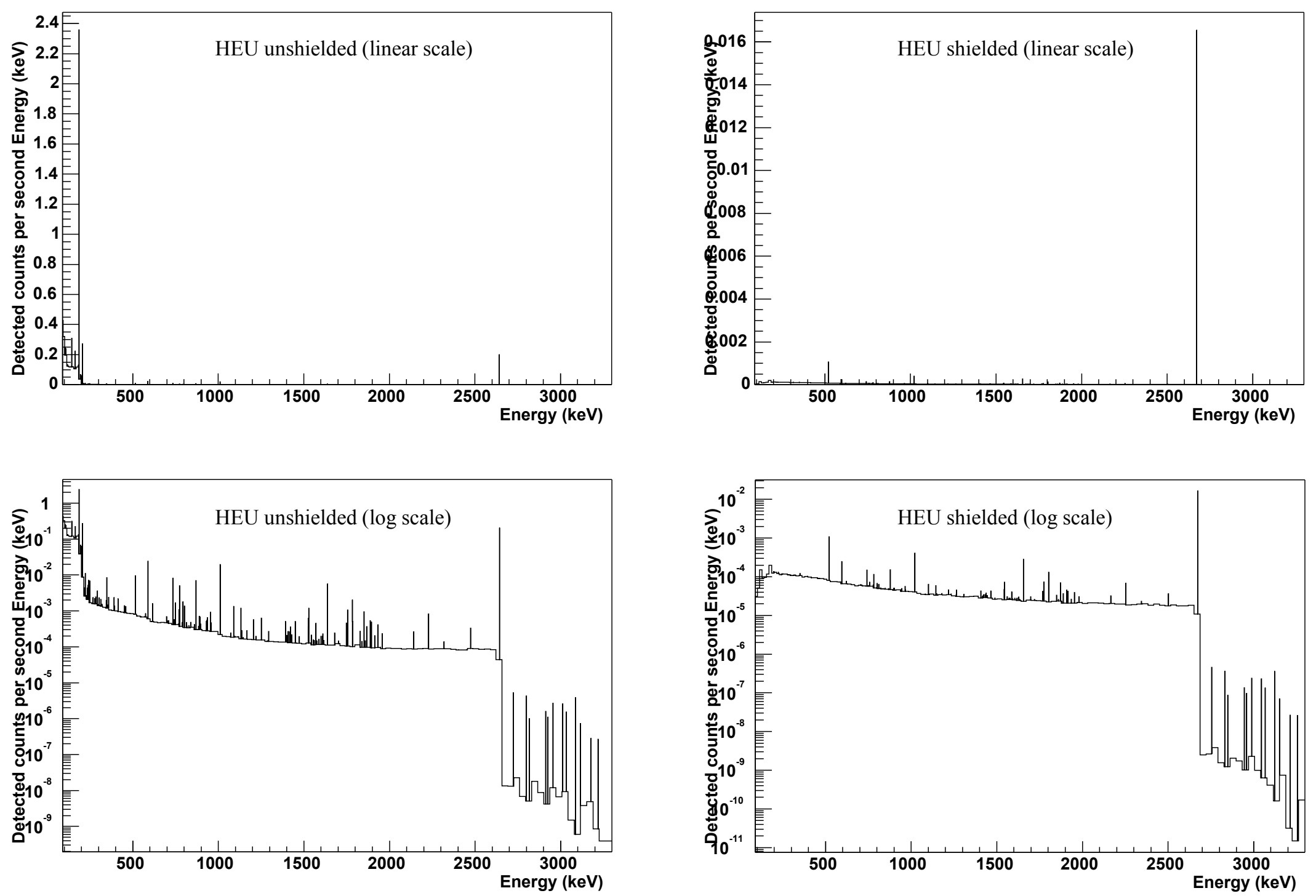

Figure 1: Counts per second of unshielded (left) and shielded (right) HEU as detected by a $1 \mathrm{~m}^{3}$ detector with $30 \%$ efficiency at $50 \mathrm{~m}$ from the source. 

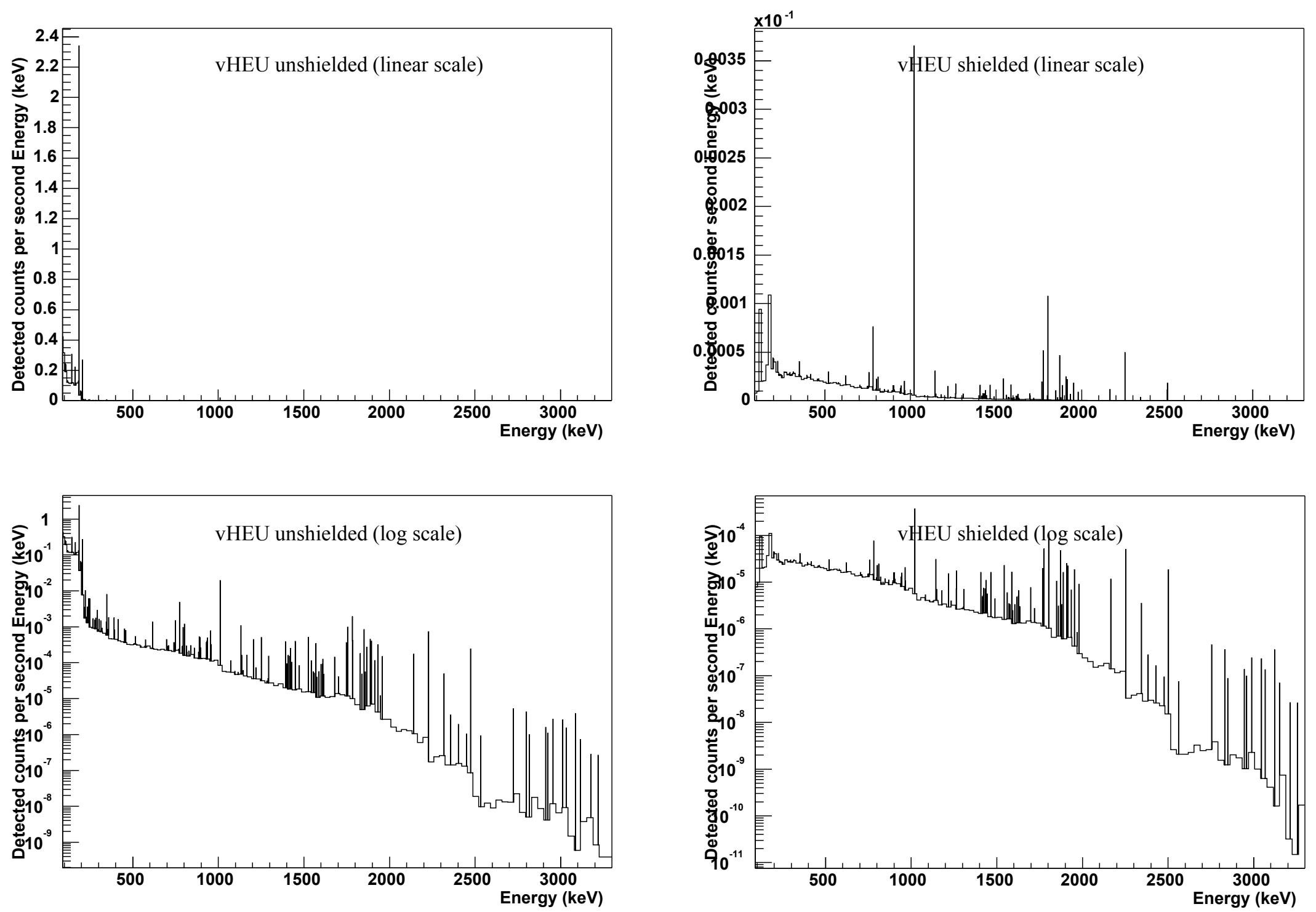

Figure 2: Counts per second of unshielded (left) and shielded (right) vHEU as detected by a $1 \mathrm{~m}^{3}$ detector with $30 \%$ efficiency at $50 \mathrm{~m}$ from the source. 

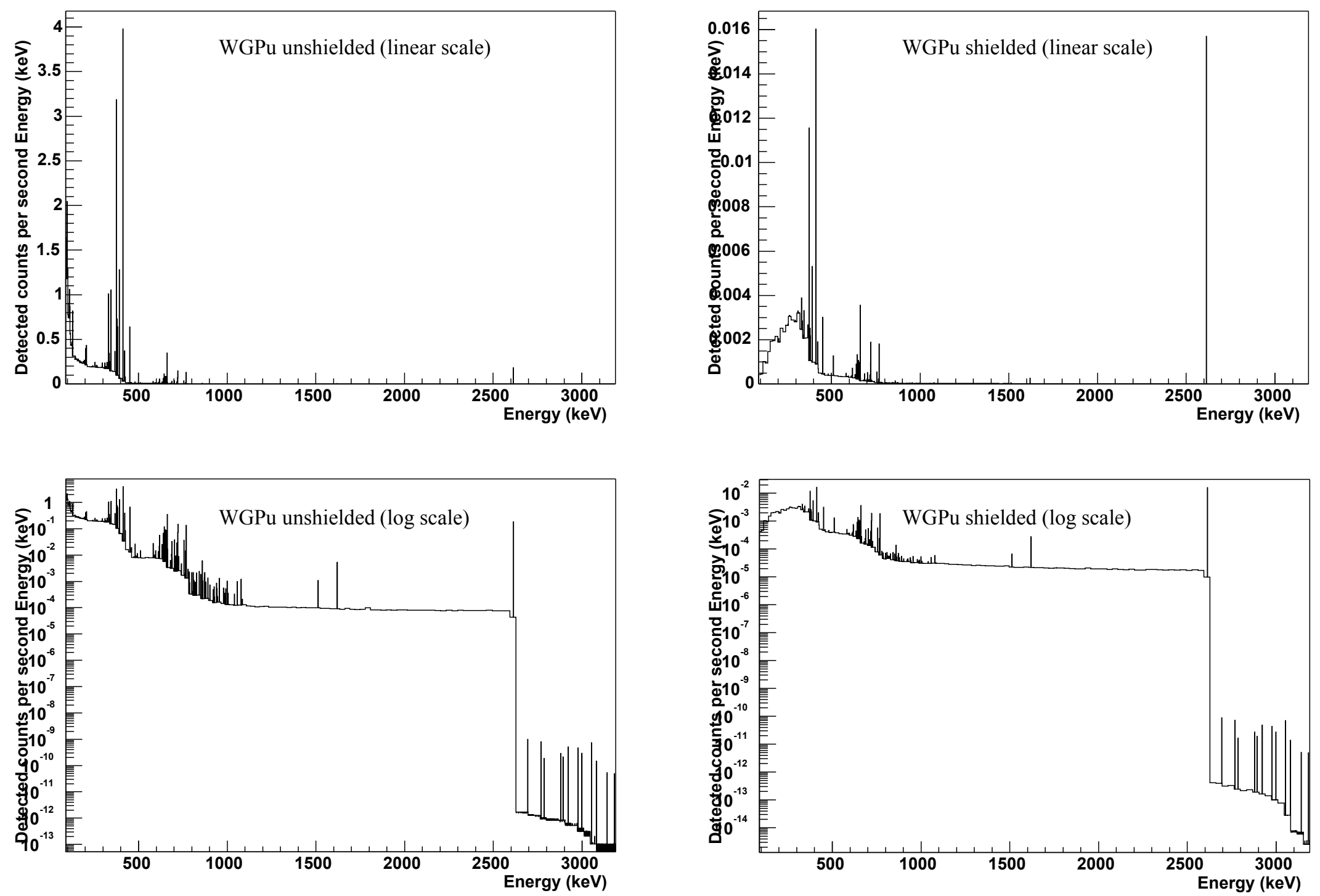

Figure 3: Counts per second of unshielded (left) and shielded (right) WGPu as detected by a $1 \mathrm{~m}^{3}$ detector with $30 \%$ efficiency at $50 \mathrm{~m}$ from the source. 

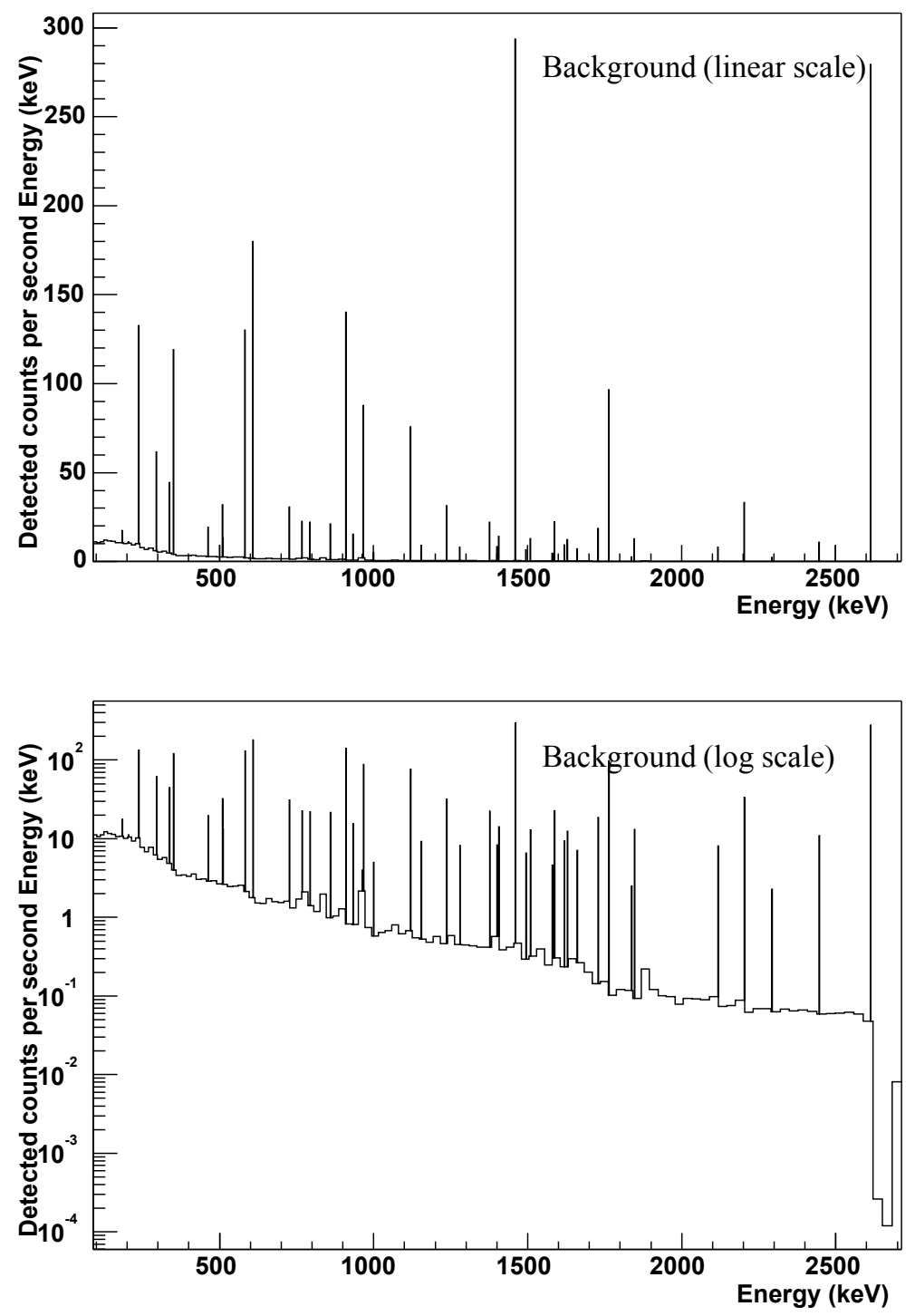

Figure 4: Counts per second of background as detected by a $1 \mathrm{~m}^{3}$ detector with $30 \%$ efficiency. 


\title{
Gamma-Ray Imaging Performance Analysis
}

\author{
Simon Labov, Lynn Pleasance, Jeff Gronberg, Stephen Johnson, David Lange, \\ Doug Wright, Ron Wurtz
}

\section{Introduction}

To detect a gamma-ray or neutron signal from radiological and nuclear weapons or weapons materials, we need detection systems that are sensitive enough to measure a signal and can discriminate that signal from non-threatening sources of radiation. Sensitivity can be achieved with large-area high-efficiency detectors. Discrimination can be achieved with spectroscopy and imaging. In this section we focus on gamma-ray imaging where we size as a signature of a real threat. Gamma-ray emissions from soil, concrete, building materials and large quantities of produce, fertilizer, kitty litter etc. are typically extended since they originate from large areas. Gamma-ray imaging can be used to discriminate these benign sources of radiation from more threatening sources which are more compact.

An example of the power of imaging is shown in Figure 1 where we present spectra from a highly enriched uranium (HEU) source (which has been reprocessed and contaminated with $232 \mathrm{U}$ as is typical of HEU produced in the U.S. and Russia). In this example, we model the source measured by a $10 \mathrm{~cm}$ x $10 \mathrm{~cm}$ high spectral resolution detector located $5 \mathrm{~m}$ away. The black line shows the measured spectrum from the source and from typical background emission, whereas the red line shows just the background emission. In Figure 1a the background emissions from all directions is included as would be measured by an uncollimated non-imaging detector. In this case the source is almost completely buried in

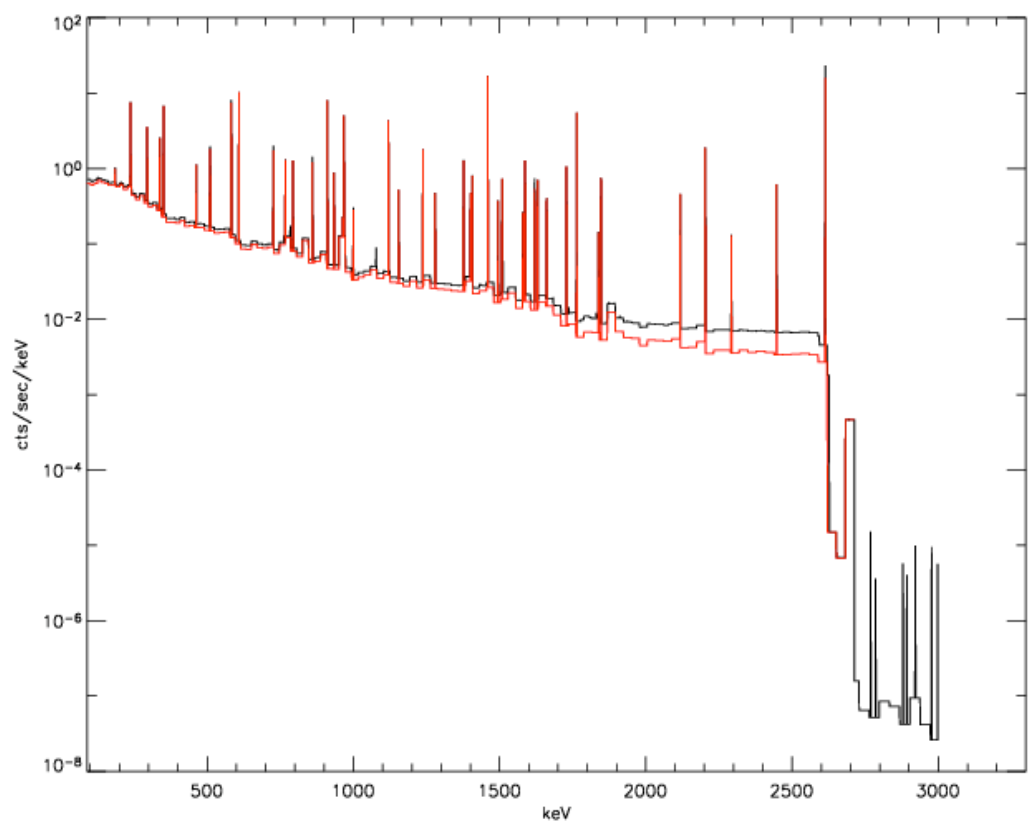

Figure 1a. Simulated emission from HEU measured by a $10 \mathrm{~cm} \times 10 \mathrm{~cm}$ detector at $5 \mathrm{~m}$ without any collimation or imaging capability. The black curve shows the observed source and background together and the red curve shows the emission from just the background. 
the gamma-ray background. In Figure $1 \mathrm{~b}$ only the emissions measured with a gamma-ray imager with 1-degree resolution. In this case the background is greatly reduced allowing the source to be measured directly.

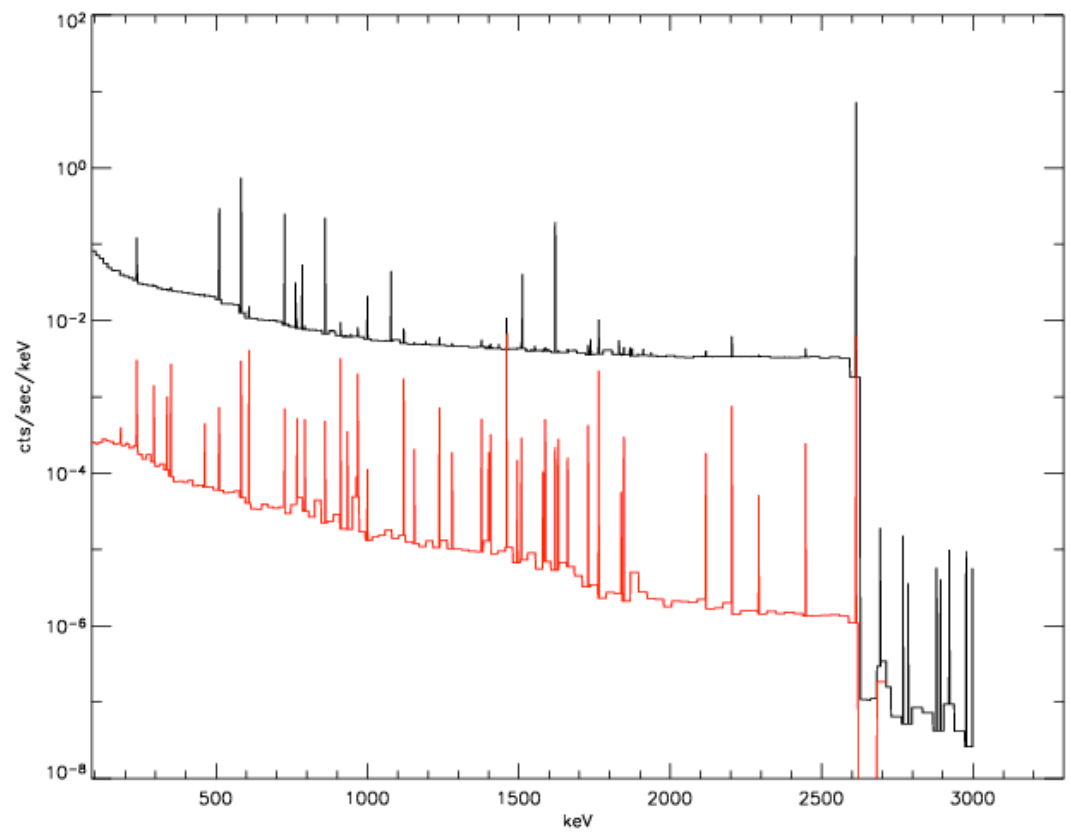

Figure 1b. Simulated emission from HEU measured by a $10 \mathrm{~cm} \times 10 \mathrm{~cm}$ detector at $5 \mathrm{~m}$ with an imaging system having 1-degree angular resolution. The black curve shows the observed source and background together and the red curve shows the emission from just the background.

\section{Modeling Overview}

To model the performance of detector systems, we crated an "end-to-end" tracking photons from their generation at the source, interacting with the environment including absorption, scattering and nuclear interactions, and then interacting with the detector. Background radiation and other sources can also be added. The model produces detector "measurements" showing the energy and position measured by the detector for each interaction. These detector output files are in the same format as real detector output files so they can be analyzed in the same way, and by the same programs, as those used for actual detectors. An overview of the model photon generation is show in Figure 2. The detector models were created using GEANT4, a Monte Carlo radiation transport code developed for high energy physics applications. All the necessary physics are included in this code, and code enhancements were found and incorporated that account for the motion of the electrons in the detector material, which gives rise to the so-called "Doppler broadening." In this study, two different classes of gamma-ray imaging systems were analyzed. 


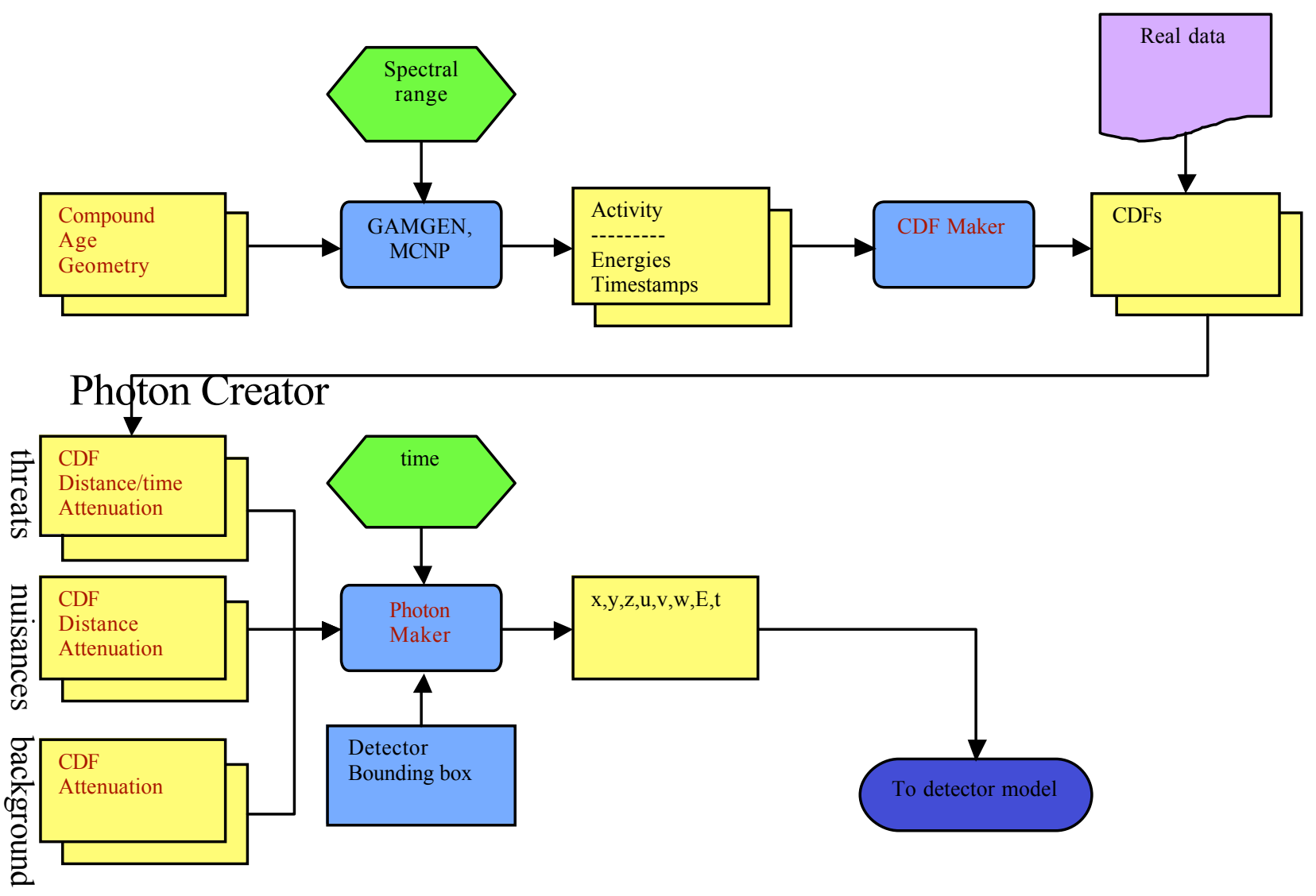

Figure 2. Overview of the photon generation modeling used in this study.

\section{Compton Ring Imagers}

The first class of Compton imagers analyzed here uses position-sensitive semiconductorbased detectors to measure the energy and location of each interaction. These semiconductor-based system can measure the energy of each interaction very precisely, typically better than $0.5 \%$, but cannot measure the direction of the electron recoil. This allows the direction of the incoming photon to be determined from within a ring of angles as shown in Figure 3. To solve for the direction of this ring, the location and energy of at least two interactions must be measured, and if only two interactions are measured, the total energy of the incoming photon must be known, or assumed to equal the total energy measured. Since this is not always true, not all interactions measured in this type of system will be reconstructed properly. It is also necessary to know the order in which the interactions took place, and in some cases there will be more than one option physically possible. Both of these effects can reduce the efficiency and accuracy of this type of imaging system. Note, however, that the high precision of the energy measurement typically results in a narrow "ring" while also providing high-resolution spectra that can also be used to discriminate threats from other sources.

For the purposes of this study we analyzed devices made from silicon and germanium. An example of an image using this modeling process is shown in Figure 4. In this case, a Pu 


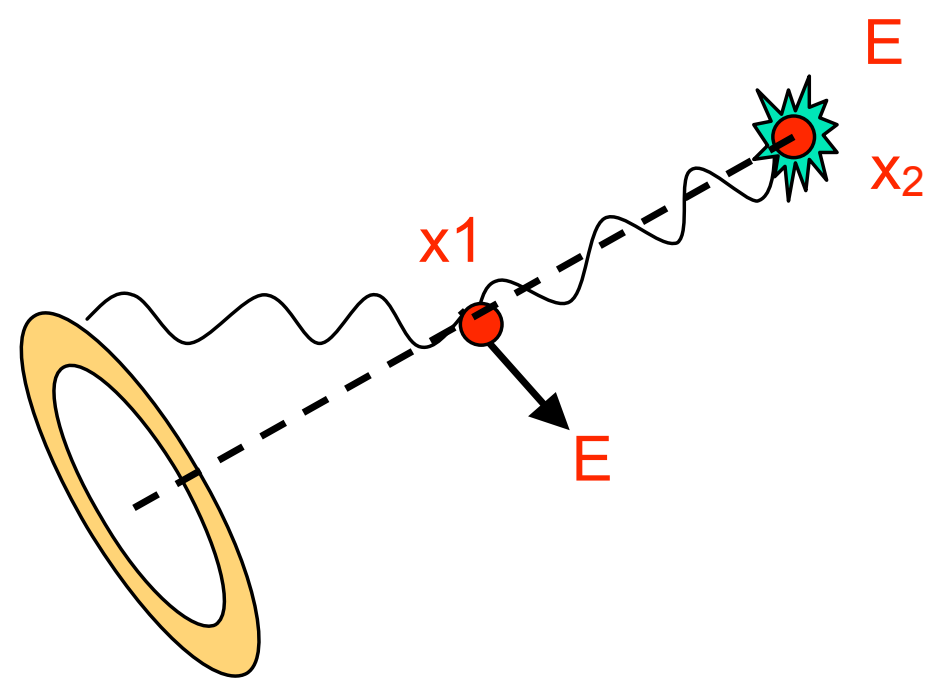

Figure 4. The semiconductor-based Compton imager device can located the direction of each photon to within a ring of angles.

threat source was combined with a isotropic background and a simulated measurement was made with a 3-dimensional position-sensitive Ge detector. The image in Figure 4 uses the photons at $414+/-3 \mathrm{keV}$ to capture the brightest $\mathrm{Pu}$ emission line. The image is made by a simple "back projection" where the ring for each set of interactions is calculated and drawn on the map. Note that due to the limited statistics artifacts from the point source make the background appear uneven.

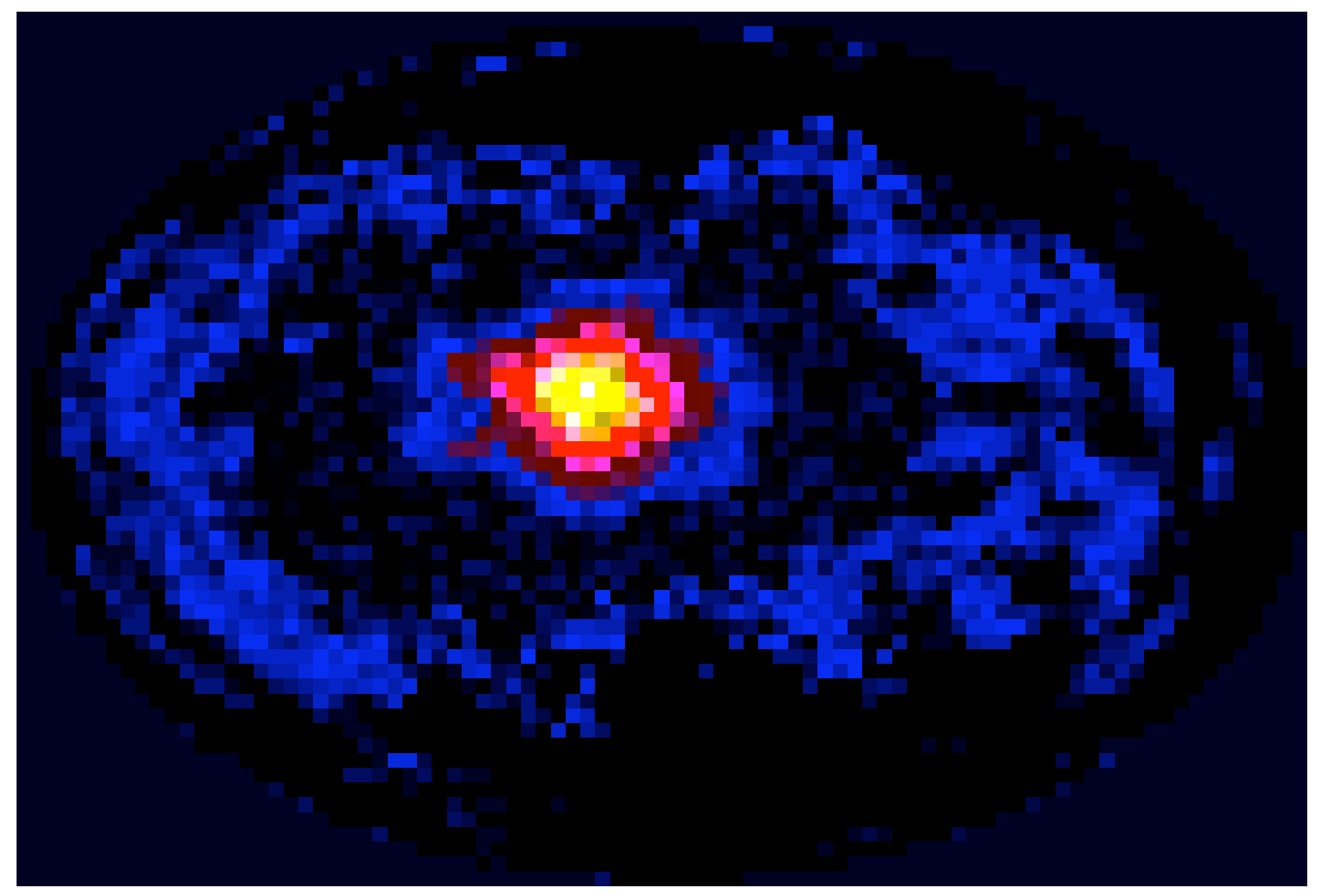

Figure 4. Simulated image of a Pu threat souce in an isotropic background measured by a Ge-based Compton imager 


\section{Electron Tracking Compton Imagers}

The second class of imaging systems studied here uses Compton scattering but also tracks the direction of the scattered electron (Figure 5). In this case the location of the Compton scattering is measured, along with the energy and direction of the Compton scattered electron. When the direction of the scattered electron can be measured, only the location of the second interaction need be measured in order to reconstruct the direction from which the gamma-ray photon originated and its energy. The energy deposited in the second interaction does not need to be measured, and when only two interactions are recorded, it is not necessary to assume the total energy of the photon was deposited. Measuring the direction of the Compton scattered electron also provides additional information that can reduce the number of possible permutations in the order of interactions. Thus these "electron recoil" Compton imagers can use more of the photons that interact in the detector and provide higher resolution than systems that cannot measure the scattering direction. When the electron recoil is measured, the direction of the incoming photon can be determined directly, so each set of interactions can be mapped to a spot or arc on the sky from which it could have come, rather than a ring. If the solid angle of the spot is smaller than the ring for a Compton ring imager, than the background could be suppressed further.

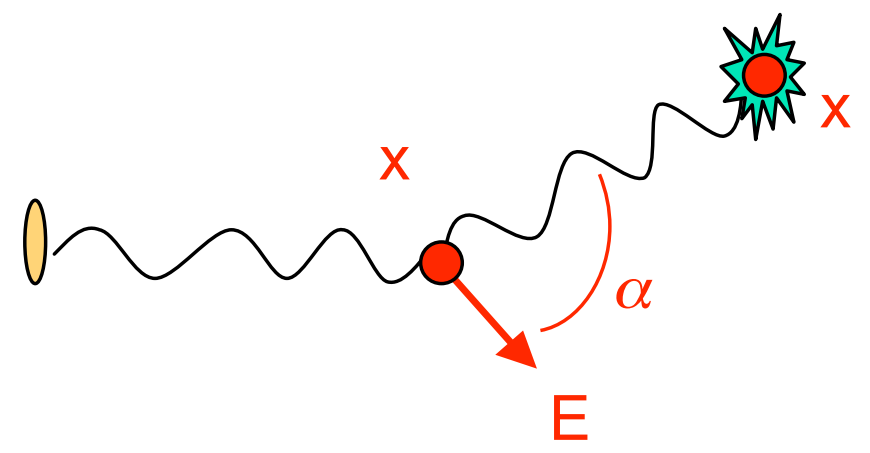

Figure 5. The electron-tracking Compton imager device can locate the direction of each photon to within an arc or spot.

Building an electron-tracking Compton imager is intrinsically difficult. To measure the direction of the electron recoil in a typical solid, the detector must have position measuring capability on the order of $100 \mu \mathrm{m}$. throughout a detector on the order of $10 \mathrm{~cm}$ in length. For a detector only $1 \mathrm{~cm}$ thick, this requires at least 108 voxels. Many different techniques have been suggested to conquer the difficulty of measuring some many locations simultaneously. The implementation we considered uses scintillating fibers of plastic or doped glass to measure the location, energy and direction of the electron recoil during the Compton scattering. The fiber-based system measures the recoil, but provides only a very crude measurement of the energy, typically $20 \%$ based on the measured length of the electron tracks. As can be seen in Figure 6a, this produces very crude imaging at energies below $1 \mathrm{meV}$, although very sharp images can be obtained at higher energies (Figure 6b). These figures were created by modeling the electron-tracking imager response to a singleline point source with no background. 


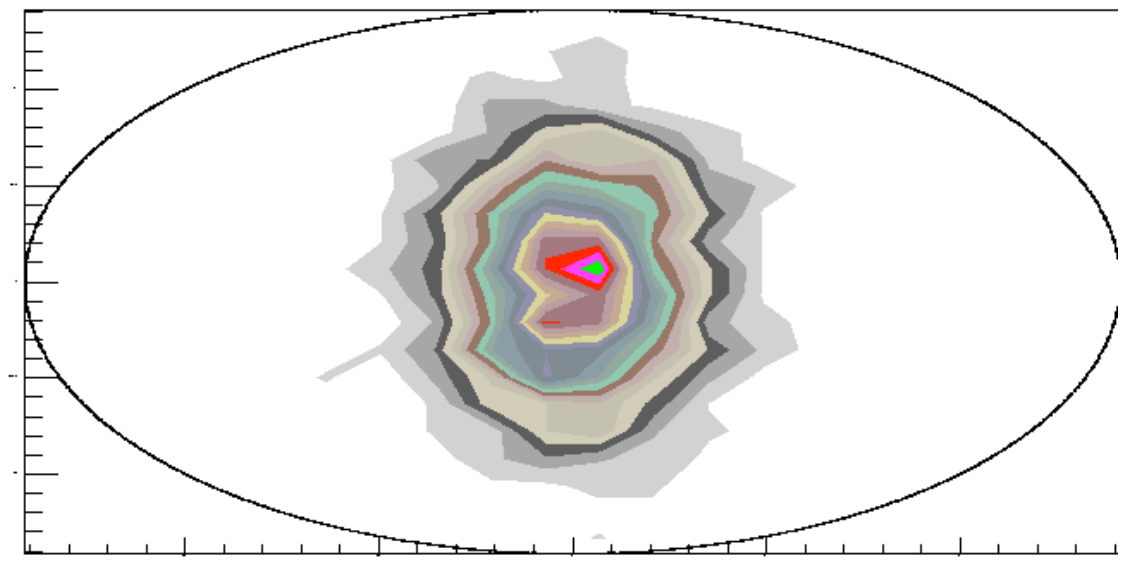

Figure $6 \mathrm{a}$. Simulated image of a point source emitting $500 \mathrm{keV}$ radiation measured by an electron-tracking Compton imager. The imager uses a low-Z plastic scintillator measuring the electron tracks with $500 \mu \mathrm{m}$ resolution and $40 \%$ energy resolution.

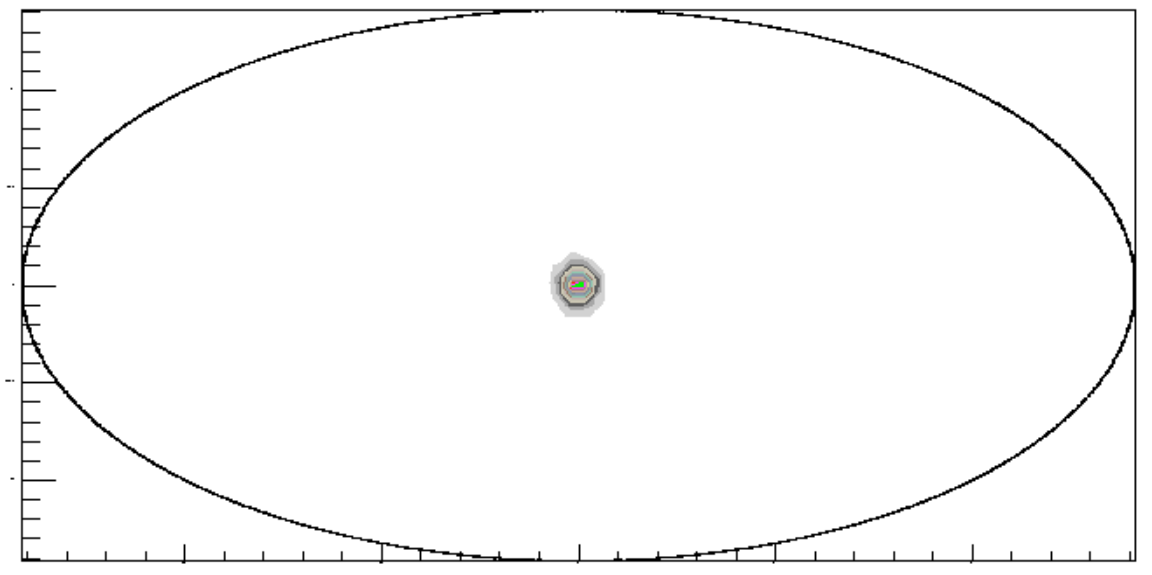

Figure $6 \mathrm{~b}$. Simulated image of a point source emitting $3 \mathrm{MeV}$ radiation measured by an electron-tracking Compton imager. The imager uses a low-Z plastic scintillator measuring the electron tracks with $300 \mu \mathrm{m}$ resolution and $20 \%$ energy resolution. 


\section{Modeling Tools for Detector Development and Comparison}

In many applications, such as searching for weak emissions from nuclear materials against a varying radioactive background, even poor imaging can improve performance when compared to a non-imaging detector. The work done here demonstrates the modeling capability which includes source models, detector analysis tools, and a mechanism to determine how well a given system can detect threat sources in the presence of real-world conditions. The source models and analysis tools are now available to anyone who wishes to determine the sensitivity of any specific detection system. These tools will now be used to optimize the next generation of imaging detectors, and determine the gain in sensitivity that is likely to be realized over a non-imaging system.

In the next several sections, we describe detailed analysis of both types of imaging systems and describe their performance characteristics such as efficiency, energy and spatial resolution as a function of energy, material etc. 
$\mathrm{h} 4 \mathrm{doc} 2 \mathrm{v} 1$

\title{
Study of Compton vs. Photoelectric Interactions
}

\author{
Jeff Gronberg, Stephen Johnson, David Lange, Doug Wright \\ High Energy Physics Group, N Division
}

September 3, 2003

\section{Introduction}

We have studied how often incoming photons interact via a Compton interaction and/or a photoelectric interaction as a function of energy and detector material Results are using a $1 \mathrm{~m}^{3}$ detector, and discrete energy photons from $0.1 \mathrm{MeV}$ up to $10 \mathrm{MeV}$. Essentially all of the lower energy photons interact at least once in a detector of this size. This is not the case at higher energies. Each detector, photon energy combination was simulated with 2000 photons.

The following pages contain plots and tables showing how the following quantities vary with photon energy and detector material:

1. Fraction of photons that interact via the Compton interaction at least once.

2. Fraction of photons that do not interact via the Compton interaction, but rather are absorbed via a photoelectric interaction.

3. Fraction of photons that interact via the Compton interaction exactly once and are then absorbed via a photoelectric interaction.

4. Fraction of photons that interact via the Compton interaction at least twice.

Table 0: Summary of detector properties. The "Plastic" is polystyene scintillator. The "Glass" is a proprietary mixture used by Arno Ledebuhr from Collimated Holes, Inc. It has a LKH-6 glass core and borosilicate glass cladding.

\begin{tabular}{cccc}
\hline Detector & Radiation length $(\mathrm{cm})$ & Density $\left(\mathrm{g} / \mathrm{cm}^{3}\right)$ & Effective Z \\
\hline \hline Germanium & 2.30 & 5.32 & 32 \\
Glass & 4.48 & 3.27 & $\approx 31$ \\
Silicon & 9.37 & 2.33 & 14 \\
Plastic & 42.55 & 1.03 & $\approx 4$ \\
\hline
\end{tabular}




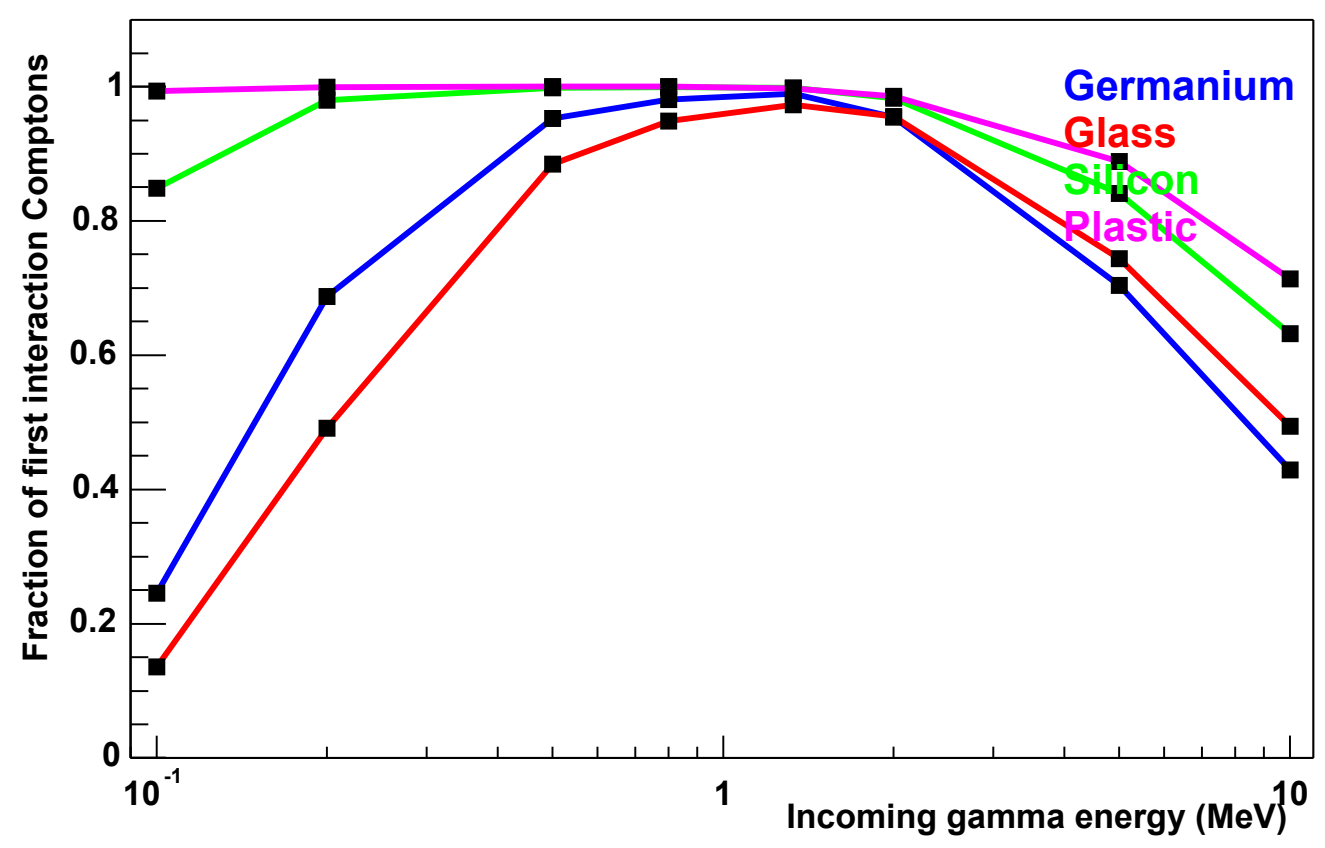

Figure 1: Fraction of gammas that undergo a Compton interaction at least once in a $1 \mathrm{~m}^{3}$ detector. Results are shown for the glass, plastic, silicon, and germanium based detectors.

Table 1: Percentage of gammas that undergo a Compton interaction as their first interaction in a $1 \mathrm{~m}^{3}$ detector.

\begin{tabular}{ccccc}
\hline Energy (MeV) & Germanium & Glass & Silicon & Plastic \\
\hline \hline 0.1 & $24.55 \pm 0.43$ & $13.59 \pm 0.34$ & $84.89 \pm 0.36$ & $99.35 \pm 0.08$ \\
0.2 & $68.74 \pm 0.46$ & $49.11 \pm 0.50$ & $97.98 \pm 0.14$ & $99.90 \pm 0.03$ \\
0.5 & $95.27 \pm 0.21$ & $88.49 \pm 0.32$ & $99.83 \pm 0.04$ & $99.97 \pm 0.02$ \\
0.8 & $98.04 \pm 0.14$ & $94.83 \pm 0.22$ & $99.93 \pm 0.03$ & $99.96 \pm 0.02$ \\
1.33 & $98.96 \pm 0.10$ & $97.30 \pm 0.16$ & $99.82 \pm 0.04$ & $99.71 \pm 0.05$ \\
2 & $95.46 \pm 0.21$ & $95.57 \pm 0.21$ & $98.22 \pm 0.13$ & $98.57 \pm 0.12$ \\
5 & $70.37 \pm 0.46$ & $74.38 \pm 0.44$ & $84.12 \pm 0.37$ & $88.88 \pm 0.31$ \\
10 & $42.90 \pm 0.49$ & $49.44 \pm 0.50$ & $63.17 \pm 0.48$ & $71.30 \pm 0.45$ \\
\hline
\end{tabular}




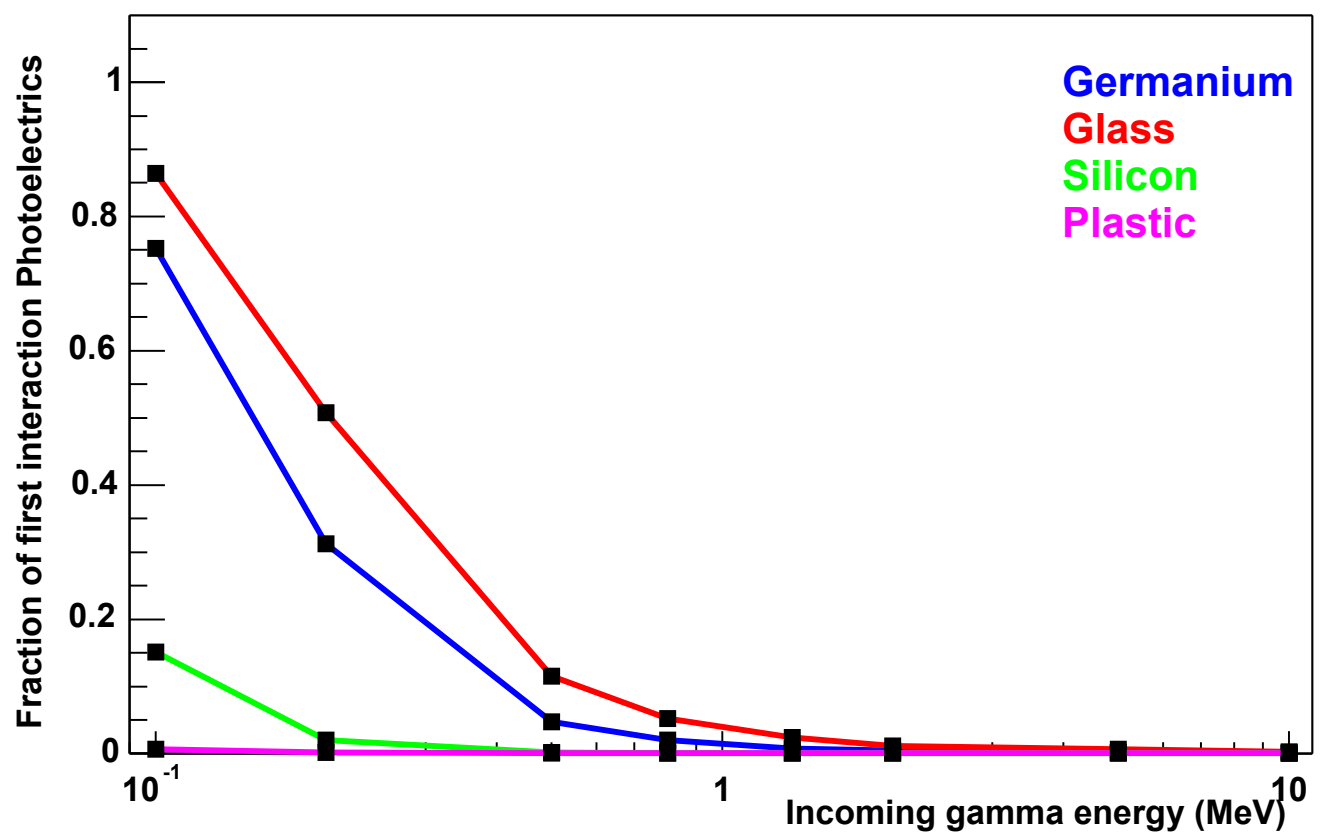

Figure 2: Fraction of gammas that undergo a photoelectric interaction as their first interaction in a $1 \mathrm{~m}^{3}$ detector. Results are shown for the glass, plastic, silicon, and germanium based detectors.

Table 2: Percentage of gammas that undergo a photoelectric interaction as their first interaction in a $1 \mathrm{~m}^{3}$ detector.

\begin{tabular}{ccccc}
\hline Energy $(\mathrm{MeV})$ & Germanium & Glass & Silicon & Plastic \\
\hline \hline 0.1 & $75.3 \pm 0.43$ & $86.4 \pm 0.34$ & $15.1 \pm 0.36$ & $0.7 \pm 0.08$ \\
0.2 & $31.3 \pm 0.46$ & $50.8 \pm 0.50$ & $2.0 \pm 0.14$ & $0.1 \pm 0.03$ \\
0.5 & $4.7 \pm 0.21$ & $11.5 \pm 0.32$ & $0.2 \pm 0.04$ & $0.0 \pm 0.01$ \\
0.8 & $2.0 \pm 0.14$ & $5.2 \pm 0.22$ & $0.1 \pm 0.03$ & $0.0 \pm 0.00$ \\
1.33 & $0.7 \pm 0.08$ & $2.3 \pm 0.15$ & $0.1 \pm 0.02$ & $0.0 \pm 0.00$ \\
2 & $0.5 \pm 0.07$ & $1.1 \pm 0.10$ & $0.1 \pm 0.03$ & $0.0 \pm 0.00$ \\
5 & $0.2 \pm 0.04$ & $0.6 \pm 0.08$ & $0.0 \pm 0.01$ & $0.0 \pm 0.00$ \\
10 & $0.1 \pm 0.03$ & $0.3 \pm 0.05$ & $0.0 \pm 0.01$ & $0.0 \pm 0.00$ \\
\hline
\end{tabular}




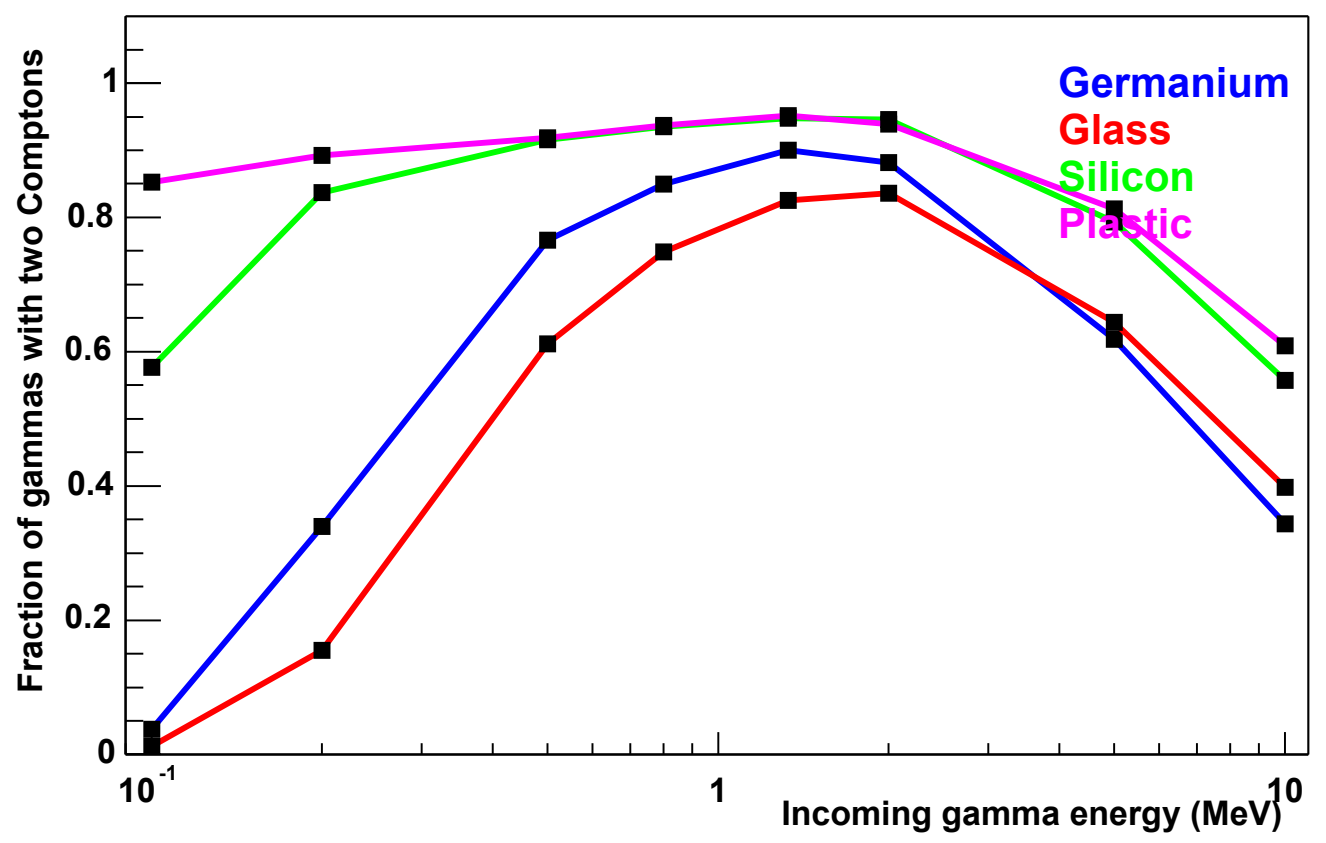

Figure 3: Fraction of gammas that undergo two Compton interactions as their first two interactions in a $1 \mathrm{~m}^{3}$ detector. Results are shown for the glass, plastic, silicon, and germanium based detectors.

Table 3: Percentage of gammas that undergo a two Compton interactions as their first two interactions in a $1 \mathrm{~m}^{3}$ detector.

\begin{tabular}{ccccc}
\hline Energy $(\mathrm{MeV})$ & Germanium & Glass & Silicon & Plastic \\
\hline \hline 0.1 & $3.7 \pm 0.19$ & $1.2 \pm 0.11$ & $57.6 \pm 0.49$ & $85.2 \pm 0.36$ \\
0.2 & $33.9 \pm 0.47$ & $15.5 \pm 0.36$ & $83.7 \pm 0.37$ & $89.3 \pm 0.31$ \\
0.5 & $76.6 \pm 0.42$ & $61.1 \pm 0.49$ & $91.5 \pm 0.28$ & $91.9 \pm 0.27$ \\
0.8 & $84.9 \pm 0.36$ & $74.8 \pm 0.43$ & $93.5 \pm 0.25$ & $93.7 \pm 0.24$ \\
1.33 & $90.0 \pm 0.30$ & $82.5 \pm 0.38$ & $94.8 \pm 0.22$ & $95.1 \pm 0.22$ \\
2 & $88.1 \pm 0.32$ & $83.6 \pm 0.37$ & $94.6 \pm 0.23$ & $93.8 \pm 0.24$ \\
5 & $61.8 \pm 0.49$ & $64.4 \pm 0.48$ & $79.3 \pm 0.41$ & $81.3 \pm 0.39$ \\
10 & $34.3 \pm 0.47$ & $39.8 \pm 0.49$ & $55.7 \pm 0.50$ & $60.8 \pm 0.49$ \\
\hline
\end{tabular}




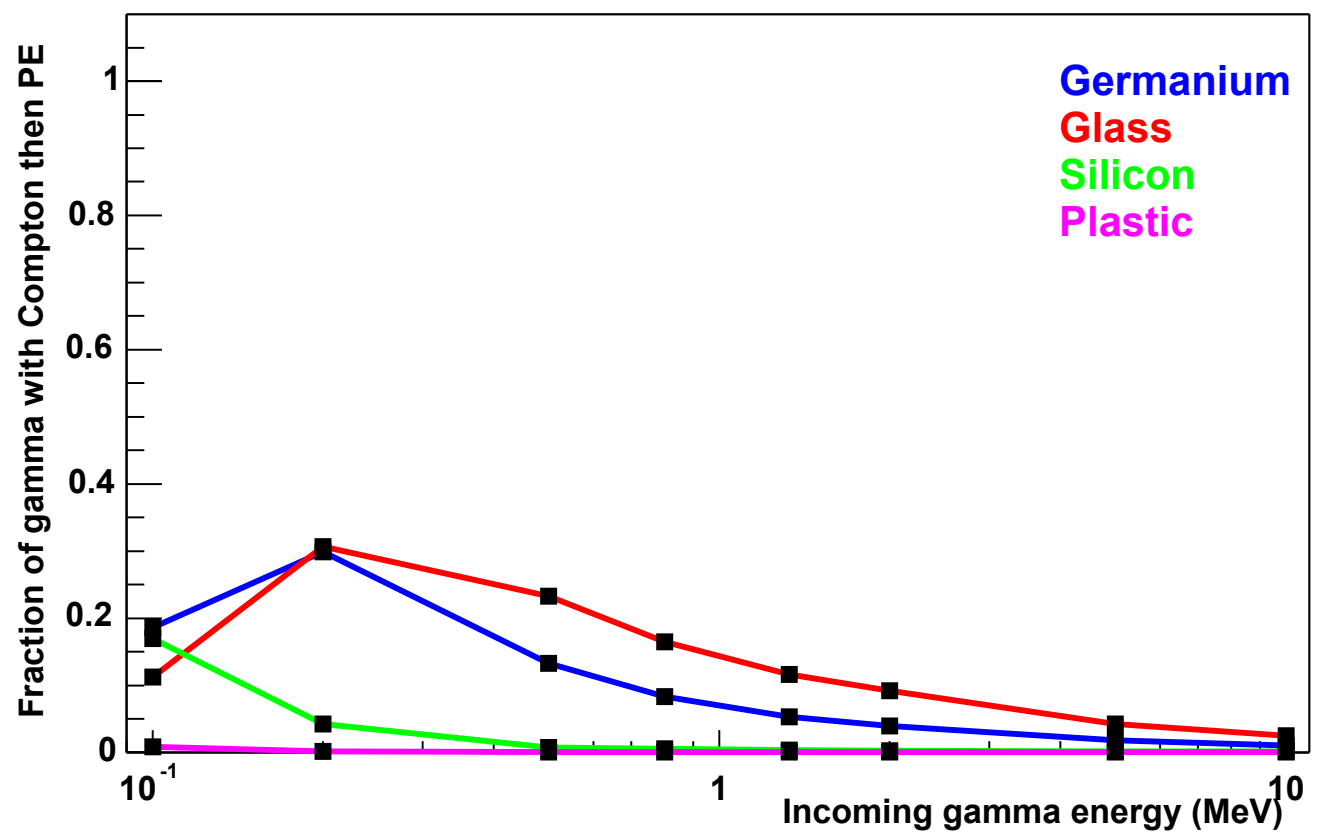

Figure 4: Fraction of gammas that undergo one Compton interaction followed by a photoelectric interaction in a $1 \mathrm{~m}^{3}$ detector. Results are shown for the glass, plastic, silicon, and germanium based detectors.

Table 4: Percentage of gammas that undergo a Compton interaction followed by a photoelectric interaction in a $1 \mathrm{~m}^{3}$ detector.

\begin{tabular}{ccccc}
\hline Energy $(\mathrm{MeV})$ & Germanium & Glass & Silicon & Plastic \\
\hline \hline 0.1 & $18.6 \pm 0.39$ & $11.3 \pm 0.32$ & $17.0 \pm 0.38$ & $0.8 \pm 0.09$ \\
0.2 & $29.9 \pm 0.46$ & $30.6 \pm 0.46$ & $4.2 \pm 0.20$ & $0.2 \pm 0.04$ \\
0.5 & $13.3 \pm 0.34$ & $23.2 \pm 0.42$ & $0.7 \pm 0.09$ & $0.0 \pm 0.02$ \\
0.8 & $8.3 \pm 0.28$ & $16.5 \pm 0.37$ & $0.5 \pm 0.07$ & $0.0 \pm 0.00$ \\
1.33 & $5.2 \pm 0.22$ & $11.6 \pm 0.32$ & $0.3 \pm 0.06$ & $0.0 \pm 0.01$ \\
2 & $4.0 \pm 0.20$ & $9.2 \pm 0.29$ & $0.2 \pm 0.05$ & $0.0 \pm 0.01$ \\
5 & $1.8 \pm 0.13$ & $4.3 \pm 0.20$ & $0.2 \pm 0.04$ & $0.0 \pm 0.01$ \\
10 & $1.0 \pm 0.10$ & $2.5 \pm 0.16$ & $0.1 \pm 0.03$ & $0.0 \pm 0.00$ \\
\hline
\end{tabular}




\section{Appendix}

For reference, we include the PDG plots of photon total cross sections for carbon and lead.

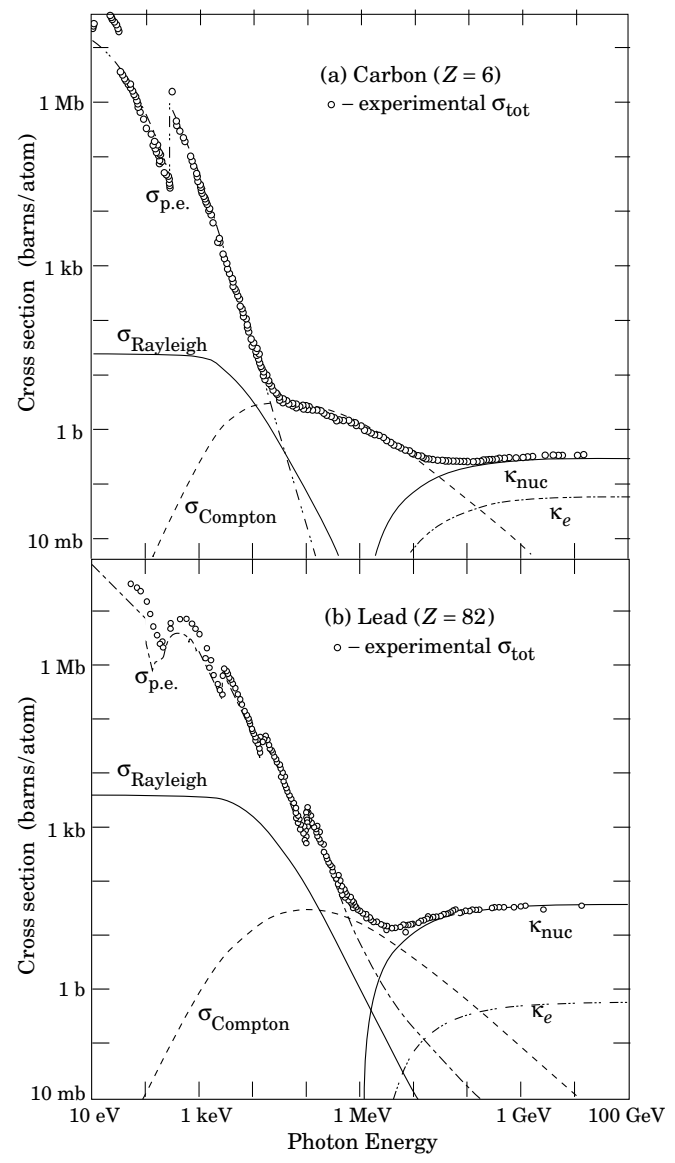

Figure 26.13: Photon total cross sections as a function of energy in carbon and lead, showing the contributions of different processes:

$\sigma_{\text {p.e. }}=$ Atomic photoelectric effect (electron ejection, photon absorption)

$\sigma_{\text {Rayleigh }}=$ Coherent scattering (Rayleigh scattering - atom neither ionized nor excited)

$\sigma_{\text {Compton }}=$ Incoherent scattering (Compton scattering off an electron)

$\kappa_{\text {nuc }}=$ Pair production, nuclear field

$\kappa_{e}=$ Pair production, electron field

Data from Hubbell, Gimm, and Øverbø, J. Phys. Chem. Ref. Data 9, 1023 (1980). Curves for these and other elements, compounds, and mixtures may be obtained from

http://physics.nist.gov/PhysRefData. The photon total cross section is approximately flat for at least two decades beyond the energy range shown. Original figures courtesy J.H. Hubbell (NIST). 


\title{
Study of the uncertainty on Compton ring reconstruction
}

\author{
Jeff Gronberg, Stephen Johnson, David Lange, Doug Wright \\ High Energy Physics group, N Division
}

September 24, 2003

\section{Introduction}

The purpose of this study was to estimate the effect of measurement errors in the position and energy of the observed interactions on the angular resolution of a Compton-ring imaging type detector.

In a Compton interaction, if one can measure the energy of the scattered photon and the energy of the Compton electron, then one can determine the scattering angle between the incoming and outgoing photon using the well-known Compton formula (see below for the derivation of this formula).

$$
\cos \theta=1+\frac{m_{e}}{E_{\gamma}}-\frac{m_{e}}{E_{\gamma^{\prime}}},
$$

where $E_{\gamma}=E_{\gamma^{\prime}}+E_{e}-m_{e}$.

If one assumes small Gaussian errors $\left(\delta E_{e}, \delta E_{\gamma}\right)$ for the observed energies $\left(E_{e}, E_{\gamma^{\prime}}\right)$, then one can derive an analytic expression for the error on the reconstruction angle $\theta$

$$
\delta \theta^{2}=\frac{m_{e}{ }^{2}}{\sin ^{2} \theta E_{\gamma}{ }^{4}}\left[\delta E_{e}{ }^{2}+\left(1-\frac{E_{\gamma^{2}}{ }^{2}}{E_{\gamma^{\prime}}{ }^{2}}\right)^{2} \delta E_{\gamma^{\prime}}{ }^{2}\right] .
$$

See Appendix A.2 (page 7) for the derivation of this result and a numerical cross-check. Note that this error function approaches infinity if $\theta$ is near zero or $\pi$. A detailed discussion on how to avoid this is given in Appendix A.2.1 (page 7) and the impact on the reconstruction of the simulated events is described in Section 4 (page 2).

\section{Error in the determination of the scattered photon direction}

There is an additional error on the determined Compton ring due to the uncertainty in the scattered photon direction. This is due to the finite precision on the two measured interaction points. In order to estimate this contribution to the Compton angle error, we use the following empirical procedure.

For each measured coordinate, we displace the nominal value by the expected measurement error in that coordinate and determine the change in the direction of the scattered photon, i.e., the opening angle between the nominal direction and direction determined with one coordinate shifted. We repeat this procedure for each of the six coordinates and add, in quadrature, the calculated angle shifts.

We have checked that this procedure agrees numerically with an analytic formulation of the error, see Equation 7 in Appendix B on page 9. The analytic formulation has the advantage that it is statistically meaningful. The input and output variables will have Gaussian distributions and thus one can calculate confidence limits. On the other hand, the results are Gaussian only when the fractional errors are less than about $50 \%$.

We combine, in quadrature, the angular error derived from the position measurement uncertainty (using the empirical method) with the error determined from Eq. 2 for the total $d \theta$ of the Compton ring.

\section{Simulation}

Using this information, we have determined how $d \theta$ depends on the detector resolution. We use a detector geometry consiting of a silicon slab that is $8 \mathrm{~cm}$ by $8 \mathrm{~cm}$ by $4 \mathrm{~cm}$. We use a point source of photons that is placed on an axi perpendicular to one of the $8 \mathrm{~cm}$ by $8 \mathrm{~cm}$ faces of this detector. We use Geant 4 to determine the event sample, considering only events where the full energy of the incoming photon has 


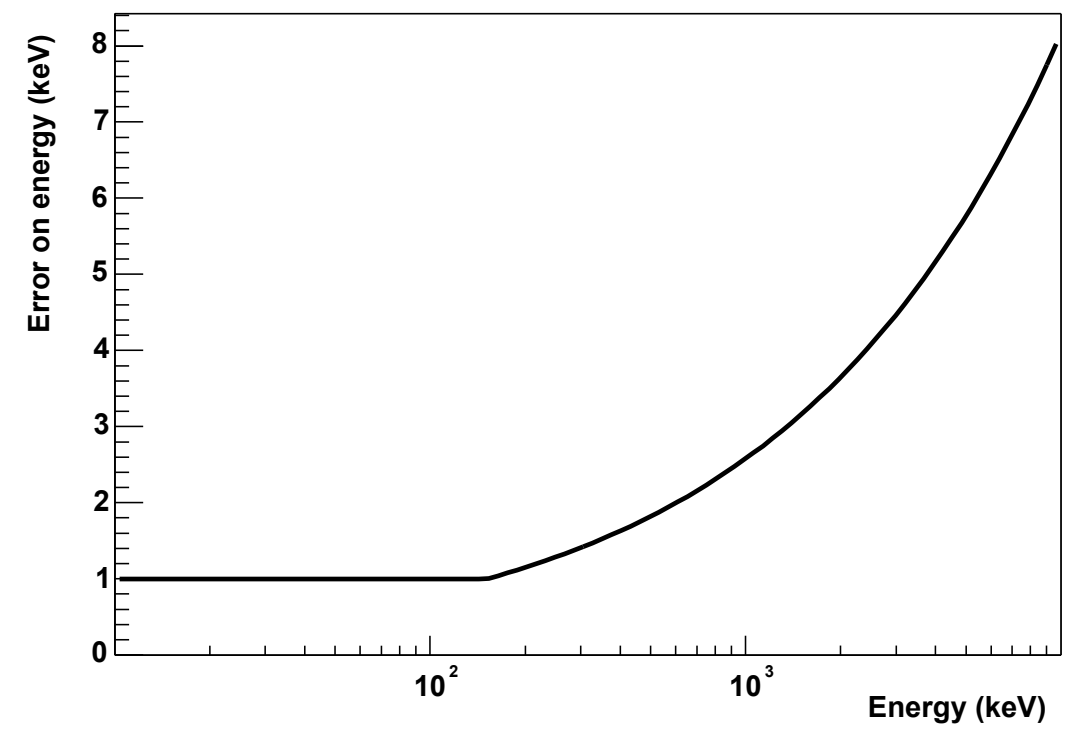

Figure 1: The simulated detector energy resolution as a function of energy deposited.

been deposited. We assume that we know the correct order of the photon interactions, and consider both photoelectric and Compton processes for the second interaction. We sum the total energy deposited in the detector to determine the incoming photon energy.

The assumed energy resolution is $2 \mathrm{keV}$ at $600 \mathrm{keV}$ with a square root energy dependence. The minimum energy resolution is $1 \mathrm{keV}$. This function is shown in Figure 1 and can be summarized as

$$
\begin{array}{r}
\delta E=1 \mathrm{keV}(E<150 \mathrm{keV}) \\
\delta E=\sqrt{E / 150 \mathrm{keV}}(E \geq 150 \mathrm{keV})
\end{array}
$$

This energy resolution is used to smear the energy deposited at each interaction site in the detector.

We then vary the position resolution from $0.1 \mathrm{~mm}$ to $10 \mathrm{~mm}$, assumed to be the same in each dimension. Given the kinematics of the generated event, the determined $d \theta$ varies considerably. For example, Figure 2 shows the $d \theta$ distribution for an input of $0.5 \mathrm{MeV}$ photons and a spatial resolution of $1 \mathrm{~mm}$. Figures 3 to 6 show the median value of $d \theta$ for initial photon energies of $0.1 \mathrm{MeV}, 0.5 \mathrm{MeV}, 1.0 \mathrm{MeV}$, and $10.0 \mathrm{MeV}$.

We observe that for spatial resolutions of the order of $1 \mathrm{~mm}$, the spatial resolution is the dominating factor in $d \theta$ for this detector configuration.

\section{Effect of events near $\theta=0$ or $\pi$}

The results reported in the previous section include all of the events, even those near $\theta=0$ or $\pi$, where the analytic expression may be overestimating the error in the Compton formula (see below).

Here we show the effect of excluding these events from the analysis. Table 1 gives the fraction of events for $\theta \sim 0$ where the electron kinetic energy is within one $\sigma$ of zero $\left(E_{e}-m_{e}<\delta E_{e}\right)$, the fraction for $\theta \sim \pi$ where the scattered photon energy is with one $\sigma$ of its minimum possible energy $\left(E_{\gamma^{\prime}}<E_{\gamma^{\prime}}(\min )+\delta E_{\gamma^{\prime}}\right)$, and the median $d \theta$ with or without these events, for various incoming gammay-ray energies.

The contribution from events near $\theta=\pi$ dominates over those near $\theta=0$. Given the size of the detector, and the requirement that the events be fully contained, then the events with low energy photons are more likely to be detected. The total number of affected events is energy dependent and is approximately $5 \%$, but grows to about $20 \%$ for $100 \mathrm{keV}$ incident gamma rays. Excluding these events shifts the median $d \theta$ by less than $8 \%$ and the effect is essentially negligible for energies above about $1 \mathrm{MeV}$. 
Table 1: The effect of excluding events with $\theta \sim 0$ or $\pi$ in the calculation of $d \theta$. Shown for discrete generated energies are the fraction of events in each region and the median $d \theta$ determined including or excluding those events.

\begin{tabular}{ccccc}
\hline $\begin{array}{c}\text { Energy } \\
(\mathrm{MeV})\end{array}$ & $\begin{array}{c}\theta \sim 0 \\
(\%)\end{array}$ & $\begin{array}{c}\theta \sim \pi \\
(\%)\end{array}$ & $\begin{array}{c}d \theta \\
\text { All } \\
\text { (radians) }\end{array}$ & $\begin{array}{c}\text { Excluding } \theta \sim 0, \pi \\
\text { (radians) }\end{array}$ \\
\hline \hline 0.1 & 2.5 & 20.3 & 0.081 & 0.075 \\
0.2 & 0.5 & 11.6 & 0.037 & 0.034 \\
0.5 & 0.03 & 5.7 & 0.021 & 0.020 \\
0.8 & 0.06 & 5.3 & 0.018 & 0.017 \\
1.0 & 0 & 4.9 & 0.016 & 0.015 \\
1.33 & 0 & 3.8 & 0.016 & 0.015 \\
2.0 & 0 & 4.5 & 0.013 & 0.013 \\
3.0 & 0 & 5.0 & 0.013 & 0.013 \\
5.0 & 0 & 3.1 & 0.011 & 0.011 \\
10.0 & 0 & 3.1 & 0.011 & 0.011 \\
\hline
\end{tabular}

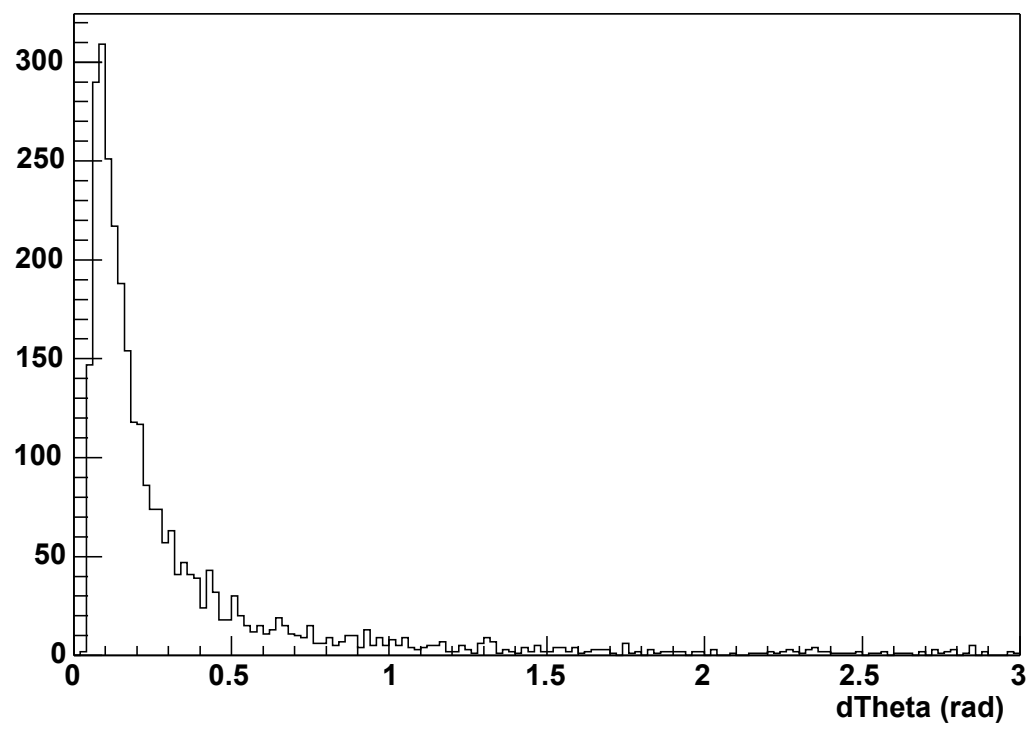

Figure 2: The $d \theta$ distribution from our simulation. The initial photon energy is $0.5 \mathrm{MeV}$ and the detector spatial resolution is simulated to be $1 \mathrm{~mm}$ in each dimension. 


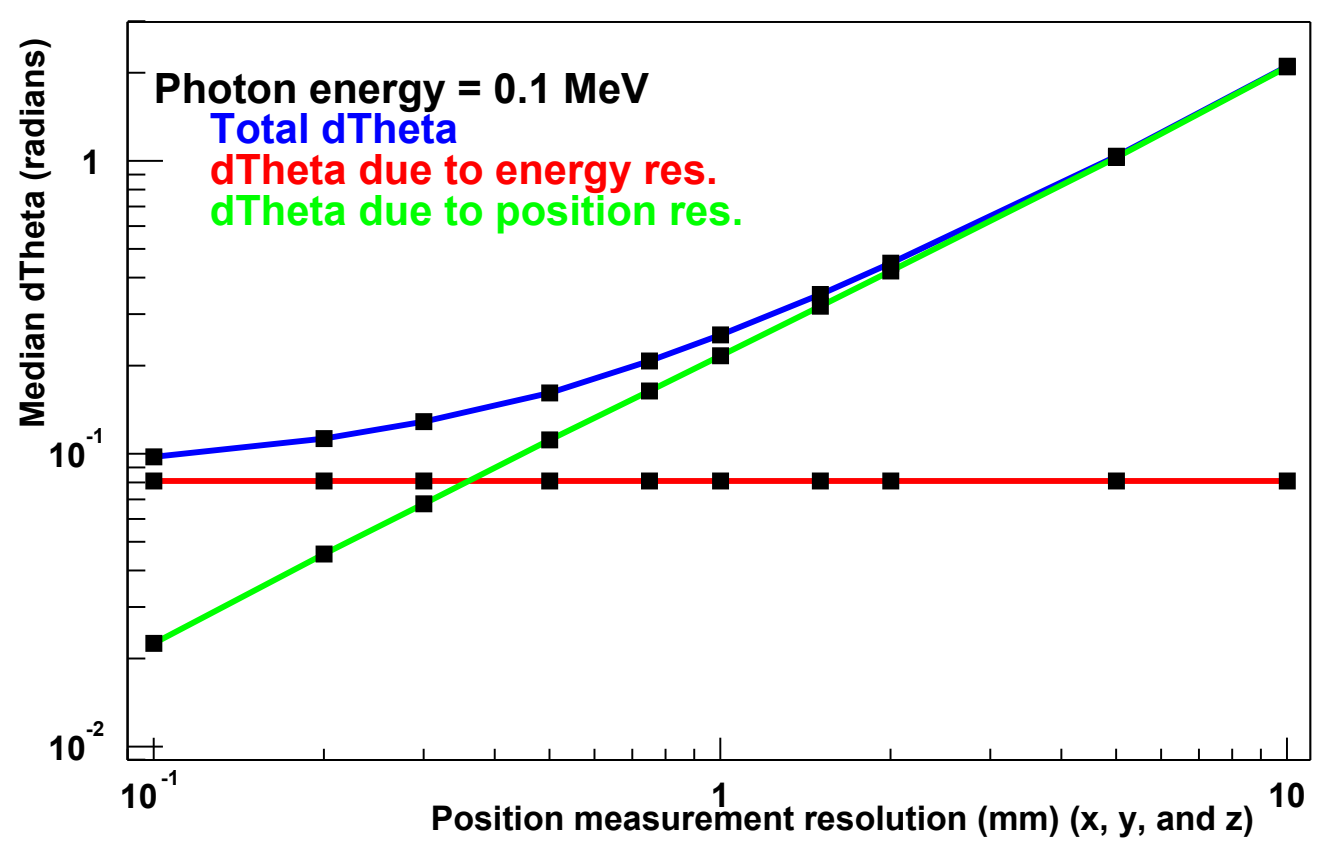

Figure 3: $d \theta$ as a function of detector spatial resolution. Plotted are the contribution to $d \theta$ due to energy resolution (red), due to spatial resolution (green), and the total $d \theta$ (blue). The input photon energy is $0.1 \mathrm{MeV}$.

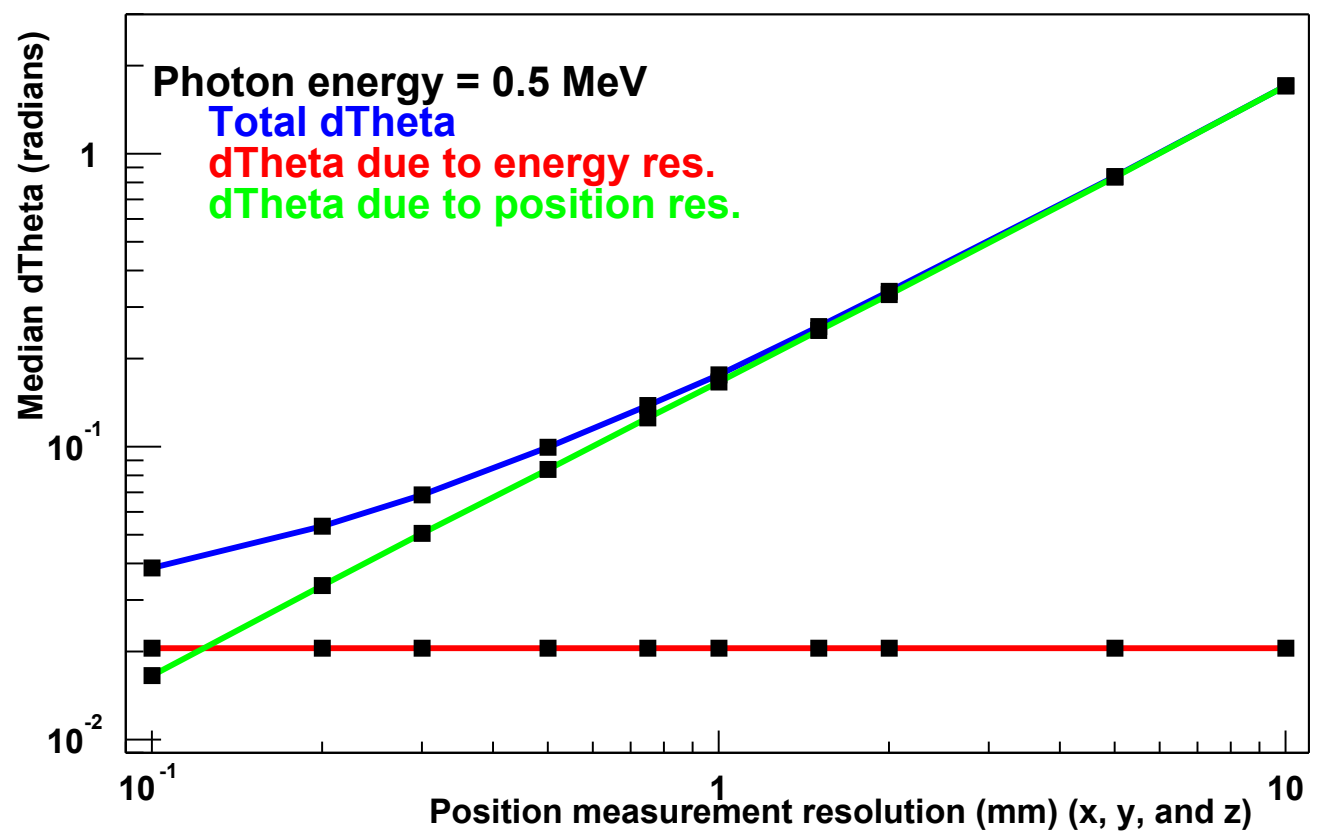

Figure 4: $d \theta$ as a function of detector spatial resolution. Plotted are the contribution to $d \theta$ due to energy resolution (red), due to spatial resolution (green), and the total $d \theta$ (blue). The input photon energy is $0.5 \mathrm{MeV}$. 


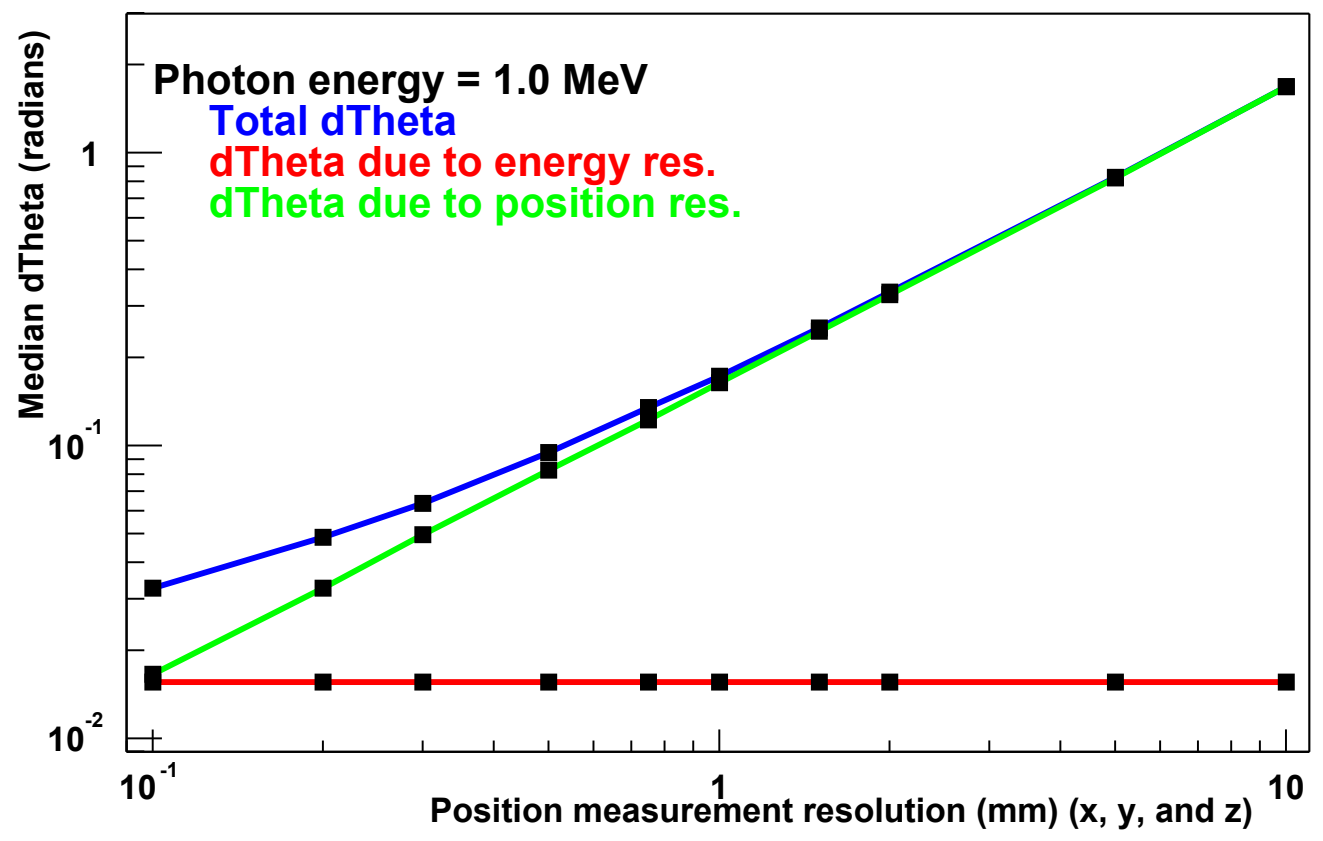

Figure 5: $d \theta$ as a function of detector spatial resolution. Plotted are the contribution to $d \theta$ due to energy resolution (red), due to spatial resolution (green), and the total $d \theta$ (blue). The input photon energy is 1 . MeV.

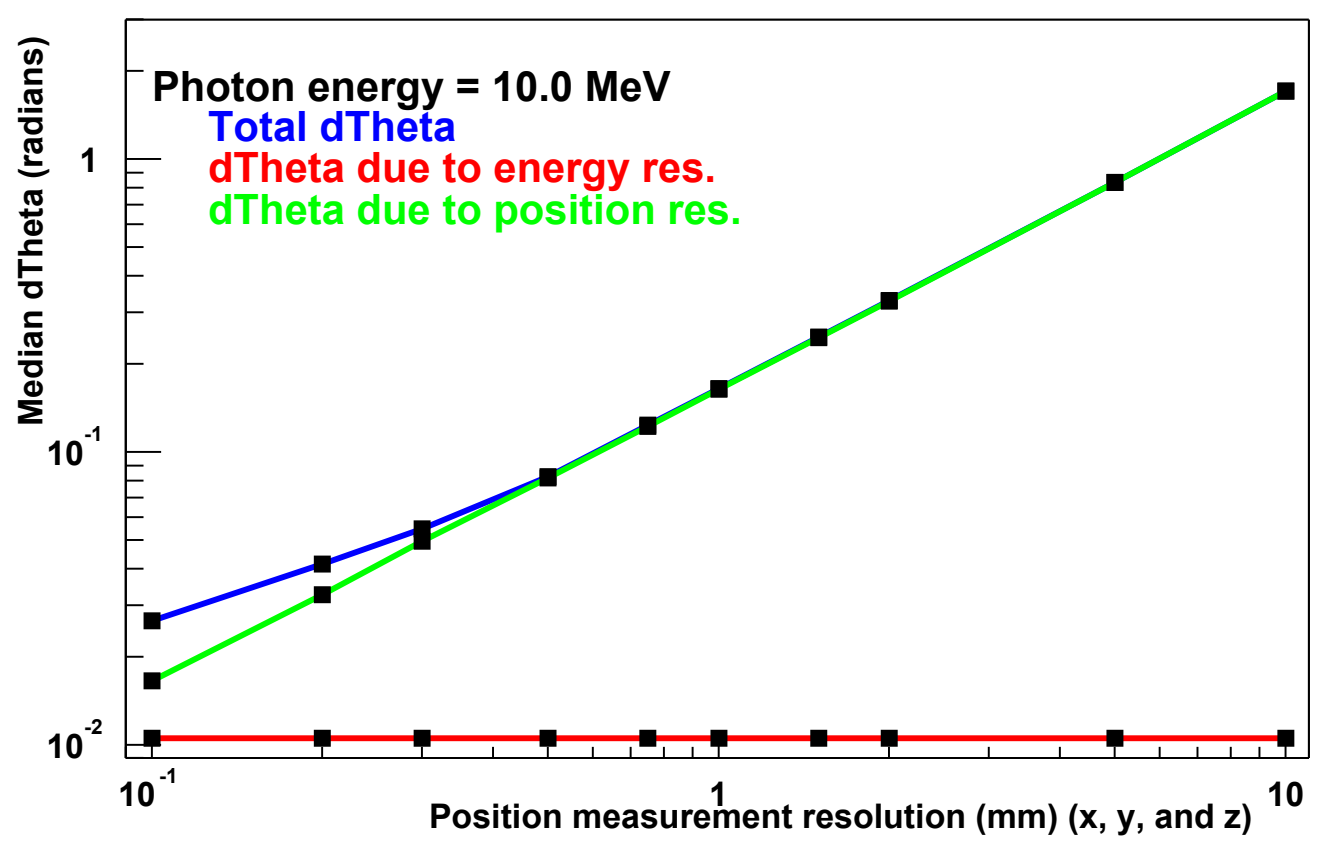

Figure 6: $d \theta$ as a function of detector spatial resolution. Plotted are the contribution to $d \theta$ due to energy resolution (red), due to spatial resolution (green), and the total $d \theta$ (blue). The input photon energy is 10. $\mathrm{MeV}$. 


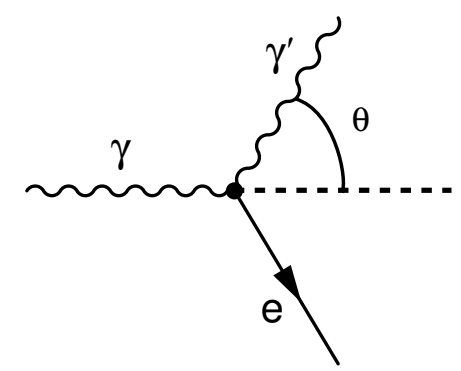

Figure 7: Compton interaction.

\section{Derivation of the Compton formula}

Assume that the inital electron is at rest, then from conservation of momentum, the electron recoils from the scattered photon (see Figure 7):

$$
\vec{p}_{e}=\vec{p}_{\gamma}-\vec{p}_{\gamma^{\prime}}
$$

Taking the square of the momentum and using $E_{\gamma}=p_{\gamma}$ and $E_{\gamma^{\prime}}=p_{\gamma^{\prime}}$ (since photons are massless and we are using units where $\mathrm{c}=1$ ), results in

$$
p_{e}{ }^{2}=E_{\gamma}{ }^{2}+E_{\gamma^{\prime}}{ }^{2}-2 E_{\gamma} E_{\gamma^{\prime}} \cos \theta .
$$

Using the invariant mass relation $p_{e}{ }^{2}=E_{e}{ }^{2}-m_{e}{ }^{2}$ and conservation of energy $\left(E_{\gamma}+m_{e}=E_{e}+E_{\gamma^{\prime}}\right)$ to eliminate the electron variables $p_{e}$ and $E_{e}$ results in

$$
\begin{array}{r}
E_{\gamma^{\prime}}+2 m_{e} E_{\gamma}+m_{e}{ }^{2}-2 E_{\gamma} E_{\gamma^{\prime}}-2 m E_{\gamma^{\prime}}+E_{\gamma^{\prime}}{ }^{2}-m_{e}{ }^{2} \\
=E_{\gamma}{ }^{2}+E_{\gamma^{\prime}}{ }^{2}-2 E_{\gamma} E_{\gamma^{\prime}} \cos \theta,
\end{array}
$$

which reduces to

$$
m_{e}\left(E_{\gamma}-E_{\gamma^{\prime}}\right)=E_{\gamma} E_{\gamma^{\prime}}(1-\cos \theta) .
$$

This is often rewritten as

$$
\cos \theta=1+\frac{m_{e}}{E_{\gamma}}-\frac{m_{e}}{E_{\gamma^{\prime}}} .
$$

\section{Compton formula implications}

Note that the angle $\theta$ approaches zero as the electron kinetic energy $\left(E_{e}-m_{e}\right)$ approaches zero. Obviously at zero angle there is no scattering and the outgoing photon has the same energy as the incoming photon.

In contrast, the minimum outgoing photon energy can not be zero, but is instead

$$
E_{\gamma^{\prime}}(\min )=\frac{m_{e}}{2+m_{e} / E_{\gamma}},
$$

which corresponds to $\theta=\pi$, i.e., the case where the photon scatters back in the direction opposite to that of the incoming photon $\left(E_{\gamma^{\prime}}=E_{\gamma}\right)$.

Using consevation of energy $\left(E_{\gamma}=E_{\gamma^{\prime}}+E_{e}-m_{e}\right)$, one can rewrite Equation 5 in terms of the electron kinetic energy $\left(K_{e}=E_{e}-m_{e}\right)$

$$
E_{\gamma^{\prime}}(\min )=\frac{1}{2}\left(\sqrt{2 m_{e} K_{e}+K_{e}^{2}}-K_{e}\right) .
$$




\section{Analytic expression for the error in the Compton angle}

The Compton angle $\theta$ in Equation 4 depends only on the energy of the incoming $\left(E_{\gamma}=E_{\gamma^{\prime}}+E_{e}-m_{e}\right)$ and outgoing $\left(E_{\gamma^{\prime}}\right)$ gamma rays. If one considers only small Gaussian errors for the observables $\left(E_{e}\right.$ and $\left.E_{\gamma^{\prime}}\right)$, then one can derive an analytic expression for the error on $\theta$ as follows.

Taking the differential of Equation 4 yields:

$$
d(\cos \theta)=-\sin \theta d \theta=-\frac{m_{e}}{E_{\gamma}{ }^{2}} d E_{\gamma}+\frac{m_{e}}{E_{\gamma^{\prime}}{ }^{2}} d E_{\gamma^{\prime}},
$$

which can be rewritten as

$$
d \theta=\frac{m_{e}}{\sin \theta E_{\gamma}{ }^{2}}\left[d E_{\gamma}-\frac{E_{\gamma}{ }^{2}}{E_{\gamma^{\prime}}{ }^{2}} d E_{\gamma^{\prime}}\right] .
$$

Since $E_{\gamma}=E_{e}+E_{\gamma^{\prime}}-m_{e}$, we have $d E_{\gamma}=d E_{e}+d E_{\gamma^{\prime}}$, and so

$$
\begin{aligned}
d \theta & =\frac{m_{e}}{\sin \theta E_{\gamma}{ }^{2}}\left[d E_{e}+d E_{\gamma^{\prime}}-\frac{E_{\gamma}{ }^{2}}{{E_{\gamma^{\prime}}}^{2}} d E_{\gamma^{\prime}}\right] \\
& =\frac{m_{e}}{\sin \theta E_{\gamma}{ }^{2}}\left[d E_{e}+\left(1-\frac{E_{\gamma}{ }^{2}}{E_{\gamma^{\prime}}{ }^{2}}\right) d E_{\gamma^{\prime}}\right] .
\end{aligned}
$$

This equation defines the partial derivatives $\partial \theta / \partial E_{e}$ and $\partial \theta / \partial E_{\gamma^{\prime}}$. So for Gaussian errors $\delta E_{e}$ and $\delta E_{\gamma^{\prime}}$, the error estimate for $\theta$ is

$$
\delta \theta^{2}=\frac{m_{e}{ }^{2}}{\sin ^{2} \theta E_{\gamma}{ }^{4}}\left[\delta E_{e}{ }^{2}+\left(1-\frac{E_{\gamma^{2}}{ }^{2}}{E_{\gamma^{\prime}}{ }^{2}}\right)^{2} \delta E_{\gamma^{\prime}}{ }^{2}\right] .
$$

This equation is valid as long as the fractional errors $\left(\delta E_{e} / E e\right.$ and $\left.\delta E_{\gamma^{\prime}} / E_{\gamma^{\prime}}\right)$ are not too large. Furthermore, one must be careful when applying this equation in situations near $\theta=0$ or $\pi$.

\section{Error in Compton Angle near $\theta=0$ or $\pi$}

The case where $\theta$ is exactly zero occurs only when the electron kinetic energy $\left(K_{e}=E_{e}-m_{e}\right)$ is zero. Thus there is no detected first interaction and the formula would not be applied. Arbitrarily small values of $K_{e}$ do not cause a problem for Equation 6 until they approach the magnitude of the energy resolution itself.

For arbitrarily small values of the kinetic energy, when $K_{e} / E_{\gamma^{\prime}} \ll 1$ Equation 6 can be approximated as

$$
\delta \theta^{2} \sim \frac{m_{e} E_{\gamma^{\prime}}{ }^{2}}{2 E_{\gamma}{ }^{4} K_{e}}\left[\delta K_{e}^{2}+\frac{4 K_{e}^{2}}{E_{\gamma^{\prime}}{ }^{4}} \delta E_{\gamma^{\prime}}{ }^{2}\right] .
$$

From this one can see that $\delta \theta^{2}$ begins to blow up when the the kinetic energy becomes smaller than the energy resolution $\delta K_{e}$, but is well behaved otherwise. Note that the second term $\left(\delta E_{\gamma^{\prime}}\right)$ is suppressed for small $K_{e}$. When $K_{e}$ is smaller than about twice the energy resolution, the Gaussian approximation itself breaks down and Equation 6 will begin to overestimate the error in $\theta$. To apply this formula, one should impose a lower cutoff on the electron energy based on the energy resolution.

For the case where $\theta$ is near $\pi$, the scattered photon energy is near its minimum (but can not be zero, see Appendix A.1.1). Because of finite measurement resolution, the observed photon energy can fluctuate downward and even be lower than the minimumally allowed energy. These kinematically unallowed events will be explicitly rejected since the Compton formula itself fails. However, the case where the photon energy reaches its minimum is kinematically allowed and can also occur when events near the minimum fluctuate downard due to the detector resolution. For these events at or very near the minimum, the error formula, Equation 6, approaches infinity. To highlight the behavior near the minimum energy, we can rewrite the equation using $E_{\gamma^{\prime}}=E_{\gamma^{\prime}}(\min )(1+\delta)$, where $\delta \ll 1$.

$$
\delta \theta^{2} \sim \frac{m_{e} E_{\gamma^{\prime}}(\min )}{2 E_{\gamma}{ }^{4} \delta}\left[\delta E_{e}{ }^{2}+\left(1-\frac{E_{\gamma}{ }^{2}}{E_{\gamma^{\prime}}(\min )^{2}}(1-2 \delta)\right)^{2} \delta E_{\gamma^{\prime}}{ }^{2}\right] .
$$

Neither term $\left(\delta E_{e}, \delta E_{\gamma^{\prime}}\right)$ is suppressed, so in order to avoid this infinity, one should apply a cut to eliminate events that are less than at least one standard deviation (in terms of the energy resolution) away from the minimum photon energy. 


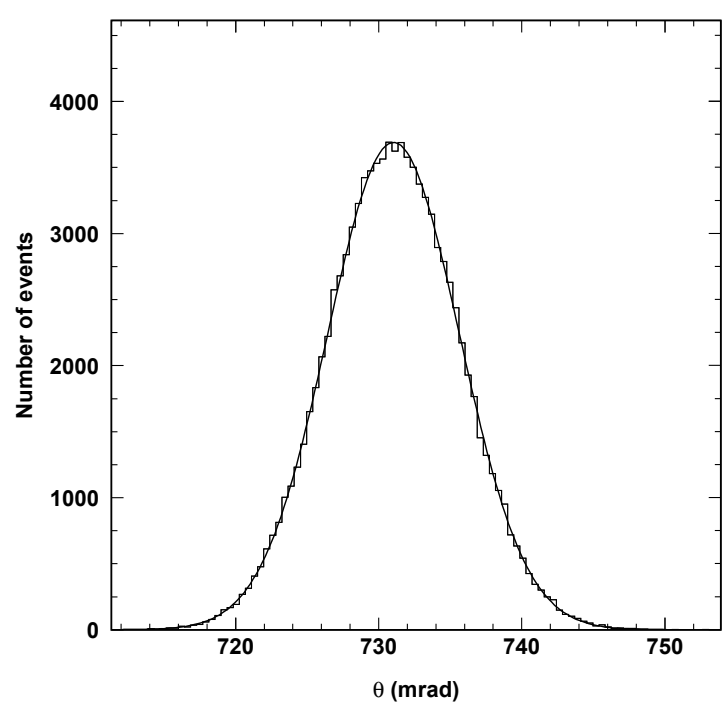

Figure 8: Reconstructed Compton angle $\theta$ from a simulation of the same physical state sampled 100,000 times with Gaussian errors for the observed energies. The fit result is superimposed on the distribution.

Table 2: Compton angle and error comparison between fit to simulation and analytic calculation. Sample of 100,000 events where the Electron kinetic energy is $100 \mathrm{keVwith}$ an RMS error of $1 \mathrm{keV}$, and scattered gamma ray energy of $400 \mathrm{keV}$ with and RMS error of $2 \mathrm{keV}$.

\begin{tabular}{|l|l|l|}
\hline Variable & Fit Result & Analytic Result \\
\hline$\theta(\mathrm{mrad})$ & $731.03 \pm 0.02$ & 731.01 \\
$\delta \theta(\mathrm{mrad})$ & $4.61 \pm 0.01$ & 4.61 \\
\hline
\end{tabular}

\section{Numerical cross-check of Equation 6}

To check the validity of Equation 6 we performed a simple Monte Carlo analysis by generating Gaussian distributions for the input variables $E_{e}$ and $E_{\gamma^{\prime}}$ and then compared the resulting distribution of $\theta$ with the analytic expression. We generated 100,000 events where the Compton electron kinetic energy $\left(E_{e}-m_{e}\right)$ was sampled from a Gaussian distribution with a mean of $100 \mathrm{keV}$ and RMS of $1 \mathrm{keV}$, and the scattered gamma ray energy distribution had a mean of $400 \mathrm{keV}$ with an RMS of $2 \mathrm{keV}$.

Using Equation 4 we reconstruct the Compton angle $\theta$ from the simulated observables. Figure 8 shows the reconstructed $\theta$ distribution. The result of a Gaussian fit to this distribution is given in Table 2 along with the results from the analytic expressions 4 and 6 . The agreement between the fit and the analytic expression is excellent. Be aware that the $\theta$ distribution is no longer Gaussian if the error on the energy measurement is too large, and thus Equation 6 would no longer apply. 


\section{Angular error from position resolution}

The direction of the outgoing photon is determined from the measured positions of the two interaction points. The measurement uncertainty of these positions creates an associated uncertainty in the direction of the photon. One can derive an analytic expression that relates these uncertainties as follows.

If the unit vector $\hat{n}$ defines the direction of the incoming photon, and the two points $v_{1}=\left(x_{1}, y_{1}, z_{1}\right)$, $v_{2}=\left(x_{2}, y_{2}, z_{2}\right)$ define the direction of the outgoing photon $\vec{v}=v_{2}-v_{1}$, then the angle between the incoming and outgoing photons is

$$
\hat{n} \cdot \vec{v}=v \cos \theta=v_{\|},
$$

where $v_{\|}$is the component of $\vec{v}$ parallel to $\hat{n}$. Taking the differential of the above equation gives

$$
d v \cos \theta-\sin \theta v d \theta=d v_{\|} .
$$

Taking the differential of $v^{2}=v_{\|}^{2}+v_{\perp}^{2}$ and substituting in the above, results in

$$
v^{2} \tan \theta d \theta=v_{\perp} d v_{\perp}+v_{\|} d v_{\|}-\frac{v d v_{\|}}{\cos \theta}=v_{\perp} d v_{\perp}+\frac{\left(v_{\|} \cos \theta-v\right) d v_{\|}}{\cos \theta} .=v_{\perp} d v_{\perp}-\frac{v \sin ^{2} \theta d v_{\|}}{\cos \theta} .
$$

The error in $\theta$ is then

$$
\delta \theta^{2}=\frac{1}{v^{2} \tan ^{2} \theta}\left[v_{\perp}^{2} d v_{\perp}^{2}+\frac{v^{2} \sin ^{4} \theta}{\cos ^{2} \theta} d v_{\|}^{2}\right] .
$$

If the position resolution for each dimension is $\delta d$, then

$$
d v_{\perp}=d v_{\|}=\sqrt{2} \delta d .
$$

So $\delta \theta$ becomes

$$
\delta \theta^{2}=\frac{1}{v^{2} \tan ^{2} \theta} \frac{\left(v_{\perp}^{2} \cos ^{2} \theta+v^{2} \sin ^{4} \theta\right)}{\cos ^{2} \theta} \delta d,
$$

which reduces to

$$
\delta \theta=\frac{\sqrt{2}\left|v_{\perp}\right|}{v\left|v_{\|}\right| \tan \theta} \delta d,
$$

where $v_{\perp}\left(v_{\|}\right)$is the component of scattered photon direction, $\vec{v}$, perpendicular (parallel) to $\hat{n}$, and $\delta d$ is the position error in each measured dimension. For the special case where the incoming photon is in the $\hat{z}$ direction $(\hat{n}=(0,0,1))$, then

$$
\delta \theta=\frac{\sqrt{2} \sqrt{v_{x}^{2}+v_{y}^{2}}}{v\left|v_{z}\right| \tan \theta} \delta d .
$$

This relation holds, meaning that the resulting distribution of $\theta$ is approximately Gaussian, as long as $\delta d / p_{i}$, where $p_{i}$ is the distance between the two points in each dimension, is less than about $50 \%$. 


\title{
Electron tracking device angular and energy resolution
}

\author{
Jeff Gronberg, Stephen Johnson, David Lange, Doug Wright \\ High Energy Physics group, N Division
}

October 14, 2003

\section{Introduction}

The purpose of this study was to estimate the intrinsic limitations and effect of detector measurement errors on the angular and energy resolution of a electron-tracking type gamma-ray detectors.

In a Compton interaction, if one can measure the energy and direction of the scattered electron, and the direction of the scattered photon, then one can determine completely the direction and energy of the incoming gamma ray (without measuring the scattered photon's energy). Multiple scattering of the Compton electron will quickly destroy the information of the electron's initial direction, so practical devices must be able to resolve the original electron direction, i.e., have tracking resolution much smaller than the typical radiation length in the material.

\section{Detectors}

The detectors considered are a $1 \mathrm{~m}^{3}$ block of either germanium, "glass", or "scintillator". The properties for these materials are given in Table 1.

\section{Simulation and Reconstruction}

We simulate events using a mono-energitic source of photons that hit the detector in its midplane, with momentum perpendicular to the detector plane. Events with two or more interactions in the detector are considered in our analysis.

We measure the electron energy by using the path length of the electron. We find that the energy is proportional to the path length, with a spread of approximately $15 \%$, in the range of energies of interest in this study. We determine the reconstructed electron energy by smearing the true electron energy according to a Gaussian with $\sigma=0.15 E$.

We measure the electron direction using the first portion of the simulated electron track. The length considered is denoted as the "electron track detection metric", and we currently assume that the measurement error using a given portion of the electron track is small compared to how well the electron track direction measures the initial direction of the electron. If the electron path length is less than the detection metric, the event is considered to be unreconstructable. We find that even for a $50 \mu \mathrm{m}$ detection metric, electron tracking is impossible for photon energies below $1 \mathrm{MeV}$ except in the scintillator detector. We further assume that we know head from tail for the measured electron momentum.

Finally, we measure the scattered photon direction using the the true position of the two detected interactions. We assume that given the detector requirements for electron tracking that the measurement error on

Table 1: Summary of detector properties. "Scintillator" is polystyrene scintillator. "Glass" is a proprietary mixture used by Arno Ledebuhr from Collimated Holes, Inc. It has a LKH-6 glass core and borosilicate glass cladding.

\begin{tabular}{cccc}
\hline Detector & Radiation length $(\mathrm{cm})$ & Density $\left(\mathrm{g} / \mathrm{cm}^{3}\right)$ & Effective Z \\
\hline \hline Germanium & 2.30 & 5.32 & 32 \\
Glass & 4.48 & 3.27 & $\approx 31$ \\
Scintillator & 42.55 & 1.03 & $\approx 4$ \\
\hline
\end{tabular}


these points is small compared to the distance traveled by the photon between the first and second interaction points.

We then determine the angular (energy) resolution on the initial photon using distribution of reconstructed photon directions (energies) with the true photon direction (energy). We fit these distributions using a Gaussian, and report the $\sigma$ of the Gaussian as the resolution. For the angular resolution, we take out the $\sin \theta$ phase space factor which comes in because we are comparing the opening angle between the true and reconstructed photon directions by weighting events by $1 / \sin \theta$ before performing the fit. We find that the angular resolution is quite insensitive to the energy resolution assumed.

\section{Results}

Figures 1 and 2 show the angular and energy resolutions as a function of photon energy for a $50 \mu \mathrm{m}$ electron detection metric, while Figures 3 and 4 show the equivalent for a $500 \mu$ m electron detection metric.

Figures 5 through 8 show the angular and energy resolution for $1 \mathrm{MeV}$ and $2.7 \mathrm{MeV}$ initial photon energies as a function of the electron track detection metric. 


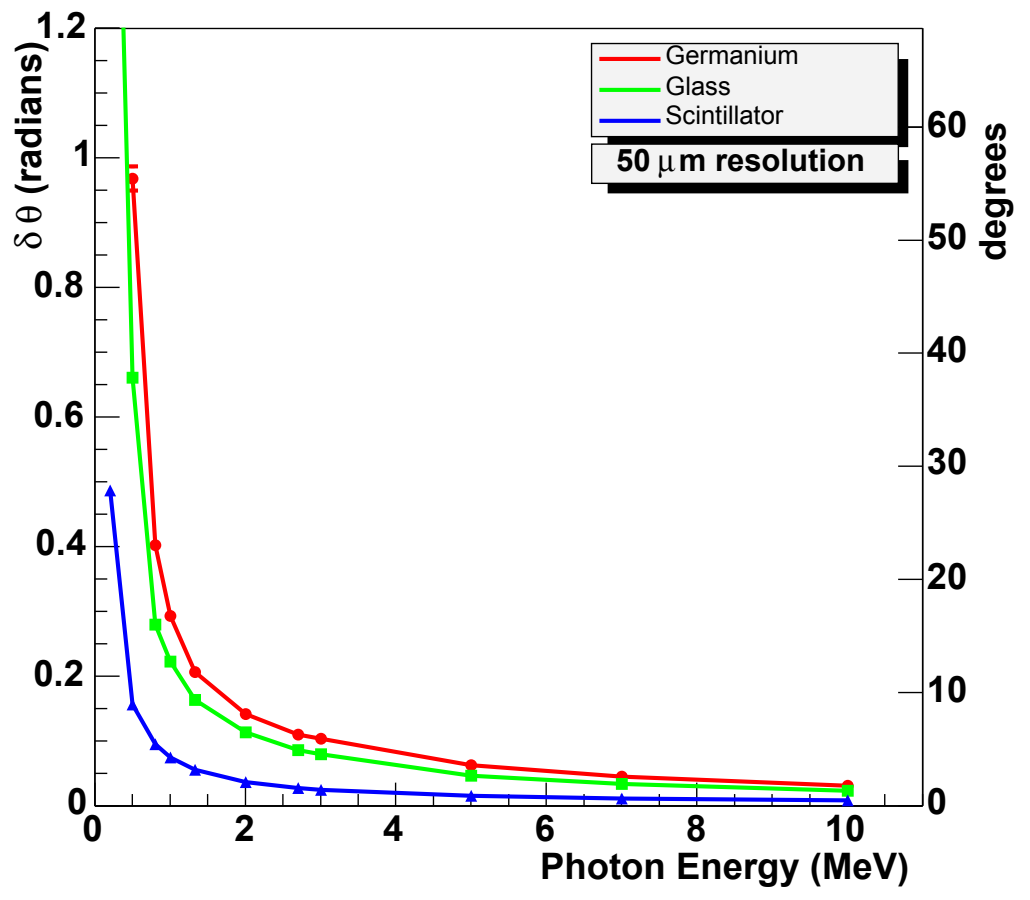

Figure 1: Angular resolution of incoming photon vs. photon energy for a $50 \mu \mathrm{m}$ electron track detection metric for germanium, glass, and scintillator detectors.

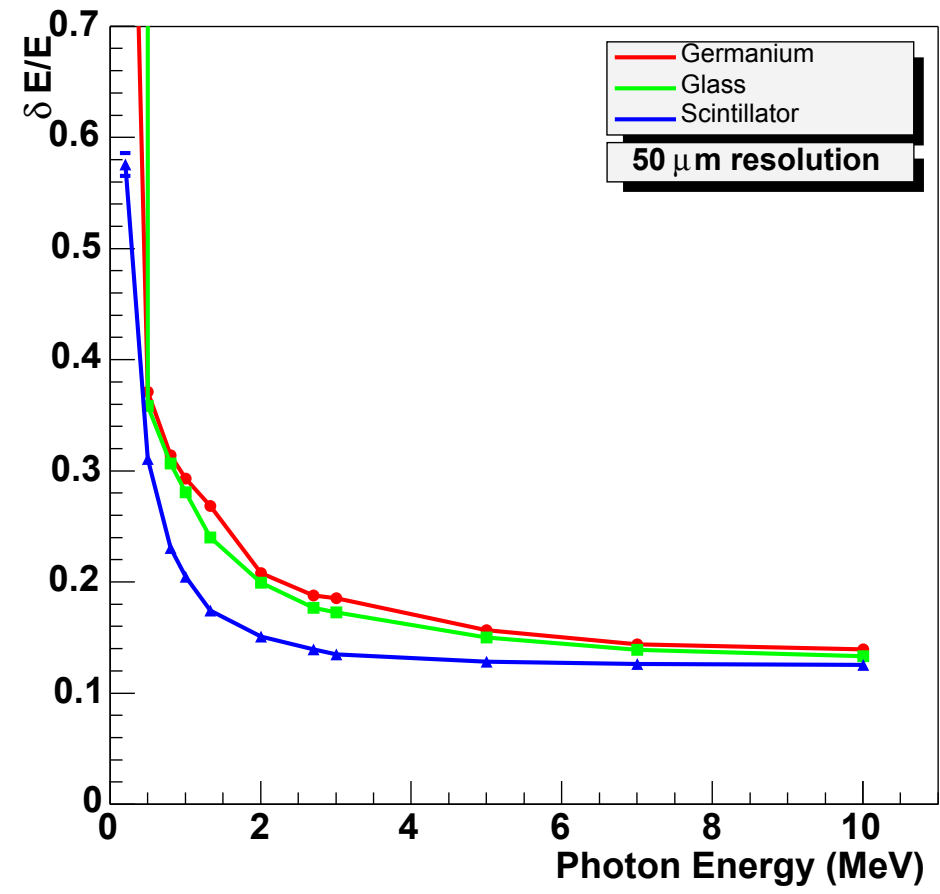

Figure 2: Energy resolution of incoming photon vs. photon energy for a $50 \mu \mathrm{m}$ electron track detection metric for germanium, glass, and scintillator detectors. 


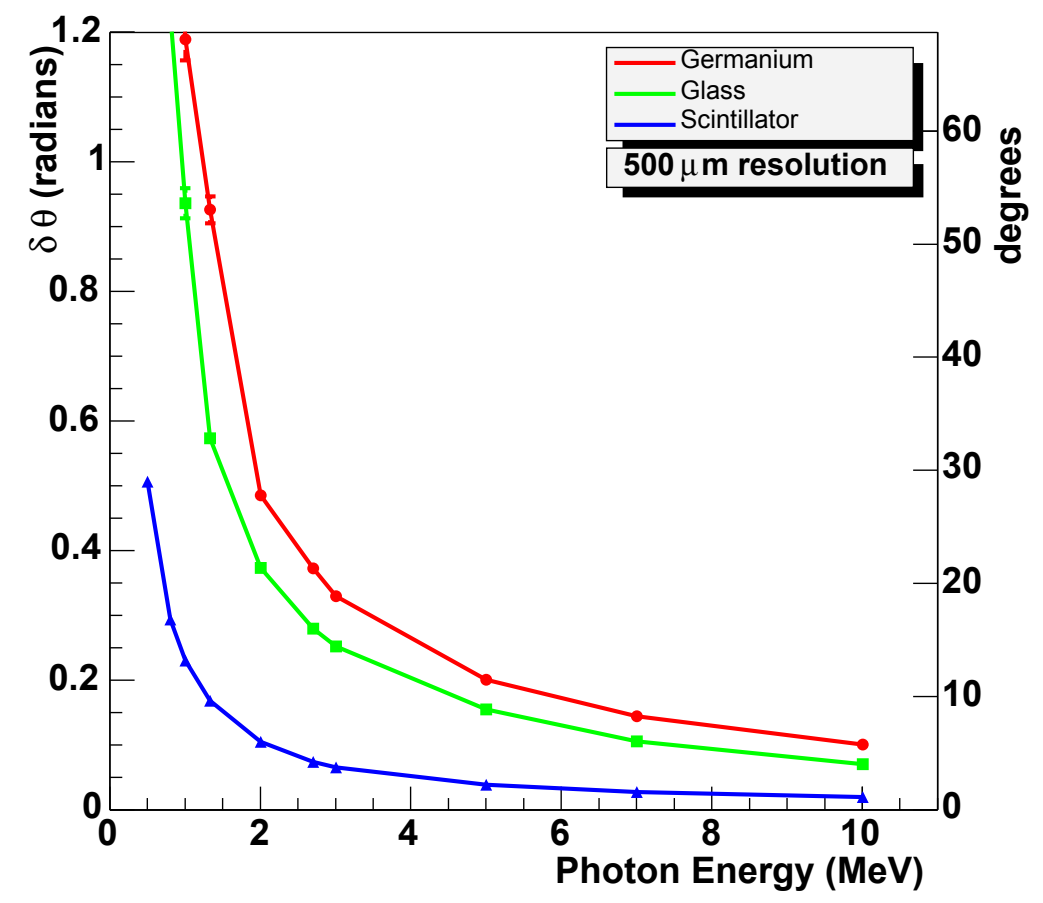

Figure 3: Angular resolution of incoming photon vs. photon energy for a $500 \mu \mathrm{m}$ electron track detection metric for germanium, glass, and scintillator detectors.

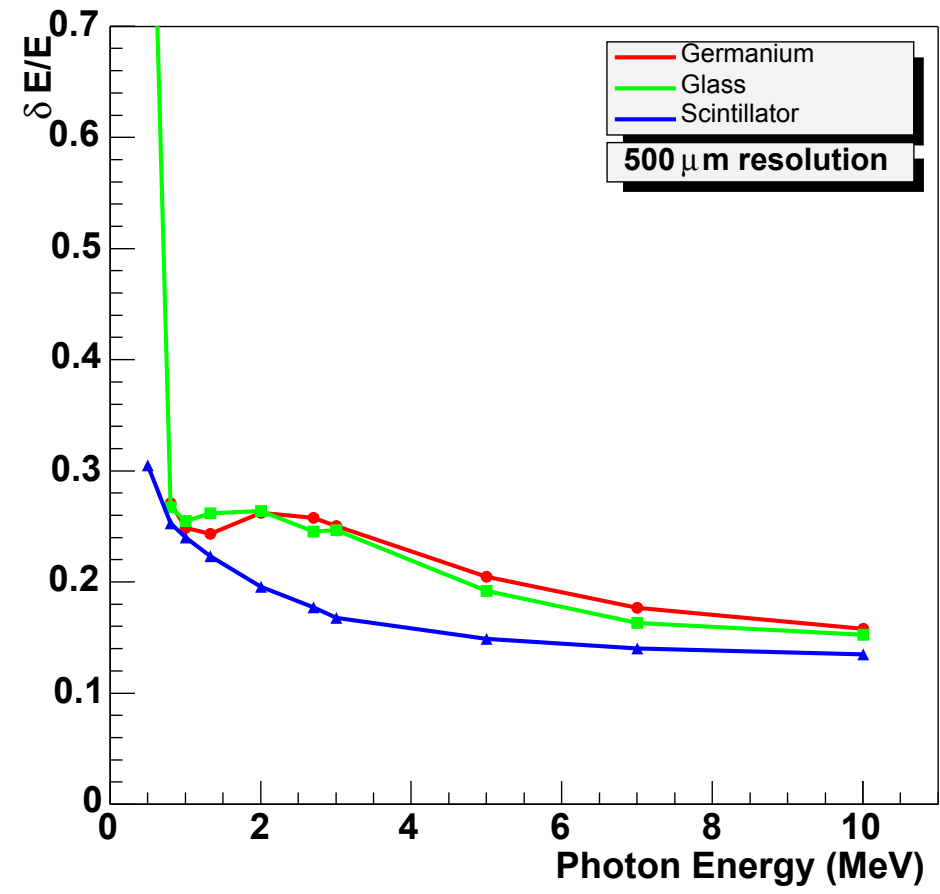

Figure 4: Energy resolution of incoming photon vs. photon energy for a $500 \mu \mathrm{m}$ electron track detection metric for germanium, glass, and scintillator detectors. 


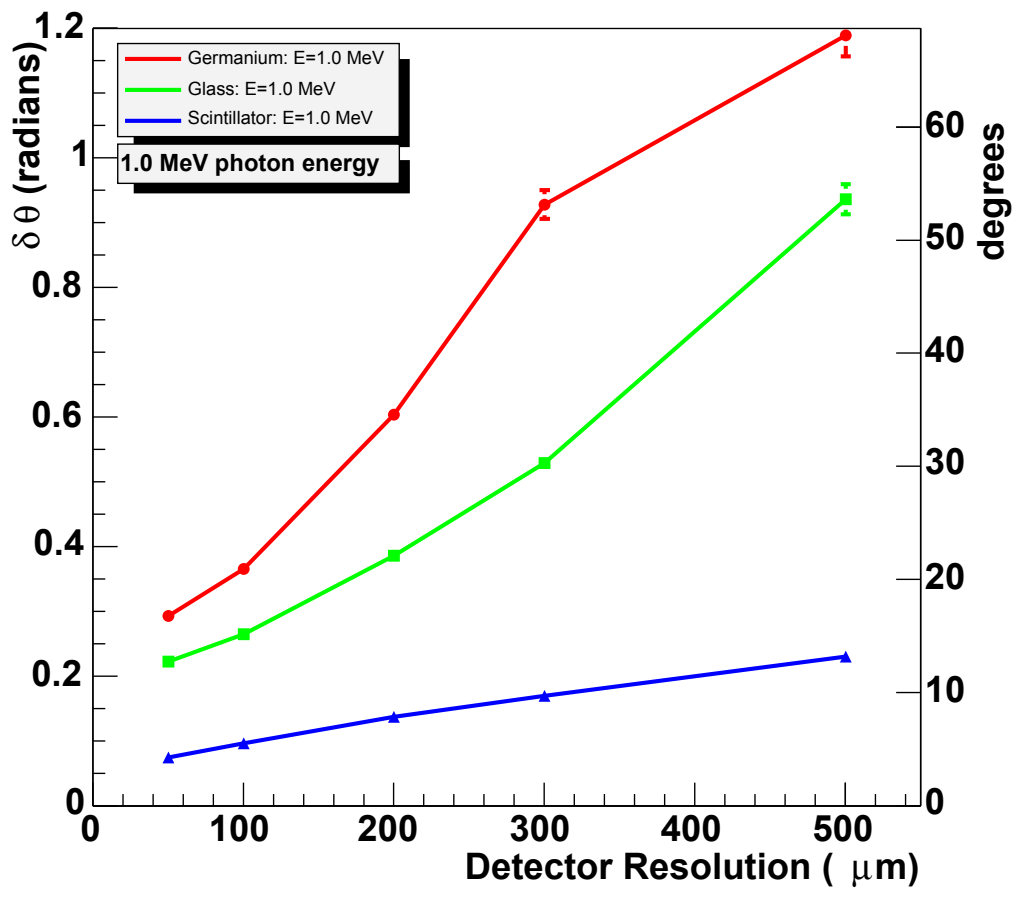

Figure 5: Reconstructed photon angular resolution vs. electron track detection metric for a $1.0 \mathrm{MeV}$ electron track detection metric for germanium, glass, and scintillator detectors.

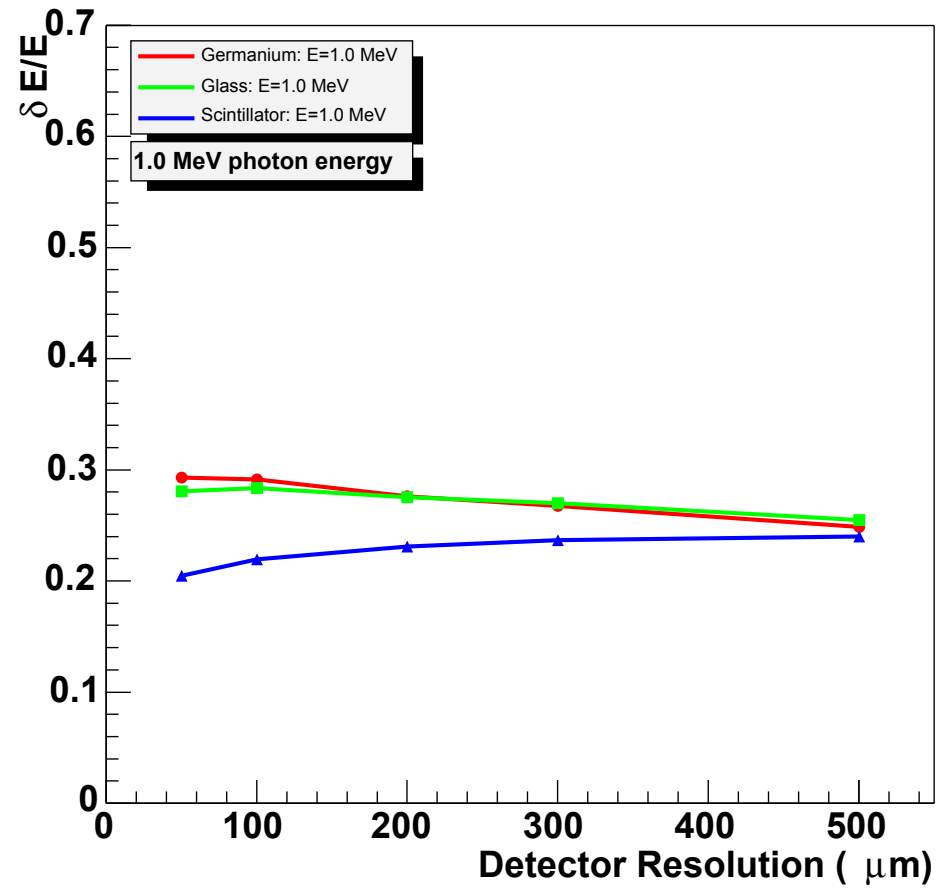

Figure 6: Reconstructed photon energy resolution vs. electron track detection metric for a $1.0 \mathrm{MeV}$ electron track detection metric for germanium, glass, and scintillator detectors. 


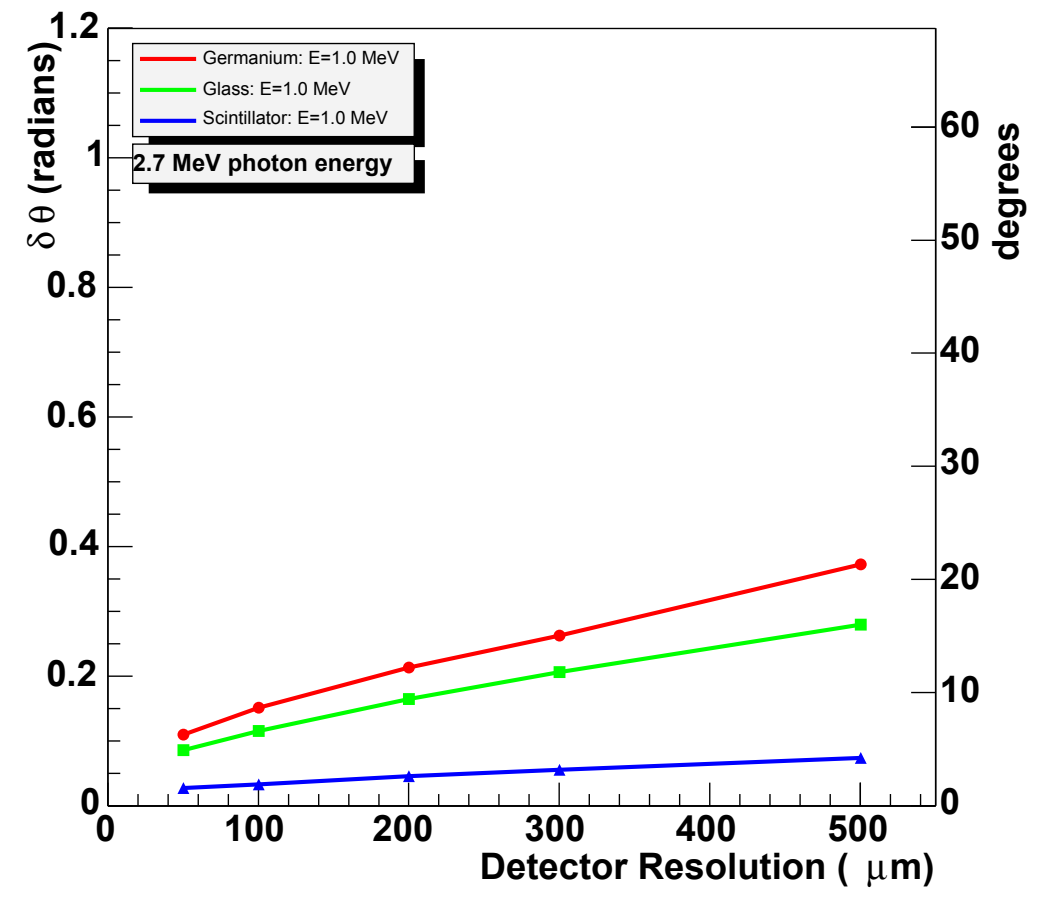

Figure 7: Reconstructed photon angular resolution vs. electron track detection metric for a $2.7 \mathrm{MeV}$ electron track detection metric for germanium, glass, and scintillator detectors.

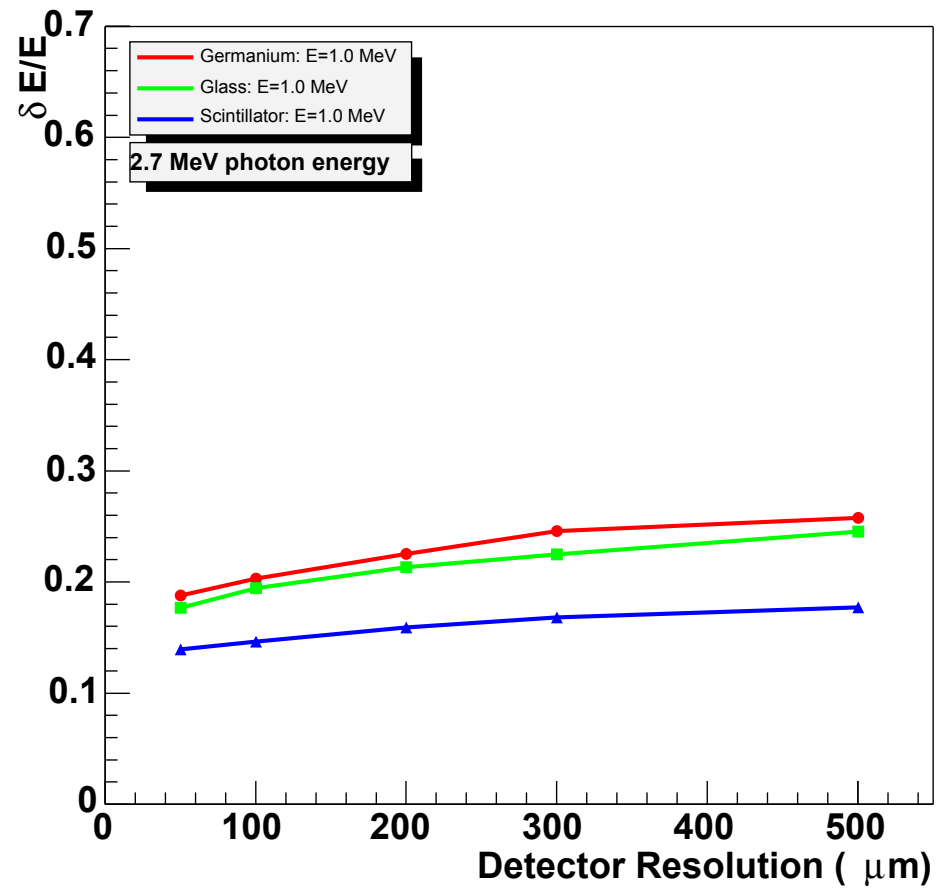

Figure 8: Reconstructed photon energy resolution vs. electron track detection metric for a $2.7 \mathrm{MeV}$ electron track detection metric for germanium, glass, and scintillator detectors. 


\title{
Evaluation of the Effectiveness of Algorithms for Source Identification
}

\author{
Karl Nelson and Padmini Sokkappa
}

\section{Executive summary}

In addition to detecting the presence of radioactive materials, it is also important to be able to determine the nature of the material. In this study, two automated techniques for isotope identification using gamma-ray spectra from $\mathrm{NaI}$ detectors were quickly developed and compared to each other and to GADRAS fittodb, an isotope identification algorithm already in use. GADRAS is an estimation technique based on multiple linear regression while the other two "toy" algorithms evaluated were classifiers, one based on principal component analysis and one based on maximum likelihood estimation. All three techniques require the use of an isotope library. The GADRAS library was used for all three in order to evaluate differences in the capabilities of the algorithms themselves rather than differences in the quality of the libraries.

Each algorithm was evaluated against a verification set and a test set and performance was evaluated using several different metrics. The verification set consisted of random samples selected from the library elements. The test set consisted of a set of inputs representing isotopes and shielding configurations that might be expected in real world situations. The metrics used for evaluation included measures of how well the output of the algorithms matched the input as well as a "threat class" metric which measured the accuracy with which the algorithms placed the inputs into one of three threat classes.

On the verification tests, GADRAS fittodb performed significantly worse than either of the other two algorithms. It produced by far the most false positive and false negative results. The larger number of false positives is expected due to the nature of estimators as opposed to classifiers, but the large number of false negatives is disturbing. Examining the cross correlation matrix between inputs and outputs, we can see, for all of the techniques, that the errors are not evenly distributed throughout the tests. The errors break roughly into two classes, isotopes for which there is another isotope with almost identical spectra, and isotopes whose spectra match a linear combination of the spectra of other isotopes. The second category of errors occurs only in estimators and contributes to the higher error rate of GADRAS.

Results from test set exhibited the same two types of errors seen for the verification set plus a third type of error that occurs when the input to the algorithm is not covered by the span of the library. In this case, the algorithm produces an erroneous result or returns the result "unknown." The algorithms were quite consistent in their performance despite the range of techniques employed. From this, we conclude that problems come primarily from deficiencies in the library and from deficiencies in the resolution of $\mathrm{NaI}$ detectors.

Based on our analysis, we draw a number of conclusions about both GADRAS fittodb and the underlying problem of isotopic identification. First, because our "toy" algorithms performed better than GADRAS on the verification set and similarly to GADRAS on the test set, we conclude that GADRAS could be improved upon with reasonable effort. Second, NaI detectors do not provide sufficient resolution to deal with certain isotopes and mixtures. They may have sufficient resolution to reject some fraction of innocent sources from threats, but, unless considerable improvement is possible, will still result in frequent false alarms. Third, the greatest bulk of future effort should be in building and tuning an isotopic library to provide the best performance for its particular 
application. One must properly survey the set isotopes to be encountered and those of concern and tune the library and the algorithm towards it.

\section{Introduction}

In many radiation detection applications, identification of the nature of the radioactive source present is equally important to its detection. This is particularly true of homeland security applications where we desire to detect radioactive sources that impose a threat and to distinguish them from the vast number innocent radiation sources. In many of these applications, the identification must be done very quickly and only very low false alarm rates are tolerable. Furthermore, the source may be moving past the detector at speeds and distances that results in relatively low counts above background being observed in the detector. The ability to detect sources based on low counts above background has been observed to be significantly higher than the ability to identify such sources.

The purpose of this study was to evaluate the capability to identify radioactive sources or to distinguish between classes of sources in a low count environment using currently available automated techniques. A second purpose was to provide insights as to where future efforts in improving isotope identification capability should be focused.

\section{Survey of existing methods}

An extensive survey was made of unclassified commercial, public domain, and DoE-internal gamma-ray spectrum analysis software. This group of packages encompasses a diverse set of methods and features. This diversity makes it hard to perform one-on-one comparisons. Most automated isotope identification is performed inside units whose software is not available to be run separately, and is optimized for the detector and geometry of the system. Meanwhile, there are a number of codes for expert users that, because they have no automated option, cannot be evaluated. Table 1 provides information about each of the packages identified. The first three are the only packages found to be automated and separable from a commercial system, but the PCA package was never completed, and the ISO ID package is unavailable for general distribution.

Table 1. Catalog of existing isotope ID tools.

\begin{tabular}{|c|c|c|c|}
\hline Technologies & $\begin{array}{l}\text { Point of } \\
\text { Contact }\end{array}$ & Detector & Comments \\
\hline \multicolumn{4}{|l|}{ Automated Tools } \\
\hline $\begin{array}{l}\text { Principle Components } \\
\text { Analysis (PCA) }\end{array}$ & $\begin{array}{l}\text { Tom Gosnell, } \\
\text { LLNL }\end{array}$ & $\begin{array}{l}\text { Ge, NaI, } \\
\text { Plastic }\end{array}$ & $\begin{array}{l}\text { Not yet finished. Need to complete the } \\
\text { template library }\end{array}$ \\
\hline ISO ID & $\begin{array}{l}\text { Mark } \\
\text { Rowland, } \\
\text { LLNL }\end{array}$ & $\mathrm{Ge}$ & Limited only to germanium detectors \\
\hline GADRAS fittodb & $\begin{array}{l}\text { Dean } \\
\text { Mitchell, SNL }\end{array}$ & $\begin{array}{l}\text { Ge, NaI, } \\
\text { Plastic }\end{array}$ & \\
\hline \multicolumn{4}{|l|}{ Tools } \\
\hline $\begin{array}{l}\text { Gamma Vision } \\
\text { Gamma Designer }\end{array}$ & $\begin{array}{l}\text { Mark } \\
\text { Rowland, } \\
\text { LLNL }\end{array}$ & $\begin{array}{l}\text { Ge, NaI, } \\
\text { Plastic }\end{array}$ & $\begin{array}{l}\text { Requires expert to get correct answer. } \\
\text { Can not be automated }\end{array}$ \\
\hline Fit2DB & Dean & Ge, NaI, & Requires expert to get correct answer. \\
\hline
\end{tabular}




\begin{tabular}{|c|c|c|c|}
\hline & $\begin{array}{l}\text { Mitchell, } \\
\text { LANL }\end{array}$ & Plastic & $\begin{array}{l}\text { Can not be automated; template } \\
\text { matching }\end{array}$ \\
\hline Shamon & & $\begin{array}{l}\text { Ge, NaI, } \\
\text { Plastic }\end{array}$ & $\begin{array}{l}\text { Requires expert to get correct answer. } \\
\text { Can not be automated }\end{array}$ \\
\hline \multicolumn{4}{|l|}{ Interpreting Language } \\
\hline $\begin{array}{l}\text { FITEK } \\
\text { (FITEK uses the methodology } \\
\text { of GAMANAL, but is more } \\
\text { general) }\end{array}$ & $\begin{array}{l}\text { Wolfgang } \\
\text { Stoeffl, LLNL }\end{array}$ & $\begin{array}{l}\text { Ge, NaI, } \\
\text { Plastic }\end{array}$ & $\begin{array}{l}\text { Automatic version does not exist but } \\
\text { can possible be created }\end{array}$ \\
\hline \multicolumn{4}{|l|}{ Line Pickers } \\
\hline FRAM & LANL & $\mathrm{Ge}$ & Not Automated - Offered by Canberra \\
\hline MGAU & $\begin{array}{l}\text { Wayne } \\
\text { Ruhter, LLNL }\end{array}$ & $\mathrm{Ge}$ & Automated for $\mathrm{Pu}$ and $\mathrm{U}$ \\
\hline GRPANL & $\begin{array}{l}\text { Wayne } \\
\text { Ruhter, LLNL }\end{array}$ & $\mathrm{Ge}$ & Automated similar spectra \\
\hline GAMANAL & $\begin{array}{l}\text { Ray Gunnink, } \\
\text { LLNL }\end{array}$ & Ge & $\begin{array}{l}\text { Not Automated - Technique and } \\
\text { software used in commercial products }\end{array}$ \\
\hline \multicolumn{4}{|l|}{ Others } \\
\hline \multicolumn{4}{|l|}{ ORIGEN } \\
\hline \multicolumn{4}{|l|}{ IAEA } \\
\hline \multicolumn{4}{|l|}{ Big Fit } \\
\hline \multicolumn{4}{|l|}{ Iso Fit } \\
\hline Gauss & INEEL & $\mathrm{Ge}$ & For reactor products \\
\hline Fit Peak & Commercial & $\mathrm{Ge}$ & \\
\hline \multicolumn{4}{|l|}{ Commercial Products } \\
\hline Sampo & $\begin{array}{l}\text { Stan Prussin } \\
\text { originally }\end{array}$ & $\mathrm{Ge}$ & Software from Finland \\
\hline \multicolumn{4}{|l|}{ Exploreanium } \\
\hline \multicolumn{4}{|l|}{ Radtech } \\
\hline Maestro Gammavision & & $\mathrm{Ge}$ & OTEC - software \\
\hline GammaW SODIGAM & Westmeier & $\mathrm{Ge}, \mathrm{NaI}$ & software \\
\hline Berkeley Nucleonics & & $\mathrm{NaI}$ & $\begin{array}{l}\text { Hardware - they have a different } \\
\text { method for analyzing NaI in software }\end{array}$ \\
\hline Inspector & & $\mathrm{Ge}$ & Canberra - hardware and software \\
\hline Genie 2000 & & & $\begin{array}{l}\text { Canberra - Suite of spectroscopy } \\
\text { packages }\end{array}$ \\
\hline ISOCS & & & Canberra - Calibration Tool \\
\hline LABSOCS & & & Canberra - Calibration Tool \\
\hline MGA & $\begin{array}{l}\text { Wayne } \\
\text { Ruhter, LLNL }\end{array}$ & $\mathrm{Ge}, \mathrm{CZT}$ & $\begin{array}{l}\text { Canberra - Tools that work with } \mathrm{Pu} \\
\text { and its daughters }\end{array}$ \\
\hline $\mathrm{MGA} / \mathrm{U}$ & $\begin{array}{l}\text { Wayne } \\
\text { Ruhter, LLNL }\end{array}$ & $\mathrm{Ge}, \mathrm{CZT}$ & $\begin{array}{l}\text { Canberra - Tools that work with } \mathrm{U} \\
\text { and its daughters }\end{array}$ \\
\hline
\end{tabular}


Eventually it became clear that only a single automated public domain package was available, "fittodb" inside the GADRAS package. After investigating the fittodb mode of operation, it was possible, for comparison's sake, to distinguish other methods of performing isotope ID, namely principal component analysis (PCA) and maximum likelihood (ML). These methods could be quickly implemented in software using the GADRAS spectral libraries, so that the outcome of the GADRAS routines could be compared against at least a strawman ID method. However, the three techniques are not exactly comparable because GADRAS is an estimator, PCA is a classifier and ML is implemented as a hybrid. A complete description of the search for available isotope ID packages and the framework for evaluating them appear in Appendix A3.

\section{Methods selected for evaluation}

\subsection{Common Elements}

Each of the isotopic identification methods investigated share a set of common elements. Each of the algorithms requires a preprocessing step to remove environmental influences, a library of isotopes, and a metric to determine the quality of fit.

The first step in each of the algorithms is to transform the input to a common energy scale. To accomplish this a gain estimator algorithm is employed. It is necessary to perform this transformation because the gain of the system from the physical medium to digitally recorded data is subject to a number of environmental influences. For NaI crystals, the light efficiency is a function of the temperature and pressure the crystals are exposed to. Further, the electronic collection system such as the phototubes have gain that is a function of high voltage that may change with the available supply voltage or temperature. Other technologies such a CZT or Ge have similar effects, though not as extreme as those found in NaI. Rather than attempting to account for each of these influences individually, the net effect is modeled as a total system gain. The locations of calibration peaks either intentionally placed or naturally occurring in the reference background collected prior to a sample spectrum are used to estimate this gain. In the process of estimating the gain, it is common to decimate the data into predefined width bins. The process of decimating improves the signal to noise ratio by combining channels with the same information together. As the resolution of $\mathrm{NaI}$ crystals is a function of the energy of an incoming photon, it is natural to represent the higher energy channels with fewer channels.

A second common element to the evaluated algorithms is each algorithm requires a library of isotopes from which a match will be made. The library consists of a set of isotopes representing potential inputs. If the identification is to be made both on the photo peaks and Compton down scatter, the library must expand to contain isotope-shielding pairs. The library can either be computed statically prior to evaluation or built dynamically. In the case of a dynamic library, all possible shielding of isotopes can be represented. However, use of a dynamic library would mean a very large search space. The alternative, use of a static library, requires the isotope shielding pairs be selected such that either the actual shielding of the observed is close enough to a precomputed library element or can be interpolated from library elements. Much of the work in defining an isotopic algorithm is the careful selection of this library. Use of unsupervised learning algorithms such as clustering were not explored in our investigation. The library must be complete in the sense of spanning all of the likely inputs. Further, it must not contain 
redundant elements that would increase the computational expense and potentially prevent convergence. As the focus of this study is evaluation of known techniques, we will use the library chosen for the fielded GADRAS code for all algorithms evaluated. As we are using the same basic library for all algorithms, our results will be able to distinguish differences in the algorithms where those differences might otherwise be a function of the input library.

The last common element to all of the algorithms is a quality of fit. In order to determine the output of each of the algorithms, a metric will be defined. This metric can be based on the underlying Poisson statistics of radiation or heuristic based on observed performance of the algorithm as the parameters are tuned. For a single algorithm like multiple linear regression, the outcome of the identification can vary wildly based on the choice of metrics. . How the metric is applied varies depending on the algorithm. In the case of a classifier, the metric is used for sorting the results of each of the evaluated library elements. In the case of estimator, the metric is applied at each iteration and directs the algorithm toward convergence. For our study, we had three different metrics. The cosine of the angle between vectors was used in the PCA algorithm. GADRAS employed a chi-squared estimator of variance. Finally, the group estimator used maximum likelihood of model from observation based on Poisson statistics.

\subsection{Approaches to Isotopic Identification}

For NaI detectors, we can divide the approaches to isotopic identification into two general categories of algorithms, classifiers and estimators. These categories do not cover techniques such as peak finders and other algorithms that are more applicable to high-resolution detectors. Such methods are often heuristics using both classification and estimation techniques.

\subsubsection{Classifier}

A classifier is an algorithm that labels an unknown sample as belonging to a set. The set can represent anything from broad categories such as threatening versus unthreatening isotopes to specific isotopic species and shielding. Loosely speaking, classifiers segment a high dimensional space of features into classes. These algorithms cover a broad class in both supervised and unsupervised learning. Examples include clustering, nearest neighbor, neutral networks, and genetic algorithms. For our example of a classifier, we will employ a nearest neighbor algorithm that is reasonable representative of the body of techniques as a whole.

A nearest neighbor algorithm does not have an iterative stage and simply evaluates a distance measure from the sample to the library elements. Nearest neighbor does not consider combinations of isotopes. Further, it may conclude the sample is outside of the library if the nearest neighbor is greater than some specified distance from the sample. For our application, we wish to identify the isotope (though not necessarily the specific shielding) to which the unknown sample belongs. We also wish to classify the sample into a set of threat classes based on the isotope identified. Our classifier will require a library with dense coverage of isotope shielding, as it cannot use linear combinations to simulate shielding configurations not represented in the library. Further, if the unknown sample can be a mixture, the library must contain a representative set of those mixtures including different relative strengths. These larger library requirements can overcome the advantage of avoiding iterative solvers. 


\subsubsection{Estimator}

Estimators approach the problem of identification by estimating the most likely weights from a fixed set of vectors that best represent the sample. An estimator seeks to ask what linear combination of library elements could have produced this output. The identification result is library elements that contribute significantly to the solution.

Estimators are generally iterative unless the problem can be reduced to a linear estimation that can be completed in one step. Estimators also can have a vast range of problems including nonuniqueness of the solution, biases from noise, local minima and failure to converge. Nonuniqueness of the solution occurs when there is a linear dependence in the library set. Local minima and failure to converge are both problems resulting from incorrectly set convergence parameters or a misbehaving gradient surface. Dealing with these problems requires a number of techniques such as increasing constraints to force unique solutions and imposing bounds to limit the size of the steps between iterations. It may not be possible to predict the number of steps required for convergence nor whether convergence will ever occur for iterative techniques. Thus, they may be slower than non-iterative classifiers.

Given the number of problems an estimator would seem to impose, it may not be immediately clear what benefits an estimator could provide. In practice, an estimator may be faster than a classifier if the library required to represent the expected range of inputs for the estimator is considerably smaller for the estimator. Further, an estimator better represents the real world where a variety of isotopes may be seen in combination either by chance or design.

\subsection{Description of methods selected for evaluation}

As GADRAS fittodb was the only automated public domain algorithm available for study, we invested a small amount of time to develop alternative algorithms based on the recommendations of others in the field. These "toy" algorithms do not represent fully formed isotopic identifiers, as they do not contain nearly the knowledge nor development time invested in GADRAS. However, as we will discuss later they allow insight into where and why GADRAS fittodb failed on certain test sets. We will now describe the methods employed by GADRAS and our "toy" algorithms.

\subsubsection{GADRAS (fittodb)}

GADRAS is a collection of tools for estimating, viewing and evaluating the output of a variety of different detector technologies including NaI, CZT, and Ge. This tool set contains three different isotopic identification algorithms, including single regressing, multiple regression and a heuristic called fittodb. Of these algorithms, only fittodb has been transformed into a fully automated identification tool called calana. The tool was automated for use in a portal monitor that uses a $2 \times 4 \times 16 \mathrm{NaI}$ crystal. The physical characteristics of the detector are built into the binary code. There would be little cost to recalibrate the algorithm to other systems; however, we had neither the source code nor sufficient knowledge of the required parameters to adjust at the start of the study. Thus all of our evaluations were done using the model that was precompiled into the binary.

As explained previously, the first step in the algorithm is to compute the gain and decimate the channels. To perform this task, fittodb estimates the location of all peaks in the background spectrum. It then matches them to a known input and computes the system gain. The two 
predefined input sets it uses are ${ }^{232}$ Th plus natural ${ }^{40} \mathrm{~K}$ or ${ }^{40} \mathrm{~K}$ plus ${ }^{242} \mathrm{Am}$. For our study, we selected the default with is to include ${ }^{232} \mathrm{Th}$ in each of the spectra. The result of this portion of the algorithm was written to a calibration file. As part of our evaluation, we verified that the gain computed matched the known gain of our inputs. For all cases observed, the gain appeared to be within $1 \%$ of the actual value except when the total count rate of the background was low at which point the algorithm failed. In investigating this problem, we found that presenting the same data spectrum with a shorter live time allowed the gain algorithm to succeed. We concluded that there was a minimum acceptable background count rate for the deployed system. To account for this, we scale our count rate to keep it inside of the acceptable range.

The fittodb algorithm is, at heart, an estimator using a multiple linear regression algorithm. It performs its analysis by reducing the spectra to 62 channels of information. This corresponds to the energy resolution of the input library. The multiple linear regression is performed to estimate the best match set of library elements. Quality of fit is based on a custom chi squared estimator. Factors used to form the chi squared fit for each energy channel include the Poisson variations of the input and the measured background, estimated uncertainty of the gain estimator, and down scatter based on the observed counts with energy greater than the channel energy. The library elements will be chosen based on the isotopes expected to be observed at the detector. For our evaluation, 143 isotope/shielding pairs were employed covering a set of observed isotopes seen in previous gate deployments. These 143 isotope/shielding pairs are divided into four subgroups: natural, industrial, medical and SNM.

A multiple linear regression algorithm was modified with heuristics to detect and remove soft solutions by dropping elements from the library. Solutions also combine library elements to form a single representative shielding for a given isotope. However, the methods used to accomplish these two tasks have not been documented and we did not attempt to extract this algorithm form the surrounding code. Also the multiple linear regression is held to positive constraints for the background, industrial, SNM, and medical sources. Natural sources are not constrained to represent the possibility of partial shielding of the background by a source vehicle. The method by which positive constraints have been applied was not documented.

Estimation of library members is performed in a five-step process. Each step is identical save for the library inputs used by the estimator. The first solution is performed using the full input library and thus is highly under constrained. Three additional sets of inputs consisting of one subgroup (medical, industrial, SNM) plus natural isotopes are solved. With these four solutions a final isotopic solution set is formed by weighting the solutions from each set by the chi squared figure of merit computed at that stage. Running it through the multiple linear regression algorithm one last time refines this composite solution.

Results from the algorithms consist of the list of isotopes produced by the refined composite solution with cut off selected on a per isotope basis. Thus insignificant contributors may not appear on the output list even if they were in the composite solution. Each isotope is given a weight of certainty consisting of low, fair and high. The exact meaning of these terms is not clearly defined but is loosely given as $50 \%, 75 \%$ and $90 \%$ percent certainty. Additionally, the goodness of fit for the composite output (including unreported isotopes) and the goodness of fit from the SNM plus natural solution are given. For the automated isotopic identification, fitness is 
reported as the reduced chi squared value, which is the chi squared value divided by the degrees of freedom. For the non-automated fittodb algorithm, the chi squared is reported without dividing by the degrees of freedom. The algorithm gives no concept of "unknown" or "outside of library." However, roughly speaking, a reduced chi squared greater than 3 would indicate a failure to converge and thus no confidence in the solution, even though the solution may have a high certainty of a specific isotope. This output state is highly confusing and we will disregard it as all inputs presented in our study are within a reasonable span of the library.

Several problems were encountered during the evaluation of this algorithm. The author of GADRAS provided numerous versions of the algorithm; however, none contained a revision number or other version defining information. As different versions of the algorithm seemed to have been hard coded for different detectors and there was no way of enquiring as to what detector was compiled into a particular version, we requested a detector model from the author and then evaluated each version against the supplied detector model. The version that performed best was not the latest and lacked neutron capability. Fittodb source code in Microsoft Visual Fortran was provided but the source code did not appear to match any of the automated versions we had. Additionally, binary codes provided were compiled for the DOS 16 virtual machine that is not stable on any of the machines we had available. After some undetermined number of runs, the result of the next execution will simply fail without reason. Those bad runs may have been inadvertently counted in the total, as there were no outward signs as to what had failed. Fittodb is currently the subject of licensing discussions and thus information pertaining to specifics of the algorithms is not available.

\subsubsection{PCA - Principle Component Analysis}

Our second evaluation algorithm was the principle component analysis. In the principle component analysis, the library is extracted from a larger basis set that spans the known input set by selecting the principle components. Principle components are the eigenvectors corresponding to the largest eigenvalues that represent the components with the greatest diversity. Reducing the input to these principle components should reduce noise that is not in the direction of the principle components. This would improve the signal to noise ratio. However, doing so comes at the cost that the differences between library elements may be reduced with the number of principle components. If too few principle components are used, library elements may become inseparable.

The algorithm functions exclusively as a classifier by taking the unknown input, transforming it along the principle components and then comparing it to each element in the library. As this is a classifier, it cannot handle a mixture nor can it form unexpected shielding configurations by combining two library elements. Thus it requires a larger library set than that used by an estimator such as fittodb. To account for this and to allow the algorithm to choose its own basis set without being restricted to the 62 channels selected by GADRAS, we increase the sampling of the library from 62 to 128 . From this larger library 72 principle components were selected representing over $99 \%$ of the diversity of the original library. Further, we increase the number of isotope/shielding pairs from 143 to over 500, as the original fittodb library was too sparse for the PCA algorithm. It is not clear whether this was the correct choice for the number of principle components as too many basis vectors may simple cause the algorithm to over represent noise 
with no benefit to matching. Further evaluation would be required to optimize the number of principle components to fully represent the input and minimize the representation of noise.

The principle component algorithm does not specify a particular fitness function for use and there are a number of potential choices. The simplest would be simply to compute the Mahalanobis distance from each library element to the sample. However, this method would imply that the strength of the sample was known in advance. This limitation can be avoided by leaving the signal strength as a free parameter and estimate it as a nuisance parameter using maximum likelihood. Doing so results in a fixed offset based in the sample alone and the dot product of the library element and the sample. We can drop the fixed offset, as it is the same for all evaluations and simply use the dot product of the sample and a library element if the library element has been fixed to have a norm of one. This method will be called a shape-based fitness. Its output is in effect the cosine of the angle between the sample and each library element. Thus this algorithm is a nearest neighbor where the distance metric is the angle between the sample and library element.

As this is a one element fit to a library element without a background, evaluation of fitness can be performed only on background-subtracted samples. As this fixes the background to a known level it may not be desirable. Using the fitness algorithm, we can sort the library elements based on their fit to the sample. During development it was found that frequently the correct element appeared somewhere near the top of the ranking, but was not the best-ranked element. The correct answer failed to appear in the top 10 less than one in 1000 times. We surmised that this behavior might have been the result of the fixed background subtraction performed earlier. To improve the results of the algorithm, we followed the ranking process by a second, more computationally expensive, operation where both the background and the sample were of unknown strength. This step was done on the full 128 channel spectra so that positive constraints could be imposed. The resulting composite algorithm was used in our evaluation.

In addition to the ranking and the selection of the best fit, the algorithm also computed the probability of producing the sample based on background alone. Unless producing a sample from the background plus the library element was significantly more probable that producing the sample from background alone it was reported as "nothing," representing the fact that insufficient counts result in poor quality of identification. Further, if the absolute probability of producing the output from the best library element was too low it was labeled an unknown sample. As an additional output, the algorithm indicated the best matched shielding for the sample. As fittodb did not have such an output, we did not use this output for scoring even though the shielding would be of aid in determining the threat class.

\subsubsection{Maximum Likelihood Estimator}

To present an alternative to the PCA classifier, we developed an algorithm based on a similar functionality to GADRAS fittodb. The algorithm finds the most likely model for which a Poisson random draw would yield the given sample. We define the probability of getting a sample from a model as

$$
\Lambda=P(O \mid \theta)=\prod_{i} \frac{e^{\lambda_{i}} \lambda_{i}^{c_{i}}}{c_{i} !}
$$


where $c_{i}$ is the counts observed in channel $i$ and $\lambda_{i}$ is the expected counts in model $\theta . \lambda_{i}$ is given by

$$
\lambda_{i}=\sum_{j} a_{j} e_{i j}
$$

where $a_{j}$ is the weight for library element $j$, and $e_{i j}$ is the expected mean for channel $i$, library element $j$.

To convert this to an iterative algorithm, we take the log of the probability of producing the sample and take the derivative to find the maximum. This yields the equation that the observed divided by the expected should equal 1 . As this is the same equation, save for the weighting function per channel, as we would find in multiple linear regression, we can conclude that these algorithms are equivalent for this application. We know the weight of each channel should be based on the expected variance of the channel. However, the expected variance is a function of the model. Thus, we need to approximate it by the square root of the observed value. This should be a good match, as the expected value of the model at convergence should equal the observation.

To extend this to an iterative algorithm, we simply apply an iterative linear solver to the described equations. Unfortunately, this will not yield the expected result. As the number of library elements exceeds the number of constraints, the problem is over specified and there are an infinite number of solutions. Further, the solutions are mostly non-physical as many have both positive and negative contributions. To correct this problem, we increased the constraints with the aid of a logarithmic barrier function. A logarithmic barrier adds to the constraining equation the $\log$ of a set of parameters times a set of weighting functions. The $\log$ of $a_{j}$ increases as $1 / a_{j}$. As the estimate approaches zero, this prevents the weight on any isotope from being negative. As a library element weight is increased, the log of that weight flattens out. Thus, the derivative of its contribution to the iterative algorithm is made insignificant. The weighting function, a constant in this case, is selected such that the total contribution of each library element is insignificant to the estimation problem when it reaches its minimum value. Thus we have increased the number of constraints on the problem from 62, the number of channels, to over 143 the number of library elements. The exact number of constraints depends on how many library elements are participating in a positive fashion. However, adding this nonlinear constraint increases the complexity of the iterative solution from a multilinear regression, to a nonlinear Gauss-Newton algorithm.

The increase in constraints should be sufficient to ensure a unique solution if the library elements do not have linear dependence when combined with positive weighting functions. However, this point proved not to be true. The background that is appended to the library can be formed from the library elements with positive weights. Thus, the algorithm driven is by noise in the sample and will converge to a solution in which both the desired isotopic decomposition of the excess in the sample and the isotopic decomposition of the background are present. This leads to an excessive number of false positives in our verification of the algorithm. We reduced, but could not alleviate, this problem by adding the constraint that the background should be at or near the expected level based on the previous background. 
This problem is not unprecedented. The GADRAS fittodb algorithm was noted to have a similar problem. The solution implemented in that code was a heuristic to identify components that created this condition and drop them from the regression. We attempted similar solutions by dropping the least contributing isotope until the solution settled, but this did not seem to improve the overall performance. Much additional work beyond the scope of this project would be required to address this issue.

\subsubsection{Maximum Likelihood of Subgroups (MLG)}

Although the unconstrained maximum likelihood estimator created excessive false positives, this did not mean that it was not possible to use it as an isotopic identification method. As the chief problem with the method was that multiple solutions could be found if all isotopes were presented simultaneously, one solution to address this was to present only a subgroup of the library at a time. We chose subgroups representing a single isotope. By doing this we are running the estimator as a classifier. It is still estimating the most likely mixture of shielding and strengths amongst the library elements but it is constrained to do so only one isotope at a time with the addition of the background.

As no single element could decompose the background by itself, this virtually eliminated the false positive in our testing. However, it does constrain the output significantly in ways that could damage its real world performance. As many isotopes are seen in combination with both the environment and other sources, the algorithm will need to be instructed about what mixture sets it can try. If a mixture is presented that it was not told to try, the best result it can produce is "unknown."

Like our PCA algorithm, the MLG algorithm produces a list of the isotopes sorted by their likelihood. Unlike the PCA, sorting of the list requires the additional step of estimating the likelihood with a varying number of freedoms.

Further, MLG was given thresholds for returning "unknown" and "insufficient counts." Unfortunately, the insufficient count mechanism was not functioning properly at the time of our evaluation. In computing the minimum, the stopping conditions do not require convergence to the absolute minimum but rather within some predefined distance from that minimum. If a sample consisting of background and an isotope were evaluated, there existed the possibility that the iterations yielded a solution containing only background with a higher likelihood than had been calculated in the iteration of background alone. Thus the algorithm was incorrectly labeling the sample as background plus an insignificant quantity of the isotope. Adding a threshold of significance to the isotope quantity solves this problem, but this solution was discovered during the preparation of this report. Further, this would also be required in the case of mixture evaluation as it would be incorrect to label the sample as a mixture if it only contained one of the isotopes.

\subsubsection{Comparison of the characteristics of each method}

We can summarize the differences between algorithms in Table 2 . 
Table 2 Summary of Algorithms

\begin{tabular}{|l|c|c|c|}
\hline & GADRAS & PCA & MLG \\
\hline Type & Estimator & Classifier & Hybrid \\
\hline Algorithm & MLR & PCA/ML & Newton ML/LBF \\
\hline Metric & Chi Squared & Poisson Likelihood & AIC \\
\hline Groups/Trials & 5 & 528 & 25 \\
\hline Isotopic Output & Composition & 1 Library Element & $\begin{array}{c}\text { 1 Isotope } \\
\text { Group }\end{array}$ \\
\hline Allows No Detection & Yes & Yes & No (broken) \\
\hline Allows Unknown & No & Yes & Yes \\
\hline Additional Output & SNM probability & Sorted Library List & $\begin{array}{c}\text { Fitness of each } \\
\text { Isotope Group }\end{array}$ \\
\hline
\end{tabular}

\section{Testing Procedures}

\subsection{Input Sets}

As part of our evaluation, we present an algorithm with an input and observe the resulting output. However, the span of the input set and the interpretation changes with each stage of evaluation. We can break these input sets into three groups.

\subsubsection{Training Set}

The training set consists of a set of inputs that may or may not be in the library and is used to tune input parameters and develop the algorithm. They represent the inputs that an expert observer has selected as representative of all valid inputs. For classifiers with the ability to learn, these are the presented with the correct labels, allowing the algorithm to learn the differences between elements. However, for our algorithms there is no learning process as the library is essentially a fixed representation. We did use the training set to revise the parameters such that we could determine reasonable limits for what should constitute an unknown sample and a background sample. For the PCA and MLG algorithms, a training set consisting of synthetic spectra from ${ }^{133} \mathrm{Ba}$ at different source strengths was used. This isotope was selected because of its ambiguity with ${ }^{131} \mathrm{I}$. As we did not develop the GADRAS code, it is not known what the range of the training set employed during its development was.

\subsubsection{Verification Set}

The verification set consists of a set of unlabeled training elements. For our evaluation this was made up by random samples of varying source intensity selected from the library elements. The use of a verification set attempts to assess how well the algorithm can distinguish samples that are in its known library. Testing with a verification set can reveal important insight into the underlying problem as linear dependence and inseparability between classes can become apparent. It should be noted that good performance of an algorithm on the verification set does not necessarily translate to good real world performance. 


\subsubsection{Test Set}

The test set consists of a set of inputs that the algorithm was neither trained on nor has in its library set. These inputs are presented in an unlabeled fashion and attempt to represent real world inputs. For our test set, we produced synthetic spectra for a $2 \times 4 \times 16 \mathrm{NaI}$ detector using both GADRAS and MCNP. The spectra represent inputs with shielding configurations between or outside the set of library elements. Some test elements also contain some trace levels of contaminants such as potash.

\subsection{Test Methods}

Creation of random samples was performed in a straightforward fashion. For each channel of the sample an expected mean was computed and a random Poisson sample was drawn from that mean. The background was drawn from a MCNP created dirt ball model and had additional ${ }^{232} \mathrm{Th}$ isotope added to aid in autocalibration. Background samples were drawn to have a total live time of 5 minutes. The sample was drawn to have a live time of 1 second, representative of samples taken from a vehicle traveling on a road or a package moving rapidly on a conveyer belt. None of the algorithms required either list mode or spatial distribution data.

The verification test presented 100 random samples for each isotope/shielding pair in the library. Samples for the verification testing were drawn from an expected mean of 334 counts from the dirt ball model, 65 counts from the ${ }^{232} \mathrm{Th}$ model, and 300 counts from the desired source.

Evaluation testing using the test set was drawn with a background level of 334 counts of background and 65 counts of ${ }^{232} \mathrm{Th}$. Samples were given an expected count of 50, 100, 200, 300, 500,750 , and 1000 counts. Thus the signal to noise ratio ranged from 2.5 to 50 . Signal to noise ratios above 50 are outside our range of interest as we expect that other algorithms such as peak finders would perform better.

\subsection{Scoring systems}

In selecting a scoring system to judge algorithms being tested, a number of factors were considered. Above all is the overall use of the algorithm. If the algorithm was to be used to alarm on certain classes of sources, then mistakes between two elements within the same class would not be penalized. However, if the algorithm was to be used to verify that the contents of a box are as indicated on a shipping document, then results would need to be scored based on each individual isotope identified. To address these divergent needs, we scored the algorithms' performance using a number of metrics. The most appropriate metric can be selected based on the algorithm's intended use. For each evaluation 100 trials were performed.

\subsubsection{Input verses Output}

In this basic scoring system, we check whether the output matches the input. To score a set, we count up the number of times the output matches the input exactly. Partially correct outputs, such as producing a mixture with one of the isotopes matching the input, are not counted. This type of scoring system tends to favor a classifier over an estimator because a classifier, by definition, is producing only one output. The degree to which the estimator erroneously produced mixtures 
will be represented in this score. This scoring system is not appropriate for inputs of mixtures as the only appropriate response a classifier can provide for a mixture is "unknown".

\subsubsection{Intersection verses Union}

Alternatively, we provide a score that sacrifices the requirement of absolute accuracy with the requirement that the output include the input. However, we do not want to give a high score to an algorithm that simply returns all elements. Thus, we calculate a score based on the number of isotopes in the intersection between the input and the output divided by the number of isotopes in the union of the input and the output. A score of one would result only from a perfect match between the input and the output. A zero would result if the input and output did not share any isotopes. The score for a partial match would reflect both the extra isotopes included in the output and the isotopes that were missing from the output. Under this scoring system, a classifier could at best score a 0.5 in the case of a mixture as it could only name one of the isotopic components at a time. An estimator could do better provided that it does not include too many extraneous isotopes in the output.

\subsubsection{Class based (Alarm Levels)}

Finally, to represent the classic alarm based usage, we assign each member of the library to a threat class. Short-lived medical and natural isotopes are placed in the lowest threat class, as they could not represent a threat unless introduced directly into a body such as by poisoning a water supply. Industrial and long-lived medical sources are potential RDD threats and thus assigned to a middle threat category. Finally, SNM and potential bombs are placed in the highest threat category.

Under this scoring system, errors in isotope identification are penalized only if they result in an incorrect threat class. When the output is a mixture, we assign it the highest threat class represented in the mixture. Thus an estimator that erroneously produces extra isotopes would only suffer if those extra isotopes belong to a higher threat class. We tabulated the results for each isotope such that we could observe both promotions and demotions in threat class.

\subsubsection{Cross Correlation Matrix}

Although it's not a scoring system in itself, we also produced a cross correlation matrix between inputs and resulting outputs. This correlation matrix allows us to examine the reasons for poor scores and identify the nature of the problems. Each time an isotope was observed in the result, the corresponding matrix element for the input and that isotope were marked. In the case of an estimator that produces multiple outputs, the total number of entries in the correlation matrix may be significantly larger that the number of trials. As a classifier produces only one isotope per trial, the correlation matrix contains all of the information needed to assess any of the previously described scoring functions. This is not the case for an estimator as an additional matrix showing the frequency that one output appears with another would be required.

\subsection{Limitations of study}

We should note a number of limitations in this system study. There were relatively few mixtures presented to the algorithms. Potash, uranium, and plutonium all have trace amounts of different 
isotopes. Thus our testing conditions would favor a classifier over an estimator as the classifier is choosing from a smaller dimensional space. A more thorough study would identify mixtures commonly seen under the desired field conditions and test the algorithms against it. Further, a classifier would be required to expand its library automatically based on its analysis in order to determine the mixtures as well as an estimator does.

The test cases may not include the full set of isotopes of interest, as we could not find a definitive threat document to target our selection. For example, potential RDDs such as ${ }^{60} \mathrm{Co}$ were not covered. However, as our inputs do represent a reasonable subset of common isotopes seen in the field covering both high and low energy components, we feel that our results are reasonably probative.

Only one background model was used for all testing purposes. It was taken from a MCNP dirt ball model in a $2 \times 4 \times 16$ " NaI crystal. Smaller crystals would be expected to have a different background and correspondingly different efficiencies for different energy gamma rays. Also, the resolution of our detector was limited to that of the standard crystal used in Sandia roadside detectors. A smaller crystal use in a handheld device would have better resolution and thus should be able to resolve peaks better. Our reference crystal has a resolution of about $8 \%$ at $662 \mathrm{keV}$, while the ideal NaI would have a resolution closer to $5 \%$. However, in examining synthetic spectra produced by GADRAS for different sizes and resolutions of detectors, we feel that a resolution of better than $2 \%$ would be required to properly separate the inputs that we found to cause significant problems in our tests. The relatively modest improvement found in a handheld detector would probably not significantly change the results.

Further, the studies performed were aimed specifically at evaluation of a system used as a driveby portal monitor, as that was the nature of the GADRAS fittodb tool. As such, the range of counts per sample used in the evaluation and the length of the background sample time were confined to be within ranges found in that application. We assumed a low count sample taken in a short amount of time. The background is assumed to be isotropic such that the total isotopic contributions from the entire view are the same. The vehicle being screened does not block such a large fraction of the field of view as to change the total background contribution. Other applications such as package screening may have parameters outside of our test range. For vehicle searches and other applications where the length of the measurement period can be extended, other algorithms, such as peak finders, that require high-count data might be a better choice. Further, we did not evaluate the case where severe depression of the background or an unknown background occurs. We also did not evaluate the case where the source was present in the background, aside from the case of natural products containing ${ }^{40} \mathrm{~K}$. Such situations would occur when the source is present in the area. A measurement of background would be taken a distance away from the source but would still contain contributions from the source. 


\section{Evaluation results}

\subsection{Verification results}

As each of the isotopes in the verification set is directly represented in the library, we expected reasonably strong performance from each of the algorithms. The results of the verification test are given in Table 3.

Table 3 Verification summary.

\begin{tabular}{|l|r|r|r|}
\hline & $\begin{array}{c}\text { GADRAS } \\
\text { fittodb }\end{array}$ & \multicolumn{1}{|c|}{ PCA } & ML group \\
\hline No Detection & 1093 & 56 & 0 \\
\hline Total Correct & 10159 & 12851 & 13234 \\
\hline Actual trials & 14300 & 14300 & 14300 \\
\hline False Negative & 4141 & 1449 & 1066 \\
\hline False Positive & 4932 & 1393 & 903 \\
\hline
\end{tabular}

Surprisingly, the GADRAS fittodb algorithm performed significantly worse than either of the two "toy" algorithms for this verification set. Fittodb produced by far the most false positive and false negative results and correctly matched the least number of isotopes. Some of this behavior is expected as an estimator is using a larger search space and can produce multiple outputs. However, the large false negative score is extremely disturbing, especially given that it produced $13 \%$ more outputs than either of the classifiers.

Examining the individual scores in the cross correlation matrix presented in Appendix A4, we can see that the errors are not distributed evenly throughout the tests. A summary of the false positive scores is shown in Table 4. The table shows the isotope that was falsely being identified in the samples, which isotopes produced the majority of those errors, and the number of occurrences of these errors in each algorithm.

Table 4 Verification false positive scores.

\begin{tabular}{|c|l|r|r|r|}
\hline $\begin{array}{l}\text { False } \\
\text { Positive }\end{array}$ & $\begin{array}{l}\text { GAducers } \\
\text { Fittodb }\end{array}$ & PCA & ML Group \\
\hline${ }^{40} \mathrm{~K}$ & ${ }^{235} \mathrm{U},{ }^{152} \mathrm{Eu}$ & 178 & 6 & 6 \\
\hline${ }^{226} \mathrm{Ra}$ & ${ }^{60} \mathrm{Co},{ }^{88} \mathrm{Y},{ }^{152} \mathrm{Eu}$ & 493 & 54 & 60 \\
\hline${ }^{232} \mathrm{Th}$ & ${ }^{228} \mathrm{Th},{ }^{232} \mathrm{U}$ & 809 & 69 & 80 \\
\hline${ }^{228} \mathrm{Th}$ & ${ }^{232} \mathrm{Th}$ & 26 & 152 & 80 \\
\hline${ }^{232} \mathrm{U}$ & ${ }^{232} \mathrm{Th},{ }^{228 \mathrm{Th}}$ & 0 & 47 & 12 \\
\hline${ }^{60} \mathrm{Co}$ & ${ }^{152} \mathrm{Eu}$ & 4 & 36 & 34 \\
\hline${ }^{133} \mathrm{Ba}$ & ${ }^{239} \mathrm{Pu},{ }^{131} \mathrm{I}$ & 333 & 70 & 89 \\
\hline${ }^{137} \mathrm{Cs}$ & ${ }^{166 m} \mathrm{Ho}$ & 76 & 11 & 3 \\
\hline${ }^{54} \mathrm{Mn}$ & ${ }^{238} \mathrm{U},{ }^{166 m} \mathrm{Ho}$ & 355 & 25 & 7 \\
\hline${ }^{57} \mathrm{Co}$ & ${ }^{201} \mathrm{Tl},{ }^{99 \mathrm{~m}} \mathrm{Tc}$ & 39 & 0 & 0 \\
\hline${ }^{139} \mathrm{Ce}$ & ${ }^{201} \mathrm{Tl}$ & 548 & 239 & 77 \\
\hline${ }^{252} \mathrm{Cf}$ & ${ }^{60} \mathrm{Co},{ }^{238} \mathrm{U}$ & 292 & 73 & 108 \\
\hline
\end{tabular}




\begin{tabular}{|c|c|r|r|r|}
\hline${ }^{238} \mathrm{U}$ & ${ }^{152} \mathrm{Eu}$ & 36 & 49 & 58 \\
\hline${ }^{235} \mathrm{U}$ & ${ }^{67} \mathrm{Ga}$ & 51 & 2 & 1 \\
\hline${ }^{239} \mathrm{Pu}$ & ${ }^{131} \mathrm{I},{ }^{192} \mathrm{Ir}$ & 63 & 116 & 34 \\
\hline${ }^{237} \mathrm{~Np}$ & ${ }^{192} \mathrm{Ir},{ }^{133} \mathrm{Ba},{ }^{131} \mathrm{I}$ & 600 & 68 & 12 \\
\hline${ }^{131} \mathrm{I}$ & ${ }^{133} \mathrm{Ba},{ }^{239} \mathrm{Pu}$ & 197 & 61 & 32 \\
\hline${ }^{99 \mathrm{~m}} \mathrm{Tc}$ & ${ }^{201} \mathrm{Tl}$ & 94 & 14 & 2 \\
\hline${ }^{67} \mathrm{Ga}$ & ${ }^{239} \mathrm{Pu},{ }^{192} \mathrm{Ir},{ }^{133} \mathrm{Ba}$ & 613 & 42 & 27 \\
\hline${ }^{201} \mathrm{Tl}$ & ${ }^{139} \mathrm{Ce}$ & 112 & 87 & 69 \\
\hline
\end{tabular}

As expected, the incorrectly identified isotopes corresponded to an isotope that produces similar spectra. However, there is additional information we can gain by examining the correlation matrix. The error matrix breaks roughly into two classes of errors, isotopes that have another isotope that matches and isotopes that match a combination of other isotopes. Those that directly match another isotope in the library are essentially inseparable such as a shielding of ${ }^{201} \mathrm{Tl}$ and a different shielding of ${ }^{139} \mathrm{Ce}$ as shown in Figure 1.

Figure $1201 \mathrm{Tl}\{26,10\}$ verses $139 \mathrm{Ce}\{13,10\}$

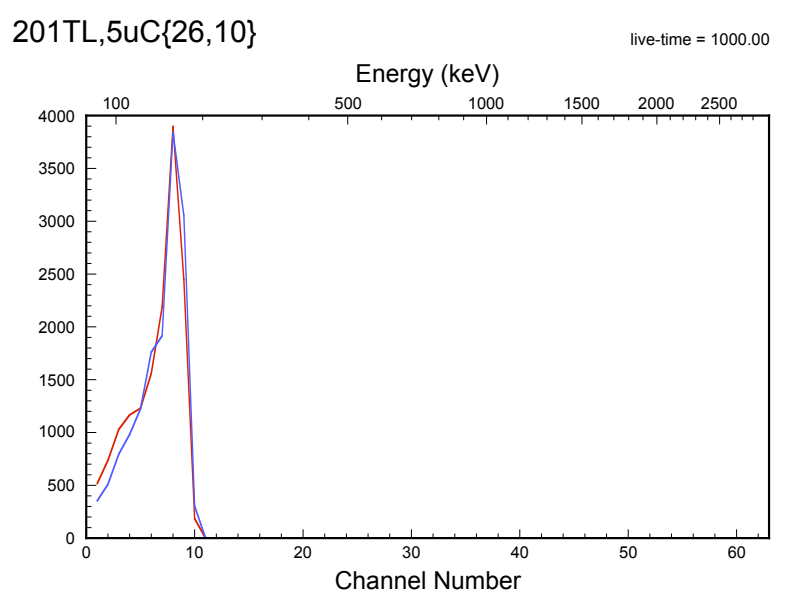

The second category of errors occurred only in estimators. In an estimator, a spectrum from an isotope could be formed from a mixture of other isotopes. Such is the case of I131,10UC $\{10,30\}$ for which GADRAS produced 277 isotope labels for 100 trials or 2.7 labels per sample. One isotope in error that was produced was ${ }^{133} \mathrm{Ba}$ that is a near match for ${ }^{131} \mathrm{I}$. However, the others produced included ${ }^{239} \mathrm{Pu},{ }^{239} \mathrm{~Np}$, and ${ }^{67} \mathrm{Ga}$. Such a combination of isotopes would be exceedingly unlikely to be found in nature. The classifiers on the other hand classified only 5 of these samples as ${ }^{239} \mathrm{Pu}$ and labeled none as ${ }^{239} \mathrm{~Np}$ or ${ }^{67} \mathrm{Ga}$. This behavior is typical of an estimator when given two or more linear solutions; it will select an arbitrary mixture of those linear solutions as its output. One can view this behavior as being the results of a null space in the input problem, or equivalently a linear dependence in the library. 


\subsection{Test set results}

\subsubsection{Common errors}

From our verification set, we identified two classes of errors inherent in the library. Further, the test set may contain items that are not possible to cover by the span of the library. Thus to interpret the test set results we will label the results by an error type. Error types have common characteristics in the results.

1. Inseparable (IS) - two or more isotopes in the library matched the sample, but the results did not produce a mixture in the output but rather the algorithm chose one or the other.

2. Linear dependence (LD) - two or more solutions are equivalent and thus the output is a random mixture. Errors of this type produced two or more labels for the majority of the trials.

3. Unknown (UNK) - there was no corresponding isotope in the library, and thus the algorithms merely chose the closest appropriate item. Errors of this type have poor quality of fit.

Inseparable and Linear Dependence both result from problems in the structure of the library though the mechanism may be different. Errors of type Unknown may be addressed by increasing the size of the library.

\subsubsection{Summary of Errors}

From the results of the test set, we produced Table 5 summarizing the errors that the algorithms made and indicating which algorithm produced the best results for each isotope. We indicate the performance of each algorithm by the number of counts required to produce $90 \%$ correct results on the basis of inclusion. For isotopes for which the algorithm did not produce a $90 \%$ result or the $90 \%$ result was achieved at an unreasonable signal to noise ratio, the error type and most frequent misidentification is indicated. The "Best" algorithm is the one that produced the most correct outputs at the lowest signal to noise ratio.

Table 5 Summary of Errors

\begin{tabular}{|c|c|c|c|c|}
\hline Isotope & GADRAS & PCA & MLG & Best \\
\hline${ }^{99 m} \mathrm{Tc}$ & 100 & 100 & 100 & PCA \\
\hline${ }^{131} \mathrm{I}$ & $1000 \mathrm{IS}^{133} \mathrm{Ba}$ & $1000 \mathrm{IS}^{133} \mathrm{Ba}$ & $1000 \mathrm{IS}^{133} \mathrm{Ba}$ & PCA \\
\hline${ }^{201} \mathrm{Tl}$ & $\mathrm{LD}^{139} \mathrm{Ce}$ & 300 & 200 & MLG \\
\hline${ }^{67} \mathrm{Ga}$ & 300 & $\mathrm{IS}^{237} \mathrm{~Np} 32 \%$ & 300 & MLG \\
\hline${ }^{137} \mathrm{Cs}$ & 200 & 100 & 100 & MLG \\
\hline Bananas & 300 & 500 & 300 & GAD \\
\hline Wood & 300 & 500 & 500 & GAD \\
\hline Potash & 500 & ${ }^{I S}{ }^{152} \mathrm{Eu} 70 \%$ & IS ${ }^{152} \mathrm{Eu} 30 \%$ & GAD \\
\hline HEU & 300 & 200 & 200 & GAD \\
\hline Virgin HEU & 300 & 200 & 300 & GAD \\
\hline $\mathrm{RG} \mathrm{Pu}$ & $1000 \mathrm{LD}{ }^{137} \mathrm{Cs}$ & $\mathrm{IS}^{40} \mathrm{~K} 30 \%$ & IS ${ }^{137} \mathrm{Cs} 12 \%$ & MLG \\
\hline WG Pu & 500 & 300 & 500 & PCA \\
\hline HEU 1" $\mathrm{Pb}$ & $\mathrm{UNK}^{232} \mathrm{U} 84 \%$ & UNK ${ }^{232}$ U 55\% & $\mathrm{UNK}^{232} \mathrm{U} 55 \%$ & None \\
\hline
\end{tabular}




\begin{tabular}{|l|l|l|l|l|}
\hline \multicolumn{1}{|c|}{ Isotope } & GADRAS & \multicolumn{1}{c|}{ PCA } & MLG & Best \\
\hline \hline Virgin $\mathrm{HEU} \mathrm{1"} \mathrm{Pb}$ & $\mathrm{UNK}^{238} \mathrm{U} 100 \%$ & $\mathrm{UNK}^{238} \mathrm{U} 100 \%$ & $\mathrm{UNK}^{238} \mathrm{U} 100 \%$ & None \\
\hline RG Pu 1" $\mathrm{Pb}$ & $\mathrm{UNK}^{232} \mathrm{Th} 79 \%$ & $\mathrm{UNK}^{232} \mathrm{Th} 79 \%$ & $\mathrm{UNK}^{252} \mathrm{Cf} 40 \%$ & None \\
\hline WG Pu 1" Pb & $\mathrm{UNK}^{137} \mathrm{Cs} 70 \%$ & $\mathrm{UNK}^{252} \mathrm{Cf} 33 \%$ & $\mathrm{UNK}^{252} \mathrm{Cf} 90 \%$ & None \\
\hline
\end{tabular}

\subsubsection{Threat class results}

The threat class scores are presented in Appendix A4. Each of the algorithms improved their class scores as the number of counts received increased. Further, all of the algorithms demoted the shielded fissile materials to less dangerous classes corresponding to the inability to recognize heavily shielded material from their input library. MLG performed the best at preventing promotion of isotopes at the highest signal level with a notable trouble spot for the fertilizer with potash test input. The potash sample was in fact a mixture and, as none of the library elements represented such a mixture, this large number of errors is expected. PCA had similar problems but additionally it confused the ${ }^{67} \mathrm{Ga}$ medical patient with ${ }^{239} \mathrm{Pu}$. Such behavior would be a serious problem in a field deployment. GADRAS fittodb performed reasonably well despite its apparent poor performance on the verification set. However, this may be because none of our real world samples corresponded to library elements for which the linear dependence problem occurs.

\subsection{Discussion of results}

\subsubsection{Library}

As we can see in Table 5, the algorithms were quite consistent in performance for our samples. This consistency existed despite the range of methods employed. As there were few differences between the results, we can only conclude the problems that occurred come from the one thing shared by all of the algorithms, the library of isotopes. The library fails in a number of ways.

First, it contains a number of isotope shielding pairs that are very nearly identical. This is not a result of poor library design but rather nature working against us. The NaI scintillator design does not have sufficient resolution to separate these isotopes in the low count environment. Outside of these investigations, we found that in order to separate the most difficult cases, a detector would need to have no worse than $2 \%$ resolution at $662 \mathrm{KeV}$. The detector used in our evaluation had $8 \%$ resolution. The $2 \%$ requirement is outside the reach of current $\mathrm{NaI}$ scintillators for which the best achievable resolution is $5 \%$.

Second, the library did not cover the full set of test cases. In particular, it lacked reactor grade material as well a heavily shielded potential weapon sources. This fault is correctable with time. In order to use an automated isotopic identifier in the field, the library must be tuned to cover those isotopes that might be seen. As new cases where the algorithm is found to fail are fed back to the developer of the library, more refined and better libraries will be developed for the detector as it is situated.

Finally, the library may be failing in one last aspect of the design. The choice of isotope shielding pairs is based on the assumption that any isotope shielding arrangement can be found 
by a linear sum of those library elements. However, we found this to work poorly for our algorithm in the verification step. In particular, the PCA and MLG algorithm both performed far better than GADRAS in verification but failed on real data where the samples represented isotopes with shielding between library elements. We would recommend that this assumption of linearity be checked. Assuming that linearity does hold, the number of the isotope shielding pairs may need to be increased to improve coverage, especially as the count rate increases.

\subsubsection{Linear dependence of elements}

We have already discussed the fact that many of the library elements were linear dependent with other library elements. There were however some algorithmic differences in how the algorithms responded to this effect. Two isotopes in our test set were largely indistinguishable from another isotope in the library. These cases were ${ }^{201} \mathrm{Tl}$ and ${ }^{131} \mathrm{I}$. In the case of ${ }^{131} \mathrm{I}$, all of the algorithms

produced a ${ }^{133} \mathrm{Ba}$ result. For NaI spectroscopy, the only differences between these ${ }^{131} \mathrm{I}$ and ${ }^{133} \mathrm{Ba}$ would be the presence or absence of a line around $637 \mathrm{keV}$. As this peak appears only at a very high signal level, the poor performance of the algorithms is reasonably explained. On the other hand, ${ }^{201} \mathrm{Tl}$ is spectrally similar to ${ }^{139} \mathrm{Ce}$, but produced poor results in only one algorithm, GADRAS. We find that GADRAS performed poorly on this isotope in the verification tests as well.

Why is it that the other algorithms performed much better if the library elements are linearly dependent? A number of possibilities exist. The primary difference is that the algorithms that performed better were both operating as classifiers. It may be that the possibility of mixtures increased the probability of misidentifying the sample. There is another possible explanation; the output of ${ }^{201} \mathrm{Tl}$ was more likely than ${ }^{139} \mathrm{Ce}$ simply by design of the library. There were 8 library elements representing ${ }^{201} \mathrm{Tl}$ and only 2 representing ${ }^{139} \mathrm{Ce}$. Assuming that the samples were inseparable and the algorithms were picking at random they had 4 times as many representative samples of ${ }^{201} \mathrm{Tl}$ and thus would by random be more likely to select it. This effect of the number of representative samples needs to be examined in greater detail.

\subsubsection{Impact of signal strength}

Increased signal strength improved the performance of the identifiers. However, the improvement and required source strength for each isotope varied. Some common trends did emerge through the testing. To demonstrate the effect of signal strength on identification, Figure 2 shows, for a number of different isotopes, the number of trials for which GADRAS included the correct isotope in its output as a function of signal strength. 
Figure 2 GADRAS scores as a function of isotope and strength

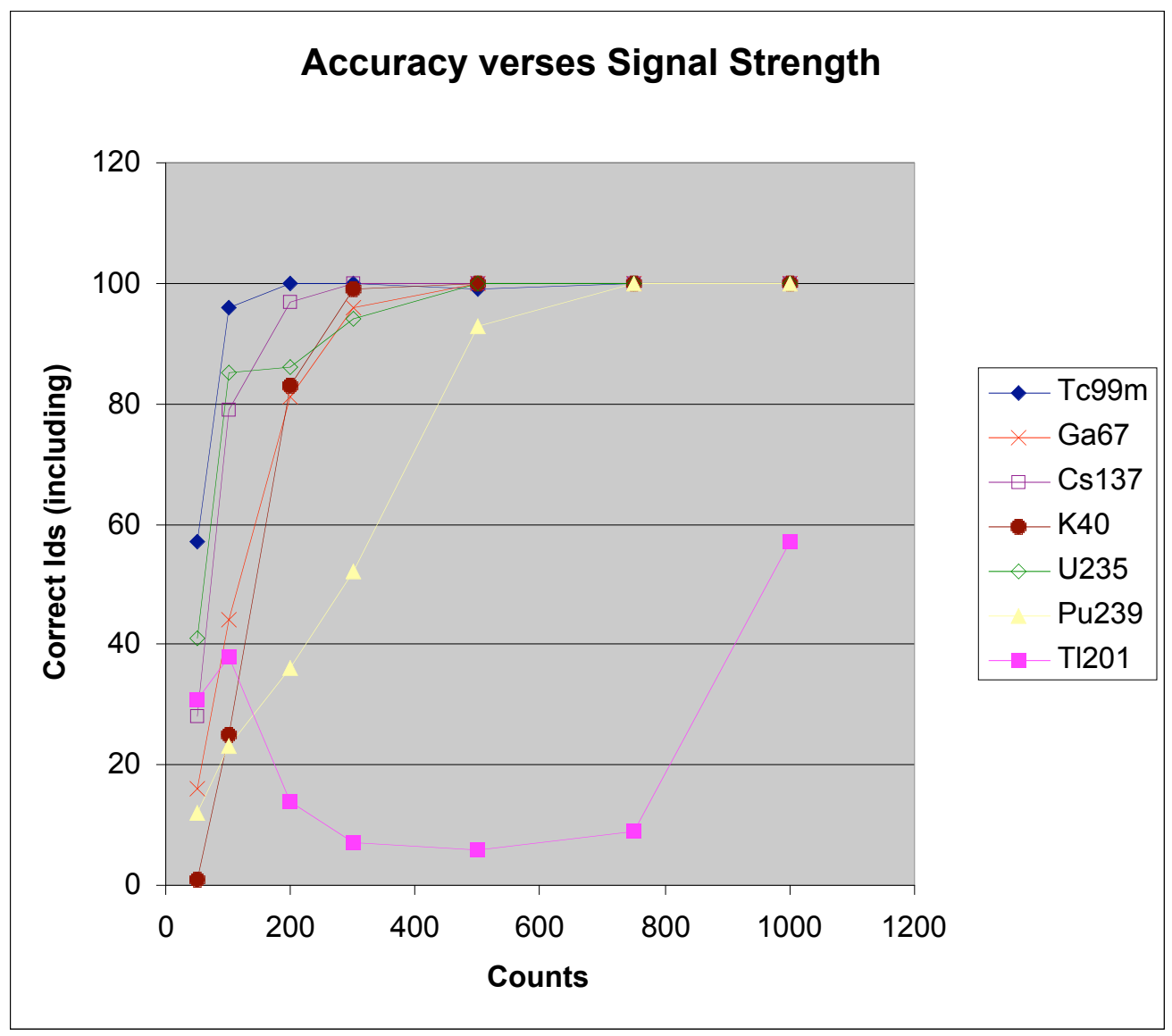

Lower energy sources were generally easier to identify compared to higher energy sources. This is particularly apparent in ${ }^{99 \mathrm{~m}} \mathrm{Tc}$. With as few as 50 counts, each of the algorithms managed to identify ${ }^{99 \mathrm{~m}} \mathrm{Tc}$ over $50 \%$ of the time. Similar behavior was seen for ${ }^{235} \mathrm{U} .{ }^{99 \mathrm{~m}} \mathrm{Tc}$ has a dominant $140 \mathrm{keV}$ line and ${ }^{235} \mathrm{U}$ has a dominant $185 \mathrm{keV}$ line. Higher energy producing isotopes such as ${ }^{137} \mathrm{Cs}(662 \mathrm{keV})$ and ${ }^{40} \mathrm{~K}(1460 \mathrm{keV})$ required more counts to produce this same performance in the algorithms. This seems to dominate the performance of the identifiers particularly with mono-energetic species. This apparent trend is easily explained. Isotopes that produced low energy lines had less scattering in the detector and thus more signal for a given number of counts in the photo-peaks that the algorithms use to perform their identification.

We can speculate that there is an additional factor affecting the improvement in identifications. Just as low energy spectra improved the ability of the algorithms to identify the isotope, the number of peaks in the spectra affects the improvement with signal strength. Isotopes with particularly complex spectra such as ${ }^{67} \mathrm{Ga}$ and ${ }^{239} \mathrm{Pu}$ tended to improve more slowly with signal strength. This is likely because the greater the number of photo-peaks, the less signal in each of them. This means that we can expect much better performance on mono-energetic species or species that are dominated by a single energy line. A much larger set of isotopes would be required to confirm this speculation. 
The final trend was that isotopes that had competing isotopes with similar spectra required higher count rates for identification. As an extreme case of this effect, ${ }^{201} \mathrm{Tl}$ that was competing with ${ }^{139} \mathrm{Ce}$ never obtained a $90 \%$ identification rate with fittodb. For these isotopes, the general trend was that signal strength does not improve the rate of identification until those minor lines such as the $637 \mathrm{keV}$ line in ${ }^{131} \mathrm{I}$ allow for identification.

\subsubsection{Best match}

For our scoring of the classifiers, we chose to use the best match as the result rather than accept the result "unknown." This was done for a very good reason. As we conducted the testing phase we found a disturbing trend in our algorithms that did not exist in the GADRAS results. That is, after reaching a reasonable identification rate in the test set, the number of correct identifications dropped as the signal to noise ratio increased. At first glance this performance does not make much sense, as an increase in signal should mean a better result. However, when we examined the algorithms it became clear what had gone wrong. Those incorrect results were entirely in the category of "unknown." As the signal to noise ratio increased, the "best match" remained at the $100 \%$ correct level but the actual distance measure between the best-matched isotope and the sample was increasing. This is illustrated in Figure 3. In the case of the PCA algorithm, the nearest neighbor did not exactly match the test sample and, thus, as the signal to noise ratio increased, the magnitude of these differences increased in comparison with the statistical sample variance. In the case of the MLG algorithm, there was no mixture of library elements that was near enough to the sample to explain it. As the algorithms produced both a best match and its statistically confident match, we chose to select the best match.

Figure 3 Results from the MLG algorithm showing the discrepancy between acceptable fit and best match

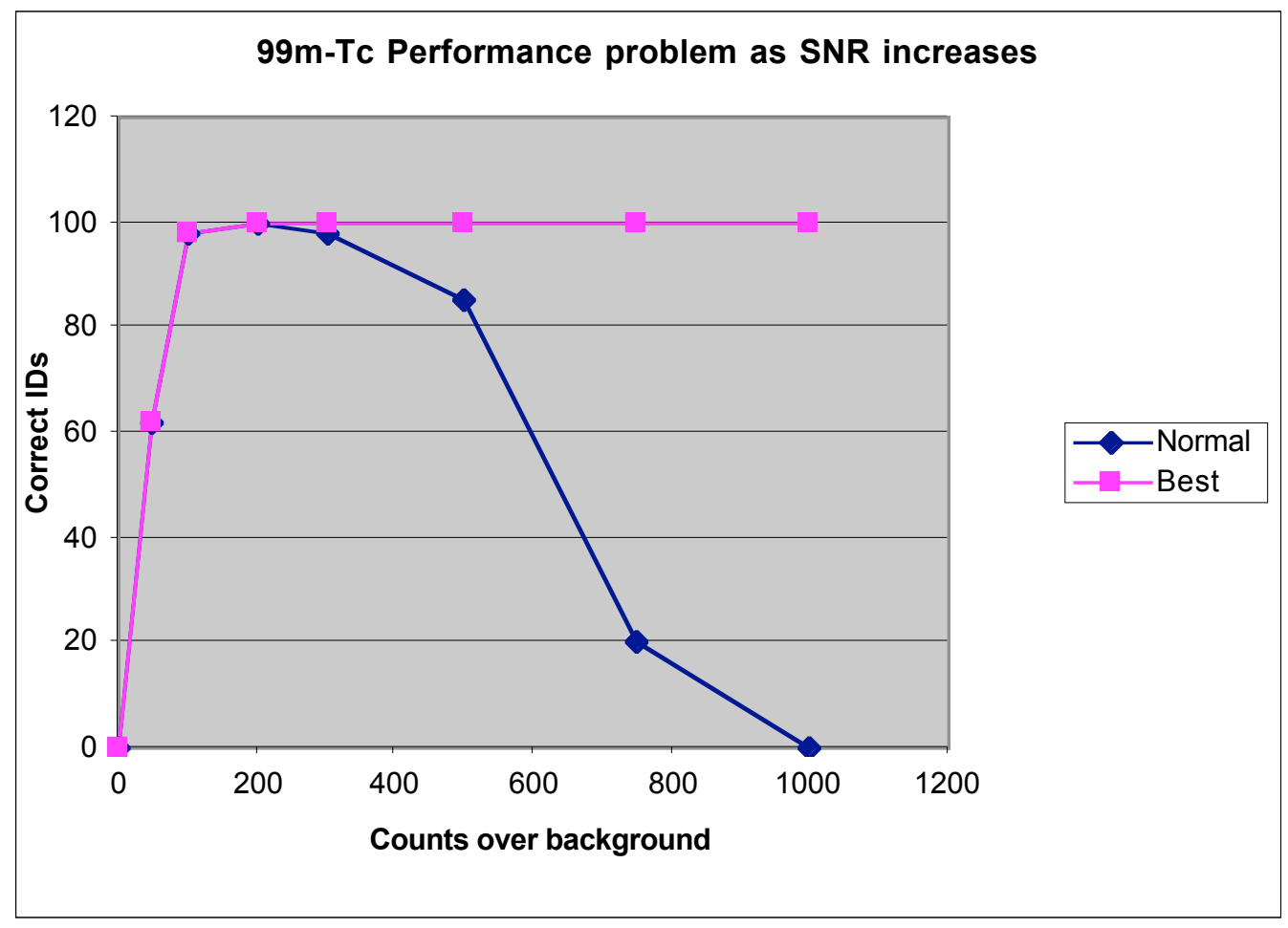


Our scoring of the best result is reasonable in that GADRAS did not produce an "unknown" condition and the conditions that resulted in our algorithms producing this result were based on the fact that the library's design was optimized for GADRAS and not our methods. However, this leaves the question as to why report the non-best-match results at all. In real world situations, if the algorithm always relies on the best match in its library, then sources outside the library will never be indicated as such. Thus, if the algorithm was used to screen packages, it could be defeated simply by mixing the substance that one wishes to hide with enough of another isotope to cause the algorithm to become confused. In practice, no library constructed on the basis of past observations at a detector installation will ever be fully complete to cover all cases that might be seen in the future. Thus the algorithm must have some reasonable means to call out the fact that the sample could not be explained by anything within its library.

It is our opinion that with improvements in the coverage of the library, the number of these "unknown" results would decrease in our "toy" algorithms, though not necessarily to the same extent. For the PCA classifier, an "unknown" will occur whenever a mixture with the correct ratio of isotopes and shieldings is not found. In the MLG, if the library properly covers the shieldings, then only a mixture that is not in the library would result in an "unknown" result. .

\subsubsection{Speed of Algorithms}

One critical consideration when an algorithm is to be used in a real-time application is how much computation resources will be required. Thus we measured the run time of each of the algorithms in identifying an unknown sample. All run times are measured on a Dell LATITUDE C840 $2 \mathrm{GHz}$ Pentium 4 system. Each code was run 100 times with the same input and the resulting processor time recorded.

\begin{tabular}{|l|r|}
\hline Algorithm & Runtime \\
\hline GADRAS fittodb & $22.7 \mathrm{~s}$ \\
\hline PCA & $72.0 \mathrm{~s}$ \\
\hline MLG & $4.9 \mathrm{~s}$ \\
\hline
\end{tabular}

The run times vary quite largely. This is only a course measurement as the repeated restarting of the program will included the overhead of loading the program and its associated data files. Surprisingly, the nonlinear MLG ran considerably faster than all others despite the relatively computationally expensive nonlinear iterative loop. Moreover, the PCA algorithm, which only requires one iteration to compute each sample, was by far the slowest. It should be noted, though, that PCA has the largest library and hence the largest loading time. GADRAS fittodb is burdened heavily by running in the virtual machine. Thus, we can conclude that the perceived speed disadvantage associated with estimators was not present in our testing. 


\subsubsection{Classifier vs. estimator}

In analyzing the results, we find a number of properties associated with classifiers and estimators held true for our problem. Estimators cannot differentiate when multiple linear combinations are all equally satisfactory. Thus, they produce a random result that may or may not contain the actual isotope. This tendency is apparent in the ${ }^{201} \mathrm{Tl}$ and ${ }^{139} \mathrm{Ce}$ confusion found in fittodb. Further, as there is no assigned likelihood of a mixture being seen in the field, estimators tend to produce mixtures that are highly unlikely to be seen in nature. For example, a frequent output of fittodb was ${ }^{237} \mathrm{~Np}$ with ${ }^{67} \mathrm{Ga}$. This results from statistic noise in the peaks of ${ }^{67} \mathrm{Ga}$ being confused with ${ }^{237} \mathrm{~Np}$. However, ${ }^{237} \mathrm{~Np}$ is an isotope that may be found in plutonium and thus a very high threat class. To find ${ }^{237} \mathrm{~Np}$ without characteristic plutonium or one of its decay chains would be quite unlikely especially given the known confusion between it and ${ }^{67} \mathrm{Ga}$. Thus such a mixture should be rejectable and produce the correct identification of ${ }^{67} \mathrm{Ga}$. It has been reported that some commercial vendors apply such rule-based systems to the output of the isotopic algorithms for exactly this purpose.

Pure estimators that do not limit to subgroups perform poorly. We found this in our original maximum likelihood algorithm. Of the 14300 trials in the verification stage, our original maximum likelihood algorithm produced 25753 false positives or nearly two extra isotopes per sample. GADRAS fittodb avoided this fate through the use of a heuristic to eliminate noncontributing components. Further, a pure estimator can decompose background into natural isotopes that results in additional false positives.

However, in spite of all the problems with estimators, classifiers have an even bigger problem. All samples in nature will be a mixture of, at minimum, the background and the sample. Further, although our test was limited in the number of mixtures, some isotopes are frequently used in medical treatments in the form of a mixture. This presents a severe challenge to a classifier as it would require all linear combinations of these isotopes to be represented as library elements. Given that the classifier was already slower than an estimator functioning in the same capacity, this will present a severe hurdle for the use of a classifier in the real world.

\section{Conclusions and recommendations}

Based on our analysis, we can draw a number of conclusions about both GADRAS fittodb and the underlying problem of isotopic identification. First, our "toy" algorithms provided better performance than GADRAS fittodb on the verification set and reasonably similar performance on the test set. Thus, we will conclude that GADRAS fittodb is not especially tuned for its application and could be replaced with reasonable effort. More effort is required for GADRAS fittodb to perform better on elements taken from its own library.

$\mathrm{NaI}$ detectors have inherent difficulties with certain linearly dependent isotopes and mixtures. They do not provide sufficient resolution to provide unambiguous isotopic identification when used in the field. Their resolution is capable of distinguishing some fraction of innocent sources from threats but, unless considerable improvement in performance is possible beyond the results we demonstrated with our case study, NaI scintillators will result in frequent false alarms. 
Further, from our study of isotopic algorithms we can make some recommendations for future development of isotopic algorithms. The greatest bulk of future effort must be in building and tuning an isotopic library to provide the best performance. That is, one must properly survey the isotopes to be encountered in the operation of the detector and tune the library and the algorithm towards it. The most general problem of unambiguously identifying isotopes is impossible with $\mathrm{NaI}$, thus focused development on an application should be preferred. The algorithms must have a library that fully spans the expected input set. Thus, data from the field must be acquired in order to build and test such a library.

Second, nature presents us with mixtures. At this time, we do not have sufficient knowledge of what mixtures are likely to be seen in the field other than that they are quite common. All dangerous sources have varying isotopic composition based on their design and age. Thus the algorithm must be able to handle a very diverse input. Covering this space by individually representing each unique design, age and isotopic mixture will likely result in an algorithm that is much slower than the generalized solution of representing these inputs as a mixture of parts. Further, individually covering points in the vast sample space will likely result in poor coverage of new and novel things, such as an improvised weapon, that might be identified as threatening when using an estimator.

If an estimator is used, it must be adapted with some logic to compute the likelihood of mixtures and thus reduce false positives caused by reporting an unlikely mixture. This means the algorithm should have logic to recognize ambiguous cases, report multiple valid solutions where they exist and restrict those valid solutions to reasonable occurrences seen in the field. Arbitrary mixtures should be accepted, but only if reasonable solutions are exhausted or if that mixture contains statistically significant probability of hiding a potential weapon.

Outside of this current study, these algorithms were applied to real world data. Performance on the real world data was found to be even worse than that reported in this study. Automatic methods failed to identify at least $25 \%$ of the cases that were identifiable easily when a human was guiding the process. However, the cause of these failures was not the methods themselves but rather lack of adaptability to real world conditions. In all of our tested algorithms, the detector was known perfectly and does not change over time save for the gain parameter. However, in the field we found that additional changes occurred. Although modeling the gain of the system is sufficient to realign peaks into their proper energy bins, none of the algorithms adjusted for the edge effects caused by the lower level discriminator (LLD) as the gain changes. A lower level discriminator is set in order to prevent noise in the amplifiers from swamping the detector. As this is a fixed electronic level it does not change with temperature. Thus the amplitude of the lower energy bins that are subject to being rejected by the LLD changes as the gain of the system shifts. As the library remains fixed and the changes caused by the LLD are not accounted for, the algorithms produced poor results. We would recommend additional preprocessing stages to account for these effects prior to performing identification. 


\title{
Spectral Analysis of Gamma-ray Event List Data Using K-S Tests
}

\author{
Ron Wurtz
}

\begin{abstract}
Lists of gamma-ray events, where each event is marked by a timestamp and an energy, are to be contrasted with binned data, usually binned in the temporal and spectral domain. When list data are available, an analysis avoiding the use of binning is suggested. Tests like the KolmogorovSmirnov (K-S) test can be used to measure consistency of discretely sampled data with putative parent populations. We simulated lists of gamma-ray events drawn from one of two parents, looking only at the spectrum in the range containing an emission line that signified the presence of a non-background source. We simulated the events for a range of counts in continuum and in line. We refined the test by using a variation on the K-S test-the Kuiper test (K-S-K). We constructed and compared receiver operating characteristic $(\mathrm{ROC})$ curves from distributions of the probabilities of consistency with each parent. We then compared the K-S-K ROC curves with those obtained simply by looking at excess count rates in the same energy range. We present a range of realistic cases where the KSK's ROC shows better performance than the excess count rate method.
\end{abstract}

\section{List Data}

An individual gamma ray can be detected by creating an electronic pulse in a circuit containing a material sensitive to incident gamma rays. The pulses are filtered and shaped, and electronics can be built to sort them and count them. It has been customary to store and analyze them as histograms corresponding to spectra. The bins of such histograms are somehow matched to the width of the distribution of pulse heights as presented by the electronics.

It is not necessary to acquire the data as spectra. When the flux is matched to the speed of the data acquisition and storage system, electronics can be built to store every event separately. End users are accustomed to analyzing the data as spectra, but histograms are technically a guide for the eye, not the essence of the information. The goal of this investigation is to build a ROC curve for a non-binned test and compare it to a binned test to determine if the non-binned test is an improvement over existing binned tests for spectral variations.

If the data are stored as lists, it seems likely that the best analysis of such data should avoid the use of binning. The Kolmogorov-Smirnov (K-S) test and its kin have a low number of statistical prerequisites for the data to be analyzed. The K-S test compares two distribution functions of a single independent variable. The empirical distribution function for a list of $N$ measurements $x$ is the function $S_{N}(x)$, which is the fraction of data points less than a given value $x$. For every possible set of $x, S_{N}(x)$ has a range from 0 at the lowest value of $x$, to 1 at the highest. Two distributions can be directly measured using the K-S statistic $D \equiv \max \left|S_{N_{1}}(x)-S_{N_{2}}(x)\right|$ or a distribution may be compared against a cumulative probability distribution function (CDF) $P(x), D \equiv \max \left|P(x)-S_{N_{2}}(x)\right|$. When 
two measured distributions are compared, it is a two-sided K-S test, when one measured distribution is compared against a theoretical distribution, it is one-sided. We used a variation called the Kuiper test [4] which looks at

$$
V \equiv D_{+}+D_{-}=\max \left[S_{N}(x)-P(x)\right]+\max \left[P(x)-S_{N}(x)\right]
$$

We implemented this test, hereinafter referred to as KSK, for this analysis. The V statistic has a known probability distribution $P\left(V_{K S K}>\right.$ observed $)$ that measures probability of consistency between the two distributions being compared that, for fixed $\mathrm{N}$, is monotonically decreasing with increasing $\mathrm{V}$.

\section{Simulations}

\section{Simulation of Data}

We restricted the comparison to seeing if we can alarm on the presence of a single line of interest at $\mathrm{E}$ on the interval $E_{0}<x<E_{1}$. Note that if the count rate is low and the restricted energy band contains very few counts, it may take awhile to obtain enough events to begin running the test, though that would also be a limitation to a binned test in the interval.

To simulate the data, we used a nonzero continuum for the "line absent" model, and a Gaussian plus continuum for the "line present" model. We constructed a discretely sampled distribution function from each of these simulated spectra. Because the parent functions were originally constructed from real data, we simulated continuously-sampled energy values using a spline with tension $=0.1$ and generated random numbers on the interval 0 to 1 . This yields a set of discrete events with the same energy distribution as the original spectrum. In a future version, it might be better to use an analytic distribution function because the simulating function is analytic.

To simulate a single set of events obtained in a fixed time, the user selects an exposure time, and the expected total number of photons in that time interval is computed. The actual number of photons measured is obtained as a single random deviate drawn from a Poisson distribution with the expected number as the mean. Finally, that total number of photons is simulated using the method described above. Any number of event sets can be created this way for each exposure time.

\section{Running the Tests}

We ran a series of trials in a 20 unit wide band containing a 2 unit FWHM line centered on the middle of the band. The total continuum and line photons could be varied. So that we can then compare the distribution of the trials' sample variable, an equal number of trials are made for event sets corresponding to the line-present and line-absent parent models.

The one-sided K-S comparison functions are ordered pairs interpolated with a spline. This code, like that for the simulated trial spectra, uses a spline so that comparison 
functions based on real data could be modeled. For the comparison distribution function, we used a pure continuum when comparing against the possibility of "line absent", and a pure Gaussian with the same input FWHM as the simulated data and with no continuum when comparing against "line present". The difference between the simulated events' $\mathrm{CDF}$ and the comparison spline is computed for every event in the trial, the maximum differences $\mathrm{D}_{-}$and $\mathrm{D}_{+}$are found, and the parameter $\mathrm{V}$ is computed. At the same time, the binned parameter "total counts in energy band" is recorded for each trial. We ran 54 trials, varying the total number of continuum and line photons, each in a range 2-1000 counts, comparable to the total background photons obtained in that band in less than a minute with a centimeter-scale Ge or CZT detector.

\section{Figure of Merit}

Because we are chiefly interested in finding an parameter from unbinned data, we built a ROC curve for each comparison statistic for each set of trials. A ROC curve is a plot of one minus the probability distribution function (PDF) of a variable in a no-threat situation versus the PDF of the same variable in the threat situation. The horizontal axis is the probability of a false alarm $\left(P_{f . a}\right)$ in the no-threat situation, and the vertical axis is the probability of actually detecting a threat $\left(P_{\text {det }}\right)$. If a family of ROC curves is built by varying input parameters of the simulation, one can estimate under what conditions the test is useful. Likewise, if a set of variables from different tests is measured from trials with the same input parameters, one can find the test that gives the best ROC curve under those conditions.

The ROC curve measures how capable the variable is of making distinctions between the two possible hypotheses by comparing the rate of misidentification between the two distributions parameterized by a threshold. One can also measure the change in steepness of the ROC curve as some observational parameter is varied. In this case, the hypotheses are whether the events are drawn from a spectrum with or without an extra line (or set of lines), the measured variable is the K-S probability of the significance, and the varied observational parameter is the exposure time. In this report, ROC curves built from the distributions of different sample variables for the same set of trials will be used to compare the utility of the alarms relying on those variables.

ROC curves also have a figure of merit: the integral under the curve. The closer the integral to 1, the better the measured parameter is for distinguishing a problem condition from a false alarm. An integral of 0.5 is equivalent to guessing. Integrals below 0.95 describe a situation where a large number of false alarms will occur when the alarm threshold is catching a large percent of the "true" threats that come through.

\section{Results}

\section{Outcome Of Simulations}

The outcome of the simulation we present in Figure 1 corresponds to the "no threat" case with 50 continuum counts expected in the energy band 400-420 keV in some short time period. In the threat case, an additional 5 counts in a $2 \mathrm{keV}$ FWHM Gaussian at $410 \mathrm{keV}$ 
have been added. There are 1000 trials of each of the "continuum only" and "line plus continuum" cases.
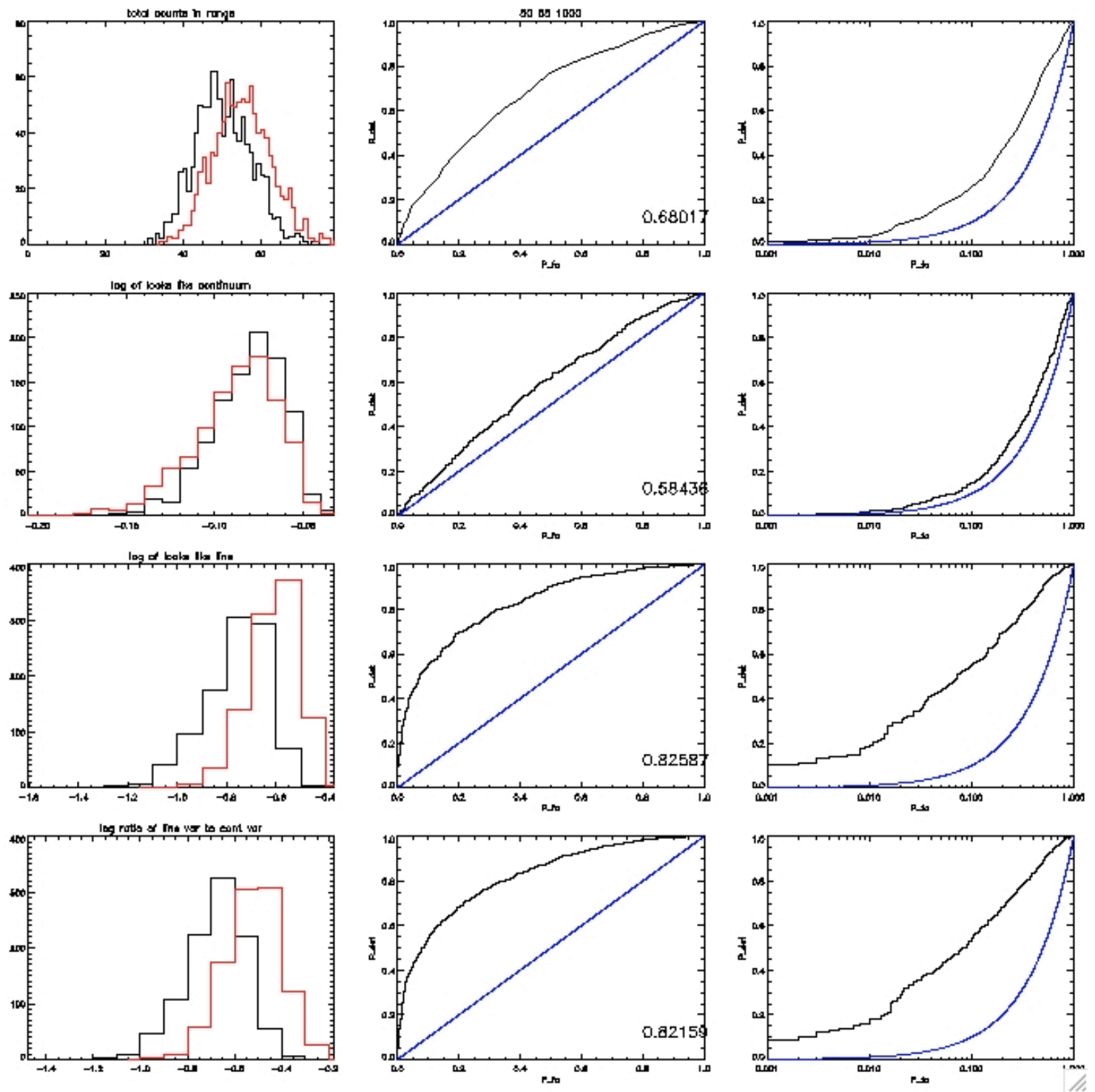

Figure 1: Distributions and ROC curves for four different variables. See text for details.

In the first column of Figure 1, we present binned distributions of four sample variables derived from 1000 Monte Carlo-simulated continuum-only (black) and continuum-plus-line (red) photon-energy lists. The sample variable in the first row is simply total counts in the energy band. The variable in the second row is the logarithm base 10 of the KSK variable $\mathrm{V}$ that the distribution of energy values is not inconsistent with a flat distribution, i.e. continuum only. The variable in the third row is the logarithm of the KSK variable $\mathrm{V}$ that the distribution of energy values is not inconsistent with a distribution drawn entirely from a Gaussian with the same width and center as the modeled events. The variable in the fourth row is the logarithm of the ratio of the Gaussian KSK V to flat KSK V, $\log \left(V_{K S K, \text { gaussian }} / V_{K S K, \text { flat }}\right)$. We used the distribution of the variable $\mathrm{V}$ instead of the probability $P\left(V_{K S K}>\right.$ observed $)$ because the tail of $\mathrm{P}$ as $\mathrm{V}$ 
approaches one gets into very small numbers. $\mathrm{P}(\mathrm{V})$ depends on the number of photons in each trial, but for nearly constant $\mathrm{N}$, the rank of $\mathrm{V}$ is nearly the same as the rank of $\mathrm{P}$.

The second column shows the ROC curve for the two distributions in the first column, in its lower right hand corner is the integral under the ROC. The third column is the same ROC curve plotted log-log. The blue line on the ROC curves corresponds to "just guessing", i.e. $P_{\text {f.a. }}=P_{\mathrm{det}}$.

\section{Comparison with Monitoring Excess Counts In A Bin}

The first row represents "looking for an excess above expected rate" for a binned distribution of photon energies. Even with only a few counts in the band, the difference between Poisson-distributed 50 and 55 counts could be used to set an alarm under conditions where the false alarm rate is not as high as the rate of detection. At these rates, such an alarm would be of little practical value, since to get a detection rate of $95 \%$, almost every non-threat source is also a false alarm. However, the fourth row indicates that, even without foreknowledge of the expected rate in the band, an alarm based only on unbinned expected spectra shape in the energy band - and independent of total counts - does better. The integral under the ROC for the countrate alarm is 0.68 and under the $\log$ ratio $\mathrm{ROC}$ is 0.82 .

We ran these trials for a range of counts in continuum and line. Figure 2 shows difference between the figures of merit of the two methods. The highest values are where the integral under the ROC for the unbinned method is much higher than the same integral for the binned method. The tickmarks show the local downhill grade on each contour. The peak of the difference is where continuum counts is in the high hundreds and the line counts are in the low tens. The unbinned test is most similar to the countrate test when there are comparatively few continuum counts compared to line counts.

We discovered that the ROC figure of merit for the unbinned spectra tests was comparable or better than the excess count test's ROC figure of merit in the entire range we checked: continuum counts in the range $2-1000$ with line counts in the range $2-$ 100. The excess counts figure of merit was only 4 parts in a thousand better for any the whole range, and the unbinned method was nearly a $20 \%$ improvement at best. 


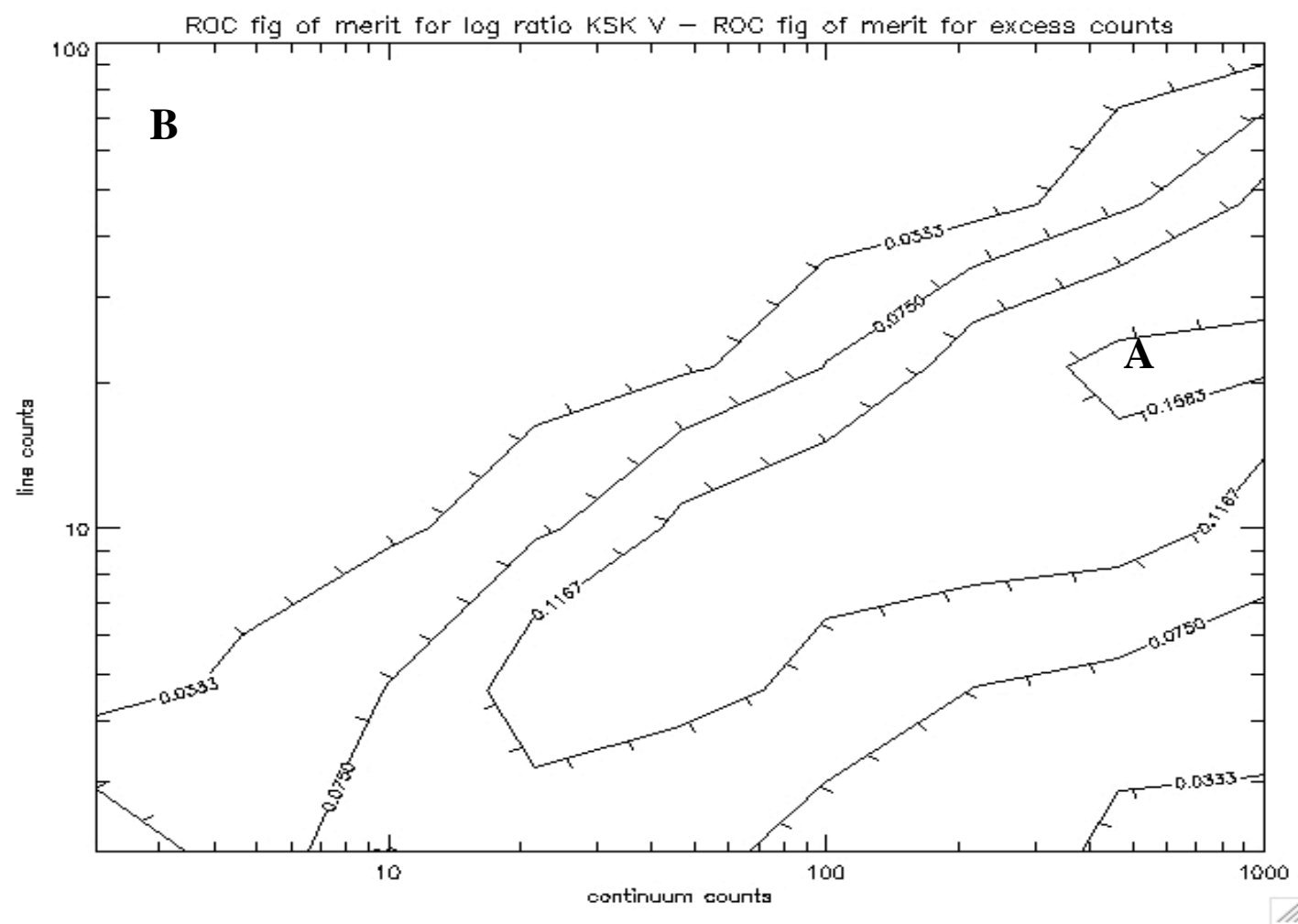

Figure 2: Contours of the ROC figure of merit for the log ratio of the V parameter for line versus continuum minus the figure of merit for countrate alarming. (A) The region with the greatest improvement in figure of merit for KSK over excess count method. (B) Low count region where KSK and excess counts perform equivalently.

\section{Continuous Monitoring with Sequential Tests}

These simulations were performed as if the events are collected for a fixed time period and then analyzed. It is possible this method may be further improved by looking at the time evolution of the log ratio of KSK probabilities; however, that simulation has yet to be done.

\section{Conclusion}

The spectral shape of a set of individual photons can be measured for consistency with a flat continuum or a pure Gaussian with only a few photons. In the simulations where one is attempting to set an alarm in a band on small count rates in both continuum and line, the method is better than looking for small increases in countrate. 


\title{
A New Method for the Modeling of Gamma-ray Spectra
}

\author{
J. Garrett Jernigan ${ }^{1}$, William Craig ${ }^{2}$, R. Wurtz ${ }^{2}$
}

\begin{abstract}
A new method for the analysis of a Gamma-ray spectrum is presented. This numerical algorithm, the Photon Clean Method (PCM), is an inverse Monte Carlo approach which successfully fits a model of a high number of degrees of freedom biased by a set of template spectra for both the source and background components. This approach is particularly well suited to real world situations in which traditional methods of fitting models of low dimensionality fail (for example Chi-sq fitting).
\end{abstract}

\section{Method of Analysis}

The analysis of the Gamma-ray Spectra taken with solid state detectors with modest energy resolution is based on an alternative method, the Photon Clean Method (PCM), first introduced in Jernigan \& Vezie 1996. This method is further developed in Jernigan, Peterson \& Kahn 2003 as an approach to "image processing". Image processing is defined as any algorithm that reduces data to an "image" which is a model with a large number of degrees of freedom typically as large as the number of degrees of freedom in the observational data. For Gamma-ray spectra individual observations are often composed of hundreds to thousands of detected events each specified by a few measured parameters (time, energy, location, etc). PCM has also been adapted for the traditional situation of fitting models of low dimensionality by Peterson, Jernigan \& Kahn, S.M. 2003b (also see Peterson et al. 2001 and Peterson 2003c).

The PCM method is particularly well suited for the analysis of multi-dimensional Gamma-ray spectra. In this paper we address the special case of a modest resolution spectrum in one dimension typical of a single integration at one location. The spectral resolution is sufficient to resolve brighter Gamma-ray lines that can indicate the presense of particular isotopes. The approach is an inverse Monte Carlo method which starts with a list of raw events of imperfect information and ends with a finite list of model photons of perfect information. The most significant feature of the method is that all mathematical steps are carried out without the creation of any spectral bins of events (or photons). PCM is different than other list-mode methods since both the raw data and the inverted model are represented in event (list-mode) form. Other list-mode algorithms do not

\footnotetext{
${ }^{1}$ Space Sciences Laboratory, UC Berkeley

${ }^{2}$ Lawrence Livermore National Laboratory
} 
represent the model in list-mode. The practical utility of PCM required the invention of a new version (first illustrated in Jernigan \& Vezie 1996) of a multi-dimensional Kolmogorov-Smirnov (KS) test (Smirnov 1948). This extended two sample KS test is uniquely different from prior efforts to extend the KS test to higher than one dimension (see Peacock, 1993 and Fasano \& Franceschini 1987). In particular the PCM extension of the KS test actually reduces the higher dimensional KS test to a simple one dimensional KS test which is easily numerically computed. Such an approach was a required invention for the practical implementation of the numerical analysis method PCM.

The PCM method as applied to this example of the analysis of a one dimensional Gamma-ray spectrum is an "inverse Monte Carlo" application which is summarized as a sequence of procedures which are iterated until convergence.

1. Select an initial guess for the trial model: $N$ events $(E)$ where $E$ is the energy of each single Gamma-ray taken from an arbitrary Gamma-ray spectrum. This input model has $\mathrm{N}$ degrees of freedom equal to the number of Gamma-rays in the spectrum. This selection of an initial guess is biased by a template of a background spectrum.

2. For each photon (E) in the model space ("image") compute an event (G) in the space of the detected Gamma-ray events measured as a pulse height proportional to the detected charge in a solid state detector. Each event $\mathrm{G}$ will have a variation that is computed as a Monte Carlo simulation of the detector response. For the example in this paper we assumed that a simple Gaussian variation in the measured charge has a fixed RMS independent of the energy $\mathrm{E}$. This is typical for a solid state detector that has noise dominated by the electronics and not the intrinsic properties of the detector material. The method is easily extended to situations in which the Monte Carlo model of the detector is more complex. The procedure in this step is a full Monte Carlo simulation of the integrated Gamma-ray Spectrum.

3. At the end of step (2) there are $N$ photons (E) and $N$ events $(G)$ which simulate a version of the raw Gamma-ray spectrum. This list of $N$ events $(G)$ can then be compared to the actual $\mathrm{N}$ events that were detected by the solid state detector. The comparison uses a two sample $\mathrm{KS}$ test to determine if the current image (E) is consistent with the observations.

4. Slightly modify the list of $N$ photons (E) and repeat steps (2) and (3). The modification of the list of photons (E) can be biased by selection from a library of templates of Gamma-ray spectra of likely sources and background. If the modification improves the KS difference computed in step (3) then keep the modifications of step (4). If the KS difference computed in step (3) indicates that the modifications are not an improvement replace the modified photons (E) with their prior values. In either case repeat steps (2), (3) and (4) until the process converges to an acceptable low value of the KS test. At that point the current list of photons (E) is a possible "image" or model comprised of $\mathrm{N}$ free parameters. 
The PCM image analysis is just an inverse Monte Carlo approach that reachs a solution via feedback using a two sample KS test as a figure of merit (see Jernigan \& Vezie 1996 for a simple example).

\section{Results for a Gamma-ray Spectrum taken with a Ge Detector}

Figures 1 through 4 show the results of PCM applied to analysis of a real Gamma-ray spectrum. Two raw spectra are used to set up a test example for analysis by PCM. The first spectrum is background only. The second spectrum is source and background. This real data was taken with a high performance cooled Ge detector. A third raw spectrum is created by differencing the first two spectra. This provides a source only spectrum. The background only and source only spectra are used to create a two template library of "truth" spectra of excellent energy resolution. These two spectra are then combined into a linear combination of source and background. This combined spectrum is then degraded by convolving with a gaussion function of RMS $3 \mathrm{keV}$ to simulate a modest resolution spectrum typical of a CZT room temperature device with less than optimum electronic performance. This produces a simulated "real" Gamma-ray spectrum with a companion "truth" high resolution spectrum at Ge energy resolution (less than 1 keV RMS). We further degrade the simulated CZT spectrum by multiplication by a function one plus a low and high period sine wave as a function of energy. Each sine wave has an amplitude 0.2 peak to peak. This ensures that the simulated "raw" spectrum can not be fit by a simple linear combination of the source and background templates and mimics likely real world complications of Gamma-ray spectra such as slow variations in the continuum and line ratios that do not match theoretical predictions. PCM is shown to successfully fit this corrupted example raw spectrum in a situation in which a simple Chi-SQ fit to a linear combination of the templates can not possibly fit the data. This property of PCM shows the most important feature of the approach. PCM can fit models with a large number of degrees of freedom in those situations in which other methods fail. This robust feature of PCM as illustrated by the example presented is the reason why PCM has potential to be the best approach for modeling real world Gamma-ray spectra with realistic complications (arbitrary mixtures of isotopes, scattering, shielding, etc). The real example explained in this paper demonstrates the reality of this important property of PCM. However this example is restricted to a "library" of Gamma-ray spectra that includes only two templates, one source and one background template. A full evaluation of PCM for a real world situation will require a uniform library of hundreds of source and background spectra. If PCM is shown to work well for a large real world dataset then the application of PCM can be extended to multi-dimensional data that includes event (list-mode) data with coordinates in energy(1D), time(1D), location(2D) and velocity(2D). PCM is uniquely applicable for the analysis of collections of events in such a six dimensional space. 


\section{REFERENCES}

Fasano \& Franceschini, 1987, MNRAS, 225

Jernigan, J.G. \& Vezie, M. 1996, Astronom. Data Analysis Software and Systems V,

Jernigan, J.G., Peterson, J.R. \& Kahn, S.M., 2003, to be submitted ApJ

Peterson, J.R., et al. , 2001, A+A, 365, 104

Peterson, J.R., Jernigan, J.G., \& Kahn, S.M., 2003, to be submitted ApJ

Peterson, J. R., 2003, Ph.D. Thesis, Physics, Columbia University

Peacock, 1993, MNRAS, 202

Smirniv, N., 1948, Annals of Mathematical Statistics, Vol 19, Issue 2, 279-281

This preprint was prepared with the AAS $\mathrm{IA}_{\mathrm{E}} \mathrm{X}$ macros v4.0. 
Fig. 1.- This display shows an example of the convergence of a model for a Gamma-ray spectrum biased by a library of source and background spectra. The initial guess is at the bottom of the plot. The top panel shows the final converged solution. The whole plot is amovie as a function of iterations from bottom to top. The bottom plot shows the inital guess which is a background spectrum which has a single bright line near $60 \mathrm{keV}$ and a broad continuum spectrum. As the PCM method iterates the biased model selects some of the photons from a template source spectrum hence the appearance of a half dozen lines that are emitted by the source isotopes. The final top panel has converged to an low value of the KS parameter and is therefore known to be an acceptable model or "image" of the Gamma-ray spectrum computed via feeback inherent in the PCM.

Fig. 2.- This figure shows the details of the initial guess for the image inversion. The top panel is a histogram of those photons that are derived from the source template (two initially or nearly zero). The next panel is a histogram of those photons that comprise the initial guess. By definition this histogram is consistent with the background template alone. The third panel is a difference between the fourth and fifth panels and shows that the initial guess is not a correct "image" or model. The fourth panel is the simulated raw Gamma-ray spectrum that contains both the source and background. It is derived from a high quality Ge spectrum (truth input) which has been degraded for a resolution of $3 \mathrm{keV}$ RMS typical of a room temperature array of CZT detectors with modest resolution dominated by electronic noise. The bottom or fifth panel is the histogram of the Monte Carlo events (G) derived from the initial guess ( $\mathrm{E}$ - second panel). The goal of PCM is to vary the second panel until the bottom two panels agree. The movie shown in figure 1 is the sequence of second panel histograms or the current "image" or model for each step of the iteration. Keep in mind that even though all of these panels show histograms for presentation only. The PCM algorithm does all internal calculations in list-mode.

Fig. 3.- The same as figure 2 except that this shows the state of PCM for an intermediate stage of iteration. The histograms show that the model or "image" has improved but still has significant changes needed for convergence. Inspection of the middle panel shows that the differences are significantly larger than Poisson noise.

Fig. 4.- The same as figures 2 and 3 except that this shows the state of PCM for an final stage of iteration. The histogram shows that the model or "image" has converged. Inspection of the middle panel shows that the differences are now consistent with Poisson noise. 

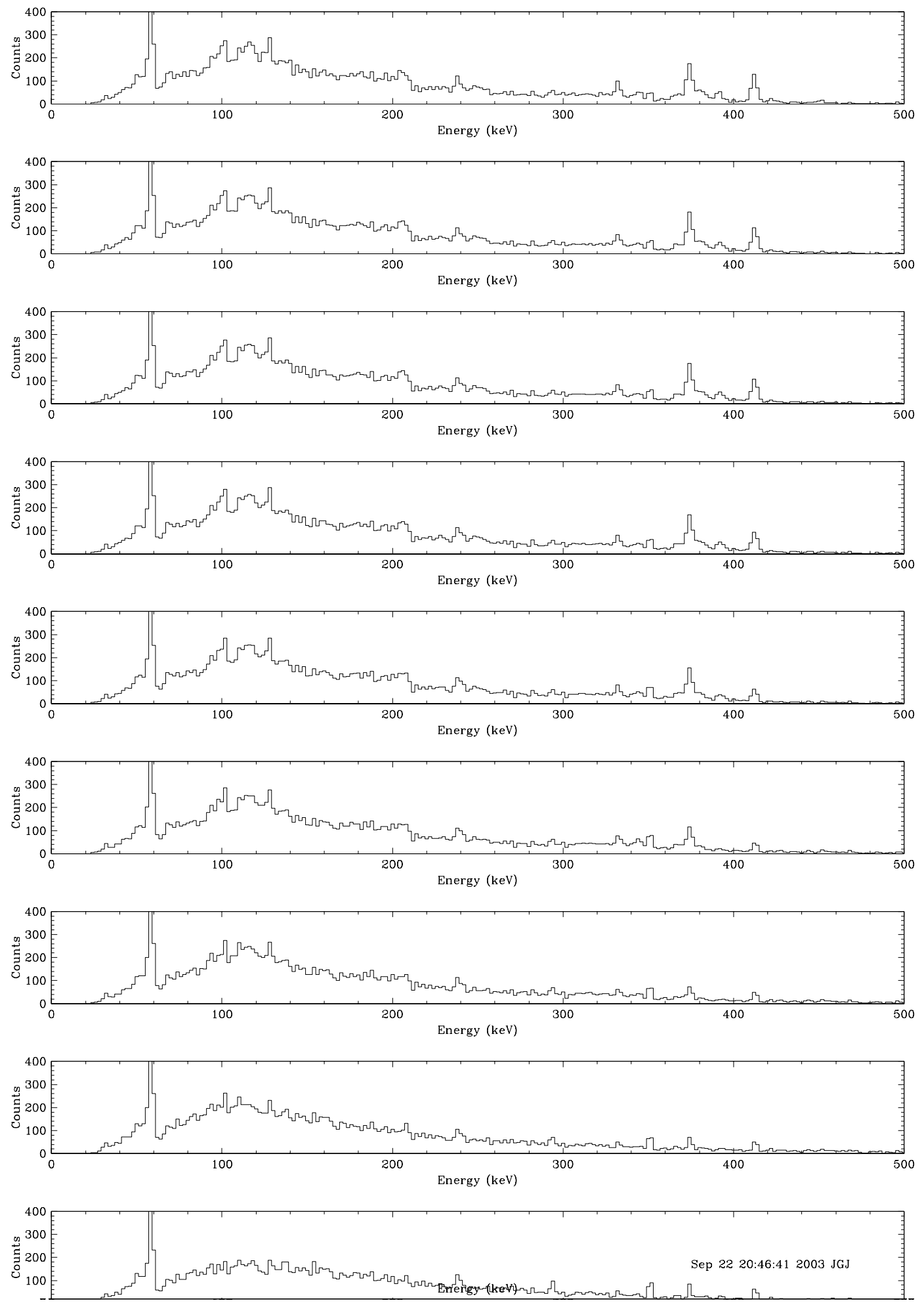

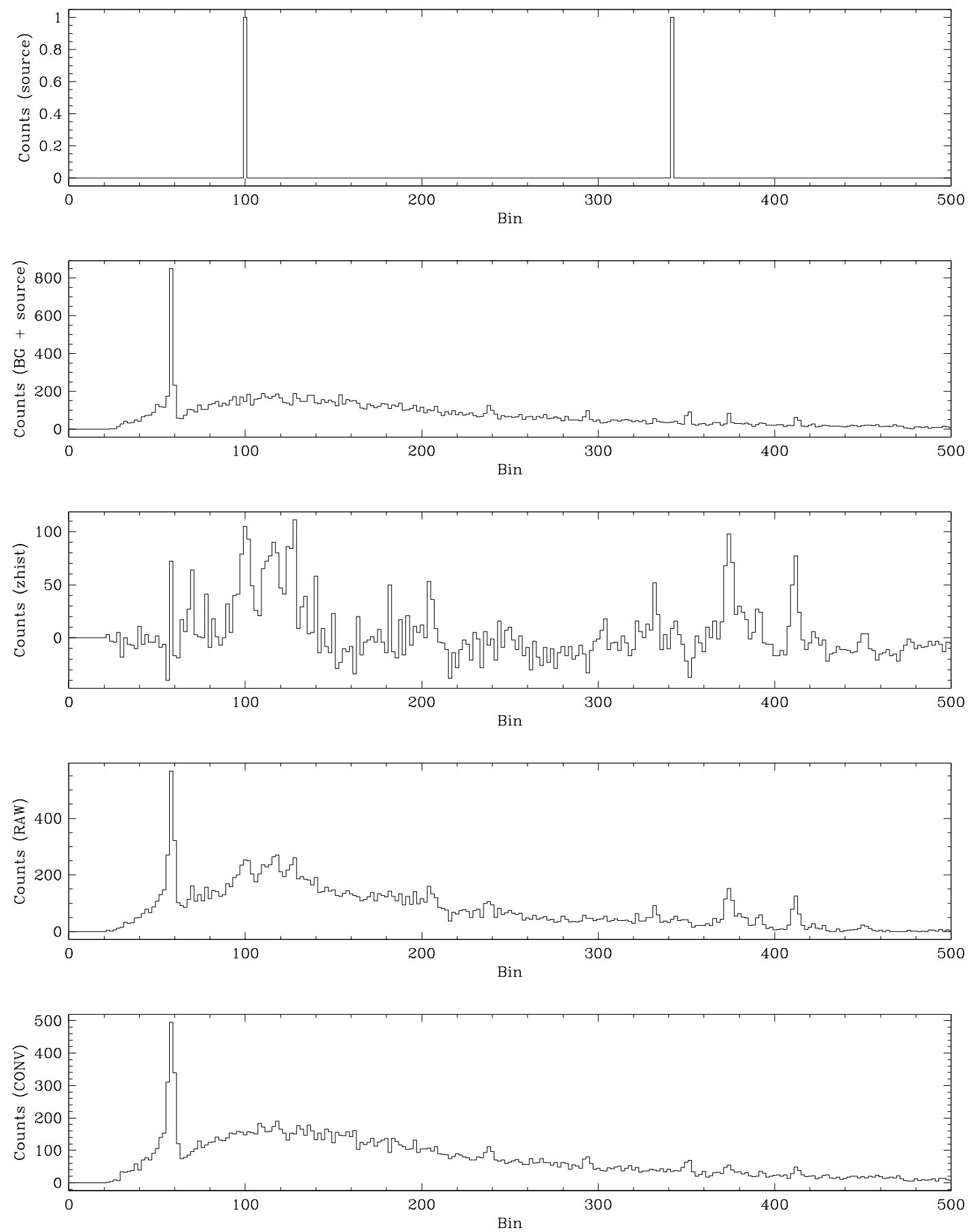

Sep 22 20:55:23 2003 JGJ 

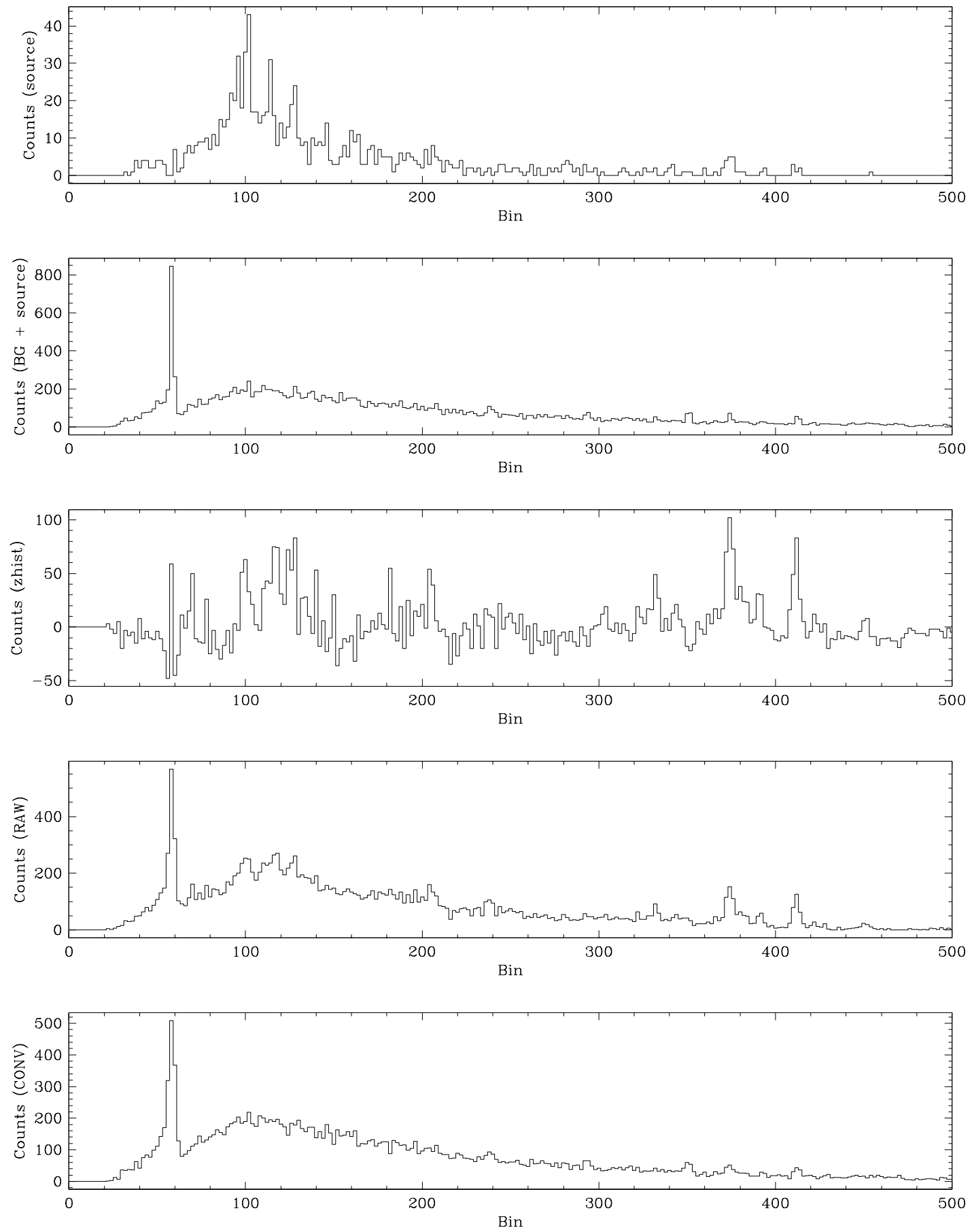

Sep 22 20:55:25 2003 JGJ 

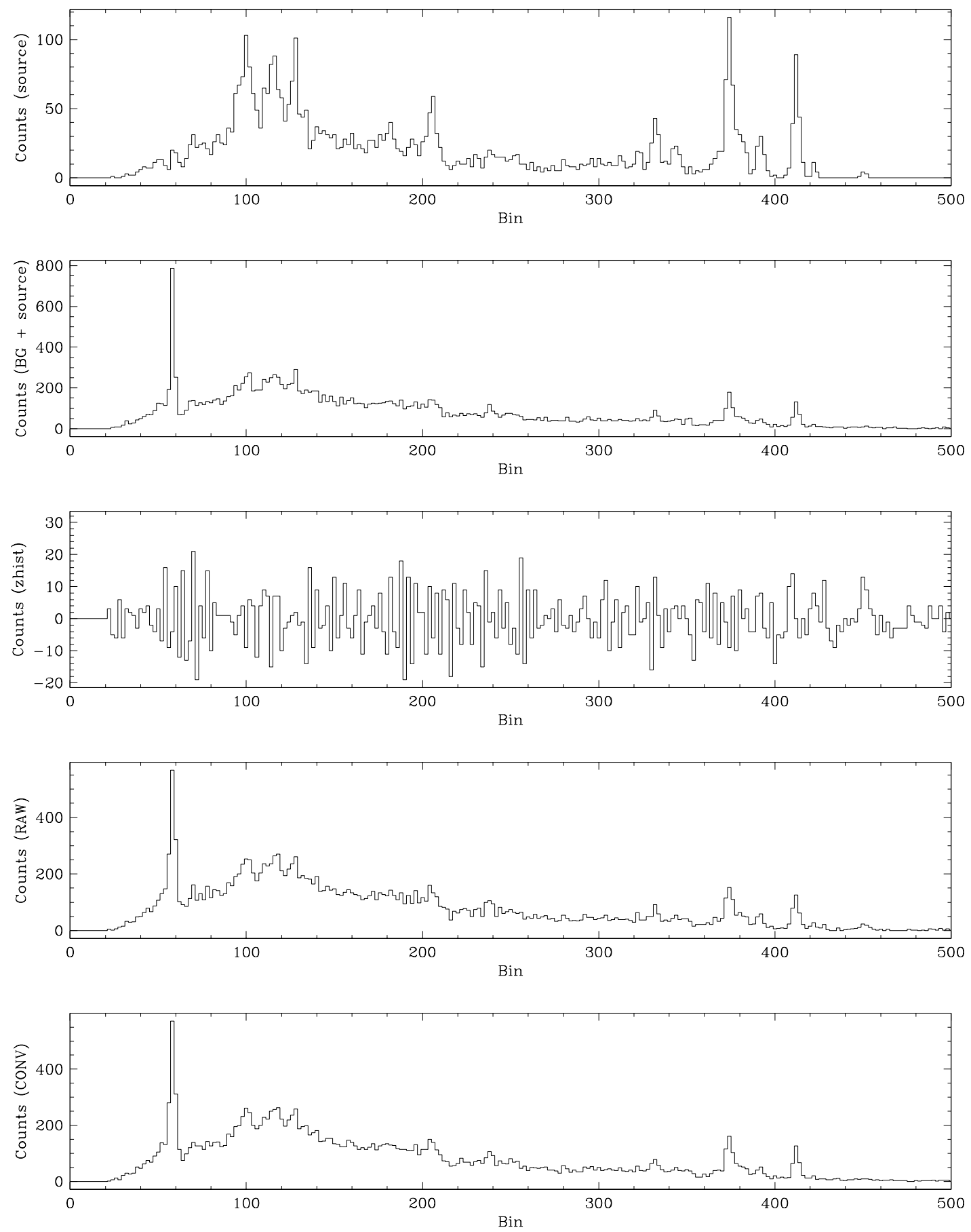

Sep 22 20:49:12 2003 JGJ 


\section{Appendix A1: Gamma-Ray Spectra of Surrogate Threat Sources}
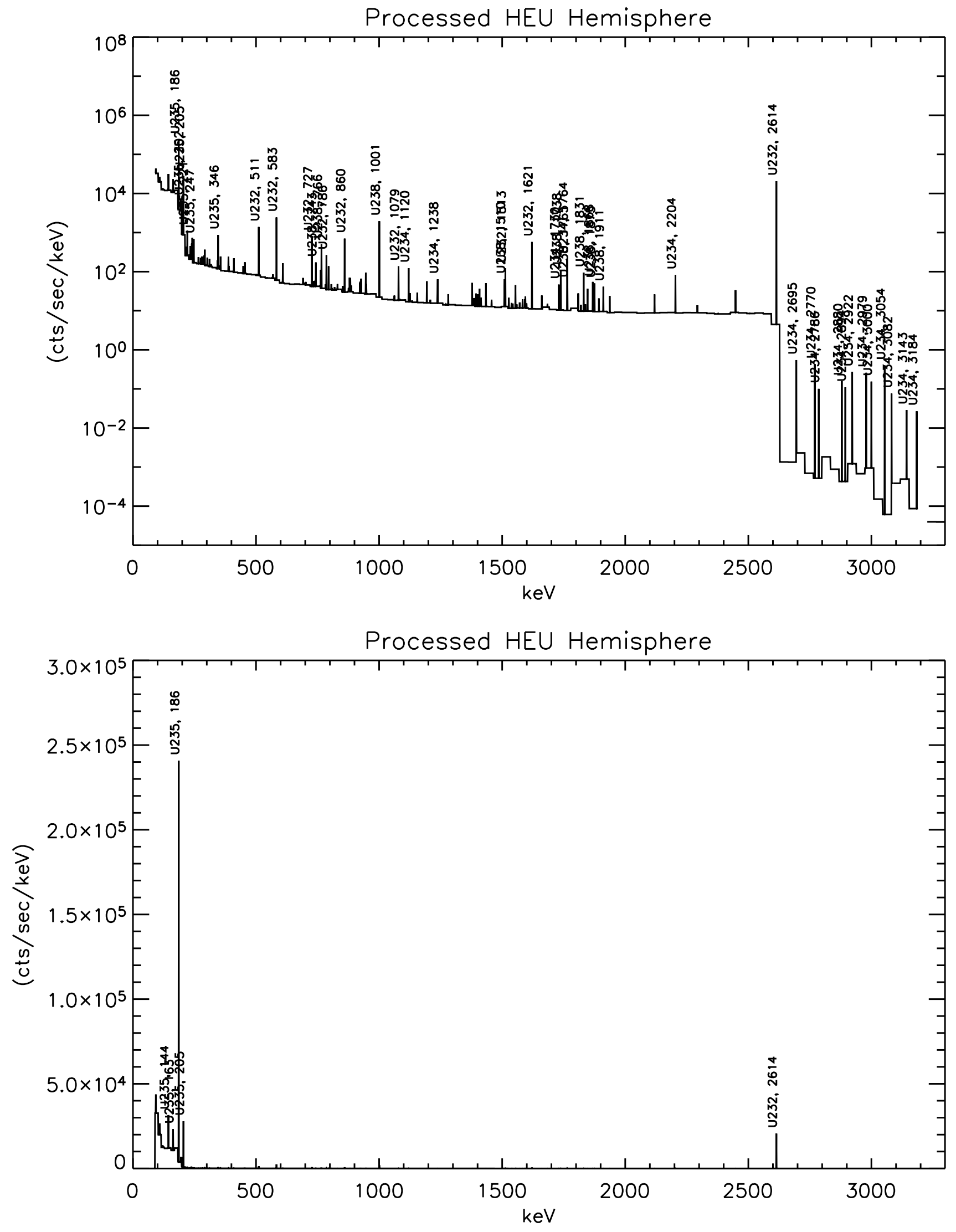

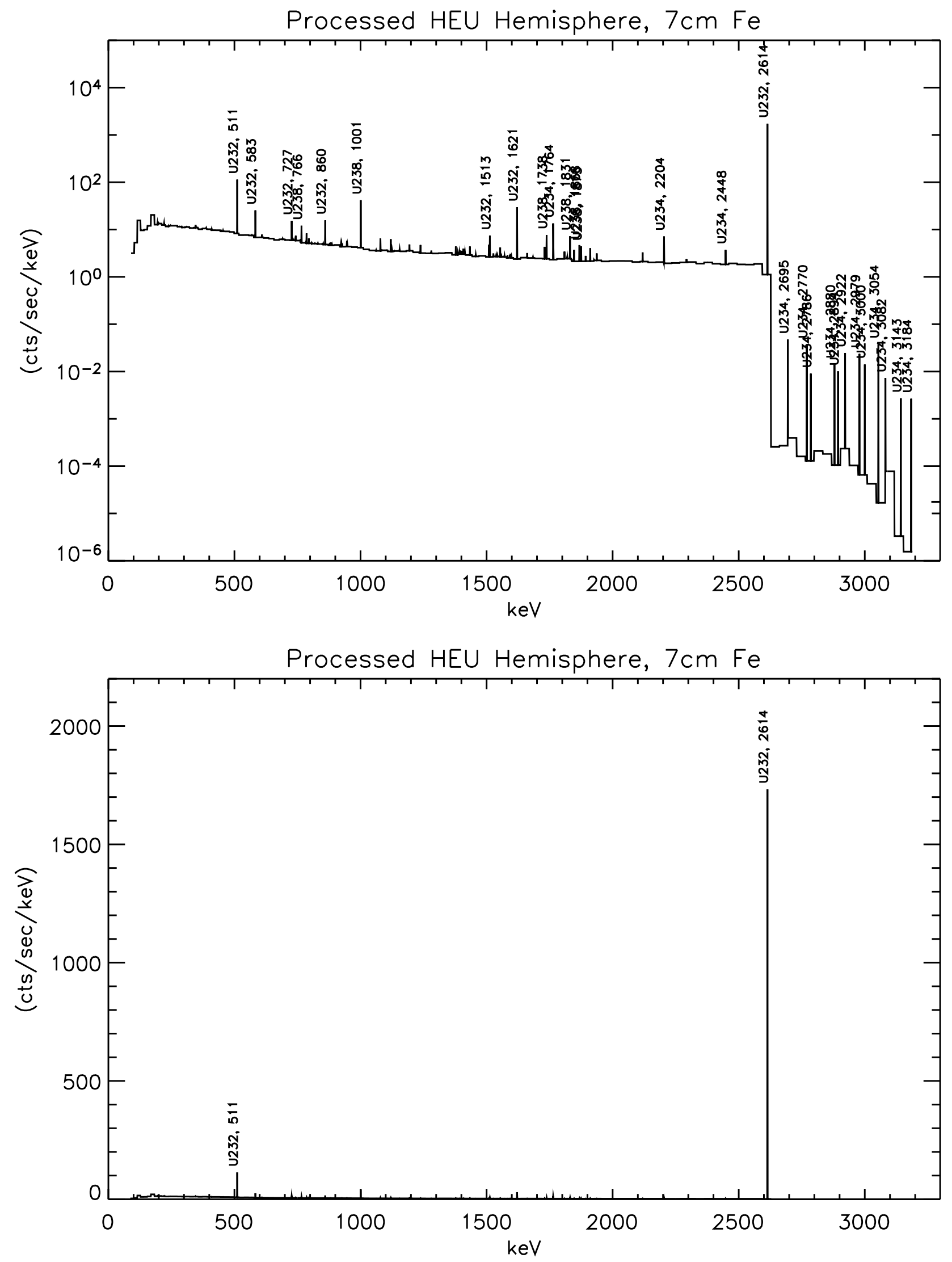


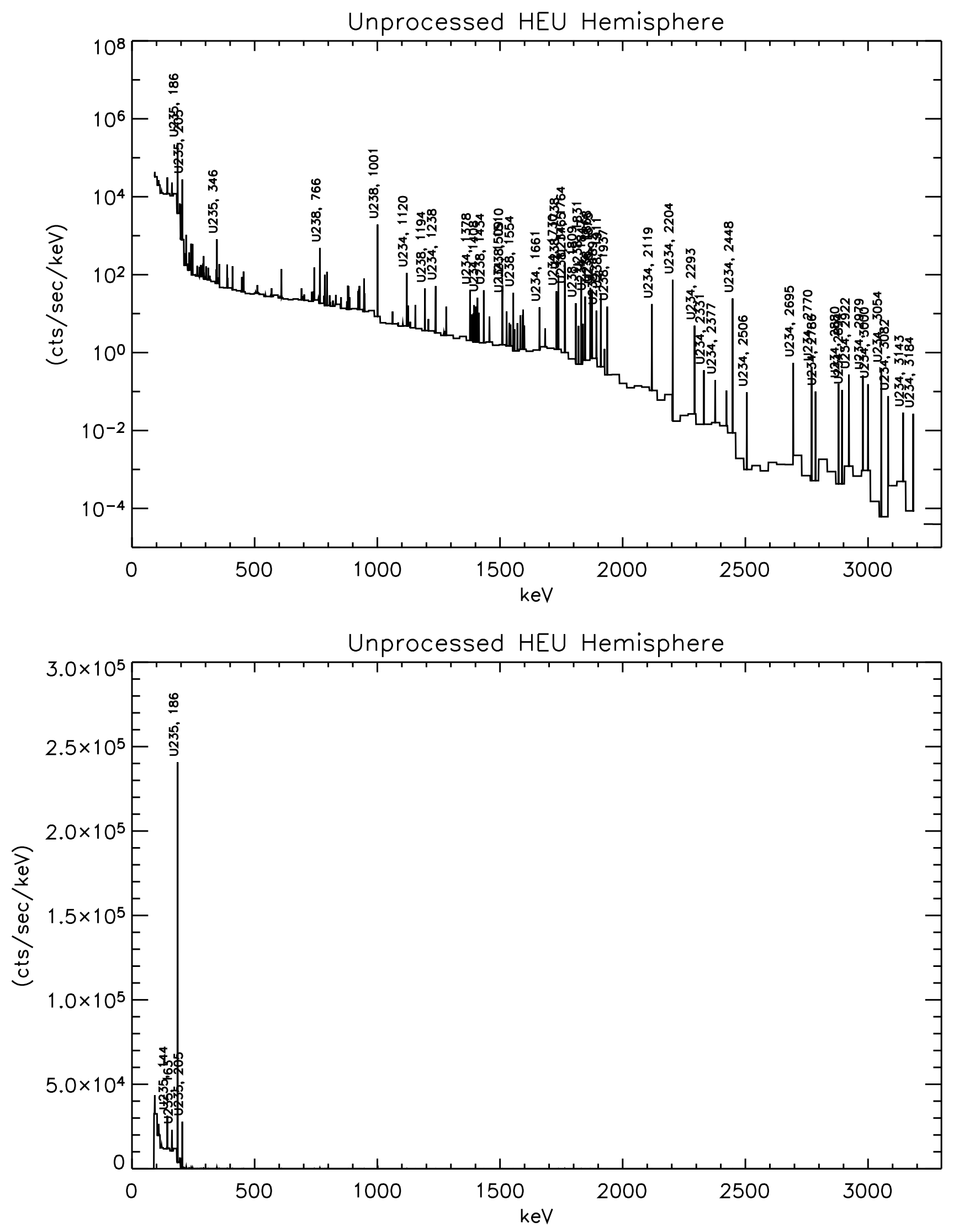



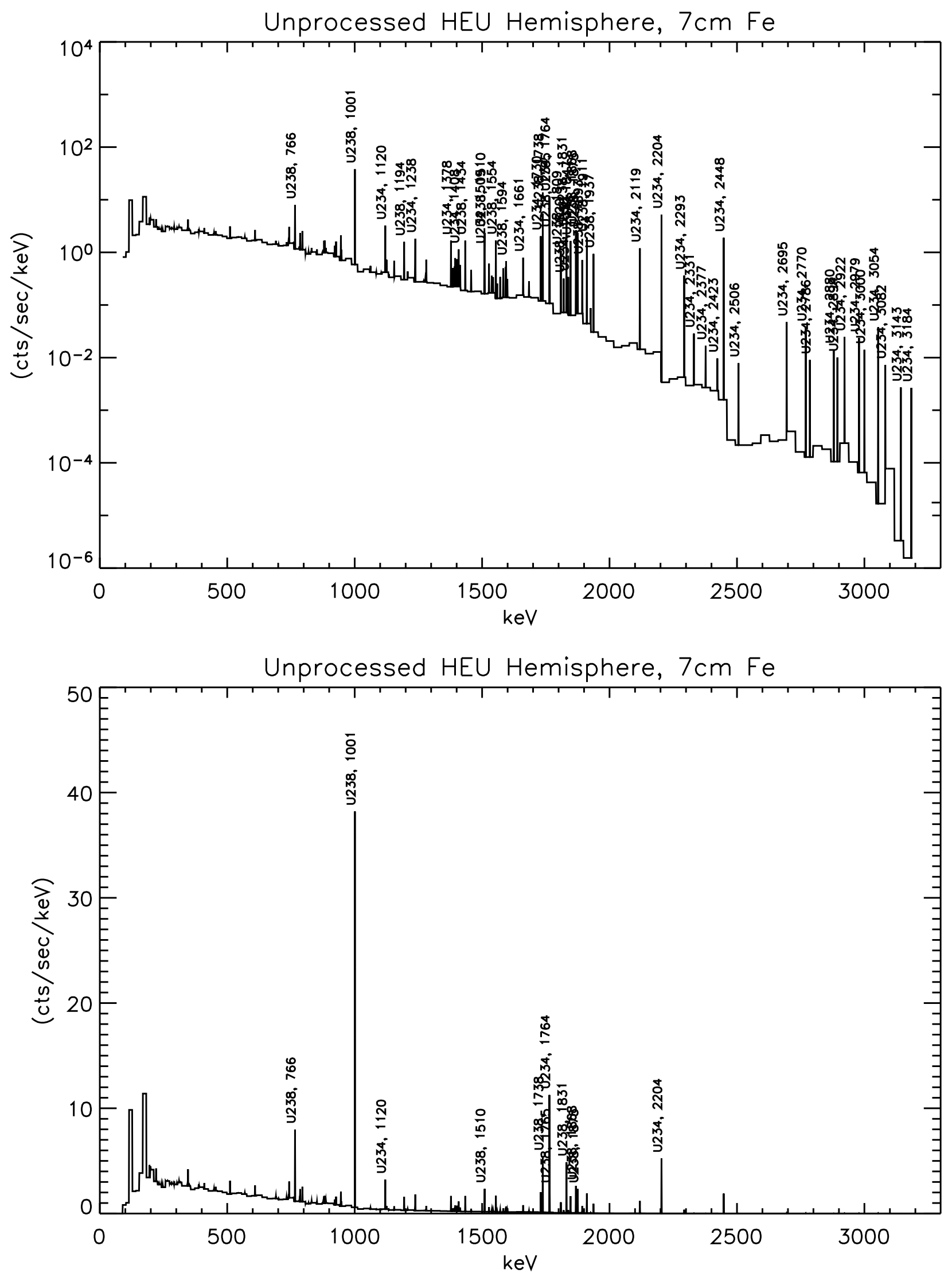

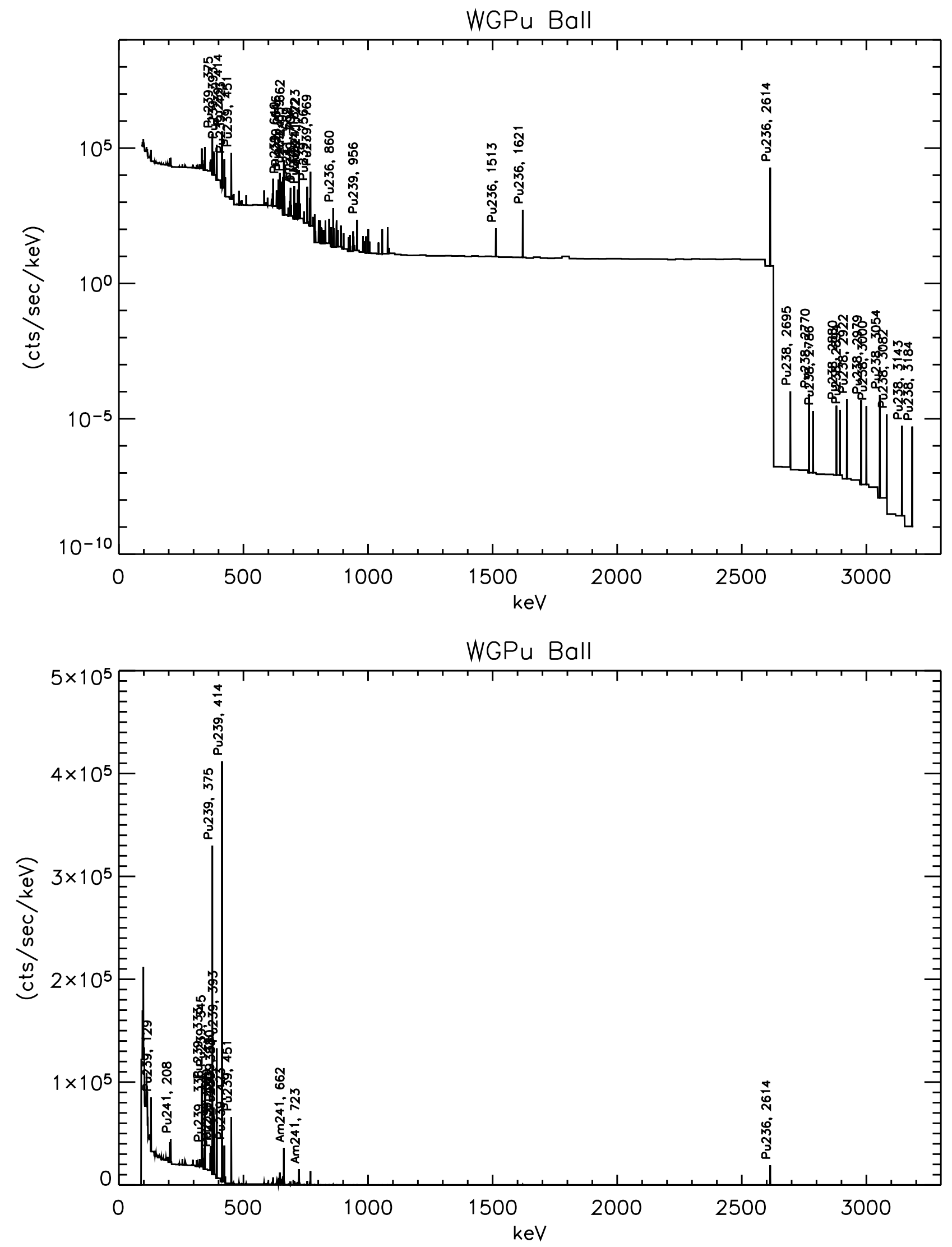

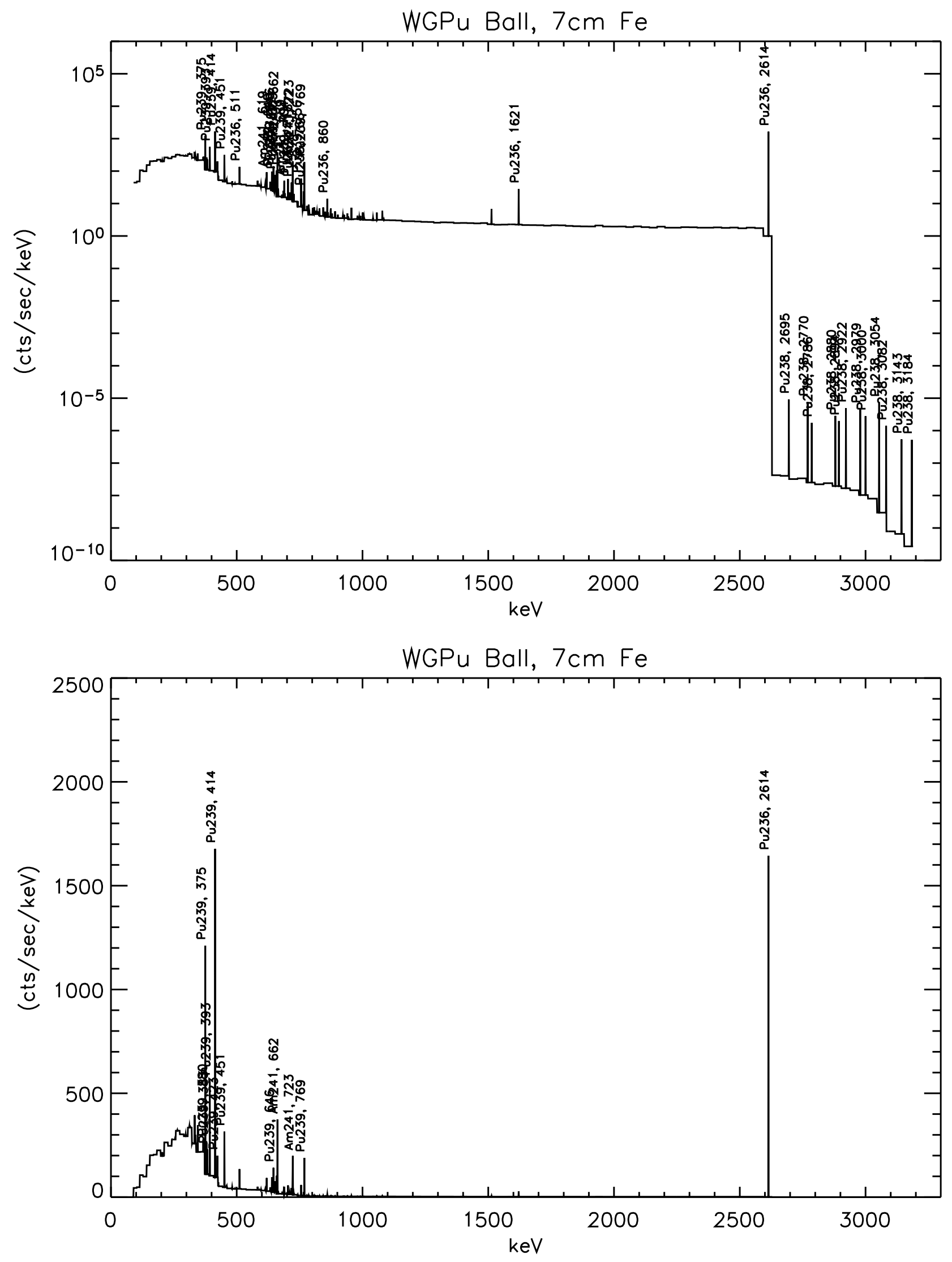

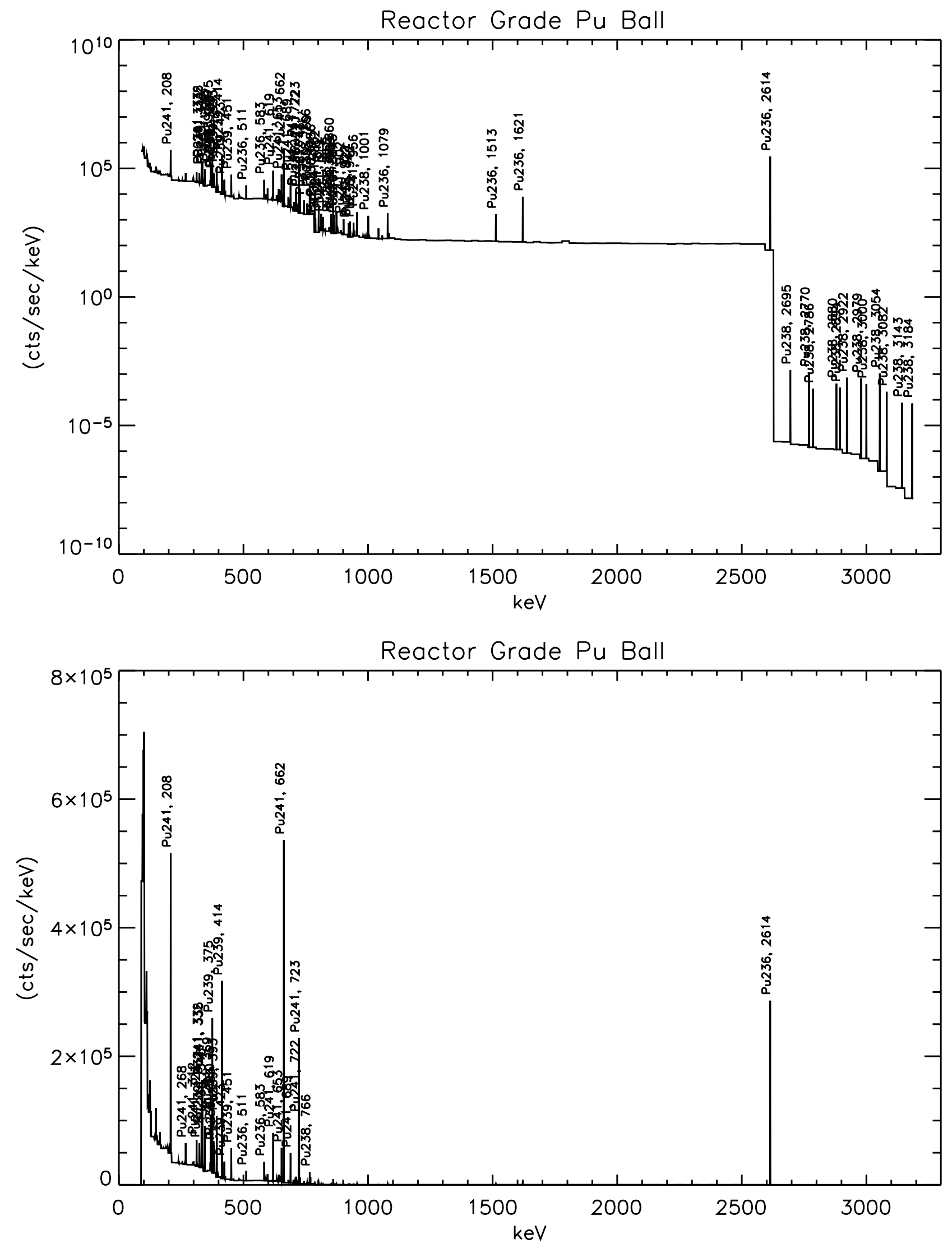

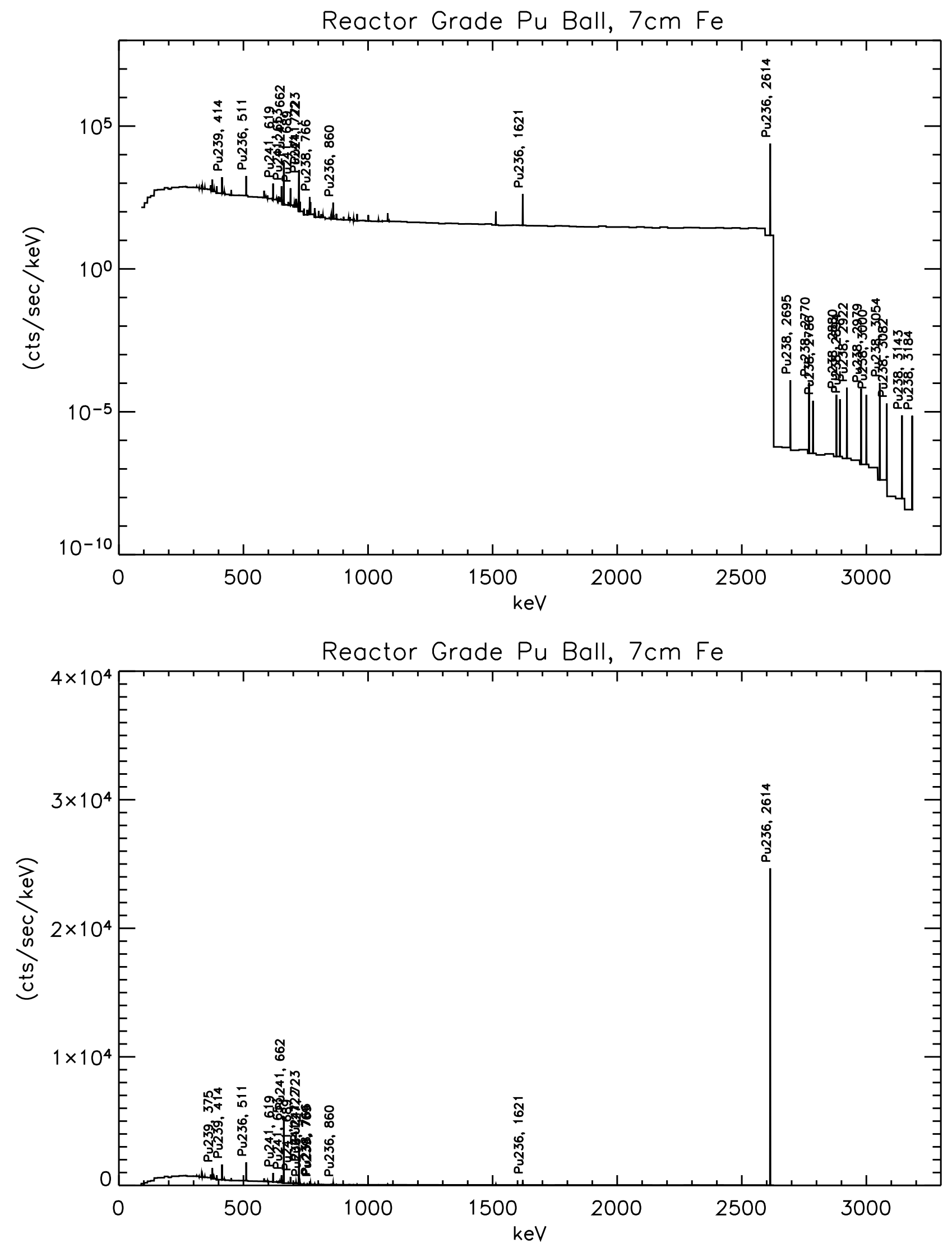

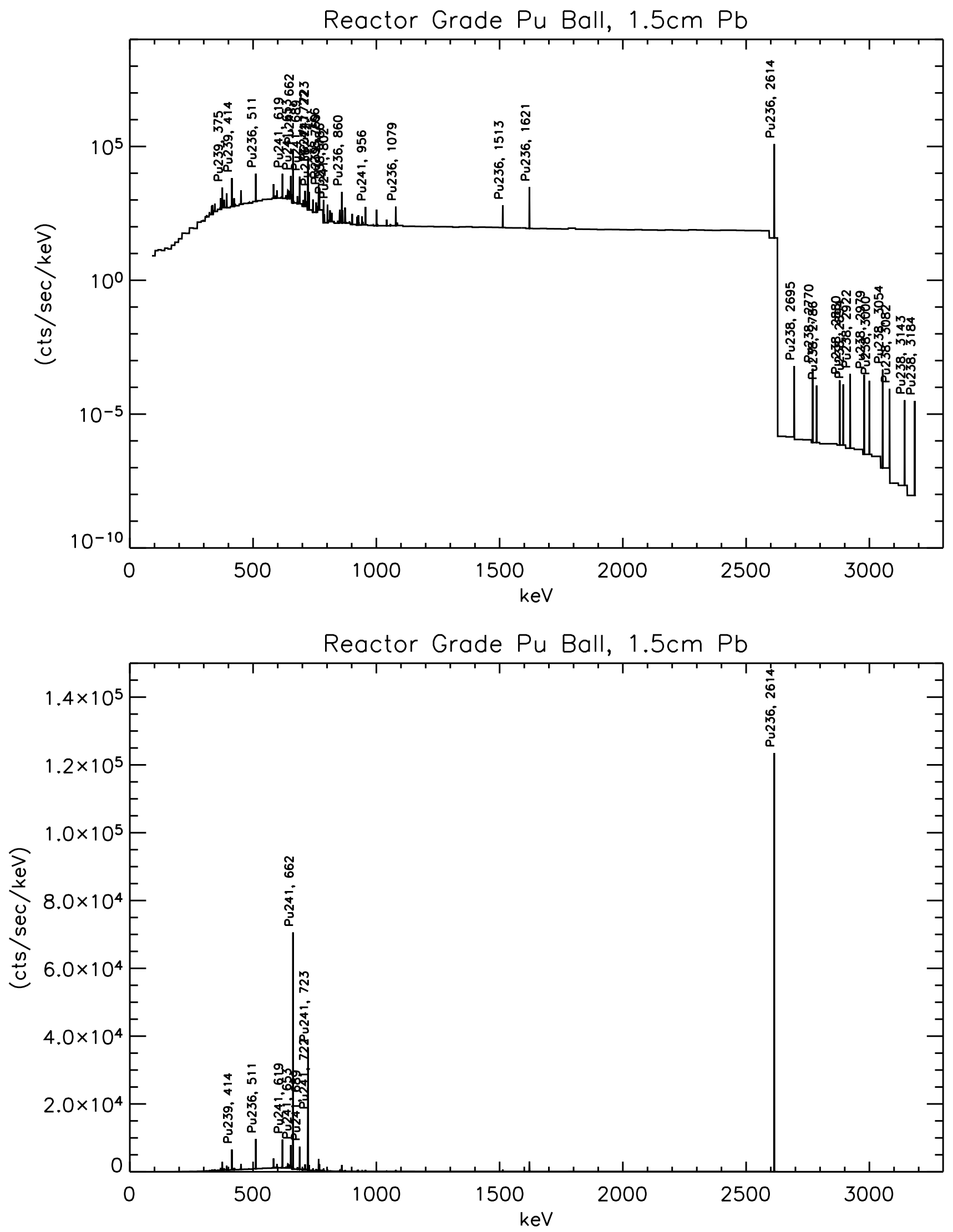

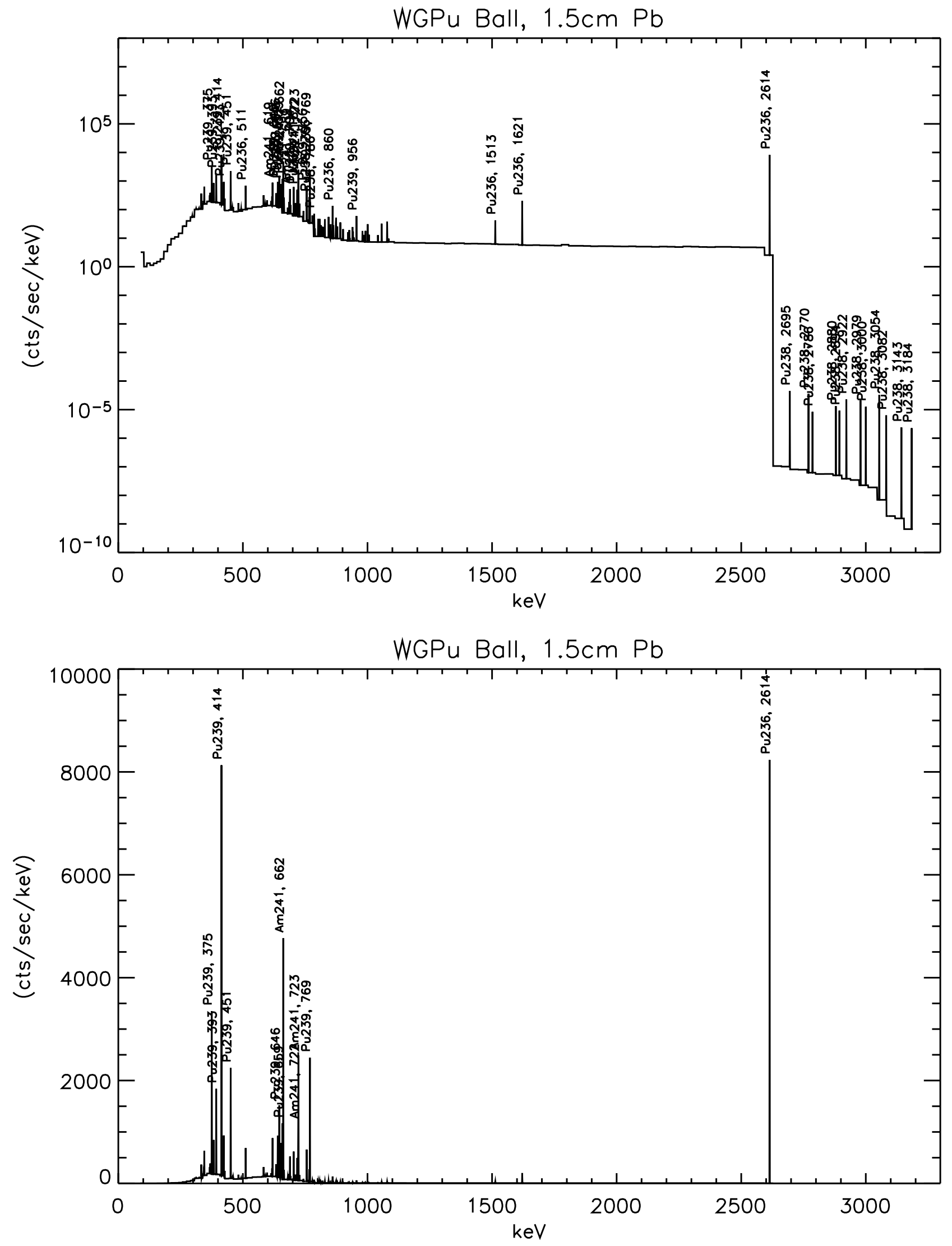


\title{
Appendix A2
}

h4doc 6v1

\section{Analytic Expressions for the Angular Resolution of Compton Gamma-ray Detectors}

\author{
Douglas Wright \\ High Energy Physics Group, N Division \\ Lawrence Livermore National Laboratory
}

September 23, 2003

Revised January 9, 2004

\section{Introduction}

This paper describes the derivation of analytic expressions for the angular resolution of reconstructing gamma rays detected via Compton interactions. We consider two types of gamma-ray detectors: Compton-ring and electron-tracking devices.

In Compton-ring devices, the direction of the scattered electron is not resolved, only the total energy (electron and scattered photon) and the scattered photon direction are measured. The measured quantities define a cone about the axis of the scattered photon direction (see Figure 1). The initial photon direction lies along this cone. Thus for single events there is a ring-like ambiguity in the photon direction. By combining multiple events, the intersection of the reconstructed rings will resolve the initial direction of the photon source. In this paper, we derive the resolution of the cone angle for individual rings.

Electron-tracking type devices resolve the electron path. Although the scattered electron subsequently undergoes multiple-Coulomb scattering, it is possible to measure the initial electron direction with sufficiently high tracking resolution. By measuring the direction and energy of the electron and the direction of the scattered photon, the initial photon direction can be uniquely determined. The challenge for this type of detector is achieving the high tracking resolution.

In Section 2 we derive the well-known Compton formula for Compton-ring devices, an analytic expression for the angular resolution of the cone angle, and discuss the limits for applying the error formula. In Section 3 we repeat the derivation of the error function for the algebraically more complicated electron-tracking device. In the final section (Section 4) we derive the effect of position measurement error on the angular resolution, which applies to both detector types. All of the analytic results are cross-checked against empirical fits to a simple Monte Carlo simulation (Sections 2.5, 3.3, and 4.2).

Doppler broadening, the effect due to the initial (and intrinsically unknown) momentum of the atomic electron, can be ignored for gamma rays with initial energy greater than a few hundred $\mathrm{keV}$. For all kinematic calculations in this paper, the electron is taken to be initially at rest. 


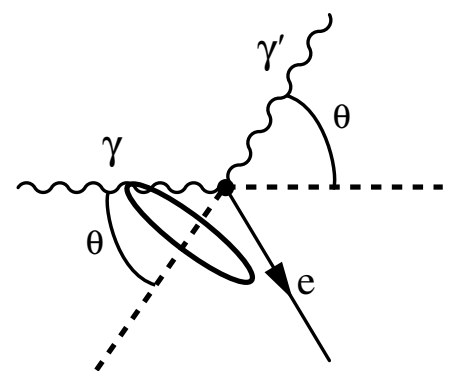

Figure 1: Compton interaction in which an incoming photon $(\gamma)$ scatters off of an atomic electron. Unless the electron direction is resolved, there is a $\phi$-like ambiguity (represented by the ring in the drawing) for the reconstructed initial photon direction.

\section{Compton-ring devices}

\subsection{Derivation of the Compton formula}

Assume that the initial electron is at rest, then from conservation of momentum, the electron recoils from the scattered photon (see Figure 1):

$$
\vec{p}_{e}=\vec{p}_{\gamma}-\vec{p}_{\gamma^{\prime}}
$$

Taking the square of the momentum and using $E_{\gamma}=p_{\gamma}$ and $E_{\gamma^{\prime}}=p_{\gamma^{\prime}}$ (since photons are massless and we are using units where $c=1$ ), results in

$$
p_{e}^{2}=E_{\gamma}^{2}+E_{\gamma^{\prime}}^{2}-2 E_{\gamma} E_{\gamma^{\prime}} \cos \theta \text {. }
$$

Using the invariant mass relation $p_{e}{ }^{2}=E_{e}{ }^{2}-m_{e}{ }^{2}$ and conservation of energy $\left(E_{\gamma}+m_{e}=E_{e}+E_{\gamma^{\prime}}\right)$ to eliminate the electron variables $p_{e}$ and $E_{e}$ results in

$$
\begin{array}{r}
E_{\gamma^{\prime}}+2 m_{e} E_{\gamma}+m_{e}{ }^{2}-2 E_{\gamma} E_{\gamma^{\prime}}-2 m E_{\gamma^{\prime}}+E_{\gamma^{\prime}}{ }^{2}-m_{e}{ }^{2} \\
=E_{\gamma^{\prime}}{ }^{2}+E_{\gamma^{\prime}}{ }^{2}-2 E_{\gamma} E_{\gamma^{\prime}} \cos \theta,
\end{array}
$$

which reduces to

$$
m_{e}\left(E_{\gamma}-E_{\gamma^{\prime}}\right)=E_{\gamma} E_{\gamma^{\prime}}(1-\cos \theta) .
$$

This is often rewritten as

$$
\cos \theta=1+\frac{m_{e}}{E_{\gamma}}-\frac{m_{e}}{E_{\gamma^{\prime}}}
$$

\subsection{Compton formula implications}

As the electron kinetic energy, $K_{e}=E_{e}-m_{e}$, approaches zero the scattering angle $\theta$ also goes to zero. Obviously at zero angle there is no scattering and the outgoing photon has the same energy as the incoming photon $\left(E_{\gamma}=E_{\gamma^{\prime}}\right)$. 
At the other extreme, the maximum electron energy (and minimum outgoing photon energy) occurs at $\theta=\pi$, i.e., when the photon scatters back in the direction opposite to that of the incoming photon. The maximum electron kinetic energy is

$$
K_{e}(\max )=\frac{E_{\gamma}^{2}}{E_{\gamma}+m_{e} / 2}
$$

while the minimum outgoing photon energy is not zero, but is instead

$$
E_{\gamma^{\prime}}(\min )=\frac{m_{e}}{2+m_{e} / E_{\gamma}}=\frac{1}{2} K_{e}(\max )\left(\sqrt{2 m_{e} / K_{e}(\max )+1}-1\right) .
$$

\subsection{Derivation of Compton angle error}

The Compton angle $\theta$ in Equation 1 depends only on the energy of the incoming $\left(E_{\gamma}=E_{\gamma^{\prime}}+E_{e}-m_{e}\right)$ and outgoing $\left(E_{\gamma^{\prime}}\right)$ gamma rays. If one considers only small Gaussian errors for the observables $E_{e}$ and $E_{\gamma^{\prime}}$, then one can derive an analytic expression for the error on $\theta$ as follows.

Taking the differential of Equation 1 yields

$$
d(\cos \theta)=-\sin \theta d \theta=-\frac{m_{e}}{E_{\gamma}{ }^{2}} d E_{\gamma}+\frac{m_{e}}{E_{\gamma^{\prime}}{ }^{2}} d E_{\gamma^{\prime}},
$$

which can be rewritten as

$$
d \theta=\frac{m_{e}}{\sin \theta E_{\gamma}^{2}}\left[d E_{\gamma}-\frac{E_{\gamma^{2}}{ }^{2}}{E_{\gamma^{\prime}}{ }^{2}} d E_{\gamma^{\prime}}\right] .
$$

Since $E_{\gamma}=E_{e}+E_{\gamma^{\prime}}-m_{e}$, we have $d E_{\gamma}=d E_{e}+d E_{\gamma^{\prime}}$. Applying this to the previous equation for $d \theta$ results in

$$
d \theta=\frac{m_{e}}{\sin \theta E_{\gamma}^{2}}\left[d E_{e}+\left(1-\frac{E_{\gamma^{2}}^{2}}{E_{\gamma^{\prime}}{ }^{2}}\right) d E_{\gamma^{\prime}}\right] .
$$

This equation defines the partial derivatives $\partial \theta / \partial E_{e}$ and $\partial \theta / \partial E_{\gamma^{\prime}}$. So for Gaussian errors $\delta E_{e}$ and $\delta E_{\gamma^{\prime}}$, the error estimate for $\theta$ is

$$
\delta \theta=\frac{m_{e}}{\sin \theta E_{\gamma}{ }^{2}} \sqrt{\delta E_{e}{ }^{2}+\left(1-\frac{E_{\gamma}{ }^{2}}{{E_{\gamma^{\prime}}}^{2}}\right)^{2} \delta E_{\gamma^{\prime}}{ }^{2}} .
$$

This equation is valid as long as the fractional errors $\left(\delta E_{e} / E_{e}\right.$ and $\left.\delta E_{\gamma^{\prime}} / E_{\gamma^{\prime}}\right)$ are not too large. Furthermore, one must be careful when applying this equation in situations near $\theta=0$ or $\pi$.

\subsection{Caveats in error function near $\theta=0$ or $\pi$}

The case where $\theta$ is exactly zero is not a problem since this only occurs when the electron kinetic energy $\left(K_{e}=E_{e}-m_{e}\right)$ is zero. Thus there is no detected first interaction and one would not apply the Compton formula. Arbitrarily small values of $K_{e}$ also do not cause a problem for Equation 3 until they approach the magnitude of the energy resolution itself. 
For arbitrarily small values of the kinetic energy, i.e., $K_{e} / E_{\gamma} \ll 1$, the Compton formula (Equation 1) and energy conservation $\left(E_{\gamma^{\prime}}=E_{\gamma}-K_{e}\right)$ imply

$$
\sin ^{2} \theta \simeq \frac{2 m_{e} K_{e}}{E_{\gamma}^{2}}
$$

to lowest order in $K_{e} / E_{\gamma}$. Thus to lowest order, the square of Equation 3 can be approximated as

$$
\delta \theta^{2} \simeq \frac{m_{e}}{2 E_{\gamma}{ }^{2}}\left[\frac{\delta K_{e}^{2}}{K_{e}}+\frac{4 K_{e}}{E_{\gamma}{ }^{2}} \delta E_{\gamma^{\prime}}{ }^{2}\right] .
$$

From this one can see that $\delta \theta^{2}$ begins to blow up when the the kinetic energy becomes smaller than the square of the energy resolution $\delta K_{e}$, but is well behaved otherwise. Note that the second term $\left(\delta E_{\gamma^{\prime}}{ }^{2}\right)$ is suppressed for small $K_{e}$. When $K_{e}$ is smaller than about twice the energy resolution, the Gaussian approximation itself breaks down and Equation 3 will begin to overestimate the error in $\theta$. To apply this formula, one should impose a lower cutoff on the electron energy based on the energy resolution.

For the case where $\theta$ is near $\pi$, the scattered photon energy is near its minimum (but can not be zero - see Section 2.2). Because of finite detector resolution, the observed photon energy can fluctuate downward and even be lower than the minimally allowed energy. These kinematically unallowed events will be explicitly rejected since the Compton formula itself fails. However, the case where the photon energy reaches its minimum is kinematically allowed. For these events at or very near the minimum, the error formula approaches infinity. To highlight the behavior near the minimum energy, we can rewrite the equation using $E_{\gamma^{\prime}}=E_{\gamma^{\prime}}(\min )(1+\epsilon)$, where $\epsilon \ll 1$. In this limit, the Compton formula (Equation 1) implies

$$
\sin ^{2} \theta \simeq \frac{2 \epsilon m_{e}}{E_{\gamma}}
$$

Thus to lowest order, the square of Equation 3 can be approximated as

$$
\delta \theta^{2} \simeq \frac{m_{e}}{2 \epsilon E_{\gamma}^{3}}\left[\delta E_{e}{ }^{2}+\left(1-\frac{(1-2 \epsilon) E_{\gamma}{ }^{2}}{E_{\gamma^{\prime}}{ }^{2}(\min )}\right)^{2} \delta E_{\gamma^{\prime}}{ }^{2}\right] .
$$

Neither term ( $\delta E_{e}$ or $\left.\delta E_{\gamma^{\prime}}\right)$ is suppressed, so in order to avoid this infinity (when $\epsilon \rightarrow 0$ ), one should eliminate events that are less than at least one standard deviation (in terms of the energy resolution) away from the minimum photon energy.

\subsection{Numerical cross-check of Compton-ring error function}

To check the validity of Equation 3 we performed a simple Monte Carlo analysis by generating Gaussian distributions for the input variables $E_{e}$ and $E_{\gamma^{\prime}}$ and compared the resulting distribution of $\theta$ with the analytic expression. We generated 100,000 events where the Compton electron kinetic energy $\left(E_{e}-m_{e}\right)$ was sampled from a Gaussian distribution with a mean of $100 \mathrm{keV}$ and RMS of $1 \mathrm{keV}$, and the scattered gamma ray energy distribution had a mean of $400 \mathrm{keV}$ with an RMS of $2 \mathrm{keV}$.

Using Equation 1 we reconstruct the Compton angle $\theta$ from the simulated observables. Figure 2 shows the reconstructed $\theta$ distribution. We fit a simple Gaussian function to this distribution. The parameters from the fit along with the results from the analytic expressions (Equations 1 and 3) are given in Table 1 The agreement between the fit and the analytic expression is excellent. 


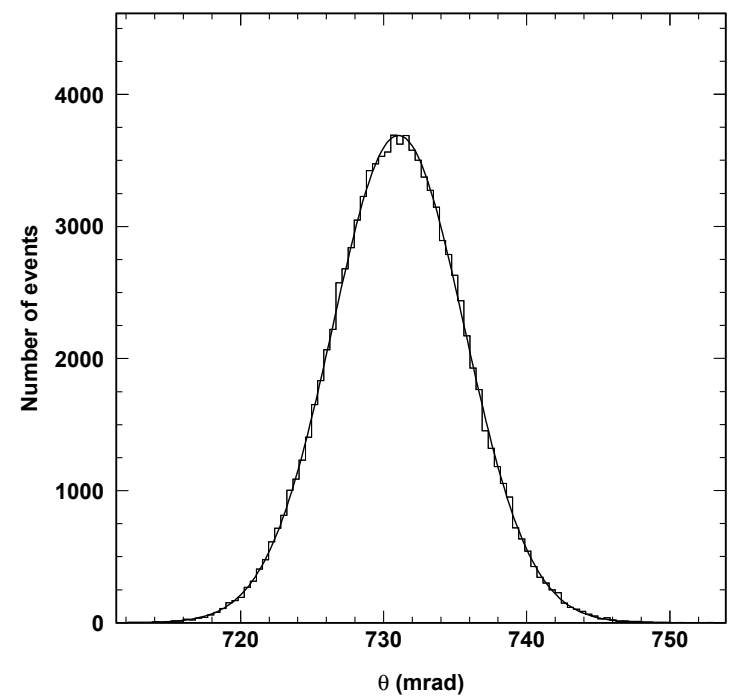

Figure 2: Compton-ring detector. Reconstructed Compton angle $\theta$ from a simulation of a single physical event sampled 100,000 times with Gaussian errors for the observed energies. The fit result is superimposed on the distribution.

Table 1: Compton-ring detector. Compton angle and error comparison between a fit to the simulation and an analytic calculation. Sample of 100,000 events where the electron kinetic energy is $100 \mathrm{keV}$ with an RMS error of $1 \mathrm{keV}$, and scattered gamma-ray energy of $400 \mathrm{keV}$ with an RMS error of $2 \mathrm{keV}$.

\begin{tabular}{|r|r|r|}
\hline Variable & Fit Result & Analytic Result \\
\hline$\theta(\mathrm{mrad})$ & $731.03 \pm 0.02$ & 731.01 \\
$\delta \theta(\mathrm{mrad})$ & $4.61 \pm 0.01$ & 4.61 \\
\hline
\end{tabular}




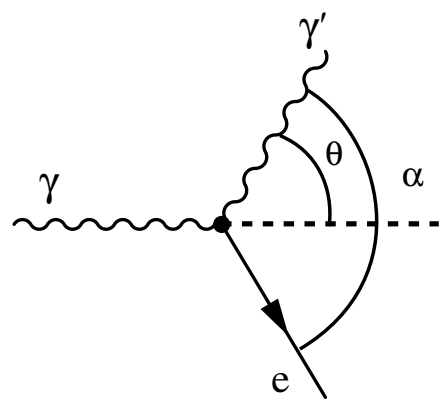

Figure 3: Compton interaction. Same as Figure 1 with the scattered photon and electron opening angle labeled as $\alpha$.

\section{Electron-tracking devices}

\subsection{Derivation of kinematic relations (à la Compton formula)}

The kinematic relations derived in Section 2 also apply to the electron-tracking type detector. Since the electron-tracking devices resolve the direction of both the electron and photon, it is useful to derive some additional relations in terms of the opening angle $\alpha$ (defined in Figure 3) of the observed particles. From conservation of momentum $\vec{p}_{\gamma}=\vec{p}_{\gamma^{\prime}}+\vec{p}_{e}$ and using $E_{\gamma}=p_{\gamma}$ and $E_{\gamma^{\prime}}=p_{\gamma^{\prime}}$ (since photons are massless), the square of the initial photon momentum is

$$
E_{\gamma}{ }^{2}=E_{\gamma^{\prime}}{ }^{2}+p_{e}{ }^{2}+2 E_{\gamma^{\prime}} p_{e} \cos \alpha .
$$

Using the invariant mass relation $p_{e}{ }^{2}=E_{e}{ }^{2}-m_{e}{ }^{2}$ and conservation of energy $\left(E_{\gamma}=E_{\gamma^{\prime}}+E_{e}-m_{e}\right)$ to eliminate the incoming photon energy $E_{\gamma}$ from the previous equations gives

$$
E_{\gamma^{\prime}}{ }^{2}+2 E_{\gamma^{\prime}}\left(E_{e}-m_{e}\right)+\left(E_{e}-m_{e}\right)^{2}=E_{\gamma^{\prime}}{ }^{2}+p_{e}{ }^{2}+2 E_{\gamma^{\prime}} p_{e} \cos \alpha .
$$

Solving for $E_{\gamma^{\prime}}$ results in

$$
E_{\gamma^{\prime}}=\frac{m_{e}\left(E_{e}-m_{e}\right)}{E_{e}-m_{e}-p_{e} \cos \alpha} .
$$

Applying conservation of energy $\left(E_{\gamma}=E_{\gamma^{\prime}}+E_{e}-m_{e}\right)$ to the above equation gives

$$
E_{\gamma}=\frac{\left(E_{e}-m_{e}\right)\left(E_{e}-p_{e} \cos \alpha\right)}{E_{e}-m_{e}-p_{e} \cos \alpha} .
$$

Note that the ratio of Equation 4 and 5 is

$$
\frac{E_{\gamma}}{E_{\gamma^{\prime}}}=\frac{E_{e}-p_{e} \cos \alpha}{m_{e}} .
$$

Combing the Compton formula (Equation 1) with Equations 4 and 5 yields a relation between the Compton angle $\theta$ and the angle between the electron and scattered photon $\alpha$ :

$$
\cos \theta=1-\frac{\left(E_{e}-m_{e}-p_{e} \cos \alpha\right)^{2}}{\left(E_{e}-m_{e}\right)\left(E_{e}-p_{e} \cos \alpha\right)} .
$$




\subsection{Derivation of electron-tracking angular error function}

The error function derived in Section 2 also applies for electron tracking devices with one important distinction: since the electron trajectory is measured, there is no ambiguity in determining the direction of the initial photon. One component of the photon direction is completely determined by the plane formed by the scattered electron and scattered photon. The error on the angular component within that plane is calculated below.

We start with the analytic expression for the angular error $\mathrm{d} \theta$ given by Equation 2. For electron tracking devices we measure the electron energy $\left(E_{e}\right)$ but not the scattered photon energy $\left(E_{\gamma^{\prime}}\right)$. Using Equation 4 we can find the relationship between the error on $E_{\gamma^{\prime}}$ with the error on the measured quantities $E_{e}$ and $\alpha$. We can then combine this relation with the previously derived error function to find an equation in terms of measured quantities only.

Taking the differential of Equation 4 gives

$$
d E_{\gamma^{\prime}}=\frac{m_{e} d E_{e}}{E_{e}-m_{e}-p_{e} \cos \alpha}-\frac{m_{e}\left(E_{e}-m_{e}\right)}{\left(E_{e}-m_{e}-p_{e} \cos \alpha\right)^{2}}\left[d E_{e}-\cos \alpha d p_{e}+p_{e} \sin \alpha d \alpha\right]
$$

Using $E_{e} d E_{e}=p_{e} d p_{e}$ (which follows from $E_{e}{ }^{2}=p_{e}{ }^{2}-m_{e}{ }^{2}$ ) and Equations 4 and 5 to simplify the expression, we get

$$
d E_{\gamma^{\prime}}=\left(\frac{E_{\gamma^{\prime}}}{E_{e}-m_{e}}-\frac{E_{\gamma^{\prime}}^{2}\left(p_{e}-E_{e} \cos \alpha\right)}{m_{e} p_{e}\left(E_{e}-m_{e}\right)}\right) d E_{e}-\frac{E_{\gamma^{\prime}}{ }^{2} p_{e} \sin \alpha}{m_{e}\left(E_{e}-m_{e}\right)} d \alpha
$$

Substituting this into Equation 2 and regrouping in terms of $\mathrm{d} E_{e}$ and $\mathrm{d} \alpha$ gives

$$
d \theta=\frac{\left(E_{\gamma}^{2}-E_{\gamma^{\prime}}{ }^{2}\right)}{E_{\gamma}{ }^{2}\left(E_{e}-m_{e}\right) \sin \theta}\left[\left(1-\frac{m_{e}}{E_{\gamma^{\prime}}}-\frac{E_{e}}{p_{e}} \cos \alpha+\frac{m_{e}\left(E_{e}-m_{e}\right)}{E_{\gamma}{ }^{2}-E_{\gamma^{\prime}}{ }^{2}}\right) d E_{e}+p_{e} \sin \alpha d \alpha\right] .
$$

This equation defines the partial derivatives $\partial \theta / \partial E_{e}$ and $\partial \theta / \partial \alpha$. So for Gaussian errors $\delta E_{e}$ and $\delta \alpha$, the error estimate for $\theta$ is

$$
\delta \theta=\frac{\left(E_{\gamma}{ }^{2}-E_{\gamma^{\prime}}{ }^{2}\right)}{E_{\gamma}{ }^{2}\left(E_{e}-m_{e}\right)^{2} \sin \theta} \sqrt{\left(1-\frac{m_{e}}{E_{\gamma^{\prime}}}-\frac{E_{e}}{p_{e}} \cos \alpha+\frac{m_{e}\left(E_{e}-m_{e}\right)}{E_{\gamma}{ }^{2}-E_{\gamma^{\prime}}{ }^{2}}\right)^{2} \delta E_{e}{ }^{2}+p_{e}{ }^{2} \sin ^{2} \alpha \delta \alpha^{2}} .
$$

This equation is valid as long as the fractional errors in the measured quantities are not too large. As in the Compton-ring case, one must be careful when applying this error function near $\theta=0$ or $\pi$. Also note that this is just the error of one component of the angle. The orthogonal component and its associated error are completely determined by the plane defined by the observed electron and photon trajectories.

\subsection{Numerical cross-check of electron-tracking error function}

To check the validity of Equation 7 we performed a simple Monte Carlo analysis by generating Gaussian distributions for the input variables $E_{e}$ and $\alpha$ and compared the resulting distribution of $\theta$ with the analytic expression. We generated 100,000 events where the Compton electron kinetic energy $\left(E_{e}-m_{e}\right)$ was sampled from a Gaussian distribution with a mean of $1000 \mathrm{keV}$ and RMS of $10 \mathrm{keV}$, 


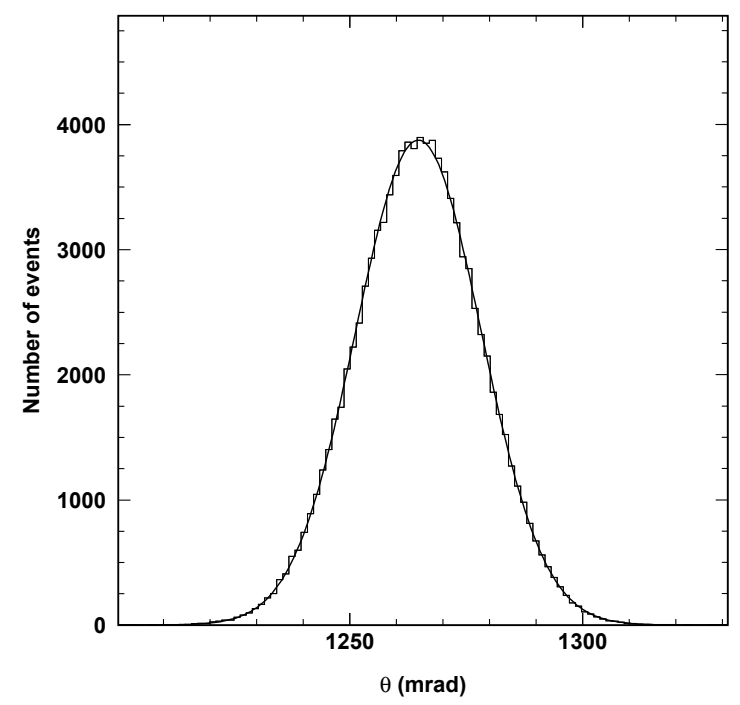

Figure 4: Reconstructed Compton angle $\theta$ from a simulation of a single physical event sampled 100,000 times with Gaussian errors for the observed energies. The fit result is superimposed on the distribution.

Table 2: Electron-tracking detector. Compton angle and error comparison between a fit to simulation and an analytic calculation. Sample of 100,000 events where the Electron kinetic energy is $1000 \mathrm{keV}$ with an RMS error of $10 \mathrm{keV}$, and the opening angle between the scattered electron and gamma ray is $1600 \mathrm{mrad}$ with an RMS of $10 \mathrm{mrad}$. with and RMS error of $2 \mathrm{keV}$.

\begin{tabular}{|r|r|r|}
\hline Variable & Fit Result & Analytic Result \\
\hline$\theta(\mathrm{mrad})$ & $1264.70 \pm 0.04$ & 1264.75 \\
$\delta \theta(\mathrm{mrad})$ & $13.47 \pm 0.03$ & 13.49 \\
\hline
\end{tabular}

and the opening angle between the scattered electron and gamma ray had a mean of 1600 mrad with an RMS of 10 mrad.

Using Equation 7 we reconstruct the angle $\theta$ from the simulated observables. Figure 4 shows the reconstructed $\theta$ distribution. We fit a simple Gaussian function to this distribution. The parameters from the fit along with the results from the analytic expressions (Equations 6 and 7) are given in Table 2. The agreement between the fit and the analytic expression is excellent. 


\section{Generic effects}

\subsection{Derivation of angular error due to position resolution}

The direction of the outgoing photon is determined from the measured positions of the two interaction points. The measurement uncertainty of these positions creates an associated uncertainty in the direction of the photon. One can derive an analytic expression for the angular error due to the position resolution as follows.

If two points $v_{1}=\left(x_{1}, y_{1}, z_{1}\right), v_{2}=\left(x_{2}, y_{2}, z_{2}\right)$ define the direction of the outgoing photon $\vec{v}=v_{2}-v_{1}$, then the angle between the photon and some arbitrary unit vector $\hat{n}$ is

$$
\hat{n} \cdot \vec{v}=v \cos \theta=v_{\|},
$$

where $v_{\|}$is the component of $\vec{v}$ parallel to $\hat{n}$. The component perpendicular to $\hat{n}$ is then $v_{\perp}=v \sin \theta$. Taking the differential of $v \cos \theta=v_{\|}$gives

$$
d v \cos \theta-\sin \theta v d \theta=d v_{\|} .
$$

Using $v d v=v_{\perp} d v_{\perp}+v_{\|} d v_{\|}$(which comes from the differential of $v^{2}=v_{\|}^{2}+v_{\perp}{ }^{2}$ ) and substituting into the equation above, results in

$$
\cos \theta\left(\frac{v_{\perp}}{v} d v_{\perp}+\frac{v_{\|}}{v} d v_{\|}\right)-\sin \theta v d \theta=d v_{\|}
$$

Using the $\sin \theta$ and $\cos \theta$ relations, this can be simplified to

$$
d \theta=\frac{1}{v^{2}}\left(v_{\|} d v_{\perp}-v_{\perp} d v_{\|}\right) .
$$

For Gaussian measurement errors, this gives an error estimate of

$$
\delta \theta=\frac{1}{v^{2}} \sqrt{v_{\|}^{2} \delta v_{\perp}^{2}+v_{\perp}^{2} \delta v_{\|}^{2}}
$$

If one wants the error estimator for just the unit vector parallel to the photon direction, i.e., $\hat{n}=\vec{v} / v$, then the above equation reduces to $\delta \theta=\delta v / v$.

If $\delta d$ is the measurement error for each coordinate $(x, y, z)$ of each point $v_{1}$ and $v_{2}$, then the measurement error for the coordinates of the vector $\vec{v}$ are

$$
\delta v_{\perp}=\delta v_{\|}=\sqrt{2} \delta d
$$

Then Equation 8 simplifies to

$$
\delta \theta=\frac{\sqrt{2}}{v} \delta d
$$

This relation holds as long as the separation between the two interaction points $v$ is significantly larger than the measurement resolution $\delta d$. 


\subsection{Numerical cross-check of error function due to position resolution}

To check the validity of Equation 9 we performed a simple Monte Carlo analysis by generating Gaussian distributions for the individual coordinates of the two interaction points that define the photon direction. We generated 100,000 events where each coordinate had an RMS of $0.01 \mathrm{~mm}$. The mean starting and ending locations were $(0,0,0)$ and $(0,0,1) \mathrm{mm}$.

For each event we calculate the opening angle between the photon direction and the expected direction. Figure 4 shows the opening angle $\theta$ distribution. We fit a simple Gaussian times a $\sin \theta$ function (to account for the geometric solid angle effect) to this distribution. The parameters from the fit along with the results from the analytic expression (Equation 9) are given in Table 3. The agreement between the fit and the analytic expression is excellent. 


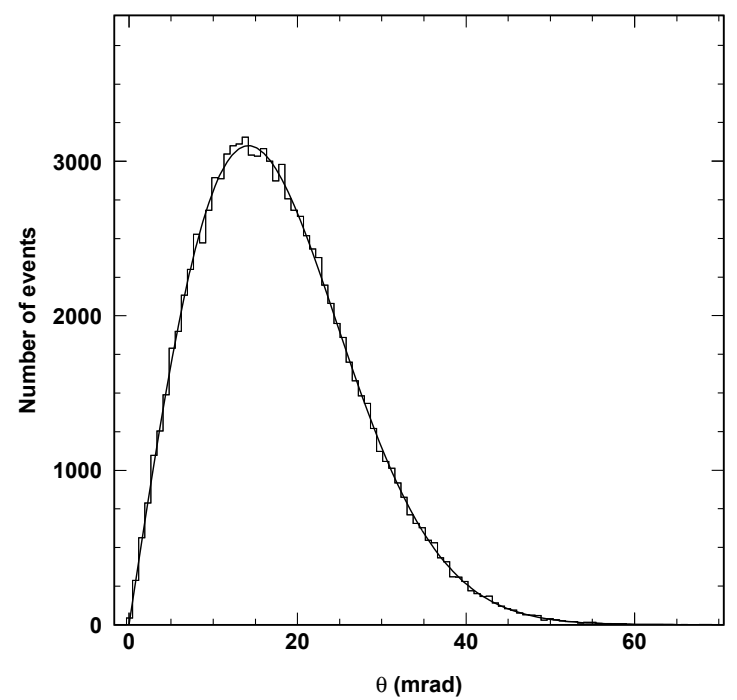

Figure 5: Angular deviation of the reconstructed photon direction from a simulation of a single physical event sampled 100,000 times with Gaussian errors for the measured interaction locations. The fit result is superimposed on the distribution.

Table 3: Opening angle comparison between the analytic calculation and the fit to the simulation. Sample of 100,000 events where the starting and ending coordinates have an RMS error of $0.01 \mathrm{~mm}$. The separation between the coordinates is $1.00 \mathrm{~mm}$

\begin{tabular}{|r|r|r|}
\hline Variable & Fit Result & Analytic Result \\
\hline$\theta(\mathrm{mrad})$ & $0.16 \pm 0.49$ & 0.00 \\
$\delta \theta(\mathrm{mrad})$ & $14.10 \pm 0.17$ & 14.14 \\
\hline
\end{tabular}




\title{
Appendix A3: Comparison of Tools to Determine Isotopic Content
}

\author{
Alan W. Meyer, James V. CAndy and Michael Axelrod
}

\section{SUMMARY}

We were asked to help perform a non-laboratory comparison of state-of-the-art tools for identifying the isotopic content of unknown gamma ray sources. First, we contacted recognized experts in the field; they are listed in Table 1. We then cataloged the existing tools and determined their principles of operation and status of development as shown in Table 2. Finally, we defined a framework to make a meaningful and fair comparison of these tools.

We found that the tools differ in significant ways as to both what they report and how they work. This makes a comparison between and among these tools very difficult. For example, it appears that only GADRAS/FIT2B can run on "automatic pilot." All the rest require an expert to run the software and interpret the output. The novice user will almost surely fail. Another problem we found is a severe lack of documentation. It's hard to know what a given tool is supposed to do and exactly how it does it, and what the output means. For example, we found that GADRAS is actually doing a form of regression analysis known, as "subset selection." This fact emerged only after an extended discussion with the author Dean Mitchell of Sandia National Laboratory. It also appears that no tool has been verified beyond the claims of its developer.

To cope with these problems we decided to define a general framework for comparison by considering the isotope identification problem as a classification problem where the measured spectrum is put into a unique category such as: "Plutonium." Alternatively, and even more simply, "SNM." Now we can use the statistical theory of supervised learning to understand how the tools work and how well they perform. With a common framework, we can compare the tools by appropriately scoring their output errors.

\section{DETAILS}

Our technical approach initially proceeded along three sequential tasks. The first task identified the significant spectral identification tools that currently exist. Concurrently, we developed an evaluation methodology. Finally, since an inadequate number of viable algorithms were identified, the third task, to execute the test plan, was over come by events. This unanticipated turn of events compelled a revision of these initial three tasks. The initial scope of three tasks was modified and expanded to become the following five tasks: The location and cataloging of existing source identification tools; The analysis of the algorithm approach and structure of these tools; The development of a methodology that ensures equitable comparison of these tools; The analysis comparing the capabilities of each candidate tool; and The development of a classification post processing framework that enables an equitable comparison without modifying any of the algorithms. 
A. We reviewed existing source identification tools and constructed a comprehensive table cataloging our results

The first task in this project conducted a methodical search to identify all existing isotope identification tools, and understand the functional principles and operations of each of these tools. To facilitate this search, we interviewed five leading experts in the field of isotope identification tools. This panel of experts is enumerated in Table 1. Table 2 catalogs the tools identified by this panel of experts with their comments summarized in the right most column. The point of contact is the individual, or, in the case of commercial tools it is the company, currently developing or maintaining the codes. The third column indicates the type of physical detector with which the code is designed to work.

Table 1: Our panel of experts.

\begin{tabular}{|l|l|l|}
\hline Dean Mitchel & GADRAS/FIT2DB & SNL \\
\hline Mark Rowland & GAMMA DESIGNER & LLNL \\
\hline Tom Gosnell & Principle Components & LLNL \\
\hline Wolfgang Stoeffl & FITEK & LLNL \\
\hline Arden Dougan & Recognized Expert & LLNL \\
\hline
\end{tabular}

Table 2: The catalog of existing source identification tools.

\begin{tabular}{|c|c|c|c|}
\hline Technologies & POC & Detector & Comments \\
\hline \multicolumn{4}{|l|}{ Automated Tools } \\
\hline $\begin{array}{l}\text { Principle Components } \\
\text { Analysis (PCA) }\end{array}$ & Gosnell & $\begin{array}{l}\text { Ge, NaI, } \\
\text { Plastic }\end{array}$ & $\begin{array}{l}\text { Not yet finished. Need to complete the } \\
\text { template library }\end{array}$ \\
\hline ISO ID & Rowland & $\mathrm{Ge}$ & Limited to only germanium detectors \\
\hline FIT2DB & Mitchell & $\begin{array}{l}\text { Ge, NaI, } \\
\text { Plastic }\end{array}$ & \\
\hline \multicolumn{4}{|l|}{ Tools } \\
\hline $\begin{array}{l}\text { Gamma Vision } \\
\text { Gamma Designer }\end{array}$ & Rowland & $\begin{array}{l}\text { Ge, NaI, } \\
\text { Plastic }\end{array}$ & $\begin{array}{l}\text { Requires expert to get correct answer. } \\
\text { Can not be automated }\end{array}$ \\
\hline Fit2DB & Mitchell & $\begin{array}{l}\text { Ge, NaI, } \\
\text { Plastic }\end{array}$ & $\begin{array}{l}\text { Requires expert to get correct answer. } \\
\text { Can not be automated; template } \\
\text { matching }\end{array}$ \\
\hline Shamon & & $\begin{array}{l}\text { Ge, NaI, } \\
\text { Plastic }\end{array}$ & $\begin{array}{l}\text { Requires expert to get correct answer. } \\
\text { Can not be automated }\end{array}$ \\
\hline \multicolumn{4}{|l|}{ Interpreting Language } \\
\hline $\begin{array}{l}\text { FITEK } \\
\text { ( FITEK uses the } \\
\text { methodology of GAMANAL, } \\
\text { but is more general) }\end{array}$ & Stoeffl & $\begin{array}{l}\text { Ge, NaI, } \\
\text { Plastic }\end{array}$ & $\begin{array}{l}\text { Automatic version does not exist but } \\
\text { can possible be created }\end{array}$ \\
\hline \multicolumn{4}{|l|}{ Line Pickers } \\
\hline FRAM & LANL & $\mathrm{Ge}$ & Not Automated - Offered by Canberra \\
\hline MGAU & Wayne Ruhter & $\mathrm{Ge}$ & Automated for $\mathrm{Pu}$ and $\mathrm{U}$ \\
\hline GRPANL & Wayne Ruhter & $\mathrm{Ge}$ & Automated similar spectra \\
\hline GAMANAL & Ray Gunnink & $\mathrm{Ge}$ & Not Automated, Technique and \\
\hline
\end{tabular}




\begin{tabular}{|l|l|l|l|}
\hline & & & software used in commercial products \\
\hline Others & & & \\
\hline ORIGEN & & & \\
\hline IAEA & & & \\
\hline Big Fit & & & \\
\hline Iso Fit & INEEL & Ge & For reactor products \\
\hline Gauss & Commercial & Ge & \\
\hline Fit Peak & & & \\
\hline Commercial Products & $\begin{array}{l}\text { Stan Prussin } \\
\text { originally }\end{array}$ & Ge & Software from Finland \\
\hline Sampo & & & \\
\hline Exploreanium & & & Ge \\
\hline Radtech & & OTEC - software \\
\hline Maestro Gammavision & Westmeier & software \\
\hline GammaW SODIGAM & & NaI & $\begin{array}{l}\text { Hardware - they have a different } \\
\text { method for analyzing NaI in software }\end{array}$ \\
\hline Berkeley Nucleonics & & Ge & Canberra - hardware and software \\
\hline Inspector & & & $\begin{array}{l}\text { Canberra - Suite of spectroscopy } \\
\text { packages }\end{array}$ \\
\hline Gennie 2000 & & & Canberra - Calibration Tool \\
\hline ISOCS & & & $\begin{array}{l}\text { Canberra - Calibration Tool } \\
\text { and its daughters }\end{array}$ \\
\hline LABSOCS & & & $\begin{array}{l}\text { Canberra - Tools that work with U } \\
\text { and its daughters }\end{array}$ \\
\hline MGA & & & \\
\hline ???? & & & \\
\hline
\end{tabular}

B. We analyzed algorithmic components of these source identification tools and made recommendations for their improvement

Through the analysis of these various algorithms we were asked to identify and comment on the algorithms merits. This naturally precipitated the identification of more effective algorithms or implementations of these algorithms and we made technical recommendations that will improve the efficiency and/or the effectiveness of these algorithms. We were able to develop a generalized framework that captures the functionality of the source identification tools (Figure 1). This framework enabled us to qualify our analytical comparison of the various tools. 


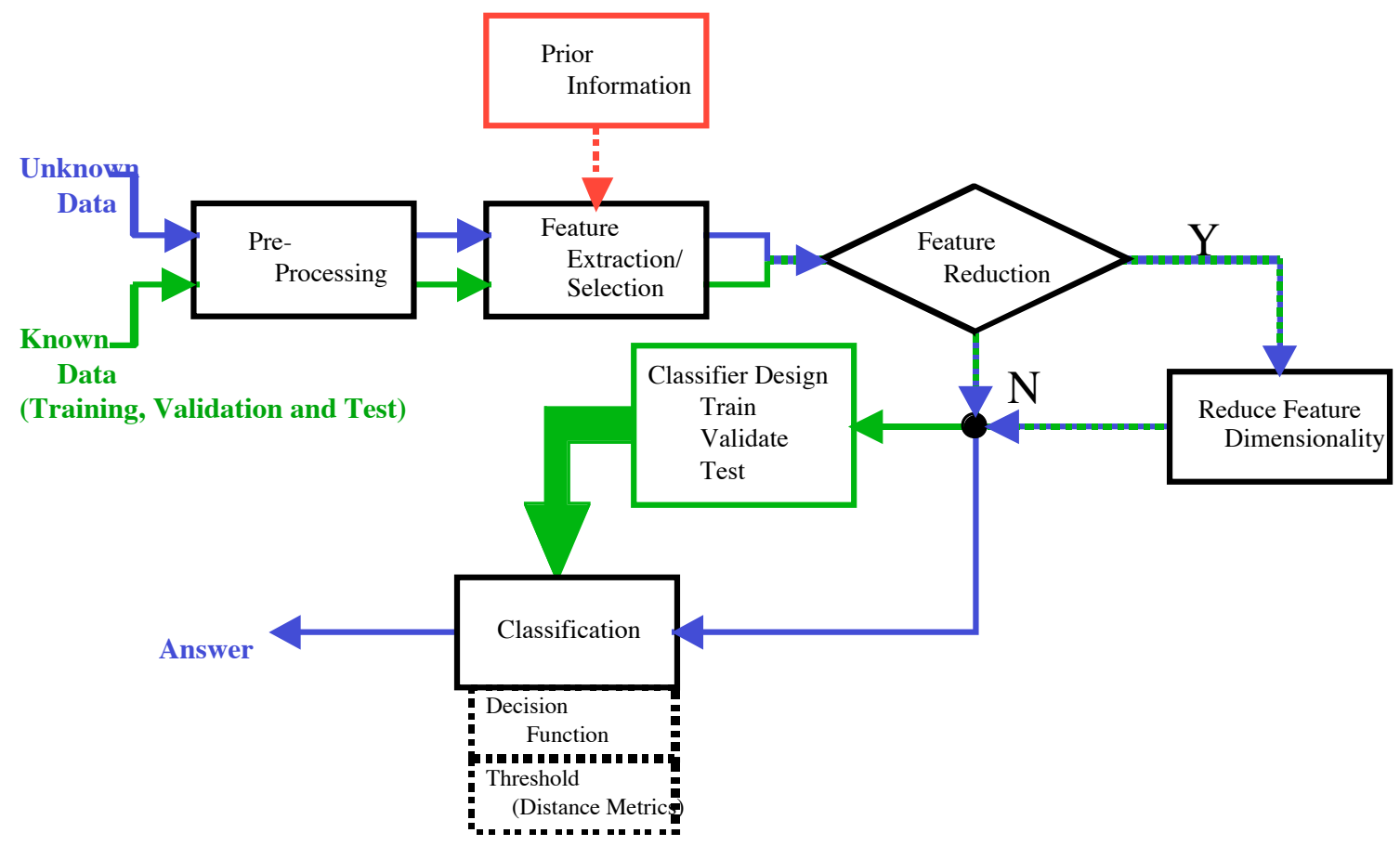

Figure 1: General framework for the source identification tools

C. We formulated a classification approach for source identification and developed a methodology for comparing source identification tools

A comparison methodology was developed based upon the using a Monte Carlo approach and ROC curves that compared each of the identified algorithms with equanimity. This approach was inhibited by the various output formulations of the different tools. Consequently, a subsequent task was added to develop an approach for transforming the various output formulations and enable the use of this comparison methodology.

Establishing a quantifiable measure of each tools performance is paramount to this effort. This performance metric is the measure of a tool's capability to correctly identify a test isotope by its gamma emission in the presence of background emissions. This performance metric is quantified by the probabilities of correctly and incorrectly identifying the test isotope.

Test isotopes and backgrounds will be drawn from a set of source species and a set of competing backgrounds, respectively. Developing these two sets of prototypical spectra is beyond the scope of this project and will be provided by another effort. For convenience, the volume of captured gamma ray emissions originating from a test isotope will be referred to as isotope counts. Likewise, the volume of captured gamma ray emissions due to the background will be referred to as background counts.

The probabilities needed to derive the performance metric statistics will be developed from multiple realizations of each source to background ratio (SBR). By randomly selecting a different sub-set from the specie isotope under consideration, a distribution will be developed for both the probability of correctly identifying the isotope as well as 
for incorrectly identifying the isotope. The Receiver Operating Characteristic (ROC) curve is a convenient way to graphically display these performance metrics. The ROC curve plots the probability of correct identification versus the probability of false alarm as a function of the SBR.

A Monte Carlo approach will be used to quantify the performance metrics. At each value for SBR, a statistically significant number $(M)$ of representations of the test isotope will be generated. The SBR determines the number $(N)$ of emissions to draw at random from the source specie. These $N$ randomly selected emissions are selected to preserve the Poisson properties of the Event-mode Sequence or time history of the energy emitted. See Figure 2.

Each representation of the test isotope is processed sequentially by a candidate tool resulting in a determination of a predicted isotope. In the case where multiple isotopes are identified and ranked, the highest ranking or most likely isotope will be selected. A count of the number of correct identifications and the number of incorrect identifications is accumulated for each tool and for all $M$ representations. These counts are then used to develop the probabilities of correct identification and the probabilities of incorrect identification for each tool. This process is then repeated for each of the designated SBRs. See Figure 3.

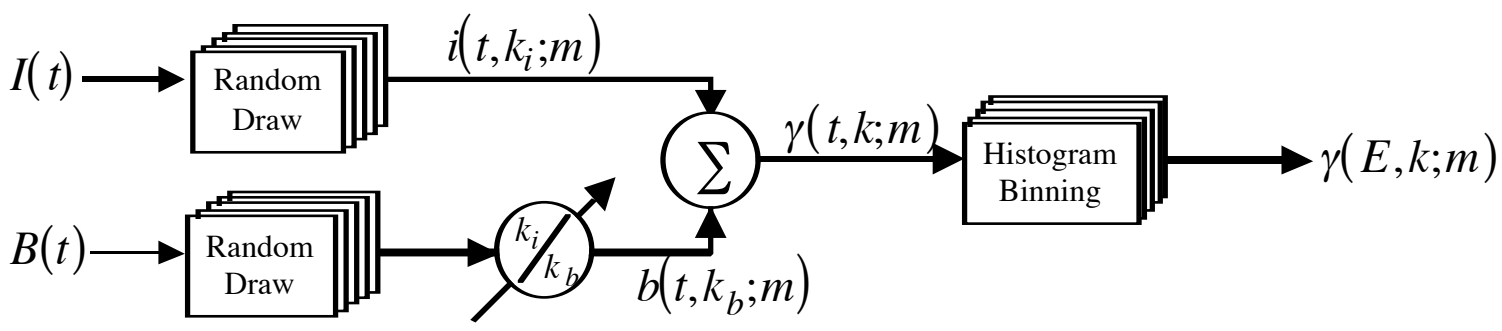

Figure 2. A block diagram of the Monte Carlo approach. This illustration indicates the multidimensional nature of this process.

D. We concluded that existing source identification tools can not be effectively compared due to their respective approaches to reporting results

No consistency for reporting results exists between the various source identification tools. This lack of consistency prevents us from directly comparing the performance of the different tools. Some tools indicate the single most probable source for the isotope. Others indicate a ranking of the most $n$ most probable isotopes. Yet others assume that all sources are mixtures of isotopes and report the proportionality of the $n$ most probable isotopes in the mixture. It is not possible to make direct comparisons between tools when their products are inconsistent. 


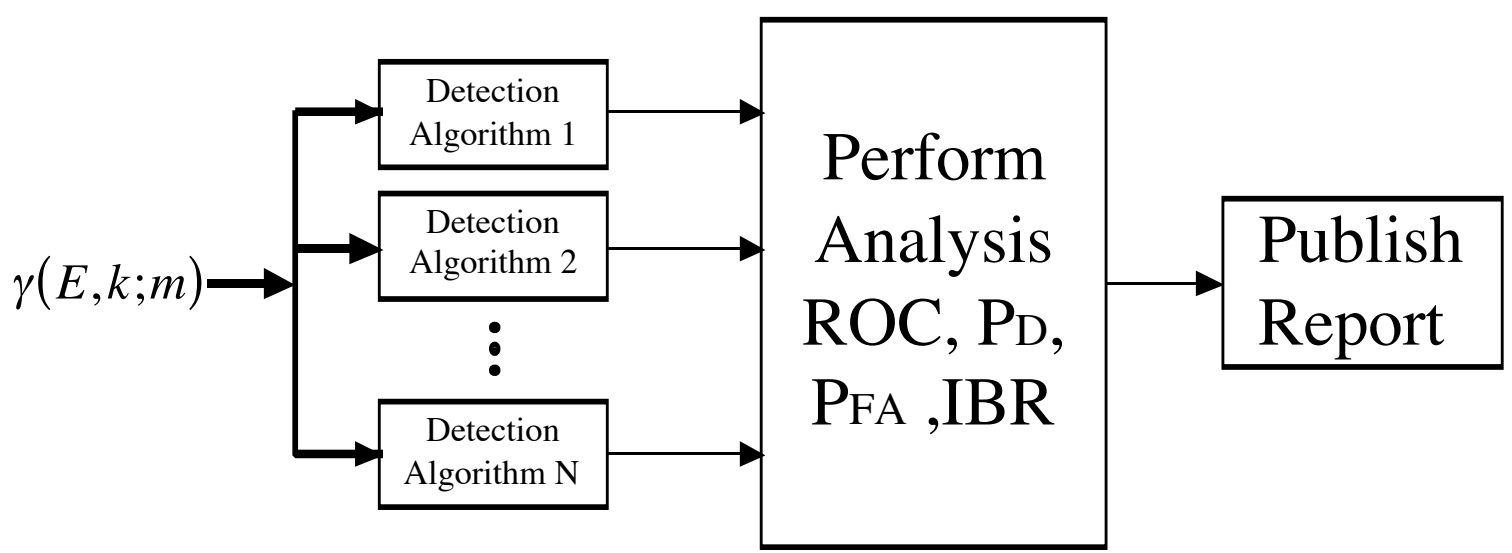

Figure 3: Block diagram illustrating the data flow through the detection algorithms, the analysis and concluding with a final report.

E. We recommended a classification framework that will transform their reporting approaches thus enabling a comparison

The inconsistency between reporting approaches or the various tools compelled the development of an approach to transforming the outputs of these tools. After careful study, we recommend the adoption of a classification framework for comparing the performance of the subject tools. This classification framework compels the selection of a single isotope or specific mixture of isotopes as the output of each tool.

We then specified an unique "decision function" that converts or transforms the output of each tool to the standard output stipulated by the classification framework. Figure 4 depicts the necessary modifications to our evaluation methodology to implement the classification framework. The blue boxes indicate functionality that is either modified or added to the initial procedure. This figure indicates the three most probable technologies for consideration and identifies the addition of unique decision functions for each tool.

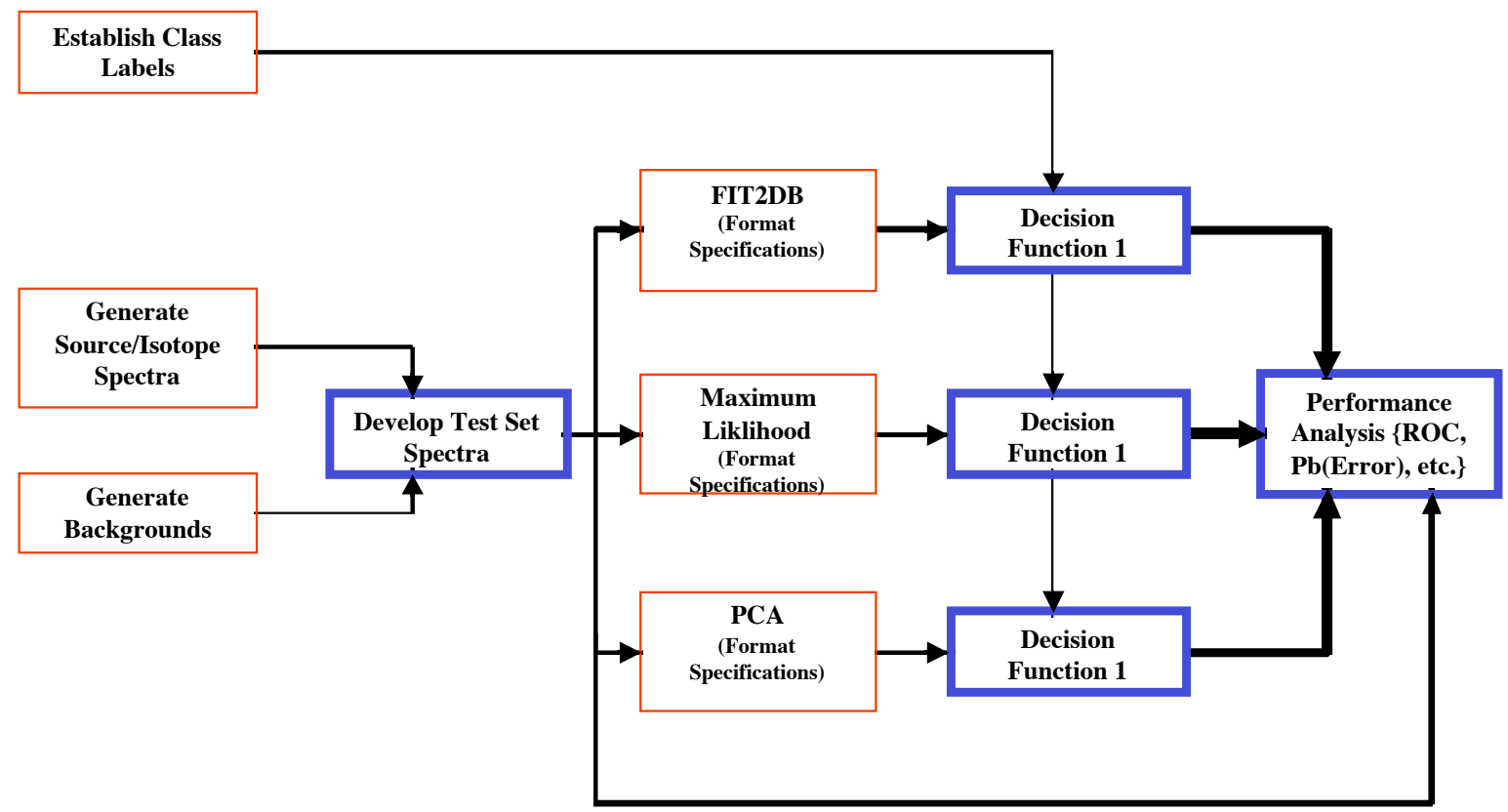

Figure 4: Methodology modifications to implement the classification framework. 


\section{ConcLuSions}

We conclude that a comparison quantifying the performance of source identification tools is not possible with out first transforming the tool output to a common framework. Given the requirement for source identification, the classification framework is most reasonable and consequently recommended. A decision function needs to be created for each tool under consideration. This decision function will convert the tool output format to the classification framework where an equitable and quantifiable comparison can be realized. We strongly recommend that this approach be taken in order to perform an equitable and effective comparison of these source identification tools 


\section{A.4 Appendix: Details of Isotope Identification Evaluation}

\section{A.4.1 GADRAS fittodb}

\section{A.4.1.1 GADRAS fittodb Isotopic Score}

Column definitions

- Sample - name of the sample in the library file

- Label - expert label algorithm is trying to match

- Includes - number of trials solution included expert label

- Exact - number of trials solution was exactly equal to expert label

- Score - Sum of scores for 100 trials, where a score is defined as intersection of solution and expert label divided by union.

\begin{tabular}{|c|c|c|c|c|c|}
\hline $\begin{array}{l}50 \text { Counts/400 Bkg } \\
\text { Sample }\end{array}$ & Label & Includes & Exact & \multicolumn{2}{|c|}{ Score } \\
\hline 99TcM-625uc+200mm-H2O+5mmFe & ${ }^{99 \mathrm{~m}} \mathrm{Tc}$ & 57 & & 23 & 38.42 \\
\hline $131 \mathrm{l}-370 \mathrm{uc}+200 \mathrm{~mm}-\mathrm{H} 2 \mathrm{O}+5 \mathrm{mmFe}$ & ${ }^{131}$ I & 12 & & 0 & 4.5 \\
\hline $201 \mathrm{TI}-250 \mathrm{uc}+200 \mathrm{~mm}-\mathrm{H} 2 \mathrm{O}+5 \mathrm{mmFe}$ & ${ }^{201} \mathrm{Tl}$ & 31 & & 6 & 17.17 \\
\hline $67 \mathrm{Ga}-350 \mathrm{uc}+200 \mathrm{~mm}-\mathrm{H} 2 \mathrm{O}+5 \mathrm{mmFe}$ & ${ }^{67} \mathrm{Ga}$ & 16 & & 5 & 8.92 \\
\hline $137 \mathrm{Cs}-8 \mathrm{mc}+24 \mathrm{~mm}-$ Lead+5mmFe & ${ }^{137} \mathrm{Cs}$ & 28 & & 27 & 27.5 \\
\hline 14Kg-of-Bananas $+5 \mathrm{mmFe}$ & ${ }^{40} \mathrm{~K}$ & 1 & & 1 & 1 \\
\hline $5 \mathrm{Kg}$-of-Wood $+5 \mathrm{mmFe}$ & ${ }^{40} \mathrm{~K}$ & 1 & & 1 & 1 \\
\hline $5 \mathrm{Kg}$-of-Potash-Fertilizer $+5 \mathrm{mmFe}$ & ${ }^{40} \mathrm{~K}$ & 0 & & 0 & 0 \\
\hline HEU & ${ }^{235} U$ & 41 & & 18 & 27.92 \\
\hline Virgin HEU & ${ }^{235} \mathrm{U}$ & 44 & & 21 & 30.25 \\
\hline Reactor Pu & ${ }^{239} \mathrm{Pu}$ & 8 & & 3 & 5 \\
\hline Weapon Pu & ${ }^{239} \mathrm{Pu}$ & 12 & & 4 & 7 \\
\hline HEU with shielding & ${ }^{235} \mathrm{U}$ & 0 & & 0 & 0 \\
\hline Virgin HEU with shielding & ${ }^{235} \mathrm{U}$ & 0 & & 0 & 0 \\
\hline Reactor Pu with shielding & ${ }^{239} \mathrm{Pu}$ & 0 & & 0 & 0 \\
\hline Weapon Pu with shielding & ${ }^{239} \mathrm{Pu}$ & 0 & & 0 & 0 \\
\hline
\end{tabular}




\begin{tabular}{|c|c|c|c|c|c|}
\hline 100 Counts/400 Bkg & & & & & \\
\hline Sample & Label & Includes & Exact & & \\
\hline 99TcM-625uc+200mm-H2O+5mmFe & ${ }^{99 \mathrm{~m}} \mathrm{Tc}$ & 96 & & 66 & 80.67 \\
\hline $131 \mathrm{l}-370 \mathrm{uc}+200 \mathrm{~mm}-\mathrm{H} 2 \mathrm{O}+5 \mathrm{mmFe}$ & ${ }^{131}$ & 34 & & 16 & 23.5 \\
\hline $201 \mathrm{TI}-250 \mathrm{uc}+200 \mathrm{~mm}-\mathrm{H} 2 \mathrm{O}+5 \mathrm{mmFe}$ & ${ }^{201} \mathrm{Tl}$ & 38 & & 10 & 22.33 \\
\hline $67 \mathrm{Ga}-350 \mathrm{uc}+200 \mathrm{~mm}-\mathrm{H} 2 \mathrm{O}+5 \mathrm{mmFe}$ & ${ }^{67} \mathrm{Ga}$ & 44 & & 25 & 31.75 \\
\hline $137 \mathrm{Cs}-8 \mathrm{mc}+24 \mathrm{~mm}-$ Lead $+5 \mathrm{mmFe}$ & ${ }^{137} \mathrm{Cs}$ & $7 c$ & & 71 & 75 \\
\hline $14 \mathrm{Kg}$-of-Bananas+5mmFe & ${ }^{40} \mathrm{~K}$ & 25 & & 25 & 25 \\
\hline $5 \mathrm{Kg}$-of-Wood+5mmFe & ${ }^{40} \mathrm{~K}$ & 24 & & 24 & 24 \\
\hline $5 \mathrm{Kg}$-of-Potash-Fertilizer $+5 \mathrm{mmFe}$ & ${ }^{40} \mathrm{~K}$ & 1 & & 1 & 1 \\
\hline HEU & ${ }^{235} U$ & 85 & & 53 & 65.83 \\
\hline Virgin HEU & ${ }^{235} U$ & 86 & & 52 & 65.17 \\
\hline Reactor Pu & ${ }^{239} \mathrm{Pu}$ & 5 & & 1 & 2.83 \\
\hline Weapon Pu & ${ }^{239} \mathrm{Pu}$ & 23 & & 8 & 13.5 \\
\hline HEU with shielding & ${ }^{235} U$ & c & & 0 & 0 \\
\hline Virgin HEU with shielding & ${ }^{235} U$ & $\mathrm{c}$ & & 0 & 0 \\
\hline Reactor Pu with shielding & ${ }^{239} \mathrm{Pu}$ & 2 & & 1 & 1.5 \\
\hline Weapon Pu with shielding & ${ }^{239} \mathrm{Pu}$ & $\varepsilon$ & & 2 & 5 \\
\hline
\end{tabular}

\begin{tabular}{|c|c|c|c|c|c|}
\hline $\begin{array}{l}200 \text { Counts/400 Bkg } \\
\text { Sample }\end{array}$ & Label & Includes & Exact & \multicolumn{2}{|c|}{ Score } \\
\hline 99TcM-625uc+200mm-H2O+5mmFe & ${ }^{99 \mathrm{~m}} \mathrm{Tc}$ & 100 & & 78 & 86.33 \\
\hline $131 \mathrm{l}-370 \mathrm{uc}+200 \mathrm{~mm}-\mathrm{H} 2 \mathrm{O}+5 \mathrm{mmFe}$ & ${ }^{131}$ & 52 & & 27 & 35.17 \\
\hline $201 \mathrm{TI}-250 \mathrm{uc}+200 \mathrm{~mm}-\mathrm{H} 2 \mathrm{O}+5 \mathrm{mmFe}$ & ${ }^{201} \mathrm{TI}$ & 14 & & 1 & 4.92 \\
\hline $67 \mathrm{Ga}-350 \mathrm{uc}+200 \mathrm{~mm}-\mathrm{H} 2 \mathrm{O}+5 \mathrm{mmFe}$ & ${ }^{67} \mathrm{Ga}$ & 81 & & 73 & 76.67 \\
\hline $137 \mathrm{Cs}-8 \mathrm{mc}+24 \mathrm{~mm}-$ Lead+5mmFe & ${ }^{137} \mathrm{Cs}$ & 97 & & 90 & 93.5 \\
\hline 14Kg-of-Bananas $+5 \mathrm{mmFe}$ & ${ }^{40} \mathrm{~K}$ & 83 & & 82 & 82.5 \\
\hline $5 \mathrm{Kg}$-of-Wood $+5 \mathrm{mmFe}$ & ${ }^{40} \mathrm{~K}$ & 83 & & 83 & 83 \\
\hline $5 \mathrm{Kg}$-of-Potash-Fertilizer $+5 \mathrm{mmFe}$ & ${ }^{40} \mathrm{~K}$ & $1<$ & & 14 & 14 \\
\hline HEU & ${ }^{235} \mathrm{U}$ & 86 & & 54 & 69.83 \\
\hline Virgin HEU & ${ }^{235} \mathrm{U}$ & 82 & & 60 & 71 \\
\hline Reactor $\mathrm{Pu}$ & ${ }^{239} \mathrm{Pu}$ & 1 & 1 & 0 & 0.5 \\
\hline Weapon Pu & ${ }^{239} \mathrm{Pu}$ & 36 & & 14 & 23.25 \\
\hline HEU with shielding & ${ }^{235} \mathrm{U}$ & $c$ & 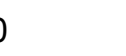 & 0 & 0 \\
\hline Virgin HEU with shielding & ${ }^{235} \mathrm{U}$ & c & 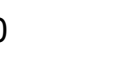 & 0 & 0 \\
\hline Reactor Pu with shielding & ${ }^{239} \mathrm{Pu}$ & c & 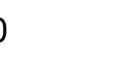 & 0 & 0 \\
\hline Weapon Pu with shielding & ${ }^{239} \mathrm{Pu}$ & 2 & 2 & 1 & 1.5 \\
\hline
\end{tabular}




\begin{tabular}{|lrrrrrr|}
\hline 300 Counts/400 Bkg & & & & & \\
Sample & Label & Includes & Exact & Score & Runs \\
\hline $99 \mathrm{TcM}-625 \mathrm{uc}+200 \mathrm{~mm}-\mathrm{H} 2 \mathrm{O}+5 \mathrm{mmFe}$ & ${ }^{99 \mathrm{~m}} \mathrm{Tc}$ & 100 & 98 & 99 & 100 \\
$131 \mathrm{l}-370 \mathrm{uc}+200 \mathrm{~mm}-\mathrm{H} 2 \mathrm{O}+5 \mathrm{mmFe}$ & ${ }^{131} \mathrm{I}$ & 67 & 17 & 31.33 & 100 \\
$201 \mathrm{Tl}-250 \mathrm{uc}+200 \mathrm{~mm}-\mathrm{H} 2 \mathrm{O}+5 \mathrm{mmFe}$ & ${ }^{201} \mathrm{TI}$ & 7 & 0 & 2.75 & 100 \\
$67 \mathrm{Ga}-350 \mathrm{uc}+200 \mathrm{~mm}-\mathrm{H} 2 \mathrm{O}+5 \mathrm{mmFe}$ & ${ }^{67} \mathrm{Ga}$ & 96 & 86 & 90.42 & 100 \\
$137 \mathrm{Cs}-8 \mathrm{mc}+24 \mathrm{~mm}-\mathrm{Le} \mathrm{Cad}+5 \mathrm{mmFe}$ & ${ }^{137} \mathrm{Cs}$ & 100 & 98 & 99 & 100 \\
$14 \mathrm{Kg}-$ of-Bananas+5mmFe & ${ }^{40} \mathrm{~K}$ & 99 & 97 & 98 & 100 \\
$5 \mathrm{Kg}-$-of-Wood+5mmFe & ${ }^{40} \mathrm{~K}$ & 99 & 98 & 98.5 & 100 \\
$5 \mathrm{Kg}-$-of-Potash-Fertilizer+5mmFe & ${ }^{40} \mathrm{~K}$ & 48 & 45 & 46.5 & 100 \\
HEU & ${ }^{235} \mathrm{U}$ & 94 & 81 & 87.33 & 100 \\
Virgin HEU & ${ }^{235} \mathrm{U}$ & 96 & 87 & 90.92 & 100 \\
Reactor Pu & ${ }^{239} \mathrm{Pu}$ & 2 & 0 & 1 & 100 \\
Weapon Pu & ${ }^{239} \mathrm{Pu}$ & 52 & 13 & 26.17 & 100 \\
HEU with shielding & ${ }^{235} \mathrm{U}$ & 0 & 0 & 0 & 100 \\
Virgin HEU with shielding & ${ }^{235} \mathrm{U}$ & 0 & 0 & 0 & 100 \\
Reactor Pu with shielding & ${ }^{239} \mathrm{Pu}$ & 0 & 0 & 0 & 100 \\
Weapon Pu with shielding & ${ }^{239} \mathrm{Pu}$ & 0 & 0 & 0 & 100 \\
\hline
\end{tabular}

\begin{tabular}{|c|c|c|c|c|c|}
\hline $\begin{array}{l}500 \text { Counts } / 400 \text { Bkg } \\
\text { Sample }\end{array}$ & Label & Includes & Exact & Score & Runs \\
\hline 99TcM-625uc+200mm-H2O+5mmFe & ${ }^{99 \mathrm{~m}} \mathrm{Tc}$ & 99 & 98 & 98.5 & 99 \\
\hline $131 \mathrm{l}-370 \mathrm{uc}+200 \mathrm{~mm}-\mathrm{H} 2 \mathrm{O}+5 \mathrm{mmFe}$ & ${ }^{131}$ I & 65 & 47 & 55.42 & 100 \\
\hline $201 \mathrm{TI}-250 \mathrm{uc}+200 \mathrm{~mm}-\mathrm{H} 2 \mathrm{O}+5 \mathrm{mmFe}$ & ${ }^{201} \mathrm{TI}$ & 6 & 0 & 2.83 & 100 \\
\hline $67 \mathrm{Ga}-350 \mathrm{uc}+200 \mathrm{~mm}-\mathrm{H} 2 \mathrm{O}+5 \mathrm{mmFe}$ & ${ }^{67} \mathrm{Ga}$ & 100 & 100 & 100 & 100 \\
\hline $137 \mathrm{Cs}-8 \mathrm{mc}+24 \mathrm{~mm}-$ Lead $+5 \mathrm{mmFe}$ & ${ }^{137} \mathrm{Cs}$ & 100 & 100 & 100 & 100 \\
\hline $14 \mathrm{Kg}$-of-Bananas $+5 \mathrm{mmFe}$ & ${ }^{40} \mathrm{~K}$ & 100 & 100 & 100 & 100 \\
\hline $5 \mathrm{Kg}$-of-Wood $+5 \mathrm{mmFe}$ & ${ }^{40} \mathrm{~K}$ & 100 & 100 & 100 & 100 \\
\hline $5 \mathrm{Kg}$-of-Potash-Fertilizer $+5 \mathrm{mmFe}$ & ${ }^{40} \mathrm{~K}$ & 93 & 88 & 90.33 & 100 \\
\hline HEU & ${ }^{235} \mathrm{U}$ & 100 & 100 & 100 & 100 \\
\hline Virgin HEU & ${ }^{235} U$ & 100 & 99 & 99.5 & 100 \\
\hline Reactor Pu & ${ }^{239} \mathrm{Pu}$ & 62 & 23 & 40.92 & 100 \\
\hline Weapon Pu & ${ }^{239} \mathrm{Pu}$ & 93 & 83 & 87.25 & 100 \\
\hline HEU with shielding & ${ }^{235} U$ & 0 & 0 & 0 & 100 \\
\hline Virgin HEU with shielding & ${ }^{235} U$ & 0 & 0 & 0 & 100 \\
\hline Reactor Pu with shielding & ${ }^{239} \mathrm{Pu}$ & 0 & 0 & 0 & 100 \\
\hline Weapon Pu with shielding & ${ }^{239} \mathrm{Pu}$ & 0 & 0 & 0 & 100 \\
\hline
\end{tabular}




\begin{tabular}{|lrrrrrr|}
\hline 750 Counts/400 Bkg & & & & & \\
Sample & Label & Includes & Exact & Score & Runs \\
\hline $99 \mathrm{TcM}-625 \mathrm{uc}+200 \mathrm{~mm}-\mathrm{H} 2 \mathrm{O}+5 \mathrm{mmFe}$ & ${ }^{99 \mathrm{~m}} \mathrm{Tc}$ & 100 & 100 & 100 & 100 \\
$131 \mathrm{l}-370 \mathrm{uc}+200 \mathrm{~mm}-\mathrm{H} 2 \mathrm{O}+5 \mathrm{mmFe}$ & ${ }^{131} \mathrm{I}$ & 81 & 59 & 68.67 & 100 \\
$201 \mathrm{Tl}-250 \mathrm{uc}+200 \mathrm{~mm}-\mathrm{H} 2 \mathrm{O}+5 \mathrm{mmFe}$ & ${ }^{201} \mathrm{TI}$ & 9 & 2 & 5.17 & 100 \\
$67 \mathrm{Ga}-350 \mathrm{uc}+200 \mathrm{~mm}-\mathrm{H} 2 \mathrm{O}+5 \mathrm{mmFe}$ & ${ }^{67} \mathrm{Ga}$ & 100 & 99 & 99.5 & 100 \\
$137 \mathrm{Cs}-8 \mathrm{mc}+24 \mathrm{~mm}-\mathrm{Lead}+5 \mathrm{mmFe}$ & ${ }^{137} \mathrm{Cs}$ & 100 & 100 & 100 & 100 \\
$14 \mathrm{Kg}-$ of-Bananas+5mmFe & ${ }^{40} \mathrm{~K}$ & 100 & 100 & 100 & 100 \\
$5 \mathrm{Kg}-$ of-Wood+5mmFe & ${ }^{40} \mathrm{~K}$ & 100 & 100 & 100 & 100 \\
$5 \mathrm{Kg}-$-of-Potash-Fertilizer+5mmFe & ${ }^{40} \mathrm{~K}$ & 100 & 99 & 99.5 & 100 \\
HEU & ${ }^{235} \mathrm{U}$ & 100 & 100 & 100 & 100 \\
Virgin HEU & ${ }^{235} \mathrm{U}$ & 100 & 100 & 100 & 100 \\
Reactor Pu & ${ }^{239} \mathrm{Pu}$ & 89 & 26 & 53.62 & 100 \\
Weapon Pu & ${ }^{239} \mathrm{Pu}$ & 100 & 95 & 97.5 & 100 \\
HEU with shielding & ${ }^{235} \mathrm{U}$ & 0 & 0 & 0 & 100 \\
Virgin HEU with shielding & ${ }^{235} \mathrm{U}$ & 0 & 0 & 0 & 100 \\
Reactor Pu with shielding & ${ }^{239} \mathrm{Pu}$ & 0 & 0 & 0 & 100 \\
Weapon Pu with shielding & ${ }^{239} \mathrm{Pu}$ & 3 & 0 & 1.33 & 100 \\
\hline
\end{tabular}

\begin{tabular}{|c|c|c|c|c|c|}
\hline $\begin{array}{l}1000 \text { Counts } / 400 \text { Bkg } \\
\text { Sample }\end{array}$ & Label & Includes & Exact & Score & Runs \\
\hline 99TcM-625uc+200mm-H2O+5mmFe & ${ }^{99 \mathrm{~m}} \mathrm{Tc}$ & 100 & 100 & 100 & 100 \\
\hline $131 \mathrm{l}-370 \mathrm{uc}+200 \mathrm{~mm}-\mathrm{H} 2 \mathrm{O}+5 \mathrm{mmFe}$ & ${ }^{131}$ & 9 & 68 & 78.83 & 100 \\
\hline $201 \mathrm{Tl}-250 \mathrm{uc}+200 \mathrm{~mm}-\mathrm{H} 2 \mathrm{O}+5 \mathrm{mmFe}$ & ${ }^{201} \mathrm{TI}$ & 57 & 0 & 22.75 & 100 \\
\hline $67 \mathrm{Ga}-350 \mathrm{uc}+200 \mathrm{~mm}-\mathrm{H} 2 \mathrm{O}+5 \mathrm{mmFe}$ & ${ }^{67} \mathrm{Ga}$ & 100 & 96 & 98 & 100 \\
\hline 137Cs-8mc+24mm-Lead+5mmFe & ${ }^{137} \mathrm{Cs}$ & 100 & 99 & 99.5 & 100 \\
\hline 14Kg-of-Bananas $+5 \mathrm{mmFe}$ & ${ }^{40} \mathrm{~K}$ & 100 & 100 & 100 & 100 \\
\hline $5 \mathrm{Kg}$-of-Wood+5mmFe & ${ }^{40} \mathrm{~K}$ & 100 & 100 & 100 & 100 \\
\hline $5 \mathrm{Kg}$-of-Potash-Fertilizer $+5 \mathrm{mmFe}$ & ${ }^{40} \mathrm{~K}$ & 100 & 98 & 99 & 100 \\
\hline HEU & ${ }^{235} \mathrm{U}$ & 100 & 100 & 100 & 100 \\
\hline Virgin HEU & ${ }^{235} \mathrm{U}$ & 100 & 100 & 100 & 100 \\
\hline Reactor $\mathrm{Pu}$ & ${ }^{239} \mathrm{Pu}$ & 95 & 36 & 59.87 & 100 \\
\hline Weapon Pu & ${ }^{239} \mathrm{Pu}$ & 100 & 99 & 99.5 & 100 \\
\hline HEU with shielding & ${ }^{235} \mathrm{U}$ & C & 0 & 0 & 100 \\
\hline Virgin HEU with shielding & ${ }^{235} \mathrm{U}$ & c & 0 & 0 & 100 \\
\hline Reactor Pu with shielding & ${ }^{239} \mathrm{Pu}$ & c & 0 & 0 & 100 \\
\hline Weapon Pu with shielding & ${ }^{239} \mathrm{Pu}$ & c & 0 & 0 & 100 \\
\hline
\end{tabular}




\section{A.4.1.2 GADRAS fittodb Class Results}

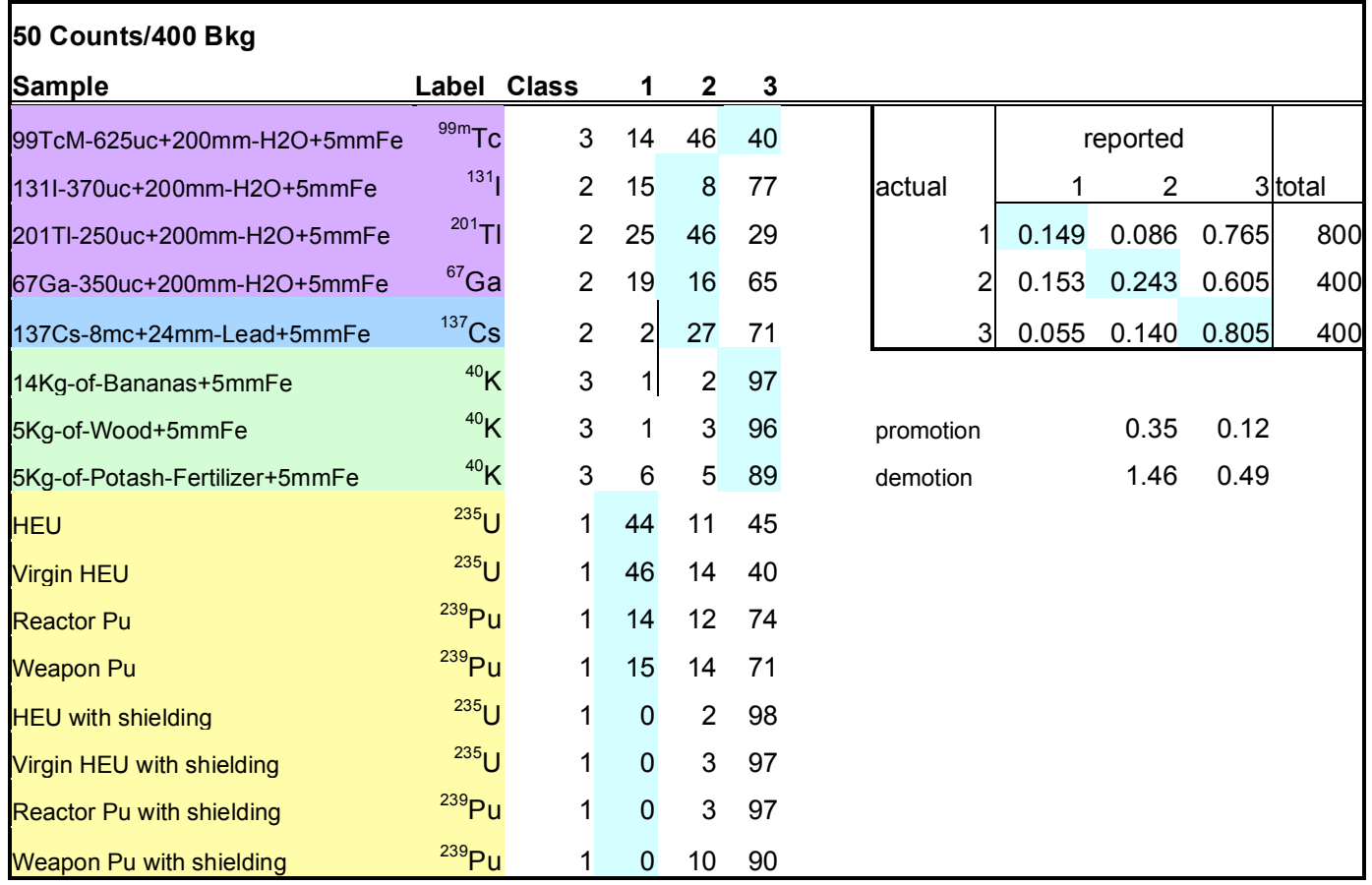

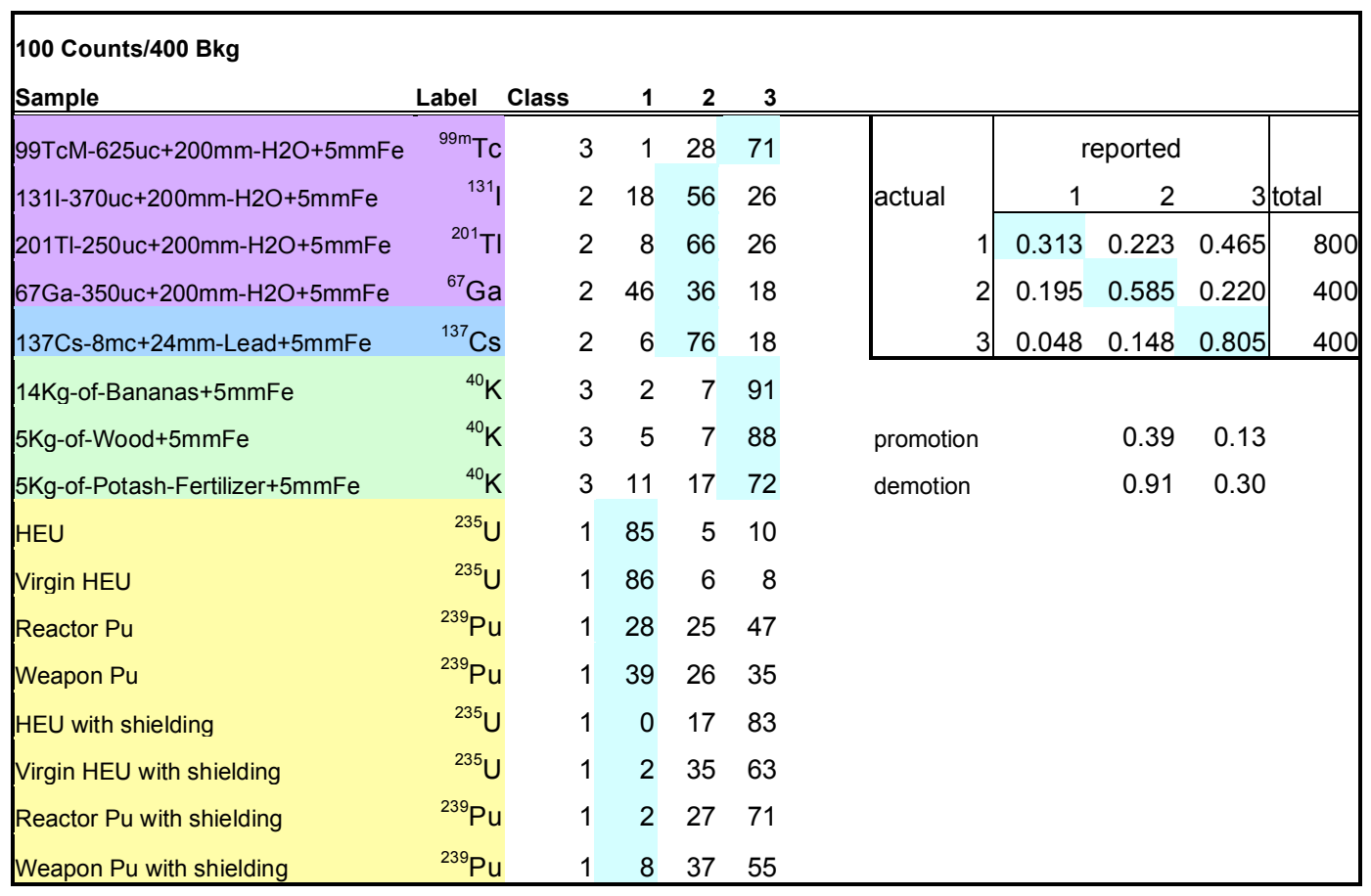




\begin{tabular}{|c|c|c|c|c|c|c|c|c|c|c|}
\hline $\begin{array}{l}200 \text { Counts/400 Bkg } \\
\text { Sample }\end{array}$ & Label & Class & 1 & 2 & 3 & & & & & \\
\hline 99TcM-625uc+200mm-H2O +5mmFe & ${ }^{99 \mathrm{~m}} \mathrm{Tc}$ & 3 & 0 & 21 & 79 & \multirow{5}{*}{ actual } & \multirow{2}{*}{\multicolumn{4}{|c|}{ Reported }} \\
\hline $131 \mathrm{l}-370 \mathrm{uc}+200 \mathrm{~mm}-\mathrm{H} 2 \mathrm{O}+5 \mathrm{mmFe}$ & 131 & 2 & 20 & 70 & 10 & & & & & \\
\hline $201 \mathrm{TI}-250 \mathrm{uc}+200 \mathrm{~mm}-\mathrm{H} 2 \mathrm{O}+5 \mathrm{mmFe}$ & ${ }^{201} \mathrm{~T}$ & 2 & 7 & 85 & 8 & & 0.270 & 0.246 & 0.484 & 800 \\
\hline $67 \mathrm{Ga}-350 \mathrm{uc}+200 \mathrm{~mm}-\mathrm{H} 2 \mathrm{O}+5 \mathrm{mmFe}$ & ${ }^{67} \mathrm{Ga}$ & 2 & 21 & 77 & 2 & & 0.120 & 0.830 & 0.050 & 400 \\
\hline $137 \mathrm{Cs}-8 \mathrm{mc}+24 \mathrm{~mm}-$ Lead $+5 \mathrm{mmFe}$ & ${ }^{137} \mathrm{Cs}$ & 2 & 0 & 100 & 0 & & 0.008 & 0.078 & 0.915 & 400 \\
\hline $14 \mathrm{Kg}$-of-Bananas $+5 \mathrm{mmFe}$ & ${ }^{40} \mathrm{~K}$ & 3 & 0 & 0 & 100 & \multirow{11}{*}{$\begin{array}{l}\text { promotion } \\
\text { demotion }\end{array}$} & & & & \\
\hline $5 \mathrm{Kg}$-of-Wood $+5 \mathrm{mmFe}$ & ${ }^{40} \mathrm{~K}$ & 3 & 0 & 0 & 100 & & & 0.21 & 0.07 & \\
\hline $5 \mathrm{Kg}$-of-Potash-Fertilizer $+5 \mathrm{mmFe}$ & ${ }^{40} \mathrm{~K}$ & 3 & 3 & 10 & 87 & & & 0.78 & 0.26 & \\
\hline $\mathrm{HEU}$ & ${ }^{235} \mathrm{U}$ & 1 & 86 & 7 & 7 & & & & & \\
\hline Virgin HEU & ${ }^{235} \mathrm{U}$ & 1 & 82 & 5 & 13 & & & & & \\
\hline Reactor Pu & ${ }^{239} \mathrm{Pu}$ & 1 & 8 & 15 & 77 & & & & & \\
\hline Weapon Pu & ${ }^{239} \mathrm{Pu}$ & 1 & 38 & 18 & 44 & & & & & \\
\hline HEU with shielding & ${ }^{235} \mathrm{U}$ & 1 & 0 & 19 & 81 & & & & & \\
\hline Virgin HEU with shielding & ${ }^{235} U$ & 1 & 0 & 66 & 34 & & & & & \\
\hline Reactor Pu with shielding & ${ }^{239} \mathrm{Pu}$ & 1 & 0 & 21 & 79 & & & & & \\
\hline Weapon Pu with shielding & ${ }^{239} \mathrm{Pu}$ & 1 & 2 & 46 & 52 & & & & & \\
\hline
\end{tabular}

\begin{tabular}{|c|c|c|c|c|c|c|c|c|c|c|}
\hline $\begin{array}{l}300 \text { Counts } / 400 \text { Bkg } \\
\text { Sample }\end{array}$ & Label & Class & 1 & 2 & 3 & & & & & \\
\hline 99TcM-625uc+200mm-H2O+5mmFe & ${ }^{99 \mathrm{~m}} \mathrm{Tc}$ & 3 & 0 & 2 & 98 & \multirow{5}{*}{ actual } & \multicolumn{3}{|c|}{ Reported } & \multirow[b]{2}{*}{ total } \\
\hline $131 \mathrm{l}-370 \mathrm{uc}+200 \mathrm{~mm}-\mathrm{H} 2 \mathrm{O}+5 \mathrm{mmFe}$ & 131 & 2 & 38 & 60 & 2 & & 1 & 2 & & \\
\hline $201 \mathrm{Tl}-250 \mathrm{uc}+200 \mathrm{~mm}-\mathrm{H} 2 \mathrm{O}+5 \mathrm{mmFe}$ & ${ }^{201} \mathrm{~T}$ & 2 & 0 & 100 & 0 & & 0.323 & 0.318 & 0.360 & 800 \\
\hline $67 \mathrm{Ga}-350 \mathrm{uc}+200 \mathrm{~mm}-\mathrm{H} 2 \mathrm{O}+5 \mathrm{mmFe}$ & ${ }^{67} \mathrm{G}$ & 2 & 8 & 91 & 1 & & 0.115 & 0.878 & 0.008 & 400 \\
\hline $137 \mathrm{Cs}-8 \mathrm{mc}+24 \mathrm{~mm}-\mathrm{Lead}+5 \mathrm{mmFe}$ & ${ }^{137} \mathrm{Cs}$ & 2 & 0 & 100 & 0 & & 0.000 & 0.015 & 0.985 & 400 \\
\hline $14 \mathrm{Kg}$-of-Bananas $+5 \mathrm{mmFe}$ & ${ }^{40} \mathrm{~K}$ & 3 & 0 & 0 & 100 & & & & & \\
\hline $5 \mathrm{Kg}-\mathrm{of}-\mathrm{Wood}+5 \mathrm{mmFe}$ & ${ }^{40} \mathrm{~K}$ & 3 & 0 & 1 & 99 & promotion & & 0.13 & 0.04 & \\
\hline $5 \mathrm{Kg}$-of-Potash-Fertilizer $+5 \mathrm{mmFe}$ & ${ }^{40} \mathrm{~K}$ & 3 & 0 & 3 & 97 & demotion & & 0.69 & 0.23 & \\
\hline HEU & ${ }^{235} \mathrm{~L}$ & 1 & 94 & 6 & 0 & & & & & \\
\hline Virgin HEU & ${ }^{235} \mathrm{~L}$ & 1 & 96 & 4 & 0 & & & & & \\
\hline Reactor Pu & ${ }^{239} \mathrm{Pl}$ & 1 & 7 & 20 & 73 & & & & & \\
\hline Weapon Pu & ${ }^{239} \mathrm{Pl}$ & 1 & 61 & 18 & 21 & & & & & \\
\hline HEU with shielding & ${ }^{235} \mathrm{~L}$ & 1 & 0 & 25 & 75 & & & & & \\
\hline Virgin HEU with shielding & ${ }^{235} \mathrm{~L}$ & 1 & 0 & 85 & 15 & & & & & \\
\hline Reactor Pu with shielding & ${ }^{239} \mathrm{Pl}$ & 1 & 0 & 34 & 66 & & & & & \\
\hline Weapon Pu with shielding & ${ }^{239} \mathrm{PL}$ & 1 & 0 & 62 & 38 & & & & & \\
\hline
\end{tabular}




\begin{tabular}{|c|c|c|c|c|c|c|c|c|c|c|}
\hline $\begin{array}{l}500 \text { Counts } / 400 \text { Bkg } \\
\text { Sample }\end{array}$ & Label & Class & 1 & 2 & 3 & & & & & \\
\hline $99 \mathrm{TcM}-625 \mathrm{uc}+200 \mathrm{~mm}-\mathrm{H} 2 \mathrm{O}+5 \mathrm{mmFe}$ & ${ }^{99 \mathrm{~m}} \mathrm{Tc}$ & 3 & 0 & 1 & 98 & \multirow{5}{*}{ actual } & \multirow{2}{*}{\multicolumn{4}{|c|}{ Reported }} \\
\hline $131 \mathrm{l}-370 \mathrm{uc}+200 \mathrm{~mm}-\mathrm{H} 2 \mathrm{O}+5 \mathrm{mmFe}$ & ${ }^{131}$ & 2 & 1 & 99 & 0 & & & & & \\
\hline $201 \mathrm{TI}-250 \mathrm{uc}+200 \mathrm{~mm}-\mathrm{H} 2 \mathrm{O}+5 \mathrm{mmFe}$ & ${ }^{201} \mathrm{TI}$ & 2 & 0 & 100 & 0 & & 0.450 & 0.385 & 0.165 & 800 \\
\hline $67 \mathrm{Ga}-350 \mathrm{uc}+200 \mathrm{~mm}-\mathrm{H} 2 \mathrm{O}+5 \mathrm{mmFe}$ & ${ }^{67} \mathrm{Ga}$ & 2 & 0 & 100 & 0 & & 0.003 & 0.998 & 0.000 & 400 \\
\hline $137 \mathrm{Cs}-8 \mathrm{mc}+24 \mathrm{~mm}$-Lead+5mmFe & ${ }^{137} \mathrm{Cs}$ & 2 & 0 & 100 & 0 & & 0.003 & 0.018 & 0.980 & 399 \\
\hline $14 \mathrm{Kg}$-of-Bananas $+5 \mathrm{mmFe}$ & ${ }^{40} \mathrm{~K}$ & 3 & 0 & 0 & 100 & & & & & \\
\hline $5 \mathrm{Kg}$-of-Wood $+5 \mathrm{mmFe}$ & ${ }^{40} \mathrm{~K}$ & 3 & 0 & 0 & 100 & promotion & & 0.02 & 0.01 & \\
\hline $5 \mathrm{Kg}$-of-Potash-Fertilizer $+5 \mathrm{mmFe}$ & ${ }^{40} \mathrm{~K}$ & 3 & 1 & 6 & 93 & demotion & & 0.55 & 0.18 & \\
\hline HEU & ${ }^{235} U$ & 1 & 100 & 0 & 0 & & & & & \\
\hline Virgin HEU & ${ }^{235} U$ & 1 & 100 & 0 & 0 & & & & & \\
\hline Reactor $\mathrm{Pu}$ & ${ }^{239} \mathrm{Pu}$ & 1 & 64 & 23 & 13 & & & & & \\
\hline Weapon Pu & ${ }^{239} \mathrm{Pu}$ & 1 & 96 & 4 & 0 & & & & & \\
\hline HEU with shielding & ${ }^{235} U$ & 1 & 0 & 55 & 45 & & & & & \\
\hline Virgin HEU with shielding & ${ }^{235} U$ & 1 & 0 & 99 & 1 & & & & & \\
\hline Reactor Pu with shielding & ${ }^{239} \mathrm{Pu}$ & 1 & 0 & 42 & 58 & & & & & \\
\hline Weapon Pu with shielding & ${ }^{239} \mathrm{Pu}$ & 1 & 0 & 85 & 15 & & & & & \\
\hline
\end{tabular}

\begin{tabular}{|c|c|c|c|c|c|c|c|c|c|c|}
\hline $\begin{array}{l}750 \text { Counts/400 Bkg } \\
\text { Sample }\end{array}$ & Label & Class & 1 & 2 & 3 & & & & & \\
\hline 99TcM-625uc $+200 m m-H 2 O+5 m m F e$ & ${ }^{99 \mathrm{~m}} \mathrm{Tc}$ & 3 & 0 & 0 & 100 & \multirow{5}{*}{ actual } & \multicolumn{3}{|c|}{ Reported } & \multirow[b]{2}{*}{ total } \\
\hline $131 \mathrm{I}-370 \mathrm{uc}+200 \mathrm{~mm}-\mathrm{H} 2 \mathrm{O}+5 \mathrm{mmFe}$ & ${ }^{131}$ & 2 & 0 & 100 & 0 & & 1 & 2 & & \\
\hline $201 \mathrm{TI}-250 \mathrm{uc}+200 \mathrm{~mm}-\mathrm{H} 2 \mathrm{O}+5 \mathrm{mmFe}$ & ${ }^{201} \mathrm{TI}$ & 2 & 0 & 100 & 0 & & 0.490 & 0.391 & 0.119 & 800 \\
\hline $67 \mathrm{Ga}-350 \mathrm{uc}+200 \mathrm{~mm}-\mathrm{H} 2 \mathrm{O}+5 \mathrm{mmFe}$ & ${ }^{67} \mathrm{Ga}$ & 2 & 0 & 100 & 0 & & 0.000 & 1.000 & 0.000 & 400 \\
\hline $137 \mathrm{Cs}-8 \mathrm{mc}+24 \mathrm{~mm}-\mathrm{Lead}+5 \mathrm{mmFe}$ & ${ }^{137} \mathrm{Cs}$ & 2 & 0 & 100 & 0 & & 0.000 & 0.000 & 1.000 & 400 \\
\hline $14 \mathrm{Kg}$-of-Bananas $+5 \mathrm{mmFe}$ & ${ }^{40} \mathrm{~K}$ & 3 & 0 & 0 & 100 & & & & & \\
\hline $5 \mathrm{Kg}$-of-Wood $+5 \mathrm{mmFe}$ & ${ }^{40} \mathrm{~K}$ & 3 & 0 & 0 & 100 & promotion & & 0.00 & 0.00 & \\
\hline $5 \mathrm{Kg}$-of-Potash-Fertilizer $+5 \mathrm{mmFe}$ & ${ }^{40} \mathrm{~K}$ & 3 & 0 & 0 & 100 & demotion & & 0.51 & 0.17 & \\
\hline HEU & ${ }^{235} \mathrm{U}$ & 1 & 100 & 0 & 0 & & & & & \\
\hline Virgin HEU & ${ }^{235} \mathrm{U}$ & 1 & 100 & 0 & 0 & & & & & \\
\hline Reactor $\mathrm{Pu}$ & ${ }^{239} \mathrm{Pu}$ & 1 & 89 & 9 & 2 & & & & & \\
\hline Weapon Pu & ${ }^{239} \mathrm{Pu}$ & 1 & 100 & 0 & 0 & & & & & \\
\hline HEU with shielding & ${ }^{235} \mathrm{U}$ & 1 & 0 & 71 & 29 & & & & & \\
\hline Virgin HEU with shielding & ${ }^{235} \mathrm{U}$ & 1 & 0 & 100 & 0 & & & & & \\
\hline Reactor Pu with shielding & ${ }^{239} \mathrm{Pu}$ & 1 & 0 & 43 & 57 & & & & & \\
\hline Weapon Pu with shielding & ${ }^{239} \mathrm{Pu}$ & 1 & 3 & 90 & 7 & & & & & \\
\hline
\end{tabular}




\begin{tabular}{|c|c|c|c|c|c|c|c|c|c|c|c|c|}
\hline $\begin{array}{l}1000 \text { Counts/400 Bkg } \\
\text { Sample }\end{array}$ & Label & Class & & 1 & 2 & 3 & & & & & & \\
\hline 99TcM-625uc+200mm-H2O+5mmFe & ${ }^{99 \mathrm{~m}} \mathrm{TC}$ & & 3 & 0 & 0 & 100 & \multirow{5}{*}{ actual } & & & eported & \multirow{2}{*}{\multicolumn{2}{|c|}{3 total }} \\
\hline $131 \mathrm{I}-370 \mathrm{uc}+200 \mathrm{~mm}-\mathrm{H} 2 \mathrm{O}+5 \mathrm{mmFe}$ & 131 & & 2 & 0 & 100 & 0 & & & 1 & 2 & & \\
\hline $201 \mathrm{TI}-250 \mathrm{uc}+200 \mathrm{~mm}-\mathrm{H} 2 \mathrm{O}+5 \mathrm{mmFe}$ & ${ }^{201} \mathrm{~T}$ & & 2 & 0 & 100 & 0 & & 1 & 0.494 & 0.401 & 0.105 & 800 \\
\hline $67 \mathrm{Ga}-350 \mathrm{uc}+200 \mathrm{~mm}-\mathrm{H} 2 \mathrm{O}+5 \mathrm{mmFe}$ & ${ }^{67} \mathrm{G}$ & & 2 & 4 & 96 & 0 & & 2 & 0.010 & 0.990 & 0.000 & 400 \\
\hline $137 \mathrm{Cs}-8 \mathrm{mc}+24 \mathrm{~mm}-$ Lead $+5 \mathrm{mmFe}$ & ${ }^{137} \mathrm{Cs}$ & & 2 & 0 & 100 & 0 & & 3 & 0.000 & 0.005 & 0.995 & 400 \\
\hline $14 \mathrm{Kg}$-of-Bananas $+5 \mathrm{mmFe}$ & ${ }^{40} \mathrm{~K}$ & & 3 & 0 & 0 & 100 & \multirow{11}{*}{\multicolumn{2}{|c|}{$\begin{array}{l}\text { promotion } \\
\text { demotion }\end{array}$}} & & & & \\
\hline $5 \mathrm{Kg}$-of-Wood $+5 \mathrm{mmFe}$ & ${ }^{40} \mathrm{~K}$ & & 3 & 0 & 0 & 100 & & & & 0.02 & 0.01 & \\
\hline $5 \mathrm{Kg}$-of-Potash-Fertilizer $+5 \mathrm{mmFe}$ & ${ }^{40} \mathrm{~K}$ & & 3 & 0 & 2 & 98 & & & & 0.51 & 0.17 & \\
\hline HEU & ${ }^{235} \mathrm{~L}$ & & 1 & 100 & 0 & 0 & & & & & & \\
\hline Virgin HEU & ${ }^{235} \mathrm{~L}$ & & 1 & 100 & 0 & 0 & & & & & & \\
\hline Reactor Pu & ${ }^{239} \mathrm{Pl}$ & & 1 & 95 & 5 & 0 & & & & & & \\
\hline Weapon Pu & ${ }^{239} \mathrm{Pl}$ & & 1 & 100 & 0 & 0 & & & & & & \\
\hline HEU with shielding & ${ }^{235} \mathrm{~L}$ & & 1 & 0 & 84 & 16 & & & & & & \\
\hline Virgin HEU with shielding & ${ }^{235} \mathrm{~L}$ & & 1 & 0 & 100 & 0 & & & & & & \\
\hline Reactor Pu with shielding & ${ }^{239} \mathrm{Pl}$ & & 1 & 0 & 45 & 55 & & & & & & \\
\hline Weapon Pu with shielding & ${ }^{239} \mathrm{PL}$ & & 1 & 0 & 87 & 13 & & & & & & \\
\hline
\end{tabular}

\section{A.4.1.3 GADRAS fittodb Correlation Matrix}

\section{Column Definitions}

- Sample - Sample name of input.

- Label - Expert label.

- ND - number of trials were "no detection" was declared.

- Sum - total number of isotopic labels assigned to samples. 


\begin{tabular}{|c|c|c|c|c|c|c|c|c|c|c|c|c|c|c|c|c|c|c|c|c|c|c|c|c|c|c|c|c|}
\hline Sample & abel & ND & $\begin{array}{l}40 \\
\mathrm{~K} \\
\end{array}$ & $\begin{array}{l}226 \\
\mathrm{Ra} \\
\end{array}$ & $\begin{array}{c}232 \\
\text { Th } \\
\end{array}$ & $\begin{array}{c}2282 \\
\text { Th } \\
\end{array}$ & $\begin{array}{c}232 \\
U\end{array}$ & $\begin{array}{l}60 \\
\text { Co } \\
\end{array}$ & $\begin{array}{l}133 \\
\mathrm{Ba}\end{array}$ & $\begin{array}{c}137 \\
\text { Cs } \\
\end{array}$ & $\begin{array}{c}54 \\
M n \\
\end{array}$ & $\begin{array}{l}57 \\
\text { Co } \\
\end{array}$ & $\begin{array}{c}1392 \\
\mathrm{Ce}\end{array}$ & $\begin{array}{c}2522 \\
\text { Cf }\end{array}$ & $\begin{array}{c}2382 \\
U\end{array}$ & $\begin{array}{c}235 \\
\mathrm{U}\end{array}$ & $\begin{array}{r}2392 \\
\mathrm{Pu} \\
\end{array}$ & $\mathrm{Np}$ & 1 & $\begin{array}{l}99^{\mathrm{m}} \\
\text { Tc }\end{array}$ & $\begin{array}{l}672 \\
\mathrm{Ga} \\
\end{array}$ & $\begin{array}{c}201 \\
\mathrm{TI} \\
\end{array}$ & $\begin{array}{l}88 \\
Y \\
\end{array}$ & $\begin{array}{l}22 \\
\mathrm{Na} \\
\end{array}$ & $\begin{array}{l}166^{\mathrm{m}} \\
\mathrm{Ho} \\
\end{array}$ & $\begin{array}{c}152 \\
\mathrm{Eu}\end{array}$ & $\begin{array}{c}192 \\
\text { Ir } \\
\end{array}$ & sum \\
\hline \multicolumn{29}{|l|}{50 Counts/400 Bkg } \\
\hline $99 \mathrm{TcM}-625 \mathrm{uc}+200 \mathrm{~mm}-\mathrm{H} 2 \mathrm{O}+5 \mathrm{mmFe}$ & $\mathrm{s}$ & 17 & 0 & 0 & 0 & 0 & 0 & 0 & 2 & 0 & 0 & 49 & 7 & 0 & 0 & 12 & 1 & 1 & 0 & 57 & 1 & 5 & 0 & 0 & 0 & 0 & & 153 \\
\hline 1311-370uc+200mm-H2O+5mmFe & & 77 & 0 & 0 & 0 & 0 & 0 & 0 & 17 & 2 & 0 & 0 & 2 & 0 & 0 & 1 & 9 & 5 & 12 & 1 & 2 & 3 & 0 & 0 & 0 & 0 & & 133 \\
\hline $201 \mathrm{TI}-250 \mathrm{uc}+200 \mathrm{~mm}-\mathrm{H} 2 \mathrm{O}+5 \mathrm{mmFe}$ & & 26 & 0 & 0 & 0 & 0 & 0 & 0 & 1 & 1 & 0 & 7 & 48 & 0 & 0 & 22 & 2 & 1 & 1 & 14 & 0 & 31 & 0 & 0 & 0 & 0 & & 155 \\
\hline $37 \mathrm{Ga}-350 \mathrm{uc}+200 \mathrm{~mm}-\mathrm{H} 2 \mathrm{O}+5 \mathrm{mmFe}$ & & 64 & 0 & 0 & 0 & 0 & 0 & 0 & 12 & 0 & 0 & 0 & 10 & 0 & 0 & 10 & 2 & 7 & 1 & 2 & 16 & 7 & 0 & 0 & 0 & 0 & & 136 \\
\hline $137 \mathrm{Cs}-8 \mathrm{mc}+24 \mathrm{~mm}-\mathrm{Lead}+5 \mathrm{mmFe}$ & ${ }^{137} \mathrm{Cs}$ & 71 & 0 & 0 & 0 & 0 & 0 & 0 & 1 & 28 & 0 & 0 & 0 & 0 & 0 & 0 & 2 & 0 & 0 & 0 & 0 & 0 & 0 & 0 & 0 & 0 & & 102 \\
\hline $14 \mathrm{Kg}$-of-Bananas $+5 \mathrm{mmFe}$ & & 96 & 1 & 0 & 0 & 0 & 0 & 0 & 1 & 1 & 0 & 0 & 0 & 0 & 0 & 0 & 1 & 0 & 0 & 0 & 0 & 0 & 0 & 1 & 0 & 0 & & 102 \\
\hline 5Kg-of-Wood+5mmFe & & 95 & 1 & 0 & 0 & 0 & 0 & 0 & 1 & 0 & 1 & 0 & 0 & 0 & 0 & 0 & 1 & 0 & 0 & 0 & 0 & 0 & 0 & 1 & 0 & 0 & & 10 \\
\hline $5 \mathrm{Kg}$-of-Potash-Fertilizer+5mmFe & & 89 & 0 & 0 & 0 & 0 & 0 & 0 & 1 & 1 & 0 & 0 & 2 & 0 & 0 & 1 & 4 & 1 & 0 & 0 & 4 & 1 & 0 & 1 & 0 & 0 & & 107 \\
\hline HEU & & 42 & 0 & 0 & 0 & 0 & 0 & 0 & 6 & 1 & 0 & 6 & 15 & 0 & 0 & 41 & 2 & 1 & 0 & 6 & 5 & 14 & 0 & 0 & 0 & 0 & & 140 \\
\hline Virgin HEU & & 38 & 0 & 0 & 0 & 0 & 0 & 0 & 6 & 1 & 0 & 7 & 17 & 0 & 0 & 44 & 1 & 1 & 1 & 7 & 4 & 15 & 0 & 0 & 0 & 0 & & 144 \\
\hline Reactor Pu & & 72 & 0 & 0 & 0 & 0 & 0 & 0 & 4 & 4 & 0 & 4 & 4 & 0 & 0 & 4 & 8 & 4 & 3 & 6 & 5 & 2 & 0 & 0 & 0 & 0 & & 121 \\
\hline Weapon Pu & & 68 & 0 & 0 & 0 & 0 & 0 & 0 & 10 & 2 & 0 & 3 & 2 & 0 & 0 & 1 & 12 & 3 & 8 & 5 & 5 & 3 & 0 & 0 & 0 & 0 & & 126 \\
\hline HEU with shielding & & 98 & 0 & 0 & 0 & 0 & 0 & 0 & 0 & 0 & 0 & 0 & 0 & 0 & 0 & 0 & 0 & 0 & 0 & 0 & 0 & 0 & 0 & 1 & 0 & 0 & & 100 \\
\hline Virgin HEU with shielding & & 97 & 0 & 0 & 0 & 0 & 0 & 0 & 0 & 1 & 0 & 0 & 0 & 0 & 0 & 0 & 0 & 0 & 0 & 0 & 0 & 0 & 0 & 1 & 1 & 0 & & $10 c$ \\
\hline Reactor Pu with shielding & & 97 & 0 & 0 & 0 & 0 & 0 & 0 & 0 & 3 & 0 & 0 & 0 & 0 & 0 & 0 & 0 & 0 & 0 & 0 & 0 & 0 & 0 & 0 & 0 & 0 & & 100 \\
\hline Weapon Pu with shielding & & 90 & 0 & 0 & 0 & 0 & 0 & 0 & 0 & 5 & 0 & 0 & 0 & 0 & 1 & 0 & 0 & 0 & 0 & 0 & 1 & 0 & 0 & 1 & 1 & 0 & & 100 \\
\hline \multicolumn{29}{|l|}{100 Counts $/ 400$ Bkg } \\
\hline $99 \mathrm{TcM}-625 \mathrm{uc}+200 \mathrm{~mm}-\mathrm{H} 2 \mathrm{O}+5 \mathrm{~m}$ & & 1 & 3 & 0 & 1 & 0 & 0 & 0 & 0 & 0 & 0 & 28 & 1 & 0 & 0 & 1 & 0 & 0 & 0 & 96 & 0 & & 0 & 0 & 0 & 0 & & 132 \\
\hline $1311-370 \mathrm{uc}+200 \mathrm{~mm}-\mathrm{H} 2 \mathrm{O}+5 \mathrm{mmFe}$ & & 25 & 0 & 2 & 0 & 0 & 0 & 0 & 43 & 3 & 0 & 2 & 0 & 0 & 0 & 2 & 12 & 4 & 34 & 2 & 8 & 1 & c & 0 & 0 & 0 & & $\begin{array}{l}3 \\
3\end{array}$ \\
\hline $201 \mathrm{TI}-250 \mathrm{uc}+200 \mathrm{~mm}-\mathrm{H} 2 \mathrm{O}+5 \mathrm{mmFe}$ & & 13 & 6 & 0 & 0 & 0 & 0 & 0 & 1 & 1 & 0 & 8 & 61 & 0 & 0 & 8 & 0 & 0 & 0 & 15 & 0 & 38 & 0 & 0 & 0 & 0 & 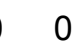 & 151 \\
\hline $67 \mathrm{Ga}-350 \mathrm{uc}+200 \mathrm{~mm}-\mathrm{H} 2 \mathrm{O}+5 \mathrm{mmFe}$ & & 18 & 0 & 0 & 0 & 0 & 0 & 0 & 30 & 0 & 0 & 2 & 5 & 0 & 0 & 33 & 2 & 11 & 1 & 3 & 44 & 6 & 0 & 0 & 0 & 0 & & 159 \\
\hline 137 Cs- $8 m c+24 m m-L e a d+5 m$ & & 17 & 0 & 0 & 0 & 0 & 0 & 0 & 0 & 79 & 0 & 0 & 0 & 0 & 0 & 1 & 5 & 0 & 1 & 2 & 1 & 0 & $c$ & 0 & 1 & 0 & & 109 \\
\hline $14 \mathrm{Kg}$-of-Bananas $+5 \mathrm{mmFe}$ & & 63 & 25 & 1 & 1 & 0 & 0 & 0 & 1 & 2 & 1 & 0 & 1 & 0 & 1 & 0 & 1 & 1 & 0 & 1 & 0 & 2 & 0 & 0 & 0 & 0 & & 103 \\
\hline $5 \mathrm{Kg}$-of-Wood +5mmFe & & 60 & 24 & 1 & 1 & 0 & 0 & 0 & 3 & 1 & 1 & 0 & 1 & 0 & 1 & 1 & 3 & 1 & 1 & 3 & 2 & 1 & 0 & 0 & 1 & 1 & & 109 \\
\hline $5 \mathrm{Kg}$-of- & & 70 & 1 & 0 & 0 & 1 & 0 & 0 & 5 & 4 & 0 & 0 & 0 & 0 & 1 & 7 & 2 & 2 & 1 & 3 & 9 & 2 & 0 & 0 & 0 & 1 & & 114 \\
\hline HEU & & 5 & 2 & 0 & 0 & 0 & 0 & 0 & 3 & 1 & 0 & 7 & 23 & 0 & 0 & 85 & 0 & 0 & 0 & 14 & 3 & 11 & 0 & 0 & 0 & 0 & & 15 \\
\hline Virgin HEU & & 4 & 2 & 0 & 0 & 0 & 0 & 0 & 2 & 0 & 0 & 10 & 29 & 0 & 0 & 86 & 0 & 0 & 0 & 11 & 2 & 14 & 0 & 0 & 0 & 0 & & 160 \\
\hline Reactor $\mathrm{Pl}$ & & 42 & 1 & 0 & 0 & 0 & 0 & 0 & 17 & 6 & 1 & 13 & 1 & 0 & 0 & 21 & 5 & 2 & 6 & 11 & 8 & 1 & 0 & 0 & 0 & 0 & & 135 \\
\hline Neapon Pu & ${ }^{239} \mathrm{Pu}$ & 34 & 1 & 0 & 0 & 0 & 0 & 0 & 23 & 5 & 1 & 4 & 3 & 0 & 0 & 10 & 23 & 6 & 16 & 6 & 15 & 1 & 0 & 0 & 1 & 0 & & \begin{tabular}{|l|l|}
2 & 151 \\
\end{tabular} \\
\hline
\end{tabular}




\begin{tabular}{|c|c|c|c|c|c|c|c|c|c|c|c|c|c|c|c|c|c|c|c|c|c|c|c|c|c|c|c|c|}
\hline Sample & & ND & $\begin{array}{c}40 \\
\mathrm{~K}\end{array}$ & $\begin{array}{c}2262 \\
\mathrm{Ra}\end{array}$ & $\begin{array}{c}232 \\
\text { Th }\end{array}$ & $\begin{array}{c}228 \\
\text { Th }\end{array}$ & $\begin{array}{c}232 \\
\text { U }\end{array}$ & $\begin{array}{l}60 \\
\text { Co }\end{array}$ & $\begin{array}{c}133 \\
\mathrm{Ba} \\
\end{array}$ & $\begin{array}{c}137 \\
\text { Cs }\end{array}$ & $\begin{array}{c}54 \\
\mathrm{Mn} \\
\end{array}$ & $\begin{array}{l}57 \\
\text { Co }\end{array}$ & $\begin{array}{c}139 \\
\mathrm{Ce}\end{array}$ & Cf & $\begin{array}{c}238 \\
\mathrm{U}\end{array}$ & $\begin{array}{c}235 \\
\mathrm{U}\end{array}$ & $\begin{array}{l}239 \\
\mathrm{Pu}\end{array}$ & $\begin{array}{l}231 \\
\mathrm{~Np}\end{array}$ & $\begin{array}{c}131 \\
1\end{array}$ & $\begin{array}{l}99^{\mathrm{m}} \\
\text { Tc }\end{array}$ & $\begin{array}{l}67 \\
\mathrm{Ga}\end{array}$ & $\begin{array}{c}201 \\
\text { TI }\end{array}$ & $\begin{array}{c}88 \\
Y\end{array}$ & $\begin{array}{l}22 \\
\mathrm{Na}\end{array}$ & $\begin{array}{c}166^{\mathrm{m}} \\
\text { Ho }\end{array}$ & $\begin{array}{c}152 \\
\mathrm{Eu}\end{array}$ & $\begin{array}{c}192 \\
\text { Ir }\end{array}$ & 2 sum \\
\hline HEU with shielding & & 66 & 1 & 0 & 16 & 0 & 10 & 0 & 1 & 0 & 0 & 0 & 0 & 0 & 1 & 0 & 0 & 0 & 0 & 0 & 0 & 0 & 3 & 1 & 1 & 0 & 0 & \begin{tabular}{l|l}
101 \\
1
\end{tabular} \\
\hline Virgin HEU with shielding & & 56 & 0 & 2 & 5 & 0 & 0 & 1 & 0 & 1 & 2 & 0 & 0 & 2 & 23 & 0 & 2 & 0 & 0 & 0 & 0 & 0 & 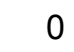 & 2 & 7 & 0 & D & 2105 \\
\hline Reactor Pu with shielding & & 57 & 0 & 3 & 10 & 1 & 0 & 0 & 0 & 21 & 1 & 0 & 0 & 0 & 1 & 0 & 2 & 0 & 0 & 1 & 0 & 0 & 0 & 0 & 3 & 0 & 0 & 1101 \\
\hline Weapon Pu with shielding & & 48 & 0 & 1 & 7 & 0 & 0 & 0 & 0 & 27 & 1 & 0 & 0 & 2 & 1 & 0 & 8 & 0 & 0 & 0 & 0 & 0 & 0 & 0 & 8 & 0 & o & $\begin{array}{ll}6 & 109 \\
\end{array}$ \\
\hline \multicolumn{29}{|l|}{200 Counts $/ 400$ Bkg } \\
\hline $99 \mathrm{TcM}-625 \mathrm{uc}+200 \mathrm{~mm}-\mathrm{H} 2 \mathrm{O}+5 \mathrm{mmFe}$ & & 0 & 1 & 0 & 0 & 0 & 0 & 0 & 0 & 0 & 0 & 21 & 0 & 0 & 0 & 0 & 0 & 0 & 0 & 100 & 0 & 16 & 0 & 0 & 0 & 0 & 0 & $0 \mid 138$ \\
\hline 1311-370uc+200mm-H2O+5mmFe & & 10 & 0 & 0 & 0 & 0 & 0 & 0 & 57 & 0 & 0 & 1 & 0 & 0 & 0 & 0 & 16 & 4 & 52 & 0 & 29 & 0 & 0 & 0 & 0 & 0 & 0 & 169 \\
\hline $201 \mathrm{TI}-250 \mathrm{uc}+200 \mathrm{~mm}-\mathrm{H} 2 \mathrm{O}+5 \mathrm{mmFe}$ & & 0 & 14 & 0 & 0 & 0 & 0 & 0 & 0 & 0 & 0 & 10 & 88 & 0 & 0 & 7 & 0 & 0 & 0 & 13 & 0 & 14 & 0 & 0 & 0 & 0 & 0 & 146 \\
\hline $67 \mathrm{Ga}-350 \mathrm{uc}+200 \mathrm{~mm}-\mathrm{H} 2 \mathrm{O}+5 \mathrm{mmFe}$ & & 1 & 2 & 0 & 0 & 0 & 0 & 0 & 3 & 0 & 0 & 0 & 2 & 0 & 0 & 17 & 0 & 4 & 0 & 0 & 81 & 1 & 0 & 0 & 0 & 0 & 0 & 111 \\
\hline $137 \mathrm{Cs}-8 \mathrm{mc}+24 \mathrm{~mm}$-Lead $+5 \mathrm{mmFe}$ & & 0 & 0 & 1 & 6 & 0 & 0 & 0 & 0 & 97 & 0 & 0 & 0 & 0 & 0 & 0 & 0 & 0 & 0 & 0 & 0 & 0 & 0 & 0 & 0 & 0 & 0 & 107 \\
\hline 14Kg-of-Bananas $+5 \mathrm{mmFe}$ & & 17 & 83 & 1 & 0 & 0 & 0 & 0 & 0 & 0 & 0 & 0 & 0 & 0 & 0 & 0 & 0 & 0 & 0 & 0 & 0 & 0 & 0 & 0 & 0 & 0 & 0 & 10 \\
\hline $5 \mathrm{Kg}$-of-Wood+5mmFe & & 16 & 83 & 0 & 1 & 0 & 0 & 0 & 0 & 0 & 0 & 0 & 0 & 0 & 0 & 0 & 0 & 0 & 0 & 0 & 0 & 0 & 0 & 0 & 0 & 0 & 0 & 100 \\
\hline $5 \mathrm{Kg}$-of-Po & & 68 & 14 & 2 & 3 & 0 & 0 & 0 & 1 & 1 & 3 & 0 & 1 & 4 & 0 & 3 & 0 & 0 & 0 & 0 & 0 & 0 & 0 & 0 & 0 & 0 & 0 & 101 \\
\hline HEU & & 0 & 36 & 0 & 2 & 0 & 0 & 0 & 0 & 0 & 0 & 1 & 6 & 0 & 0 & 86 & 0 & 0 & 0 & 2 & 0 & 3 & 0 & 0 & 0 & 0 & 0 & 136 \\
\hline Virgin HEU & & 1 & 32 & 0 & 1 & 0 & 0 & 0 & 0 & 0 & 0 & 0 & 3 & 0 & 0 & 82 & 0 & 0 & 0 & 2 & 0 & 2 & 0 & 0 & 0 & 0 & 0 & 123 \\
\hline Reactor Pu & & 50 & 20 & 0 & 1 & 0 & 0 & 0 & 1 & 3 & 0 & 6 & 1 & 0 & 0 & 6 & 1 & 1 & 0 & 7 & 5 & 0 & 0 & 0 & 0 & 0 & 0 & 102 \\
\hline Weapon PL & & 39 & 3 & 0 & 0 & 0 & 0 & 0 & 7 & 2 & 0 & 1 & 1 & 0 & 0 & 1 & 36 & 1 & 23 & 3 & 20 & $c$ & $\mathrm{c}$ & 0 & 0 & 0 & 0 & 137 \\
\hline HEU with & & 14 & 1 & 16 & 51 & 1 & 18 & 0 & 0 & 0 & 0 & 0 & 0 & 1 & 0 & 0 & 0 & 0 & 0 & 0 & 0 & 0 & 0 & 0 & 0 & 0 & 0 & 102 \\
\hline Virgin HEU w & & 14 & 0 & 17 & 14 & 0 & 0 & 0 & 0 & 0 & 0 & 0 & 0 & 0 & 61 & 0 & 0 & 0 & 0 & 1 & 0 & 0 & 0 & 0 & 0 & 5 & 5 & 112 \\
\hline Reactor PL & & 18 & 0 & 3 & 58 & 1 & 3 & 0 & 0 & 5 & 0 & 0 & 0 & 10 & 0 & 0 & 0 & 0 & 0 & 0 & 0 & & & 0 & 2 & 0 & 0 & 100 \\
\hline Weapon Pu with shielding & & 23 & 0 & 4 & 31 & 0 & 0 & 0 & 0 & 25 & 0 & 0 & 0 & 6 & 5 & 0 & 2 & 0 & 0 & 0 & 0 & 0 & 0 & 0 & 10 & 0 & b & 108 \\
\hline \multicolumn{29}{|l|}{300 Counts/400 Bkg } \\
\hline $99 \mathrm{TcM}-625 \mathrm{uc}+200 \mathrm{~mm}-\mathrm{H} 2 \mathrm{O}+5 \mathrm{~m}$ & & 0 & 0 & 0 & 0 & 0 & 0 & 0 & 0 & 0 & 0 & 2 & 0 & 0 & 0 & 0 & 0 & 0 & & 100 & 0 & & & 0 & 0 & 0 & 0 & 0 \\
\hline $1311-370 \mathrm{uc}+200 \mathrm{~mm}-\mathrm{H} 2 \mathrm{O}+5 \mathrm{mmFe}$ & & 0 & 0 & 2 & 0 & 0 & 0 & 0 & 79 & 1 & 0 & 0 & 0 & 0 & 0 & 0 & 21 & 17 & 67 & 0 & 55 & 0 & 0 & 0 & 0 & 0 & 0 & 242 \\
\hline $201 \mathrm{Tl}-250 \mathrm{uc}+200 \mathrm{~mm}-\mathrm{H} 2 \mathrm{O}+$ & & 0 & 0 & 0 & 0 & 0 & 0 & 0 & 0 & 0 & 0 & & 100 & 0 & 0 & 0 & 0 & 0 & 0 & 5 & 0 & 7 & 0 & 0 & 0 & 0 & 0 & 113 \\
\hline 77G & & 1 & 4 & 0 & 0 & 0 & 0 & 0 & 5 & 0 & 0 & 0 & 1 & 0 & 0 & 3 & 0 & 5 & 0 & 0 & 96 & 0 & 0 & 0 & 0 & 0 & 0 & 115 \\
\hline $137 \mathrm{Cs}-8 \mathrm{mc}+24 \mathrm{~mm}$-Lead $+5 \mathrm{mmFe}$ & ${ }^{137} \mathrm{Cs}$ & 0 & 0 & 0 & 2 & 0 & 0 & 0 & & 100 & 0 & 0 & 0 & 0 & 0 & 0 & 0 & 0 & 0 & 0 & 0 & 0 & 0 & 0 & 0 & 0 & 0 & 102 \\
\hline $14 \mathrm{Kg}$-of-Bananas $+5 \mathrm{mmFe}$ & & 1 & 99 & 2 & 0 & 0 & 0 & 0 & 0 & 0 & 0 & 0 & 0 & 0 & 0 & 0 & 0 & 0 & 0 & 0 & 0 & 0 & 0 & 0 & 0 & 0 & 0 & 102 \\
\hline $5 \mathrm{Kg}$-of-Wo & & 0 & 99 & 1 & 0 & 0 & 0 & 0 & 0 & 0 & 0 & 0 & 0 & 0 & 0 & 0 & 0 & 0 & 0 & 0 & 0 & 0 & 0 & 0 & 0 & 1 & 1 & 101 \\
\hline 5Kg-of-Potash-Fertilizer $+5 \mathrm{mmFe}$ & ${ }^{40} \mathrm{~K}$ & 42 & 48 & 2 & 7 & 0 & 0 & 0 & 0 & 0 & 0 & 0 & 0 & 1 & 0 & 0 & 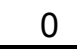 & 0 & 0 & 2 & 2 & 0 & 0 & 0 & 0 & $\underline{0}$ & b & 104 \\
\hline
\end{tabular}




\begin{tabular}{|c|c|c|c|c|c|c|c|c|c|c|c|c|c|c|c|c|c|c|c|c|c|c|c|c|c|c|c|c|}
\hline Sample & & ND & $\begin{array}{c}40 \\
\mathrm{~K}\end{array}$ & $\begin{array}{c}226 \\
\mathrm{Ra}\end{array}$ & $\begin{array}{l}2322 \\
\text { Th } \\
\end{array}$ & $\begin{array}{l}228 \\
\text { Th }\end{array}$ & $\begin{array}{c}232 \\
\mathrm{U}\end{array}$ & $\begin{array}{l}60 \\
\text { Co }\end{array}$ & $\begin{array}{c}1331 \\
\mathrm{Ba}\end{array}$ & $\begin{array}{c}137 \\
\text { Cs }\end{array}$ & $\begin{array}{l}54 \\
M n\end{array}$ & $\begin{array}{ll}57 & 1 \\
\text { Co } & \end{array}$ & $\begin{array}{c}139 \\
\mathrm{Ce}\end{array}$ & $\begin{array}{c}252 \\
\text { Cf }\end{array}$ & $\begin{array}{c}238 \\
U\end{array}$ & $\begin{array}{c}2352 \\
\mathrm{U}\end{array}$ & $\begin{array}{ll}239 \\
\mathrm{Pu}\end{array}$ & $\mathrm{Np}$ & $\begin{array}{c}131 \mathrm{~s} \\
\mathrm{I}\end{array}$ & $\begin{array}{c}99^{\mathrm{m}} \\
\text { Tc }\end{array}$ & $\begin{array}{l}672 \\
\mathrm{Ga}\end{array}$ & $\begin{array}{c}201 \\
\text { TI }\end{array}$ & $\begin{array}{c}88 \\
Y\end{array}$ & $\begin{array}{l}22 \\
\mathrm{Na}\end{array}$ & $\begin{array}{l}166^{\mathrm{m}} \\
\mathrm{Ho}\end{array}$ & $\begin{array}{c}152 \\
\mathrm{Eu}\end{array}$ & $\begin{array}{c}192 \\
\text { Ir }\end{array}$ & sum \\
\hline HEU & & 0 & 12 & 0 & 0 & 0 & 0 & 0 & 0 & 0 & 0 & 0 & 8 & 0 & 0 & 94 & 0 & 0 & 0 & 1 & 0 & 0 & 0 & 0 & 0 & 0 & & 115 \\
\hline Virgin HEU & & 0 & 4 & 0 & 0 & 0 & 0 & 0 & 0 & 0 & 0 & 0 & 11 & 0 & 0 & 96 & 0 & 0 & 0 & 0 & 0 & 3 & 0 & 0 & 0 & 0 & & 114 \\
\hline Reactor Pu & & 21 & 46 & 0 & 9 & 0 & 0 & 0 & 1 & 7 & 0 & 0 & 1 & 0 & 0 & 5 & 2 & 0 & 0 & 7 & 12 & 0 & 0 & 0 & 0 & 0 & & 111 \\
\hline Weapon Pu & & 15 & 5 & 0 & 2 & 0 & 0 & 0 & 31 & 11 & 0 & 0 & 0 & 0 & 0 & 1 & 52 & 9 & 40 & 1 & 39 & 0 & 0 & 0 & 0 & 0 & & 208 \\
\hline HEU with shielding & & 0 & 1 & 31 & 60 & 0 & 24 & 0 & 0 & 0 & 0 & 0 & 0 & 2 & 0 & 0 & 0 & 0 & 0 & 0 & 0 & 0 & 0 & 0 & 0 & 0 & & 118 \\
\hline Virgin HEU with shielding & & 3 & 0 & 39 & 10 & 0 & 0 & 0 & 0 & 0 & 0 & 0 & 0 & 0 & 82 & 0 & 0 & 0 & 0 & 0 & 0 & 0 & 0 & 0 & 0 & 4 & & 138 \\
\hline Reactor Pu with shielding & & 5 & 0 & 10 & 70 & 0 & 6 & 0 & 0 & 22 & 0 & 0 & 0 & 12 & 0 & 0 & 0 & 0 & 0 & 0 & 0 & 0 & 0 & 0 & 6 & 0 & & 131 \\
\hline Weapon Pu with shielding & & 4 & 0 & 9 & 55 & 0 & 0 & 0 & 0 & 46 & 0 & 0 & 0 & 7 & 4 & 0 & 0 & 0 & 0 & 0 & 0 & 0 & 0 & 0 & 11 & 0 & & 138 \\
\hline \multicolumn{29}{|l|}{500 Counts/400 Bkg } \\
\hline $99 \mathrm{TcM}-625 \mathrm{uc}+200 \mathrm{~mm}-\mathrm{H} 2 \mathrm{O}+5 \mathrm{mmFe}$ & & 0 & 0 & 0 & 0 & 0 & 0 & 0 & 0 & 0 & 0 & 1 & 0 & 0 & 0 & 0 & 0 & 0 & 0 & 99 & 0 & 0 & 0 & 0 & 0 & 0 & & 100 \\
\hline $1311-370 \mathrm{uc}+200 \mathrm{~mm}-\mathrm{H} 2 \mathrm{O}+5 \mathrm{mmFe}$ & & 0 & 0 & 0 & 0 & 0 & 0 & 0 & 52 & 0 & 0 & 0 & 0 & 0 & 0 & 0 & 1 & 0 & 65 & 0 & 5 & 0 & 0 & 0 & 0 & 0 & & 123 \\
\hline $201 \mathrm{TI}-250 \mathrm{uc}+200 \mathrm{~mm}-\mathrm{H} 2 \mathrm{O}+5 \mathrm{mmFe}$ & & 0 & 0 & 0 & 0 & 0 & 0 & 0 & 0 & 0 & 0 & & 100 & 0 & 0 & 0 & 0 & 0 & 0 & 6 & 0 & 6 & 0 & 0 & 0 & 0 & & 113 \\
\hline $57 \mathrm{Ga}-350 \mathrm{uc}+2 \mathrm{C}$ & & 0 & 0 & 0 & 0 & 0 & 0 & 0 & 0 & 0 & 0 & 0 & 0 & 0 & 0 & 0 & 0 & 0 & 0 & & 100 & 0 & 0 & 0 & 0 & 0 & & 100 \\
\hline $137 \mathrm{Cs}-8 \mathrm{mc}+24 \mathrm{~mm}$-Lead+5mmFe & & 0 & 0 & 0 & 0 & 0 & 0 & 0 & & 100 & 0 & 0 & 0 & 0 & 0 & 0 & 0 & 0 & 0 & 0 & 0 & 0 & 0 & 0 & 0 & 0 & & 100 \\
\hline 14Kg-of-Bananas $+5 \mathrm{mmFe}$ & & & 100 & 0 & 0 & 0 & 0 & 0 & 0 & 0 & 0 & 0 & 0 & 0 & 0 & 0 & 0 & 0 & 0 & 0 & 0 & 0 & 0 & 0 & 0 & 0 & & 100 \\
\hline $5 \mathrm{Kg}$-of-Wood+5mmFe & & & 100 & 0 & 0 & 0 & 0 & 0 & 0 & 0 & 0 & 0 & 0 & 0 & 0 & 0 & 0 & 0 & 0 & 0 & 0 & 0 & 0 & 0 & 0 & 0 & & 100 \\
\hline 5Kg-of-Potash-Fertilizer- & & 2 & 93 & 1 & 2 & 0 & 0 & 0 & 0 & 0 & 0 & 0 & 0 & 1 & 1 & 0 & 1 & 0 & 0 & 0 & 3 & 0 & 0 & 0 & 1 & 0 & & 106 \\
\hline HEU & & 0 & 0 & 0 & 0 & 0 & 0 & 0 & 0 & 0 & 0 & 0 & 0 & 0 & & 100 & 0 & 0 & 0 & 0 & 0 & & $\mathrm{c}$ & 0 & 0 & 0 & & 100 \\
\hline Virgin HEU & & 0 & 0 & 0 & 0 & 0 & 0 & 0 & 0 & 0 & 0 & 0 & 1 & 0 & & 100 & 0 & 0 & 0 & 0 & 0 & 0 & 0 & 0 & 0 & 0 & & 101 \\
\hline Reactor Pu & & 0 & 33 & 0 & 14 & 0 & 0 & 0 & 9 & 8 & 0 & 1 & 0 & 0 & 0 & 4 & 62 & 1 & 7 & 3 & 30 & & & 0 & 0 & 0 & & 172 \\
\hline & & 0 & 1 & 0 & 0 & 0 & 0 & 0 & 13 & 0 & 0 & 0 & 0 & 0 & 0 & 0 & 93 & 3 & 4 & 0 & 10 & 0 & 0 & 0 & 0 & 0 & & 124 \\
\hline HEU with shiel & & 1 & 0 & 50 & 53 & 0 & 55 & 0 & 0 & 0 & 0 & 0 & 0 & 0 & 0 & 0 & 0 & 0 & 0 & 0 & 0 & 0 & 0 & 0 & 0 & 0 & & 159 \\
\hline Virgin HEU wit & & 0 & 0 & 18 & 0 & 0 & 0 & 0 & 0 & 0 & 0 & 0 & 0 & 0 & 97 & 0 & 0 & 0 & 0 & 0 & 0 & & 0 & 0 & 1 & 1 & & 117 \\
\hline Reactor P & & 5 & 0 & 10 & 74 & 0 & 8 & 0 & 0 & 35 & 0 & 0 & 0 & 9 & 0 & 0 & 0 & 0 & 0 & 0 & 0 & 0 & 0 & 0 & 5 & 0 & & 1147 \\
\hline Weapon Pu with shielding & & 6 & 0 & 7 & 53 & 0 & 1 & 0 & 0 & 73 & 0 & 0 & 0 & 9 & 2 & 0 & 0 & 0 & 0 & 0 & 0 & & 0 & 0 & 11 & 0 & 10 & 172 \\
\hline \multicolumn{29}{|l|}{750 Counts/400 Bkg } \\
\hline $99 \mathrm{TcM}-625 \mathrm{uc}+200 \mathrm{~mm}-\mathrm{H} 2 \mathrm{O}+5 \mathrm{mmFe}$ & & 0 & 0 & 0 & 0 & 0 & 0 & 0 & 0 & 0 & 0 & 0 & 0 & 0 & 0 & 0 & 0 & 0 & 01 & 100 & 0 & 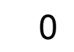 & 0 & 0 & 0 & 0 & & 100 \\
\hline $1311-370 \mathrm{uc}+200 \mathrm{~mm}-\mathrm{H} 2 \mathrm{O}+5 \mathrm{mmFe}$ & & 0 & 0 & 0 & 0 & 0 & 0 & 0 & 39 & 0 & 0 & 0 & 0 & 0 & 0 & 0 & 0 & 0 & 81 & 0 & 10 & 0 & 0 & 0 & 0 & 0 & & 130 \\
\hline $201 \mathrm{TI}-250 \mathrm{uc}+200 \mathrm{~mm}-\mathrm{H} 2 \mathrm{O}+5 \mathrm{mmFe}$ & & 0 & 0 & 0 & 0 & 0 & 0 & 0 & 0 & 0 & 0 & 6 & 98 & 0 & 0 & 0 & 0 & 0 & 0 & 28 & 0 & 9 & 0 & 0 & 0 & 0 & & 141 \\
\hline $67 \mathrm{Ga}-350 \mathrm{uc}+200 \mathrm{~mm}-\mathrm{H} 2 \mathrm{O}+5 \mathrm{mmFe}$ & ${ }^{67} \mathrm{Ga}$ & 0 & 0 & 0 & 0 & 0 & 0 & 0 & 0 & 0 & 0 & 0 & 0 & 0 & 0 & 0 & 0 & 0 & 0 & & 100 & 1 & 0 & 0 & 0 & 0 & & 101 \\
\hline
\end{tabular}




\begin{tabular}{|c|c|c|c|c|c|c|c|c|c|c|c|c|c|c|c|c|c|c|c|c|c|c|c|c|c|c|c|c|}
\hline Sample & & ND & $\begin{array}{l}40 \\
K\end{array}$ & $\begin{array}{c}226 \\
\mathrm{Ra}\end{array}$ & $\begin{array}{c}232 \\
\text { Th }\end{array}$ & $\begin{array}{l}228 \\
\text { Th }\end{array}$ & $\begin{array}{c}232 \\
U\end{array}$ & $\begin{array}{ll}60 & 1 \\
\text { Co } & \end{array}$ & $\begin{array}{c}133 \\
\mathrm{Ba}\end{array}$ & $\begin{array}{c}137 \\
\text { Cs }\end{array}$ & $\begin{array}{c}54 \\
M n\end{array}$ & $\begin{array}{ll}57 & 1 \\
\text { Co }\end{array}$ & $\begin{array}{c}139 \\
\mathrm{Ce}\end{array}$ & $\begin{array}{c}2522 \\
C f\end{array}$ & $\begin{array}{c}238 \\
U\end{array}$ & $\begin{array}{c}235 \\
U\end{array}$ & $\begin{array}{l}239 \\
\mathrm{Pu}\end{array}$ & $\begin{array}{l}237 \\
\mathrm{~Np}\end{array}$ & $\begin{array}{c}1315 \\
1\end{array}$ & $\begin{array}{l}99^{\prime \prime \prime} \\
\text { Tc }\end{array}$ & $\begin{array}{l}67 \\
\mathrm{Ga}\end{array}$ & $\begin{array}{c}201 \\
\text { TI }\end{array}$ & $\begin{array}{l}88 \\
Y\end{array}$ & $\begin{array}{l}22 \\
\mathrm{Na}\end{array}$ & $\begin{array}{c}166^{m} \\
\text { Ho }\end{array}$ & $\begin{array}{l}152 \\
\mathrm{Eu}\end{array}$ & $\begin{array}{l}192 \\
\text { Ir }\end{array}$ & 2 |sum \\
\hline $137 \mathrm{Cs}-8 \mathrm{mc}+24 \mathrm{~mm}-\mathrm{Lead}+5 \mathrm{mmFe}$ & & 0 & 0 & 0 & 0 & 0 & 0 & 0 & & 100 & 0 & 0 & 0 & 0 & 0 & 0 & 0 & 0 & 0 & 0 & 0 & 0 & 0 & 0 & 0 & & 0 & \begin{tabular}{l|l}
0 & 100
\end{tabular} \\
\hline $14 \mathrm{Kg}$-of-Bananas $+5 \mathrm{mmFe}$ & & & 100 & 0 & 0 & 0 & 0 & 0 & 0 & 0 & 0 & 0 & 0 & 0 & 0 & 0 & 0 & 0 & 0 & 0 & 0 & 0 & 0 & 0 & 0 & & 0 & 100 \\
\hline $5 \mathrm{Kg}$-of-Wood+5mmFe & & 0 & 100 & 0 & 0 & 0 & 0 & 0 & 0 & 0 & 0 & 0 & 0 & 0 & 0 & 0 & 0 & 0 & 0 & 0 & 0 & 0 & 0 & 0 & 0 & & 0 & 100 \\
\hline 5Kg-of-Potash-Fertilizer+5mmFe & & & 100 & 1 & 0 & 0 & 0 & 0 & 0 & 0 & 0 & 0 & 0 & 0 & 0 & 0 & 0 & 0 & 0 & 0 & 0 & 0 & 0 & 0 & 0 & & 0 & 101 \\
\hline HEU & & 0 & 0 & 0 & 0 & 0 & 0 & 0 & 0 & 0 & 0 & 0 & 0 & 0 & & 100 & 0 & 0 & 0 & 0 & 0 & 0 & 0 & 0 & 0 & & 0 & 100 \\
\hline Virgin HEU & & 0 & 0 & 0 & 0 & 0 & 0 & 0 & 0 & 0 & 0 & 0 & 0 & 0 & & 100 & 0 & 0 & 0 & 0 & 0 & 0 & 0 & 0 & 0 & & 0 & 100 \\
\hline Reactor Pu & & 0 & 24 & 0 & 8 & 0 & 0 & 0 & 13 & 20 & 1 & 2 & 0 & 0 & 0 & 4 & 89 & 1 & 6 & 0 & 29 & 0 & 0 & 0 & 0 & & 0 & 198 \\
\hline Weapon Pu & & 0 & 0 & 0 & 0 & 0 & 0 & 0 & 2 & 0 & 0 & 0 & 0 & 0 & 0 & & 100 & 0 & 0 & 0 & 3 & 0 & 0 & 0 & 0 & & 0 & 105 \\
\hline HEU with shielding & & 0 & 0 & 68 & 46 & 0 & 71 & 0 & 0 & 0 & 0 & 0 & 0 & 0 & 0 & 0 & 0 & 0 & 0 & 0 & 0 & 0 & 0 & 0 & 0 & & 0 & 185 \\
\hline Virgin HEU with shielding & & 0 & 0 & 5 & 0 & 0 & 0 & 0 & 0 & 0 & 0 & 0 & 0 & & 100 & 0 & 0 & 0 & 0 & 0 & 0 & 0 & 0 & 0 & 0 & & 0 & 105 \\
\hline Reactor Pu wi & & 0 & 0 & 6 & 80 & 0 & 10 & 0 & 0 & 38 & 0 & 0 & 0 & 17 & 1 & 0 & 0 & 0 & 0 & 0 & 0 & 0 & 0 & 0 & 6 & & 0 & 158 \\
\hline Weapon Pu with shielding & & 2 & 0 & 7 & 52 & 2 & 4 & 0 & 0 & 85 & 0 & 0 & 0 & 15 & 2 & 0 & 3 & 0 & 0 & 0 & 0 & 0 & 0 & 0 & 15 & & 0 & 191 \\
\hline 1000 Counts/400 Bk & & & & & & & & & & & & & & & & & & & & & & & & & & & & \\
\hline $99 \mathrm{TcM}-625 \mathrm{uc}+200 \mathrm{~mm}-\mathrm{H} 2 \mathrm{O}+5 \mathrm{mmFe}$ & & 0 & 0 & 0 & 0 & 0 & 0 & 0 & 0 & 0 & 0 & 0 & 0 & 0 & 0 & 0 & 0 & 0 & & 100 & 0 & 0 & 0 & 0 & 0 & & 0 & 0100 \\
\hline $1311-370 \mathrm{uc}+200 \mathrm{~mm}-\mathrm{H} 2 \mathrm{O}+5 \mathrm{mmFe}$ & & 0 & 0 & 0 & 0 & 0 & 0 & 0 & 32 & 0 & 0 & 0 & 0 & 0 & 0 & 0 & 0 & 0 & 91 & 0 & 4 & 0 & 0 & 0 & 0 & 0 & & 127 \\
\hline $201 \mathrm{TI}-250 \mathrm{uc}+200 \mathrm{~mm}-\mathrm{H}$ & & 0 & 0 & 0 & 0 & 0 & 0 & 0 & 0 & 0 & 0 & 45 & 99 & 0 & 0 & 0 & 0 & 0 & 0 & 18 & 0 & 57 & 0 & 0 & 0 & 0 & & 219 \\
\hline $37 \mathrm{Ga}-350 \mathrm{uc}+20$ & & 0 & 0 & 0 & 0 & 0 & 0 & 0 & 0 & 0 & 0 & 0 & 0 & 0 & 0 & 0 & 0 & 4 & 0 & \multicolumn{2}{|c|}{0100} & 0 & 0 & 0 & 0 & 0 & & 104 \\
\hline 137 Cs-8mc+24 & & 0 & 0 & 0 & 0 & 0 & 0 & 0 & & 100 & 0 & 0 & 0 & 0 & 0 & 0 & 0 & 0 & 0 & 0 & 0 & & c & 0 & 0 & & & \\
\hline $14 \mathrm{Kg}$-of-Bananas $+5 \mathrm{mmFe}$ & & & 100 & 0 & 0 & 0 & 0 & 0 & 0 & 0 & 0 & 0 & 0 & 0 & 0 & 0 & 0 & 0 & 0 & 0 & 0 & 0 & 0 & 0 & 0 & 0 & 0 & 100 \\
\hline $5 \mathrm{Kg}$-of-Wood+5mmFe & & & 100 & 0 & 0 & 0 & 0 & 0 & 0 & 0 & 0 & 0 & 0 & 0 & 0 & 0 & 0 & 0 & 0 & 0 & 0 & 0 & 0 & 0 & 0 & 0 & & 100 \\
\hline $5 \mathrm{Kg}$-of & & & 100 & 0 & 0 & 0 & 0 & 0 & 0 & 0 & 0 & 0 & 0 & 0 & 2 & 0 & 0 & 0 & 0 & 0 & 0 & & & 0 & 0 & & & 102 \\
\hline HEU & & 0 & 0 & 0 & 0 & 0 & 0 & 0 & 0 & 0 & 0 & 0 & 0 & 0 & \multicolumn{2}{|c|}{0100} & 0 & 0 & 0 & 0 & 0 & 0 & 0 & 0 & 0 & 0 & & 100 \\
\hline Virgin HEU & & 0 & 0 & 0 & 0 & 0 & 0 & 0 & 0 & 0 & 0 & 0 & 0 & 0 & \multicolumn{2}{|c|}{0100} & 0 & 0 & 0 & 0 & 0 & 0 & 0 & 0 & 0 & 0 & & 100 \\
\hline Reactor Pu & & 0 & 8 & 0 & 11 & 0 & 0 & 0 & 14 & 38 & 0 & 7 & 0 & 0 & 0 & 1 & 95 & 1 & 3 & 0 & 23 & 0 & 0 & 0 & 1 & 0 & & 202 \\
\hline Weapon Pu & & 0 & 0 & 0 & 0 & 0 & 0 & 0 & 0 & 0 & 0 & 0 & 0 & 0 & 0 & \multicolumn{2}{|c|}{0100} & 0 & 0 & 0 & 1 & 0 & 0 & 0 & 0 & 0 & & 10 \\
\hline HEU with & & 0 & 0 & 82 & 32 & 0 & 84 & 0 & 0 & 0 & 0 & 0 & 0 & 0 & 0 & 0 & 0 & 0 & 0 & 0 & 0 & 0 & 0 & 0 & 0 & 0 & & 198 \\
\hline Virgin HEL & & 0 & 0 & 2 & 0 & 0 & 0 & 0 & 0 & 0 & 0 & 0 & 0 & \multicolumn{2}{|c|}{0100} & 0 & 0 & 0 & 0 & 0 & 0 & 0 & 0 & 0 & 0 & 0 & 0 & 102 \\
\hline Reactor Pu & & 0 & 0 & 6 & 83 & 0 & 5 & 0 & 0 & 40 & 0 & 0 & 0 & 17 & 0 & 0 & 0 & 0 & 0 & 0 & 0 & 0 & 0 & 0 & 4 & 0 & & 155 \\
\hline Veapon Pu with shielding & ${ }^{239} \mathrm{Pu}$ & 0 & 0 & 12 & 65 & 0 & 5 & 0 & 0 & 78 & 0 & 0 & 0 & 12 & 0 & 0 & 0 & 0 & 0 & 0 & 0 & 0 & 0 & 0 & 20 & 0 & & 219 \\
\hline
\end{tabular}




\section{A.4.2 PCA}

\section{A.4.2.1 PCA Isotopic Score}

Column definitions

- Sample - name of the sample in the library file

- Label - expert label algorithm is trying to match

- Score - Number of trials in which the output matched the label.

- Best - Number of trials in which the output matched the score when taking best guess.

As a classifier produces only one output, the "Score" column here is equivelent to the "Included" and "Exact" column for GADRAS fittodb.

\begin{tabular}{|lrrr|}
\hline 50 Counts/400 Bkg & & & \\
Sample & Label & Score & Best \\
\hline $99 \mathrm{TcM}-625 \mathrm{uc}+200 \mathrm{~mm}-\mathrm{H} 2 \mathrm{O}+5 \mathrm{mmFe}$ & ${ }^{99 \mathrm{~m}} \mathrm{Tc}$ & 62 & 62 \\
$131 \mathrm{l}-370 \mathrm{uc}+200 \mathrm{~mm}-\mathrm{H} 2 \mathrm{O}+5 \mathrm{mmFe}$ & ${ }^{131} \mathrm{I}$ & 4 & 4 \\
$201 \mathrm{Tl}-250 \mathrm{uc}+200 \mathrm{~mm}-\mathrm{H} 2 \mathrm{O}+5 \mathrm{mmFe}$ & ${ }^{201} \mathrm{TI}$ & 34 & 34 \\
$67 \mathrm{Ga}-350 \mathrm{uc}+200 \mathrm{~mm}-\mathrm{H} 2 \mathrm{O}+5 \mathrm{mmFe}$ & ${ }^{67} \mathrm{Ga}$ & 4 & 4 \\
$137 \mathrm{Cs}-8 \mathrm{mc}+24 \mathrm{~mm}-\mathrm{Lead}+5 \mathrm{mmFe}$ & ${ }^{137} \mathrm{Cs}$ & 26 & 26 \\
$14 \mathrm{Kg}-$ of-Bananas+5mmFe & ${ }^{40} \mathrm{~K}$ & 1 & 1 \\
$5 \mathrm{Kg}-$-of-Wood+5mmFe & ${ }^{40} \mathrm{~K}$ & 1 & 1 \\
$5 \mathrm{Kg}-$-of-Potash-Fertilizer+5mmFe & ${ }^{40} \mathrm{~K}$ & 0 & 0 \\
HEU & ${ }^{235} \mathrm{U}$ & 12 & 12 \\
Virgin HEU & ${ }^{235} \mathrm{U}$ & 18 & 18 \\
Reactor Pu & ${ }^{239} \mathrm{Pu}$ & 2 & 2 \\
Weapon Pu & ${ }^{239} \mathrm{Pu}$ & 3 & 3 \\
HEU with shielding & ${ }^{235} \mathrm{U}$ & 0 & 0 \\
Virgin HEU with shielding & ${ }^{235} \mathrm{U}$ & 0 & 0 \\
Reactor Pu with shielding & ${ }^{239} \mathrm{Pu}$ & 0 & 0 \\
Weapon Pu with shielding & ${ }^{239} \mathrm{Pu}$ & 0 & 0 \\
\hline
\end{tabular}




\begin{tabular}{|c|c|c|c|c|}
\hline $\begin{array}{l}100 \text { Counts/400 Bkg } \\
\text { Sample }\end{array}$ & Label & Score & & \\
\hline 99TcM-625uc+200mm-H2O+5mmFe & ${ }^{99 m} \mathrm{Tc}$ & & 98 & 98 \\
\hline $131 \mathrm{l}-370 \mathrm{uc}+200 \mathrm{~mm}-\mathrm{H} 2 \mathrm{O}+5 \mathrm{mmFe}$ & ${ }^{131}$ & & 32 & 32 \\
\hline $201 \mathrm{TI}-250 \mathrm{uc}+200 \mathrm{~mm}-\mathrm{H} 2 \mathrm{O}+5 \mathrm{mmFe}$ & ${ }^{201} \mathrm{TI}$ & & 73 & 73 \\
\hline $67 \mathrm{Ga}-350 \mathrm{uc}+200 \mathrm{~mm}-\mathrm{H} 2 \mathrm{O}+5 \mathrm{mmFe}$ & ${ }^{67} \mathrm{Ga}$ & & 49 & 49 \\
\hline $137 \mathrm{Cs}-8 \mathrm{mc}+24 \mathrm{~mm}-$ Lead+5mmFe & ${ }^{137} \mathrm{Cs}$ & & 95 & 95 \\
\hline $14 \mathrm{Kg}$-of-Bananas+5mmFe & ${ }^{40} \mathrm{~K}$ & & 22 & 22 \\
\hline $5 \mathrm{Kg}$-of-Wood+5mmFe & ${ }^{40} \mathrm{~K}$ & & 20 & 20 \\
\hline $5 \mathrm{Kg}$-of-Potash-Fertilizer $+5 \mathrm{mmFe}$ & ${ }^{40} \mathrm{~K}$ & & 1 & 1 \\
\hline HEU & ${ }^{235} U$ & & 68 & 68 \\
\hline Virgin HEU & ${ }^{235} \mathrm{U}$ & & 72 & 72 \\
\hline Reactor Pu & ${ }^{239} \mathrm{Pu}$ & & 14 & 14 \\
\hline Weapon $\mathrm{Pu}$ & ${ }^{239} \mathrm{Pu}$ & & 26 & 26 \\
\hline HEU with shielding & ${ }^{235} \mathrm{U}$ & & 0 & 0 \\
\hline Virgin HEU with shielding & ${ }^{235} U$ & & 0 & 0 \\
\hline Reactor Pu with shielding & ${ }^{239} \mathrm{Pu}$ & & 1 & 1 \\
\hline Weapon Pu with shielding & ${ }^{239} \mathrm{Pu}$ & & 7 & 7 \\
\hline
\end{tabular}

\begin{tabular}{|c|c|c|c|c|}
\hline $\begin{array}{l}200 \text { Counts/400 Bkg } \\
\text { Sample }\end{array}$ & Label & Score & Best & \\
\hline 99TcM-625uc+200mm-H2O+5mmFe & ${ }^{99 \mathrm{~m}} \mathrm{Tc}$ & 100 & & 100 \\
\hline $131 \mathrm{l}-370 \mathrm{uc}+200 \mathrm{~mm}-\mathrm{H} 2 \mathrm{O}+5 \mathrm{mmFe}$ & ${ }^{131}$ I & 67 & & 67 \\
\hline $201 \mathrm{Tl}-250 \mathrm{uc}+200 \mathrm{~mm}-\mathrm{H} 2 \mathrm{O}+5 \mathrm{mmFe}$ & ${ }^{201} \mathrm{TI}$ & 85 & & 85 \\
\hline $67 \mathrm{Ga}-350 \mathrm{uc}+200 \mathrm{~mm}-\mathrm{H} 2 \mathrm{O}+5 \mathrm{mmFe}$ & ${ }^{67} \mathrm{Ga}$ & 70 & & 70 \\
\hline $137 \mathrm{Cs}-8 \mathrm{mc}+24 \mathrm{~mm}$-Lead+5mmFe & ${ }^{137} \mathrm{Cs}$ & 100 & & 100 \\
\hline 14Kg-of-Bananas $+5 \mathrm{mmFe}$ & ${ }^{40} \mathrm{~K}$ & 83 & & 83 \\
\hline $5 \mathrm{Kg}$-of-Wood $+5 \mathrm{mmFe}$ & ${ }^{40} \mathrm{~K}$ & 77 & & 77 \\
\hline $5 \mathrm{Kg}$-of-Potash-Fertilizer $+5 \mathrm{mmFe}$ & ${ }^{40} \mathrm{~K}$ & 28 & & 28 \\
\hline HEU & ${ }^{235} \mathrm{U}$ & 87 & & 90 \\
\hline Virgin HEU & ${ }^{235} \mathrm{U}$ & 87 & & 91 \\
\hline Reactor $\mathrm{Pu}$ & ${ }^{239} \mathrm{Pu}$ & 34 & & 35 \\
\hline Weapon $\mathrm{Pu}$ & ${ }^{239} \mathrm{Pu}$ & 78 & & 78 \\
\hline HEU with shielding & ${ }^{235} \mathrm{U}$ & 0 & & 0 \\
\hline Virgin HEU with shielding & ${ }^{235} \mathrm{U}$ & 0 & & 0 \\
\hline Reactor Pu with shielding & ${ }^{239} \mathrm{Pu}$ & 0 & & 0 \\
\hline Weapon Pu with shielding & ${ }^{239} \mathrm{Pu}$ & 5 & & 5 \\
\hline
\end{tabular}




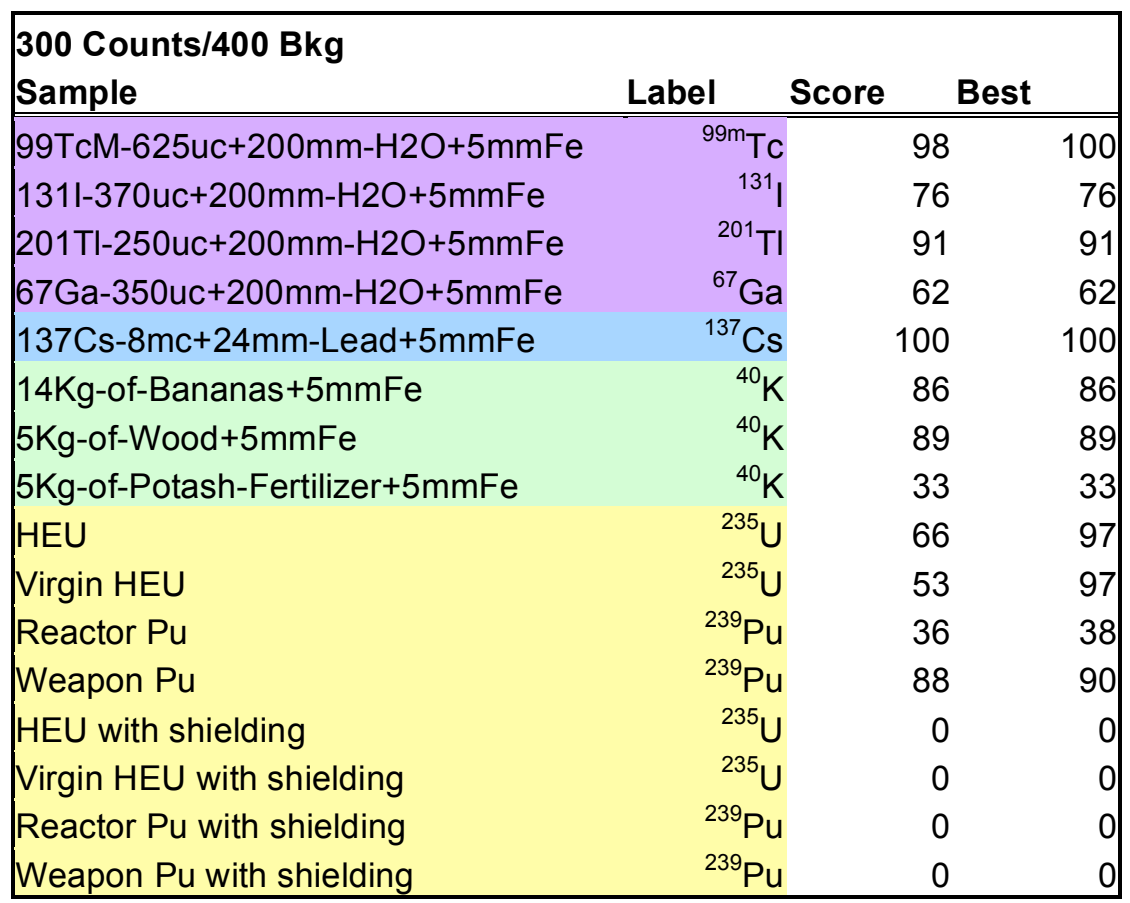

\begin{tabular}{|c|c|c|c|c|}
\hline $\begin{array}{l}500 \text { Counts/400 Bkg } \\
\text { Sample }\end{array}$ & Label & Score & Best & \\
\hline 99TcM-625uc+200mm-H2O+5mmFe & ${ }^{99 \mathrm{~m}} \mathrm{Tc}$ & 85 & & 100 \\
\hline $131 \mathrm{l}-370 \mathrm{uc}+200 \mathrm{~mm}-\mathrm{H} 2 \mathrm{O}+5 \mathrm{mmFe}$ & ${ }^{131}$ I & 73 & & 73 \\
\hline $201 \mathrm{TI}-250 \mathrm{uc}+200 \mathrm{~mm}-\mathrm{H} 2 \mathrm{O}+5 \mathrm{mmFe}$ & ${ }^{201} \mathrm{TI}$ & 74 & & 95 \\
\hline $67 \mathrm{Ga}-350 \mathrm{uc}+200 \mathrm{~mm}-\mathrm{H} 2 \mathrm{O}+5 \mathrm{mmFe}$ & ${ }^{67} \mathrm{Ga}$ & 60 & & 66 \\
\hline $137 \mathrm{Cs}-8 \mathrm{mc}+24 \mathrm{~mm}$-Lead+5mmFe & ${ }^{137} \mathrm{Cs}$ & 100 & & 100 \\
\hline $14 \mathrm{Kg}$-of-Bananas $+5 \mathrm{mmFe}$ & ${ }^{40} \mathrm{~K}$ & 94 & & 94 \\
\hline $5 \mathrm{Kg}$-of-Wood+5mmFe & ${ }^{40} \mathrm{~K}$ & 95 & & 97 \\
\hline $5 \mathrm{Kg}$-of-Potash-Fertilizer $+5 \mathrm{mmFe}$ & ${ }^{40} \mathrm{~K}$ & 30 & & 30 \\
\hline HEU & ${ }^{235} \mathrm{U}$ & 1 & 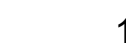 & 100 \\
\hline Virgin HEU & ${ }^{235} \mathrm{U}$ & 0 & ) & 100 \\
\hline Reactor Pu & ${ }^{239} \mathrm{Pu}$ & 21 & & 49 \\
\hline Weapon Pu & ${ }^{239} \mathrm{Pu}$ & 78 & & 97 \\
\hline HEU with shielding & ${ }^{235} \mathrm{U}$ & 0 & & 0 \\
\hline Virgin HEU with shielding & ${ }^{235} \mathrm{U}$ & 0 & ) & 0 \\
\hline Reactor Pu with shielding & ${ }^{239} \mathrm{Pu}$ & 0 & & 0 \\
\hline Weapon Pu with shielding & ${ }^{239} \mathrm{Pu}$ & 0 & ) & 0 \\
\hline
\end{tabular}




\begin{tabular}{|c|c|c|c|c|}
\hline $\begin{array}{l}750 \text { Counts/400 Bkg } \\
\text { Sample }\end{array}$ & Label & Score & Best & \\
\hline 99TcM-625uc+200mm-H2O+5mmFe & ${ }^{99 m} \mathrm{Tc}$ & 20 & & 100 \\
\hline $131 \mathrm{l}-370 \mathrm{uc}+200 \mathrm{~mm}-\mathrm{H} 2 \mathrm{O}+5 \mathrm{mmFe}$ & ${ }^{131}$ & 83 & & 86 \\
\hline $201 \mathrm{Tl}-250 \mathrm{uc}+200 \mathrm{~mm}-\mathrm{H} 2 \mathrm{O}+5 \mathrm{mmFe}$ & ${ }^{201} \mathrm{TI}$ & 19 & & 99 \\
\hline $67 \mathrm{Ga}-350 \mathrm{uc}+200 \mathrm{~mm}-\mathrm{H} 2 \mathrm{O}+5 \mathrm{mmFe}$ & ${ }^{67} \mathrm{Ga}$ & 24 & & 64 \\
\hline 137Cs-8mc+24mm-Lead+5mmFe & ${ }^{137} \mathrm{Cs}$ & 100 & & 100 \\
\hline 14Kg-of-Bananas+5mmFe & ${ }^{40} \mathrm{~K}$ & 94 & & 100 \\
\hline $5 \mathrm{Kg}$-of-Wood+5mmFe & ${ }^{40} \mathrm{~K}$ & 94 & & 100 \\
\hline $5 \mathrm{Kg}$-of-Potash-Fertilizer $+5 \mathrm{mmFe}$ & ${ }^{40} \mathrm{~K}$ & 22 & & 23 \\
\hline HEU & ${ }^{235} U$ & 0 & 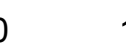 & 100 \\
\hline Virgin HEU & ${ }^{235} U$ & 0 & b & 100 \\
\hline Reactor Pu & ${ }^{239} \mathrm{Pu}$ & 1 & 1 & 62 \\
\hline Weapon $\mathrm{Pu}$ & ${ }^{239} \mathrm{Pu}$ & 9 & 9 & 100 \\
\hline HEU with shielding & ${ }^{235} \mathrm{U}$ & 0 & 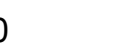 & 0 \\
\hline Virgin HEU with shielding & ${ }^{235} U$ & 0 & 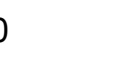 & 0 \\
\hline Reactor Pu with shielding & ${ }^{239} \mathrm{Pu}$ & 0 & 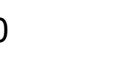 & 0 \\
\hline Weapon Pu with shielding & ${ }^{239} \mathrm{Pu}$ & 0 & 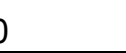 & 0 \\
\hline
\end{tabular}

\begin{tabular}{|c|c|c|c|c|}
\hline $\begin{array}{l}1000 \text { Counts } / 400 \text { Bkg } \\
\text { Sample }\end{array}$ & Label & Score & \multicolumn{2}{|c|}{ Best } \\
\hline 99TcM-625uc+200mm-H2O+5mmFe & ${ }^{99 \mathrm{~m}} \mathrm{Tc}$ & & 0 & 100 \\
\hline $131 \mathrm{l}-370 \mathrm{uc}+200 \mathrm{~mm}-\mathrm{H} 2 \mathrm{O}+5 \mathrm{mmFe}$ & ${ }^{131}$ & & 74 & 92 \\
\hline $201 \mathrm{Tl}-250 \mathrm{uc}+200 \mathrm{~mm}-\mathrm{H} 2 \mathrm{O}+5 \mathrm{mmFe}$ & ${ }^{201} \mathrm{TI}$ & & 3 & 100 \\
\hline $67 \mathrm{Ga}-350 \mathrm{uc}+200 \mathrm{~mm}-\mathrm{H} 2 \mathrm{O}+5 \mathrm{mmFe}$ & ${ }^{67} \mathrm{Ga}$ & & 3 & 66 \\
\hline $137 \mathrm{Cs}-8 \mathrm{mc}+24 \mathrm{~mm}-\mathrm{Lead}+5 \mathrm{mmFe}$ & ${ }^{137} \mathrm{Cs}$ & & 98 & 100 \\
\hline 14Kg-of-Bananas $+5 \mathrm{mmFe}$ & ${ }^{40} \mathrm{~K}$ & & 66 & 99 \\
\hline $5 \mathrm{Kg}$-of-Wood+5mmFe & ${ }^{40} \mathrm{~K}$ & & 66 & 99 \\
\hline $5 \mathrm{Kg}$-of-Potash-Fertilizer $+5 \mathrm{mmFe}$ & ${ }^{40} \mathrm{~K}$ & & 18 & 23 \\
\hline HEU & ${ }^{235} U$ & & 0 & 100 \\
\hline Virgin HEU & ${ }^{235} U$ & & 0 & 100 \\
\hline Reactor Pu & ${ }^{239} \mathrm{Pu}$ & & 0 & 62 \\
\hline Weapon Pu & ${ }^{239} \mathrm{Pu}$ & & 0 & 100 \\
\hline HEU with shielding & ${ }^{235} U$ & & 0 & 0 \\
\hline Virgin HEU with shielding & ${ }^{235} U$ & & 0 & 0 \\
\hline Reactor Pu with shielding & ${ }^{239} \mathrm{Pu}$ & & 0 & 0 \\
\hline Weapon Pu with shielding & ${ }^{239} \mathrm{Pu}$ & & 0 & 0 \\
\hline
\end{tabular}




\section{A.4.2.2 PCA Class Results}

Class scoring was only performed on the "best match" result.

\begin{tabular}{|c|c|c|c|c|c|c|c|c|c|c|c|}
\hline $\begin{array}{l}50 \text { Counts } / 400 \text { Bkg } \\
\text { Sample }\end{array}$ & Label & Class & 1 & 2 & 3 & & & & & & \\
\hline 99TcM-625uc+200mm-H2O+5mmFe & ${ }^{99 \mathrm{~m}} \mathrm{Tc}$ & 3 & 0 & 15 & 85 & \multirow{5}{*}{ actual } & & \multicolumn{3}{|c|}{ reported } & \multirow[b]{2}{*}{ total } \\
\hline $131 \mathrm{I}-370 \mathrm{uc}+200 \mathrm{~mm}-\mathrm{H} 2 \mathrm{O}+5 \mathrm{mmFe}$ & ${ }^{131}$ & 2 & 4 & 6 & 90 & & & 1 & 2 & & \\
\hline $201 \mathrm{TI}-250 \mathrm{uc}+200 \mathrm{~mm}-\mathrm{H} 2 \mathrm{O}+5 \mathrm{mmFe}$ & ${ }^{201} \mathrm{~T}$ & 2 & 2 & 42 & 56 & & 1 & 0.045 & 0.049 & 0.906 & 800 \\
\hline $67 \mathrm{Ga}-350 \mathrm{uc}+200 \mathrm{~mm}-\mathrm{H} 2 \mathrm{O}+5 \mathrm{mmFe}$ & ${ }^{67} \mathrm{Ga}$ & 2 & 3 & 5 & 92 & & 2 & 0.023 & 0.198 & 0.780 & 400 \\
\hline $137 \mathrm{Cs}-8 \mathrm{mc}+24 \mathrm{~mm}-$ Lead $+5 \mathrm{mmFe}$ & ${ }^{137} \mathrm{Cs}$ & 2 & 0 & 26 & 74 & & 3 & 0.000 & 0.038 & 0.963 & 400 \\
\hline 14Kg-of-Bananas $+5 \mathrm{mmFe}$ & ${ }^{40} \mathrm{~K}$ & 3 & 0 & 0 & 100 & & & & & & \\
\hline $5 \mathrm{Kg}$-of-Wood $+5 \mathrm{mmFe}$ & ${ }^{40} \mathrm{~K}$ & 3 & 0 & 0 & 100 & promotion & & & 0.06 & 0.02 & \\
\hline $5 \mathrm{Kg}$-of-Potash-Fertilizer $+5 \mathrm{mmFe}$ & ${ }^{40} \mathrm{~K}$ & 3 & 0 & 0 & 100 & demotion & & & 1.74 & 0.58 & \\
\hline HEU & ${ }^{235} U$ & 1 & 12 & 5 & 83 & & & & & & \\
\hline Virgin HEU & ${ }^{235} U$ & 1 & 19 & 8 & 73 & & & & & & \\
\hline Reactor Pu & ${ }^{239} \mathrm{Pu}$ & 1 & 2 & 1 & 97 & & & & & & \\
\hline Weapon $\mathrm{Pu}$ & ${ }^{239} \mathrm{Pu}$ & 1 & 3 & 1 & 96 & & & & & & \\
\hline HEU with shielding & ${ }^{235} U$ & 1 & 0 & 8 & 92 & & & & & & \\
\hline Virgin HEU with shielding & ${ }^{235} \mathrm{U}$ & 1 & 0 & 6 & 94 & & & & & & \\
\hline Reactor Pu with shielding & ${ }^{239} \mathrm{Pu}$ & 1 & 0 & 4 & 96 & & & & & & \\
\hline Weapon Pu with shielding & ${ }^{239} \mathrm{Pu}$ & 1 & 0 & 6 & 94 & & & & & & \\
\hline
\end{tabular}

\begin{tabular}{|c|c|c|c|c|c|c|c|c|c|c|}
\hline \multicolumn{11}{|l|}{100 Counts/400 Bkg } \\
\hline Sample & Label & Class & 1 & 2 & 3 & & & & & \\
\hline 99TcM-625uc+200mm-H2O+5mmFe & ${ }^{99 \mathrm{~m}} \mathrm{Tc}$ & 3 & 11 & 59 & 30 & \multirow{5}{*}{ actual } & \multicolumn{3}{|c|}{ reported } & \multirow[b]{2}{*}{ Total } \\
\hline 131I-370uc +200mm-H2O $+5 \mathrm{mmFe}$ & 131 & 2 & 0 & 92 & 8 & & 1 & 2 & & \\
\hline $201 \mathrm{TI}-250 \mathrm{uc}+200 \mathrm{~mm}-\mathrm{H} 2 \mathrm{O}+5 \mathrm{mmFe}$ & ${ }^{201} \mathrm{~T}$ & 2 & 18 & 67 & 15 & & 0.251 & 0.391 & 0.358 & 800 \\
\hline $67 \mathrm{Ga}-350 \mathrm{uc}+200 \mathrm{~mm}-\mathrm{H} 2 \mathrm{O}+5 \mathrm{mmFe}$ & ${ }^{67} \mathrm{Ga}$ & 2 & 0 & 97 & 3 & & 0.073 & 0.788 & 0.140 & 400 \\
\hline $137 \mathrm{Cs}-8 \mathrm{mc}+24 \mathrm{~mm}-\mathrm{Lead}+5 \mathrm{mmFe}$ & ${ }^{137} \mathrm{Cs}$ & 2 & 0 & 7 & 93 & & 0.003 & 0.045 & 0.953 & 400 \\
\hline 14Kg-of-Bananas $+5 \mathrm{mmFe}$ & ${ }^{40} \mathrm{~K}$ & 3 & 0 & 7 & 93 & & & & & \\
\hline $5 \mathrm{Kg}$-of-Wood $+5 \mathrm{mmFe}$ & ${ }^{40} \mathrm{~K}$ & 3 & 1 & 2 & 97 & promotion & & 0.12 & 0.04 & \\
\hline $5 \mathrm{Kg}$-of-Potash-Fertilizer $+5 \mathrm{mmFe}$ & ${ }^{40} \mathrm{~K}$ & 3 & 69 & 23 & 8 & demotion & & 0.89 & 0.30 & \\
\hline HEU & ${ }^{235} U$ & 1 & 73 & 21 & 6 & & & & & \\
\hline Virgin HEU & ${ }^{235} U$ & 1 & 19 & 25 & 56 & & & & & \\
\hline Reactor Pu & ${ }^{239} \mathrm{Pu}$ & 1 & 31 & 26 & 43 & & & & & \\
\hline Weapon Pu & ${ }^{239} \mathrm{Pu}$ & 1 & 0 & 52 & 48 & & & & & \\
\hline HEU with shielding & ${ }^{235} U$ & 1 & 1 & 67 & 32 & & & & & \\
\hline Virgin HEU with shielding & ${ }^{235} U$ & 1 & 1 & 48 & 51 & & & & & \\
\hline Reactor Pu with shielding & ${ }^{239} \mathrm{Pu}$ & 1 & 7 & 51 & 42 & & & & & \\
\hline Weapon Pu with shielding & ${ }^{239} \mathrm{Pu}$ & 1 & 11 & 59 & 30 & & & & & \\
\hline
\end{tabular}




\begin{tabular}{|c|c|c|c|c|c|c|c|c|c|c|}
\hline \multicolumn{11}{|l|}{200 Counts/400 Bkg } \\
\hline Sample & Label & Class & 1 & 2 & 3 & & & & & \\
\hline 99TcM-625uc+200mm-H2O $+5 \mathrm{mmFe}$ & ${ }^{99 \mathrm{~m}} \mathrm{Tc}$ & 3 & 0 & 0 & 100 & \multirow{5}{*}{ actual } & \multicolumn{3}{|c|}{ reported } & \multirow[b]{2}{*}{ total } \\
\hline $1311-370 u c+200 m m-H 2 O+5 m m F e$ & 131 & 2 & 8 & 92 & 0 & & 1 & 2 & & \\
\hline $201 \mathrm{Tl}-250 \mathrm{uc}+200 \mathrm{~mm}-\mathrm{H} 2 \mathrm{O}+5 \mathrm{mmFe}$ & ${ }^{201} \mathrm{~T}$ & 2 & 0 & 98 & 2 & & 0.380 & 0.469 & 0.151 & 800 \\
\hline $67 \mathrm{Ga}-350 \mathrm{uc}+200 \mathrm{~mm}-\mathrm{H} 2 \mathrm{O}+5 \mathrm{mmFe}$ & ${ }^{67} \mathrm{Ga}$ & 2 & 16 & 84 & 0 & & 0.060 & 0.935 & 0.005 & 400 \\
\hline $137 \mathrm{Cs}-8 \mathrm{mc}+24 \mathrm{~mm}-\mathrm{Lead}+5 \mathrm{mmFe}$ & ${ }^{137} \mathrm{Cs}$ & 2 & 0 & 100 & 0 & & 0.008 & 0.220 & 0.773 & 400 \\
\hline $14 \mathrm{Kg}$-of-Bananas $+5 \mathrm{mmFe}$ & ${ }^{40} \mathrm{~K}$ & 3 & 0 & 14 & 86 & & & & & \\
\hline $5 \mathrm{Kg}$-of-Wood $+5 \mathrm{mmFe}$ & ${ }^{40} \mathrm{~K}$ & 3 & 0 & 19 & 81 & promotion & & 0.29 & 0.10 & \\
\hline $5 \mathrm{Kg}$-of-Potash-Fertilizer $+5 \mathrm{mmFe}$ & ${ }^{40} \mathrm{~K}$ & 3 & 3 & 55 & 42 & demotion & & 0.63 & 0.21 & \\
\hline HEU & ${ }^{235} \mathrm{U}$ & 1 & 90 & 9 & 1 & & & & & \\
\hline Virgin HEU & ${ }^{235} \mathrm{U}$ & 1 & 91 & 8 & 1 & & & & & \\
\hline Reactor $\mathrm{Pu}$ & ${ }^{239} \mathrm{Pl}$ & 1 & 40 & 41 & 19 & & & & & \\
\hline Weapon $\mathrm{Pu}$ & ${ }^{239} \mathrm{Pl}$ & 1 & 78 & 21 & 1 & & & & & \\
\hline HEU with shielding & ${ }^{235} \mathrm{U}$ & 1 & 0 & 65 & 35 & & & & & \\
\hline Virgin HEU with shielding & ${ }^{235} \mathrm{U}$ & 1 & 0 & 90 & 10 & & & & & \\
\hline Reactor Pu with shielding & ${ }^{239} \mathrm{Pl}$ & 1 & 0 & 56 & 44 & & & & & \\
\hline Weapon Pu with shielding & ${ }^{239} \mathrm{PL}$ & 1 & 5 & 85 & 10 & & & & & \\
\hline
\end{tabular}

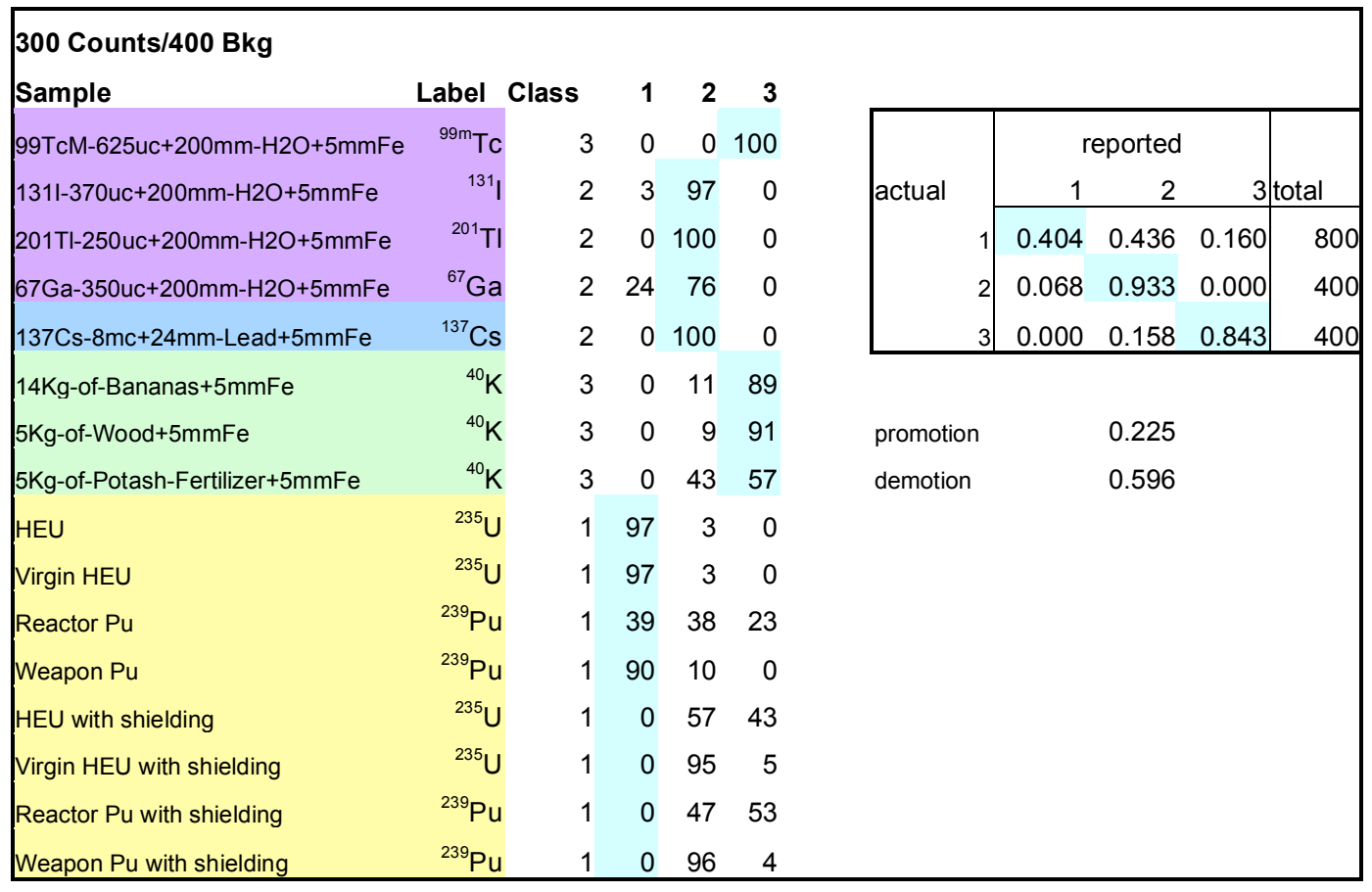




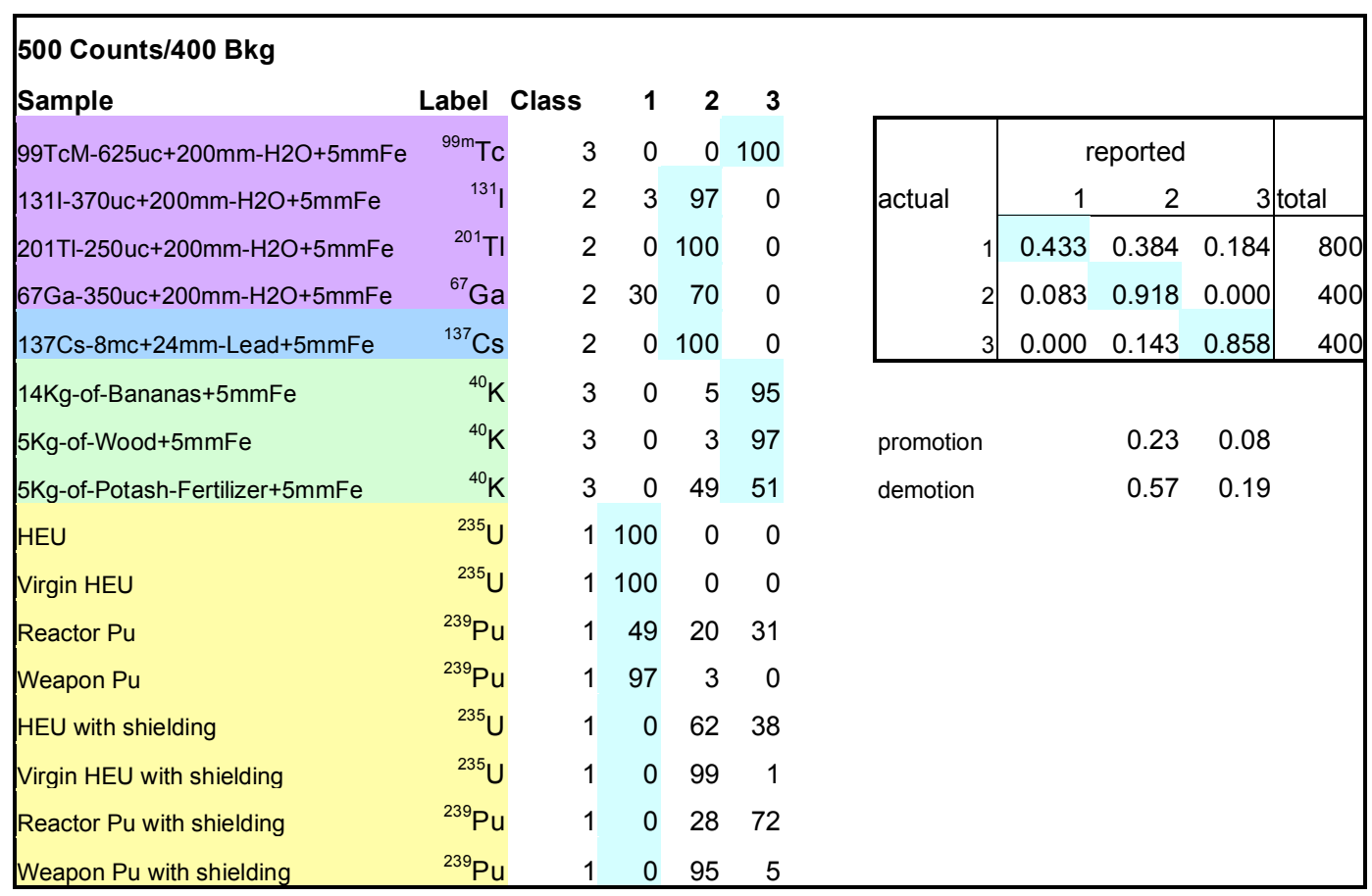

\begin{tabular}{|c|c|c|c|c|c|c|c|c|c|c|}
\hline \multicolumn{11}{|l|}{750 Counts/400 Bkg } \\
\hline Sample & Label & Class & 1 & 2 & 3 & & & & & \\
\hline 99TcM-625uc+200mm-H2O +5mmFe & ${ }^{99 \mathrm{~m}} \mathrm{Tc}$ & 3 & 1 & 2 & 3 & \multirow{5}{*}{ actual } & \multicolumn{3}{|c|}{ reported } & \multirow[b]{2}{*}{ |total } \\
\hline 1311-370uc $+200 \mathrm{~mm}-\mathrm{H} 2 \mathrm{O}+5 \mathrm{mmFe}$ & ${ }^{131}$ & 2 & 0 & 0 & 100 & & 1 & 2 & & \\
\hline $201 \mathrm{Tl}-250 \mathrm{uc}+200 \mathrm{~mm}-\mathrm{H} 2 \mathrm{O}+5 \mathrm{mmFe}$ & ${ }^{201} \mathrm{TI}$ & 2 & 0 & 100 & 0 & & 0.453 & 0.338 & 0.210 & 800 \\
\hline $67 \mathrm{Ga}-350 \mathrm{uc}+200 \mathrm{~mm}-\mathrm{H} 2 \mathrm{O}+5 \mathrm{mmFe}$ & ${ }^{67} \mathrm{Ga}$ & 2 & 0 & 100 & 0 & & 0.090 & 0.910 & 0.000 & 400 \\
\hline $137 \mathrm{Cs}-8 \mathrm{mc}+24 \mathrm{~mm}-\mathrm{Lead}+5 \mathrm{mmFe}$ & ${ }^{137} \mathrm{Cs}$ & 2 & 36 & 64 & 0 & & 0.000 & 0.175 & 0.825 & 400 \\
\hline $14 \mathrm{Kg}$-of-Bananas $+5 \mathrm{mmFe}$ & ${ }^{40} \mathrm{~K}$ & 3 & 0 & 100 & 0 & & & & & \\
\hline $5 \mathrm{Kg}$-of-Wood $+5 \mathrm{mmFe}$ & ${ }^{40} \mathrm{~K}$ & 3 & 0 & 0 & 100 & promotion & & 0.27 & 0.09 & \\
\hline $5 \mathrm{Kg}$-of-Potash-Fertilizer $+5 \mathrm{mmFe}$ & ${ }^{40} \mathrm{~K}$ & 3 & 0 & 0 & 100 & demotion & & 0.55 & 0.18 & \\
\hline HEU & ${ }^{235} U$ & 1 & 0 & 70 & 30 & & & & & \\
\hline Virgin HEU & ${ }^{235} U$ & 1 & 100 & 0 & 0 & & & & & \\
\hline Reactor Pu & ${ }^{239} \mathrm{Pu}$ & 1 & 100 & 0 & 0 & & & & & \\
\hline Weapon $\mathrm{Pu}$ & ${ }^{239} \mathrm{Pu}$ & 1 & 62 & 5 & 33 & & & & & \\
\hline HEU with shielding & ${ }^{235} \mathrm{U}$ & 1 & 100 & 0 & 0 & & & & & \\
\hline Virgin HEU with shielding & ${ }^{235} \mathrm{U}$ & 1 & 0 & 53 & 47 & & & & & \\
\hline Reactor Pu with shielding & ${ }^{239} \mathrm{Pu}$ & 1 & 0 & 100 & 0 & & & & & \\
\hline Weapon Pu with shielding & ${ }^{239} \mathrm{Pu}$ & 1 & 0 & 16 & 84 & & & & & \\
\hline
\end{tabular}




\begin{tabular}{|c|c|c|c|c|c|c|c|c|c|c|}
\hline \multicolumn{11}{|l|}{1000 Counts/400 Bkg } \\
\hline Sample & Label & Class & 1 & 2 & 3 & & & & & \\
\hline $99 \mathrm{TcM}-625 \mathrm{uc}+200 \mathrm{~mm}-\mathrm{H} 2 \mathrm{O}+5 \mathrm{mmFe}$ & ${ }^{99 \mathrm{~m}} \mathrm{Tc}$ & 3 & 0 & 0 & 100 & \multirow{5}{*}{ actual } & \multicolumn{3}{|c|}{ reported } & \multirow[b]{2}{*}{ total } \\
\hline $131 \mathrm{l}-370 \mathrm{uc}+200 \mathrm{~mm}-\mathrm{H} 2 \mathrm{O}+5 \mathrm{mmFe}$ & ${ }^{131}$ & 2 & 0 & 100 & 0 & & 1 & 2 & & \\
\hline $201 \mathrm{TI}-250 \mathrm{uc}+200 \mathrm{~mm}-\mathrm{H} 2 \mathrm{O}+5 \mathrm{mmFe}$ & ${ }^{201} \mathrm{TI}$ & 2 & 0 & 100 & 0 & & 0.453 & 0.345 & 0.203 & 800 \\
\hline $67 \mathrm{Ga}-350 \mathrm{uc}+200 \mathrm{~mm}-\mathrm{H} 2 \mathrm{O}+5 \mathrm{mmFe}$ & ${ }^{67} \mathrm{Ga}$ & 2 & 32 & 68 & 0 & & 0.080 & 0.920 & 0.000 & 400 \\
\hline $137 \mathrm{Cs}-8 \mathrm{mc}+24 \mathrm{~mm}-\mathrm{Lead}+5 \mathrm{mmFe}$ & ${ }^{137} \mathrm{Cs}$ & 2 & 0 & 100 & 0 & & 0.000 & 0.185 & 0.815 & 400 \\
\hline 14Kg-of-Bananas $+5 \mathrm{mmFe}$ & ${ }^{40} \mathrm{~K}$ & 3 & 0 & 1 & 99 & & & & & \\
\hline $5 \mathrm{Kg}$-of-Wood $+5 \mathrm{mmFe}$ & ${ }^{40} \mathrm{~K}$ & 3 & 0 & 1 & 99 & promotion & & 0.27 & 0.09 & \\
\hline $5 \mathrm{Kg}$-of-Potash-Fertilizer $+5 \mathrm{mmFe}$ & ${ }^{40} \mathrm{~K}$ & 3 & 0 & 72 & 28 & demotion & & 0.55 & 0.18 & \\
\hline HEU & ${ }^{235} U$ & 1 & 100 & 0 & 0 & & & & & \\
\hline Virgin HEU & ${ }^{235} \mathrm{U}$ & 1 & 100 & 0 & 0 & & & & & \\
\hline Reactor Pu & ${ }^{239} \mathrm{Pu}$ & 1 & 62 & 7 & 31 & & & & & \\
\hline Weapon Pu & ${ }^{239} \mathrm{Pu}$ & 1 & 100 & 0 & 0 & & & & & \\
\hline HEU with shielding & ${ }^{235} U$ & 1 & 0 & 55 & 45 & & & & & \\
\hline Virgin HEU with shielding & ${ }^{235} U$ & 1 & 0 & 100 & 0 & & & & & \\
\hline Reactor Pu with shielding & ${ }^{239} \mathrm{Pu}$ & 1 & 0 & 17 & 83 & & & & & \\
\hline Weapon Pu with shielding & ${ }^{239} \mathrm{Pu}$ & 1 & 0 & 97 & 3 & & & & & \\
\hline
\end{tabular}

\section{A.4.2.3 PCA Correlation Matrix}

\section{Column Definitions}

- Sample - Sample name of input.

- Label - Expert label.

- ND - number of trials where "no detection" was declared.

- UNK - number of trials where "unknown" was declared

- Sum - total number of isotopic labels assigned to samples. 


\section{A.4.2.3.1 Normal Result}

\begin{tabular}{|c|c|c|c|c|c|c|c|c|c|c|c|c|c|c|c|c|c|c|c|c|c|c|c|c|c|c|c|c|c|c|}
\hline Sample & Label & ND 1 & UNK & $\begin{array}{l}40 \\
\mathrm{~K} \\
\end{array}$ & $\begin{array}{r}226 \\
\mathrm{Ra} \\
\end{array}$ & $\begin{array}{c}232 \\
\mathrm{Th} \\
\end{array}$ & $\begin{array}{c}228 \\
\text { Th } \\
\end{array}$ & $\begin{array}{c}232 \\
\mathrm{U} \\
\end{array}$ & $\begin{array}{l}60 \\
\text { Co } \\
\end{array}$ & $\begin{array}{c}133 \\
\mathrm{Ba} \\
\end{array}$ & $\begin{array}{c}137 \\
\text { Cs }\end{array}$ & $\begin{array}{l}54 \\
\mathrm{Mn} \\
\end{array}$ & $\begin{array}{l}57 \\
\text { Co } \\
\end{array}$ & $\begin{array}{c}139 \\
\mathrm{Ce} \\
\end{array}$ & $\begin{array}{r}252 \\
\mathrm{Cf} \\
\end{array}$ & $\begin{array}{c}238 \\
\mathrm{U} \\
\end{array}$ & $\begin{array}{c}235 \\
\mathrm{U} \\
\end{array}$ & $\begin{array}{r}23 \\
\mathrm{PI} \\
\end{array}$ & & $\begin{array}{l}237 \\
\mathrm{~Np} \\
\end{array}$ & $\begin{array}{c}131 \\
1 \\
\end{array}$ & $\begin{array}{l}99^{\mathrm{m}} \\
\text { Tc } \\
\end{array}$ & $\begin{array}{l}67 \\
\mathrm{Ga} \\
\end{array}$ & $\begin{array}{c}201 \\
\mathrm{TI} \\
\end{array}$ & $\begin{array}{l}88 \\
Y \\
\end{array}$ & $\begin{array}{l}22 \\
\mathrm{Na} \\
\end{array}$ & $\begin{array}{r}166^{\mathrm{m}} \\
\mathrm{Ho} \\
\end{array}$ & $\begin{array}{r}15 \\
\mathrm{Eu} \\
\end{array}$ & $\begin{array}{rr}2 & 19 \\
1 & \mathrm{Ir} \\
\end{array}$ & $r^{2}$ SUM \\
\hline \multicolumn{31}{|l|}{50 Counts/400 Bkg } \\
\hline $99 \mathrm{TcM}-625 \mathrm{uc}+200 \mathrm{~mm}-\mathrm{H} 2 \mathrm{O}+5 \mathrm{mmFe}$ & ${ }^{99 m} \mathrm{Tc}$ & 23 & 0 & 0 & 0 & 0 & 0 & 0 & 0 & 1 & 0 & 0 & 11 & 1 & 0 & 0 & 0 & 0 & 0 & 0 & 0 & 62 & 0 & 2 & 0 & 0 & 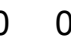 & & 0 & 0100 \\
\hline $131 \mathrm{I}-370 \mathrm{uc}+200 \mathrm{~mm}-\mathrm{H} 2 \mathrm{O}+5 \mathrm{mmFe}$ & ${ }^{131}$ & 90 & 0 & 0 & 0 & 0 & 0 & 0 & 0 & 1 & 0 & 0 & 0 & 0 & 0 & 0 & 0 & 0 & 4 & 0 & 4 & 0 & 1 & 0 & 0 & 0 & 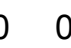 & & 0 & 0100 \\
\hline $201 \mathrm{Tl}-250 \mathrm{uc}+200 \mathrm{~mm}-\mathrm{H} 2 \mathrm{O}+5 \mathrm{mmFe}$ & ${ }^{201} \mathrm{TI}$ & 47 & 0 & 0 & 0 & 0 & 0 & 0 & 0 & 0 & 0 & 0 & 1 & 6 & 0 & 0 & 2 & 2 & 0 & 0 & 0 & 9 & 1 & 34 & 0 & 0 & b & & 0 & 0100 \\
\hline $67 \mathrm{Ga}-350 \mathrm{uc}+200 \mathrm{~mm}-\mathrm{H} 2 \mathrm{O}+5 \mathrm{mmFe}$ & ${ }^{67} \mathrm{Ga}$ & 91 & 0 & 0 & 0 & 0 & 0 & 0 & 0 & 0 & 0 & 0 & 0 & 0 & 0 & 0 & 2 & 2 & 1 & 0 & 0 & 1 & 4 & 1 & 0 & 0 & b & & 0 & 0100 \\
\hline $137 \mathrm{Cs}-8 \mathrm{mc}+24 \mathrm{~mm}-$ Lead $+5 \mathrm{mmFe}$ & ${ }^{137} \mathrm{Cs}$ & 74 & 0 & 0 & 0 & 0 & 0 & 0 & 0 & 0 & 26 & 0 & 0 & 0 & 0 & 0 & 0 & 0 & 0 & 0 & 0 & 0 & 0 & 0 & 0 & 0 & b & & 0 & 0100 \\
\hline $14 \mathrm{Kg}$-of-Bananas $+5 \mathrm{mmFe}$ & ${ }^{40} \mathrm{~K}$ & 99 & 0 & 1 & 0 & 0 & 0 & 0 & 0 & 0 & 0 & 0 & 0 & 0 & 0 & 0 & 0 & 0 & 0 & 0 & 0 & 0 & 0 & 0 & 0 & 0 & 0 & & 0 & 0100 \\
\hline $5 \mathrm{Kg}$-of-Wood+5mmFe & ${ }^{40} \mathrm{~K}$ & 99 & 0 & 1 & 0 & 0 & 0 & 0 & 0 & 0 & 0 & 0 & 0 & 0 & 0 & 0 & 0 & 0 & 0 & 0 & 0 & 0 & 0 & 0 & 0 & 0 & 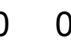 & & 0 & 0100 \\
\hline $5 \mathrm{Kg}$-of-Potash-Fertilizer $+5 \mathrm{mmFe}$ & ${ }^{40} \mathrm{~K}$ & 100 & 0 & 0 & 0 & 0 & 0 & 0 & 0 & 0 & 0 & 0 & 0 & 0 & 0 & 0 & 0 & 0 & 0 & 0 & 0 & 0 & 0 & 0 & 0 & 0 & ) & & 0 & 0100 \\
\hline HEU & ${ }^{235} \mathrm{U}$ & 79 & 0 & 0 & 0 & 0 & 0 & 0 & 0 & 0 & 0 & 0 & 0 & 2 & 0 & 0 & 12 & & 0 & 0 & 0 & 4 & 1 & 2 & 0 & 0 & b & & 0 & 0100 \\
\hline Virgin HEU & ${ }^{235} \mathrm{U}$ & 69 & 0 & 0 & 0 & 0 & 0 & 0 & 0 & 0 & 0 & 0 & 0 & 2 & 0 & 0 & 18 & & 1 & 0 & 0 & 4 & 2 & 4 & 0 & 0 & 0 & & 0 & 0100 \\
\hline Reactor Pu & ${ }^{239} \mathrm{Pu}$ & 97 & 0 & 0 & 0 & 0 & 0 & 0 & 0 & 0 & 0 & 0 & 0 & 0 & 0 & 0 & 0 & 0 & 2 & 0 & 0 & 0 & 0 & 1 & 0 & 0 & ) & & 0 & 0100 \\
\hline Weapon Pu & ${ }^{239} \mathrm{Pu}$ & 96 & 0 & 0 & 0 & 0 & 0 & 0 & 0 & 0 & 0 & 0 & 0 & 0 & 0 & 0 & 0 & 0 & 3 & 0 & 0 & 0 & 1 & 0 & 0 & 0 & 0 & & 0 & 0100 \\
\hline HEU with shielding & ${ }^{235} \mathrm{U}$ & 91 & 0 & 0 & 1 & 0 & 2 & 3 & 1 & 0 & 0 & 0 & 0 & 0 & 1 & 0 & 0 & 0 & 0 & 0 & 0 & 0 & 0 & 0 & 0 & 1 & 1 & & 0 & 0100 \\
\hline Virgin HEU with shielding & ${ }^{235} \mathrm{U}$ & 94 & 0 & 0 & 0 & 0 & 0 & 0 & 0 & 0 & 0 & 1 & 0 & 0 & 0 & 2 & 0 & 0 & 0 & 0 & 0 & 0 & 0 & 0 & 0 & 1 & 1 & & 1 & 0100 \\
\hline Reactor Pu with shielding & ${ }^{239} \mathrm{Pu}$ & 96 & 0 & 0 & 0 & 0 & 0 & 0 & 0 & 0 & 3 & 0 & 0 & 0 & 0 & 0 & 0 & 0 & 0 & 0 & 0 & 0 & 0 & 0 & 0 & 1 & 1 & & 0 & 0100 \\
\hline Weapon Pu with shielding & ${ }^{239} \mathrm{Pu}$ & 94 & 0 & 0 & 0 & 0 & 0 & 0 & 0 & 0 & 4 & 1 & 0 & 0 & 0 & 0 & 0 & 0 & 0 & 0 & 0 & 0 & 0 & 0 & 0 & 0 & 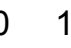 & & 0 & 0100 \\
\hline \multicolumn{31}{|l|}{100 Counts/400 Bkg } \\
\hline 99TcM-625uc+200mm-H2O+5mmFe & ${ }^{99 \mathrm{~m}} \mathrm{Tc}$ & 0 & 0 & 0 & 0 & 0 & 0 & 0 & 0 & 0 & 0 & 0 & 2 & 0 & 0 & 0 & 0 & 0 & 0 & 0 & 0 & 98 & 0 & 0 & 0 & 0 & b & & 0 & 0100 \\
\hline 131I-370uc+200mm-H2O+5mmFe & ${ }^{131}$ & 30 & 0 & 0 & 0 & 0 & 0 & 0 & 0 & 25 & 0 & 0 & 0 & 0 & 0 & 0 & 0 & 01 & 10 & 1 & 32 & 0 & 2 & 0 & 0 & 0 & כ & & 0 & 010 \\
\hline $201 \mathrm{TI}-250 \mathrm{uc}+200 \mathrm{~mm}-\mathrm{H} 2 \mathrm{O}+5 \mathrm{mmFe}$ & ${ }^{201} \mathrm{Tl}$ & 0 & 0 & 0 & 0 & 0 & 0 & 0 & 0 & 1 & 0 & 0 & 0 & 18 & 0 & 0 & 0 & 0 & 0 & 0 & 0 & 8 & 0 & 73 & 0 & 0 & 0 & & 0 & 0100 \\
\hline $67 \mathrm{Ga}-350 \mathrm{uc}+200 \mathrm{~mm}-\mathrm{H} 2 \mathrm{O}+5 \mathrm{mmFe}$ & ${ }^{67} \mathrm{Ga}$ & 14 & 0 & 0 & 0 & 0 & 0 & 0 & 0 & 7 & 0 & 0 & 0 & 1 & 0 & 0 & $\varsigma$ & 9 & 3 & 6 & 1 & 1 & 49 & 3 & 0 & 0 & 0 & & 0 & 6100 \\
\hline $137 \mathrm{Cs}-8 \mathrm{mc}+24 \mathrm{~mm}-\mathrm{Lead}+5 \mathrm{mmFe}$ & ${ }^{137} \mathrm{Cs}$ & 3 & 0 & 0 & 0 & 0 & 0 & 0 & 0 & 0 & 95 & 0 & 0 & 0 & 0 & 0 & C & 0 & 0 & 0 & 0 & 0 & 0 & 0 & 0 & 0 & ? & & 0 & 1100 \\
\hline 14Kg-of-Bananas $+5 \mathrm{mmFe}$ & ${ }^{40} \mathrm{~K}$ & 71 & 0 & 22 & 0 & 0 & 0 & 0 & 0 & 0 & 1 & 0 & 0 & 0 & 0 & 1 & $\mathrm{C}$ & 0 & 0 & 0 & 0 & 0 & 0 & 0 & 1 & 0 & 0 & & 4 & 0100 \\
\hline $5 \mathrm{Kg}-\mathrm{of}-\mathrm{Wood}+5 \mathrm{mmFe}$ & ${ }^{40} \mathrm{~K}$ & 73 & 0 & 20 & 0 & 0 & 0 & 0 & 0 & 0 & 0 & 0 & 0 & 0 & 0 & 1 & c & 0 & 0 & 0 & 0 & 0 & 0 & 0 & 1 & 0 & 0 & & 5 & 0100 \\
\hline $5 \mathrm{Kg}$-of-Potash-Fertilizer $+5 \mathrm{mmFe}$ & ${ }^{40} \mathrm{~K}$ & 96 & 0 & 1 & 0 & 0 & 0 & 0 & 0 & 0 & 1 & 0 & 0 & 0 & 1 & 0 & $c$ & 0 & 1 & 0 & 0 & 0 & 0 & 0 & 0 & 0 & 0 & & 0 & 0100 \\
\hline $\mathrm{HEU}$ & ${ }^{235} U$ & 3 & 0 & 0 & 0 & 0 & 0 & 0 & 0 & 2 & 0 & 0 & 2 & 0 & 0 & 0 & 68 & & 0 & 1 & 0 & 5 & 7 & 11 & 0 & 0 & 0 & & 0 & 1100 \\
\hline
\end{tabular}




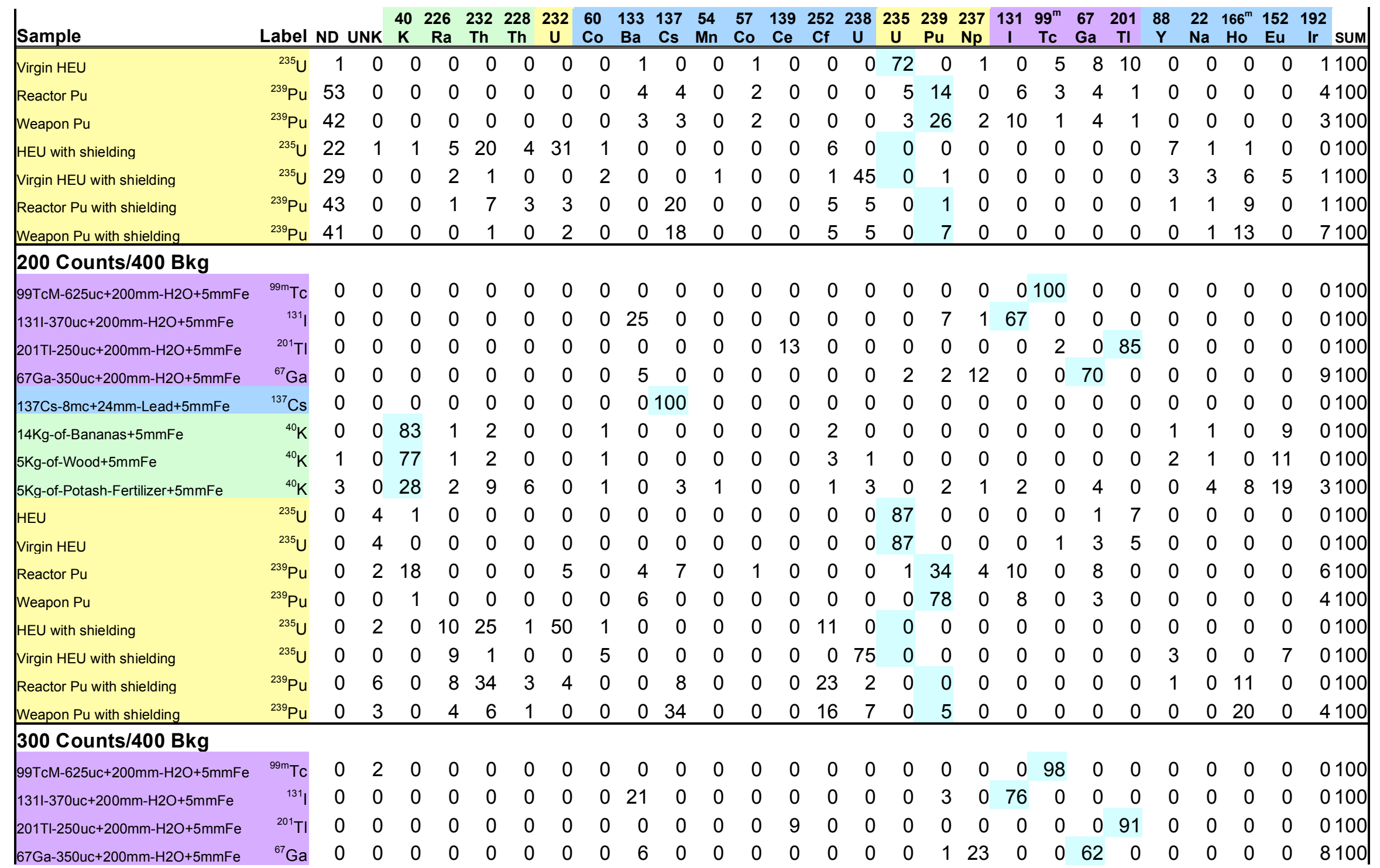




\begin{tabular}{|c|c|c|c|c|c|c|c|c|c|c|c|c|c|c|c|c|c|c|c|c|c|c|c|c|c|c|c|c|c|c|}
\hline Sample & Label & ND U & & $\begin{array}{l}40 \\
\mathrm{~K} \\
\end{array}$ & $\begin{array}{r}226 \\
\mathrm{Ra} \\
\end{array}$ & $\begin{array}{l}232 \\
\text { Th } \\
\end{array}$ & $\begin{array}{l}228 \\
\text { Th } \\
\end{array}$ & $\begin{array}{c}232 \\
U \\
\end{array}$ & & & $\begin{array}{rr}33 & 1 \\
B a & C \\
\end{array}$ & & $\begin{array}{l}54 \\
\mathrm{Mn} \\
\end{array}$ & $\begin{array}{l}57 \\
\text { Co } \\
\end{array}$ & $\begin{array}{l}139 \\
\mathrm{Ce} \\
\end{array}$ & $\begin{array}{c}252 \\
\mathrm{Cf} \\
\end{array}$ & & $\begin{array}{r}23 \\
\mathrm{U} \\
\end{array}$ & & & $\begin{array}{ll}237 & 1 \\
\mathrm{~Np} \\
\end{array}$ & $\begin{array}{cc}1319 \\
1 \quad 7 \\
\end{array}$ & $\begin{array}{l}99^{\mathrm{m}} \\
\text { Tc } \\
\end{array}$ & $\begin{array}{l}67 \\
\mathrm{Ga} \\
\end{array}$ & $\begin{array}{c}201 \\
\text { TI } \\
\end{array}$ & $\begin{array}{c}88 \\
Y \\
\end{array}$ & $\begin{array}{l}22 \\
\mathrm{Na} \\
\end{array}$ & $\begin{array}{l}166^{m} \\
\text { Ho }\end{array}$ & $\begin{array}{c}152 \\
\mathrm{Eu} \\
\end{array}$ & $\begin{array}{l}192 \\
\text { Ir SUM } \\
\end{array}$ \\
\hline $137 \mathrm{Cs}-8 \mathrm{mc}+24 \mathrm{~mm}$-Lead $+5 \mathrm{mmFe}$ & ${ }^{137} \mathrm{Cs}$ & 0 & 0 & 0 & 0 & 0 & 0 & 0 & & 0 & & 100 & 0 & 0 & 0 & 0 & & 0 & 0 & 0 & 0 & 0 & 0 & 0 & 0 & 0 & 0 & 0 & 0 & 0100 \\
\hline 14Kg-of-Bananas+5mmFe & ${ }^{40} \mathrm{~K}$ & 0 & 0 & 86 & 2 & 1 & 0 & 0 & ) & 1 & 0 & 0 & 0 & 0 & 0 & 0 & & 0 & 0 & 0 & 0 & 0 & 0 & 0 & 0 & 0 & 1 & 0 & 9 & 0100 \\
\hline $5 \mathrm{Kg}$-of-Wood+5mmFe & ${ }^{40} \mathrm{~K}$ & 0 & 0 & 89 & 2 & 0 & 0 & 0 & & 3 & 0 & 0 & 0 & 0 & 0 & 0 & & 0 & 0 & 0 & 0 & 0 & 0 & 0 & 0 & 0 & 1 & 0 & 5 & 100 \\
\hline HEU & ${ }^{235} \mathrm{U}$ & 0 & 33 & 0 & 0 & 0 & 0 & 0 & & 0 & 0 & 0 & 0 & 0 & 0 & 0 & & 0 & 36 & 0 & 0 & 0 & 0 & 0 & 1 & 0 & 0 & 0 & 0 & 100 \\
\hline Virgin HEU & ${ }^{235} \mathrm{U}$ & 0 & 45 & 0 & 0 & 0 & 0 & 0 & & 0 & 0 & 0 & 0 & 0 & 0 & 0 & & 05 & 53 & 0 & 0 & 0 & 0 & 0 & 2 & 0 & 0 & 0 & 0 & 0100 \\
\hline Reactor Pu & ${ }^{239} \mathrm{Pu}$ & 0 & 3 & 22 & 0 & 0 & 0 & 4 & & 0 & 5 & 6 & 0 & 0 & 0 & 0 & & 0 & 1 & 36 & 0 & 10 & 0 & 6 & 0 & 0 & 0 & 0 & 0 & 7100 \\
\hline HEU with shiel & ${ }^{235} \mathrm{U}$ & 0 & 10 & 0 & 1 & 35 & 0 & 51 & & 0 & 0 & 0 & 0 & 0 & 0 & 3 & & & 0 & 0 & 0 & 0 & 0 & 0 & 0 & 0 & 0 & 0 & 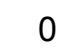 & 0100 \\
\hline Virgin HEU with shielding & ${ }^{235} \mathrm{U}$ & 0 & 1 & 0 & 5 & 0 & 0 & 0 & & 2 & 0 & 0 & 0 & 0 & 0 & 0 & 82 & & 0 & 0 & 0 & 0 & 0 & 0 & 0 & 5 & 0 & 0 & 5 & 0100 \\
\hline Reactor Pu with shielding & ${ }^{239} \mathrm{Pu}$ & 0 & 32 & 0 & 2 & 40 & 0 & 1 & & 0 & 0 & 4 & 0 & 0 & 0 & 19 & & 1 & 0 & 0 & 0 & 0 & 0 & 0 & 0 & 0 & 0 & 1 & & 0100 \\
\hline Weapon Pu with shielding & ${ }^{239} \mathrm{Pu}$ & 0 & 23 & 0 & 1 & 3 & 0 & 0 & & 0 & 0 & 34 & 0 & 0 & 0 & 17 & & 5 & 0 & 0 & 0 & 0 & 0 & 0 & 0 & 0 & 0 & 16 & ( & 1100 \\
\hline \multicolumn{31}{|l|}{500 Counts $/ 400$ Bkg } \\
\hline 99TcM-625uc+200mm-H2O+5mmFe & ${ }^{99 \mathrm{~m}} \mathrm{Tc}$ & 0 & 15 & 0 & 0 & 0 & 0 & 0 & D & 0 & 0 & 0 & 0 & 0 & 0 & & & 0 & 0 & 0 & 0 & 0 & 85 & 0 & 0 & 0 & 0 & 0 & 0 & 0100 \\
\hline $14 \mathrm{Kg}$-of-Bananas $+5 \mathrm{mmFe}$ & ${ }^{40} \mathrm{~K}$ & 0 & 0 & 94 & 1 & 0 & 0 & 0 & & 0 & 0 & 0 & 0 & c & c & & & & 0 & 0 & 0 & 0 & 0 & 0 & & 0 & & 0 & & 0100 \\
\hline 5Kg-of-Wood- & ${ }^{40} \mathrm{~K}$ & 0 & 2 & 95 & 0 & 0 & 0 & 0 & & 0 & 0 & 0 & 0 & 0 & 0 & & & 0 & 0 & 0 & 0 & 0 & 0 & 0 & & 0 & & 0 & 3 & 0100 \\
\hline 5Kg-of-Potash-Fertilizer+5mmFe & ${ }^{40} \mathrm{~K}$ & 0 & 4 & 30 & 13 & 6 & 1 & 0 & & 0 & 0 & 0 & 0 & 0 & 0 & 2 & & & 0 & 0 & 0 & 0 & 0 & 0 & 0 & 0 & 0 & 0 & 43 & 0100 \\
\hline HEU & ${ }^{235} \mathrm{U}$ & 0 & 99 & 0 & 0 & 0 & 0 & 0 & & 0 & 0 & 0 & 0 & 0 & 0 & & & & 1 & 0 & 0 & 0 & 0 & 0 & & 0 & & 0 & & 0100 \\
\hline Virgin HEU & ${ }^{235} \mathrm{U}$ & & 100 & 0 & 0 & 0 & 0 & 0 & & 0 & 0 & 0 & 0 & 0 & 0 & & & & 0 & 0 & 0 & 0 & 0 & 0 & & 0 & & 0 & & 0100 \\
\hline Reactor Pu & ${ }^{239} \mathrm{Pu}$ & 0 & 72 & 1 & 0 & 0 & 0 & 0 & & 0 & 0 & 1 & 0 & 0 & 0 & & & & 0 & 21 & 0 & 3 & 0 & 1 & & 0 & & 0 & & 1100 \\
\hline Weapon Pu & ${ }^{239} \mathrm{Pu}$ & 0 & 19 & 0 & 0 & 0 & 0 & 0 & & 0 & 0 & 0 & 0 & 0 & 0 & C & & & 0 & 78 & 0 & 2 & 0 & 0 & 0 & 0 & ) & 0 & ) & 1100 \\
\hline HEU with shi & ${ }^{235} \mathrm{U}$ & 0 & 64 & 0 & 0 & 7 & 0 & 29 & & 0 & 0 & 0 & 0 & 0 & 0 & 0 & & 0 & 0 & 0 & 0 & 0 & 0 & 0 & & 0 & & 0 & & 0100 \\
\hline Virgin HEU with & ${ }^{235} \mathrm{U}$ & 0 & 3 & 0 & 1 & 0 & 0 & 0 & & 0 & 0 & 0 & 0 & 0 & 0 & 0 & 9 & & 0 & 0 & 0 & 0 & 0 & 0 & b & 2 & & 0 & & 0100 \\
\hline Reactor Pu w & ${ }^{239} \mathrm{Pu}$ & 0 & 80 & 0 & 0 & 17 & 0 & 0 & & 0 & 0 & 0 & 0 & 0 & 0 & ) & & & 0 & 0 & 0 & 0 & 0 & 0 & 0 & 0 & 0 & 0 & 0 & 0100 \\
\hline eapon Pu with shielding & ${ }^{239} \mathrm{Pu}$ & 0 & 92 & 0 & 0 & 0 & 0 & 0 & & 0 & 0 & 3 & 0 & 0 & 0 & ) & & 2 & 0 & 0 & 0 & 0 & 0 & 0 & 0 & 0 & 0 & 1 & 0 & 0100 \\
\hline
\end{tabular}




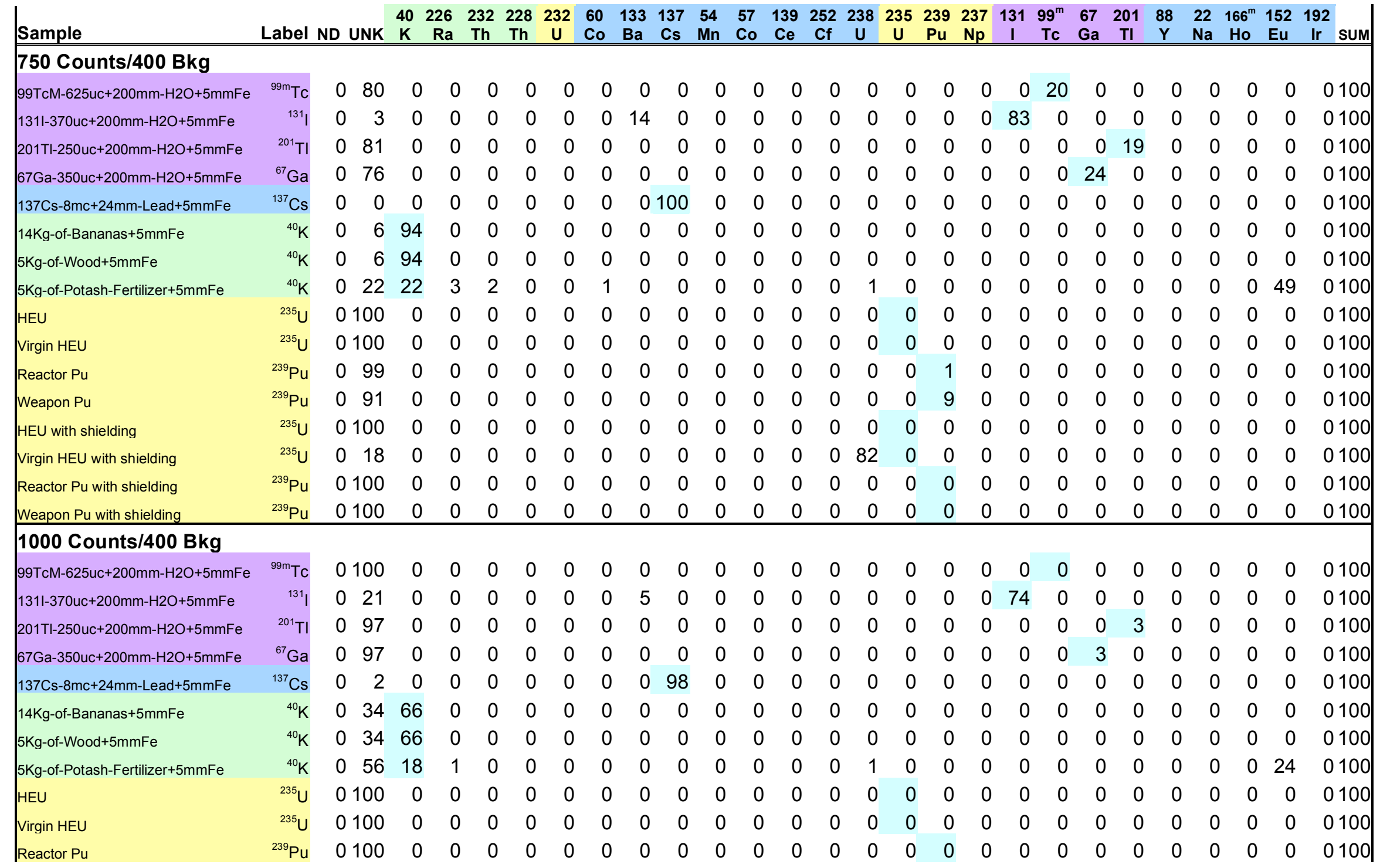




\begin{tabular}{|c|c|c|c|c|c|c|c|c|c|c|c|c|c|c|c|c|c|c|c|c|c|c|c|c|c|c|c|}
\hline Sample & Label & ND UNK & $\begin{array}{l}402 \\
\mathrm{~K} \\
\end{array}$ & $\begin{array}{r}226 \\
\mathrm{Ra} \\
\end{array}$ & $\begin{array}{c}232 \\
\text { Th } \\
\end{array}$ & $\begin{array}{c}228 \\
\text { Th } \\
\end{array}$ & $\begin{array}{c}232 \\
U \\
\end{array}$ & $\begin{array}{l}60 \\
\text { Co } \\
\end{array}$ & $\begin{array}{l}133 \\
\mathrm{Ba} \\
\end{array}$ & $\begin{array}{l}137 \\
\text { Cs } \\
\end{array}$ & $\begin{array}{l}54 \\
\mathrm{Mn} \\
\end{array}$ & $\begin{array}{l}57 \\
\text { Co } \\
\end{array}$ & $\begin{array}{l}139 \\
\mathrm{Ce} \\
\end{array}$ & $\begin{array}{r}252 \\
\text { Cf } \\
\end{array}$ & $\begin{array}{c}238 \\
\mathrm{U}\end{array}$ & $\begin{array}{c}235 \\
\mathrm{U} \\
\end{array}$ & $\begin{array}{ll}239 & 2 \\
\mathrm{Pu} & 1 \\
\end{array}$ & $\begin{array}{l}237 \\
\mathrm{~Np} \\
\end{array}$ & $\begin{array}{cc}131 & 9 \\
\quad \quad \mathrm{~T} \\
\end{array}$ & $\begin{array}{l}99^{\mathrm{m}} \\
\text { Tc } \\
\end{array}$ & $\begin{array}{ll}67 & 2 \\
\mathrm{Ga} & \\
\end{array}$ & $\begin{array}{c}201 \\
\text { TI } \\
\end{array}$ & $\begin{array}{c}88 \\
Y \\
\end{array}$ & & $\begin{array}{l}166^{\mathrm{m}} \\
\text { Ho }\end{array}$ & $\begin{array}{l}152 \\
\mathrm{Eu} \\
\end{array}$ & $\begin{array}{l}192 \\
\text { Ir sum }\end{array}$ \\
\hline Weapon Pu & ${ }^{239} \mathrm{Pu}$ & 0100 & 0 & 0 & 0 & 0 & 0 & 0 & 0 & 0 & 0 & 0 & 0 & 0 & 0 & 0 & 0 & 0 & 0 & 0 & 0 & 0 & 0 & 0 & 0 & 0 & 0100 \\
\hline HEU with sh & ${ }^{235} \mathrm{U}$ & 0100 & 0 & 0 & 0 & 0 & 0 & 0 & 0 & 0 & 0 & 0 & 0 & 0 & 0 & 0 & 0 & 0 & 0 & 0 & 0 & 0 & 0 & 0 & 0 & 0 & 0100 \\
\hline Virgin HEU with shielding & ${ }^{235} \mathrm{U}$ & $0 \quad 34$ & 0 & 0 & 0 & 0 & 0 & 0 & 0 & 0 & 0 & 0 & 0 & 0 & 66 & 0 & 0 & 0 & 0 & 0 & 0 & 0 & 0 & 0 & 0 & 0 & 0100 \\
\hline leapon Pu with shielding & ${ }^{239} \mathrm{Pu}$ & 0100 & 0 & 0 & 0 & 0 & 0 & 0 & 0 & 0 & 0 & 0 & 0 & 0 & 0 & 0 & 0 & 0 & 0 & 0 & 0 & 0 & 0 & 0 & 0 & 0 & 0100 \\
\hline
\end{tabular}

\section{A.4.2.3.2 "Best” Match Result}

\begin{tabular}{|c|c|c|c|c|c|c|c|c|c|c|c|c|c|c|c|c|c|c|c|c|c|c|c|c|c|c|c|c|c|c|}
\hline Sample & Label & ND & UNK & $\begin{array}{r}40 \\
\mathrm{~K} \\
\end{array}$ & $\begin{array}{r}226 \\
\mathrm{Ra} \\
\end{array}$ & $\begin{array}{c}232 \\
\text { Th } \\
\end{array}$ & $\begin{array}{c}228 \\
\text { Th } \\
\end{array}$ & $\begin{array}{c}232 \\
\mathrm{U} \\
\end{array}$ & $\begin{array}{l}60 \\
\text { Co } \\
\end{array}$ & $\begin{array}{c}133 \\
\mathrm{Ba} \\
\end{array}$ & $\begin{array}{c}137 \\
\text { Cs } \\
\end{array}$ & $\begin{array}{c}54 \\
\mathrm{Mn} \\
\end{array}$ & $\begin{array}{l}57 \\
\text { Co } \\
\end{array}$ & $\begin{array}{c}139 \\
\mathrm{Ce} \\
\end{array}$ & $\begin{array}{r}252 \\
\mathrm{Cf} \\
\end{array}$ & $\begin{array}{r}238 \\
\mathrm{U} \\
\end{array}$ & & $\begin{array}{cc}235 \\
\mathrm{U}\end{array}$ & $\begin{array}{l}239 \\
\mathrm{Pu} \\
\end{array}$ & $\begin{array}{l}237 \\
\mathrm{~Np} \\
\end{array}$ & $\begin{array}{c}131 \\
1 \\
\end{array}$ & $\begin{array}{c}99^{\mathrm{m}} \\
\text { Tc } \\
\end{array}$ & $\begin{array}{l}67 \\
\mathrm{Ga} \\
\end{array}$ & $\begin{array}{c}201 \\
\mathrm{TI} \\
\end{array}$ & $\begin{array}{l}88 \\
\mathrm{Y} \\
\end{array}$ & $\begin{array}{l}22 \\
\mathrm{Na} \\
\end{array}$ & $\begin{array}{c}166^{\mathrm{m}} \\
\text { Ho } \\
\end{array}$ & $\begin{array}{c}152 \\
\mathrm{Eu} \\
\end{array}$ & & \\
\hline \multicolumn{31}{|l|}{50 Counts/400 Bkg } \\
\hline $9 \mathrm{TcM}-625 \mathrm{uc}+200 \mathrm{~mm}-\mathrm{H} 2 \mathrm{O}+5 \mathrm{mmFe}$ & ${ }^{\mathrm{m}} \mathrm{Tc}$ & 23 & 0 & 0 & 0 & 0 & 0 & 0 & 0 & 1 & 0 & 0 & 11 & 1 & 0 & & 0 & 0 & 0 & 0 & 0 & 62 & 0 & 2 & 0 & 0 & 0 & 0 & 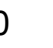 & 0100 \\
\hline $311-370 \mathrm{uc}+200 \mathrm{~mm}-\mathrm{H} 2 \mathrm{O}+5 \mathrm{mmFe}$ & ${ }^{131} \mid$ & 90 & 0 & 0 & b & 0 & 0 & 0 & 0 & 1 & 0 & 0 & 0 & 0 & 0 & & 0 & 0 & 4 & 0 & 4 & 0 & 1 & 0 & 0 & 0 & 0 & 0 & כ & 0100 \\
\hline $01 \mathrm{TI}-250 \mathrm{uc}+200 \mathrm{~mm}-\mathrm{H} 2 \mathrm{O}+5 \mathrm{mmFe}$ & ${ }^{201} \mathrm{Tl}$ & 47 & 0 & 0 & 0 & b & 0 & b & 0 & 0 & 0 & 0 & 1 & 6 & 0 & & 0 & 2 & 0 & 0 & 0 & 9 & 1 & 34 & 0 & 0 & 0 & 0 & b & 0100 \\
\hline $7 \mathrm{Ga}-350 \mathrm{uc}+200 \mathrm{~mm}-\mathrm{H} 2 \mathrm{O}+5 \mathrm{mmFe}$ & ${ }^{67} \mathrm{Ga}$ & 91 & 0 & 0 & 0 & 0 & 0 & 0 & 0 & 0 & 0 & 0 & 0 & 0 & 0 & & 0 & 2 & 1 & 0 & 0 & 1 & 4 & 1 & 0 & 0 & 0 & 0 & כ & 100 \\
\hline $137 \mathrm{Cs}-8 \mathrm{mc}+24 \mathrm{~mm}-\mathrm{Lead}+5 \mathrm{mmFe}$ & ${ }^{137} \mathrm{Cs}$ & 74 & 0 & 0 & 0 & 0 & 0 & 0 & 0 & 0 & 26 & 0 & 0 & 0 & 0 & & 0 & 0 & 0 & 0 & 0 & 0 & 0 & 0 & 0 & 0 & 0 & 0 & D & 100 \\
\hline $4 \mathrm{Kg}$-of-Bananas+5mmFe & ${ }^{40} \mathrm{~K}$ & 99 & 0 & 1 & 1 & 0 & 0 & 0 & 0 & 0 & 0 & 0 & 0 & 0 & 0 & & 0 & 0 & 0 & 0 & 0 & 0 & 0 & 0 & 0 & 0 & ) & 0 & D & 100 \\
\hline $5 \mathrm{Kg}$-of-Wood+5mmFe & ${ }^{40} \mathrm{~K}$ & 99 & 0 & 1 & 1 & 0 & 0 & 0 & 0 & 0 & 0 & 0 & 0 & 0 & 0 & & 0 & 0 & 0 & 0 & 0 & 0 & 0 & 0 & 0 & 0 & 0 & 0 & D & 0100 \\
\hline $5 \mathrm{Kg}$-of-Potash-Fertilizer+5mmFe & ${ }^{40} \mathrm{~K}$ & 100 & 0 & 0 & 0 & 0 & 0 & 0 & 0 & 0 & 0 & 0 & 0 & 0 & 0 & & 0 & 0 & 0 & 0 & 0 & 0 & 0 & 0 & 0 & 0 & 0 & 0 & D & 0100 \\
\hline HEU & ${ }^{235} U$ & 79 & 0 & 0 & 0 & 0 & 0 & 0 & 0 & 0 & 0 & 0 & 0 & 2 & 0 & & 0 & 12 & 0 & 0 & 0 & 4 & 1 & 2 & 0 & 0 & 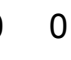 & 0 & J & 010 \\
\hline irgin HEU & ${ }^{235} \mathrm{U}$ & 69 & 0 & 0 & 0 & 0 & 0 & 0 & 0 & 0 & 0 & 0 & 0 & 2 & 0 & & 0 & 18 & 1 & 0 & 0 & 4 & 2 & 4 & 0 & 0 & 0 & & J & 0100 \\
\hline Reactor Pu & ${ }^{239} \mathrm{Pu}$ & 97 & 0 & 0 & 0 & 0 & 0 & b & 0 & 0 & 0 & 0 & 0 & 0 & 0 & & 0 & 0 & 2 & 0 & 0 & 0 & 0 & 1 & 0 & 0 & 0 & 0 & J & 010 \\
\hline Weapon Pu & ${ }^{239} \mathrm{Pu}$ & 96 & 0 & 0 & 0 & 0 & 0 & b & 0 & 0 & 0 & 0 & 0 & 0 & 0 & & 0 & 0 & 3 & 0 & 0 & 0 & 1 & 0 & 0 & 0 & 0 & & כ & 10 \\
\hline HEU with shielding & ${ }^{235} U$ & 91 & 0 & 0 & 0 & 0 & 2 & 2 & 1 & 0 & 0 & 0 & 0 & 0 & 1 & & 0 & 0 & 0 & 0 & 0 & 0 & 0 & 0 & 0 & 1 & 0 & & J & 0 \\
\hline Virgin HEU with shielding & ${ }^{235} \mathrm{U}$ & 94 & 0 & 0 & 0 & 0 & 0 & 0 & 0 & 0 & 0 & 1 & 0 & 0 & 0 & & 2 & 0 & 0 & 0 & 0 & 0 & 0 & 0 & 0 & 1 & 1 & & 1 & 010 \\
\hline Reactor Pu with shielding & ${ }^{239} \mathrm{Pu}$ & 96 & 0 & 0 & 0 & 0 & 0 & 0 & 0 & 0 & 3 & 0 & 0 & 0 & 0 & & 0 & 0 & 0 & 0 & 0 & 0 & 0 & 0 & 0 & 1 & 0 & 0 & כ & 0 \\
\hline Weapon Pu w & ${ }^{239} \mathrm{Pu}$ & 94 & 0 & 0 & 0 & 0 & 0 & 0 & 0 & 0 & 4 & 1 & 0 & 0 & 0 & & 0 & 0 & 0 & 0 & 0 & 0 & 0 & 0 & 0 & 0 & 1 & 0 & כ & 010 \\
\hline \multicolumn{31}{|l|}{100 Counts/400 Bkg } \\
\hline $99 \mathrm{TcM}-625 \mathrm{uc}+200 \mathrm{~mm}-\mathrm{H} 2 \mathrm{O}+5 \mathrm{mmFe}$ & ${ }^{99 \mathrm{~m}} \mathrm{Tc}$ & 0 & 0 & 0 & 0 & 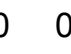 & 0 & כ & 0 & 0 & 0 & 0 & 2 & 0 & $v$ & & 0 & 0 & 0 & 0 & 0 & 98 & 0 & 0 & 0 & 0 & ) & & 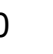 & \\
\hline 1311-370uc+200mm-H2O+5mmFe & ${ }^{131}$ & 30 & 0 & 0 & 0 & 0 & 0 & 0 & 0 & 25 & 0 & 0 & 0 & 0 & 0 & & 0 & 0 & 10 & 1 & 32 & 0 & 2 & 0 & 0 & 0 & 0 & 0 & 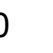 & 010 \\
\hline $01 \mathrm{TI}-250 \mathrm{uc}+200 \mathrm{~mm}-\mathrm{H} 2 \mathrm{O}+5 \mathrm{mmFe}$ & ${ }^{201} \mathrm{TI}$ & 0 & 0 & 0 & 0 & 0 & 0 & 0 & 0 & 1 & 0 & 0 & 0 & 18 & 0 & & 0 & 0 & U & 0 & 0 & 8 & 0 & 73 & 0 & 0 & 0 & 0 & 0 & 010 \\
\hline
\end{tabular}




\begin{tabular}{|c|c|c|c|c|c|c|c|c|c|c|c|c|c|c|c|c|c|c|c|c|c|c|c|c|c|c|c|c|c|c|}
\hline Sample & Label & ND u & & $\begin{array}{l}40 \\
K \\
\end{array}$ & $\begin{array}{r}226 \\
\mathrm{Ra} \\
\end{array}$ & $\begin{array}{l}232 \\
\text { Th } \\
\end{array}$ & $\begin{array}{l}228 \\
\text { Th } \\
\end{array}$ & $\begin{array}{c}232 \\
\mathrm{U} \\
\end{array}$ & $\begin{array}{l}60 \\
\text { Co } \\
\end{array}$ & $\begin{array}{l}133 \\
\mathrm{Ba} \\
\end{array}$ & $\begin{array}{l}137 \\
\text { Cs } \\
\end{array}$ & $\begin{array}{r}54 \\
\mathrm{Mn} \\
\end{array}$ & & $\mathrm{Ce}$ & & & $\begin{array}{l}238 \\
U \\
\end{array}$ & $\begin{array}{c}235 \\
U \\
\end{array}$ & $\begin{array}{l}239 \\
\mathrm{Pu} \\
\end{array}$ & $\begin{array}{l}237 \\
\text { Np } \\
\end{array}$ & $\begin{array}{c}131 \\
1 \\
\end{array}$ & $\begin{array}{l}99^{\mathrm{m}} \\
\text { Tc } \\
\end{array}$ & $\begin{array}{l}67 \\
\mathrm{Ga} \\
\end{array}$ & $\begin{array}{c}201 \\
\mathrm{TI} \\
\end{array}$ & $\begin{array}{l}88 \\
Y \\
\end{array}$ & $\begin{array}{l}22 \\
\mathrm{Na} \\
\end{array}$ & $\begin{array}{c}166^{\mathrm{m}} \\
\mathrm{Ho} \\
\end{array}$ & $\begin{array}{c}152 \\
\mathrm{Eu} \\
\end{array}$ & $\begin{array}{c}192 \\
\text { Ir }\end{array}$ & 2 sum \\
\hline $57 \mathrm{Ga}-350 \mathrm{uc}+200 \mathrm{~mm}-\mathrm{H} 2 \mathrm{O}+5 \mathrm{mmFe}$ & ${ }^{67} \mathrm{Ga}$ & 14 & 0 & 0 & 0 & 0 & 0 & 0 & 0 & 7 & 0 & 0 & 0 & ) & 1 & 0 & 0 & 9 & 3 & 6 & 1 & 1 & 49 & 3 & 0 & 0 & 0 & 0 & & 6100 \\
\hline 137 Cs-8mc+24mm-Lead+ & ${ }^{137} \mathrm{Cs}$ & 3 & 0 & 0 & 0 & 0 & 0 & 0 & 0 & 0 & 95 & 0 & 0 & ) & 0 & 0 & 0 & 0 & 0 & 0 & 0 & 0 & 0 & 0 & 0 & 0 & 1 & 0 & & 100 \\
\hline 14Kg-of-Bananas+5mmF & ${ }^{40} \mathrm{~K}$ & 71 & 0 & 22 & 0 & 0 & 0 & 0 & 0 & 0 & 1 & 0 & C & ) & 0 & 0 & 1 & 0 & 0 & 0 & 0 & 0 & 0 & 0 & 1 & 0 & 0 & 4 & & 100 \\
\hline 5Kg-of-Po & ${ }^{40} \mathrm{~K}$ & 96 & 0 & 1 & 0 & 0 & 0 & 0 & 0 & 0 & 1 & 0 & c & ) & 0 & 1 & 0 & 0 & 1 & 0 & 0 & 0 & 0 & 0 & 0 & 0 & 0 & 0 & & 0100 \\
\hline HEU & ${ }^{235} \mathrm{U}$ & 3 & 0 & 0 & 0 & 0 & 0 & 0 & 0 & 2 & 0 & 0 & 2 & 2 & 0 & 0 & 0 & 68 & 0 & 1 & 0 & 5 & 7 & 11 & 0 & 0 & 0 & 0 & & 100 \\
\hline Virgin HEU & ${ }^{235} \mathrm{U}$ & 1 & 0 & 0 & 0 & 0 & 0 & 0 & 0 & 1 & 0 & 0 & 1 & 1 & 0 & 0 & 0 & 72 & 0 & 1 & 0 & 5 & 8 & 10 & 0 & 0 & 0 & 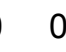 & & 1100 \\
\hline Neapon Pu & ${ }^{239} \mathrm{Pu}$ & 42 & 0 & 0 & 0 & 0 & 0 & 0 & 0 & 3 & 3 & 0 & 2 & ? & 0 & 0 & 0 & 3 & 26 & 2 & 10 & 1 & 4 & 1 & 0 & 0 & 0 & 0 & & 3100 \\
\hline HEU wit & ${ }^{235} \mathrm{U}$ & 22 & 0 & 1 & 5 & 20 & 4 & 31 & 1 & 0 & 0 & 0 & 0 & 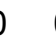 & 0 & 6 & 1 & 0 & 0 & 0 & 0 & 0 & 0 & 0 & 7 & 1 & 1 & 0 & & 0100 \\
\hline Virgin HEU w & ${ }^{235} \mathrm{U}$ & 29 & 0 & 0 & 2 & 1 & 0 & 0 & 2 & 0 & 0 & 1 & 0 & ) & 0 & 1 & 45 & 0 & 1 & 0 & 0 & 0 & 0 & 0 & 3 & 3 & 6 & 5 & & 1100 \\
\hline Reactor Pu with & ${ }^{239} \mathrm{Pu}$ & 43 & 0 & 0 & 1 & 7 & 3 & 3 & 0 & 0 & 20 & 0 & C & 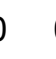 & 0 & 5 & 5 & 0 & 1 & 0 & 0 & 0 & 0 & 0 & 1 & 1 & 9 & 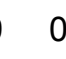 & & 1100 \\
\hline Weapon Pu with shielding & ${ }^{239} \mathrm{Pu}$ & 41 & 0 & 0 & 0 & 1 & 0 & 2 & 0 & 0 & 18 & - & 0 & 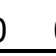 & 0 & 5 & 5 & 0 & 7 & 0 & 0 & 0 & 0 & 0 & 0 & 1 & 13 & 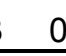 & 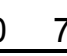 & 7100 \\
\hline \multicolumn{31}{|l|}{200 Counts/400 Bkg } \\
\hline 137 Cs-8mct & ${ }^{137} \mathrm{Cs}$ & 0 & 0 & 0 & c & 0 & 0 & 0 & 0 & & 100 & 0 & & & 0 & 0 & c & 0 & 0 & 0 & 0 & 0 & 0 & 0 & 0 & 0 & 0 & ) & & 0100 \\
\hline $14 \mathrm{Kg}-\mathrm{of}-\mathrm{I}$ & ${ }^{40} \mathrm{~K}$ & 0 & 0 & 83 & 1 & 2 & 0 & 0 & 1 & 0 & 0 & 0 & & & & & c & 0 & & 0 & c & 0 & 0 & & & 1 & 0 & 9 & & 0100 \\
\hline 5Kg-of-Wood & ${ }^{40} \mathrm{~K}$ & 1 & 0 & 77 & 1 & 2 & 0 & 0 & 1 & 0 & 0 & 0 & c & ) & 0 & 3 & 1 & 0 & $c$ & 0 & 0 & 0 & 0 & 0 & 2 & 1 & 0 & 11 & & 0100 \\
\hline 5Kg-of-Pc & ${ }^{40} \mathrm{~K}$ & 3 & 0 & 28 & 2 & 9 & 6 & 0 & 1 & 0 & 3 & 1 & & & 0 & 1 & 3 & 0 & 2 & 1 & 2 & 0 & 4 & 0 & 0 & 4 & 8 & 19 & & 3100 \\
\hline HEU & ${ }^{235} \mathrm{U}$ & 0 & 0 & 1 & 0 & 0 & 0 & 0 & 0 & 0 & 0 & $\mathrm{c}$ & & & C & & 0 & 90 & & 0 & c & 0 & 2 & 7 & 0 & 0 & 0 & 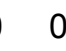 & & 0100 \\
\hline Virg & ${ }^{235} \mathrm{U}$ & 0 & 0 & 0 & 0 & 0 & 0 & 0 & 0 & 0 & 0 & 0 & & & 0 & 0 & 0 & 91 & 0 & 0 & 0 & 1 & 3 & 5 & 0 & 0 & 0 & 0 & & 0100 \\
\hline Reactor P & ${ }^{239} \mathrm{Pu}$ & 0 & 0 & 19 & 0 & 0 & 0 & 5 & 0 & 4 & 7 & 0 & & & O & 0 & 0 & 1 & 35 & 4 & 10 & 0 & 8 & 0 & 0 & 0 & 0 & 0 & & 6100 \\
\hline Weapon & ${ }^{239} \mathrm{Pu}$ & 0 & 0 & 1 & 0 & 0 & 0 & 0 & 0 & 6 & 0 & 0 & & & 0 & 0 & 0 & 0 & 78 & 0 & 8 & 0 & 3 & 0 & 0 & 0 & 0 & 0 & & 4100 \\
\hline HEU witt & ${ }^{235} \mathrm{U}$ & 0 & 0 & 0 & 10 & 25 & 1 & 50 & 2 & 0 & 0 & 0 & c & & 0 & 12 & 0 & 0 & 0 & 0 & 0 & 0 & 0 & 0 & 0 & 0 & 0 & 0 & & 0100 \\
\hline /irgin HEU with & ${ }^{235} \mathrm{U}$ & 0 & 0 & 0 & 9 & 1 & 0 & 0 & 5 & 0 & 0 & & & & 0 & 0 & 75 & 0 & & 0 & 0 & 0 & 0 & 0 & 3 & 0 & 0 & 7 & & 0100 \\
\hline eactor Pu with shielding & ${ }^{239} \mathrm{Pu}$ & 0 & 0 & 0 & 8 & 36 & 3 & 4 & 0 & 0 & 9 & 0 & c & & 0 & 23 & 3 & 0 & 0 & 0 & 0 & 0 & 0 & 0 & 1 & 0 & 13 & 0 & & 0100 \\
\hline
\end{tabular}




\begin{tabular}{|c|c|c|c|c|c|c|c|c|c|c|c|c|c|c|c|c|c|c|c|c|c|c|c|c|c|c|c|c|c|}
\hline Sample & Label & VD U & & $\begin{array}{r}40 \\
K \\
\end{array}$ & $\begin{array}{r}226 \\
\mathrm{Ra} \\
\end{array}$ & $\begin{array}{l}232 \\
\text { Th } \\
\end{array}$ & $\begin{array}{l}228 \\
\text { Th } \\
\end{array}$ & $\begin{array}{c}232 \\
\mathrm{U} \\
\end{array}$ & $\begin{array}{l}60 \\
\text { Co } \\
\end{array}$ & $\begin{array}{l}133 \\
\mathrm{Ba}\end{array}$ & $\begin{array}{l}137 \\
\text { Cs }\end{array}$ & $\begin{array}{r}54 \\
\mathrm{Mn} \\
\end{array}$ & $\begin{array}{l}57 \\
\text { Co } \\
\end{array}$ & $\begin{array}{c}139 \\
\mathrm{Ce} \\
\end{array}$ & $\begin{array}{c}252 \\
\mathrm{Cf} \\
\end{array}$ & $\begin{array}{c}238 \\
U \\
\end{array}$ & $\begin{array}{c}235 \\
U \\
\end{array}$ & $\begin{array}{c}239 \\
\mathrm{Pu} \\
\end{array}$ & $\begin{array}{l}237 \\
\mathrm{~Np} \\
\end{array}$ & $\begin{array}{r}131 \\
1 \\
\end{array}$ & & & & $\begin{array}{c}201 \\
\mathrm{TI} \\
\end{array}$ & $\begin{array}{c}88 \\
Y \\
\end{array}$ & $\begin{array}{r}22 \\
\mathrm{Na} \\
\end{array}$ & $\begin{array}{c}166^{\mathrm{m}} \\
\mathrm{Ho} \\
\end{array}$ & $\begin{array}{l}152 \\
\mathrm{Eu} \\
\end{array}$ & $\begin{array}{l}192 \\
\text { Ir SUM }\end{array}$ \\
\hline Weapon Pu with shielding & ${ }^{239} \mathrm{Pu}$ & 0 & 0 & 0 & 4 & 6 & 1 & 0 & 0 & 0 & 36 & 0 & 0 & 0 & 16 & 7 & 0 & 5 & 0 & ( & 0 & 0 & 0 & 0 & 0 & 0 & 20 & 0 & 5100 \\
\hline \multicolumn{30}{|l|}{300 Counts/400 Bkg } \\
\hline $9 \mathrm{TcM}-625 \mathrm{uc}+200 \mathrm{~mm}-\mathrm{H} 2 \mathrm{O}+5 \mathrm{mmFe}$ & ${ }^{99 \mathrm{~m}} \mathrm{Tc}$ & 0 & 0 & 0 & 0 & 0 & 0 & 0 & 0 & 0 & 0 & 0 & 0 & 0 & 0 & 0 & 0 & 0 & 0 & \multicolumn{3}{|c|}{0100} & 0 & 0 & 0 & 0 & 0 & 0 & 0100 \\
\hline $01 \mathrm{Tl}-250 \mathrm{uc}+200 \mathrm{~mm}-\mathrm{H} 2 \mathrm{O}+5 \mathrm{mmFe}$ & ${ }^{201} \mathrm{TI}$ & 0 & 0 & 0 & 0 & 0 & 0 & 0 & 0 & 0 & 0 & 0 & 0 & 9 & 0 & 0 & 0 & 0 & 0 & 0 & 0 & 0 & 0 & 91 & 0 & 0 & 0 & 0 & 0100 \\
\hline $67 \mathrm{Ga}-350 \mathrm{uc}+200 \mathrm{~mm}-\mathrm{H} 2 \mathrm{O}+5$ & ${ }^{67} \mathrm{Ga}$ & 0 & 0 & 0 & 0 & 0 & 0 & 0 & 0 & 6 & 0 & 0 & 0 & 0 & 0 & 0 & 0 & 1 & 23 & 3 & 0 & 0 & 62 & 0 & 0 & 0 & 0 & 0 & 8100 \\
\hline $137 \mathrm{Cs}-8 \mathrm{mc}+24 \mathrm{~mm}-\mathrm{Lead}+5 \mathrm{mmFe}$ & ${ }^{137} \mathrm{Cs}$ & 0 & 0 & 0 & 0 & 0 & 0 & 0 & 0 & \multicolumn{2}{|c|}{0100} & 0 & 0 & 0 & 0 & 0 & 0 & 0 & 0 & ( & 0 & 0 & 0 & $c$ & 0 & 0 & 0 & 0 & 0100 \\
\hline $5 \mathrm{Kg}$-of-Wood $+5 \mathrm{mmFe}$ & ${ }^{40} \mathrm{~K}$ & 0 & 0 & 89 & 2 & 0 & 0 & 0 & 3 & 0 & 0 & 0 & 0 & 0 & 0 & 0 & 0 & 0 & 0 & b & 0 & 0 & 0 & ( & 0 & 1 & 0 & 5 & 0100 \\
\hline 5Kg-of-Potash-Fertilizer & ${ }^{40} \mathrm{~K}$ & 0 & 0 & 33 & 16 & 8 & 2 & 0 & 3 & 0 & 0 & 0 & 0 & 0 & 2 & 3 & 0 & 0 & 0 & ( & 0 & 0 & 0 & $\mathrm{c}$ & 1 & 2 & 12 & 18 & 0100 \\
\hline HEU & ${ }^{235} \mathrm{U}$ & 0 & 0 & 0 & 0 & 0 & 0 & 0 & 0 & 0 & 0 & 0 & 0 & 0 & 0 & 0 & 97 & 0 & 0 & ( & 0 & O & 1 & & 0 & 0 & 0 & 0 & 0100 \\
\hline Virgin HEU & ${ }^{235} \mathrm{U}$ & 0 & 0 & 0 & 0 & 0 & 0 & 0 & 0 & 0 & 0 & 0 & 0 & 0 & 0 & 0 & 97 & 0 & 0 & 0 & 0 & 0 & 0 & 3 & 0 & 0 & 0 & 0 & 0100 \\
\hline Reactor Pu & ${ }^{239} \mathrm{Pu}$ & 0 & 0 & 23 & 0 & 0 & 0 & 4 & 0 & 5 & 6 & 0 & 0 & 0 & 0 & 0 & 1 & 38 & 0 & 10 & & 0 & 6 & ( & 0 & 0 & 0 & 0 & 7100 \\
\hline Weapon Pu & ${ }^{239} \mathrm{Pu}$ & 0 & 0 & 0 & 0 & 0 & 0 & 0 & 0 & 2 & 1 & 0 & 0 & 0 & 0 & 0 & 0 & 90 & 0 & 0 & 6 & 0 & 1 & & 0 & & 0 & 0 & 0100 \\
\hline \multicolumn{30}{|l|}{500 Counts/400 Bkg } \\
\hline 99TсM & ${ }^{9 \mathrm{~m}} \mathrm{Tc}$ & 0 & 0 & 0 & 0 & 0 & 0 & 0 & 0 & 0 & 0 & 0 & 0 & 0 & 0 & 0 & 0 & 0 & 0 & \multicolumn{3}{|c|}{0100} & 0 & & 0 & & 0 & 0 & 0100 \\
\hline $1311-370 u c+2$ & & 0 & 0 & 0 & 0 & 0 & 0 & 0 & 0 & 24 & 0 & 0 & 0 & 0 & 0 & 0 & 0 & 3 & 0 & 7 & & 0 & 0 & 0 & 0 & 0 & 0 & 0 & 0100 \\
\hline 201TI-250uc & & 0 & 0 & 0 & 0 & 0 & 0 & 0 & 0 & 0 & 0 & 0 & 0 & 5 & 0 & 0 & 0 & 0 & 0 & ) & 0 & 0 & 0 & 95 & 0 & & 0 & $v$ & 0100 \\
\hline $67 \mathrm{Ga}-3$ & ${ }^{67} \mathrm{Ga}$ & 0 & 0 & 0 & 0 & 0 & 0 & 0 & 0 & 2 & 0 & 0 & 0 & 0 & 0 & 0 & 0 & 0 & 30 & 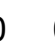 & 0 & 0 & 66 & 0 & 0 & & 0 & 0 & 2100 \\
\hline 137 Cs-8m & ${ }^{137} \mathrm{Cs}$ & 0 & 0 & 0 & 0 & 0 & 0 & 0 & 0 & & 100 & 0 & 0 & 0 & 0 & 0 & 0 & 0 & 0 & 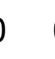 & 0 & 0 & 0 & ( & 0 & & 0 & 0 & 0100 \\
\hline 14Kg-of-Banar & ${ }^{40} \mathrm{~K}$ & 0 & 0 & 94 & 1 & 0 & 0 & 0 & 0 & 0 & 0 & 0 & 0 & 0 & 0 & 0 & 0 & 0 & 0 & 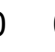 & 0 & 0 & 0 & . & 0 & & 0 & 5 & 0100 \\
\hline 5Kg-of-Wood & ${ }^{40} \mathrm{~K}$ & 0 & 0 & 97 & 0 & 0 & 0 & 0 & 0 & 0 & 0 & 0 & 0 & 0 & 0 & 0 & 0 & 0 & 0 & 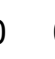 & 0 & & 0 & & 0 & 0 & 0 & 3 & 0100 \\
\hline 5Kg-of-Potas! & ${ }^{40} \mathrm{~K}$ & 0 & 0 & 30 & 15 & 6 & 1 & 0 & 0 & 0 & 0 & 0 & c & 0 & 2 & 1 & 0 & 0 & 0 & 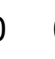 & 0 & & 0 & & 0 & & 0 & 45 & 0100 \\
\hline HEU & ${ }^{235} \mathrm{U}$ & 0 & 0 & 0 & 0 & 0 & 0 & 0 & 0 & 0 & 0 & 0 & 0 & 0 & 0 & & 100 & 0 & 0 & 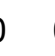 & 0 & & 0 & & 0 & & 0 & 0 & 0100 \\
\hline irgin HEU & ${ }^{235} \mathrm{U}$ & 0 & 0 & 0 & 0 & 0 & 0 & 0 & 0 & 0 & 0 & 0 & 0 & 0 & 0 & & 100 & 0 & 0 & ) & 0 & 0 & 0 & 0 & 0 & 0 & 0 & 0 & 0100 \\
\hline
\end{tabular}




\begin{tabular}{|c|c|c|c|c|c|c|c|c|c|c|c|c|c|c|c|c|c|c|c|c|c|c|c|c|c|c|c|c|}
\hline Sample & Label & ND & & $\begin{array}{r}40 \\
K \\
\end{array}$ & $\begin{array}{r}226 \\
\mathrm{Ra} \\
\end{array}$ & $\begin{array}{c}232 \\
\text { Th } \\
\end{array}$ & $\begin{array}{l}228 \\
\text { Th } \\
\end{array}$ & $\begin{array}{c}232 \\
\mathrm{U} \\
\end{array}$ & $\begin{array}{l}60 \\
\text { Co } \\
\end{array}$ & $\begin{array}{l}133 \\
\mathrm{Ba} \\
\end{array}$ & $\begin{array}{l}137 \\
\text { Cs } \\
\end{array}$ & $\begin{array}{l}54 \\
\mathrm{Mn} \\
\end{array}$ & $\begin{array}{l}57 \\
\text { Co } \\
\end{array}$ & $\begin{array}{c}139 \\
\mathrm{Ce} \\
\end{array}$ & $\begin{array}{c}252 \\
\text { Cf } \\
\end{array}$ & $\begin{array}{c}238 \\
\mathrm{U}\end{array}$ & $\begin{array}{c}235 \\
\mathrm{U} \\
\end{array}$ & $\begin{array}{l}239 \\
\mathrm{Pu} \\
\end{array}$ & $\begin{array}{l}237 \\
\mathrm{~Np} \\
\end{array}$ & $\begin{array}{c}131 \\
1 \\
\end{array}$ & $\begin{array}{l}99^{\mathrm{m}} \\
\text { Tc } \\
\end{array}$ & $\begin{array}{l}67 \\
\text { Ga } \\
\end{array}$ & $\begin{array}{c}201 \\
\mathrm{TI} \\
\end{array}$ & $\begin{array}{c}88 \\
Y \\
\end{array}$ & $\begin{array}{l}22 \\
\mathrm{Na} \\
\end{array}$ & $\begin{array}{c}166^{\mathrm{m}} \\
\text { Ho } \\
\end{array}$ & $\begin{array}{c}152 \\
\mathrm{Eu} \\
\end{array}$ & $\begin{array}{l}192 \\
\text { Ir sum }\end{array}$ \\
\hline Reactor Pu & ${ }^{239} \mathrm{Pu}$ & 0 & 0 & 31 & 0 & 0 & 0 & 1 & 0 & 2 & 2 & 0 & 0 & 0 & 0 & 0 & 0 & 49 & 0 & 8 & 0 & 3 & 0 & 0 & 0 & 0 & 0 & 4100 \\
\hline eapon Pu & ${ }^{239} \mathrm{Pu}$ & 0 & 0 & 0 & 0 & 0 & 0 & 0 & 0 & 0 & 0 & 0 & 0 & 0 & 0 & 0 & 0 & 97 & 0 & 2 & 0 & 0 & c & 0 & 0 & 0 & 0 & 1100 \\
\hline EU with shielding & ${ }^{235} \mathrm{U}$ & 0 & 0 & 0 & 1 & 37 & 0 & 61 & 0 & 0 & 0 & 0 & 0 & 0 & 0 & 0 & 0 & 0 & 0 & 0 & 0 & 0 & c & 1 & 0 & 0 & 0 & 0100 \\
\hline Reactor Pu with shielding & ${ }^{239} \mathrm{Pu}$ & 0 & 0 & 0 & 6 & 66 & 0 & 0 & 0 & 0 & 4 & 0 & 0 & 0 & 21 & 0 & 0 & 0 & 0 & 0 & 0 & 0 & 0 & 0 & 0 & 3 & 0 & 0100 \\
\hline Weapon Pu with shielding & ${ }^{239} \mathrm{Pu}$ & 0 & 0 & 0 & 2 & 3 & 0 & 0 & 0 & 0 & 35 & 0 & 0 & 0 & 24 & 13 & 0 & 0 & 0 & 0 & 0 & 0 & $\mathrm{c}$ & ( & 0 & 19 & 0 & 4100 \\
\hline \multicolumn{29}{|l|}{750 Counts/400 Bkg } \\
\hline $1311-370 u c+200 n$ & & 0 & 0 & 0 & 0 & 0 & 0 & 0 & 0 & 14 & 0 & 0 & 0 & 0 & 0 & 0 & 0 & 0 & 0 & 86 & 0 & 0 & 0 & 0 & 0 & 0 & 0 & 0100 \\
\hline $201 \mathrm{Tl}-250 \mathrm{uc}+200 \mathrm{~mm}-\mathrm{H} 2 \mathrm{O}+5 \mathrm{mmFe}$ & 20 & 0 & 0 & 0 & 0 & 0 & 0 & 0 & 0 & 0 & 0 & 0 & 0 & 1 & 0 & 0 & 0 & 0 & 0 & 0 & 0 & 0 & 99 & 0 & 0 & 0 & 0 & 0100 \\
\hline $37 \mathrm{Ga}-350 \mathrm{uc}+200 \mathrm{~mm}-\mathrm{H} 2 \mathrm{O}+5 \mathrm{mmFe}$ & ${ }^{67} \mathrm{Ga}$ & 0 & 0 & 0 & 0 & 0 & 0 & 0 & 0 & 0 & 0 & 0 & 0 & 0 & 0 & 0 & 0 & 0 & 36 & 0 & 0 & 64 & & 0 & 0 & 0 & & 0100 \\
\hline $137 \mathrm{Cs}-8 \mathrm{mc}+24 \mathrm{~mm}-\mathrm{Lead}+5 \mathrm{mmFe}$ & ${ }^{137} \mathrm{Cs}$ & 0 & 0 & 0 & 0 & 0 & 0 & 0 & 0 & \multicolumn{2}{|c|}{0100} & 0 & 0 & 0 & 0 & 0 & 0 & 0 & 0 & 0 & 0 & 0 & & 0 & 0 & 0 & 0 & 0100 \\
\hline $14 \mathrm{Kg}$-of-Bananas $+5 \mathrm{mmFe}$ & ${ }^{40} \mathrm{~K}$ & 0 & \multicolumn{2}{|c|}{0100} & 0 & 0 & 0 & 0 & 0 & 0 & 0 & 0 & 0 & 0 & 0 & 0 & $c$ & 0 & 0 & 0 & $\mathrm{C}$ & 0 & & 0 & 0 & 0 & 0 & 0100 \\
\hline $5 \mathrm{Kg}$-of-Wood+5mmFe & ${ }^{40} \mathrm{~K}$ & 0 & \multicolumn{2}{|c|}{0100} & 0 & 0 & 0 & 0 & c & 0 & $c$ & 0 & c & 0 & 0 & 0 & ( & 0 & 0 & 0 & & 0 & & & & 0 & & 0100 \\
\hline Weapon $\mathrm{F}$ & ${ }^{239} \mathrm{Pu}$ & 0 & 0 & 0 & 0 & 0 & 0 & 0 & 0 & ( & 0 & 0 & 0 & 0 & 0 & 0 & & 100 & 0 & 0 & & 0 & & 0 & & 0 & & 0100 \\
\hline HEU wit & ${ }^{235} \mathrm{U}$ & 0 & 0 & 0 & 0 & 47 & 0 & 53 & 0 & 0 & 0 & 0 & 0 & 0 & 0 & 0 & 0 & 0 & 0 & 0 & & 0 & & 0 & & 0 & & 0100 \\
\hline Virgin HEU with & ${ }^{235} \mathrm{U}$ & 0 & 0 & 0 & 0 & 0 & 0 & 0 & 0 & 0 & 0 & 0 & 0 & 0 & \multicolumn{2}{|c|}{0100} & 0 & 0 & 0 & 0 & 0 & 0 & & 0 & 0 & 0 & 0 & 010 \\
\hline Reactor Pu with & ${ }^{239} \mathrm{Pu}$ & 0 & 0 & 0 & 3 & 81 & 0 & 0 & 0 & 0 & 0 & 0 & 0 & 0 & 14 & 1 & 0 & 0 & 0 & 0 & 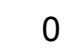 & 0 & c & 0 & 0 & 1 & 0 & 0100 \\
\hline Weapon Pu wit & ${ }^{239} \mathrm{Pu}$ & 0 & 0 & 0 & 1 & 3 & 0 & 0 & 0 & 0 & 25 & 0 & 0 & 0 & 38 & 17 & 0 & 0 & 0 & 0 & 0 & 0 & 0 & U & 0 & 16 & 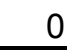 & 0100 \\
\hline \multicolumn{29}{|l|}{1000 Counts/400 Bkg } \\
\hline $99 \mathrm{TcM}-625 \mathrm{uc}+200 \mathrm{~mm}-\mathrm{H} 2 \mathrm{O}+5 \mathrm{mmFe}$ & & 0 & 0 & 0 & 0 & 0 & 0 & 0 & 0 & & 0 & & 0 & 0 & 0 & 0 & 0 & 0 & 0 & \multicolumn{2}{|c|}{0100} & 0 & 0 & 0 & 0 & 0 & & 0100 \\
\hline$n-\mathrm{H} 2 \mathrm{O}+5 \mathrm{mmFe}$ & & 0 & 0 & 0 & 0 & 0 & 0 & 0 & 0 & 8 & 0 & 0 & 0 & 0 & 0 & 0 & 0 & 0 & 0 & 92 & 0 & 0 & 0 & 0 & 0 & 0 & & 0100 \\
\hline $1 \mathrm{Tl}-250 \mathrm{uc}+2$ & ${ }^{201} \mathrm{TI}$ & 0 & 0 & 0 & 0 & 0 & 0 & 0 & 0 & 0 & 0 & 0 & 0 & 0 & 0 & 0 & 0 & 0 & 0 & 0 & 0 & \multicolumn{2}{|c|}{0100} & 0 & 0 & 0 & & 0100 \\
\hline $7 \mathrm{Ga}-350 \mathrm{a}$ & ${ }^{67} \mathrm{Ga}$ & 0 & 0 & 0 & 0 & 0 & 0 & 0 & 0 & 0 & 0 & 0 & 0 & 0 & 0 & 0 & 0 & 0 & 32 & 0 & 0 & 66 & 0 & 0 & 0 & 0 & 0 & 2100 \\
\hline $37 \mathrm{Cs}-8 \mathrm{mc}+24 \mathrm{~mm}-\mathrm{Lead}+5 \mathrm{mmFe}$ & ${ }^{137} \mathrm{Cs}$ & 0 & 0 & 0 & 0 & 0 & 0 & 0 & 0 & & 100 & 0 & 0 & 0 & 0 & 0 & 0 & 0 & 0 & 0 & 0 & 0 & 0 & 0 & 0 & 0 & 0 & 0100 \\
\hline
\end{tabular}




\begin{tabular}{|c|c|c|c|c|c|c|c|c|c|c|c|c|c|c|c|c|c|c|c|c|c|c|c|c|c|c|c|c|}
\hline Sample & abel & ND 1 & & $\begin{array}{c}40 \\
\mathrm{~K} \\
\end{array}$ & $\begin{array}{r}226 \\
\mathrm{Ra} \\
\end{array}$ & $\begin{array}{c}232 \\
\text { Th } \\
\end{array}$ & $\begin{array}{c}228 \\
\text { Th } \\
\end{array}$ & $\begin{array}{c}232 \\
U \\
\end{array}$ & $\begin{array}{l}60 \\
\text { Co } \\
\end{array}$ & $\begin{array}{l}133 \\
\mathrm{Ba} \\
\end{array}$ & $\begin{array}{l}137 \\
\text { Cs } \\
\end{array}$ & $\begin{array}{l}54 \\
\mathrm{Mn} \\
\end{array}$ & $\begin{array}{l}57 \\
\text { Co } \\
\end{array}$ & $\begin{array}{l}139 \\
\mathrm{Ce} \\
\end{array}$ & $\begin{array}{c}252 \\
\text { Cf } \\
\end{array}$ & $\begin{array}{c}238 \\
\mathrm{U}\end{array}$ & $\begin{array}{cc}235 & 23 \\
\mathrm{U} & \mathrm{P} \\
\end{array}$ & $\begin{array}{ll}239 & 2 \\
\mathrm{Pu} & 1 \\
\end{array}$ & $\begin{array}{l}237 \\
\mathrm{~Np} \\
\end{array}$ & $\begin{array}{rr}131 & 9 \\
\mathrm{I} & \mathrm{T} \\
\end{array}$ & $\begin{array}{l}99^{\mathrm{m}} \\
\text { Tc } \\
\end{array}$ & $\begin{array}{ll}67 & 2 \\
\mathrm{Ga} & \\
\end{array}$ & $\begin{array}{l}201 \\
\text { TI } \\
\end{array}$ & $\begin{array}{c}88 \\
Y \\
\end{array}$ & $\begin{array}{l}22 \\
\mathrm{Na} \\
\end{array}$ & $\begin{array}{c}166^{\mathrm{m}} \\
\text { Ho }\end{array}$ & $\begin{array}{l}152 \\
\mathrm{Eu} \\
\end{array}$ & $\begin{array}{l}192 \\
\text { Ir SUM }\end{array}$ \\
\hline $14 \mathrm{Kg}$-of-Bananas $+5 \mathrm{mmFe}$ & ${ }^{40} \mathrm{~K}$ & 0 & 0 & 99 & 0 & 0 & 0 & 0 & 0 & 0 & 0 & 0 & 0 & 0 & 0 & 0 & 0 & 0 & 0 & 0 & 0 & 0 & 0 & 0 & 0 & 0 & 1 & 0100 \\
\hline $5 \mathrm{Kg}$-of-Wood $+5 \mathrm{mmFe}$ & ${ }^{40} \mathrm{~K}$ & 0 & 0 & 99 & 0 & 0 & 0 & 0 & 0 & 0 & 0 & 0 & 0 & 0 & 0 & 0 & 0 & 0 & 0 & 0 & 0 & 0 & 0 & 0 & 0 & 0 & 1 & 0100 \\
\hline 5Kg-of-Potash- & ${ }^{40} \mathrm{~K}$ & 0 & 0 & 23 & 4 & 1 & 0 & 0 & 0 & 0 & 0 & 0 & 0 & 0 & 0 & 1 & 0 & 0 & 0 & 0 & 0 & 0 & c & 0 & 0 & 0 & 71 & 0100 \\
\hline IEU & ${ }^{235} \mathrm{U}$ & 0 & 0 & 0 & 0 & 0 & 0 & 0 & $c$ & 0 & 0 & 0 & 0 & 0 & 0 & & 100 & 0 & 0 & 0 & 0 & 0 & c & 0 & 0 & 0 & & 0100 \\
\hline Reactor Pu & ${ }^{239} \mathrm{Pu}$ & 0 & 0 & 31 & 0 & 0 & 0 & 0 & 0 & 1 & 1 & 0 & 0 & 0 & 0 & 0 & 0 & 62 & 0 & 4 & 0 & 0 & 0 & 0 & 0 & 0 & 0 & 1100 \\
\hline Neapon Pu & ${ }^{239} \mathrm{Pu}$ & 0 & 0 & 0 & 0 & 0 & 0 & 0 & 0 & 0 & 0 & 0 & 0 & 0 & 0 & 0 & & 100 & 0 & 0 & 0 & 0 & ( & 0 & 0 & 0 & & 0100 \\
\hline Virgin HEU wi & ${ }^{235} \mathrm{U}$ & 0 & 0 & 0 & 0 & 0 & 0 & 0 & 0 & 0 & 0 & 0 & 0 & 0 & & 100 & 0 & 0 & 0 & 0 & 0 & 0 & 0 & 0 & 0 & 0 & 0 & 0100 \\
\hline Reacto & ${ }^{239} \mathrm{Pu}$ & 0 & 0 & 0 & 4 & 79 & 0 & 0 & 0 & 0 & 0 & 0 & 0 & 0 & 17 & 0 & 0 & 0 & 0 & 0 & 0 & 0 & 0 & 0 & 0 & 0 & 0 & 0100 \\
\hline eapon Pu with shielding & ${ }^{239} \mathrm{Pu}$ & 0 & 0 & 0 & 2 & 1 & 0 & 0 & $\mathrm{c}$ & 0 & 24 & 0 & 0 & 0 & 33 & 28 & 0 & 0 & 0 & 0 & 0 & 0 & $c$ & 0 & 0 & 12 & 0 & 0100 \\
\hline
\end{tabular}




\section{A.4.3 ML Group}

\section{A.4.3.1 ML Group Isotopic Score}

Column definitions

- Sample - name of the sample in the library file

- Label - expert label algorithm is trying to match

- Score - Number of trials in which the output matched the label.

- Best - Number of trials in which the output matched the score when taking best guess.

As a classifier produces only one output, the "Score" column here is equivelent to the "Included" and "Exact" column for GADRAS fittodb.

\begin{tabular}{|lrrr|}
\hline Sample & Label & Score & Best \\
50 Counts/400 Bkg & & & \\
99TcM-625uc+200mm-H2O+5mmFe & ${ }^{99 m} \mathrm{Tc}$ & 74 & 76 \\
131I-370uc+200mm-H2O+5mmFe & ${ }^{131} \mathrm{I}$ & 24 & 24 \\
201TI-250uc+200mm-H2O+5mmFe & ${ }^{201} \mathrm{TI}$ & 53 & 53 \\
67Ga-350uc+200mm-H2O+5mmFe & ${ }^{67} \mathrm{Ga}$ & 20 & 20 \\
137Cs-8mc+24mm-Lead+5mmFe & ${ }^{137} \mathrm{Cs}$ & 69 & 69 \\
14Kg-of-Bananas+5mmFe & ${ }^{40} \mathrm{~K}$ & 11 & 11 \\
5Kg-of-Wood+5mmFe & ${ }^{40} \mathrm{~K}$ & 12 & 12 \\
5Kg-of-Potash-Fertilizer+5mmFe & ${ }^{40} \mathrm{~K}$ & 4 & 4 \\
HEU & ${ }^{235} \mathrm{U}$ & 40 & 40 \\
Virgin HEU & ${ }^{235} \mathrm{U}$ & 48 & 48 \\
Reactor Pu & ${ }^{239} \mathrm{Pu}$ & 13 & 13 \\
Weapon Pu & ${ }^{239} \mathrm{Pu}$ & 18 & 18 \\
HEU with shielding & ${ }^{235} \mathrm{U}$ & 2 & 2 \\
Virgin HEU with shielding & ${ }^{235} \mathrm{U}$ & 1 & 1 \\
Reactor Pu with shielding & ${ }^{239} \mathrm{Pu}$ & 3 & 3 \\
Weapon Pu with shielding & ${ }^{239} \mathrm{Pu}$ & 8 & 8 \\
100 Counts/400 Bkg & & & \\
99TcM-625uc+200mm-H2O+5mmFe & ${ }^{99 m} \mathrm{Tc}$ & 95 & 96 \\
131I-370uc+200mm-H2O+5mmFe & ${ }^{131} \mathrm{I}$ & 30 & 30 \\
201TI-250uc+200mm-H2O+5mmFe & ${ }^{201} \mathrm{TI}$ & 78 & 80 \\
67Ga-350uc+200mm-H2O+5mmFe & ${ }^{67} \mathrm{Ga}$ & 58 & 58 \\
137Cs-8mc+24mm-Lead+5mmFe & ${ }^{137} \mathrm{Cs}$ & 96 & 96 \\
14Kg-of-Bananas+5mmFe & ${ }^{40} \mathrm{~K}$ & 43 & 44 \\
5Kg-of-Wood+5mmFe & ${ }^{40} \mathrm{~K}$ & 46 & 47 \\
5 Kg-of-Potash-Fertilizer+5mmFe & ${ }^{40} \mathrm{~K}$ & 11 & 11 \\
HEU & ${ }^{235} \mathrm{U}$ & 71 & 73 \\
Virgin HEU & ${ }^{235} \mathrm{U}$ & 69 & 72 \\
Reactor Pu & ${ }^{239} \mathrm{Pu}$ & 24 & 24 \\
Weapon Pu & ${ }^{239} \mathrm{Pu}$ & 38 & 38 \\
HEU with shielding & ${ }^{235} \mathrm{U}$ & 0 & 0 \\
\hline & & & \\
\hline
\end{tabular}




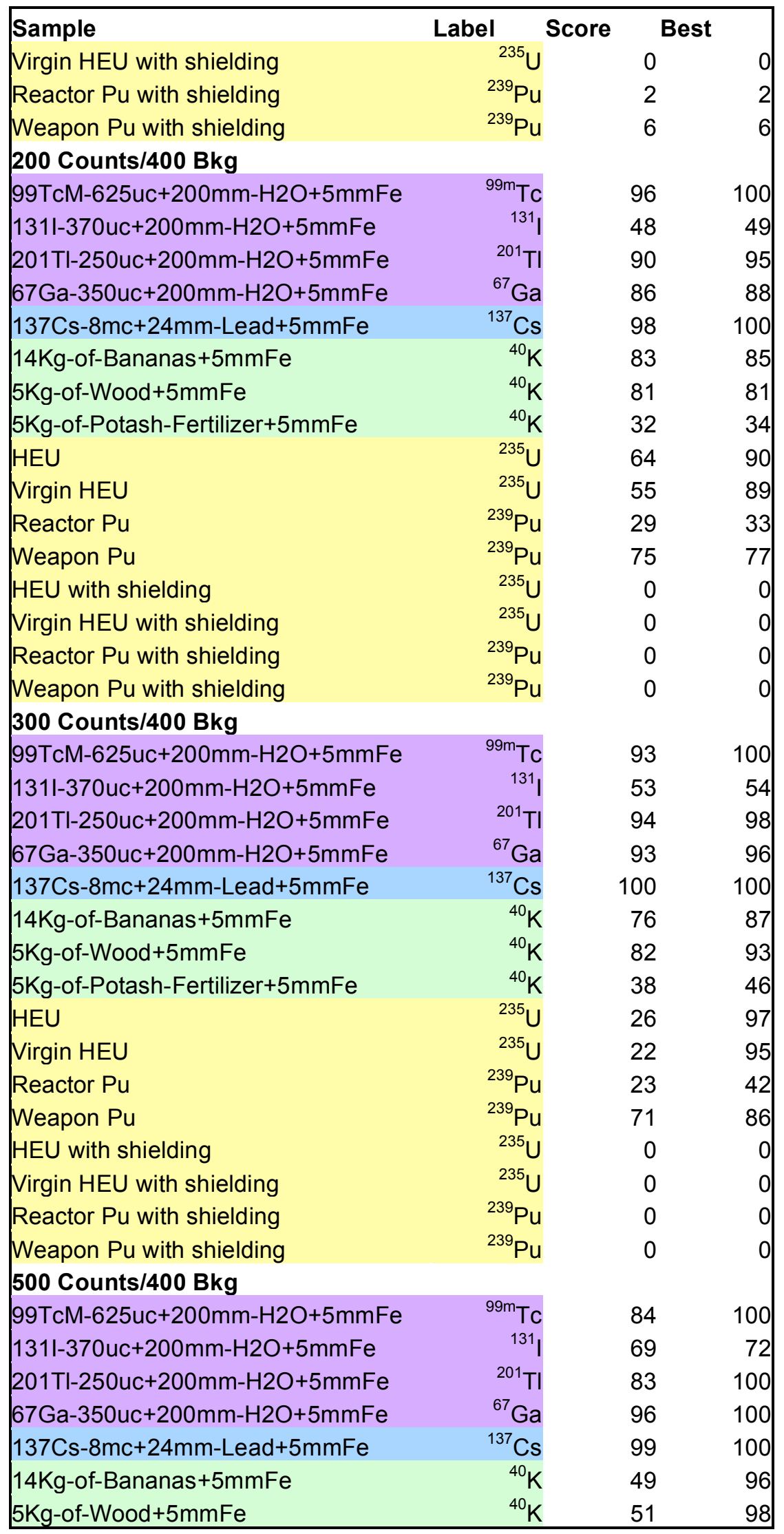




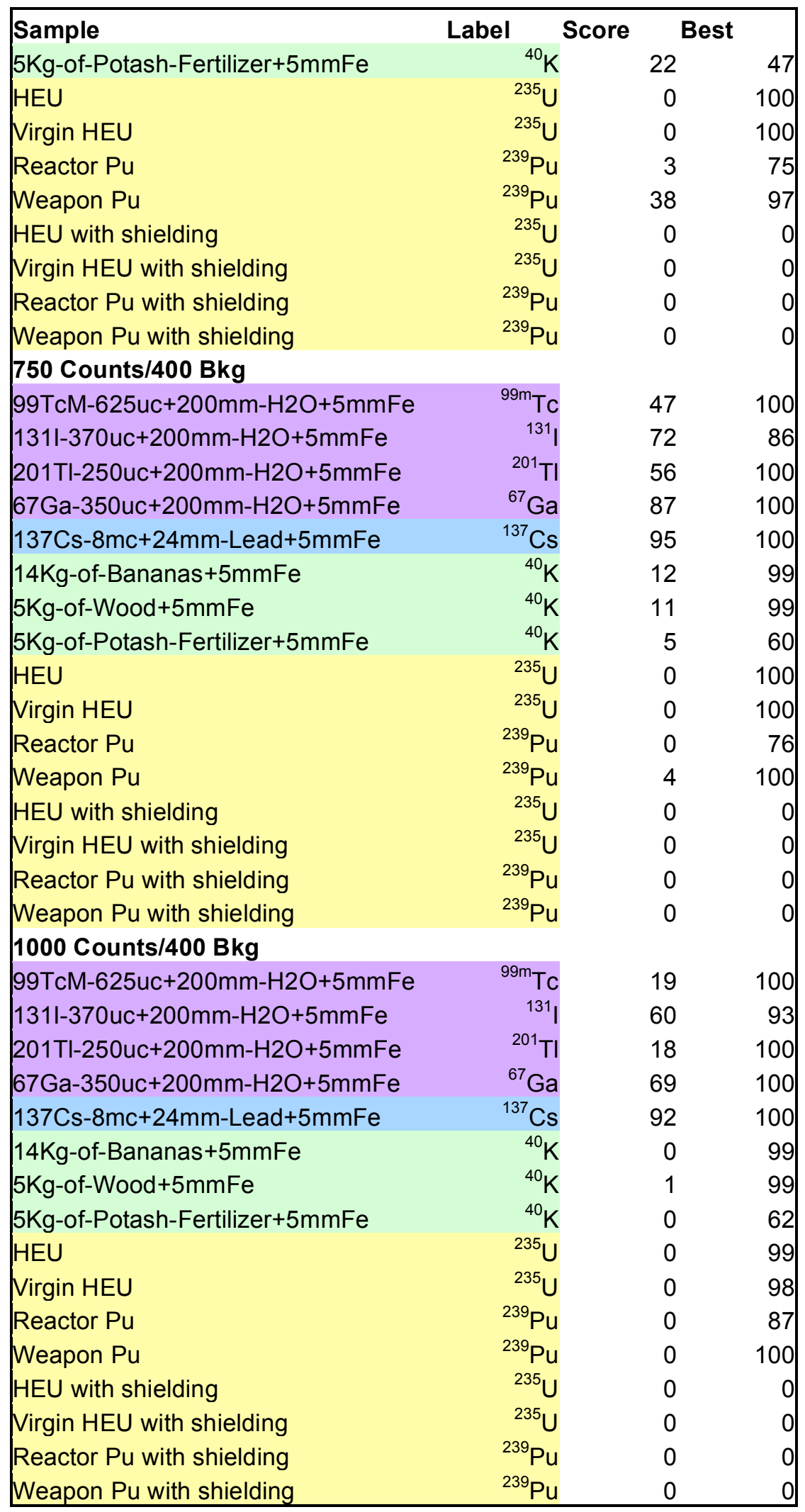




\section{A.4.3.2 ML Group Class Results}

Class scoring was only performed on the "best match" result.

\begin{tabular}{|c|c|c|c|c|c|c|c|c|c|c|}
\hline $\begin{array}{l}50 \text { Counts } / 400 \text { Bkg } \\
\text { Sample }\end{array}$ & Label & Class & 1 & 2 & 3 & & & & & \\
\hline 99TcM-625uc+200mm-H2O+5mmFe & ${ }^{99 \mathrm{~m}} \mathrm{Tc}$ & 3 & 1 & 23 & 76 & \multirow{5}{*}{ actual } & \multicolumn{3}{|c|}{ reported } & \multirow[b]{2}{*}{ total } \\
\hline $131 \mathrm{l}-370 \mathrm{uc}+200 \mathrm{~mm}-\mathrm{H} 2 \mathrm{O}+5 \mathrm{mmFe}$ & 131 & 2 & 19 & 74 & 7 & & 1 & 2 & & \\
\hline $201 \mathrm{Tl}-250 \mathrm{uc}+200 \mathrm{~mm}-\mathrm{H} 2 \mathrm{O}+5 \mathrm{mmFe}$ & ${ }^{201} \mathrm{~T}$ & 2 & 8 & 75 & 17 & & 0.263 & 0.672 & 0.066 & 1200 \\
\hline $67 \mathrm{Ga}-350 \mathrm{uc}+200 \mathrm{~mm}-\mathrm{H} 2 \mathrm{O}+5 \mathrm{mmFe}$ & ${ }^{67} \mathrm{Ge}$ & 2 & 22 & 71 & 7 & & 0.145 & 0.778 & 0.078 & 400 \\
\hline $137 \mathrm{Cs}-8 \mathrm{mc}+24 \mathrm{~mm}-$ Lead $+5 \mathrm{mmFe}$ & ${ }^{137} \mathrm{Cs}$ & 2 & 9 & 91 & 0 & & 0.125 & 0.585 & 0.290 & 400 \\
\hline 14Kg-of-Bananas $+5 \mathrm{mmFe}$ & ${ }^{40} \mathrm{~K}$ & 3 & 14 & 71 & 15 & & & & & \\
\hline $5 \mathrm{Kg}$-of-Wood $+5 \mathrm{mmFe}$ & ${ }^{40} \mathrm{~K}$ & 3 & 14 & 71 & 15 & promotion & & 0.86 & 0.29 & \\
\hline $5 \mathrm{Kg}$-of-Potash-Fertilizer $+5 \mathrm{mmFe}$ & ${ }^{40} \mathrm{~K}$ & 3 & 21 & 69 & 10 & demotion & & 0.82 & 0.27 & \\
\hline HEU & ${ }^{235} \mathrm{U}$ & 1 & 42 & 46 & 12 & & & & & \\
\hline Virgin HEU & ${ }^{235} \mathrm{U}$ & 1 & 50 & 35 & 15 & & & & & \\
\hline Reactor Pu & ${ }^{239} \mathrm{Pl}$ & 1 & 21 & 69 & 10 & & & & & \\
\hline Weapon $\mathrm{Pu}$ & ${ }^{239} \mathrm{Pl}$ & 1 & 24 & 71 & 5 & & & & & \\
\hline HEU with shielding & ${ }^{235} \mathrm{U}$ & 1 & 5 & 89 & 6 & & & & & \\
\hline Virgin HEU with shielding & ${ }^{235} \mathrm{U}$ & 1 & 4 & 94 & 2 & & & & & \\
\hline Reactor Pu with shielding & ${ }^{239} \mathrm{Pl}$ & 1 & 5 & 91 & 4 & & & & & \\
\hline Weapon Pu with shielding & ${ }^{239} \mathrm{PL}$ & 1 & 10 & 88 & 2 & & & & & \\
\hline
\end{tabular}

\begin{tabular}{|c|c|c|c|c|c|c|c|c|c|c|}
\hline \multicolumn{11}{|l|}{100 Counts/400 Bkg } \\
\hline Sample & Label & Class & 1 & 2 & 3 & & & & & \\
\hline 99TcM-625uc+200mm-H2O+5mmFe & ${ }^{99 \mathrm{~m}} \mathrm{Tc}$ & 3 & 0 & 4 & 96 & \multirow{5}{*}{ actual } & \multicolumn{3}{|c|}{ reported } & \multirow[b]{2}{*}{ Total } \\
\hline $131 \mathrm{I}-370 \mathrm{uc}+200 \mathrm{~mm}-\mathrm{H} 2 \mathrm{O}+5 \mathrm{mmFe}$ & 131 & 2 & 14 & 86 & 0 & & 1 & 2 & & \\
\hline $201 \mathrm{TI}-250 \mathrm{uc}+200 \mathrm{~mm}-\mathrm{H} 2 \mathrm{O}+5 \mathrm{mmFe}$ & ${ }^{201} \mathrm{~T}$ & 2 & 0 & 93 & 7 & & 0.396 & 0.544 & 0.060 & 1200 \\
\hline $67 \mathrm{Ga}-350 \mathrm{uc}+200 \mathrm{~mm}-\mathrm{H} 2 \mathrm{O}+5 \mathrm{mmFe}$ & ${ }^{67} \mathrm{Ga}$ & 2 & 17 & 80 & 3 & & 0.078 & 0.898 & 0.025 & 400 \\
\hline $137 \mathrm{Cs}-8 \mathrm{mc}+24 \mathrm{~mm}-\mathrm{Lead}+5 \mathrm{mmFe}$ & ${ }^{137} \mathrm{Cs}$ & 2 & 0 & 100 & 0 & & 0.075 & 0.413 & 0.513 & 400 \\
\hline $14 \mathrm{Kg}$-of-Bananas $+5 \mathrm{mmFe}$ & ${ }^{40} \mathrm{~K}$ & 3 & 5 & 50 & 45 & & & & & \\
\hline $5 \mathrm{Kg}$-of-Wood+5mmFe & ${ }^{40} \mathrm{~K}$ & 3 & 7 & 44 & 49 & promotion & & 0.57 & 0.19 & \\
\hline $5 \mathrm{Kg}$-of-Potash-Fertilizer $+5 \mathrm{mmFe}$ & ${ }^{40} \mathrm{~K}$ & 3 & 18 & 67 & 15 & demotion & & 0.63 & 0.21 & \\
\hline HEU & ${ }^{235} U$ & 1 & 73 & 22 & 5 & & & & & \\
\hline Virgin HEU & ${ }^{235} U$ & 1 & 72 & 24 & 4 & & & & & \\
\hline Reactor Pu & ${ }^{239} \mathrm{Pu}$ & 1 & 35 & 60 & 5 & & & & & \\
\hline Weapon $\mathrm{Pu}$ & ${ }^{239} \mathrm{Pu}$ & 1 & 45 & 54 & 1 & & & & & \\
\hline HEU with shielding & ${ }^{235} \mathrm{U}$ & 1 & 0 & 80 & 20 & & & & & \\
\hline Virgin HEU with shielding & ${ }^{235} U$ & 1 & 1 & 91 & 8 & & & & & \\
\hline Reactor Pu with shielding & ${ }^{239} \mathrm{Pu}$ & 1 & 3 & 81 & 16 & & & & & \\
\hline Weapon Pu with shielding & ${ }^{239} \mathrm{Pu}$ & 1 & 6 & 90 & 4 & & & & & \\
\hline
\end{tabular}




\begin{tabular}{|c|c|c|c|c|c|c|c|c|c|c|}
\hline \multicolumn{11}{|l|}{200 Counts/400 Bkg } \\
\hline Sample & Label & Class & 1 & 2 & 3 & & & & & \\
\hline $99 \mathrm{TcM}-625 \mathrm{uc}+200 \mathrm{~mm}-\mathrm{H} 2 \mathrm{O}+5 \mathrm{mmFe}$ & ${ }^{99 \mathrm{~m}} \mathrm{Tc}$ & 3 & 0 & 0 & 100 & \multirow{5}{*}{ actual } & \multicolumn{3}{|c|}{ reported } & \multirow[b]{2}{*}{ total } \\
\hline $131 \mathrm{l}-370 \mathrm{uc}+200 \mathrm{~mm}-\mathrm{H} 2 \mathrm{O}+5 \mathrm{mmFe}$ & 131 & 2 & 15 & 85 & 0 & & 1 & 2 & & \\
\hline $201 \mathrm{Tl}-250 \mathrm{uc}+200 \mathrm{~mm}-\mathrm{H} 2 \mathrm{O}+5 \mathrm{mmFe}$ & ${ }^{201} \mathrm{~T}$ & 2 & 0 & 100 & 0 & & 0.478 & 0.412 & 0.111 & 1200 \\
\hline $67 \mathrm{Ga}-350 \mathrm{uc}+200 \mathrm{~mm}-\mathrm{H} 2 \mathrm{O}+5 \mathrm{mmFe}$ & ${ }^{67} \mathrm{G}$ & 2 & 1 & 99 & 0 & & 0.040 & 0.960 & 0.000 & 400 \\
\hline $137 \mathrm{Cs}-8 \mathrm{mc}+24 \mathrm{~mm}-\mathrm{Lead}+5 \mathrm{mmFe}$ & ${ }^{137} \mathrm{Cs}$ & 2 & 0 & 100 & 0 & & 0.003 & 0.210 & 0.788 & 400 \\
\hline 14Kg-of-Bananas $+5 \mathrm{mmFe}$ & ${ }^{40} \mathrm{~K}$ & 3 & 0 & 13 & 87 & & & & & \\
\hline $5 \mathrm{Kg}$-of-Wood $+5 \mathrm{mmFe}$ & ${ }^{40} \mathrm{~K}$ & 3 & 0 & 16 & 84 & promotion & & 0.25 & 0.08 & \\
\hline $5 \mathrm{Kg}$-of-Potash-Fertilizer $+5 \mathrm{mmFe}$ & ${ }^{40} \mathrm{~K}$ & 3 & 1 & 55 & 44 & demotion & & 0.52 & 0.17 & \\
\hline HEU & ${ }^{235} \mathrm{U}$ & 1 & 90 & 10 & 0 & & & & & \\
\hline Virgin HEU & ${ }^{235} \mathrm{U}$ & 1 & 89 & 11 & 0 & & & & & \\
\hline Reactor $\mathrm{Pu}$ & ${ }^{239} \mathrm{Pl}$ & 1 & 35 & 55 & 10 & & & & & \\
\hline Weapon Pu & ${ }^{239} \mathrm{Pl}$ & 1 & 77 & 23 & 0 & & & & & \\
\hline HEU with shielding & ${ }^{235} \mathrm{U}$ & 1 & 0 & 72 & 28 & & & & & \\
\hline Virgin HEU with shielding & ${ }^{235} U$ & 1 & 0 & 94 & 6 & & & & & \\
\hline Reactor Pu with shielding & ${ }^{239} \mathrm{Pl}$ & 1 & 0 & 46 & 54 & & & & & \\
\hline Weapon Pu with shielding & ${ }^{239} \mathrm{Pu}$ & 1 & 0 & 84 & 16 & & & & & \\
\hline
\end{tabular}

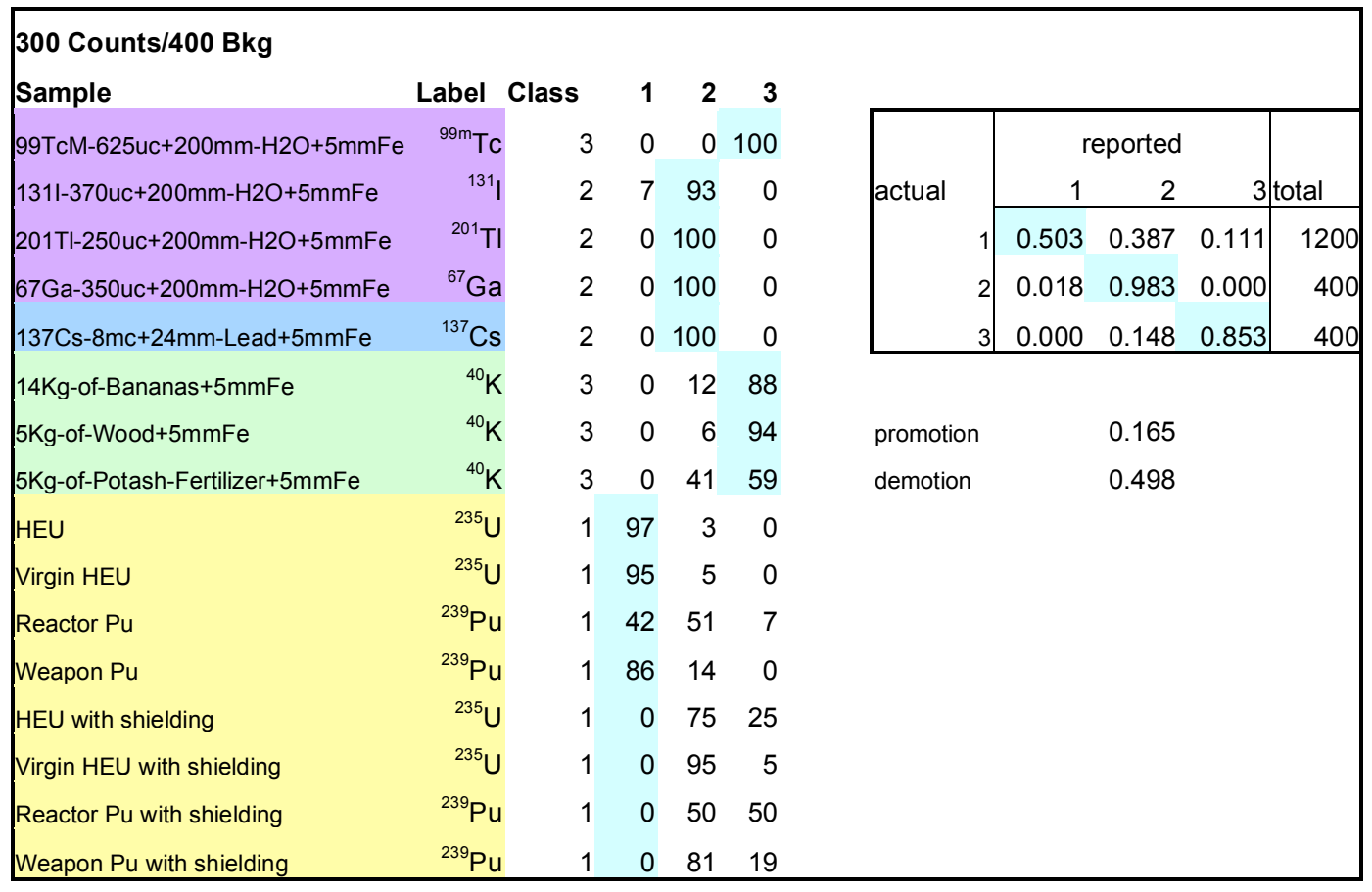




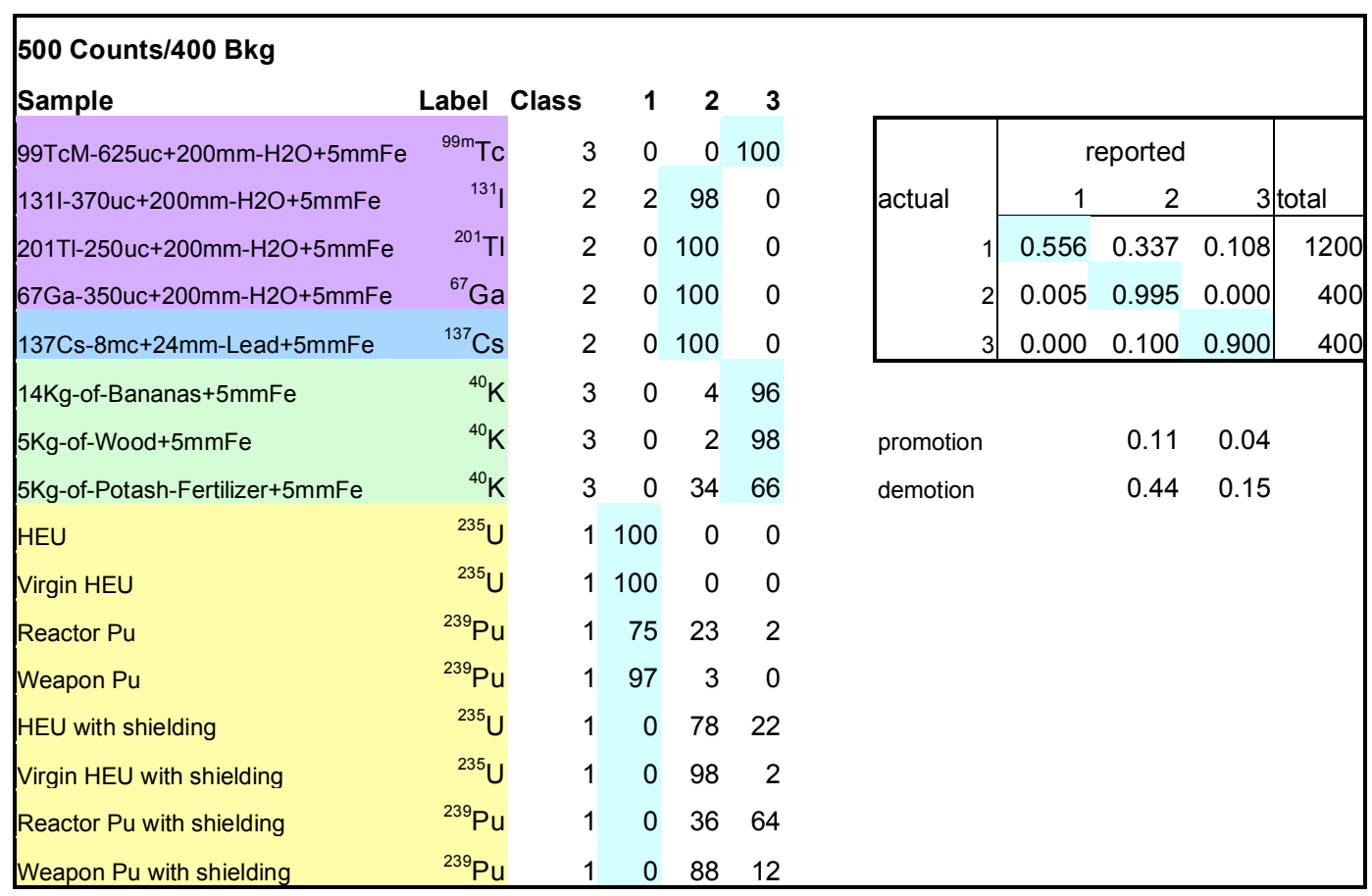

\begin{tabular}{|c|c|c|c|c|c|c|c|c|c|c|}
\hline \multicolumn{11}{|l|}{750 Counts $/ 400$ Bkg } \\
\hline Sample & Label & Class & 1 & 2 & 3 & & & & & \\
\hline 99TcM-625uc+200mm-H2O +5mmFe & ${ }^{99 \mathrm{~m}} \mathrm{Tc}$ & 3 & 0 & 0 & 100 & \multirow{5}{*}{ actual } & \multicolumn{3}{|c|}{ reported } & \multirow[b]{2}{*}{ total } \\
\hline 1311-370uc $+200 \mathrm{~mm}-\mathrm{H} 2 \mathrm{O}+5 \mathrm{mmFe}$ & ${ }^{131} \mid$ & 2 & 0 & 100 & 0 & & 1 & 2 & 3 & \\
\hline $201 \mathrm{Tl}-250 \mathrm{uc}+200 \mathrm{~mm}-\mathrm{H} 2 \mathrm{O}+5 \mathrm{mmFe}$ & ${ }^{201} \mathrm{Tl}$ & 2 & 0 & 100 & 0 & & 0.563 & 0.332 & 0.106 & 1200 \\
\hline $67 \mathrm{Ga}-350 \mathrm{uc}+200 \mathrm{~mm}-\mathrm{H} 2 \mathrm{O}+5 \mathrm{mmFe}$ & ${ }^{67} \mathrm{Ga}$ & 2 & 0 & 100 & 0 & & 0.000 & 1.000 & 0.000 & 400 \\
\hline $137 \mathrm{Cs}-8 \mathrm{mc}+24 \mathrm{~mm}-\mathrm{Lead}+5 \mathrm{mmFe}$ & ${ }^{137} \mathrm{Cs}$ & 2 & 0 & 100 & 0 & & 0.000 & 0.078 & 0.923 & 400 \\
\hline $14 \mathrm{Kg}$-of-Bananas $+5 \mathrm{mmFe}$ & ${ }^{40} \mathrm{~K}$ & 3 & 0 & 1 & 99 & & & & & \\
\hline $5 \mathrm{Kg}$-of-Wood $+5 \mathrm{mmFe}$ & ${ }^{40} \mathrm{~K}$ & 3 & 0 & 0 & 100 & promotion & & 0.08 & 0.03 & \\
\hline $5 \mathrm{Kg}$-of-Potash-Fertilizer+5mmFe & ${ }^{40} \mathrm{~K}$ & 3 & 0 & 30 & 70 & demotion & & 0.44 & 0.15 & \\
\hline HEU & ${ }^{235} U$ & 1 & 100 & 0 & 0 & & & & & \\
\hline Virgin HEU & ${ }^{235} U$ & 1 & 100 & 0 & 0 & & & & & \\
\hline Reactor Pu & ${ }^{239} \mathrm{Pu}$ & 1 & 76 & 23 & 1 & & & & & \\
\hline Weapon $\mathrm{Pu}$ & ${ }^{239} \mathrm{Pu}$ & 1 & 100 & 0 & 0 & & & & & \\
\hline HEU with shielding & ${ }^{235} U$ & 1 & 0 & 65 & 35 & & & & & \\
\hline Virgin HEU with shielding & ${ }^{235} \mathrm{U}$ & 1 & 0 & 100 & 0 & & & & & \\
\hline Reactor Pu with shielding & ${ }^{239} \mathrm{Pu}$ & 1 & 0 & 37 & 63 & & & & & \\
\hline Weapon Pu with shielding & ${ }^{239} \mathrm{Pu}$ & 1 & 0 & 88 & 12 & & & & & \\
\hline
\end{tabular}




\begin{tabular}{|c|c|c|c|c|c|c|c|c|c|c|c|}
\hline \multicolumn{12}{|l|}{1000 Counts/400 Bkg } \\
\hline Sample & Label & Class & 1 & 2 & 3 & & & & & & \\
\hline $99 \mathrm{TcM}-625 \mathrm{uc}+200 \mathrm{~mm}-\mathrm{H} 2 \mathrm{O}+5 \mathrm{mmFe}$ & ${ }^{99 \mathrm{~m}} \mathrm{Tc}$ & 3 & 0 & 0 & 100 & \multirow{5}{*}{ actual } & & \multicolumn{3}{|c|}{ reported } & \multirow[b]{2}{*}{ total } \\
\hline $131 \mathrm{I}-370 \mathrm{uc}+200 \mathrm{~mm}-\mathrm{H} 2 \mathrm{O}+5 \mathrm{mmFe}$ & ${ }^{131}$ & 2 & 0 & 100 & 0 & & & 1 & 2 & & \\
\hline $201 \mathrm{TI}-250 \mathrm{uc}+200 \mathrm{~mm}-\mathrm{H} 2 \mathrm{O}+5 \mathrm{mmFe}$ & ${ }^{201} \mathrm{TI}$ & 2 & 0 & 100 & 0 & & 1 & 0.569 & 0.313 & 0.118 & 1200 \\
\hline $67 \mathrm{Ga}-350 \mathrm{uc}+200 \mathrm{~mm}-\mathrm{H} 2 \mathrm{O}+5 \mathrm{mmFe}$ & ${ }^{67} \mathrm{Ga}$ & 2 & 0 & 100 & 0 & & 2 & 0.000 & 1.000 & 0.000 & 400 \\
\hline $137 \mathrm{Cs}-8 \mathrm{mc}+24 \mathrm{~mm}-\mathrm{Lead}+5 \mathrm{mmFe}$ & ${ }^{137} \mathrm{Cs}$ & 2 & 0 & 100 & 0 & & 3 & 0.000 & 0.080 & 0.920 & 400 \\
\hline 14Kg-of-Bananas $+5 \mathrm{mmFe}$ & ${ }^{40} \mathrm{~K}$ & 3 & 0 & 1 & 99 & & & & & & \\
\hline $5 \mathrm{Kg}$-of-Wood $+5 \mathrm{mmFe}$ & ${ }^{40} \mathrm{~K}$ & 3 & 0 & 1 & 99 & promotion & & & 0.08 & 0.03 & \\
\hline $5 \mathrm{Kg}$-of-Potash-Fertilizer $+5 \mathrm{mmFe}$ & ${ }^{40} \mathrm{~K}$ & 3 & 0 & 30 & 70 & demotion & & & 0.43 & 0.14 & \\
\hline HEU & ${ }^{235} U$ & 1 & 99 & 0 & 1 & & & & & & \\
\hline Virgin HEU & ${ }^{235} U$ & 1 & 98 & 0 & 2 & & & & & & \\
\hline Reactor Pu & ${ }^{239} \mathrm{Pu}$ & 1 & 87 & 13 & 0 & & & & & & \\
\hline Weapon Pu & ${ }^{239} \mathrm{Pu}$ & 1 & 100 & 0 & 0 & & & & & & \\
\hline HEU with shielding & ${ }^{235} U$ & 1 & 0 & 56 & 44 & & & & & & \\
\hline Virgin HEU with shielding & ${ }^{235} U$ & 1 & 0 & 100 & 0 & & & & & & \\
\hline Reactor Pu with shielding & ${ }^{239} \mathrm{Pu}$ & 1 & 0 & 33 & 67 & & & & & & \\
\hline Weapon Pu with shielding & ${ }^{239} \mathrm{Pu}$ & 1 & 0 & 95 & 5 & & & & & & \\
\hline
\end{tabular}

\section{A.4.3.3 ML Group Correlation Matrix}

\section{Column Definitions}

- Sample - Sample name of input.

- Label - Expert label.

- ND - number of trials where "no detection" was declared.

- UNK - number of trials where "unknown" was declared

- Sum - total number of isotopic labels assigned to samples. 


\section{A.4.3.3.1 Normal Result}

\begin{tabular}{|c|c|c|c|c|c|c|c|c|c|c|c|c|c|c|c|c|c|c|c|c|c|c|c|c|c|c|c|c|c|}
\hline Sample & Label & ND & UNK & $\begin{array}{l}40 \\
\mathrm{~K} \\
\end{array}$ & $\begin{array}{r}226 \\
\mathrm{Ra} \\
\end{array}$ & $\begin{array}{c}232 \\
\text { Th } \\
\end{array}$ & $\begin{array}{c}228 \\
\text { Th } \\
\end{array}$ & $\begin{array}{c}232 \\
\mathrm{U} \\
\end{array}$ & $\begin{array}{l}60 \\
\text { Co } \\
\end{array}$ & $\begin{array}{l}133 \\
\mathrm{Ba} \\
\end{array}$ & $\begin{array}{l}137 \\
\text { Cs } \\
\end{array}$ & $\begin{array}{c}54 \\
\mathrm{Mn} \\
\end{array}$ & $\begin{array}{l}57 \\
\text { Co } \\
\end{array}$ & $\begin{array}{c}139 \\
\mathrm{Ce} \\
\end{array}$ & $\begin{array}{r}252 \\
\mathrm{Cf} \\
\end{array}$ & $\begin{array}{c}238 \\
\mathrm{U}\end{array}$ & $\begin{array}{c}235 \\
\mathrm{U}\end{array}$ & $\begin{array}{l}239 \\
\mathrm{Pu} \\
\end{array}$ & $\begin{array}{l}237 \\
\mathrm{~Np} \\
\end{array}$ & $\begin{array}{c}131 \\
1 \\
\end{array}$ & $\begin{array}{c}99^{\mathrm{m}} \\
\text { Tc } \\
\end{array}$ & $\begin{array}{l}67 \\
\mathrm{Ga} \\
\end{array}$ & $\begin{array}{c}201 \\
\text { TI } \\
\end{array}$ & $\begin{array}{c}88 \\
\mathrm{Y} \\
\end{array}$ & $\begin{array}{l}22 \\
\mathrm{Na} \\
\end{array}$ & $\begin{array}{c}166^{\mathrm{m}} \\
\mathrm{Ho}\end{array}$ & $\begin{array}{c}152 \\
\mathrm{Eu} \\
\end{array}$ & $\begin{array}{c}192 \\
\text { Ir } \\
\end{array}$ & Sum \\
\hline \multicolumn{30}{|l|}{50 Counts/400 Bkg } \\
\hline $99 \mathrm{TcM}-625 \mathrm{uc}+200 \mathrm{~mm}-\mathrm{H} 2 \mathrm{O}+5 \mathrm{mmFe}$ & ${ }^{99 \mathrm{~m}} \mathrm{Tc}$ & 0 & 2 & 0 & 0 & 0 & 0 & 0 & 0 & 0 & 0 & 0 & 14 & 1 & 0 & 0 & 1 & 0 & 0 & 0 & 74 & 1 & 7 & 0 & 0 & 0 & 0 & 0 & 0 \\
\hline 1311-370uc+200mm-H2O+5mmFe & ${ }^{131}$ & 0 & 1 & 0 & 0 & 0 & 0 & 0 & 0 & 16 & 3 & 1 & 2 & 5 & 0 & 0 & 4 & 12 & 3 & 24 & 6 & 5 & 5 & 0 & 3 & 1 & 0 & 9 & \\
\hline $201 \mathrm{Tl}-250 \mathrm{uc}+200 \mathrm{~mm}-\mathrm{H} 2 \mathrm{O}+5 \mathrm{mmFe}$ & ${ }^{201} \mathrm{TI}$ & 0 & 0 & 0 & 0 & 0 & 0 & 0 & 0 & 0 & 0 & 0 & 3 & 17 & 0 & 0 & 7 & 0 & 1 & 0 & 17 & 2 & 53 & 0 & 0 & 0 & 0 & 0 & \\
\hline $67 \mathrm{Ga}-350 \mathrm{uc}+200 \mathrm{~mm}-\mathrm{H} 2 \mathrm{O}+5 \mathrm{mmFe}$ & ${ }^{67} \mathrm{Ga}$ & 0 & 0 & 1 & 0 & 0 & 0 & 0 & 0 & 11 & 5 & 0 & 2 & 10 & 0 & 0 & 11 & 4 & 7 & 4 & 6 & 20 & 11 & 0 & 0 & 1 & 0 & 7 & U \\
\hline $137 \mathrm{Cs}-8 \mathrm{mc}+24 \mathrm{~mm}-\mathrm{Lead}+5 \mathrm{mmFe}$ & ${ }^{137} \mathrm{Cs}$ & 0 & 0 & 0 & 0 & 0 & 0 & 0 & 0 & 1 & 69 & 1 & 1 & 2 & 0 & 0 & 4 & 5 & 0 & 0 & 0 & 0 & 3 & 0 & 3 & 5 & 0 & 6 & 0 \\
\hline $14 \mathrm{Kg}$-of-Bananas $+5 \mathrm{mmFe}$ & ${ }^{40} \mathrm{~K}$ & 0 & 2 & 11 & 0 & 0 & 0 & 0 & 3 & 2 & 17 & 5 & 1 & 6 & 0 & 1 & 4 & 9 & 1 & 6 & 4 & 1 & 6 & 3 & 6 & 3 & 3 & 6 & 0 \\
\hline $5 \mathrm{Kg}$-of-Wood+5mmFe & ${ }^{40} \mathrm{~K}$ & 0 & 2 & 12 & 0 & 0 & 0 & 0 & 2 & 2 & 16 & 6 & 1 & 5 & 0 & 1 & 3 & 10 & 1 & 5 & 3 & 1 & 7 & 3 & 8 & 3 & 3 & 6 & \\
\hline $5 \mathrm{Kg}$-of-Potash-Fertilizer $+5 \mathrm{mmFe}$ & ${ }^{40} \mathrm{~K}$ & 0 & 1 & 4 & 0 & 0 & 0 & 0 & 3 & 3 & 18 & 4 & 5 & 5 & 0 & 1 & 9 & 10 & 2 & 5 & 6 & 3 & 4 & 2 & 5 & 4 & 0 & 6 & \\
\hline HEU & ${ }^{235} \mathrm{U}$ & 0 & 0 & 0 & 0 & 0 & 0 & 0 & 0 & 14 & 5 & 1 & 4 & 6 & 0 & 0 & 2 & 15 & 2 & 10 & 7 & 10 & 8 & 0 & 3 & 2 & 0 & 11 & \\
\hline Virgin HEU & ${ }^{235} \mathrm{U}$ & 0 & 1 & 0 & 0 & 0 & 0 & 0 & 0 & 11 & 9 & 2 & 0 & 3 & 0 & 0 & 0 & 37 & 2 & 10 & 4 & 4 & 4 & 1 & 3 & 1 & 0 & 8 & \\
\hline Reactor Pu & ${ }^{239} \mathrm{Pu}$ & 0 & 1 & 1 & 0 & 0 & 0 & 1 & 1 & 2 & 13 & 2 & 3 & 10 & 0 & 0 & 12 & 9 & 2 & 4 & 6 & 3 & 8 & 4 & 8 & 7 & 0 & 3 & \\
\hline Weapon Pu & ${ }^{239} \mathrm{Pu}$ & 0 & 0 & 1 & 0 & 0 & 0 & 0 & 0 & 2 & 1 & 0 & 0 & 5 & 0 & 0 & 72 & 1 & 0 & 1 & 3 & 3 & 9 & 0 & 0 & 1 & 0 & 1 & \\
\hline HEU with shielding & ${ }^{235} \mathrm{U}$ & 0 & 1 & 2 & 0 & 0 & 0 & 0 & 0 & 2 & 3 & 0 & 11 & 10 & 0 & 0 & 40 & 2 & 0 & 1 & 9 & 3 & 15 & 0 & 0 & 1 & 0 & 0 & \\
\hline Virgin HEU with shielding & ${ }^{235} \mathrm{U}$ & 0 & 1 & 1 & 0 & 0 & 0 & 0 & 0 & 3 & 1 & 0 & 6 & 9 & 0 & 0 & 48 & 2 & 0 & 1 & 13 & 3 & 11 & 0 & 0 & 0 & 0 & 1 & \\
\hline Reactor Pu with shielding & ${ }^{239} \mathrm{Pu}$ & 0 & 0 & 1 & 0 & 0 & 0 & 0 & 0 & 8 & 9 & 3 & 19 & 5 & 0 & 0 & 8 & 13 & 0 & 4 & 9 & 3 & 9 & 1 & 2 & 0 & 0 & 6 & \\
\hline Weapon Pu with shielding & ${ }^{239} \mathrm{Pu}$ & 0 & 0 & 1 & 0 & 0 & 0 & 0 & 0 & 12 & 6 & 2 & 10 & 7 & 0 & 0 & 4 & 18 & 2 & 9 & 4 & 5 & 6 & 0 & 3 & 1 & 0 & 10 & \\
\hline \multicolumn{30}{|l|}{100 Counts/400 Bkg } \\
\hline 99TcM- $625 \mathrm{uc}+200 \mathrm{~mm}-\mathrm{H} 2 \mathrm{O}+5 \mathrm{mmFe}$ & ${ }^{99 \mathrm{~m}} \mathrm{Tc}$ & 0 & 1 & 0 & 0 & 0 & 0 & 0 & 0 & 0 & 0 & 0 & 4 & 0 & 0 & 0 & 0 & 0 & 0 & 0 & 95 & 0 & 0 & 0 & 0 & 0 & 0 & 0 & \\
\hline $1311-370 \mathrm{uc}+200 \mathrm{~mm}-\mathrm{H} 2 \mathrm{O}+5 \mathrm{mmFe}$ & ${ }^{131}$ & 0 & 0 & 0 & 0 & 0 & 0 & 0 & 0 & 44 & 3 & 0 & 0 & 0 & 0 & 0 & 1 & 13 & 0 & 30 & 0 & 3 & 0 & 0 & 1 & 2 & 0 & 3 & \\
\hline $201 \mathrm{TI}-250 \mathrm{uc}+200 \mathrm{~mm}-\mathrm{H} 2 \mathrm{O}+5 \mathrm{mmFe}$ & ${ }^{201} \mathrm{TI}$ & 0 & 2 & 0 & 0 & 0 & 0 & 0 & 0 & 1 & 0 & 0 & 1 & 11 & 0 & 0 & 0 & 0 & 0 & 0 & 7 & 0 & 78 & 0 & 0 & 0 & 0 & 0 & \\
\hline $67 \mathrm{Ga}-350 \mathrm{uc}+200 \mathrm{~mm}-\mathrm{H} 2 \mathrm{O}+5 \mathrm{mmFe}$ & ${ }^{67} \mathrm{Ga}$ & 0 & 0 & 1 & 0 & 0 & 0 & 0 & 0 & 9 & 1 & 0 & 0 & 0 & 0 & 0 & 10 & 2 & 5 & 2 & 2 & 58 & 2 & 0 & 0 & 1 & 0 & $r$ & \\
\hline $137 \mathrm{Cs}-8 \mathrm{mc}+24 \mathrm{~mm}-\mathrm{Lead}+5 \mathrm{mmFe}$ & ${ }^{137} \mathrm{Cs}$ & 0 & 0 & 0 & 0 & 0 & 0 & 0 & 0 & 0 & 96 & 0 & 0 & 0 & 0 & 0 & 0 & 0 & 0 & 0 & 0 & 0 & 0 & 0 & 0 & 2 & 0 & 2 & \\
\hline 14Kg-of-Bananas $+5 \mathrm{mmFe}$ & ${ }^{40} \mathrm{~K}$ & 0 & 1 & 43 & 0 & 1 & 0 & 0 & 5 & 0 & 5 & 2 & 0 & 0 & 1 & 7 & 1 & 4 & 0 & 1 & 0 & 1 & 0 & 2 & 6 & 4 & 11 & 5 & \\
\hline $5 \mathrm{Kg}$-of-Wood+5mmFe & ${ }^{40} \mathrm{~K}$ & 0 & 1 & 46 & 0 & 1 & 0 & 0 & 2 & 0 & 7 & 2 & 0 & 0 & 1 & 7 & 2 & 5 & 0 & 1 & 1 & 0 & 0 & 2 & 4 & 1 & 12 & 5 & \\
\hline $5 \mathrm{Kg}$-of-Potash-Fertilizer $+5 \mathrm{mmFe}$ & ${ }^{40} \mathrm{~K}$ & 0 & 0 & 11 & 0 & 0 & 0 & 0 & 0 & 4 & 9 & 1 & 0 & 6 & 2 & 2 & 6 & 11 & 1 & 2 & 4 & 8 & 3 & 1 & 6 & 7 & 3 & 13 & \\
\hline HEU & ${ }^{235} \mathrm{U}$ & 0 & 0 & 0 & 0 & 0 & 0 & 0 & 0 & 12 & 5 & 1 & 1 & 0 & 0 & 0 & 2 & 33 & 2 & 12 & 2 & 17 & 1 & 0 & 1 & 1 & 0 & 10 & \\
\hline
\end{tabular}




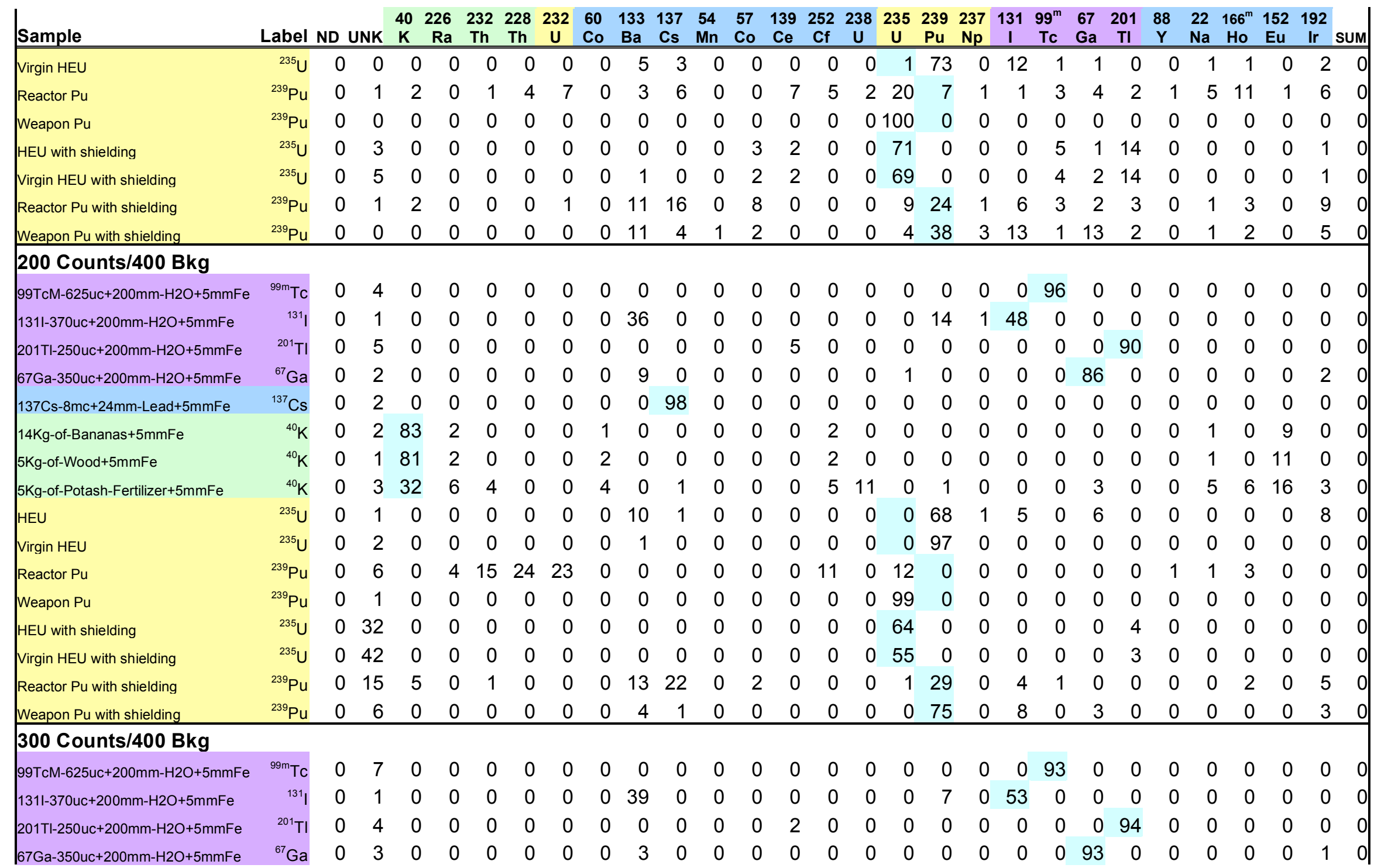




\begin{tabular}{|c|c|c|c|c|c|c|c|c|c|c|c|c|c|c|c|c|c|c|c|c|c|c|c|c|c|c|c|c|c|c|}
\hline Sample & Label & ND U & & $\begin{array}{cc}402 \\
\mathrm{~K} \\
\end{array}$ & $\begin{array}{r}226 \\
\mathrm{Ra} \\
\end{array}$ & $\begin{array}{c}232 \\
\text { Th } \\
\end{array}$ & $\begin{array}{c}228 \\
\mathrm{Th} \\
\end{array}$ & $\begin{array}{c}232 \\
U \\
\end{array}$ & $\begin{array}{l}60 \\
\text { Co } \\
\end{array}$ & $\begin{array}{l}133 \\
\mathrm{Ba} \\
\end{array}$ & $\begin{array}{l}137 \\
\text { Cs } \\
\end{array}$ & $\begin{array}{l}54 \\
\mathrm{Mn} \\
\end{array}$ & $\begin{array}{l}57 \\
\text { Co } \\
\end{array}$ & & $\begin{array}{r}25 \\
\mathrm{C} \\
\end{array}$ & & & $\begin{array}{cc}2352 \\
\mathrm{U} \\
\end{array}$ & $\begin{array}{l}239 \\
\mathrm{Pu} \\
\end{array}$ & $\begin{array}{l}237 \\
\mathrm{~Np} \\
\end{array}$ & $\begin{array}{c}1319 \\
1 \quad 1 \\
\end{array}$ & $\begin{array}{l}99^{\mathrm{m}} \\
\text { Tc } \\
\end{array}$ & $\begin{array}{l}67 \\
\mathrm{Ga} \\
\end{array}$ & $\begin{array}{c}201 \\
\text { TI } \\
\end{array}$ & $\begin{array}{c}88 \\
\mathrm{Y} \\
\end{array}$ & $\begin{array}{l}22 \\
\mathrm{Na} \\
\end{array}$ & $\begin{array}{c}166^{\mathrm{m}} \\
\mathrm{Ho} \\
\end{array}$ & $\begin{array}{c}152 \\
\mathrm{Eu} \\
\end{array}$ & & SuM \\
\hline $137 \mathrm{Cs}-8 \mathrm{mc}+24 \mathrm{~mm}$-Lead $+5 \mathrm{mmFe}$ & ${ }^{137} \mathrm{Cs}$ & 0 & 0 & 0 & 0 & 0 & 0 & 0 & 0 & & 100 & 0 & 0 & c & 0 & 0 & 0 & 0 & 0 & 0 & 0 & 0 & 0 & 0 & 0 & 0 & 0 & 0 & 0 & \\
\hline 14Kg-of-Bananas+5mmFe & ${ }^{40} \mathrm{~K}$ & 0 & 12 & 76 & 1 & 0 & 0 & 0 & 1 & 0 & 0 & 0 & 0 & c & 0 & 1 & 0 & 0 & 0 & 0 & 0 & 0 & 0 & 0 & 0 & 0 & 0 & 9 & 0 & ) \\
\hline 5Kg-of-Wood+5mmFe & ${ }^{40} \mathrm{~K}$ & 0 & 11 & 82 & 1 & 0 & 0 & 0 & 2 & 0 & 0 & 0 & 0 & c & & 1 & 0 & 0 & 0 & 0 & 0 & 0 & 0 & 0 & 0 & 0 & 0 & 3 & 0 & \\
\hline HEU & ${ }^{235} \mathrm{U}$ & 0 & 3 & 0 & 0 & 0 & 0 & 0 & 0 & 6 & 0 & 0 & 0 & $c$ & 0 & 0 & 0 & 0 & 78 & 0 & 4 & 0 & 4 & 0 & 0 & 0 & 0 & 0 & 5 & 5 \\
\hline Virgin HEU & ${ }^{235} \mathrm{U}$ & 0 & 0 & 0 & 0 & 0 & 0 & 0 & 0 & 0 & 0 & 0 & 0 & c & & 0 & 0 & & 100 & 0 & 0 & 0 & 0 & 0 & 0 & 0 & 0 & 0 & 0 & ) \\
\hline Reactor Pu & ${ }^{239} \mathrm{Pu}$ & 0 & 19 & 0 & 3 & 19 & 17 & 28 & 0 & 0 & 0 & 0 & 0 & c & 1 & 13 & 0 & 1 & 0 & 0 & 0 & 0 & 0 & 0 & 0 & 0 & 0 & 0 & 0 & ) \\
\hline HEU with shielding & ${ }^{235} \mathrm{U}$ & 0 & 74 & 0 & 0 & 0 & 0 & 0 & 0 & 0 & 0 & 0 & 0 & ( & & 0 & 0 & 26 & 0 & 0 & 0 & 0 & 0 & 0 & 0 & 0 & 0 & 0 & 0 & \\
\hline Virgin HEU with shielding & ${ }^{235} \mathrm{U}$ & 0 & 78 & 0 & 0 & 0 & 0 & 0 & 0 & 0 & 0 & 0 & 0 & ( & & 0 & 0 & 22 & 0 & 0 & 0 & 0 & 0 & 0 & 0 & 0 & 0 & 0 & 0 & \\
\hline Reactor Pu with shielding & ${ }^{239} \mathrm{Pu}$ & 0 & 46 & 4 & 0 & 0 & 0 & 0 & 0 & 8 & 16 & 0 & 0 & ( & & 0 & 0 & 0 & 23 & 0 & 1 & 0 & 1 & 0 & 0 & 0 & 0 & 0 & 1 & \\
\hline Weapon Pu with shielding & ${ }^{239} \mathrm{Pu}$ & 0 & 23 & 0 & 0 & 0 & 0 & 0 & 0 & 2 & 0 & 0 & 0 & ( & & 0 & 0 & 0 & 71 & 0 & 4 & 0 & 0 & 0 & 0 & 0 & 0 & 0 & $\underline{0}$ & ) \\
\hline \multicolumn{31}{|l|}{500 Counts $/ 400$ Bkg } \\
\hline $99 \mathrm{TcM}-625 \mathrm{uc}+200 \mathrm{~mm}-\mathrm{H} 2 \mathrm{O}+5 \mathrm{mmFe}$ & ${ }^{99 m} \mathrm{Tc}$ & 0 & 16 & 0 & 0 & 0 & 0 & 0 & 0 & 0 & 0 & 0 & 0 & & & 0 & 0 & 0 & 0 & 0 & 0 & 84 & 0 & 0 & 0 & & 0 & 0 & 0 & \\
\hline $14 \mathrm{Kg}$-of-Bananas $+5 \mathrm{mmFe}$ & ${ }^{40} \mathrm{~K}$ & 0 & 50 & 49 & 0 & 0 & 0 & 0 & 0 & 0 & 0 & 0 & c & & & 0 & 0 & 0 & 0 & 0 & 0 & 0 & 0 & & 0 & & 0 & & 0 & \\
\hline $5 \mathrm{Kg}$-of-Wood +5 & ${ }^{40} \mathrm{~K}$ & 0 & 49 & 51 & 0 & 0 & 0 & 0 & 0 & 0 & 0 & 0 & 0 & ( & & 0 & 0 & 0 & 0 & 0 & 0 & 0 & 0 & & 0 & & 0 & 0 & 0 & \\
\hline 5Kg-of-Potash-Fertilizer+5mmFe & ${ }^{40} \mathrm{~K}$ & 0 & 54 & 22 & 6 & 0 & 0 & 0 & 0 & 0 & 0 & 0 & 0 & ( & & 3 & 0 & 0 & 0 & 0 & 0 & 0 & 0 & 0 & 0 & 0 & 0 & 15 & 0 & ) \\
\hline HEU & ${ }^{235} \mathrm{U}$ & 0 & 7 & 0 & 0 & 0 & 0 & 0 & 0 & 2 & 0 & 0 & 0 & & & 0 & 0 & 0 & 88 & 0 & 3 & 0 & 0 & & 0 & & 0 & 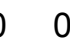 & 0 & ) \\
\hline Virgin HEU & ${ }^{235} \mathrm{U}$ & 0 & 3 & 0 & 0 & 0 & 0 & 0 & 0 & 0 & 0 & 0 & 0 & & & 0 & 0 & 0 & 97 & 0 & 0 & 0 & 0 & & 0 & 0 & 0 & 0 & 0 & ) \\
\hline Reactor Pu & ${ }^{239} \mathrm{Pu}$ & 0 & 56 & 0 & 1 & 11 & 7 & 20 & 0 & 0 & 0 & 0 & 0 & & & 5 & 0 & 0 & 0 & 0 & 0 & 0 & 0 & 0 & 0 & 0 & 0 & 0 & 0 & ) \\
\hline Weapon Pu & ${ }^{239} \mathrm{Pu}$ & 0 & 3 & 0 & 0 & 0 & 0 & 0 & 0 & 0 & 0 & 0 & 0 & & & 0 & 0 & 97 & 0 & 0 & 0 & 0 & 0 & 0 & 0 & 0 & 0 & ) & 0 & ) \\
\hline HEU with shie & ${ }^{235} \mathrm{U}$ & & 100 & 0 & 0 & 0 & 0 & 0 & 0 & 0 & 0 & 0 & 0 & & & 0 & 0 & 0 & 0 & 0 & 0 & 0 & 0 & & 0 & $U$ & 0 & 0 & 0 & ) \\
\hline Virgin HEU with s & ${ }^{235} \mathrm{U}$ & & 100 & 0 & 0 & 0 & 0 & 0 & 0 & 0 & 0 & 0 & 0 & & & 0 & 0 & 0 & 0 & 0 & 0 & 0 & 0 & 0 & 0 & 0 & 0 & ) & 0 & ) \\
\hline Reactor Pu wit & ${ }^{239} \mathrm{Pu}$ & 0 & 97 & 0 & 0 & 0 & 0 & 0 & 0 & 0 & 0 & 0 & 0 & & & 0 & 0 & 0 & 3 & 0 & 0 & 0 & 0 & 0 & 0 & 0 & 0 & 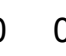 & 0 & ) \\
\hline eapon Pu with shielding & ${ }^{239} \mathrm{Pu}$ & 0 & 62 & 0 & 0 & 0 & 0 & 0 & 0 & 0 & 0 & 0 & 0 & & 0 & 0 & 0 & 0 & 38 & 0 & 0 & 0 & 0 & 0 & 0 & 0 & 0 & 0 & 0 & \\
\hline
\end{tabular}




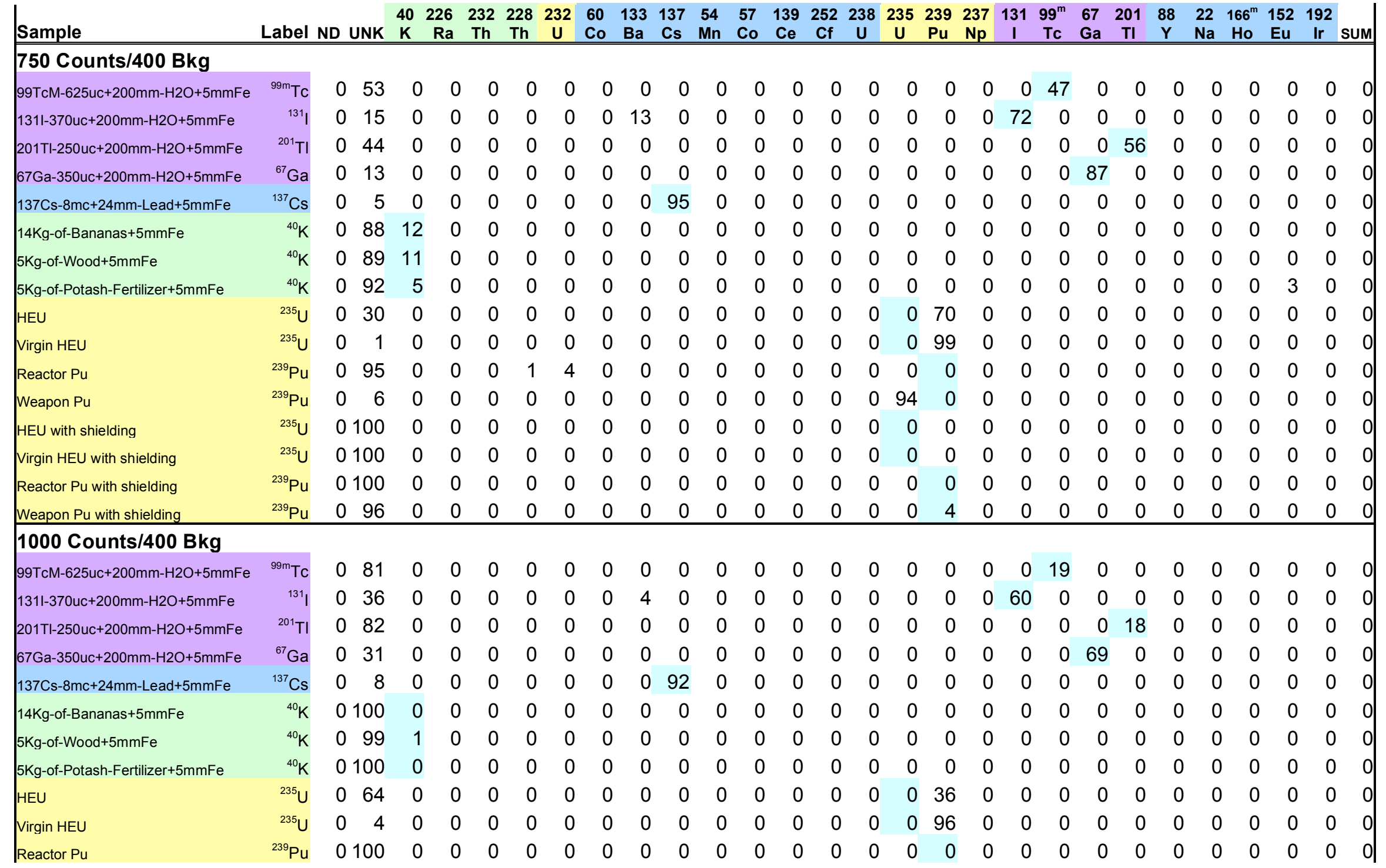




\begin{tabular}{|c|c|c|c|c|c|c|c|c|c|c|c|c|c|c|c|c|c|c|c|c|c|c|c|c|c|c|c|}
\hline Sample & Label & ID UNK & $\begin{array}{l}40 \\
\mathrm{~K} \\
\end{array}$ & $\begin{array}{r}226 \\
\mathrm{Ra} \\
\end{array}$ & $\begin{array}{c}232 \\
\text { Th } \\
\end{array}$ & $\begin{array}{l}228 \\
\text { Th } \\
\end{array}$ & $\begin{array}{c}232 \\
\mathrm{U}\end{array}$ & $\begin{array}{l}60 \\
\text { Co } \\
\end{array}$ & $\begin{array}{l}133 \\
\mathrm{Ba} \\
\end{array}$ & $\begin{array}{l}137 \\
\text { Cs }\end{array}$ & $\begin{array}{l}54 \\
\mathrm{Mn} \\
\end{array}$ & $\begin{array}{l}57 \\
\text { Co } \\
\end{array}$ & $\begin{array}{l}139 \\
\mathrm{Ce} \\
\end{array}$ & $\begin{array}{c}252 \\
\mathrm{Cf} \\
\end{array}$ & $\begin{array}{c}238 \\
\mathrm{U}\end{array}$ & $\begin{array}{c}235 \\
\mathrm{U} \\
\end{array}$ & $\begin{array}{cc}239 & 2 \\
\mathrm{Pu} & \mathrm{N} \\
\end{array}$ & $\begin{array}{l}237 \\
\mathrm{~Np} \\
\end{array}$ & $\begin{array}{rr}131 & 9 \\
\mathrm{I} & \mathrm{T} \\
\end{array}$ & $\begin{array}{l}99^{\mathrm{m}} \\
\text { Tc } \\
\end{array}$ & $\begin{array}{ll}67 & 2 \\
\mathrm{Ga} & \\
\end{array}$ & $\begin{array}{l}201 \\
\text { TI } \\
\end{array}$ & $\begin{array}{c}88 \\
Y \\
\end{array}$ & $\begin{array}{ll}22 & 1 \\
\mathrm{Na} & \mathrm{H} \\
\end{array}$ & $\begin{array}{ll}166^{\mathrm{m}} & 1 \\
\text { Ho } & \mathrm{E} \\
\end{array}$ & $\begin{array}{ll}152 & 1 \\
\text { Eu } & \\
\end{array}$ & $\begin{array}{l}192 \\
\text { Ir SUM }\end{array}$ \\
\hline Weapon Pu & ${ }^{239} \mathrm{Pu}$ & 013 & 0 & 0 & 0 & 0 & 0 & 0 & 0 & 0 & 0 & 0 & 0 & 0 & 0 & 87 & 0 & 0 & 0 & 0 & 0 & 0 & 0 & 0 & 0 & 0 & 0 \\
\hline HEU with shielding & ${ }^{235} \mathrm{U}$ & 0100 & 0 & 0 & 0 & 0 & 0 & 0 & 0 & 0 & 0 & 0 & 0 & 0 & 0 & 0 & 0 & 0 & 0 & 0 & 0 & 0 & 0 & 0 & 0 & 0 & 0 \\
\hline Virgin HEU with sh & ${ }^{235} \mathrm{U}$ & 0100 & 0 & 0 & 0 & 0 & 0 & 0 & 0 & 0 & 0 & 0 & 0 & 0 & 0 & 0 & 0 & 0 & 0 & 0 & 0 & 0 & 0 & 0 & 0 & 0 & 0 \\
\hline leapon Pu with shielding & ${ }^{239} \mathrm{Pu}$ & 0100 & 0 & 0 & 0 & 0 & 0 & 0 & 0 & 0 & 0 & 0 & 0 & 0 & 0 & 0 & 0 & 0 & 0 & 0 & 0 & 0 & 0 & 0 & 0 & 0 & 0 \\
\hline
\end{tabular}

\section{A.4.3.3.2 “Best” Match Result}

\begin{tabular}{|c|c|c|c|c|c|c|c|c|c|c|c|c|c|c|c|c|c|c|c|c|c|c|c|c|c|c|c|c|c|c|}
\hline Sample & Label & ND & UNK & $\begin{array}{l}40 \\
\mathrm{~K}\end{array}$ & $\begin{array}{r}226 \\
\mathrm{Ra} \\
\end{array}$ & $\begin{array}{c}232 \\
\text { Th } \\
\end{array}$ & $\begin{array}{c}228 \\
\text { Th } \\
\end{array}$ & $\begin{array}{c}232 \\
\mathrm{U} \\
\end{array}$ & $\begin{array}{l}60 \\
\text { Co } \\
\end{array}$ & $\begin{array}{c}133 \\
\mathrm{Ba} \\
\end{array}$ & $\begin{array}{l}137 \\
\text { Cs } \\
\end{array}$ & $\begin{array}{c}54 \\
\mathrm{Mn} \\
\end{array}$ & $\begin{array}{l}57 \\
\text { Co } \\
\end{array}$ & $\begin{array}{c}139 \\
\mathrm{Ce} \\
\end{array}$ & $\begin{array}{r}252 \\
\mathrm{Cf} \\
\end{array}$ & $\begin{array}{c}238 \\
\mathrm{U} \\
\end{array}$ & $\begin{array}{c}235 \\
\mathrm{U}\end{array}$ & $\begin{array}{c}239 \\
\mathrm{Pu}\end{array}$ & $\begin{array}{l}237 \\
\mathrm{~Np} \\
\end{array}$ & $\begin{array}{c}131 \\
1\end{array}$ & $\begin{array}{c}99^{\mathrm{m}} \\
\text { TC }\end{array}$ & $\begin{array}{l}67 \\
\mathrm{Ga}\end{array}$ & $\begin{array}{c}201 \\
\text { TI }\end{array}$ & $\begin{array}{l}88 \\
Y\end{array}$ & $\begin{array}{l}22 \\
\mathrm{Na}\end{array}$ & $\begin{array}{c}166^{\mathrm{m}} \\
\mathrm{Ho}\end{array}$ & $\begin{array}{c}152 \\
\mathrm{Eu}\end{array}$ & & & sum \\
\hline \multicolumn{31}{|l|}{50 Counts/400 Bkg } \\
\hline $99 \mathrm{TcM}-625 \mathrm{uc}+200 \mathrm{~mm}-\mathrm{H} 2 \mathrm{O}+5 \mathrm{mmFe}$ & ${ }^{99 \mathrm{~m}} \mathrm{Tc}$ & 0 & 0 & 0 & 0 & 0 & 0 & 0 & 0 & 0 & 0 & 0 & 14 & 1 & 0 & 0 & 1 & 0 & 0 & 0 & 76 & 1 & 7 & 0 & 0 & 0 & 0 & 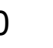 & 0 & 0 \\
\hline $1311-370 u c+200 m m-H 2 O+5 m m F e$ & ${ }^{131}$ & 0 & 0 & 0 & 0 & 0 & 0 & 0 & 0 & 16 & 3 & 1 & 2 & 5 & 0 & 0 & 4 & 12 & 3 & 24 & 7 & 5 & 5 & 0 & 3 & 1 & 0 & 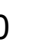 & 9 & 0 \\
\hline $201 \mathrm{TI}-250 \mathrm{uc}+200 \mathrm{~mm}-\mathrm{H} 2 \mathrm{O}+5 \mathrm{mmFe}$ & ${ }^{201} \mathrm{TI}$ & 0 & 0 & 0 & 0 & 0 & 0 & 0 & 0 & 0 & 0 & 0 & 3 & 17 & 0 & 0 & 7 & 0 & 1 & 0 & 17 & 2 & 53 & 0 & 0 & 0 & 0 & b & 0 & 0 \\
\hline $67 \mathrm{Ga}-350 \mathrm{uc}+200 \mathrm{~mm}-\mathrm{H} 2 \mathrm{O}+5 \mathrm{mmFe}$ & ${ }^{67} \mathrm{Ga}$ & 0 & 0 & 1 & 0 & 0 & 0 & 0 & 0 & 11 & 5 & 0 & 2 & 10 & 0 & 0 & 11 & 4 & 7 & 4 & 6 & 20 & 11 & 0 & 0 & 1 & 0 & b & 7 & 0 \\
\hline $137 \mathrm{Cs}-8 \mathrm{mc}+24 \mathrm{~mm}-\mathrm{Lead}+5 \mathrm{mmFe}$ & ${ }^{137} \mathrm{Cs}$ & 0 & 0 & 0 & 0 & 0 & 0 & 0 & 0 & 1 & 69 & 1 & 1 & 2 & 0 & 0 & 4 & 5 & 0 & 0 & 0 & 0 & 3 & 0 & 3 & 5 & 0 & כ & 6 & 0 \\
\hline 14Kg-of-Bananas $+5 \mathrm{mmFe}$ & ${ }^{40} \mathrm{~K}$ & 0 & 0 & 11 & 0 & 0 & 0 & 0 & 4 & 2 & 17 & 5 & 1 & 6 & 0 & 1 & 4 & 9 & 1 & 6 & 4 & 1 & 6 & 3 & 7 & 3 & 3 & 3 & 6 & 0 \\
\hline $5 \mathrm{Kg}$-of-Wood $+5 \mathrm{mmFe}$ & ${ }^{40} \mathrm{~K}$ & 0 & 0 & 12 & 0 & 0 & 0 & 0 & 3 & 2 & 16 & 6 & 1 & 5 & 0 & 1 & 3 & 10 & 1 & 5 & 3 & 1 & 7 & 3 & 9 & 3 & 3 & 3 & 6 & 0 \\
\hline $5 \mathrm{Kg}$-of-Potash-Fertilizer $+5 \mathrm{mmFe}$ & ${ }^{40} \mathrm{~K}$ & 0 & 0 & 4 & 0 & 0 & 0 & 0 & 3 & 3 & 18 & 4 & 5 & 5 & 0 & 1 & 9 & 10 & 2 & 5 & 6 & 3 & 4 & 2 & 6 & 4 & 0 & 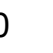 & 6 & 0 \\
\hline HEU & ${ }^{235} \mathrm{U}$ & 0 & 0 & 0 & 0 & 0 & 0 & 0 & 0 & 14 & 5 & 1 & 4 & 6 & 0 & 0 & 2 & 15 & 2 & 10 & 7 & 10 & 8 & 0 & 3 & 2 & 0 & 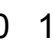 & 11 & 0 \\
\hline Virgin HEU & ${ }^{235} \mathrm{U}$ & 0 & 0 & 0 & 0 & 0 & 0 & 0 & 0 & 11 & 9 & 2 & 0 & 3 & 0 & 0 & 0 & 37 & 2 & 10 & 5 & 4 & 4 & 1 & 3 & 1 & 0 & b & 8 & 0 \\
\hline Reactor $\mathrm{Pu}$ & ${ }^{239} \mathrm{Pu}$ & 0 & 0 & 1 & 0 & 0 & 0 & 1 & 1 & 2 & 13 & 2 & 3 & 10 & 0 & 0 & 12 & 9 & 2 & 4 & 6 & 3 & 8 & 4 & 9 & 7 & 0 & ) & 3 & 0 \\
\hline Weapon Pu & ${ }^{239} \mathrm{Pu}$ & 0 & 0 & 1 & 0 & 0 & 0 & 0 & 0 & 2 & 1 & 0 & 0 & 5 & 0 & 0 & 72 & 1 & 0 & 1 & 3 & 3 & 9 & 0 & 0 & 1 & 0 & 0 & 1 & 0 \\
\hline HEU with shielding & ${ }^{235} \mathrm{U}$ & 0 & 0 & 2 & 0 & 0 & 0 & 0 & 0 & 2 & 3 & 0 & 11 & 10 & 0 & 0 & 40 & 2 & 0 & 1 & 10 & 3 & 15 & 0 & 0 & 1 & 0 & כ & 0 & 0 \\
\hline Virgin HEU with shielding & ${ }^{235} \mathrm{U}$ & 0 & 0 & 1 & 0 & 0 & 0 & 0 & 0 & 3 & 1 & 0 & 6 & 9 & 0 & 0 & 048 & 2 & 0 & 1 & 14 & 3 & 11 & 0 & 0 & 0 & 0 & 0 & 1 & 0 \\
\hline Reactor Pu with shielding & ${ }^{239} \mathrm{Pu}$ & 0 & 0 & 1 & 0 & 0 & 0 & 0 & 0 & 8 & 9 & 3 & 19 & 5 & 0 & 0 & 8 & 13 & 0 & 4 & 9 & 3 & 9 & 1 & 2 & 0 & 0 & 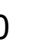 & 6 & 0 \\
\hline Weapon Pu with shielding & ${ }^{239} \mathrm{Pu}$ & 0 & 0 & 1 & 0 & 0 & 0 & 0 & 0 & 12 & 6 & 2 & 10 & 7 & 0 & 0 & 4 & 18 & 2 & 9 & 4 & 5 & 6 & 0 & 3 & 1 & & D & 10 & 0 \\
\hline \multicolumn{31}{|l|}{100 Counts/400 Bkg } \\
\hline 99TcM-625uc+200mm-H2O+5mmFe & & 0 & 0 & 0 & 0 & 0 & 0 & 0 & 0 & 0 & 0 & 0 & 4 & 0 & 0 & 0 & 0 & 0 & 0 & 0 & 96 & 0 & 0 & 0 & 0 & 0 & 0 & כ & 0 & 0 \\
\hline 1311-370uc+200mm-H2O+5mmFe & 1 & 0 & 0 & 0 & 0 & 0 & 0 & 0 & 0 & 44 & 3 & 0 & 0 & 0 & 0 & 0 & 1 & 13 & 0 & 30 & 0 & 3 & 0 & 0 & 1 & 2 & 0 & 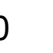 & 3 & 0 \\
\hline $201 \mathrm{TI}-250 \mathrm{uc}+200 \mathrm{~mm}-\mathrm{H} 2 \mathrm{O}+5 \mathrm{mmFe}$ & ${ }^{201} \mathrm{TI}$ & 0 & 0 & 0 & 0 & 0 & 0 & 0 & 0 & 1 & 0 & 0 & 1 & 11 & 0 & 0 & 0 & 0 & 0 & 0 & 7 & 0 & 80 & 0 & 0 & 0 & 0 & 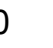 & 0 & 4 \\
\hline
\end{tabular}




\begin{tabular}{|c|c|c|c|c|c|c|c|c|c|c|c|c|c|c|c|c|c|c|c|c|c|c|c|c|c|c|c|c|c|c|c|}
\hline Sample & Label & VD L & & $\begin{array}{l}40 \\
\mathrm{~K} \\
\end{array}$ & $\begin{array}{r}226 \\
\mathrm{Ra} \\
\end{array}$ & $\begin{array}{c}232 \\
\mathrm{Th} \\
\end{array}$ & $\begin{array}{c}228 \\
\mathrm{Th} \\
\end{array}$ & $\begin{array}{c}232 \\
\mathrm{U} \\
\end{array}$ & 60 & & & & $\begin{array}{l}54 \\
\mathrm{Mn} \\
\end{array}$ & $\begin{array}{l}57 \\
\text { Co } \\
\end{array}$ & $\begin{array}{l}139 \\
\mathrm{Ce} \\
\end{array}$ & $\begin{array}{r}252 \\
\mathrm{Cf} \\
\end{array}$ & $\begin{array}{c}238 \\
\mathrm{U} \\
\end{array}$ & $\begin{array}{c}235 \\
U \\
\end{array}$ & & & $\begin{array}{lll}37 & 1 \\
\mathbf{p} & \\
\end{array}$ & $\begin{array}{l}319 \\
1 \quad 1 \\
\quad\end{array}$ & $\begin{array}{l}99^{\mathrm{m}} \\
\text { Tc } \\
\end{array}$ & $\begin{array}{l}67 \\
\mathrm{Ga} \\
\end{array}$ & $\begin{array}{c}201 \\
\mathrm{TI} \\
\end{array}$ & $\begin{array}{c}88 \\
\mathrm{Y} \\
\end{array}$ & $\begin{array}{l}22 \\
\mathrm{Na} \\
\end{array}$ & $\begin{array}{l}166^{\mathrm{m}} \\
\text { Ho } \\
\end{array}$ & $\begin{array}{l}152 \\
\mathrm{Eu} \\
\end{array}$ & $\begin{array}{c}192 \\
\text { Ir } \\
\end{array}$ & SUM \\
\hline $67 \mathrm{Ga}-350 \mathrm{uc}+200 \mathrm{~mm}-\mathrm{H} 2 \mathrm{O}+5 \mathrm{mmFe}$ & ${ }^{67} \mathrm{Ga}$ & 0 & 0 & 1 & 0 & 0 & 0 & 0 & $\mathrm{c}$ & 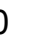 & 9 & 1 & 0 & 0 & 0 & 0 & 0 & 10 & & 2 & 5 & 2 & 2 & 58 & 2 & 0 & 0 & 1 & 0 & 7 & \\
\hline $137 \mathrm{Cs}-8 \mathrm{mc}+24 \mathrm{~mm}-\mathrm{Lead}+5 \mathrm{mmFe}$ & ${ }^{137} \mathrm{Cs}$ & 0 & 0 & 0 & 0 & 0 & 0 & 0 & $c$ & D & 0 & 96 & 0 & 0 & 0 & 0 & 0 & & 0 & 0 & 0 & 0 & 0 & 0 & 0 & 0 & 0 & 2 & 0 & 2 & \\
\hline 14Kg-of-Bananas+5mmFe & ${ }^{40} \mathrm{~K}$ & 0 & 0 & 44 & 0 & 1 & 0 & 0 & 5 & 5 & 0 & 5 & 2 & 0 & 0 & 1 & 7 & & 1 & 4 & 0 & 1 & 0 & 1 & 0 & 2 & 6 & 4 & 11 & 5 & \\
\hline $5 \mathrm{Kg}$-of-Potash-Fertilizer $+5 \mathrm{mmFe}$ & ${ }^{40} \mathrm{~K}$ & 0 & 0 & 11 & 0 & 0 & 0 & 0 & $c$ & D & 4 & 9 & 1 & 0 & 6 & 2 & 2 & 6 & 61 & 11 & 1 & 2 & 4 & 8 & 3 & 1 & 6 & 7 & 3 & 13 & \\
\hline HEU & ${ }^{235} \mathrm{U}$ & 0 & 0 & 0 & 0 & 0 & 0 & 0 & c & 1 & 2 & 5 & 1 & 1 & 0 & 0 & 0 & & 2 & 33 & 2 & 12 & 2 & 17 & 1 & 0 & 1 & 1 & 0 & 10 & \\
\hline Virgin HEU & ${ }^{235} \mathrm{U}$ & 0 & 0 & 0 & 0 & 0 & 0 & 0 & c & ) & 5 & 3 & 0 & 0 & 0 & 0 & 0 & ) & 1 & 73 & 0 & 12 & 1 & 1 & 0 & 0 & 1 & 1 & 0 & 2 & \\
\hline Weapon Pu & ${ }^{239} \mathrm{Pu}$ & 0 & 0 & 0 & 0 & 0 & 0 & 0 & c & כ & 0 & 0 & 0 & 0 & 0 & 0 & & 100 & & 0 & 0 & 0 & 0 & 0 & 0 & 0 & 0 & 0 & 0 & 0 & \\
\hline HEU with shielding & ${ }^{235} \mathrm{U}$ & 0 & 0 & 0 & 0 & 0 & 0 & 0 & c & כ & 0 & 0 & 0 & 3 & 2 & 0 & 0 & 73 & & 0 & 0 & 0 & 5 & 1 & 15 & 0 & 0 & 0 & 0 & 1 & \\
\hline Virgin HEU with shielding & ${ }^{235} \mathrm{U}$ & 0 & 0 & 0 & 0 & 0 & 0 & 0 & $c$ & D & 1 & 0 & 0 & 2 & 2 & 0 & 0 & 72 & & 0 & 0 & 0 & 4 & 2 & 16 & 0 & 0 & 0 & 0 & 1 & \\
\hline Reactor Pu with shielding & ${ }^{239} \mathrm{Pu}$ & 0 & 0 & 2 & 0 & 0 & 0 & 1 & c & 1 & 11 & 16 & 0 & 8 & 0 & 0 & 0 & & 9 & 24 & 2 & 6 & 3 & 2 & 3 & 0 & 1 & 3 & 0 & 9 & \\
\hline Weapon Pu with shielding & ${ }^{239} \mathrm{Pu}$ & 0 & 0 & 0 & 0 & 0 & 0 & c & c & 1 & 1 & 4 & 1 & 2 & 0 & 0 & 0 & & 4 & 38 & 3 & 13 & 1 & 13 & 2 & 0 & 1 & 2 & 0 & 5 & \\
\hline \multicolumn{32}{|l|}{200 Counts/400 Bk } \\
\hline $137 \mathrm{Cs}-8 \mathrm{mc}+24 \mathrm{~mm}-\mathrm{Lead}+5 \mathrm{mmFe}$ & ${ }^{137} \mathrm{Cs}$ & 0 & 0 & 0 & 0 & 0 & 0 & 0 & ( & & 010 & & 0 & 0 & 0 & 0 & 0 & & 0 & 0 & 0 & 0 & 0 & 0 & & 0 & & 0 & & 0 & \\
\hline $14 \mathrm{Kg}$-of-Bananas $+5 \mathrm{mmFe}$ & ${ }^{40} \mathrm{~K}$ & 0 & 0 & 85 & 2 & 0 & 0 & 0 & & & 0 & 0 & 0 & 0 & 0 & 2 & 0 & & 0 & 0 & 0 & 0 & 0 & 0 & & 0 & & 0 & & 0 & \\
\hline $5 \mathrm{Kg}$-of-Wood+5mmFe & ${ }^{40} \mathrm{~K}$ & 0 & 0 & 81 & 3 & 0 & 0 & 0 & 2 & $?$ & 0 & 0 & 0 & 0 & 0 & 2 & 0 & & 0 & 0 & 0 & 0 & 0 & 0 & 0 & 0 & 1 & 0 & 11 & 0 & \\
\hline 5Kg-of-Potash-Fertilizer- & ${ }^{40} \mathrm{~K}$ & 0 & 0 & 34 & 6 & 4 & 0 & 0 & 2 & & 0 & 1 & 0 & 0 & 0 & 5 & 11 & & 0 & 1 & 0 & 0 & 0 & 3 & 0 & 0 & 5 & 7 & 16 & 3 & \\
\hline HEU & ${ }^{235} \mathrm{U}$ & 0 & 0 & 0 & 0 & 0 & 0 & 0 & ( & 1 & 0 & 1 & 0 & 0 & 0 & 0 & 0 & & 0 & 99 & 1 & 5 & 0 & 6 & & 0 & & 0 & & 8 & \\
\hline Virgin HEU & ${ }^{235} \mathrm{U}$ & 0 & 0 & 0 & 0 & 0 & 0 & 0 & ( & D & 1 & 0 & 0 & 0 & 0 & 0 & 0 & C & 0 & 99 & 0 & 0 & 0 & 0 & & 0 & & 0 & & 0 & \\
\hline Reactor Pu & ${ }^{239} \mathrm{Pu}$ & 0 & 0 & 0 & 4 & 15 & 526 & 24 & ( & b & 0 & 0 & 0 & 0 & 0 & 13 & 0 & 13 & & 0 & 0 & 0 & 0 & 0 & 0 & 1 & 1 & 3 & 0 & 0 & \\
\hline Weapon Pu & ${ }^{239} \mathrm{Pu}$ & 0 & 0 & 0 & 0 & c & 0 & c & ( & & 0 & 0 & 0 & 0 & 0 & 0 & & 100 & & 0 & 0 & 0 & 0 & 0 & 0 & 0 & 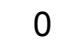 & 0 & 0 & 0 & \\
\hline HEU with sh & ${ }^{235} \mathrm{U}$ & 0 & 0 & 0 & 0 & 0 & 0 & 0 & ( & ) & 0 & 0 & 0 & 1 & 0 & 0 & 0 & 90 & & 0 & 0 & 0 & 0 & 0 & 9 & 0 & & 0 & 0 & 0 & \\
\hline Virgin HEU with sh & ${ }^{235} \mathrm{U}$ & 0 & 0 & 0 & 0 & 0 & 0 & 0 & ( & D & 0 & 0 & 0 & 1 & 0 & 0 & 0 & 89 & & 0 & 0 & 0 & 0 & 0 & 10 & 0 & 0 & 0 & 0 & 0 & \\
\hline Reactor Pu with shielding & ${ }^{239} \mathrm{Pu}$ & 0 & 0 & 8 & 0 & 1 & 0 & 1 & ( & 1 & 42 & 25 & 0 & 2 & 0 & 0 & 0 & ) & & 3 & 0 & 5 & 1 & 0 & 0 & 0 & 0 & 2 & 0 & 6 & \\
\hline
\end{tabular}




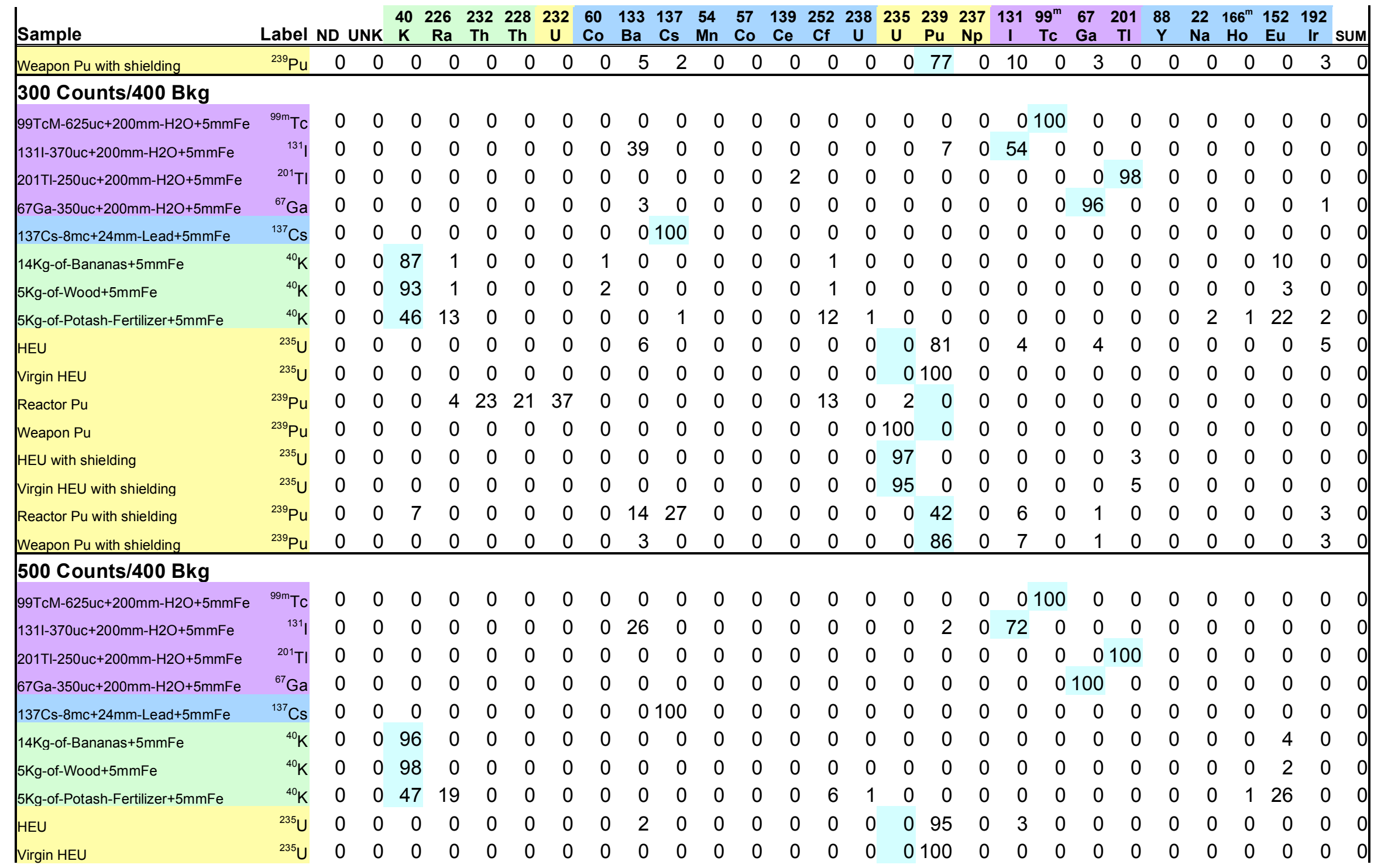




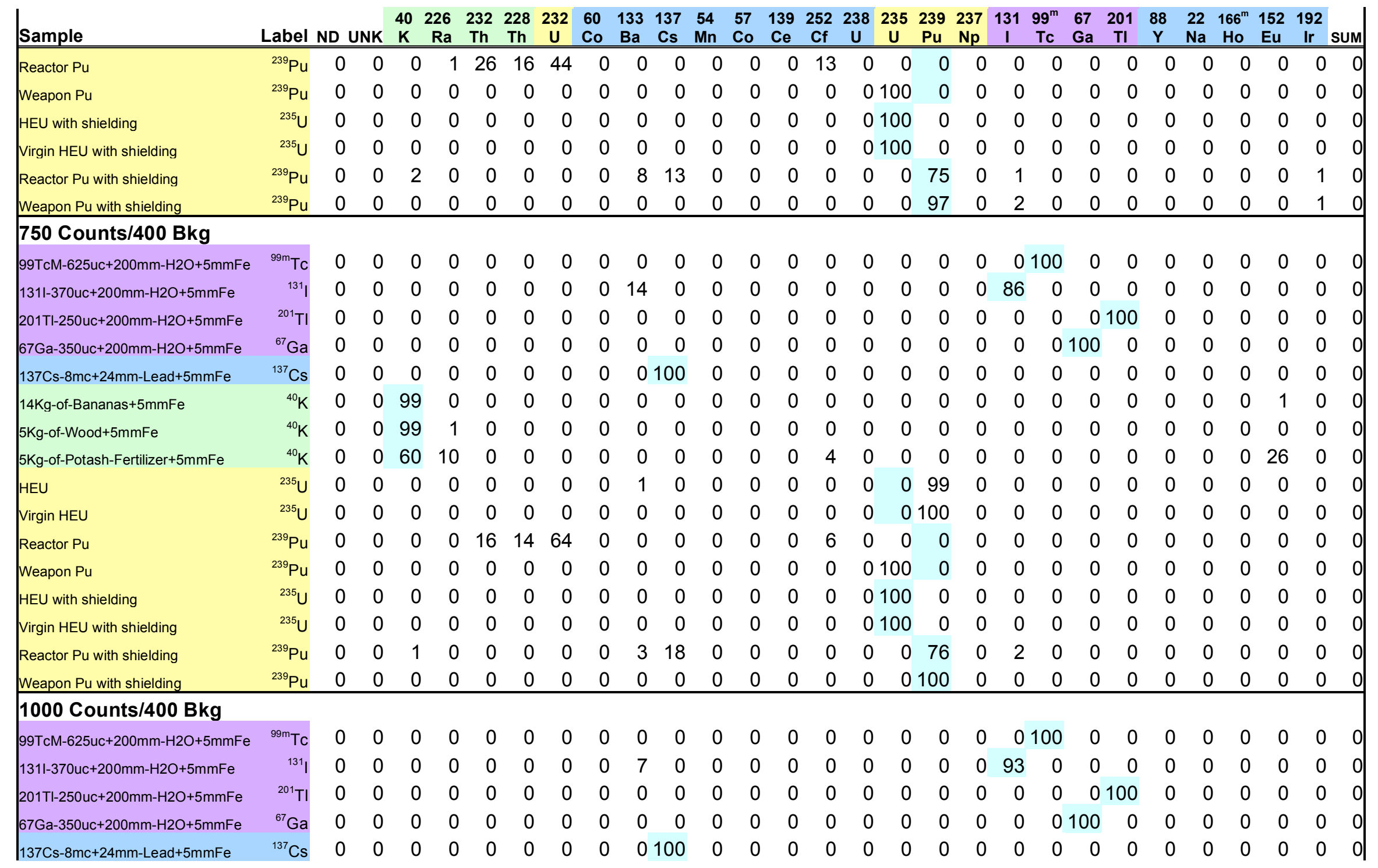




\begin{tabular}{|c|c|c|c|c|c|c|c|c|c|c|c|c|c|c|c|c|c|c|c|c|c|c|c|c|c|c|c|c|c|}
\hline Sample & Label & ND 1 & & $\begin{array}{l}40 \\
\mathrm{~K} \\
\end{array}$ & $\begin{array}{r}226 \\
\mathrm{Ra} \\
\end{array}$ & $\begin{array}{c}232 \\
\text { Th } \\
\end{array}$ & $\begin{array}{c}228 \\
\text { Th } \\
\end{array}$ & $\begin{array}{c}232 \\
\mathrm{U} \\
\end{array}$ & $\begin{array}{l}60 \\
\text { Co } \\
\end{array}$ & $\begin{array}{l}133 \\
\mathrm{Ba} \\
\end{array}$ & $\begin{array}{l}137 \\
\text { Cs } \\
\end{array}$ & $\begin{array}{c}54 \\
\mathrm{Mn} \\
\end{array}$ & $\begin{array}{l}57 \\
\text { Co } \\
\end{array}$ & $\begin{array}{l}139 \\
\mathrm{Ce} \\
\end{array}$ & $\begin{array}{r}252 \\
\text { Cf } \\
\end{array}$ & $\begin{array}{c}238 \\
\mathrm{U} \\
\end{array}$ & $\begin{array}{c}235 \\
\mathrm{U} \\
\end{array}$ & $\begin{array}{l}239 \\
\mathrm{Pu} \\
\end{array}$ & $\begin{array}{l}237 \\
\mathrm{~Np} \\
\end{array}$ & $\begin{array}{c}1319 \\
1 \quad 1 \\
\end{array}$ & $\begin{array}{l}99^{\mathrm{m}} \\
\text { Tc } \\
\end{array}$ & $\begin{array}{ll}67 & 2 \\
\mathrm{Ga}\end{array}$ & $\begin{array}{l}201 \\
\mathrm{TI} \\
\end{array}$ & $\begin{array}{l}88 \\
Y \\
\end{array}$ & $\begin{array}{l}22 \\
\mathrm{Na} \\
\end{array}$ & $\begin{array}{c}166^{\mathrm{m}} \\
\text { Ho } \\
\end{array}$ & $\begin{array}{c}152 \\
\mathrm{Eu} \\
\end{array}$ & $\begin{array}{c}192 \\
\text { Ir } \mathrm{s}\end{array}$ & sum \\
\hline $14 \mathrm{Kg}$-of-Bananas $+5 \mathrm{mmFe}$ & ${ }^{40} \mathrm{~K}$ & 0 & 0 & 99 & 0 & 0 & 0 & 0 & 0 & 0 & 0 & 0 & 0 & 0 & 0 & 0 & 0 & 0 & 0 & 0 & 0 & 0 & 0 & 0 & 0 & 0 & 1 & 0 & 0 \\
\hline $5 \mathrm{Kg}$-of-Wood+5mmFe & ${ }^{40} \mathrm{~K}$ & 0 & 0 & 99 & 0 & 0 & 0 & 0 & 0 & 0 & 0 & 0 & 0 & 0 & 0 & 0 & 0 & 0 & 0 & 0 & 0 & 0 & 0 & 0 & 0 & 0 & 1 & 0 & \\
\hline 5Kg-of-Potash-Fer & ${ }^{40} \mathrm{~K}$ & 0 & 0 & 62 & 8 & 0 & 0 & 0 & 0 & 0 & 0 & 0 & 0 & 0 & 1 & 0 & 0 & 0 & 0 & 0 & 0 & 0 & 0 & 0 & 0 & 0 & 29 & 0 & \\
\hline rgin HEU & ${ }^{235} \mathrm{U}$ & 0 & 0 & 0 & 0 & 0 & 0 & 0 & 0 & 0 & 0 & 0 & 0 & 0 & 0 & 0 & & 100 & 0 & 0 & 0 & 0 & ( & 0 & 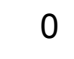 & 0 & 0 & 0 & \\
\hline Reactor Pu & ${ }^{239} \mathrm{Pu}$ & 0 & 0 & 0 & 0 & 22 & 7 & 69 & 0 & 0 & 0 & 0 & 0 & 0 & 2 & 0 & 0 & 0 & 0 & 0 & 0 & 0 & 0 & 0 & 0 & 0 & 0 & 0 & \\
\hline Neapon Pu & ${ }^{239} \mathrm{Pu}$ & 0 & 0 & 0 & 0 & 0 & 0 & 0 & 0 & 0 & 0 & 0 & 0 & 0 & 0 & & 100 & 0 & 0 & 0 & 0 & 0 & ( & 0 & 0 & 0 & c & 0 & \\
\hline Virgin HEU witl & ${ }^{235} \mathrm{U}$ & 0 & 2 & 0 & 0 & 0 & 0 & 0 & 0 & 0 & 0 & 0 & 0 & 0 & 0 & 0 & 98 & 0 & 0 & 0 & 0 & 0 & 0 & 0 & 0 & 0 & 0 & 0 & \\
\hline Reactor & ${ }^{239} \mathrm{Pu}$ & 0 & 0 & 0 & 0 & 0 & 0 & 0 & 0 & 1 & 12 & 0 & 0 & 0 & 0 & 0 & 0 & 87 & 0 & 0 & 0 & 0 & 0 & 0 & 0 & 0 & 0 & 0 & \\
\hline eapon Pu with shielding & ${ }^{239} \mathrm{Pu}$ & 0 & 0 & 0 & 0 & 0 & 0 & 0 & 0 & 0 & 0 & 0 & 0 & 0 & 0 & 0 & & 100 & 0 & 0 & 0 & 0 & 0 & 0 & 0 & 0 & 0 & 0 & \\
\hline
\end{tabular}




\section{A.4.4 Verification Set}

\section{A.4.4.1 GADRAS Verification Set Correlation Matrix}

\begin{tabular}{|c|c|c|c|c|c|c|c|c|c|c|c|c|c|c|c|c|c|c|c|c|c|c|c|c|c|c|c|c|c|}
\hline Isotope_type & Id & ND & $\begin{array}{l}40 \\
K\end{array}$ & $\begin{array}{c}226 \\
\mathrm{Ra}\end{array}$ & $\begin{array}{c}232 \\
\text { Th }\end{array}$ & $\begin{array}{c}228 \\
\text { Th }\end{array}$ & 232 & $\begin{array}{l}60 \\
\text { Co }\end{array}$ & $\begin{array}{c}133 \\
\mathrm{Ba}\end{array}$ & $\begin{array}{c}137 \\
\text { Cs }\end{array}$ & $\begin{array}{c}54 \\
\mathrm{Mn}\end{array}$ & $\begin{array}{l}57 \\
\text { Co }\end{array}$ & $\begin{array}{c}139 \\
\mathrm{Ce}\end{array}$ & $\begin{array}{r}252 \\
\text { Cf }\end{array}$ & $\begin{array}{l}238 \\
\mathrm{U}\end{array}$ & $\begin{array}{c}235 \\
\mathrm{U}\end{array}$ & $\begin{array}{c}239 \\
\text { Pu }\end{array}$ & $\mathrm{Np}$ & $\begin{array}{c}131 \\
\text { I }\end{array}$ & $\begin{array}{c}99 \mathrm{~m} \\
\text { Tc }\end{array}$ & $\begin{array}{l}67 \\
\mathrm{Ga}\end{array}$ & $\begin{array}{c}201 \\
\mathrm{TI}\end{array}$ & $\begin{array}{l}88 \\
Y\end{array}$ & $\begin{array}{l}22 \\
\mathrm{Na}\end{array}$ & $\begin{array}{c}166 m \\
\text { Ho }\end{array}$ & $\begin{array}{c}152 \\
\mathrm{Eu}\end{array}$ & $\begin{array}{l}219 \\
1\end{array}$ & $\begin{array}{l}92 \\
\text { Ir S }\end{array}$ & SUM \\
\hline Total Id & & 1093 & 753 & 1150 & 1409 & 217 & 226 & 613 & 3890 & 764 & 648 & 239 & 9647 & 387 & 421 & 632 & 856 & 1069 & 556 & 394 & 974 & 432 & 486 & 460 & 243 & 3245 & 153 & 380 & \\
\hline Total Correct & & 0 & 575 & 657 & 600 & 191 & 226 & 609 & 557 & 688 & 293 & 200 & 99 & 95 & 385 & 581 & 793 & 469 & 359 & 300 & 361 & 1320 & 486 & 459 & 242 & 240 & & 374 & 10159 \\
\hline Actual trials & & 0 & 600 & 800 & 700 & 400 & 600 & 800 & 700 & 700 & 300 & 200 & 200 & 300 & 800 & 600 & 900 & 500 & 500 & 300 & 400 & 800 & 600 & 600 & 600 & 600 & & 800 & 14300 \\
\hline False Negative & & & 25 & 143 & 100 & 209 & 374 & 191 & 143 & 12 & 7 & 0 & 101 & 205 & 415 & 19 & 107 & 31 & 141 & 0 & 39 & 480 & 114 & 141 & 1358 & 3360 & & 426 & 4141 \\
\hline False Positive & & & 178 & 493 & 809 & 26 & 0 & 4 & +333 & 76 & 355 & 39 & 9548 & 292 & 36 & 51 & 63 & 600 & 197 & 94 & 613 & 3112 & 0 & 1 & 1 & & 5 & 6 & 4932 \\
\hline $\mathrm{K} 40,1 \mathrm{MC}$ & K40 & & 100 & 0 & 0 & 0 & 0 & 0 & 0 & 0 & 0 & 0 & 0 & 0 & 0 & 0 & 0 & 0 & 0 & 0 & 0 & 0 & 0 & 0 & 0 & & 0 & 0 & 100 \\
\hline $\mathrm{K} 40,1 \mathrm{MC}\{10,10\}$ & $\mathrm{K} 40$ & & 100 & 0 & 0 & 0 & 0 & 0 & 0 & 0 & 0 & 0 & 0 & 0 & 0 & 0 & 0 & 0 & 0 & 0 & 0 & 0 & 0 & 0 & 0 & & 0 & 0 & 100 \\
\hline$K 40,1 \mathrm{MC}\{10,30\}$ & $\mathrm{K} 40$ & 5 & 95 & 0 & 1 & 0 & 0 & 0 & 0 & 0 & 0 & 0 & 0 & 0 & 0 & 0 & 0 & 0 & 0 & 0 & 0 & 0 & 0 & 0 & 0 & & 0 & 0 & 101 \\
\hline$K 40,1 M C\{10,60\}$ & $\mathrm{K} 40$ & 17 & 81 & 0 & 0 & 0 & 0 & 0 & 0 & 0 & 0 & 0 & 0 & 0 & 0 & 0 & 0 & 0 & 0 & 0 & 2 & 0 & 0 & 0 & 0 & & 0 & 0 & 100 \\
\hline $\mathrm{K} 40,1 \mathrm{MC}\{26,10\}$ & $\mathrm{K} 40$ & & 100 & 0 & 0 & 0 & 0 & 0 & 0 & 0 & 0 & 0 & 0 & 0 & 0 & 0 & 0 & 0 & 0 & 0 & 0 & 0 & 0 & 0 & 0 & & 0 & 0 & 100 \\
\hline$K 40,1 \mathrm{MC}\{26,30\}$ & $\mathrm{K} 40$ & 1 & 99 & 0 & 1 & 0 & 0 & 0 & 0 & 0 & 0 & 0 & 0 & 0 & 0 & 0 & 0 & 0 & 0 & 0 & 0 & 0 & 0 & 0 & 0 & & 0 & 0 & 101 \\
\hline Ra226,10UC & $\mathrm{Ra} 226$ & 0 & & 100 & 0 & 0 & 0 & 0 & 0 & 0 & 0 & 0 & 0 & 0 & 0 & 0 & 0 & 0 & 1 & 0 & 0 & 0 & 0 & 0 & 0 & & 0 & 0 & 101 \\
\hline $\operatorname{Ra} 226,10 \cup C\{10,10\}$ & $\mathrm{Ra} 226$ & 2 & 1 & 96 & 3 & 0 & 0 & 0 & 0 & 1 & 0 & 0 & 0 & 0 & 0 & 0 & 0 & 0 & 1 & 0 & 0 & 0 & 0 & 0 & 0 & & 0 & 0 & 104 \\
\hline $\operatorname{Ra} 226,10 \cup C\{10,30\}$ & $\mathrm{Ra} 226$ & 32 & 0 & 61 & 4 & 0 & 0 & 0 & 0 & 0 & 1 & 0 & 0 & 1 & 0 & 0 & 0 & 0 & 0 & 1 & 1 & 0 & 0 & 0 & 0 & & 0 & 0 & 101 \\
\hline $\operatorname{Ra} 226,10 \cup C\{10,60\}$ & $\mathrm{Ra} 226$ & 48 & 0 & 44 & 4 & 0 & 0 & 0 & 0 & 1 & 0 & 0 & 0 & 1 & 0 & 0 & 0 & 0 & 0 & 0 & 2 & 0 & 0 & 0 & 0 & & 0 & 0 & 100 \\
\hline $\operatorname{Ra} 226,10 \cup C\{26,10\}$ & $\mathrm{Ra} 226$ & 0 & & 100 & 1 & 0 & 0 & 0 & 0 & 0 & 0 & 0 & 0 & 0 & 0 & 0 & 0 & 0 & 0 & 0 & 0 & 0 & 0 & 0 & 0 & & 0 & 0 & 101 \\
\hline $\operatorname{Ra} 226,10 \cup C\{26,30\}$ & $\mathrm{Ra} 226$ & 9 & 0 & 88 & 2 & 0 & 0 & 0 & 0 & 0 & 0 & 0 & 0 & 1 & 0 & 0 & 1 & 0 & 0 & 0 & 0 & 0 & 0 & 0 & 0 & & 0 & 0 & 101 \\
\hline $\operatorname{Ra} 226,10 \cup C\{26,50\}$ & Ra226 & 23 & 0 & 72 & 4 & 0 & 0 & 0 & 0 & 0 & 1 & 0 & 0 & 0 & 1 & 0 & 0 & 0 & 0 & 0 & 0 & 0 & 0 & 0 & 0 & & 0 & 0 & 101 \\
\hline $\operatorname{Ra} 226,100 \cup C\{74,40\}$ & $\mathrm{Ra} 226$ & 4 & 0 & 96 & 1 & 0 & 0 & 0 & 0 & 0 & 0 & 0 & 0 & 0 & 0 & 0 & 0 & 0 & 0 & 0 & 0 & 0 & 0 & 0 & 0 & & 0 & 0 & 101 \\
\hline Th232,10UC & Th232 & 0 & 0 & 1 & 99 & 1 & 0 & 0 & 0 & 0 & 0 & 0 & 0 & 0 & 0 & 0 & 0 & 0 & 0 & 0 & 0 & 0 & 0 & 0 & 0 & c & 0 & 0 & 101 \\
\hline Th232,10UC $\{10,10\}$ & Th232 & 3 & 0 & 2 & 95 & 0 & 0 & 0 & 0 & 0 & 0 & 0 & 0 & 0 & 0 & 0 & 0 & 0 & 0 & 0 & 1 & 0 & 0 & 0 & 0 & c & 0 & 0 & 101 \\
\hline Th232,10UC $\{10,30\}$ & Th232 & 26 & 0 & 2 & 70 & 0 & 0 & 0 & 0 & 0 & 0 & 0 & 0 & 0 & 0 & 0 & 0 & 0 & 0 & 0 & 2 & 0 & 0 & 0 & 0 & c & 0 & 0 & 100 \\
\hline Th232,10UC $\{10,60\}$ & Th232 & 45 & 1 & 1 & 49 & 0 & 0 & 0 & 0 & 1 & 1 & 0 & 0 & 0 & 0 & 1 & 0 & 0 & 0 & 0 & 1 & 0 & 0 & 0 & 0 & c & 0 & 0 & 100 \\
\hline Th232,10UC $\{26,10\}$ & Th232 & 0 & 0 & & 100 & 0 & 0 & 0 & 0 & 0 & 0 & 0 & 0 & 0 & 0 & 0 & 0 & 0 & 0 & 0 & 0 & 0 & 0 & 0 & 0 & c & 0 & 0 & 100 \\
\hline Th232,10UC $\{26,30\}$ & Th232 & 10 & 0 & 2 & 88 & 0 & 0 & 0 & 0 & 0 & 0 & 0 & 0 & 0 & 0 & 0 & 0 & 0 & 0 & 0 & 0 & 0 & 0 & 0 & 0 & c & 0 & 0 & 100 \\
\hline
\end{tabular}




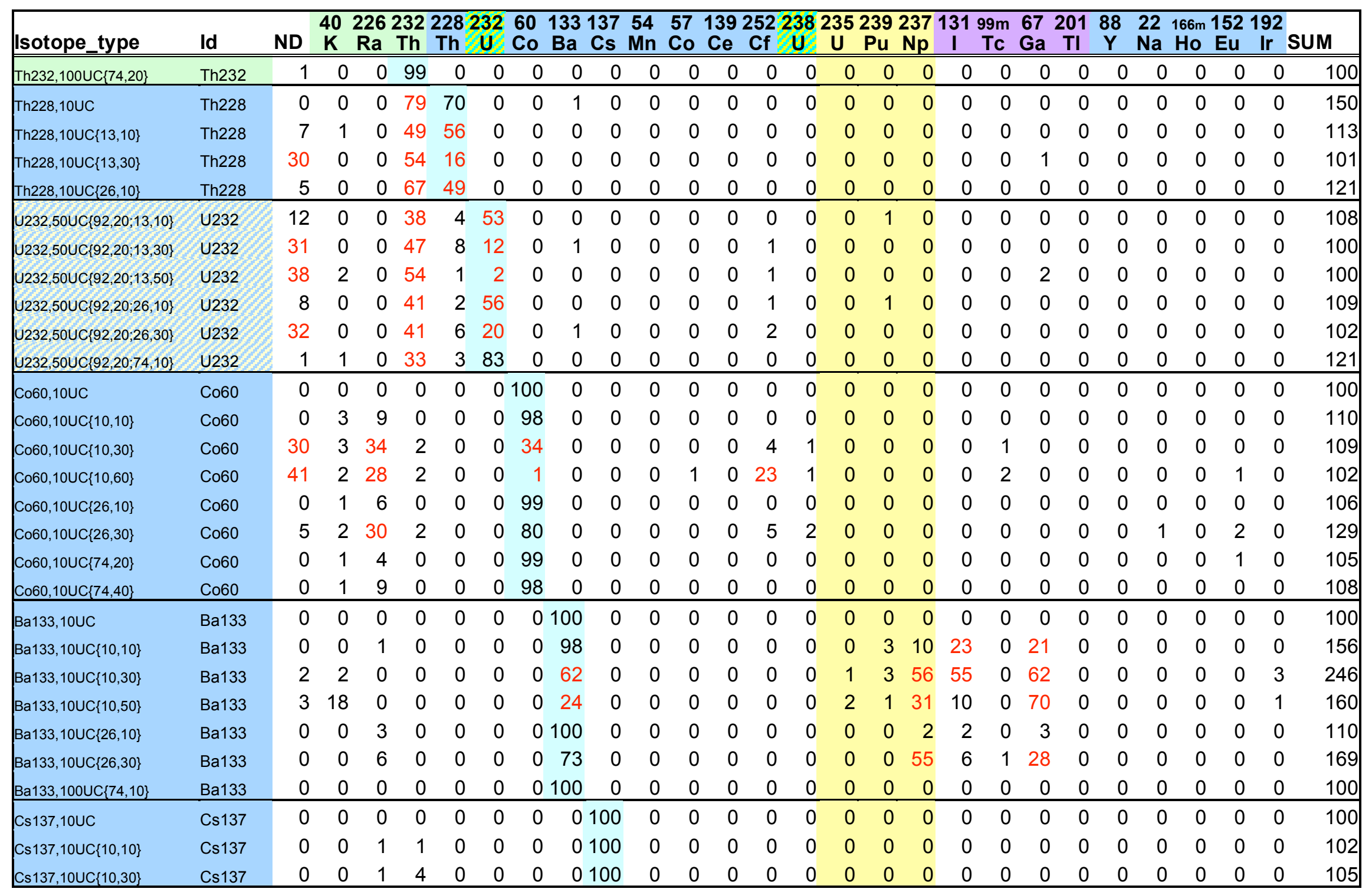




\begin{tabular}{|c|c|c|c|c|c|c|c|c|c|c|c|c|c|c|c|c|c|c|c|c|c|c|c|c|c|c|c|c|c|c|c|c|}
\hline Isotope_ & Id & ND & $\begin{array}{l}40 \\
\mathrm{~K}\end{array}$ & $\begin{array}{l}226 \\
\mathrm{Ra} \\
\end{array}$ & $\begin{array}{l}232 \\
\text { Th } \\
\end{array}$ & $\begin{array}{c}228 \\
\text { Th } \\
\end{array}$ & 232 & $\begin{array}{l}60 \\
\text { Co } \\
\end{array}$ & $\begin{array}{l}133 \\
\mathrm{Ba} \\
\end{array}$ & $\begin{array}{rr}3 & 13 \\
a & C s \\
\end{array}$ & & $\begin{array}{l}54 \\
\mathrm{Mn} \quad \\
\end{array}$ & $\begin{array}{l}57 \\
\text { Co } \\
\end{array}$ & $\begin{array}{c}139 \\
\mathrm{Ce} \\
\end{array}$ & $\begin{array}{c}252 \\
\mathrm{Cf} \\
\end{array}$ & 238 & U & $\begin{array}{l}239 \\
\mathrm{Pu}\end{array}$ & & Np & $\begin{array}{c}1319 \\
\mathrm{I}\end{array}$ & $\begin{array}{l}99 \mathrm{~m} \\
\text { Tc }\end{array}$ & $\begin{array}{l}67 \\
\mathbf{G a} \\
\end{array}$ & $\begin{array}{c}201 \\
\text { TI } \\
\end{array}$ & $\begin{array}{r}188 \\
Y \\
\end{array}$ & 1 & $\begin{array}{ll}22 & 1 \\
\mathrm{Na} & \\
\end{array}$ & $\begin{array}{l}166 m \\
\mathrm{Ho} \\
\end{array}$ & $\begin{array}{c}152 \\
\mathrm{Eu} \\
\end{array}$ & $\begin{array}{c}192 \\
\text { Ir }\end{array}$ & SUN & \\
\hline Cs $137,10 \cup C\{10,60\}$ & Cs137 & 4 & 2 & 0 & 2 & 0 & 0 & 0 & 0 & 08 & 38 & 0 & 0 & 0 & 0 & 0 & 0 & & 3 & 0 & 2 & 0 & 5 & 0 & 0 & 0 & 0 & 0 & 0 & 0 & & 106 \\
\hline Cs $137,10 \cup C\{26,10\}$ & s 137 & 0 & 0 & 0 & 0 & 0 & 0 & 0 & 0 & 010 & & 0 & 0 & 0 & 0 & 0 & 0 & & 0 & 0 & 0 & 0 & 0 & 0 & 0 & 0 & 0 & 0 & 0 & 0 & & 100 \\
\hline $\mathrm{Cs} 137,10 \cup \mathrm{U}\{26,30\}$ & s137 & 0 & 0 & 2 & 6 & 0 & 0 & 0 & 0 & 010 & & 0 & 0 & 0 & 0 & 0 & 0 & & 0 & 0 & 0 & 0 & 0 & 0 & 0 & 0 & 0 & 0 & 0 & 0 & & 108 \\
\hline$U C\{26,50\}$ & 127 & 0 & 0 & 4 & 9 & 0 & 0 & 0 & 0 & 010 & & 0 & 0 & 0 & 0 & 0 & 0 & & 0 & 0 & 0 & 0 & 0 & 0 & 0 & 0 & 0 & 0 & 0 & 0 & & 113 \\
\hline Mn54,10UC & Mn54 & 0 & 0 & 0 & 0 & 0 & 0 & 0 & 0 & 0 & 01 & 100 & 0 & 0 & 0 & 0 & 0 & & 0 & 0 & 0 & 0 & 0 & 0 & 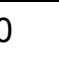 & 0 & 0 & 6 & 0 & 0 & & 100 \\
\hline $\operatorname{Mn} 54,10 \cup C\{13,10\}$ & Mn54 & 0 & 0 & 0 & 8 & 0 & 0 & 0 & c & 0 & 01 & 100 & 0 & 0 & 0 & 0 & 0 & & 0 & 0 & 0 & 0 & 0 & 0 & 0 & 0 & 0 & 0 & 0 & 0 & & 108 \\
\hline $\operatorname{Mn} 54,10 \cup C\{13,30\}$ & Mn54 & 0 & 0 & 1 & 21 & 0 & 0 & 0 & c & 0 & 0 & 93 & 0 & 0 & 0 & 0 & 0 & & 0 & 0 & 0 & 0 & 0 & 0 & 0 & 0 & 0 & 0 & 0 & 0 & & 115 \\
\hline Co57, & & 0 & 0 & 0 & 0 & 0 & 0 & 0 & 0 & 0 & 0 & & 100 & 0 & 0 & 0 & 0 & & 0 & 0 & 0 & 0 & 0 & 0 & 0 & 0 & 0 & 6 & 0 & 0 & & 100 \\
\hline Co57,10UC $\{13,10\}$ & Co57 & 0 & 0 & 0 & 0 & 0 & 0 & 0 & C & 0 & 0 & & 100 & 0 & 0 & 0 & 0 & & 0 & 0 & 0 & 0 & 0 & 0 & 0 & 0 & 0 & 0 & 0 & 0 & & 100 \\
\hline Ce139,10UC & Ce139 & 0 & 0 & 0 & 0 & 0 & 0 & 0 & c & 0 & 0 & 0 & 0 & 9 & 0 & 0 & 0 & & 0 & 0 & 0 & 0 & 0 & 98 & & 0 & 0 & 0 & 0 & 0 & & 107 \\
\hline Ce139,10UC $\{13,10\}$ & Ce139 & 0 & 0 & 0 & 0 & 0 & 0 & 0 & C & 0 & 0 & 0 & 0 & 90 & 0 & 0 & 0 & & 0 & 0 & 0 & 0 & 0 & 10 & & 0 & 0 & 0 & 0 & 0 & & 100 \\
\hline Nylon 1 & Cf252 & 48 & 0 & 15 & 11 & 1 & 0 & 0 & 0 & 0 & 0 & 0 & 1 & 0 & 24 & 0 & 0 & & $u$ & 1 & 0 & 0 & 0 & 0 & & 0 & 0 & $\sigma$ & 0 & 0 & & 101 \\
\hline Nylon1-Cf $\{90,1\}$ & Cf252 & 16 & 0 & 25 & 14 & 0 & 0 & 0 & 3 & 3 & 0 & 0 & 0 & 0 & 47 & 0 & 0 & & 0 & 0 & 1 & 0 & 0 & 0 & 0 & 0 & 0 & 0 & 0 & 0 & & 106 \\
\hline Fe1-Cf & $\mathrm{Cf252}$ & 49 & 0 & 18 & 9 & 0 & 0 & 0 & C & 0 & 0 & 0 & 0 & 1 & 24 & 1 & 0 & & 1 & 0 & 0 & 2 & 0 & 0 & 0 & 0 & 0 & 0 & 0 & 0 & & 105 \\
\hline $1 \mathrm{KGU} 238$ & $\mathrm{U} 238$ & 16 & 0 & 4 & 6 & 0 & 0 & 0 & c & 0 & 2 & 1 & 0 & 0 & 24 & 67 & 0 & & 2 & 0 & 1 & 0 & 0 & 0 & 0 & 0 & 0 & 0 & 0 & 0 & & 123 \\
\hline & U238 & 36 & 0 & 6 & 10 & 0 & 0 & 0 & 1 & 1 & 1 & 9 & 1 & 0 & 24 & 23 & 0 & & 0 & 0 & 0 & 1 & 1 & 0 & 0 & 0 & 0 & 0 & 0 & 0 & & 113 \\
\hline $1 \mathrm{KGU} 238\{13,30\}$ & 0238 & 43 & 1 & 6 & 9 & 0 & 0 & 0 & 0 & 0 & 0 & 15 & 0 & 0 & 18 & 16 & 0 & & 1 & 0 & 0 & 1 & 1 & 0 & 0 & 0 & 0 & 0 & 0 & 0 & & 111 \\
\hline $1 \mathrm{KGU} 2$ & 4238 & 31 & 0 & 3 & 6 & 0 & 0 & 0 & 0 & 0 & 0 & 40 & 0 & 0 & 9 & 26 & 0 & & 1 & 0 & 0 & 2 & 0 & 0 & 0 & 0 & 0 & 0 & 0 & 0 & & 118 \\
\hline & 0238 & 21 & 0 & 4 & 7 & 0 & 0 & 0 & 0 & 0 & 2 & 2 & 0 & 0 & 36 & 46 & 0 & & 0 & 0 & 1 & 1 & 0 & 0 & 0 & 0 & 0 & 0 & 0 & 0 & & 120 \\
\hline & $\mathrm{U} 238$ & 24 & 0 & 2 & 4 & 0 & 0 & 0 & c & 0 & 1 & 8 & 0 & 1 & 35 & 45 & 0 & & 0 & 0 & 1 & 1 & 0 & 0 & 0 & 0 & 0 & 0 & 0 & 0 & & 122 \\
\hline & & 11 & 0 & 0 & 2 & 0 & 0 & 0 & c & 0 & 0 & 21 & 0 & 0 & 18 & 62 & 0 & & 1 & 0 & 0 & 0 & 0 & 0 & 0 & 0 & 0 & 1 & 1 & 0 & & 117 \\
\hline & & 0 & 0 & 1 & 2 & 0 & 0 & 0 & c & 0 & 0 & 0 & 0 & 0 & 0 & 100 & 0 & & 0 & 0 & 0 & 0 & 0 & 0 & 0 & 0 & 0 & 0 & 0 & 0 & & 103 \\
\hline \multirow{6}{*}{$\begin{array}{l}\text { U235,25UC+UXRAY,5UC } \\
\text { U235,25UC }\{13,3\} \\
+ \text { +UXRAY,5UCC }\{13,3\} \\
\text { U235,25UC }\{13,10\} \\
+ \text { +UXRAY,5UC }\{13,10\} \\
1 \text { 1KGU235\{13,20\} } \\
+ \text { +UXRAY,5UC }\{13,10\} \\
1 \text { KGU } 235\{26,5\} \\
+ \text { +UXRAY,5UC }\{26,5\} \\
1 \text { KGU235\{26,20\} } \\
\text { +UXRAY,5UC }\{26,20\}\end{array}$} & U235 & 0 & 0 & 0 & 0 & 0 & 0 & 0 & 0 & 0 & 0 & 0 & 0 & 0 & 0 & \multicolumn{2}{|c|}{0100} & & 0 & 0 & 0 & 0 & 0 & 0 & 0 & 0 & 0 & 0 & 0 & 0 & & 100 \\
\hline & U235 & 0 & 2 & 0 & 0 & 0 & 0 & 0 & 0 & 0 & 0 & 0 & 0 & 0 & 0 & \multicolumn{2}{|c|}{0100} & & 0 & 0 & 0 & 0 & 0 & 0 & 0 & 0 & 0 & U & 0 & 0 & & 102 \\
\hline & U235 & 0 & 4 & 0 & 0 & 0 & 0 & 0 & c & 0 & 0 & 0 & 0 & 5 & 0 & \multicolumn{2}{|c|}{$0 \quad 97$} & & 0 & 0 & 0 & 0 & 0 & 0 & 0 & 0 & 0 & 0 & 0 & 0 & & 106 \\
\hline & U235 & 0 & 26 & 0 & 0 & 0 & 0 & 0 & 0 & 0 & 0 & 0 & 0 & 16 & 0 & \multicolumn{2}{|c|}{084} & & 0 & 0 & 0 & 0 & 0 & 3 & 3 & 0 & 0 & U & 0 & 0 & & 129 \\
\hline & U235 & 0 & 0 & 0 & 0 & 0 & 0 & 0 & c & 0 & 0 & 0 & 0 & 0 & 0 & \multicolumn{2}{|c|}{0100} & & 0 & 0 & 0 & U & 0 & 0 & 0 & 0 & 0 & U & 0 & 0 & & 100 \\
\hline & U235 & 0 & 0 & 0 & 0 & 0 & 0 & 0 & 0 & 0 & 0 & 0 & 0 & 0 & 0 & \multicolumn{2}{|c|}{0100} & & 0 & 0 & 0 & 0 & 0 & 0 & 0 & 0 & 0 & 0 & 0 & 0 & & 100 \\
\hline
\end{tabular}




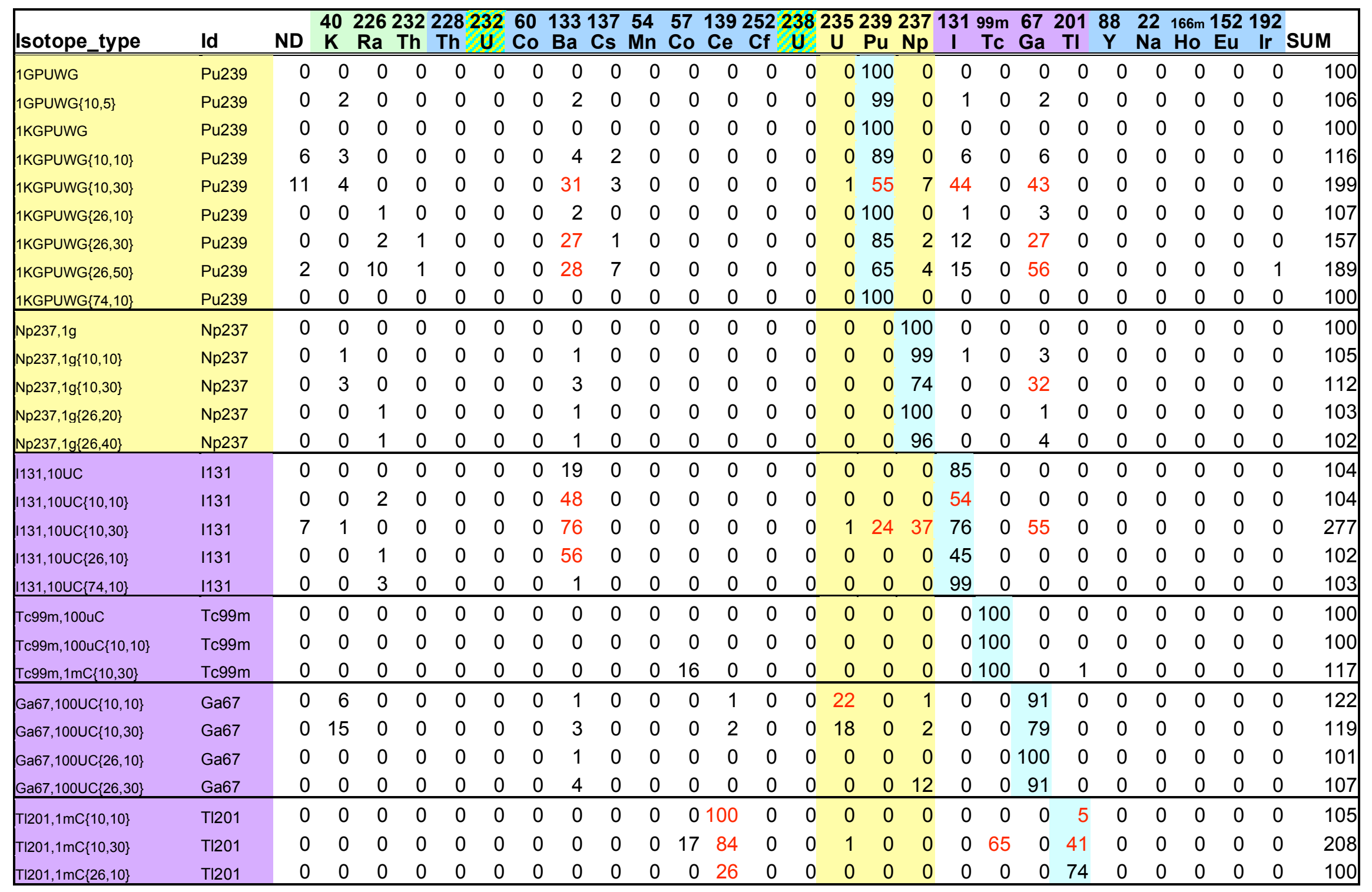




\begin{tabular}{|c|c|c|c|c|c|c|c|c|c|c|c|c|c|c|c|c|c|c|c|c|c|c|c|c|c|c|c|c|c|c|c|}
\hline Isotope_type & Id & ND & $\begin{array}{l}40 \\
\mathbf{K} \\
\end{array}$ & $\begin{array}{l}226 \\
\mathrm{Ra} \\
\end{array}$ & $\begin{array}{c}232 \\
\text { Th } \\
\end{array}$ & $\begin{array}{c}2282 \\
\mathrm{Th} \\
\end{array}$ & & $\begin{array}{l}60 \\
\text { Co } \\
\end{array}$ & $\begin{array}{c}133 \\
\mathrm{Ba} \\
\end{array}$ & $\begin{array}{r}137 \\
\mathrm{Cs} \\
\end{array}$ & & $\begin{array}{l}4 \\
\text { In } \\
\end{array}$ & $\begin{array}{l}57 \\
\text { Co } \\
\end{array}$ & $\begin{array}{c}139 \\
\mathrm{Ce} \\
\end{array}$ & $\begin{array}{c}252 \\
\mathrm{Cf} \\
\end{array}$ & 238 & $\begin{array}{c}235 \\
\mathrm{U}\end{array}$ & & $\begin{array}{ll}39 & 2 \\
\mathrm{u} \quad 1 & 1\end{array}$ & $\begin{array}{l}237 \\
\text { Np }\end{array}$ & $\begin{array}{c}1319 \\
\mathrm{I}\end{array}$ & $\begin{array}{c}99 \mathrm{~m} \\
\mathrm{TC}\end{array}$ & $\begin{array}{l}672 \\
\mathrm{Ga} \\
\end{array}$ & $\begin{array}{c}201 \\
\text { TI }\end{array}$ & $\begin{array}{l}88 \\
Y \\
\end{array}$ & $\begin{array}{l}22 \\
\mathrm{Na} \\
\end{array}$ & $\begin{array}{c}166 \mathrm{~m} \\
\mathrm{Ho}\end{array}$ & $\begin{array}{c}152 \\
\mathrm{Eu} \\
\end{array}$ & $\begin{array}{c}192 \\
\text { Ir }\end{array}$ & suI & \\
\hline \multirow{5}{*}{$\begin{array}{l}\text { TI201,1mC }\{26,30\} \\
\text { TI201,1mC }\{13,5\} \\
\text { +TL202,80NC }\{13,5\} \\
\text { TI201,1mC }\{13,20\} \\
\text { +TL202,80NC }\{13,20\} \\
\text { TI201,1mC }\{26,5\} \\
\text { +TL202,80NC }\{26,5\} \\
\text { TI201,1mC }\{26,20\} \\
\text { +TL202,80NC }\{26,20\} \\
\end{array}$} & $\mathrm{Tl} 201$ & 0 & 0 & 0 & 0 & 0 & 0 & 0 & 0 & & 0 & 0 & 0 & 45 & 0 & 0 & 0 & 0 & 0 & 0 & 0 & 0 & 0 & 55 & 0 & 0 & 0 & 0 & 0 & 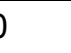 & 100 \\
\hline & TI201 & 0 & 0 & 0 & 0 & 0 & 0 & 0 & 0 & & 0 & 0 & 0 & 99 & 0 & 0 & 0 & 0 & 0 & 0 & 0 & 0 & 0 & 4 & 0 & 0 & 0 & 0 & 0 & & 103 \\
\hline & $\mathrm{Tl} 201$ & 0 & 1 & 0 & 0 & 0 & 0 & 0 & 0 & & 0 & 0 & 2 & 97 & 0 & 0 & 0 & 0 & 0 & 0 & 0 & 13 & 0 & 11 & 0 & 0 & 0 & 0 & 0 & & 124 \\
\hline & TI201 & 0 & 0 & 0 & 0 & 0 & 0 & 0 & 0 & & 0 & 0 & 0 & 31 & 0 & 0 & 0 & 0 & 0 & 0 & 0 & 0 & 0 & 69 & 0 & 0 & 0 & 0 & 0 & & 100 \\
\hline & TI201 & 0 & 0 & 0 & 0 & 0 & 0 & 0 & 0 & & 0 & 0 & 0 & 39 & 0 & 0 & 0 & $\underline{0}$ & 0 & 0 & 0 & 0 & 0 & 61 & 0 & 0 & 0 & 0 & 0 & & 100 \\
\hline Y88,10UC & Y88 & 0 & 0 & 0 & 0 & 0 & 0 & 0 & 0 & & 0 & 0 & 0 & 0 & 0 & 0 & 0 & 0 & 0 & 0 & 0 & 0 & 0 & & 100 & 0 & 0 & 0 & 0 & & 100 \\
\hline$Y 88,10 \cup C\{10,10\}$ & Y88 & 0 & 2 & 6 & 11 & 0 & 0 & 0 & 1 & & 0 & 0 & 0 & 0 & 0 & 0 & 0 & 0 & 0 & 0 & 0 & 0 & 0 & 0 & 97 & 0 & 0 & 0 & 0 & & 117 \\
\hline$Y 88,10 \cup C\{10,30\}$ & Y88 & 26 & 3 & 43 & 10 & 0 & 0 & 0 & 1 & & 0 & 0 & 0 & 0 & 0 & 0 & 0 & 0 & 1 & 0 & 0 & 0 & 0 & 0 & 22 & 0 & 0 & 0 & 0 & & 106 \\
\hline$Y 88,10 \cup C\{26,10\}$ & Y88 & 0 & 1 & 4 & 8 & 0 & 0 & 1 & 0 & & 0 & 0 & 0 & 0 & 0 & 0 & 0 & 0 & 0 & 0 & 0 & 0 & 0 & 0 & 99 & 0 & 0 & 0 & 0 & & 113 \\
\hline$Y 88,10 \cup C\{26,30\}$ & Y88 & 8 & 1 & 30 & 7 & 0 & 0 & 0 & 1 & & 1 & 0 & 0 & 0 & 1 & 1 & 0 & 0 & 0 & 0 & 0 & 0 & 0 & 0 & 69 & 0 & 0 & 0 & 0 & & 119 \\
\hline$Y 88,10 \cup C\{74,20\}$ & Y88 & 0 & 0 & 24 & 2 & 0 & 0 & 0 & 0 & & 0 & 0 & 0 & 0 & 0 & 0 & $\underline{0}$ & $\underline{0}$ & 0 & 0 & 0 & 0 & 0 & 0 & 99 & 0 & 0 & 0 & 0 & 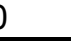 & 125 \\
\hline $\mathrm{Na} 22,10 \mathrm{UC}$ & $\mathrm{Na} 22$ & 0 & 0 & 0 & 0 & 0 & 0 & 0 & 0 & & 0 & 0 & 0 & 0 & 0 & 0 & 0 & 0 & 0 & 0 & 0 & 0 & 0 & 0 & & 100 & 0 & 0 & 0 & 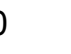 & 100 \\
\hline $\mathrm{Na} 22,10 \cup \mathrm{C}\{10,10\}$ & $\mathrm{Na} 22$ & 2 & 0 & 2 & 3 & 0 & 0 & 0 & 0 & & 0 & 0 & 0 & 0 & 2 & 1 & 0 & 0 & 0 & 0 & 0 & 0 & 0 & 0 & 0 & 93 & 0 & 0 & 0 & 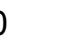 & 103 \\
\hline $\mathrm{Na} 22,10 \cup \mathrm{C}\{10,30\}$ & $\mathrm{Na} 22$ & 54 & 0 & 12 & 3 & 0 & 0 & 0 & 0 & & 4 & 0 & 0 & 0 & 9 & 0 & 0 & 0 & 0 & 0 & 0 & 1 & 0 & 0 & 0 & 18 & 0 & 0 & 0 & 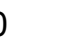 & 101 \\
\hline $\mathrm{Na} 22,10 \cup \mathrm{C}\{26,10\}$ & $\mathrm{Na} 22$ & 0 & 0 & 2 & 2 & 0 & 0 & 0 & 0 & & 0 & 0 & 0 & 0 & 1 & 0 & 0 & 0 & 0 & 0 & 0 & 0 & 0 & 0 & 0 & 98 & 0 & 0 & 0 & & 103 \\
\hline $\mathrm{Na} 22,10 \cup \mathrm{U}\{26,30\}$ & $\mathrm{Na} 22$ & 19 & 0 & 8 & 1 & 0 & 0 & 0 & 0 & & 0 & 0 & 0 & 0 & 22 & 1 & 0 & 0 & 2 & 0 & 0 & 0 & 0 & 0 & 0 & 50 & 0 & 0 & 1 & 1 & 104 \\
\hline Na22,10UC $\{74,20\}$ & $\mathrm{Na} 22$ & 0 & 0 & 1 & 1 & 0 & 0 & 1 & 0 & & 0 & 0 & 0 & 0 & 0 & 0 & 0 & $\underline{0}$ & 0 & 0 & 0 & 0 & 0 & 0 & & 100 & 0 & 0 & 0 & & 103 \\
\hline Ho166m, 10UC & Ho166m & 0 & 0 & 0 & 4 & 0 & 0 & 0 & 0 & & 1 & 25 & 0 & 0 & 0 & 1 & 1 & 1 & 0 & 0 & 0 & 0 & 1 & 0 & 0 & 0 & 75 & 0 & 0 & 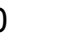 & 108 \\
\hline Ho166m, 10UC $\{10,10\}$ & Ho166m & 5 & 2 & 1 & 6 & 0 & 0 & 0 & 1 & & 1 & 73 & 0 & 0 & 2 & 0 & 1 & 1 & 0 & 0 & 0 & 1 & 3 & 0 & 0 & 0 & 9 & 0 & 0 & 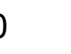 & 105 \\
\hline Ho166m, 10UC $\{10,30\}$ & Ho166m & 14 & 3 & 2 & 12 & 0 & 0 & 0 & 1 & 21 & 1 & 43 & 0 & 0 & 2 & 0 & 1 & 1 & 2 & 0 & 0 & 0 & 4 & 0 & 0 & 0 & 2 & 0 & 0 & 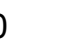 & 107 \\
\hline Ho166m, $10 \cup C\{26,10\}$ & Ho166m & 2 & 0 & 1 & 15 & 0 & 0 & 0 & 0 & & 0 & 63 & 0 & 0 & 2 & 0 & 0 & 0 & 0 & 0 & 0 & 0 & 0 & 0 & 0 & 0 & 30 & 0 & 0 & & 113 \\
\hline Ho166m, $10 \cup C\{26,30\}$ & Ho166m & 7 & 0 & 1 & 27 & 0 & 0 & 0 & 0 & 16 & 64 & 40 & 0 & 0 & 9 & 0 & 0 & 0 & 1 & 0 & 0 & 0 & 0 & 0 & 0 & 0 & 26 & 0 & 0 & & 127 \\
\hline Ho166m,10UC $\{74,20\}$ & Ho166m & 0 & 0 & 0 & 0 & 0 & 0 & 0 & 0 & & 1 & 3 & 0 & 0 & 0 & 2 & $\underline{0}$ & $\underline{0}$ & 0 & 0 & 0 & 0 & 0 & 0 & 0 & & 100 & 0 & 0 & & 106 \\
\hline Eu152,10UC & Eu152 & 9 & 12 & 3 & 0 & 0 & 0 & 0 & 3 & & 0 & 0 & 0 & 0 & 0 & 0 & 0 & 0 & 0 & 0 & 0 & 0 & 0 & 0 & 0 & 0 & 0 & 78 & 0 & & 105 \\
\hline Eu152,10uC $\{10,10\}$ & Eu152 & 44 & 16 & 17 & 3 & 0 & 0 & 0 & 1 & & 0 & 4 & 1 & 0 & 2 & 2 & 0 & 0 & 0 & 0 & 2 & 0 & 0 & 0 & 0 & 0 & 0 & 12 & 0 & & 104 \\
\hline Eu152,10UC $\{10,30\}$ & Eu152 & 48 & 9 & 19 & 8 & 0 & 0 & 0 & 0 & & 1 & 4 & 0 & 0 & 7 & 4 & 0 & 0 & 0 & 0 & 0 & 1 & 1 & 0 & 0 & 0 & 0 & 2 & 0 & 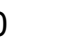 & 104 \\
\hline Eu152,10UC $\{26,10\}$ & Eu152 & 26 & 7 & 17 & 4 & 0 & 0 & 0 & 0 & & 0 & 0 & 0 & 0 & 7 & 1 & 0 & 0 & 1 & 0 & 0 & 0 & 0 & 0 & 0 & 0 & 0 & 40 & 0 & ) & 103 \\
\hline Eu152,10UC $\{26,30\}$ & Eu152 & 32 & 2 & 20 & 4 & 0 & 0 & 0 & 0 & & 1 & 1 & 0 & 1 & 22 & 9 & 0 & 0 & 1 & 0 & 2 & 0 & 0 & 0 & 0 & 0 & 0 & 17 & 0 & 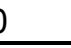 & 112 \\
\hline
\end{tabular}




\begin{tabular}{|c|c|c|c|c|c|c|c|c|c|c|c|c|c|c|c|c|c|c|c|c|c|c|c|c|c|c|c|c|c|}
\hline Isotope_type & Id & ND & $\begin{array}{l}402 \\
\mathrm{~K}\end{array}$ & $\begin{array}{l}226 \\
\mathrm{Ra}\end{array}$ & $\begin{array}{l}232 \\
\text { Th } \\
\end{array}$ & $\begin{array}{l}2282 \\
\mathrm{Th}\end{array}$ & & & $\begin{array}{l}133 \\
\mathrm{Ba} \\
\end{array}$ & & $\begin{array}{l}54 \\
\mathrm{Mn} \\
\end{array}$ & $\begin{array}{l}57 \\
\text { Co }\end{array}$ & $\begin{array}{c}139 \\
\mathrm{Ce}\end{array}$ & $\begin{array}{c}252 \\
\mathrm{Cf}\end{array}$ & $\begin{array}{r}238 \\
U\end{array}$ & $\begin{array}{r}823 \\
U \\
\end{array}$ & $\begin{array}{l}352 \\
\end{array}$ & $\begin{array}{l}392 \\
\mathrm{Pu}\end{array}$ & $\begin{array}{l}2371 \\
\text { Np }\end{array}$ & $\begin{array}{c}13199 \\
1 \quad T\end{array}$ & $\begin{array}{l}99 \mathrm{~m} \\
\text { Tc }\end{array}$ & $\begin{array}{l}672 \\
\mathrm{Ga}\end{array}$ & $\begin{array}{c}201 \\
\text { TI }\end{array}$ & $\begin{array}{c}88 \\
Y\end{array}$ & $\begin{array}{ll}22 & 1 \\
\mathrm{Na} & \\
\end{array}$ & $\begin{array}{l}166 \mathrm{~m} 1 \\
\mathrm{Ho} \\
\end{array}$ & $\begin{array}{l}1521 \\
\mathrm{Eu}\end{array}$ & $\begin{array}{c}192 \\
\text { Ir }\end{array}$ & SUM \\
\hline Ir192,10UC & Ir192 & 0 & 0 & 2 & 0 & 0 & 0 & 0 & 0 & 0 & 0 & 0 & 0 & 0 & & 0 & 0 & 0 & 28 & 0 & 0 & 0 & 0 & 0 & 0 & 0 & 0 & 87 & 117 \\
\hline Ir $192,10 \cup C\{10,30\}$ & Ir192 & 1 & 1 & 0 & 0 & 0 & 0 & 0 & 3 & 0 & 0 & 0 & 0 & 0 & & 0 & 1 & 0 & 44 & 2 & 0 & 69 & 0 & 0 & 0 & 0 & 0 & 4 & 125 \\
\hline Ir $192,10 \cup C\{26,10\}$ & Ir192 & 0 & 0 & 6 & 1 & 0 & 0 & 0 & 0 & 1 & 0 & 0 & 0 & 0 & & 0 & 0 & 0 & 98 & 0 & 0 & 2 & 0 & 0 & 0 & 0 & 0 & 19 & 127 \\
\hline Ir192,10UC $\{26,30\}$ & Ir192 & 0 & 0 & 5 & 3 & 0 & 0 & 0 & 1 & 1 & 0 & 0 & 0 & 0 & & 0 & 0 & 1 & 77 & 0 & 0 & 31 & 0 & 0 & 0 & 0 & 0 & 30 & 149 \\
\hline $\operatorname{Ir} 192,10 \mathrm{mC}\{74,60\}$ & Ir192 & 0 & 0 & 1 & 4 & 0 & 0 & 0 & 0 & 1 & 0 & 0 & 0 & 0 & & 0 & 0 & 0 & 0 & 0 & 0 & 0 & 0 & 0 & 0 & 0 & & 100 & 106 \\
\hline
\end{tabular}

\section{A.4.4.2 PCA Verification Set Correlation Matrix}

\begin{tabular}{|c|c|c|c|c|c|c|c|c|c|c|c|c|c|c|c|c|c|c|c|c|c|c|c|c|c|c|c|c|c|}
\hline Isotope_type & Id & ND & $\begin{array}{l}40 \\
K\end{array}$ & $\begin{array}{c}226 \\
\mathrm{Ra}\end{array}$ & $\begin{array}{c}232 \\
\text { Th } \\
\end{array}$ & $\begin{array}{c}228 \\
\text { Th } \\
\end{array}$ & $\begin{array}{l}232 \\
\mathrm{U}\end{array}$ & $\begin{array}{l}60 \\
\text { Co }\end{array}$ & $\begin{array}{c}133 \\
\mathrm{Ba} \\
\end{array}$ & $\begin{array}{c}137 \\
\text { Cs } \\
\end{array}$ & $\begin{array}{l}754 \\
\mathrm{Mn} \\
\end{array}$ & $\begin{array}{l}57 \\
\text { Co } \\
\end{array}$ & $\begin{array}{c}139 \\
\mathrm{Ce}\end{array}$ & $\begin{array}{r}252 \\
\mathrm{Cf} \\
\end{array}$ & $\begin{array}{l}238 \\
\mathrm{U}\end{array}$ & $\begin{array}{c}235 \\
\mathrm{U}\end{array}$ & $\begin{array}{r}239 \\
\mathrm{Pu}\end{array}$ & $\begin{array}{l}237 \\
\mathrm{~Np}\end{array}$ & $\begin{array}{c}131 \\
\text { I }\end{array}$ & $\begin{array}{c}99 \mathrm{~m} \\
\mathrm{Tc}\end{array}$ & $\begin{array}{l}67 \\
\mathrm{Ga} \\
\end{array}$ & $\begin{array}{c}201 \\
\text { TI }\end{array}$ & $\begin{array}{l}88 \\
Y \\
\end{array}$ & $\begin{array}{l}22 \\
\mathrm{Na}\end{array}$ & $\begin{array}{l}166 \mathrm{~m} \\
\mathrm{Ho}\end{array}$ & $\begin{array}{l}152 \\
\mathrm{Eu}\end{array}$ & $\begin{array}{r}211 \\
4 \quad 1 \\
\end{array}$ & $\begin{array}{l}92 \\
\text { Ir S }\end{array}$ & SUM \\
\hline Total Id & & 56 & 560 & 686 & 532 & 516 & 5668 & 810 & 703 & 700 & 0320 & 200 & 352 & 308 & 786 & 602 & 2995 & 545 & 516 & 314 & 387 & 634 & 620 & 6616 & 620 & $\overline{0604}$ & 27 & 750 & \\
\hline Jorrect & & 0 & 554 & 632 & 463 & 364 & 521 & 774 & . 633 & 689 & 9295 & 200 & 113 & 235 & 737 & 600 & 0879 & 477 & 455 & 300 & 345 & 547 & 600 & 598 & 580 & 0556 & 567 & 704 & 12851 \\
\hline Actual trials & & 0 & 600 & 800 & 700 & 400 & 600 & 800 & 700 & 700 & 0300 & 200 & 200 & 300 & 800 & 600 & 0900 & 500 & 500 & 300 & 400 & 800 & 600 & 600 & 600 & 0600 & 008 & 800 & 14300 \\
\hline False Negative & & & 46 & 168 & 237 & 36 & 79 & 26 & 67 & 11 & 5 & 0 & 87 & 65 & 63 & 0 & 21 & 23 & 45 & 0 & 55 & 253 & 0 & 2 & 20 & & 44 & 96 & 1449 \\
\hline False Positive & & & 6 & 54 & 69 & 152 & 47 & 36 & 70 & 11 & 25 & 0 & 239 & 73 & 49 & 2 & 2116 & 68 & 61 & 14 & 42 & 87 & 20 & 18 & 40 & & 48 & 46 & 1393 \\
\hline $\mathrm{K} 40,1 \mathrm{MC}$ & K40 & & 100 & 0 & 0 & 0 & 0 & 0 & 0 & 0 & 0 & 0 & 0 & 0 & 0 & 0 & 0 & 0 & 0 & 0 & 0 & 0 & 0 & U & U & 0 & 0 & 0 & 100 \\
\hline $\mathrm{K} 40,1 \mathrm{MC}\{10,10\}$ & K40 & 2 & 98 & 0 & 0 & 0 & 0 & 0 & 0 & 0 & 0 & 0 & 0 & 0 & 0 & 0 & 0 & 0 & 0 & 0 & 0 & 0 & 0 & 0 & 0 & 0 & 0 & 0 & 100 \\
\hline $\mathrm{K} 40,1 \mathrm{MC}\{10,30\}$ & K40 & 0 & 92 & 0 & 1 & 0 & 0 & 2 & 0 & 0 & 0 & 0 & 0 & 0 & 0 & 0 & 0 & 0 & 0 & 0 & 0 & 0 & 0 & 0 & 0 & 5 & 5 & 0 & 100 \\
\hline $\mathrm{K} 40,1 \mathrm{MC}\{10,60\}$ & K40 & 1 & 70 & 3 & 4 & 0 & 2 & 0 & 2 & 1 & 0 & 0 & 0 & 0 & 0 & 0 & 6 & 0 & 0 & 0 & 3 & 0 & 0 & 0 & 1 & & 0 & 7 & 100 \\
\hline $\mathrm{K} 40,1 \mathrm{MC}\{26,10\}$ & K40 & & 100 & 0 & 0 & 0 & 0 & 0 & 0 & 0 & 0 & 0 & 0 & 0 & 0 & 0 & 0 & 0 & 0 & 0 & 0 & 0 & 0 & 0 & 0 & 0 & 0 & 0 & 100 \\
\hline $\mathrm{K} 40,1 \mathrm{MC}\{26,30\}$ & $\mathrm{K} 40$ & 0 & 94 & 0 & 0 & 0 & 0 & 1 & 0 & 0 & 0 & 0 & 0 & 0 & 1 & 0 & 0 & 0 & 0 & 0 & 0 & 0 & 0 & 0 & 0 & 3 & 3 & 1 & 100 \\
\hline Ra226,10UC & Ra226 & 0 & & 100 & 0 & 0 & 0 & 0 & 0 & 0 & 0 & 0 & 0 & 0 & 0 & 0 & 0 & 0 & 0 & 0 & 0 & 0 & 0 & 0 & 0 & & 0 & 0 & 100 \\
\hline $\operatorname{Ra} 226,10 \cup C\{10,10\}$ & Ra226 & 1 & 0 & 90 & 0 & 0 & 0 & 0 & 1 & 1 & 0 & 0 & 0 & 0 & 0 & 0 & 3 & 0 & 3 & 0 & 0 & 0 & 0 & 0 & 0 & 0 & 0 & 1 & 100 \\
\hline $\operatorname{Ra} 226,10 \cup C\{10,30\}$ & $\mathrm{Ra} 226$ & 3 & 0 & 65 & 5 & 1 & 0 & 3 & 0 & 3 & 1 & 0 & 0 & 2 & 2 & 0 & 3 & 0 & 1 & 0 & 1 & 0 & 2 & 1 & 2 & 4 & 4 & 1 & 100 \\
\hline $\operatorname{Ra} 226,10 \cup C\{10,60\}$ & $\mathrm{Ra} 226$ & 4 & 2 & 48 & 8 & 3 & 0 & 4 & 0 & 1 & 1 & 0 & 0 & 4 & 4 & 0 & 3 & 0 & 0 & 0 & 0 & 0 & 1 & 2 & 3 & 8 & 8 & 4 & 100 \\
\hline $\operatorname{Ra} 226,10 \cup C\{26,10\}$ & $\mathrm{Ra} 226$ & 0 & 0 & 99 & 0 & 0 & 0 & 0 & 0 & 0 & 0 & 0 & 0 & 0 & 0 & 0 & 1 & 0 & 0 & 0 & 0 & 0 & 0 & 0 & 0 & & 0 & 0 & 100 \\
\hline
\end{tabular}




\begin{tabular}{|c|c|c|c|c|c|c|c|c|c|c|c|c|c|c|c|c|c|c|c|c|c|c|c|c|c|c|c|c|c|c|c|c|}
\hline Isotope_type & Id & ND & $\begin{array}{c}40 \\
K\end{array}$ & $\begin{array}{l}220 \\
\mathrm{Ra}\end{array}$ & Th & $\begin{array}{c}228 \\
\text { Th }\end{array}$ & & $\begin{array}{l}60 \\
\mathrm{Co} \\
\end{array}$ & $\begin{array}{c}133 \\
\mathrm{Ba} \\
\end{array}$ & Cs & & $\begin{array}{l}54 \\
\mathrm{Mn} \\
\end{array}$ & $\begin{array}{l}57 \\
\text { Co } \\
\end{array}$ & $\mathrm{Ce}$ & & 522 & $\mathrm{U}$ & $\begin{array}{c}2352 \\
\mathrm{U}\end{array}$ & $\begin{array}{l}239 \\
\mathrm{Pu}\end{array}$ & $\begin{array}{l}237 \\
\mathrm{~Np}\end{array}$ & $\begin{array}{r}13 \\
\mathrm{I}\end{array}$ & $\begin{array}{r}199 \\
T\end{array}$ & $\begin{array}{l}9 \mathrm{~m} \\
\mathrm{Tc}\end{array}$ & $\begin{array}{l}67 \\
\mathrm{Ga} \\
\end{array}$ & $\begin{array}{c}201 \\
\text { TI }\end{array}$ & $\begin{array}{l}88 \\
Y\end{array}$ & $\begin{array}{l}22 \\
\mathrm{Na}\end{array}$ & $\begin{array}{l}166 m \\
\text { Ho }\end{array}$ & $\begin{array}{l}152 \\
\text { Eu }\end{array}$ & $\begin{array}{c}192 \\
\text { Ir }\end{array}$ & SUN & \\
\hline $\operatorname{Ra} 226,10 \cup C\{26,30\}$ & Ra226 & 0 & 0 & 78 & 1 & 1 & 0 & 0 & 0 & & 0 & 0 & 0 & 0 & 01 & 11 & 1 & 0 & 2 & 0 & & 0 & 0 & 0 & 0 & 2 & 0 & 0 & 3 & 1 & & 100 \\
\hline $\operatorname{Ra} 226,10 \cup C\{26,50\}$ & Ra226 & 1 & 0 & 58 & 2 & 1 & 0 & 3 & 0 & & 1 & 1 & 0 & 0 & 2 & 21 & 1 & 0 & 2 & 0 & & 0 & 0 & 0 & 0 & 3 & 1 & 0 & 5 & 0 & & 100 \\
\hline $\operatorname{Ra} 226,100 \cup C\{74,40\}$ & $\mathrm{Ra} 226$ & 0 & 0 & 94 & 0 & 0 & 0 & 1 & 0 & & 0 & 0 & 0 & 0 & 0 & 1 & 0 & 0 & 0 & 0 & & 0 & 0 & 0 & 0 & 3 & 0 & 0 & 1 & 0 & & 100 \\
\hline Th232,10UC & Th232 & 1 & 0 & 0 & 87 & 11 & 0 & 0 & 0 & & 0 & 0 & 0 & 0 & 0 & 0 & 0 & 0 & 0 & 0 & & 0 & 0 & 0 & 0 & 1 & 0 & 0 & 0 & 0 & & 100 \\
\hline Th232,10UC $\{10,10\}$ & Th232 & 4 & 0 & 2 & 66 & 21 & 1 & 0 & 0 & & 0 & 0 & 0 & 0 & 0 & 1 & 1 & 0 & 1 & 0 & & 0 & 0 & 1 & 0 & 1 & 0 & 0 & 1 & 0 & & 100 \\
\hline Th232,10UC $\{10,30\}$ & Th232 & 6 & 0 & 4 & 44 & 19 & 6 & 1 & 0 & & 1 & 1 & 0 & 0 & 0 & 4 & 4 & 0 & 2 & 0 & & 0 & 0 & 0 & 0 & 0 & 1 & 5 & 0 & 2 & & 100 \\
\hline Th232,10UC $\{10,60\}$ & Th232 & 6 & 0 & 7 & 35 & 16 & 14 & 0 & 1 & & 3 & 1 & 0 & 0 & 0 & 2 & 0 & 0 & 2 & 0 & & 1 & 0 & 1 & 0 & 0 & 0 & 6 & 2 & 3 & & 100 \\
\hline Th232,10UC $\{26,10\}$ & Th232 & 4 & 0 & 1 & 76 & 12 & 0 & 0 & 0 & & 0 & 0 & 0 & 0 & 0 & 4 & 0 & 0 & 3 & 0 & & 0 & 0 & 0 & 0 & 0 & 0 & 0 & 0 & 0 & & 100 \\
\hline Th232,10UC $\{26,30\}$ & Th232 & 4 & 0 & 2 & 59 & 10 & 1 & 0 & 0 & & 0 & 0 & 0 & 0 & 1 & 13 & 6 & 0 & 4 & 0 & & 0 & 0 & 0 & 0 & 1 & 0 & 0 & 0 & 0 & & 100 \\
\hline Th232,100UC $\{74,20\}$ & Th232 & 0 & 0 & 0 & 96 & 0 & 1 & 0 & 0 & & 0 & 0 & 0 & 0 & 0 & 1 & 2 & 0 & 0 & 0 & & 0 & 0 & 0 & 0 & 0 & 0 & 0 & 0 & 0 & & 100 \\
\hline Th228,10UC & Th228 & 0 & 0 & 0 & 1 & 99 & 0 & 0 & 0 & & 0 & 0 & 0 & 0 & 0 & 0 & 0 & 0 & 0 & 0 & & 0 & 0 & 0 & 0 & 0 & 0 & 0 & 0 & 0 & & 100 \\
\hline Th228,10UC $\{13,10\}$ & Th228 & 1 & 0 & 0 & 2 & 96 & 1 & 0 & 0 & & 0 & 0 & 0 & 0 & 0 & 0 & 0 & 0 & 0 & 0 & & 0 & 0 & 0 & 0 & 0 & 0 & 0 & 0 & 0 & & 100 \\
\hline Th228,10UC $\{13,30\}$ & Th228 & 4 & 0 & 1 & 5 & 71 & 18 & 0 & 0 & & 0 & 0 & 0 & 0 & 0 & 1 & 0 & 0 & 0 & 0 & & 0 & 0 & 0 & 0 & 0 & 0 & 0 & 0 & 0 & & 100 \\
\hline $\operatorname{Th} 228,10 \cup C\{26,10\}$ & Th228 & 0 & 0 & 0 & 2 & 98 & 0 & 0 & 0 & & 0 & 0 & 0 & 0 & 0 & 0 & 0 & 0 & 0 & 0 & & 0 & 0 & 0 & 0 & 0 & 0 & 0 & 0 & 0 & & 100 \\
\hline$U 232,50 \cup C\{92,20 ; 13,10\}$ & $\mathrm{U} 232$ & 0 & 0 & 0 & 2 & 4 & 94 & 0 & 0 & & 0 & 0 & 0 & 0 & 0 & 0 & 0 & 0 & 0 & 0 & & 0 & 0 & 0 & 0 & 0 & 0 & 0 & 0 & 0 & & 100 \\
\hline$\cup 232,50 \cup C\{92,20 ; 13,30\}$ & $\mathrm{u} 232$ & 1 & 0 & 0 & 2 & 13 & 81 & 0 & 0 & & 0 & 0 & 0 & 0 & 0 & 1 & 0 & 0 & 1 & 0 & & 0 & 0 & 0 & 0 & 1 & 0 & 0 & 0 & 0 & & 100 \\
\hline$U 232,50 \cup C\{92,20 ; 13,50\}$ & 4232 & 4 & 0 & 0 & 6 & 17 & 66 & 0 & 0 & & 0 & 0 & 0 & 0 & 0 & 2 & 0 & 0 & 3 & 0 & & 1 & 0 & 1 & 0 & 0 & 0 & 0 & 0 & 0 & & 100 \\
\hline$\cup 232,50 \cup \subset\{\{2,20 ; 26,10\}$ & 0232 & 0 & 0 & 0 & 2 & 2 & 96 & 0 & 0 & & 0 & 0 & 0 & 0 & 0 & 0 & 0 & 0 & 0 & 0 & & 0 & 0 & 0 & 0 & 0 & 0 & 0 & 0 & 0 & & 100 \\
\hline$\cup 232,50 \cup C\{92,20 ; 26,30\}$ & 4232 & 1 & 0 & 0 & 4 & 8 & 85 & 0 & 0 & & 0 & 0 & 0 & 0 & 0 & 1 & 0 & 0 & 0 & 0 & & 0 & 0 & 0 & 0 & 1 & 0 & 0 & 0 & 0 & & 100 \\
\hline U232,50UC $\{92,20 ; 74,10\}$ & $\mathrm{u} 232$ & 0 & 0 & 0 & 0 & 1 & 99 & 0 & 0 & & 0 & 0 & 0 & 0 & 0 & 0 & 0 & 0 & 0 & 0 & & 0 & 0 & 0 & 0 & 0 & 0 & 0 & 0 & 0 & & 100 \\
\hline Co60,10UC & Co60 & 0 & 0 & 0 & 0 & 0 & & 100 & 0 & & 0 & 0 & 0 & 0 & 0 & 0 & 0 & 0 & 0 & 0 & & 0 & 0 & 0 & 0 & 0 & 0 & 0 & 0 & 0 & & 100 \\
\hline Co60,10UC $\{10,10\}$ & Co60 & 0 & 0 & 0 & 0 & 0 & & 100 & 0 & & 0 & 0 & 0 & 0 & 0 & 0 & 0 & 0 & 0 & 0 & & 0 & 0 & 0 & 0 & 0 & 0 & 0 & 0 & 0 & & 100 \\
\hline Co60,10UC $\{10,30\}$ & Co60 & 0 & 0 & 0 & 0 & 0 & 0 & 97 & 0 & & 0 & 0 & 0 & 0 & 0 & 1 & 0 & 0 & 0 & 0 & & 0 & 0 & 0 & 0 & 0 & 0 & 0 & 2 & 0 & & 100 \\
\hline Co60,10UC $\{10,60\}$ & Co60 & 1 & 1 & 5 & 0 & 0 & 0 & 79 & 0 & & 0 & 0 & 0 & 0 & 0 & 1 & 3 & 0 & 3 & 0 & & 0 & 0 & 0 & 0 & 0 & 0 & 1 & 6 & 0 & & 100 \\
\hline $\mathrm{Co} 60,10 \cup \mathrm{C}\{26,10\}$ & Co60 & 0 & 0 & 0 & 0 & 0 & & 100 & 0 & & 0 & 0 & 0 & 0 & 0 & 0 & 0 & 0 & 0 & 0 & & 0 & 0 & 0 & 0 & 0 & 0 & 0 & 0 & 0 & & 100 \\
\hline Co60,10UC $\{26,30\}$ & Co60 & 0 & 0 & 0 & 0 & 0 & 0 & 99 & 0 & & 0 & 0 & 0 & 0 & 0 & 0 & 0 & 0 & 0 & 0 & & 0 & 0 & 0 & 0 & 0 & 0 & 0 & 1 & 0 & & 100 \\
\hline Co60,10UC $\{74,20\}$ & Co60 & 0 & 0 & 0 & 0 & 0 & & 100 & 0 & & 0 & 0 & 0 & 0 & 0 & 0 & 0 & 0 & 0 & 0 & & 0 & 0 & 0 & 0 & 0 & 0 & 0 & 0 & 0 & & 100 \\
\hline Co60,10UC $\{74,40\}$ & Co60 & 0 & 0 & 0 & 0 & 0 & 0 & 99 & 0 & & 0 & 0 & 0 & 0 & 0 & 0 & 0 & 0 & 0 & 0 & & 0 & 0 & 0 & 0 & 0 & 0 & 0 & 1 & 0 & & 100 \\
\hline Ba133,10UC & Ba133 & 0 & 0 & 0 & 0 & 0 & 0 & 0 & 94 & & 0 & 0 & 0 & 0 & 0 & 0 & 0 & 0 & 0 & 0 & & 6 & 0 & 0 & 0 & 0 & 0 & 0 & 0 & 0 & & 100 \\
\hline
\end{tabular}




\begin{tabular}{|c|c|c|c|c|c|c|c|c|c|c|c|c|c|c|c|c|c|c|c|c|c|c|c|c|c|c|c|c|c|c|c|}
\hline Isotope_type & Id & ND & $\begin{array}{l}40 \\
\mathrm{~K} \\
\end{array}$ & $\begin{array}{c}226 \\
\mathrm{Ra} \\
\end{array}$ & $\begin{array}{c}232 \\
\text { Th } \\
\end{array}$ & $\begin{array}{c}228 \\
\text { Th }\end{array}$ & $\mathrm{U}$ & $\begin{array}{l}60 \\
\text { Co } \\
\end{array}$ & $\begin{array}{c}133 \\
\mathrm{Ba} \\
\end{array}$ & $\begin{array}{r}137 \\
\text { Cs } \\
\end{array}$ & $\begin{array}{l}754 \\
5 \mathrm{Mn} \\
\end{array}$ & $\begin{array}{l}57 \\
\text { Co } \\
\end{array}$ & $\begin{array}{r}139 \\
\mathrm{Ce} \\
\end{array}$ & $\begin{array}{c}252 \\
\mathrm{Cf} \\
\end{array}$ & $\begin{array}{r}238 \\
\mathrm{U}\end{array}$ & $\begin{array}{r}823 \\
U\end{array}$ & & $\begin{array}{l}392 \\
\mathrm{Pu}\end{array}$ & $\begin{array}{l}237 \\
\mathrm{~Np} \\
\end{array}$ & $\begin{array}{c}131 \\
\text { I }\end{array}$ & $\begin{array}{c}99 \mathrm{~m} \\
\mathrm{Tc}\end{array}$ & & $\begin{array}{ll}37 \\
3 a\end{array}$ & $\begin{array}{l}201 \\
\mathrm{TI}\end{array}$ & $\begin{array}{l}88 \\
Y \\
\end{array}$ & $\begin{array}{ll}22 \\
\mathrm{Na}\end{array}$ & $\begin{array}{l}166 \mathrm{~m} \\
\mathrm{Ho} \\
\end{array}$ & $\begin{array}{l}152 \\
\text { Eu } \\
\end{array}$ & $\begin{array}{c}192 \\
\text { Ir }\end{array}$ & SUn & \\
\hline $\mathrm{Ba} 133,10 \cup \mathrm{U}\{10,10\}$ & Ba133 & 0 & 0 & 0 & 0 & 0 & 0 & 0 & 88 & & $\begin{array}{ll}0 & 0\end{array}$ & 0 & 0 & 0 & 00 & 0 & 0 & 0 & 0 & 12 & 0 & 5 & 0 & 0 & 0 & 0 & 0 & 0 & 0 & 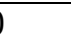 & 100 \\
\hline $\mathrm{Ba} 133,10 \cup \mathrm{C}\{10,30\}$ & Ba133 & 0 & 0 & 0 & 0 & 0 & 0 & 0 & 83 & & 0 & 0 & 0 & 0 & 0 & 0 & 0 & 1 & 1 & 12 & 0 & 0 & 2 & 0 & 0 & 0 & 0 & 0 & 1 & 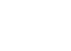 & 100 \\
\hline $\mathrm{Ba} 133,10 \cup \mathrm{C}\{10,50\}$ & Ba133 & 0 & 0 & 0 & 0 & 0 & 0 & 0 & 76 & & 0 & 0 & 0 & 0 & 0 & 0 & 0 & 1 & 12 & 7 & 0 & D & 0 & 0 & 0 & 0 & 0 & 0 & 4 & + & 100 \\
\hline $\operatorname{Ba} 133,10 \cup C\{26,10\}$ & $\mathrm{Ba} 133$ & 0 & 0 & 0 & 0 & 0 & 0 & 0 & 94 & & 0 & 0 & 0 & 0 & b & 0 & 0 & 0 & 0 & 6 & 0 & b & 0 & 0 & 0 & 0 & 0 & 0 & 0 & ) & 100 \\
\hline $\mathrm{Ba} 133,10 \cup \mathrm{C}\{26,30\}$ & $\mathrm{Ba} 13$ & 0 & 0 & 0 & 0 & 0 & 0 & & 100 & & 0 & 0 & 0 & 0 & 0 & 0 & 0 & 0 & 0 & 0 & 0 & 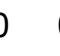 & 0 & 0 & 0 & 0 & 0 & 0 & 0 & 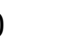 & 100 \\
\hline $\mathrm{Ba} 133,100 \cup \mathrm{C}\{74,10\}$ & $\mathrm{Ba} 133$ & 0 & 0 & 0 & 0 & 0 & 0 & 0 & 98 & & 0 & 0 & 0 & 0 & 0 & 0 & 0 & 0 & 0 & 2 & 0 & D & 0 & 0 & 0 & 0 & 0 & 0 & 0 & 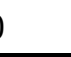 & 100 \\
\hline Cs137,10UC & Cs137 & 0 & 0 & 0 & 0 & 0 & 0 & 0 & 0 & 100 & 0 & 0 & 0 & 0 & 0 & 0 & 0 & 0 & 0 & 0 & 0 & D & 0 & 0 & 0 & 0 & 0 & 0 & 0 & 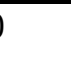 & 100 \\
\hline Cs137,10UC $\{10,10\}$ & Cs137 & 0 & 0 & 0 & 0 & 0 & 0 & 0 & 0 & 100 & 0 & 0 & 0 & 0 & 0 & 0 & 0 & 0 & 0 & 0 & 0 & b & 0 & 0 & 0 & 0 & 0 & 0 & 0 & 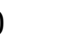 & 10 \\
\hline Cs137,10UC $\{10,30\}$ & Cs137 & 0 & 0 & 0 & 0 & 0 & 0 & 0 & 0 & 99 & 9 & 0 & 0 & 0 & 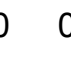 & 0 & 0 & 1 & 0 & 0 & 0 & b & 0 & 0 & 0 & 0 & 0 & 0 & 0 & 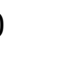 & 100 \\
\hline Cs $137,10 \cup C\{10,60\}$ & Cs137 & 0 & 1 & 0 & 0 & 0 & 0 & 0 & 0 & 90 & 0 & 0 & 0 & 0 & 0 & 0 & 0 & 6 & 0 & 0 & 0 & D & 0 & 0 & 0 & 0 & 1 & 0 & 2 & & 100 \\
\hline Cs $137,10 \cup C\{26,10\}$ & Cs137 & 0 & 0 & 0 & 0 & 0 & 0 & 0 & 0 & 100 & 0 & 0 & 0 & 0 & 0 & 0 & 0 & 0 & 0 & 0 & 0 & D & 0 & 0 & 0 & 0 & 0 & 0 & 0 & & 100 \\
\hline Cs137,10UC $\{26,30\}$ & Cs137 & 0 & 0 & 0 & 0 & 0 & 0 & 0 & 0 & 100 & 0 & 0 & 0 & 0 & 0 & 0 & 0 & 0 & 0 & 0 & 0 & b & 0 & 0 & 0 & 0 & 0 & 0 & 0 & & 100 \\
\hline Cs13 & Cs137 & 0 & 0 & 0 & 0 & 0 & 0 & 0 & 0 & 100 & 0 & 0 & 0 & 0 & 0 & 0 & 0 & 0 & 0 & 0 & 0 & ) & 0 & 0 & 0 & 0 & 0 & 0 & 0 & & 100 \\
\hline Mn54,10UC & Mn54 & 0 & 0 & 0 & 0 & 0 & 0 & 0 & 0 & 0 & 0100 & 0 & 0 & 0 & 5 & 0 & 0 & 0 & 0 & 0 & 0 & 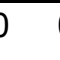 & 0 & 0 & 0 & 0 & 0 & 0 & 0 & & 100 \\
\hline $\operatorname{Mn} 54,10 \cup C\{13,10\}$ & Mn54 & 0 & 0 & 0 & 0 & 0 & 0 & 0 & 0 & 0 & 0100 & 0 & 0 & 0 & 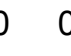 & 0 & 0 & 0 & 0 & 0 & 0 & 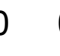 & 0 & 0 & 0 & 0 & 0 & 0 & 0 & & 100 \\
\hline $\operatorname{Mn} 54,10 \cup C\{13,30\}$ & Mn54 & 0 & 0 & 0 & 0 & 0 & 0 & 0 & 0 & 0 & 95 & 0 & 0 & 0 & 0 & 0 & 0 & 0 & 0 & 0 & 0 & 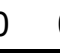 & 0 & 0 & 0 & 0 & 5 & 0 & 0 & & 100 \\
\hline Co57,10UC & Co57 & 0 & 0 & 0 & 0 & 0 & 0 & 0 & 0 & 0 & 0 & 100 & 0 & 0 & b & 0 & 0 & 0 & 0 & 0 & 0 & ) & 0 & 0 & 0 & 0 & 0 & 0 & 0 & & 100 \\
\hline $\mathrm{Co} 57,10 \cup C\{13,10\}$ & & 0 & 0 & 0 & 0 & 0 & 0 & 0 & 0 & 0 & 0 & 100 & $\begin{array}{l}0 \quad 0 \\
\end{array}$ & 0 & 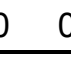 & 0 & 0 & 0 & 0 & 0 & 0 & 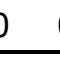 & 0 & 0 & 0 & 0 & 0 & 0 & 0 & & 100 \\
\hline Ce139,10UC & Ce139 & 0 & 0 & 0 & 0 & 0 & 0 & 0 & 0 & 0 & 0 & & 0100 & 0 & 5 & 0 & 0 & 0 & 0 & 0 & 0 & 5 & 0 & 0 & 0 & 0 & 0 & 0 & 0 & & 100 \\
\hline Ce139,10UC $\{13,10\}$ & Ce139 & 0 & 0 & 0 & 0 & 0 & 0 & 0 & 0 & 0 & 0 & 0 & 13 & 0 & b & 0 & 0 & 0 & 0 & 0 & 0 & ) & 0 & 87 & 0 & 0 & 0 & 0 & 0 & & 100 \\
\hline Nylon1-Cf & Cf252 & 0 & 0 & 10 & 8 & 8 & 3 & 0 & 0 & 0 & 0 & 0 & 0 & 69 & & 0 & 0 & 1 & 0 & 0 & 0 & D & 0 & 0 & 0 & 0 & 1 & 0 & 0 & & 100 \\
\hline Nylon1-Cf $\{90,1\}$ & Cf252 & 0 & 0 & 0 & 2 & 1 & 0 & 0 & 0 & 0 & 0 & 0 & 0 & 96 & & 0 & 0 & 0 & 0 & 0 & 0 & כ & 0 & 0 & 1 & 0 & 0 & 0 & 0 & & 100 \\
\hline $\mathrm{Fe} 1-\mathrm{Cf}$ & Cf252 & 1 & 0 & 6 & 4 & 1 & 0 & 2 & 0 & 0 & 0 & 0 & 0 & 70 & & 8 & 0 & 3 & 0 & 0 & 0 & 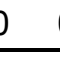 & 0 & 0 & 1 & 2 & 0 & 1 & 0 & & 100 \\
\hline $1 \mathrm{KGU} 238$ & 4238 & 0 & 0 & 0 & 0 & 0 & 0 & 0 & 0 & 0 & 0 & 0 & 0 & 0 & 5100 & & 0 & 0 & 0 & 0 & 0 & D & 0 & 0 & 0 & 0 & 0 & 0 & 0 & & 100 \\
\hline $1 \mathrm{KGU} 238\{13,10\}$ & 0238 & 1 & 0 & 2 & 1 & 0 & 0 & 3 & 0 & 0 & 0 & 0 & 0 & 0 & 89 & 9 & 0 & 1 & 0 & 0 & 0 & ) & 0 & 0 & 0 & 1 & 1 & 1 & 0 & & 100 \\
\hline $1 K G \cup 238\{13,30\}$ & $\mathrm{U} 238$ & 2 & 0 & 2 & 3 & 0 & 0 & 2 & 0 & 0 & 0 & 0 & 0 & 1 & 73 & 3 & 0 & 1 & 0 & 0 & 0 & b & 0 & 0 & 1 & 2 & 8 & 2 & 1 & & 100 \\
\hline $1 \mathrm{KGU} 238\{13,50\}$ & $\mathrm{U} 238$ & 0 & 0 & 0 & 1 & 1 & 0 & 2 & 0 & 0 & 0 & 0 & 0 & 0 & 86 & 6 & 0 & 0 & 0 & 0 & 0 & b & 0 & 0 & 0 & 1 & 2 & 1 & 1 & & 100 \\
\hline $1 \mathrm{KGU} 238\{26,10\}$ & $\mathrm{U} 238$ & 0 & 0 & 1 & 0 & 0 & 0 & 0 & 0 & 0 & 0 & 0 & 0 & 1 & 95 & 5 & 0 & 0 & 0 & 0 & 0 & b & 0 & 0 & 0 & 2 & 1 & 0 & 0 & & 100 \\
\hline $1 \mathrm{KGU} 238\{26,30\}$ & $\mathrm{U} 238$ & 0 & 0 & 0 & 0 & 0 & 0 & 0 & 0 & 0 & 0 & 0 & 0 & 0 & 94 & 4 & 0 & 0 & 0 & 0 & 0 & b & 0 & 0 & 0 & 3 & 2 & 1 & 0 & & 100 \\
\hline
\end{tabular}




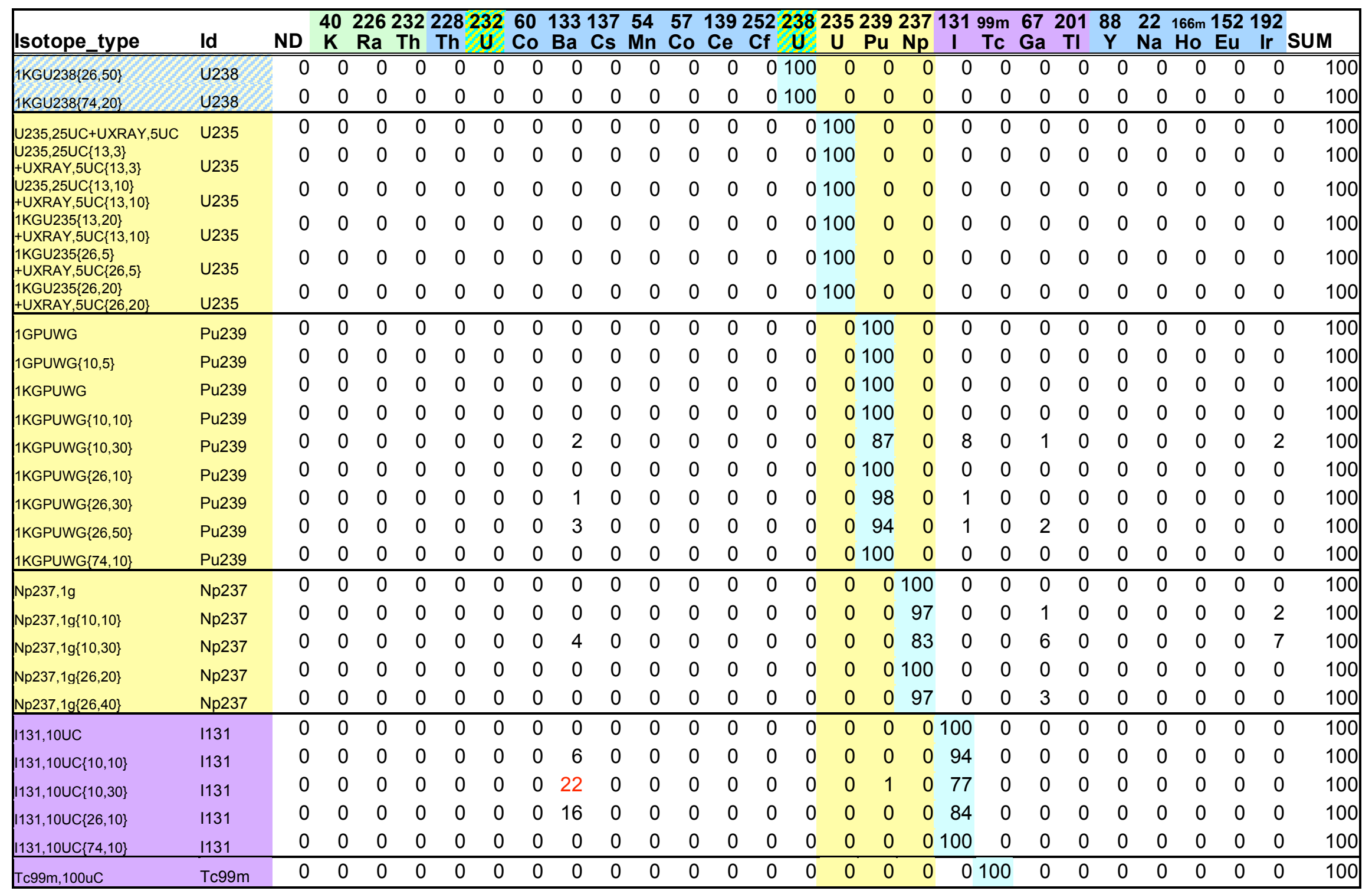




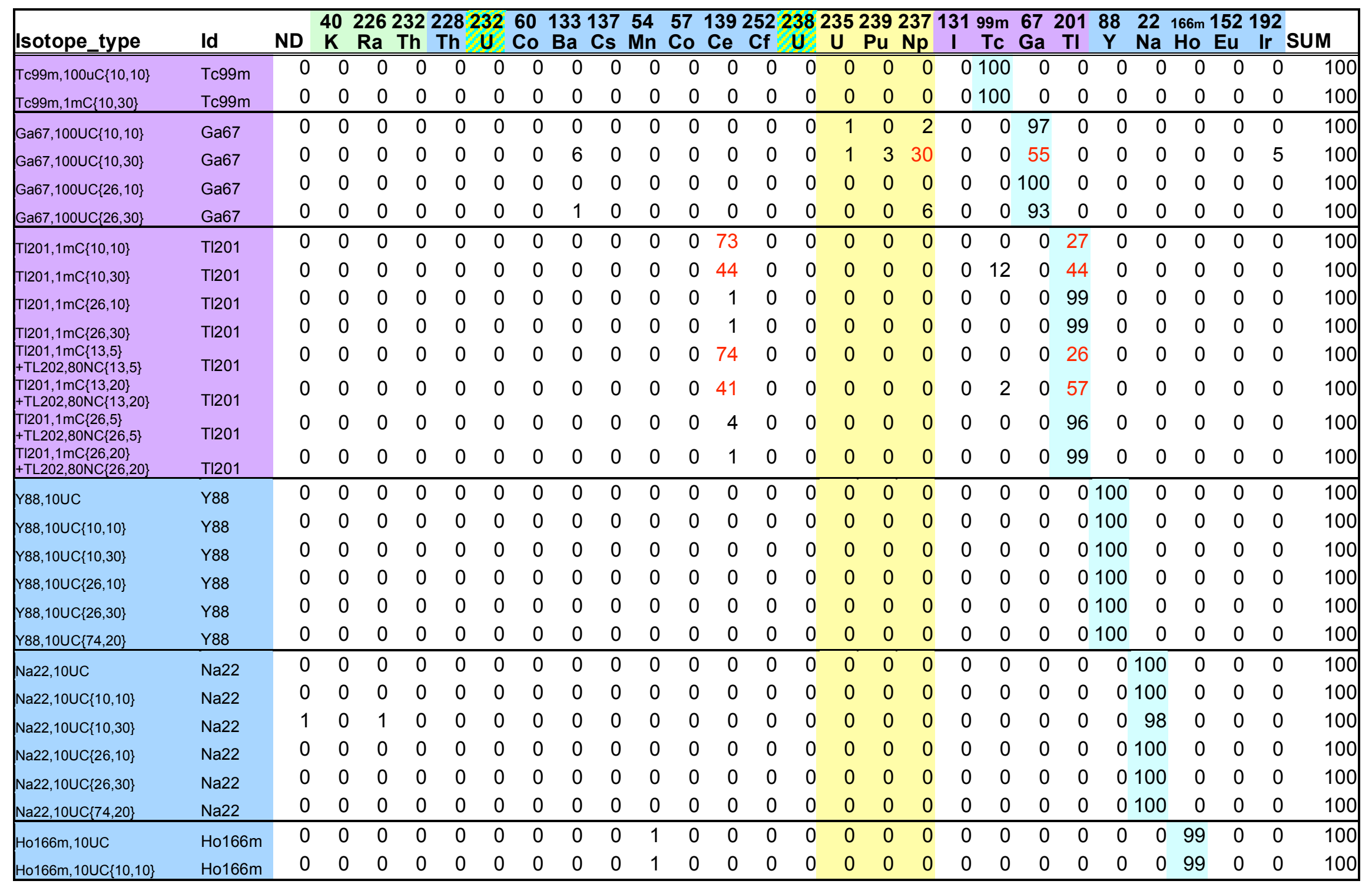




\begin{tabular}{|c|c|c|c|c|c|c|c|c|c|c|c|c|c|c|c|c|c|c|c|c|c|c|c|c|c|c|c|c|c|}
\hline Isotope_type & Id & ND & $\begin{array}{r}40 \\
K\end{array}$ & $\begin{array}{c}226 \\
\mathrm{Ra} \\
\end{array}$ & Th & $\begin{array}{c}228 \\
\mathrm{Th} \\
\end{array}$ & $\mathrm{U}$ & Co & $\begin{array}{l}133 \\
\mathrm{Ba} \\
\end{array}$ & Cs & & 4 & $\begin{array}{l}57 \\
\text { Co } \\
\end{array}$ & $\begin{array}{c}1392 \\
\mathrm{Ce}\end{array}$ & $\begin{array}{c}2522 \\
\mathrm{Cf}\end{array}$ & 238 & $\begin{array}{c}2352 \\
\mathrm{U}\end{array}$ & $\begin{array}{l}239 \\
\mathrm{Pu}\end{array}$ & $\begin{array}{l}237 \\
\mathrm{~Np}\end{array}$ & $\begin{array}{c}131 \\
I\end{array}$ & $\begin{array}{l}99 \mathrm{~m} \\
\text { Tc }\end{array}$ & $\begin{array}{l}67 \\
\mathbf{G a} \\
\end{array}$ & $\begin{array}{c}201 \\
\mathrm{TI}\end{array}$ & $\begin{array}{l}88 \\
Y \\
\end{array}$ & $\begin{array}{ll}22 & 1 \\
\mathrm{Na} & 1 \\
\end{array}$ & $\begin{array}{l}166 m \\
\mathrm{Ho}\end{array}$ & $\begin{array}{c}152 \\
\mathrm{Eu}\end{array}$ & $\begin{array}{l}192 \\
\text { Ir }\end{array}$ & SUM \\
\hline Ho166m, 10UC $\{10,30\}$ & Ho166m & 1 & 0 & 0 & 3 & 0 & 0 & 0 & 0 & & 0 & 9 & 0 & 0 & 0 & 2 & 0 & 1 & 0 & 0 & 0 & 0 & 0 & 0 & 0 & 84 & 0 & 0 & 100 \\
\hline Ho166m, 10UC $\{26,10\}$ & Ho166m & 0 & 0 & 0 & 0 & 0 & 0 & 0 & 0 & & 0 & 0 & 0 & 0 & 0 & 0 & 0 & 0 & 0 & 0 & 0 & 0 & 0 & 0 & & 100 & 0 & 0 & 100 \\
\hline & lo166m & 0 & 0 & 0 & 0 & 0 & 0 & 0 & 0 & & 0 & 0 & 0 & 0 & 0 & 1 & 0 & 0 & 0 & 0 & 0 & 0 & 0 & 0 & 1 & 98 & 0 & 0 & 100 \\
\hline Ho166m,10UC $\{74,20\}$ & Ho166m & 0 & 0 & 0 & 0 & 0 & 0 & 0 & 0 & & 0 & 0 & 0 & 0 & 0 & 0 & 0 & 0 & 0 & 0 & 0 & 0 & 0 & 0 & & 100 & 0 & 0 & 100 \\
\hline Eu152,10UC & Eu152 & 0 & 0 & 0 & 0 & 0 & 0 & 0 & 0 & & 0 & 0 & 0 & 0 & 0 & 0 & 0 & 0 & 0 & 0 & 0 & 0 & 0 & 0 & 0 & & 100 & 0 & 100 \\
\hline Eu152,10UC $\{10,10\}$ & Eu152 & 0 & 0 & 0 & 0 & 0 & 0 & 1 & 0 & & 0 & 0 & 0 & 0 & 0 & 1 & 0 & 1 & 0 & 0 & 0 & 0 & 0 & 0 & 0 & 0 & 97 & 0 & 100 \\
\hline Eu152,10UC $\{10,30\}$ & Eu152 & 1 & 2 & 6 & 0 & 1 & 0 & 8 & 0 & & 0 & 1 & 0 & 0 & 0 & 6 & 0 & 2 & 0 & 0 & 0 & 0 & 0 & 1 & 0 & 1 & 70 & 1 & 100 \\
\hline Eu152,10UC $\{26,10\}$ & u152 & 0 & 0 & 0 & 0 & 0 & 0 & 1 & 0 & & 0 & 0 & 0 & 0 & 0 & 1 & 0 & 0 & 0 & 0 & 0 & 0 & 0 & 0 & 0 & 0 & 98 & 0 & 100 \\
\hline Eu152,10UC $\{26,30\}$ & Eu152 & 0 & 0 & 1 & 0 & 0 & 0 & 2 & 0 & & 0 & 0 & 0 & 0 & 0 & 4 & 0 & 0 & 0 & 0 & 0 & 0 & 0 & 0 & 1 & 0 & 92 & 0 & 100 \\
\hline Eu152,10UC $\{74,20\}$ & Eu152 & 0 & 0 & 0 & 0 & 0 & 0 & 0 & 0 & & 0 & 0 & 0 & 0 & 0 & 1 & 0 & 0 & 0 & 0 & 0 & 0 & 0 & 0 & 0 & 0 & 99 & 0 & 100 \\
\hline Ir192,10UC & Ir192 & 0 & 0 & 0 & 0 & 0 & 0 & 0 & 0 & & 0 & 0 & 0 & 0 & 0 & 0 & 0 & 0 & 0 & 0 & 0 & 0 & 0 & 0 & 0 & 0 & \multicolumn{2}{|c|}{0100} & 100 \\
\hline $\operatorname{Ir} 192,10 \cup C\{10,10\}$ & Ir192 & 0 & 0 & 0 & 0 & 0 & 0 & 0 & 0 & & 0 & 0 & 0 & 0 & 0 & 0 & 0 & 0 & 4 & 0 & 0 & 0 & 0 & 0 & 0 & 0 & \multicolumn{2}{|c|}{096} & 100 \\
\hline $\operatorname{Ir} 192,10 \cup C\{10,30\}$ & Ir192 & 0 & 0 & 0 & 0 & 0 & 0 & 0 & 1 & & 0 & 0 & 0 & 0 & 0 & 0 & 0 & 1 & 5 & 0 & 0 & 7 & 0 & 0 & 0 & 0 & \multicolumn{2}{|c|}{086} & 100 \\
\hline Ir192,10UC $\{26,10\}$ & Ir192 & 0 & 0 & 0 & 0 & 0 & 0 & 0 & 0 & & 0 & 0 & 0 & 0 & 0 & 0 & 0 & 0 & 0 & 0 & 0 & 0 & 0 & 0 & 0 & 0 & \multicolumn{2}{|c|}{0100} & 100 \\
\hline $\operatorname{Ir} 192,10 \cup C\{26,30\}$ & $\operatorname{lr} 192$ & 0 & 0 & 0 & 0 & 0 & 0 & 0 & 2 & & 0 & 0 & 0 & 0 & 0 & 0 & 0 & 7 & 4 & 0 & 0 & 4 & 0 & 0 & 0 & 0 & \multicolumn{2}{|c|}{083} & 100 \\
\hline $\operatorname{Ir} 192,10 \cup C\{26,50\}$ & Ir192 & 0 & 0 & 0 & 0 & 0 & 0 & 0 & 2 & & 0 & 0 & 0 & 0 & 0 & 0 & 0 & 46 & 4 & 0 & 0 & 9 & 0 & 0 & 0 & 0 & \multicolumn{2}{|c|}{039} & 100 \\
\hline $\operatorname{Ir} 192,1 \mathrm{mC}\{74,20\}$ & Ir192 & 0 & 0 & 0 & 0 & 0 & 0 & 0 & 0 & & 0 & 0 & 0 & 0 & 0 & 0 & 0 & 0 & 0 & 0 & 0 & 0 & 0 & 0 & 0 & 0 & \multicolumn{2}{|c|}{0100} & 100 \\
\hline $\operatorname{Ir} 192,10 \mathrm{mC}\{74,60\}$ & Ir192 & 0 & 0 & 0 & 0 & 0 & 0 & 0 & 0 & & 0 & 0 & 0 & 0 & 0 & 0 & 0 & 0 & 0 & 0 & 0 & 0 & 0 & 0 & 0 & 0 & \multicolumn{2}{|c|}{0100} & 100 \\
\hline
\end{tabular}

\section{A.4.4.3 ML Group Verification Set Correlation Matrix}

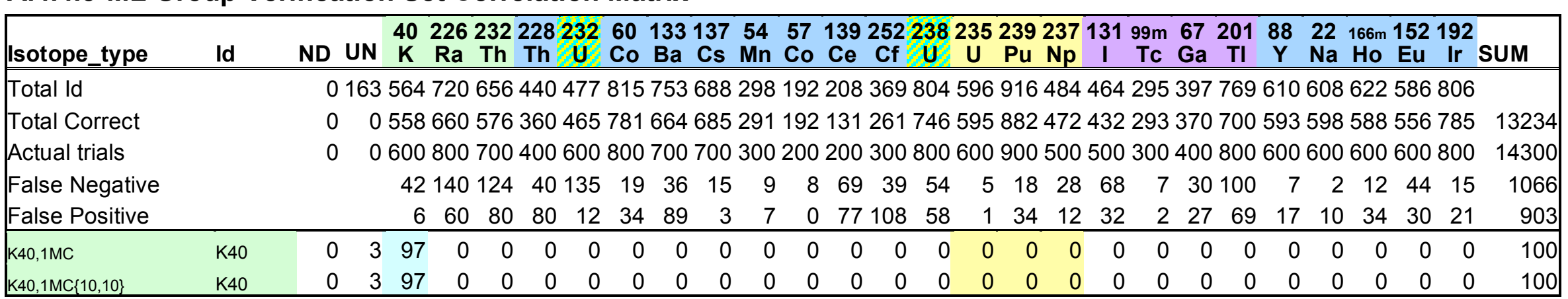




\begin{tabular}{|c|c|c|c|c|c|c|c|c|c|c|c|c|c|c|c|c|c|c|c|c|c|c|c|c|c|c|c|c|c|c|}
\hline Isotope_type & Id & ND & & $\begin{array}{l}40 \\
\mathrm{~K}\end{array}$ & $\begin{array}{l}226 \\
\mathrm{Ra}\end{array}$ & $\begin{array}{l}2322 \\
\text { Th }\end{array}$ & $\begin{array}{c}228 \\
\text { Th }\end{array}$ & U & $\begin{array}{ll}601 \\
\text { Co } \\
\end{array}$ & $\begin{array}{c}133 \\
\mathrm{Ba}\end{array}$ & $\begin{array}{c}137 \\
\text { Cs }\end{array}$ & $\begin{array}{l}54 \\
\mathrm{Mn} \\
\end{array}$ & $\begin{array}{l}571 \\
\text { Co } \\
\end{array}$ & $\begin{array}{c}139 \\
\mathrm{Ce}\end{array}$ & $\begin{array}{c}252 \\
\mathrm{Cf}\end{array}$ & $\begin{array}{r}2382 \\
\mathrm{U}\end{array}$ & $\begin{array}{c}2352 \\
U\end{array}$ & $\begin{array}{l}2392 \\
\mathrm{Pu} \quad \mathrm{I}\end{array}$ & $\begin{array}{l}237 \\
\text { Np }\end{array}$ & $\begin{array}{cc}13199 \\
1 \quad T \\
\end{array}$ & $\begin{array}{l}9 \mathrm{~m} 6 \\
\text { Tc } \mathrm{C}\end{array}$ & $\begin{array}{ll}67 & 2 \\
\mathrm{Ga} & 1\end{array}$ & $\begin{array}{l}201 \\
\mathrm{TI} \\
\end{array}$ & $\begin{array}{cc}88 & 2 \\
\mathrm{Y} & \mathrm{N} \\
\end{array}$ & $\begin{array}{ll}22 & 1 \\
\mathrm{Na} & 1\end{array}$ & $\begin{array}{l}166 \mathrm{~m} \\
\mathrm{Ho} \\
\end{array}$ & $\begin{array}{c}1521 \\
\mathrm{Eu}\end{array}$ & $\begin{array}{c}192 \\
\text { Ir }\end{array}$ & SUM & \\
\hline $\mathrm{K} 40,1 \mathrm{MC}\{10,30\}$ & K40 & 0 & 4 & 89 & 3 & 0 & 0 & 0 & 1 & 0 & 0 & 0 & 0 & 0 & 0 & 0 & 0 & 0 & 0 & 0 & 0 & 0 & 0 & 0 & 0 & 0 & 3 & 0 & & 100 \\
\hline $\mathrm{K} 40,1 \mathrm{MC}\{10,60\}$ & K40 & 0 & 1 & 86 & 5 & 2 & 0 & 0 & 0 & 0 & 0 & 0 & 0 & 0 & 0 & 1 & 0 & 1 & 0 & 1 & 0 & 0 & 0 & 0 & 0 & 1 & 1 & 1 & & 100 \\
\hline $\mathrm{K} 40,1 \mathrm{MC}\{26,10\}$ & K40 & 0 & 2 & 98 & 0 & 0 & 0 & 0 & 0 & 0 & 0 & 0 & 0 & 0 & 0 & 0 & 0 & 0 & 0 & 0 & 0 & 0 & 0 & 0 & 0 & 0 & 0 & 0 & & 100 \\
\hline$K 40,1 \mathrm{MC}\{26,30\}$ & $\mathrm{K} 40$ & 0 & 2 & 91 & 1 & 0 & 0 & 0 & 1 & 0 & 0 & 0 & 0 & 0 & 0 & 0 & 0 & 0 & 0 & 0 & 0 & 0 & 0 & 0 & 0 & 0 & 5 & 0 & & 100 \\
\hline Ra226,10UC & Ra226 & 0 & 3 & 0 & 97 & 0 & 0 & 0 & 0 & 0 & 0 & 0 & 0 & 0 & 0 & 0 & 0 & 0 & 0 & 0 & 0 & 0 & 0 & 0 & 0 & 0 & 0 & 0 & & 100 \\
\hline $\operatorname{Ra} 226,10 \cup C\{10,10\}$ & $\mathrm{Ra} 226$ & 0 & 0 & 0 & 94 & 0 & 0 & 0 & 1 & 0 & 0 & 0 & 0 & 0 & 1 & 0 & 0 & 1 & 0 & 2 & 0 & 0 & 0 & 1 & 0 & 0 & 0 & 0 & & 100 \\
\hline $\operatorname{Ra} 226,10 \cup C\{10,30\}$ & $\mathrm{Ra} 226$ & 0 & 0 & 0 & 74 & 2 & 0 & 0 & 4 & 0 & 0 & 0 & 0 & 0 & 8 & 4 & 0 & 2 & 0 & 0 & 0 & 0 & 0 & 1 & 1 & 1 & 3 & 0 & & 100 \\
\hline $\operatorname{Ra} 226,10 \cup C\{10,60\}$ & Ra226 & 0 & 1 & 1 & 65 & 8 & 0 & 0 & 3 & 0 & 0 & 0 & 0 & 0 & 6 & 8 & 0 & 1 & 0 & 0 & 0 & 0 & 0 & 1 & 1 & 0 & 4 & 1 & & 100 \\
\hline $\operatorname{Ra} 226,10 \cup C\{26,10\}$ & Ra226 & 0 & 2 & 0 & 97 & 0 & 0 & 0 & 0 & 0 & 0 & 0 & 0 & 0 & 0 & 0 & 0 & 0 & 0 & 0 & 0 & 0 & 0 & 1 & 0 & 0 & 0 & 0 & & 100 \\
\hline $\operatorname{Ra} 226,10 \cup C\{26,30\}$ & $\mathrm{Ra} 226$ & 0 & 2 & 0 & 81 & 2 & 0 & 0 & 0 & 0 & 0 & 0 & 0 & 0 & 11 & 1 & 0 & 1 & 0 & 0 & 0 & 0 & 0 & 1 & 0 & 0 & 1 & 0 & & 100 \\
\hline $\operatorname{Ra} 226,10 \cup C\{26,50\}$ & Ra226 & 0 & 0 & 0 & 65 & 2 & 0 & 0 & 1 & 0 & 0 & 0 & 0 & 0 & 24 & 4 & 0 & 0 & 0 & 0 & 0 & 0 & 0 & 2 & 0 & 0 & 1 & 1 & & 100 \\
\hline $\operatorname{Ra} 226,100 \cup C\{74,40\}$ & $\mathrm{Ra} 226$ & 0 & 3 & 0 & 87 & 0 & 0 & 0 & 3 & 0 & 0 & 0 & 0 & 0 & 1 & 0 & 0 & 0 & 0 & 0 & 0 & 0 & 0 & 6 & 0 & 0 & 0 & 0 & & 100 \\
\hline Th232,10UC & Th232 & 0 & 3 & 0 & 0 & 93 & 4 & 0 & 0 & 0 & 0 & 0 & 0 & 0 & 0 & 0 & 0 & 0 & 0 & 0 & 0 & 0 & 0 & 0 & 0 & 0 & 0 & 0 & & 100 \\
\hline Th232,10UC $\{10,10\}$ & Th232 & 0 & 0 & 0 & 2 & 93 & 3 & 0 & 0 & 0 & 0 & 0 & 0 & 0 & 1 & 0 & 0 & 1 & 0 & 0 & 0 & 0 & 0 & 0 & 0 & 0 & 0 & 0 & & 100 \\
\hline Th232,10UC $\{10,30\}$ & Th232 & 0 & 2 & 0 & 6 & 70 & 5 & 1 & 2 & 0 & 1 & 1 & 0 & 0 & 4 & 4 & 0 & 2 & 0 & 0 & 0 & 0 & 0 & 0 & 0 & 2 & 0 & 0 & & 100 \\
\hline Th232,10UC $\{10,60\}$ & Th232 & 0 & 1 & 0 & 10 & 63 & 8 & 5 & 0 & 0 & 1 & 0 & 0 & 0 & 4 & 3 & 0 & 1 & 0 & 0 & 0 & 0 & 0 & 0 & 1 & 2 & 0 & 1 & & 100 \\
\hline Th232,10UC $\{26,10\}$ & Th232 & 0 & 0 & 0 & 0 & 92 & 4 & 0 & 0 & 0 & 0 & 0 & 0 & 0 & 2 & 1 & 0 & 1 & 0 & 0 & 0 & 0 & 0 & 0 & 0 & 0 & 0 & 0 & & 100 \\
\hline Th232,10UC $\{26,30\}$ & Th232 & 0 & 0 & 0 & 0 & 69 & 4 & 0 & 0 & 0 & 0 & 1 & 0 & 0 & 18 & 6 & 0 & 1 & 0 & 0 & 0 & 0 & 0 & 1 & 0 & 0 & 0 & 0 & & 100 \\
\hline Th232,100UC $\{74,20\}$ & Th232 & 0 & 2 & 0 & 0 & 96 & 0 & 0 & 0 & 0 & 0 & 0 & 0 & 0 & 0 & 1 & 0 & 0 & 0 & 0 & 0 & 0 & 0 & 1 & 0 & 0 & 0 & 0 & & 100 \\
\hline Th228,10uC & Th228 & 0 & 4 & 0 & 0 & 0 & 96 & 0 & 0 & 0 & 0 & 0 & 0 & 0 & 0 & 0 & 0 & 0 & 0 & 0 & 0 & 0 & 0 & 0 & 0 & 0 & 0 & 0 & & 100 \\
\hline Th228,10UC $\{13,10\}$ & Th228 & 0 & 0 & 0 & 0 & 3 & 96 & 0 & 0 & 0 & 0 & 0 & 0 & 0 & 0 & 0 & 0 & 0 & 0 & 0 & 0 & 0 & 0 & 0 & 0 & 1 & 0 & 0 & & 100 \\
\hline Th228,10UC $\{13,30\}$ & Th228 & 0 & 3 & 0 & 3 & 15 & 72 & 6 & 0 & 0 & 0 & 0 & 0 & 0 & 0 & 0 & 0 & 0 & 0 & 0 & 0 & 0 & 0 & 0 & 0 & 1 & 0 & 0 & & 100 \\
\hline Th228,10UC $\{26,10\}$ & Th228 & 0 & 2 & 0 & 0 & 2 & 96 & 0 & 0 & 0 & 0 & 0 & 0 & 0 & 0 & 0 & 0 & 0 & 0 & 0 & 0 & 0 & 0 & 0 & 0 & 0 & 0 & 0 & & 100 \\
\hline U232,50UC $\{92,20 ; 13,10\}$ & $\mathrm{U} 232$ & 0 & 5 & 0 & 0 & 3 & 1 & 91 & 0 & 0 & 0 & 0 & 0 & 0 & 0 & 0 & 0 & 0 & 0 & 0 & 0 & 0 & 0 & 0 & 0 & 0 & 0 & 0 & & 100 \\
\hline U232,50UC\{92,20;13,30\} & U232 & 0 & 4 & 0 & 1 & 8 & 17 & 67 & 0 & 0 & 0 & 0 & 0 & 0 & 3 & 0 & 0 & 0 & 0 & 0 & 0 & 0 & 0 & 0 & 0 & 0 & 0 & 0 & & 100 \\
\hline U232,50uC\{92,20;13,50\} & $\mathrm{U} 232$ & 0 & 3 & 0 & 2 & 20 & 22 & 48 & 0 & 0 & 0 & 0 & 0 & 0 & 5 & 0 & 0 & 0 & 0 & 0 & 0 & 0 & 0 & 0 & 0 & 0 & 0 & 0 & & 100 \\
\hline U232,50UC\{92,20;26,10\} & U232 & 0 & 3 & 0 & 0 & 1 & 0 & 95 & 0 & 0 & 0 & 0 & 0 & 0 & 1 & 0 & 0 & 0 & 0 & 0 & 0 & 0 & 0 & 0 & 0 & 0 & 0 & 0 & & 100 \\
\hline U232,50UC\{92,20;26,30\} & 4232 & 0 & 5 & 0 & 0 & 8 & 10 & 70 & 0 & 0 & 0 & 0 & 0 & 0 & 6 & 0 & 0 & 0 & 0 & 0 & 0 & 0 & 0 & 1 & 0 & 0 & 0 & 0 & & 100 \\
\hline U232,50UC\{92,20;74,10\} & $\mathrm{U} 232$ & 0 & 6 & 0 & 0 & 0 & 0 & 94 & 0 & 0 & 0 & 0 & 0 & 0 & 0 & 0 & 0 & 0 & 0 & 0 & 0 & 0 & 0 & 0 & 0 & 0 & 0 & 0 & & 100 \\
\hline
\end{tabular}




\begin{tabular}{|c|c|c|c|c|c|c|c|c|c|c|c|c|c|c|c|c|c|c|c|c|c|c|c|c|c|c|c|c|c|c|c|c|c|}
\hline Isotope_type & Id & ND & UN & $\begin{array}{c}40 \\
\mathrm{~K} \\
\end{array}$ & $\begin{array}{r}226 \\
\mathrm{Ra}\end{array}$ & & & $\begin{array}{l}2823 \\
\mathrm{~h} \quad \mathrm{U}\end{array}$ & $\begin{array}{l}326 \\
\mathrm{U} \\
\end{array}$ & $\begin{array}{ll}60 & 1 \\
\text { Co } & \\
\end{array}$ & $\begin{array}{l}1331 \\
\mathrm{Ba} \mathrm{C}\end{array}$ & $\begin{array}{l}137 \\
\text { Cs }\end{array}$ & $\begin{array}{r}54 \\
\mathrm{Mn} \\
\end{array}$ & $\begin{array}{l}57 \\
\text { Co } \\
\end{array}$ & $\begin{array}{c}139 \\
\mathrm{Ce}\end{array}$ & & $\begin{array}{l}223 \\
\mathrm{f}\end{array}$ & $\begin{array}{l}382 \\
\mathrm{j}\end{array}$ & $\begin{array}{l}352 \\
\mathrm{U} \quad \mathrm{P}\end{array}$ & $\begin{array}{l}2392 \\
\mathrm{Pu} \quad \mathrm{I}\end{array}$ & $\begin{array}{l}237 \\
\text { Np }\end{array}$ & $\begin{array}{c}131 \\
1\end{array}$ & $\begin{array}{c}99 \mathrm{~m} \\
\text { Tc }\end{array}$ & $\begin{array}{l}67 \\
\mathrm{Ga} \\
\end{array}$ & $\begin{array}{r}20 \\
a \quad T I \\
\end{array}$ & 8 & $\begin{array}{ll}38 & 2 \\
Y & 1 \\
\end{array}$ & $\begin{array}{ll}22 & 1 \\
\mathrm{Na} & \mathrm{H} \\
\end{array}$ & $\begin{array}{l}166 \mathrm{~m} 1 \\
\text { Ho }\end{array}$ & $\begin{array}{c}1521 \\
\mathrm{Eu}\end{array}$ & $\begin{array}{l}192 \\
\text { Ir s }\end{array}$ & SUM & \\
\hline Co60,10UC & Co60 & 0 & 0 & c & 0 & c & 0 & 0 & 01 & 100 & 0 & 0 & 0 & 0 & 0 & 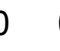 & 0 & 0 & 0 & 0 & 0 & 0 & 0 & 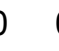 & 0 & 0 & 0 & 0 & 0 & 0 & 0 & & 100 \\
\hline Co60,10UC $\{10,10\}$ & Co60 & 0 & 0 & c & 0 & c & 0 & 0 & & 100 & 0 & 0 & 0 & 0 & 0 & & 0 & 0 & 0 & 0 & 0 & 0 & 0 & 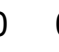 & 0 & 0 & 0 & 0 & 0 & 0 & 0 & & 100 \\
\hline $\mathrm{C} 060,10 \cup C\{10,30\}$ & Co60 & 0 & 0 & $c$ & 0 & c & 0 & 0 & 0 & 97 & 0 & 0 & 0 & 0 & 0 & & 0 & 0 & 0 & 0 & 0 & 0 & 0 & 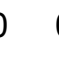 & 0 & 0 & 0 & 0 & 0 & 3 & 0 & & 100 \\
\hline Co60,10UC $\{10,60\}$ & Co60 & 0 & 0 & 1 & 4 & c & 0 & 0 & 0 & 87 & 0 & 0 & 0 & 0 & 0 & & 3 & 1 & 0 & 0 & 0 & 0 & 0 & 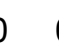 & 0 & 0 & 0 & 0 & 1 & 3 & 0 & & 100 \\
\hline Co60,10UC $\{26,10\}$ & Co60 & 0 & 0 & c & 0 & ) & 0 & 0 & & 100 & 0 & 0 & 0 & 0 & 0 & & 0 & 0 & 0 & 0 & 0 & 0 & 0 & 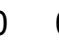 & 0 & 0 & 0 & 0 & 0 & 0 & 0 & & 100 \\
\hline Co60,10UC $\{26,30\}$ & Co60 & 0 & 1 & c & 0 & ) & 0 & 0 & $0 \subseteq$ & 97 & 0 & 0 & 0 & 0 & 0 & & 0 & 0 & 0 & 0 & 0 & 0 & 0 & 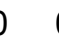 & 0 & 0 & 0 & 0 & 0 & 2 & 0 & & 100 \\
\hline Co60,10UC $\{74,20\}$ & Co60 & 0 & 0 & c & 0 & c & 0 & 0 & & 100 & 0 & 0 & 0 & 0 & 0 & & 0 & 0 & 0 & 0 & 0 & 0 & 0 & 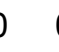 & 0 & 0 & 0 & 0 & 0 & 0 & 0 & & 100 \\
\hline Co60,10UC $\{74,40\}$ & $\mathrm{Co60}$ & 0 & $\underline{0}$ & c & $\underline{0}$ & c & $\underline{0}$ & 0 & & 100 & 0 & 0 & 0 & 0 & 0 & & 0 & 0 & 0 & 0 & 0 & 0 & 0 & 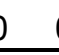 & 0 & 0 & 0 & 0 & 0 & 0 & 0 & & 100 \\
\hline Ba133,10UC & Ba133 & 0 & 0 & $c$ & 0 & c & 0 & 0 & 0 & 0 & 96 & 0 & 0 & 0 & 0 & & 0 & 0 & 0 & 0 & 0 & 4 & 0 & ( & 0 & 0 & 0 & 0 & 0 & 0 & 0 & & 100 \\
\hline $\mathrm{Ba} 133,10 \cup \mathrm{C}\{10,10\}$ & Ba133 & 0 & 0 & c & 0 & c & 0 & 0 & 0 & 0 & 92 & 0 & 0 & 0 & 0 & & 0 & 0 & 0 & 0 & 0 & 8 & 0 & 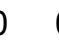 & 0 & 0 & 0 & 0 & 0 & 0 & 0 & & 100 \\
\hline $\mathrm{Ba} 133,10 \cup \mathrm{UC}\{10,30\}$ & $\mathrm{Ba} 133$ & 0 & 1 & c & 0 & c & 0 & 0 & 0 & 0 & 90 & 0 & 0 & 0 & 0 & & 0 & 0 & 0 & 1 & 0 & 7 & 0 & 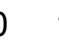 & 1 & 0 & 0 & 0 & 0 & 0 & 0 & & 100 \\
\hline $\mathrm{Ba} 133,10 \cup \mathrm{C}\{10,50\}$ & $\mathrm{Ba} 133$ & 0 & 1 & c & 0 & c & 0 & 0 & 0 & 0 & 88 & 0 & 0 & 0 & 0 & & 0 & 0 & 0 & 1 & 3 & 0 & 0 & 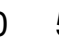 & 5 & 0 & 0 & 0 & 0 & 0 & 2 & & 100 \\
\hline $\mathrm{Ba} 133,10 \cup \mathrm{UC}\{26,10\}$ & Ba133 & 0 & 0 & c & 0 & ) & 0 & 0 & 0 & 0 & 99 & 0 & 0 & 0 & 0 & & 0 & 0 & 0 & 0 & 0 & 1 & 0 & 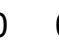 & 0 & 0 & 0 & 0 & 0 & 0 & 0 & & 100 \\
\hline $\mathrm{Ba} 133,10 \cup \mathrm{C}\{26,30\}$ & Ba133 & 0 & 0 & c & 0 & b & 0 & 0 & 0 & & 100 & 0 & 0 & 0 & 0 & & 0 & 0 & 0 & 0 & 0 & 0 & 0 & 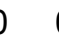 & 0 & 0 & 0 & 0 & 0 & 0 & 0 & & 100 \\
\hline Ba133,100UC $\{74,10\}$ & Ba133 & 0 & $\underline{0}$ & c & 0 & 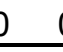 & 0 & 0 & 0 & 0 & 99 & 0 & 0 & 0 & $\underline{0}$ & & $\underline{0}$ & 0 & 0 & 0 & 0 & 1 & 0 & 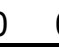 & 0 & 0 & 0 & 0 & 0 & 0 & 0 & & 100 \\
\hline Cs137,10UC & Cs137 & 0 & 0 & c & 0 & 0 & 0 & 0 & 0 & 0 & & 100 & 0 & 0 & 0 & & 0 & 0 & 0 & 0 & 0 & 0 & 0 & 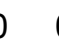 & 0 & 0 & 0 & 0 & 0 & 0 & 0 & & 100 \\
\hline $\operatorname{Cs} 137,10 \cup C\{10,10\}$ & Cs137 & 0 & 0 & c & 0 & 0 & 0 & 0 & 0 & 0 & & 100 & 0 & 0 & 0 & & 0 & 0 & 0 & 0 & 0 & 0 & 0 & 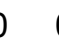 & 0 & 0 & 0 & 0 & 0 & 0 & 0 & & 100 \\
\hline Cs137,10UC $\{10,30\}$ & Cs137 & 0 & 1 & $c$ & 0 & b & 0 & 0 & 0 & 0 & 0 & 99 & 0 & 0 & 0 & & 0 & 0 & 0 & 0 & 0 & 0 & 0 & & 0 & 0 & 0 & 0 & 0 & 0 & 0 & & 100 \\
\hline Cs $137,10 \cup C\{10,60\}$ & Cs137 & 0 & 0 & 1 & 1 & 0 & 0 & 0 & 0 & 0 & 0 & 89 & 0 & 0 & 0 & & 0 & 0 & 0 & 5 & 0 & 0 & 0 & 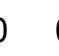 & 0 & 0 & 0 & 0 & 1 & 0 & 4 & & 100 \\
\hline $\operatorname{Cs} 137,10 \cup C\{26,10\}$ & Cs137 & 0 & 0 & c & 0 & 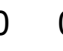 & 0 & 0 & 0 & 0 & & 100 & 0 & 0 & 0 & & 0 & 0 & 0 & 0 & 0 & 0 & 0 & 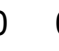 & 0 & 0 & 0 & 0 & 0 & 0 & 0 & & 100 \\
\hline Cs $137,10 \cup C\{26,30\}$ & Cs137 & 0 & 0 & c & 0 & 0 & 0 & 0 & 0 & 0 & & 100 & 0 & 0 & 0 & & 0 & 0 & 0 & 0 & 0 & 0 & 0 & & 0 & 0 & 0 & 0 & 0 & 0 & 0 & & 100 \\
\hline Cs $137,10 \cup C\{26,50\}$ & Cs137 & 0 & $\underline{0}$ & c & $\underline{0}$ & 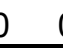 & $\underline{0}$ & 0 & 0 & 0 & 0 & 97 & 0 & 0 & $\underline{0}$ & & $\underline{0}$ & 1 & 0 & 0 & 0 & 0 & 0 & 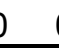 & 0 & 0 & 0 & 0 & 0 & 0 & 2 & & 100 \\
\hline Mn54,10UC & Mn54 & 0 & 0 & c & 0 & b & 0 & 0 & 0 & 0 & 0 & & 100 & 0 & 0 & & 0 & 0 & 0 & 0 & 0 & 0 & 0 & & 0 & 0 & 0 & 0 & 0 & 0 & 0 & & 100 \\
\hline $\operatorname{Mn} 54,10 \cup C\{13,10\}$ & Mn54 & 0 & 0 & $c$ & 0 & 0 & 0 & 0 & 0 & 0 & 0 & & 100 & 0 & 0 & & 0 & 0 & 0 & 0 & 0 & 0 & 0 & 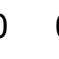 & 0 & 0 & 0 & 0 & 0 & 0 & 0 & & 100 \\
\hline Mn54,10UC $\{13,30\}$ & Mn54 & 0 & 2 & c & 0 & 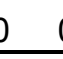 & 0 & 0 & 0 & 0 & 0 & 0 & 91 & 0 & $\underline{0}$ & & 0 & 0 & 0 & 0 & 0 & 0 & 0 & 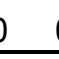 & 0 & 0 & 0 & 0 & 7 & 0 & 0 & & 100 \\
\hline Co57,10UC & Co57 & 0 & 2 & c & 0 & D & 0 & 0 & 0 & 0 & 0 & 0 & 0 & 98 & 0 & & 0 & 0 & 0 & 0 & 0 & 0 & 0 & 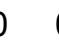 & 0 & 0 & 0 & 0 & 0 & 0 & 0 & & 100 \\
\hline Co57,10UC $\{13,10\}$ & Co57 & 0 & 6 & c & $\underline{0}$ & 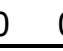 & $\underline{0}$ & 0 & 0 & 0 & 0 & 0 & 0 & 94 & $\underline{0}$ & & $\underline{0}$ & 0 & 0 & 0 & 0 & 0 & 0 & 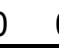 & $\underline{0}$ & 0 & 0 & 0 & 0 & 0 & 0 & & 100 \\
\hline Ce139,10UC & Ce139 & 0 & 0 & c & 0 & 0 & 0 & 0 & 0 & 0 & 0 & 0 & 0 & & 100 & & 0 & 0 & 0 & 0 & 0 & 0 & 0 & 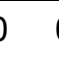 & 0 & 0 & 0 & 0 & 0 & 0 & 0 & & 100 \\
\hline Ce139,10UC $\{13,10\}$ & Ce139 & 0 & $\underline{0}$ & c & 0 & 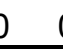 & $\underline{0}$ & 0 & 0 & 0 & 0 & 0 & 0 & 0 & 31 & & 0 & 0 & 0 & 0 & 0 & 0 & 0 & ) & $\begin{array}{ll}0 & 6 \\
\end{array}$ & 9 & 0 & 0 & 0 & 0 & 0 & & 100 \\
\hline
\end{tabular}




\begin{tabular}{|c|c|c|c|c|c|c|c|c|c|c|c|c|c|c|c|c|c|c|c|c|c|c|c|c|c|c|c|c|c|c|c|c|c|c|c|c|}
\hline Isotope_type & Id & ND & UN & $\begin{array}{r}40 \\
K \\
\end{array}$ & $\begin{array}{c}226 \\
\mathrm{Ra} \\
\end{array}$ & $\begin{array}{r}232 \\
\text { Th } \\
\end{array}$ & $\begin{array}{r}22 \\
\mathrm{Th} \\
\end{array}$ & $\begin{array}{l}82 \\
\mathrm{~h} 2\end{array}$ & 32 & $\begin{array}{l}60 \\
\mathrm{Co}\end{array}$ & $\begin{array}{c}133 \\
\mathrm{Ba} \\
\end{array}$ & & & $\begin{array}{c}54 \\
\mathrm{Mn} \\
\end{array}$ & $\begin{array}{l}57 \\
\text { Co } \\
\end{array}$ & $\begin{array}{r}13 \\
\mathrm{Ce}\end{array}$ & $\begin{array}{l}925 \\
\quad \quad C \\
\end{array}$ & $\begin{array}{l}522 \\
\mathrm{Cf}\end{array}$ & 238 & $\begin{array}{c}235 \\
U\end{array}$ & $\begin{array}{r}23 \\
\mathrm{Pu}\end{array}$ & $\begin{array}{l}392 \\
\mathbf{u} \quad \mathrm{N} \\
\end{array}$ & $\begin{array}{l}37 \\
\text { Np }\end{array}$ & $\begin{array}{c}131 \\
I \\
\end{array}$ & $\begin{array}{l}99 n \\
\text { Tc }\end{array}$ & & $\begin{array}{l}67 \\
\mathrm{Ga}\end{array}$ & $\begin{array}{c}201 \\
\text { TI }\end{array}$ & $\begin{array}{l}88 \\
Y \\
\end{array}$ & $\begin{array}{l}22 \\
\mathrm{Na}\end{array}$ & & $\begin{array}{l}36 \mathrm{~m} 1 \\
\text { to } \\
\end{array}$ & $\begin{array}{l}1521 \\
\text { Eu }\end{array}$ & $\begin{array}{c}192 \\
\text { Ir }\end{array}$ & SUN & \\
\hline Nylon1-Cf & Cf252 & 0 & 6 & 0 & 7 & 0 & 0 & 2 & 0 & 0 & 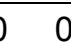 & & 0 & 0 & 0 & (2) & $0 \varepsilon$ & 85 & 0 & 0 & 0 & 0 & 0 & 0 & & 0 & 0 & 0 & 0 & & 0 & 0 & 0 & 0 & & 100 \\
\hline Nylon1-Cf $\{90,1\}$ & Cf252 & 0 & 4 & 0 & 1 & $\mathrm{c}$ & 0 & 0 & 0 & 0 & ) & & 0 & 0 & 0 & 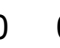 & 0 & 94 & 0 & 0 & 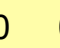 & 0 & 0 & 0 & & 0 & 0 & 0 & 1 & & 0 & 0 & 0 & 0 & & 100 \\
\hline $\mathrm{Fe} 1-\mathrm{Cf}$ & $\mathrm{Cf} 252$ & 0 & 1 & 0 & 6 & 2 & 2 & 0 & 0 & 1 & 0 & & 0 & 0 & 0 & 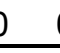 & 0 & 82 & 7 & 0 & b & 1 & 0 & 0 & & 0 & 0 & 0 & 0 & & 0 & 0 & 0 & 0 & & 100 \\
\hline $1 \mathrm{KGU} 238$ & 4238 & 0 & 0 & 0 & 0 & c & 0 & 0 & 0 & 0 & 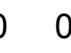 & & 0 & 0 & 0 & ) & 0 & 1 & 98 & 0 & 0 & 0 & 0 & 0 & & 0 & 0 & 0 & 0 & & 0 & 1 & 0 & 0 & & 100 \\
\hline $1 \mathrm{KGU} 238\{13,10\}$ & 0238 & 0 & 1 & 0 & 2 & 1 & 1 & 0 & 0 & 0 & 0 & & 0 & 0 & 0 & ) & 0 & 2 & 89 & 0 & כ & 1 & 0 & 0 & & 0 & 0 & 0 & 0 & & 2 & 2 & 0 & 0 & & 100 \\
\hline $1 \mathrm{KGU} 238\{13,30\}$ & $\cup 238$ & 0 & 2 & 2 & 2 & 1 & 1 & 0 & 0 & 2 & 2 & & 0 & 1 & 0 & ) & 0 & 2 & 79 & 0 & כ & 0 & 0 & 0 & & 0 & 0 & 0 & 0 & & 2 & 7 & 1 & 1 & & 100 \\
\hline $1 \mathrm{KGU} 238\{13,50\}$ & 0238 & 0 & 1 & 0 & 1 & c & כ & 0 & 0 & 1 & 0 & & 0 & 2 & 0 & ) & 0 & 0 & 92 & 0 & כ & 0 & 0 & 0 & & 0 & 0 & 0 & 0 & & 0 & 2 & 1 & 0 & & 100 \\
\hline $1 \mathrm{KGU} 238\{26,10\}$ & $\mathrm{U} 238$ & 0 & 0 & 0 & 0 & c & O & 0 & 0 & 0 & 0 & & 0 & 0 & 0 & 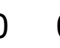 & 0 & 1 & 96 & 0 & כ & 0 & 0 & 0 & & 0 & 0 & 0 & 0 & & 1 & 1 & 1 & 0 & & 100 \\
\hline $1 K G U 238\{26,30\}$ & $\cup 238$ & 0 & 0 & 0 & 0 & C & 0 & 0 & 0 & 0 & 0 & & 0 & 0 & 0 & ) & 0 & 2 & 93 & 0 & כ & 0 & 0 & 0 & & 0 & 0 & 0 & 0 & & 1 & 3 & 1 & 0 & & 100 \\
\hline $1 \mathrm{KGU} 238\{26,50\}$ & 4238 & 0 & 0 & 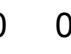 & 0 & c & 0 & 0 & 0 & 0 & 0 & & 0 & 0 & 0 & ) & 0 & 0 & 99 & 0 & 0 & 0 & 0 & 0 & & 0 & 0 & 0 & 0 & & 0 & 1 & 0 & 0 & & 100 \\
\hline $1 \mathrm{KGU} 238\{74,20\}$ & $\mathrm{U} 238$ & 0 & 0 & 0 & 0 & C & כ & 0 & 0 & 0 & 0 & & 0 & 0 & 0 & 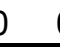 & 0 & 0 & 100 & 0 & 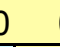 & 0 & 0 & 0 & & 0 & 0 & 0 & 0 & & 0 & 0 & 0 & 0 & & 100 \\
\hline \multirow{6}{*}{ 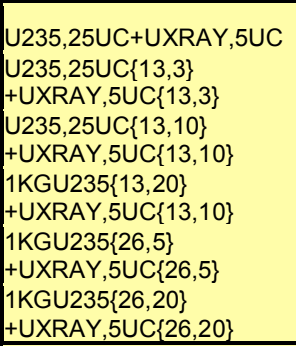 } & U235 & 0 & 0 & 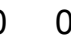 & 0 & C & 0 & 0 & 0 & 0 & 0 & & 0 & 0 & 0 & D & 0 & 0 & \multicolumn{3}{|c|}{0100} & 0 & 0 & 0 & & 0 & 0 & 0 & 0 & & 0 & 0 & 0 & 0 & & 100 \\
\hline & U235 & 0 & 1 & 0 & 0 & c & כ & 0 & 0 & 0 & 0 & & 0 & 0 & 0 & D & 0 & 0 & & 0 & 0 & 0 & & 0 & 0 & 0 & 0 & & 0 & 0 & 0 & 0 & & 100 \\
\hline & U235 & 0 & 2 & 0 & 0 & c & 0 & 0 & 0 & 0 & 0 & & 0 & 0 & 0 & ) & 0 & 0 & \multicolumn{2}{|c|}{098} & & 0 & 0 & 0 & & 0 & 0 & 0 & 0 & & 0 & 0 & 0 & 0 & & 100 \\
\hline & U235 & 0 & 1 & 0 & 0 & c & 0 & 0 & 0 & 0 & 0 & & 0 & 0 & 0 & ) & 0 & 0 & \multicolumn{3}{|c|}{099} & 0 & 0 & 0 & & 0 & 0 & 0 & 0 & & 0 & 0 & 0 & 0 & & 100 \\
\hline & U235 & 0 & 1 & 0 & 0 & c & 0 & 0 & 0 & 0 & 0 & & 0 & 0 & 0 & D & 0 & 0 & \multicolumn{3}{|c|}{099} & 0 & 0 & 0 & & 0 & 0 & 0 & 0 & & 0 & 0 & 0 & 0 & & 100 \\
\hline & U235 & 0 & 0 & 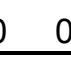 & 0 & c & כ & 0 & 0 & 0 & 0 & & 0 & 0 & 0 & D & 0 & 0 & \multicolumn{3}{|c|}{0100} & 0 & 0 & 0 & & 0 & 0 & 0 & 0 & & 0 & 0 & 0 & 0 & & 100 \\
\hline 1GPUWG & Pu239 & 0 & 0 & 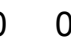 & 0 & c & 0 & 0 & 0 & 0 & 0 & & 0 & 0 & 0 & 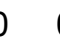 & 0 & 0 & 0 & \multicolumn{3}{|c|}{0100} & 0 & 0 & & 0 & 0 & 0 & 0 & & 0 & 0 & 0 & 0 & & 100 \\
\hline 1GPUWG $\{10,5\}$ & Pu239 & 0 & 1 & 0 & 0 & c & 0 & 0 & 0 & 0 & 0 & & 0 & 0 & 0 & ) & 0 & 0 & 0 & \multicolumn{3}{|c|}{099} & 0 & 0 & & 0 & 0 & 0 & 0 & & 0 & 0 & 0 & 0 & & 100 \\
\hline 1KGPUWG & Pu239 & 0 & 0 & 0 & 0 & c & 0 & 0 & 0 & 0 & 0 & & 0 & 0 & 0 & ) & 0 & 0 & 0 & \multicolumn{3}{|c|}{0100} & 0 & 0 & & 0 & 0 & 0 & 0 & & 0 & 0 & 0 & 0 & & 100 \\
\hline 1KGPUWG $\{10,10\}$ & Pu239 & 0 & 0 & 0 & 0 & c & 0 & 0 & 0 & 0 & 0 & & 0 & 0 & 0 & ) & 0 & 0 & 0 & \multicolumn{3}{|c|}{0100} & 0 & 0 & & 0 & 0 & 0 & 0 & & 0 & 0 & 0 & 0 & & 100 \\
\hline 1 KGPUWG $\{10,30\}$ & Pu239 & 0 & 2 & 0 & 0 & c & 0 & 0 & 0 & 0 & 0 & & 0 & 0 & 0 & ) & 0 & 0 & 0 & \multicolumn{3}{|c|}{090} & 0 & 6 & & 0 & 1 & 0 & 0 & & 0 & 0 & 0 & 1 & & 100 \\
\hline 1KGPUWG $\{26,10\}$ & Pu239 & 0 & 0 & ) & 0 & $\mathrm{c}$ & 0 & 0 & 0 & 0 & 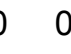 & & 0 & 0 & 0 & 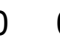 & 0 & 0 & 0 & \multicolumn{3}{|c|}{0100} & 0 & 0 & & 0 & 0 & 0 & 0 & & 0 & 0 & 0 & 0 & & 100 \\
\hline 1 KGPUWG $\{26,30\}$ & Pu239 & 0 & 0 & ) & 0 & C & 0 & 0 & 0 & 0 & ) & & 0 & 0 & 0 & ) & 0 & 0 & 0 & \multicolumn{3}{|c|}{098} & 0 & 1 & & 0 & 0 & 0 & 0 & & 0 & 0 & 0 & 0 & & 100 \\
\hline 1 KGPUWG $\{26,50\}$ & Pu239 & 0 & 0 & 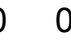 & 0 & C & 0 & 0 & 0 & 0 & 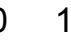 & & 0 & 0 & 0 & ) & 0 & 0 & 0 & \multicolumn{3}{|c|}{095} & 0 & 1 & & 0 & 0 & 0 & 0 & & 0 & 0 & 0 & 3 & & 100 \\
\hline 1KGPUWG $\{74,10\}$ & Pu239 & 0 & 0 & 0 & $\underline{0}$ & c & 0 & 0 & 0 & 0 & $\underline{0}$ & & 0 & 0 & 0 & 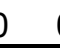 & 0 & 0 & 0 & \multicolumn{3}{|c|}{0100} & 0 & 0 & & 0 & 0 & 0 & 0 & & 0 & 0 & 0 & 0 & & 100 \\
\hline Np237,1g & Np237 & 0 & 1 & 0 & 0 & c & 0 & 0 & 0 & 0 & 0 & & 0 & 0 & 0 & 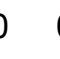 & 0 & 0 & 0 & 0 & 0 & 0 & 99 & 0 & & 0 & 0 & 0 & 0 & & 0 & 0 & 0 & 0 & & 100 \\
\hline Np237,1g $\{10,10\}$ & Np237 & 0 & 0 & 0 & 0 & C & 0 & 0 & 0 & 0 & 0 & & 0 & 0 & 0 & 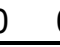 & 0 & 0 & 0 & 0 & 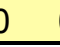 & 0 & 99 & 0 & & 0 & 1 & 0 & 0 & & 0 & 0 & 0 & 0 & & 100 \\
\hline
\end{tabular}




\begin{tabular}{|c|c|c|c|c|c|c|c|c|c|c|c|c|c|c|c|c|c|c|c|c|c|c|c|c|c|c|c|c|c|c|c|c|c|c|c|}
\hline Isotope_type & Id & ND & UN & $\begin{array}{l}40 \\
K\end{array}$ & $\begin{array}{c}226 \\
\mathrm{Ra}\end{array}$ & $\begin{array}{c}232 \\
\text { Th }\end{array}$ & $\begin{array}{c}228 \\
\text { Th }\end{array}$ & 23 & 26 & $\begin{array}{l}60 \\
\text { Co }\end{array}$ & $\begin{array}{c}133 \\
\mathrm{Ba}\end{array}$ & $\begin{array}{l}137 \\
\text { Cs }\end{array}$ & $\begin{array}{ll}7 & 5 \\
5 & M\end{array}$ & $\begin{array}{l}4 \\
\ln \end{array}$ & $\begin{array}{l}57 \\
\text { Co }\end{array}$ & $\begin{array}{c}139 \\
\mathrm{Ce}\end{array}$ & $\begin{array}{r}252 \\
\text { Cf }\end{array}$ & ${ }^{2} 23$ & $\begin{array}{l}382 \\
\mathrm{U}\end{array}$ & $\begin{array}{c}235 \\
\text { U }\end{array}$ & $\begin{array}{r}239 \\
\mathrm{Pu}\end{array}$ & $\begin{array}{l}923 \\
1 \quad N\end{array}$ & $\begin{array}{l}37 \\
\mathrm{Jp}\end{array}$ & $\begin{array}{c}131 \\
\text { I }\end{array}$ & $\begin{array}{c}99 \mathrm{~m} \\
\text { Tc }\end{array}$ & $\begin{array}{l}67 \\
\mathrm{Ga}\end{array}$ & $\begin{array}{r}201 \\
\text { TI }\end{array}$ & $\begin{array}{l}88 \\
Y\end{array}$ & $\begin{array}{l}22 \\
\mathrm{Na}\end{array}$ & $\begin{array}{l}166 \mathrm{~m} \\
\text { Ho }\end{array}$ & $\begin{array}{l}m 15 \\
\quad \mathrm{Eu}\end{array}$ & $\begin{array}{l}521 \\
\mathbf{u}\end{array}$ & $\begin{array}{l}92 \\
\text { Ir }\end{array}$ & SUN & \\
\hline $\mathrm{Np} 237,1 \mathrm{~g}\{10,30\}$ & Np237 & 0 & 0 & 0 & 0 & 0 & 0 & & 0 & 0 & 10 & & 0 & 0 & 0 & 0 & & 0 & 0 & 0 & C & 0 & 77 & 0 & 0 & 10 & 0 & 0 & 0 & C & 0 & 0 & 3 & & 100 \\
\hline$N p 237,1 \mathrm{~g}\{26,20\}$ & Np237 & 0 & 0 & 0 & 0 & 0 & 0 & & 0 & 0 & 0 & & 0 & 0 & 0 & 0 & & 0 & 0 & 0 & 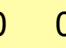 & 010 & 00 & 0 & 0 & 0 & 0 & 0 & 0 & 0 & 0 & 0 & 0 & & 100 \\
\hline $\mathrm{Np} 237,1 \mathrm{~g}\{26,40\}$ & $\mathrm{Np} 237$ & 0 & 0 & 0 & 0 & 0 & 0 & & 0 & 0 & 0 & & 0 & 0 & 0 & 0 & & 0 & 0 & 0 & 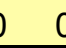 & 09 & 97 & 0 & 0 & 3 & 0 & 0 & 0 & C & 0 & 0 & 0 & & 100 \\
\hline I131,10UC & $\mid 131$ & 0 & 0 & 0 & 0 & 0 & 0 & & 0 & 0 & 1 & & 0 & 0 & 0 & 0 & & 0 & 0 & 0 & C & 0 & 0 & 99 & 0 & 0 & 0 & 0 & 0 & 0 & 0 & 0 & 0 & & 100 \\
\hline $\mid 131,10 \cup C\{10,10\}$ & $\mid 131$ & 0 & 2 & 0 & 0 & 0 & 0 & & 0 & 0 & 10 & & 0 & 0 & 0 & 0 & & 0 & 0 & 0 & c & 0 & 0 & 88 & 0 & 0 & 0 & 0 & 0 & 0 & 0 & 0 & 0 & & 100 \\
\hline $\mid 131,10 \cup C\{10,30\}$ & $\mid 131$ & 0 & 1 & 0 & 0 & 0 & 0 & & 0 & 0 & 35 & & 0 & 0 & 0 & 0 & & 0 & 0 & 0 & 5 & 5 & 0 & 59 & 0 & 0 & 0 & 0 & 0 & 0 & 0 & 0 & 0 & & 100 \\
\hline $\mid 131,10 \cup C\{26,10\}$ & $\mid 131$ & 0 & 0 & 0 & 0 & 0 & 0 & & 0 & 0 & 14 & & 0 & 0 & 0 & 0 & & 0 & 0 & 0 & C & 0 & 0 & 86 & 0 & 0 & 0 & 0 & 0 & 0 & 0 & 0 & 0 & & 100 \\
\hline $\mid 131,10 \cup C\{74,10\}$ & $\mid 131$ & 0 & 0 & 0 & 0 & 0 & 0 & & 0 & 0 & 0 & & 0 & 0 & 0 & 0 & & 0 & 0 & 0 & 0 & 0 & & 100 & 0 & 0 & 0 & 0 & 0 & 0 & 0 & 0 & 0 & & 100 \\
\hline Tc99m,100uC & Tc99m & 0 & 0 & 0 & 0 & 0 & 0 & & 0 & 0 & 0 & & 0 & 0 & 0 & 0 & & 0 & 0 & 0 & 0 & 0 & 0 & & 100 & 0 & 0 & 0 & 0 & 0 & 0 & 0 & 0 & & 100 \\
\hline Tc99m,100uC $\{10,10\}$ & Tc99m & 0 & 2 & 0 & 0 & 0 & 0 & & 0 & 0 & 0 & & 0 & 0 & 0 & 0 & & 0 & 0 & 0 & 0 & 0 & 0 & 0 & 98 & 0 & 0 & 0 & 0 & 0 & 0 & 0 & 0 & & 100 \\
\hline $\mathrm{Tc} 99 \mathrm{~m}, 1 \mathrm{mC}\{10,30\}$ & Tc99m & 0 & 5 & 0 & 0 & 0 & 0 & & 0 & 0 & 0 & & 0 & 0 & 0 & 0 & & 0 & 0 & 0 & 0 & 0 & 0 & 0 & 95 & 0 & 0 & 0 & 0 & 0 & 0 & 0 & 0 & & 100 \\
\hline Ga67,100UC $\{10,10\}$ & Ga67 & 0 & 2 & 0 & 0 & 0 & 0 & & 0 & 0 & 0 & & 0 & 0 & 0 & 0 & & 0 & 0 & 0 & 0 & 0 & 0 & 0 & 0 & 98 & 0 & 0 & 0 & 0 & 0 & 0 & 0 & & 100 \\
\hline$G a 67,100 \cup C\{10,30\}$ & Ga67 & 0 & 2 & 0 & 0 & 0 & 0 & & 0 & 0 & 15 & & 0 & 0 & 0 & 0 & & 0 & 0 & 1 & 0 & 0 & 1 & 0 & 0 & 81 & 0 & 0 & 0 & 0 & 0 & 0 & 0 & & 100 \\
\hline Ga67,100UC $\{26,10\}$ & Ga67 & 0 & 1 & 0 & 0 & 0 & 0 & & 0 & 0 & 0 & & 0 & 0 & 0 & 0 & & 0 & 0 & 0 & 0 & 0 & 0 & 0 & 0 & 99 & 0 & 0 & 0 & 0 & 0 & 0 & 0 & & 100 \\
\hline Ga67,100UC $\{26,30\}$ & Ga67 & 0 & 0 & 0 & 0 & 0 & 0 & & 0 & 0 & 1 & & 0 & 0 & 0 & 0 & & 0 & 0 & 0 & 0 & 0 & 7 & 0 & 0 & 92 & 0 & 0 & 0 & & 0 & 0 & 0 & & 100 \\
\hline $\mathrm{TI} 201,1 \mathrm{mC}\{10,10\}$ & Tl201 & 0 & 0 & 0 & 0 & 0 & 0 & & 0 & 0 & 0 & & 0 & 0 & 0 & 6 & & 0 & 0 & 0 & 0 & 0 & 0 & 0 & 0 & 0 & 94 & 0 & 0 & 0 & 0 & 0 & 0 & & 100 \\
\hline $\mathrm{TI} 201,1 \mathrm{mC}\{10,30\}$ & TI201 & 0 & 15 & 0 & 0 & 0 & 0 & & 0 & 0 & 0 & & 0 & 0 & 0 & 0 & & 0 & 0 & 0 & 0 & 0 & 0 & 0 & 2 & 0 & 83 & 0 & 0 & 0 & 0 & 0 & 0 & & 100 \\
\hline $\mathrm{TI} 201,1 \mathrm{mC}\{26,10\}$ & Tl201 & 0 & 0 & 0 & 0 & 0 & 0 & & 0 & 0 & 0 & & 0 & 0 & 0 & 8 & & 0 & 0 & 0 & 0 & 0 & 0 & 0 & 0 & 0 & 92 & 0 & 0 & 0 & 0 & 0 & 0 & & 100 \\
\hline $\mathrm{TI} \mid 201,1 \mathrm{mC}\{26,30\}$ & Tl201 & 0 & 0 & 0 & 0 & 0 & 0 & & 0 & 0 & 0 & & 0 & 0 & 0 & 1 & & 0 & 0 & 0 & 0 & 0 & 0 & 0 & 0 & 0 & 99 & 0 & 0 & & 0 & 0 & 0 & & 100 \\
\hline $\begin{array}{l}\text { TI201,1mC }\{13,5\} \\
+ \text { TL202,80NC }\{13,5\}\end{array}$ & TI201 & 0 & 1 & 0 & 0 & 0 & 0 & & 0 & 0 & 0 & & 0 & 0 & 0 & 27 & & 0 & 0 & 0 & 0 & 0 & 0 & 0 & 0 & 0 & 72 & 0 & 0 & & 0 & 0 & 0 & & 100 \\
\hline $\begin{array}{l}\text { TI201,1mC }\{13,20\} \\
+ \text { TL202,80NC }\{13,20\}\end{array}$ & Tl201 & 0 & 5 & 0 & 0 & 0 & 0 & & 0 & 0 & 0 & & 0 & 0 & 0 & 0 & & 0 & 0 & 0 & 0 & 0 & 0 & 0 & 0 & 0 & 95 & 0 & 0 & & 0 & 0 & 0 & & 100 \\
\hline $\begin{array}{l}\text { TI201,1mC }\{26,5\} \\
+ \text { +TL202,80NC }\{26,5\}\end{array}$ & Tl201 & 0 & 0 & 0 & 0 & 0 & 0 & & 0 & 0 & 0 & & 0 & 0 & 0 & 26 & & 0 & 0 & 0 & 0 & 0 & 0 & 0 & 0 & 0 & 74 & 0 & 0 & & 0 & 0 & 0 & & 100 \\
\hline $\begin{array}{l}\text { TI201,1mC }\{26,20\} \\
+ \text { TL202,80NC }\{26,20\}\end{array}$ & TI201 & 0 & 0 & 0 & 0 & 0 & 0 & & 0 & 0 & 0 & & 0 & 0 & 0 & 9 & & 0 & 0 & 0 & 0 & 0 & 0 & 0 & 0 & 0 & 91 & 0 & 0 & & 0 & 0 & 0 & & 100 \\
\hline Y88,10UC & Y88 & 0 & 0 & 0 & 0 & 0 & 0 & & 0 & 0 & 0 & & 0 & 0 & 0 & 0 & & 0 & 0 & 0 & 0 & 0 & 0 & 0 & 0 & 0 & \multicolumn{2}{|c|}{0100} & 0 & 0 & 0 & 0 & 0 & & 100 \\
\hline$Y 88,10 \cup C\{10,10\}$ & Y88 & 0 & 1 & 0 & 0 & 0 & 0 & & 0 & 0 & 0 & & 0 & 0 & 0 & 0 & & 0 & 0 & 0 & 0 & 0 & 0 & 0 & 0 & 0 & 0 & 99 & 0 & & 0 & 0 & 0 & & 100 \\
\hline$Y 88,10 \cup C\{10,30\}$ & Y88 & 0 & 1 & 0 & 0 & 0 & 0 & & 0 & 0 & 0 & & 0 & 0 & 0 & 0 & & 0 & 0 & 0 & 0 & 0 & 0 & 0 & 0 & 0 & 0 & 99 & 0 & & 0 & 0 & 0 & & 100 \\
\hline$Y 88,10 \cup C\{26,10\}$ & Y88 & 0 & 3 & 0 & 0 & 0 & 0 & & 0 & 0 & 0 & & 0 & 0 & 0 & 0 & & 0 & 0 & 0 & 0 & 0 & 0 & 0 & 0 & 0 & 0 & 97 & 0 & 0 & 0 & 0 & 0 & & 100 \\
\hline$Y 88,10 \cup C\{26,30\}$ & Y88 & 0 & 1 & 0 & 0 & 0 & 0 & & 0 & 0 & 0 & & 0 & 0 & 0 & 0 & & 0 & 0 & 0 & 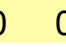 & 0 & 0 & 0 & 0 & 0 & 0 & 99 & 0 & 0 & 0 & 0 & 0 & & 100 \\
\hline
\end{tabular}




\begin{tabular}{|c|c|c|c|c|c|c|c|c|c|c|c|c|c|c|c|c|c|c|c|c|c|c|c|c|c|c|c|c|c|c|c|c|}
\hline Isotope_type & Id & ND & UN & $\begin{array}{c}40 \\
\mathrm{~K}\end{array}$ & & & & & 36 & & & & $\begin{array}{l}54 \\
\mathrm{Mn} \\
\end{array}$ & $\begin{array}{l}57 \\
\text { Co } \\
\end{array}$ & & & & $\begin{array}{ll}3823 \\
\mathrm{~J}\end{array}$ & $\begin{array}{l}352 \\
U \quad P\end{array}$ & $\begin{array}{l}2392 \\
\mathrm{Pu} \quad \mathrm{T}\end{array}$ & $\begin{array}{l}237 \\
\mathrm{~Np}\end{array}$ & $\begin{array}{c}1319 \\
1\end{array}$ & $\begin{array}{c}99 \mathrm{~m} \\
\text { Tc }\end{array}$ & $\begin{array}{l}67 \\
\mathrm{Ga} \\
\end{array}$ & $\begin{array}{r}201 \\
\mathrm{TI} \\
\end{array}$ & $\begin{array}{c}188 \\
\quad Y \\
\end{array}$ & $\begin{array}{ll}822 \\
\mathrm{Na}\end{array}$ & $\begin{array}{ll}22 & 16 \\
\mathrm{Na} & \mathrm{H} \\
\end{array}$ & $\begin{array}{c}166 m \\
\mathrm{Ho}\end{array}$ & $\begin{array}{c}1521 \\
\mathrm{Eu}\end{array}$ & $\begin{array}{c}192 \\
\text { Ir }\end{array}$ & UM \\
\hline Y88,10UC $\{74,20\}$ & Y88 & 0 & 1 & 0 & $\underline{0}$ & 0 & 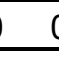 & o & 0 & 0 & 0 & 0 & 0 & 0 & $\underline{0}$ & & 0 & 0 & 0 & 0 & 0 & 0 & 0 & 0 & 0 & $0 \quad 95$ & 99 & 0 & 0 & 0 & 0 & 100 \\
\hline $\mathrm{Na22,10U \textrm {C }}$ & $\mathrm{Na} 22$ & 0 & 1 & 0 & 0 & 0 & c & 0 & 0 & 0 & 0 & 0 & 0 & 0 & 0 & & 0 & 0 & 0 & 0 & 0 & 0 & 0 & 0 & 0 & 0 & 0 & 99 & 0 & 0 & 0 & 100 \\
\hline Na22, 10UC $\{10,10\}$ & $\mathrm{Na} 22$ & 0 & 1 & 0 & 0 & 0 & c & 0 & 0 & 0 & 0 & 0 & 0 & 0 & 0 & & 0 & 0 & 0 & 0 & 0 & 0 & 0 & 0 & 0 & 0 & 09 & 99 & 0 & 0 & 0 & 100 \\
\hline $\mathrm{Na} 22,10 \cup \mathrm{C}\{10,30\}$ & $\mathrm{Na} 22$ & 0 & 0 & 0 & 0 & 0 & c & 0 & 0 & 0 & 0 & 0 & 0 & 0 & 0 & & 0 & 0 & 0 & 0 & 0 & 0 & 0 & 0 & 0 & 0 & 010 & 00 & 0 & 0 & 0 & 100 \\
\hline $\mathrm{Na} 22,10 \mathrm{UC}\{26,10\}$ & $\mathrm{Na} 22$ & 0 & 0 & 0 & 0 & 0 & c & 0 & 0 & 0 & 0 & 0 & 0 & 0 & 0 & & 0 & 0 & 0 & 0 & 0 & 0 & 0 & 0 & 0 & 0 & 010 & 00 & 0 & 0 & 0 & 100 \\
\hline $\mathrm{Na} 22,10 \cup C\{26,30\}$ & $\mathrm{Na} 22$ & 0 & 0 & 0 & 0 & 0 & c & 0 & 0 & 0 & 0 & 0 & 0 & 0 & 0 & & 0 & 0 & 0 & 0 & 0 & 0 & 0 & 0 & 0 & 0 & 010 & 00 & 0 & 0 & 0 & 100 \\
\hline $\mathrm{Na} 22,10 \cup \mathrm{C}\{74,20\}$ & $\mathrm{Na} 22$ & 0 & $\underline{0}$ & 0 & 0 & 0 & c & b & 0 & 0 & 0 & 0 & 0 & 0 & $\underline{0}$ & & $\underline{0}$ & 0 & 0 & 0 & 0 & 0 & 0 & 0 & $\underline{0}$ & $\underline{0}$ & 010 & 00 & 0 & 0 & 0 & 100 \\
\hline Ho166m,10UC & Ho166m & 0 & 1 & 0 & 0 & 0 & c & 0 & 0 & 0 & 0 & 0 & 0 & 0 & 0 & & 0 & 0 & 0 & 0 & 0 & 0 & 0 & 0 & 0 & 0 & 0 & 0 & 99 & 0 & 0 & 100 \\
\hline Ho166m, 10UC $\{10,10\}$ & Ho166m & 0 & 2 & 0 & 0 & 0 & c & 0 & 0 & 0 & 0 & 0 & 0 & 0 & 0 & & 0 & 1 & 0 & 0 & 0 & 0 & 0 & 0 & 0 & 0 & 0 & 0 & 97 & 0 & 0 & 100 \\
\hline Ho166m, 10UC $\{10,30\}$ & Ho166m & 0 & 0 & 0 & 0 & 0 & c & 0 & 0 & 0 & 0 & 1 & 1 & 0 & 0 & & 0 & 1 & 0 & 1 & 0 & 0 & 0 & 0 & 0 & 0 & 0 & 0 & 96 & 0 & 0 & 100 \\
\hline Ho166m, 10UC $\{26,10\}$ & Ho166m & 0 & 0 & 0 & 0 & 0 & c & 0 & 0 & 0 & 0 & 0 & 0 & 0 & 0 & & 0 & 1 & 0 & 0 & 0 & 0 & 0 & 0 & 0 & 0 & 0 & 0 & 99 & 0 & 0 & 100 \\
\hline Ho166m, 10UC $\{26,30\}$ & Ho166m & 0 & 0 & 0 & 0 & 0 & c & 0 & 0 & 0 & 0 & 0 & 0 & 0 & 0 & & 0 & 1 & 0 & 0 & 0 & 0 & 0 & 0 & 0 & 0 & 0 & 0 & 98 & 0 & 1 & 100 \\
\hline Ho166m, $10 \cup C\{74,20\}$ & Ho166m & 0 & 1 & 0 & 0 & 0 & c & 0 & 0 & 0 & 0 & 0 & 0 & 0 & 0 & & 0 & 0 & 0 & 0 & 0 & 0 & 0 & 0 & 0 & 0 & 0 & 0 & 99 & 0 & 0 & 100 \\
\hline Eu152,10uC & Eu152 & 0 & 1 & 0 & 0 & 0 & c & 0 & 0 & 0 & 0 & 0 & 0 & 0 & 0 & & 0 & 0 & 0 & 0 & 0 & 0 & 0 & 0 & 0 & 0 & 0 & 0 & 0 & 99 & $c$ & 100 \\
\hline Eu152,10UC $\{10,10\}$ & Eu152 & 0 & 1 & 0 & 0 & 0 & c & 0 & 0 & 3 & 0 & 0 & 0 & 0 & 0 & & 0 & 2 & 0 & 0 & 0 & 0 & 0 & 0 & 0 & 0 & 0 & 0 & 0 & 94 & 0 & 100 \\
\hline Eu152,10UC $\{10,30\}$ & Eu152 & 0 & 1 & 3 & 4 & 0 & c & 0 & 0 & 7 & 0 & 0 & 1 & 0 & 0 & & 2 & 3 & 0 & 2 & 0 & 0 & 0 & 0 & 0 & 0 & 0 & 0 & 0 & 77 & 0 & 100 \\
\hline Eu152,10UC $\{26,10\}$ & Eu152 & 0 & 2 & 0 & 0 & 0 & c & 0 & 0 & 0 & 0 & 0 & 0 & 0 & 0 & & 0 & 0 & 0 & 0 & 0 & 0 & 0 & 0 & 0 & 0 & 0 & 0 & 0 & 98 & 0 & 100 \\
\hline Eu152,10uC $\{26,30\}$ & Eu152 & 0 & 0 & 0 & 0 & 0 & c & 0 & 0 & 2 & 0 & 0 & 0 & 0 & 0 & & 0 & 6 & 0 & 0 & 0 & 0 & 0 & 0 & 0 & 0 & 0 & 1 & 0 & 91 & 0 & 100 \\
\hline Eu152,10UC $\{74,20\}$ & Eu152 & 0 & $\underline{0}$ & 0 & 0 & 0 & c & b & 0 & 2 & 0 & 0 & 0 & 0 & $\underline{0}$ & & 0 & 1 & 0 & 0 & 0 & 0 & 0 & 0 & $\underline{0}$ & 0 & 0 & 0 & 0 & 97 & 0 & 100 \\
\hline Ir192,10UC & Ir192 & 0 & 0 & 0 & 0 & 0 & c & 0 & 0 & 0 & 0 & 0 & 0 & 0 & 0 & & 0 & 0 & 0 & 0 & 0 & 0 & 0 & 0 & 0 & 0 & 0 & 0 & 0 & & 100 & 100 \\
\hline Ir192,10UC $\{10,10\}$ & Ir192 & 0 & 1 & 0 & 0 & 0 & c & 0 & 0 & 0 & 0 & 0 & 0 & 0 & 0 & & 0 & 0 & 0 & 0 & 0 & 0 & 0 & 1 & 0 & 0 & 0 & 0 & 0 & 0 & 98 & 100 \\
\hline Ir192,10UC $\{10,30\}$ & Ir192 & 0 & 1 & 0 & 0 & 0 & c & 0 & 0 & 0 & 1 & 0 & 0 & 0 & 0 & & 0 & 0 & 0 & 1 & 1 & 0 & 0 & 4 & 0 & 0 & 0 & 0 & 0 & 0 & 92 & 100 \\
\hline Ir192,10UC $\{26,10\}$ & Ir192 & 0 & 0 & 0 & 0 & 0 & c & 0 & 0 & 0 & 0 & 0 & 0 & 0 & 0 & & 0 & 0 & 0 & 0 & 0 & 0 & 0 & 0 & 0 & 0 & 0 & 0 & 0 & & 100 & 100 \\
\hline Ir192,10UC $\{26,30\}$ & Ir192 & 0 & 0 & 0 & 0 & 0 & c & 0 & 0 & 0 & 0 & 0 & 0 & 0 & 0 & & 0 & 0 & 0 & 0 & 0 & 0 & 0 & 0 & 0 & 0 & 0 & 0 & 0 & & 100 & 100 \\
\hline Ir $192,10 \cup C\{26,50\}$ & Ir192 & 0 & 0 & 0 & 0 & 0 & c & 0 & 0 & 0 & 0 & 0 & 0 & 0 & 0 & & 0 & 0 & 0 & 4 & 0 & 0 & 0 & 1 & 0 & 0 & 0 & 0 & 0 & & 95 & 100 \\
\hline Ir192,1mC\{74,20\} & Ir192 & 0 & 0 & 0 & 0 & 0 & c & 0 & 0 & 0 & 0 & 0 & 0 & 0 & 0 & & 0 & 0 & 0 & 0 & 0 & 0 & 0 & 0 & 0 & 0 & 0 & 0 & 0 & & 100 & 100 \\
\hline $\mid r 192,10 \mathrm{mC}\{74,60\}$ & Ir192 & 0 & 0 & 0 & 0 & 0 & c & כ & 0 & 0 & 0 & 0 & 0 & 0 & $\underline{0}$ & & 0 & 0 & 0 & 0 & 0 & 0 & 0 & 0 & $\underline{0}$ & $\underline{0}$ & 0 & 0 & 0 & & 100 & 100 \\
\hline
\end{tabular}




\title{
Appendix A5: Remote Muon Scanning for Nuclear Materials Inside Containerships
}

Peter Barnes, George Chapline, Dan Dietrich, Mark Rowland, Neal Snyderman

\begin{abstract}
It is proposed that all containerships be stopped at a suitable distance from US ports, and that a $2 \mathrm{GeV}$ muon beam be used to survey the ship for nuclear materials. It is estimated that within a few hours the presence of a nuclear device could be detected with high confidence.
\end{abstract}

\section{Introduction}

The problem of finding hidden nuclear materials onboard containerships is extremely difficult because of the sheer size of containerships. Passive neutron and gamma ray signals from a point source may be undetectable, particularly in the case of HEU, because of the large attenuation that these signals may suffer going through many containers. In addition, a nuclear device could be shielded with a moderator and neutron absorber. The one thing a nuclear smuggler cannot do, though, is to shield the nuclear material against muons. It might be recalled that in 1968 Louis Alvarez used cosmic ray muons to survey the interior of Chephren's pyramid for hidden chambers. Indeed given sufficient time any large structure could be surveyed using cosmic ray muons. In the case of looking for uranium or plutonium inside a large structure, surveying the structure with negative muons provides an additional benefit in the form of a unique spectrum of characteristic 1-6 MeV muonic "x-rays". Detection of this characteristic radiation with a $\gamma$-ray detector would provide unambiguous evidence for the presence of uranium or plutonium. If the $\gamma$-ray detector had sufficient energy resolution one could even determine the isotopic composition of the nuclear material, viz. whether the material contained Pu239 or U235. Although neutron probes can also initiate telltale signals, we believe that the using a negative muon beam to detect hidden nuclear material may offer significant advantages when the structure to be searched is very large.

Our operational concept for inspecting containerships for nuclear materials is to introduce off-shore interdiction points off all three coasts where containerships will be stopped and scanned with muon beams. Our initial concept for the muon source is a ship based proton accelerator which is used with a low $\mathrm{Z}$ target to generate a muon beam. The most straightforward 
way to generate the needed muon beams would be to guide a multi-GeV proton beam to the targets, and use the kinematic focusing of the pions produced as a result of nucleon - nucleon collisions to focus the muons onto the container stack. The ship carrying the accelerator would have to be positioned next to the containership in order to avoid having to send the muon beam through water while inspecting the containers below the waterline. Positioning the inspection ship next to the containership would have the added advantage of maximizing the solid angle presented by signal detectors onboard the inspection ship. In addition, proximate positioning of the inspection ship would allow the muon beams to be slanted upwards or downwards, thus taking advantage of the low average density of the containers.

\section{Estimated requirements for the muon source}

Alvarez needed rather high energy cosmic ray muons ( $\sim 50 \mathrm{Gev})$ to traverse the $100 \mathrm{~m}$ of solid limestone in Chephren's pyramid, and these would be difficult to produce in a portable source. Fortunately, a containership is mostly air; the weight limit of 50,000 lb per container translates into an average density of $0.2 \mathrm{gm} / \mathrm{cc}$. In this situation 8 containers ( i.e. halfway across current generation containerships) represent an average lateral thickness of $\sim 500 \mathrm{gm} / \mathrm{cm}^{2}$. The range of high energy muons is an almost unique function of the thickness measured in $\mathrm{gm} / \mathrm{cm}^{2}$, and $500 \mathrm{gm} / \mathrm{cm}^{2}$ corresponds to the range of $\sim 1 \mathrm{GeV}$ muon. Of course, certain paths, e.g. straight across the bottom half of a horizontal row of containers, may present a higher aerial density, but by slanting the muon beam upwards or downwards it should be possible to reach the entire cargo with muons whose energy is less than $\sim 2 \mathrm{GeV}$.

A crucial question is whether one can produce the up to $2 \mathrm{GeV}$ muons needed to survey a containership with a portable artificial source of muons. Muons with energies up to $300 \mathrm{MeV}$ muons could be copiously produced using a cyclotron that could easily fit on a ship. If some means could be invented to accelerate these muons in situ, that would solve our problem. Alternatively a proton synchrotron capable of producing $2 \mathrm{GeV}$ muons might conceivably be fitted onto a large ship. Assuming the proton source uses a 100 nanoamp $5 \mathrm{GeV}$ proton current, approximately $10^{12}$ muons a second might be produced into a cone with an opening angle of $0.1 \mathrm{rad}$.

In order to estimate the fluence of muons needed to detect nuclear material hidden on a containership, we need to make some assumption regarding the efficiency for detecting 3 to 6 
$\mathrm{MeV} \gamma$ rays from a point source. If we assume that we have a $20 \mathrm{~m}^{2}$ detector $20 \mathrm{~m}$ from the source, then the geometric efficiency is $1 / 250$. Let's imagine the detector efficiency is fairly high resulting in an overall efficiency $\sim 10^{-3}$. The attenuation of 3-6 MeV $\gamma$ rays will depend somewhat on the materials in the containers, but at an assumed average density of $0.2 \mathrm{gm} / \mathrm{cc}$ this attenuation will be on the order of $10^{-6}$. If we assume we need 100 counts to confirm the existence of muonic lines at $\sim 3$ and $6 \mathrm{MeV}$, then the number of $\mathrm{U}$ or $\mathrm{Pu}$ muonic atoms that would need to be formed would have to be greater than $100 \times 10^{6} \times 10^{3}=10^{11}$. If we assume the nuclear material forms a cube with side $10 \mathrm{~cm}$, then a muon fluence $\sim 10^{13}$ per square meter will be required with an energy in a range to stop in the nuclear material. Fortunately, the aerial density of the nuclear material is a substantial fraction of the total thickness of the containers, so only a factor $\sim 2$ larger fluence is needed for the muon beam itself.

Let us assume that the total area of one side of the container stack is $10^{3}$ square meters, and that 2 beams were used to interrogate each side of the ship. Let us further assume the average flux of muons inside the containership is $10^{12} / \mathrm{m}^{2} / \mathrm{sec}$. Then approximately 10 seconds would be needed to interrogate each square meter of the container stack. The entire search volume could be surveyed in $3 \mathrm{hrs}$. Because muon beams can be used in parallel, the search time could be reduced be reduced in proportion to the number of beams used, e.g. using 4 beams on each side could reduce the search time to 90 minutes. It should be noted that the radiation doses from the beam are substantial from the point of view of biological hazard, so the crew would have to be evacuated or at least kept away from the beam. 
Radiation Detection Technologies Program
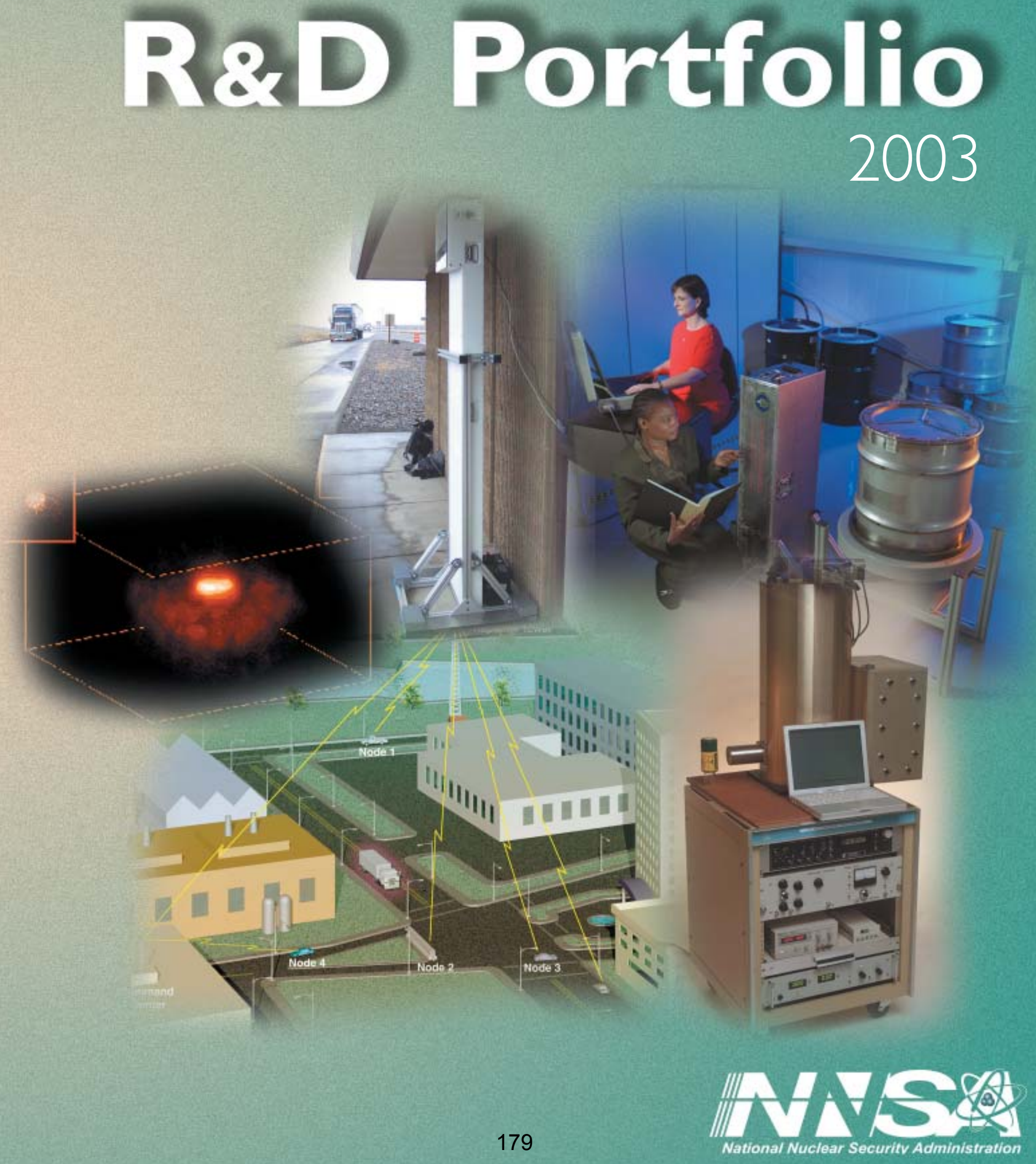


\section{Foundation To Improve Passive Detection Systems}

Simon Labov, Lawrence Livermore National Laboratory, and Garret Jernigan, University of California, Berkeley

Nuclear and radiological weapons contain radioactive materials that emit a constant stream of gamma rays, neutrons, and alpha and beta particles. In many situations, the gamma rays and neutrons can be detected remotely. Passive detection systems measure these spontaneous emissions without applying any external stimulation such as $\mathrm{x}$-ray, gamma-ray, or particle beams. Passive systems are less invasive and less likely to expose people to radiation or to trigger a booby trap.

Two factors severely limit passive detection systems: radioactivity and background radiation. The radioactivity of some sources is low or easily shielded. Background radiation varies dramatically with location, time, and viewing direction. New detection systems use imaging and spectroscopy to subtract much of or eliminate this background noise, greatly improving sensitivity. We have calculated the sensitivity of these new systems.

Models of the radiation output were created from potential threats as well as from common sources of radioactivity. For threat sources, standard "criticality test configurations" and simple spherical geometries provided unclassified estimates of the output of improvised or more sophisticated nuclear weapons.

We also modeled various detection systems to determine performance characteristics. The models tracked gamma rays from the sources and from

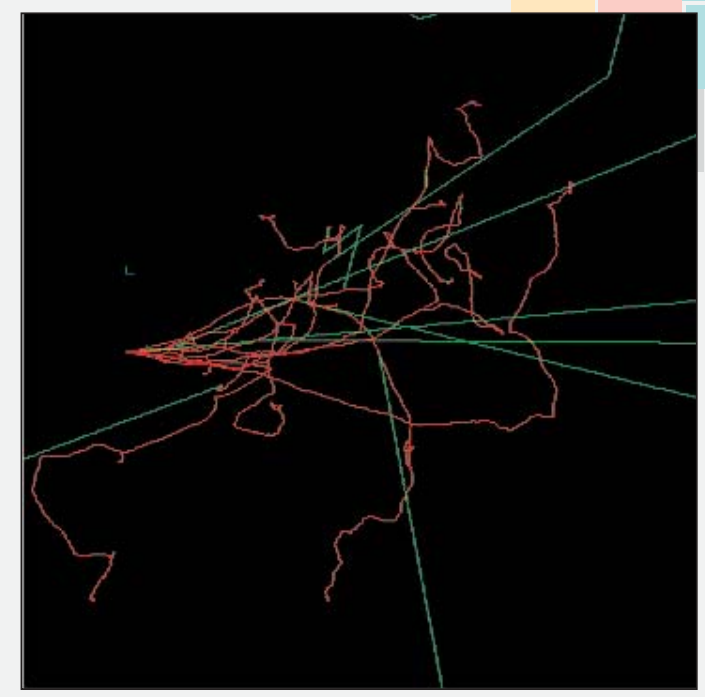

Figure I. Monte Carlo modeling of Compton scattering in a gamma-ray imaging detector shows electron (red) and gamma-ray (green) tracks. the background; these gamma rays then passed through various shielding configurations and entered the detection system. Interactions in the detector were modeled, and "measurements" of these interactions were listed in the same way as measurements from real detectors. We compared the output from various detection systems to determine the probability of detection and of triggering false alarms.

Somel gamma-ray imaging detectors measure the interactions during Compton scattering to determine some information about the direction of the incoming gamma ray (Figure I). One class of Compton imagers uses position-sensitive, semiconductor-based detectors to measure the energy and location of each interaction. The second class uses scintillating fibers to measure the location, energy, and direction of the electron recoil during Compton scattering.

The semiconductor-based system measures the energy of each interaction precisely, typically better than 0.5 percent, but not the direction of the electron recoil. The fiber-based system measures the recoil, but provided only a crude measurement of the energy, typically 20 percent.

We created source models, detector analysis tools, and a mechanism to determine how well a given system can detect threat sources under real-world conditions. The source models and analysis tools are currently available to anyone who wishes to determine the sensitivity of any specific detection system. These tools will now be used to optimize the next generation of imaging detectors.

It is also important to determine the nature of the material. Several automated routines were developed to identify isotopes from gamma-ray spectra. These routines were compared with each other and with existing routines. The routines used the same data libraries that characterize the different isotopes, but used different analysis techniques, including multiple linear regression (AutoGADRAS/Fit2B), principle component analysis, and maximum likelihood. These routines were tested against spectra produced from our source models, and the performance of each routine was evaluated.A fourth isotope identification technique was also developed using individual photon energy measurements rather than the collection of such measurements binned into a spectrum. This "event mode" technique is powerful for quickly and accurately determining the absence of a source of interest with few processor operations. $\$$ 